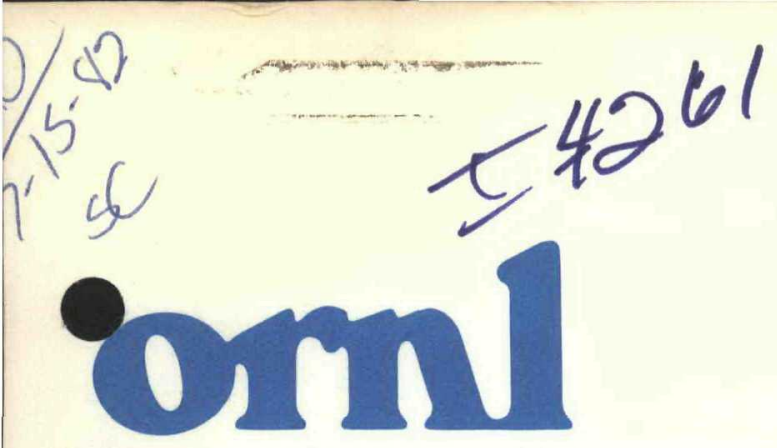

OAK

RIDGE

NATIONAL

LABORATORY

UNION

CARBIDE

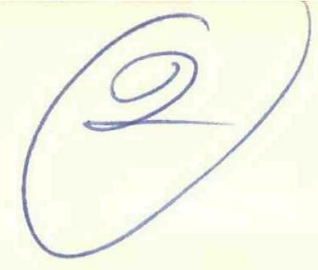

693

ORNL/TM-8303

\title{
A Transmission Electron Microscope Study of Fusion- Environment Radiation Damage in Iron and Iron-Chromium Alloys
}
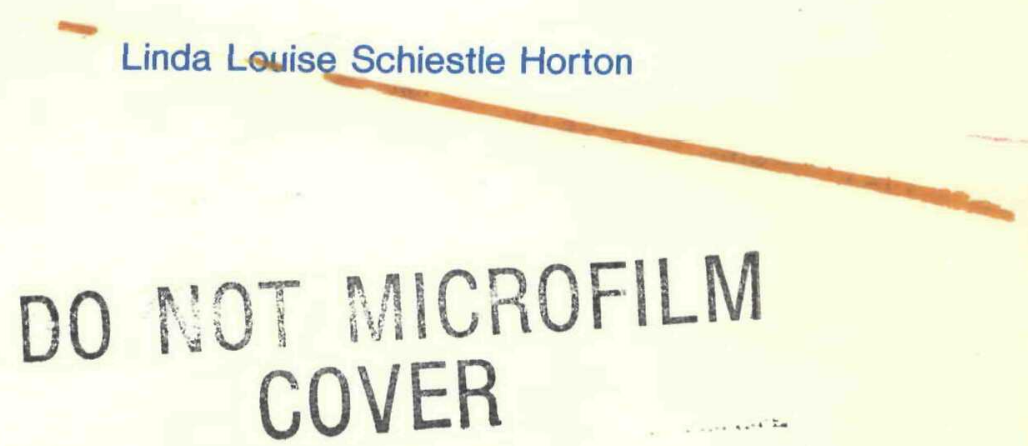

\section{OPERATED BY}

UNION CARBIDE CORPORATION FOR THE UNITED STATES DEPARTMENT OF ENERGY 
Printed in the United States of America. Available from National Technical Information Service

U.S. Department of Commerce

5285 Port Royal Road, Springfield, Virginia 22161

NTIS price codes-Printed Copy:A13 Microfiche A01

This report was prepared as an account of work sponsored by an agency of the United States Government. Neither the United States Government nor any agency thereof, nor any of their employees, makes any warranty, express or implied, or assumes any legal liability or responsibility for the accuracy, completeness, or usefulness of any information, apparatus, product, or process disclosed, or represents that its use would not infringe privately owned rights. Reference herein to any specific commercial product, process, or service by trade name, trademark, manufacturer, or otherwise, does not necessarily constitute or imply its endorsement, recommendation, or favoring by the United States Government or any agency thereof. The views and opinions of authors expressed herein do not necessarily state or reflect those of the United States Government or any agency thereof. 


\section{DISCLAIMER}

This report was prepared as an account of work sponsored by an agency of the United States Government. Neither the United States Government nor any agency Thereof, nor any of their employees, makes any warranty, express or implied, or assumes any legal liability or responsibility for the accuracy, completeness, or usefulness of any information, apparatus, product, or process disclosed, or represents that its use would not infringe privately owned rights. Reference herein to any specific commercial product, process, or service by trade name, trademark, manufacturer, or otherwise does not necessarily constitute or imply its endorsement, recommendation, or favoring by the United States Government or any agency thereof. The views and opinions of authors expressed herein do not necessarily state or reflect those of the United States Government or any agency thereof. 


\section{DISCLAIMER}

Portions of this document may be illegible in electronic image products. Images are produced from the best available original document. 
This report was ereotas DISCLAMMER

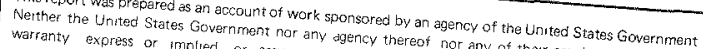

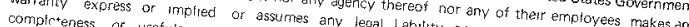
represents that its use would not any informat on dpparatus producr or procos the accuracy

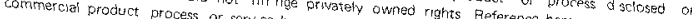
not necessarily constrtute or moly by trade rame trademark manuface herest to any speciltic

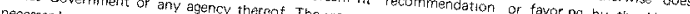

ORNL/TM-8303

Dist. Category UC-20C, -25

Contract No. W-7405-eng-26

Metals and Ceramics Division

$\operatorname{ORL} / \mathrm{TH}=-8303$

$D E 2018525$

\title{
A TRANSMISSION ELECTRON MICROSCOPE STUDY OF FUSION-ENVIRONMENT RADIATION DAMAGE IN IRON AND IRON-CHROMIUM ALLOYS
}

\section{by}

\section{Linda Louise Schiestle Horton}

\author{
Submitted in partial fulfillment of the requirements \\ for the \\ degree of Doctor of Philosophy \\ in Materials Science \\ to the \\ Faculty at the University of Virginia \\ Charlottesville, Virginia \\ 1982 \\ Date Published - July 1982
}

NOTICE This document contains information of a preliminary nature.

It is subject to revision or correction and therefore does not represent a

final report.

OAK RIDGE NATIONAL LABORATORY

Oak Ridge, Tennessee 37830

operated by

UNION CARBIDE CORPORATION

for the

U.S. DEPARTMENT OF ENERGY

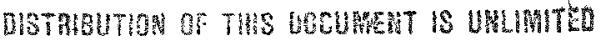

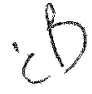


-

- 
ABSTRACT ...................... . vii

ACKNOWLEDGMENTS .................... IX

LIST OF SYMBOLS ..................... xi

1 INTRODUCTION

2 LITERATURE REVIEW ..................... 9

2.1 Experimental Investigations . . . . . . . 9

2.1.1 Neutron Irradiation Experiments . . . . 9

2.1.2 Ion Irradiation Experiments ...... 18

2.1.3 Electron Irradiation Experiments . . . 22

2.1.4 Summary of Experimental Observations . . 24

2.2 Swe11ing Suppression Mechanisms ....... 27

3 NEUTRON IRRADIATION EXPERIMENTS $\cdot \cdot \cdot \cdot \cdot \cdot \cdot \cdot \cdot \cdot \cdot \cdot \cdot 31$

3.1 Irradiation-Induced Dislocation Microstructures . 33

3.2 Irradiation-Induced Cavity Microstructures . . 52

3.3 Irradiation-Induced Halo Microstructures . . . 55

4 DAMAGE DEPTH PROFILES IN TRIPLE-BEAM IRRADIATED IRON • . 67

4.1 Electroplating Procedure ......... 69

4.2 TEM Specimen Preparation ........ 73

4.3 TEM Observations ........... 84

4.4 Discussion .............. . . 77

4.4.1 Comparison of Calculated and Experimental

Damage Profiles ....... 79

4.4.2 Sectioning Depth .......... 87

5 TRIPLE-BEAM ION IRRADIATION EXPERIMENTS . . . . . . 89

5.1 Unirradiated Microstructures . . . . . . 90

5.2 Temperature Dependence Studies . . . . . . 95

5.3 Dose Dependence Studies ............ 103

5.4 Effect of Alloy Chromium Content . . . . . 115

5.5 Effect of Helium and Deuterium ........ 118

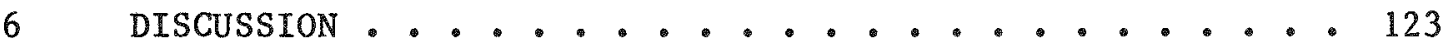

6.1 Damage Microstructures .......... 123

6.1 .1 Dislocations ........... 123

6.1 .2 Cavities .......... 125

6.2 Swelling Suppression Mechanisms ....... 133

6.3 Damage Evolution .......... 134 
CHAPTER

Page

APPENDIX

A EXPERIMENTAL DETAILS - ORR NEUTRON IRRADIATION OF IRON • 143

A.1 Specimen Preparation . . . . . . . . . 143

A.2 ORR - Specimen Loading and Assembly Details . . . 144

A.3 Irradiation Details Including Calculations of

$\mathrm{dpa}$ and Concentrations of Helium and Hydrogen . . 146

A.4 TEM Specimen Preparation ............. 151

B ELECTRON MICROSCOPY PROCEDURES . . . . . . . . 153

B.1 Calibration of the JEM 120C - AMG . . . . . . 155

B.2 Microscopy Procedures for Magnetic Specimens . . . 158

B.2.1 Microscope Alignment ........... 159

B.2.2 Specimen Loading and Stage Insertion .... 159

B.2.3 Eucentric Height : Magnetic Specimens . . . 159

B.2.4 Specimen Tilting Procedures : Magnetic

Specimens .............. 160

B.2.5 Additional Precautions .......... 161

B.3 Thickness Measurements ............. 162

B.4 Dislocation Density Measurements ......... 162

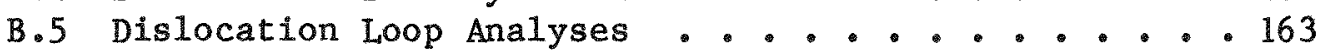

B.6 Cavity Analyses ............... 166

B.7 Error Analyses ............... 167

C PREPARATION OF IRON-CHROMIUM ALLOYS . . . . . . . . 171

D PROCEDURE - TRIPLE-BEAM IRRADTATION . . . . . . . 175

D.1 Description of the Triple-Beam Technique and the ORNL Irradiation Facility . . . . . . . . . 175

D.2 Specimen Preparation of $\mathrm{Fe}, \mathrm{Fe}-5 \% \mathrm{Cr}$, and $\mathrm{Fe}-10 \% \mathrm{Cr}$ for Triple-Beam Irradiation . . . . . . . . . 179

D.3 Specimen Arrays ................. 180

D.4 Irradiation Parameters . . . . . . . . . . 180

D.5 TEM Specimen Preparation ............ 190

E E-DEP-1 COMPUTER CODE: DEPOSITED ENERGY, DEPOSITED ION AND DPA PROFILES .................. 189 
F CAlCUlation OF THE CRitical GAVtTy RAdius . . . . . . 195

F.1 Continuity Equations and Point Defect Sink Strengths - 195

F.2 Cavity Growth Equations .............. 198

F.3 Direct Calculations of $\mathrm{r}_{\text {crit: }}$ Dislocation

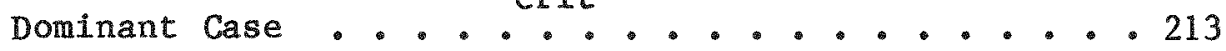

$G$ CALCULATIONS OF THE NUMBER OF HELIUM ATOMS IN AN

EQUILIBRIUM BUBBLE ... . . . . . . . . . . 215

REFERENCES ........................ 239 
-

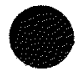


A transmission electron microscopy study of radiation damage microstructures in iron and iron-chromium alloys has been performed. This study consisted of both qualitative and quantitative characterization of the dislocation and cavity microstructures, including determination of vacancy/interstitial character and Burgers vectors for dislocation loops and analysis of the cavity morphology. The effects of irradiation temperature, fluence, helium implantation, and chromium content were investigated. Neutron irradiation (iron specimens, 1 dpa, 455 to $1000 \mathrm{~K}$ ) and triple-beam ion irradiation ( $\mathrm{Fe}-10 \% \mathrm{Cr}$ specimens, $10 \mathrm{dpa}, 725$ to $950 \mathrm{~K}$; Fe-10\% Cr specimens, $850 \mathrm{~K}, 0.3$ to $100 \mathrm{dpa}$; and $\mathrm{Fe}, \mathrm{Fe}-5 \% \mathrm{Cr}, \mathrm{Fe}-10 \% \mathrm{Cr}$ specimens, $850 \mathrm{~K}, 10 \mathrm{dpa}$ ) were employed. In the triple-beam ion irradiation procedure, simultaneous bombardment with $4 \mathrm{MeV} \mathrm{Fe}$ ions and energetic $\mathrm{He}^{+}$and $\mathrm{D}_{2}^{+}$ions was used to simulate the fusion environment (10 at. ppm He/dpa and 41 at. $\mathrm{ppm} \mathrm{D/dpa).}$ In addition, single-beam $4 \mathrm{MeV} \mathrm{Fe}$ it ion irradiations of $\mathrm{Fe}-10 \% \mathrm{Cr}$ both with and without pre-injection of helium and deuterium were performed.

Significant results from this investigation include: (1) the experimental determination of the depth profile of the damage for triple-beam ion-irradiated iron; (2) the observation of interstitial dislocation loops with predominantly a<100> Burgers vectors (in ionirradiated $\mathrm{Fe}-10 \% \mathrm{Cr}$ these loops had a convoluted shape); (3) the characterization of the dislocation evolution in $\mathrm{Fe}-10 \% \mathrm{Cr}$ which initiated with the formation of interstitial loops with $\underline{b}=a\langle 100\rangle$, followed by the development of interstitial loops with $\underline{b}=a / 2\langle 111\rangle$, and culminated in a dislocation network formed by the interaction of the two types of loops; (4) the observations in neutron-irradiated iron of pre-existing dislocation segments decorated with defect clusters and discrete clusters of small dislocation loops; (5) the determination of a truncated octahedral cavity morphology with $\{111\}$ facets and $\{100\}$ truncations for both neutron and ion-irradiated specimens; and (6) the observation 
of a dramatic increase in the cavity diameter and swelling between 30 and $100 \mathrm{dpa}$ in ion-irradiated $\mathrm{Fe}-10 \% \mathrm{Cr}$. In addition, damage halos were observed in neutron-irradiated iron and there was no cavity formation in $\mathrm{Fe}-5 \% \mathrm{Cr}$ with the same irradiation conditions that produced cavities in $\mathrm{Fe}$ and $\mathrm{Fe}-10 \% \mathrm{Cr}$.

These results were compared to similar investigations reported in the literature. Currently proposed swelling suppression mechanisms for ferritic materials were discussed. Cavity growth was also considered in detail, including rate theory calculations of the critical cavity radius for bias-driven growth and calculations of the number of helium atoms in equilibrium bubbles using a high density equation of state. In addition, a mechanism was suggested to explain the observed damage evolution in $\mathrm{Fe}-10 \% \mathrm{Cr}$. The results should direct and aid in the microstructural analyses in future studies, as well as provide a basis for understanding the mechanisms of radiation damage in ferritic materials. 


\section{ACRNOWLEDGMENTS}

Many people have assisted in the research effort and support work required for this dissertation. The guidance, interest and personal help of my research advisers, Dr. J. Bentley of ORNL and Dr. W. A. Jesser of UVA are appreciated. Special thanks are extended to Dr. Bentley for providing instruction for many of the TEM/AEM techniques used in this investigation. Discussions with Drs. L. K. Mansur and $K$. Farrell concerning the planning and interpretation of the experiments; the use of the Van de Graaff ion irradiation facility operated by Dr. M. B. Lewis; the computer progran and assistance required for utilizing his HDEOS provided by Dr. W. G. Wolfer; and the assistance of C. G. McKamey and J. T. Houston in preparing TEM specimens are gratefully acknowledged. I would also like to thank Drs. J. O. Stiegler and E. E. Bloom for their interest in this investigation and for arranging for the research to be performed at ORNL. In addition, I would like to express my gratitude to Dr. J. A. Horton for his suggestions, help and encouragement throughout this endeavor. Thanks are extended for many helpful discussions with the members of the research staff of REMAG at ORNL including: Drs. W. A. Coghlan, E. A. Kenik, E. H. Lee, M. B. Lewis, N. H. Packan, A. F. Rowcliffe, P. S. Sklad, and F. W. Wiffen. The help of Dr. Kenik with HVEM operation, Dr. Sklad with EDS and EELS operation, and Dr. Packan with the experimental procedures for the ion-irradiation experiments is also appreciated. Other contributors who were essential to this research effort included: Dr. R. A. Mckee (provided the high-purity iron); R. L. Heestand (provided the high-purity chromium); G. F. Petersen (performed the wet/dry hydrogen anneals); Dr. Y. K. Chang and H. Harmon (assisted with alloy fabrication); R. A. Buhl and S. W. Cook (helped to perform the ion irradiations); L. T. Gibson and T. Estes (helped with EDM operation); and A. T. Fisher and G. L. Lehman (assisted with the microscopes). 
I sincerely appreciate the extra time, effort and many suggestions provided by Frances Scarboro in preparing this manuscript. I am also grateful to C.K.H. DuBose, C. G. McKamey, W. Smith, and C. Zachary for printing many of the figures.

I am thankful for the many years of moral support and encouragement provided by my family, especially my mother, Winifred Schiestle.

The financial support for this research project was provided by the US Department of Energy: by the Division of Materials Sciences under contract No. W-7405-eng-26 with Union Carbide Corporation and by the Office of Fusion Energy under a contract with the University of Virginia. 


\begin{tabular}{|c|c|}
\hline Symbol & Units \\
\hline$a$ & Newton $-\mathrm{m}^{4}$-atom ${ }^{-2}$ \\
\hline$a, a_{0}$ & $\mathrm{~nm}$ \\
\hline$a_{1}, a_{2}$ & \\
\hline A & $m^{2}$ \\
\hline$A_{0}$ & $\mathrm{~K}$ \\
\hline$A_{\text {beam }}$ & $\mathrm{cm}^{2}$ \\
\hline$b$ & $\mathrm{~m}^{3}-a \operatorname{tom}^{-1}$ \\
\hline$\underline{b}$ & $\mathrm{~nm}$ \\
\hline $\bar{B}$ & $m^{3}-a \operatorname{tom}^{-1}$ \\
\hline C & \\
\hline $\mathrm{C}_{\mathrm{c}}$ & $\mathrm{m}^{-3}$ \\
\hline $\mathrm{C}_{\text {cluster }}$ & $\mathrm{m}^{-3}$ \\
\hline $\mathrm{c}_{\mathrm{H}}$ & at. $\mathrm{ppm}, \mathrm{m}^{-3}$ \\
\hline $\mathrm{C}_{\mathrm{He}}$ & at. $\mathrm{ppm}, \mathrm{m}^{-3}$ \\
\hline$c_{1}$ & $\mathrm{~m}^{-3}$ \\
\hline $\mathrm{C}_{\mathrm{L}}$ & $\mathrm{m}^{-3}$ \\
\hline $\mathrm{C}_{\mathrm{T}}$ & Coulombs \\
\hline $\mathrm{C}_{\mathrm{v}}$ & $\mathrm{m}^{-3}$ \\
\hline $\mathrm{C}_{\mathrm{v}}^{\mathrm{e}}$ & $\mathrm{m}^{-3}$ \\
\hline $\mathrm{C}_{58} \mathrm{NI}$ & at. $\mathrm{ppm}, \mathrm{m}^{-3}$ \\
\hline CVF & $\%$ \\
\hline$d$ & $\mathrm{~nm}$ \\
\hline$d_{B}$ & $\mathrm{~nm}$ \\
\hline$d_{c}$ & $\mathrm{~nm}$ \\
\hline $\mathrm{d}_{\text {cluster }}$ & $\mathrm{nm}$ \\
\hline $\mathrm{d}_{\mathrm{L}}$ & $\mathrm{nm}$ \\
\hline dpa & $\begin{array}{l}\text { displacements per } \\
\text { atom }\end{array}$ \\
\hline
\end{tabular}

Van der Waals constant

Lattice parameter

Constants

Area

Constant

Area of the fon beam

Van der Waals constant

Burgers vector

Second virial coefficient

Constant

Cavity concentration

Loop cluster concentration

Concentration of hydrogen

Concentration of helium

Interstitial concentration

Dislocation loop concentration

Total Ion beam charge

Vacancy concentration

Bulk thermal vacancy concentration

Concentration of ${ }^{58} \mathrm{NI}$

Cavity volume fraction

Effective hard sphere diameter for

an atom

Effective hard sphere diameter for

an atom without corrections

Volume-averaged cavity diameter

Average loop cluster diameter

Average dislocation loop diameter

Damage level 
Symbol Units

$\begin{array}{ll}\mathrm{dpa}_{\mathrm{N}} & \begin{array}{l}\text { displacements per } \\ \text { atom }\end{array} \\ \mathrm{D}_{\mathrm{i}} & \mathrm{m}^{2}-\mathrm{s}^{-1} \\ \mathrm{D}^{0} & \mathrm{~m}^{2}-\mathrm{s}^{-1} \\ \mathrm{D}_{0} & \mathrm{~K} \\ \mathrm{D}_{\mathrm{V}} & \mathrm{m}^{2}-\mathrm{s}^{-1} \\ \mathrm{D}^{0} & \mathrm{~m}^{2}-\mathrm{s}^{-1} \\ \mathrm{~V} & \\ \mathrm{E}^{\mathrm{i}} & \mathrm{eV} \\ \mathrm{E}_{\mathrm{d}} & \mathrm{eV} \\ \mathrm{E}^{\mathrm{m}} & \mathrm{eV} \\ 1 & \\ \mathrm{E}^{\mathrm{f}} & \mathrm{eV} \\ \mathrm{V} & \\ \mathrm{E}^{\mathrm{m}} & \mathrm{eV} \\ \mathrm{V} & \\ \mathrm{f}^{\mathrm{m}} & \end{array}$

$f(x)$

$\begin{array}{ll}g & \mathrm{~nm}^{-1} \\ G & \mathrm{~m}^{-3}-\mathrm{s}^{-1} \\ \mathrm{G}_{1} & \mathrm{~m}^{-3}-\mathrm{s}^{-1} \\ \mathrm{G}_{\mathrm{T}} & \mathrm{m}^{-3}-\mathrm{s}^{-1} \\ \mathrm{G}_{\mathrm{V}} & \mathrm{m}^{-3}-\mathrm{s}^{-1}\end{array}$

Nominal damage leve 1

Interstitial diffusion coefficient

Interstitial diffusion constant

Constant

Vacancy diffusion coefficient

Vacancy diffusion constant

Energy

Average energy required to produce one atomic displacement

Interstitial migration energy

Vacancy formation energy

Vacancy migration energy

Ratio of the generation rate of defects surviving the cascade to the displacement rate for ion bombardment

Deposited ion distribution

Diffracting vector

Atomic generation rate

Interstitial generation rate

Vacancy generation rate by thermal emission from sinks

Vacancy generation rate 
Symbol Units

$k$

$\begin{array}{ll}\mathrm{k}_{\mathrm{B}} & \mathrm{eV}-\mathrm{K}^{-1} \\ \mathrm{~K}_{\mathrm{i}} & \mathrm{s}^{-1} \\ \mathrm{~K}_{\mathrm{V}} & \mathrm{s}^{-1} \\ \mathrm{~L} & \mathrm{~m} \\ \mathrm{~L} & \mathrm{~m}^{-2} \\ \mathrm{M} & \\ \mathrm{Mag} & \\ \mathrm{n} & \\ \mathrm{n} & \\ \mathrm{n} & \end{array}$

$\mathrm{N}$

$\begin{array}{ll}N_{c} & m^{-3} \\ N_{L} & m^{-3} \\ N_{L} / \text { cluster } & \end{array}$

p $\quad \operatorname{mim}$

$\mathrm{P} \quad \mathrm{Pa}$

$P^{\infty}$

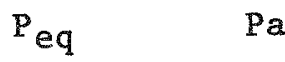

r nm

$r_{c} \quad$ nm

crit nm

$\mathrm{r}_{\mathrm{r}} \mathrm{nm}$

$\mathrm{R} \quad \mu \mathrm{m}$

R $\quad \mathrm{nm}$

R $\quad \mathrm{m}^{3}-\mathrm{s}^{-1}$
Electronic stopping power

Boltzmann's constant

Interstitial loss rate to sinks

Vacancy loss rate to sinks

Length

Dislocation density

Magnification

Magnification

Upwards loop normal

Number of helium atoms in a cavity

Number of helium atoms in an equilibrium bubble

Number of dislocation ends, number of dislocation-1ine intersections

Concentration of cavities

Dislocation loop number density

Average number of dislocation loops

per cluster

Parallax

Pressure

Helium pressure within a cavity expressed as a fraction of $\mathrm{P}_{\mathrm{eq}}$

Equilibrium bubble pressure

Bubble radius

Cavity radius

Critical cavity radius

Radius of recombination

Ion range

Interatomic distance

Coefficient of recombination 
Symbo1 Units

\begin{tabular}{|c|c|}
\hline $\mathrm{R}_{\mathrm{m}}$ & $\mathrm{nm}$ \\
\hline $\mathrm{s}_{\mathrm{g}}$ & $\mathrm{nm}^{-1}$ \\
\hline$s_{D}(x)$ & $\mathrm{MeV}-\mu \mathrm{m}^{-1}-1 \mathrm{~N}^{-1}$ \\
\hline$s_{1}$ & $m^{-2}$ \\
\hline $\begin{array}{c}\mathrm{s}^{j} \\
1\end{array}$ & $\mathrm{~m}^{-2}$ \\
\hline $\mathrm{s}_{\mathrm{v}}$ & $m^{-2}$ \\
\hline$s^{f}$ & $J-K^{-1}$ \\
\hline $\mathbf{s j}^{j}$ & $\mathrm{~m}^{-2}$ \\
\hline
\end{tabular}

$\begin{array}{ll}\mathrm{t} & \mathrm{nm} \\ \mathrm{T} & \mathrm{K} \\ \mathrm{T}_{\mathrm{I}} & \mathrm{K},{ }^{\circ} \mathrm{C} \\ \mathrm{T}_{\mathrm{M}} & \mathrm{K} \\ \mathrm{T}_{\mathrm{N}} & \mathrm{K},{ }^{\circ} \mathrm{C} \\ \mathrm{V} & \mathrm{m}^{3} \\ \mathrm{~V}(\mathrm{x}) & \mathrm{eV} \\ \mathrm{V}_{\mathrm{O}}(\mathrm{x}) & \mathrm{eV}\end{array}$

$\begin{array}{ll}\mathrm{w} & \mu \mathrm{m} \\ \mathrm{x}_{\mathrm{m}} & \mu \mathrm{m} \\ \mathrm{x}_{\max } & \mu \mathrm{m}\end{array}$

Interatomic distance at the minimum interatomic potential

Deviation from the Bragg angle Deposited energy per unit length of ion range

Interstitial sink strength

Sink strength of sink type $j$ for interstitials

Vacancy sink strength

Entropy of vacancy formation

Sink strength of sink type $J$ for vacancies

Thickness

Absolute temperature

Irradiation temperature

Absolute melting temperature

Nominal irradiation temperature

Volume

Interatomic potential

Repulsive part of the interatomic potential

Deviation from the Bragg angle

Depth from the target surface

Projected ion range

Depth of the maximum deposited energy

(Ion Irradiations)

Constants

Packing fraction

Hard sphere packing factor

Electron beam direction

Atomic number

Compressibility 
$2^{c}$

i, v

$Z^{d}$

$i, v$

$\gamma$

$\delta$

$\delta Q$

$\varepsilon$

$\varepsilon_{i}$

$\varepsilon_{\mathrm{V}}$

$\theta$

k

$\Lambda$

$\mu$

$\xi$

$\xi \mathbf{j}$

$\rho \quad \mathrm{m}^{-2}$

$\rho_{\mathrm{Fe}} \quad \mathrm{m}^{-3}$

$\rho(x)$

$\sigma$

$\sigma$

$\phi_{\text {bn }}$

$\phi_{\mathrm{zb}}$

$\phi_{\text {zn }}$

$\psi$

$\Omega$
$\mathrm{J}-\mathrm{m}^{-2}$

$\mathrm{eV}$

$m^{-2}$

$\mu \mathrm{m}$

$\mathrm{nm}$

displacements$\mu \mathrm{C}^{-1}-\mathrm{cm}^{-1}$

$\mathrm{nm}$

o

$\circ$

-

$\mathrm{m}^{3}-\mathrm{atom}^{-1}$
Cavity capture efficiency

Dislocation capture efficiency

Surface energy

Correction term for the effective hard sphere diameter for an atom

Measurement error for parameter $Q$

The minimum interatomic potential

for helium

Additional interstitial generation rate due to self-ion injection

Fraction of vacancies retained in vacancy loops

Tilt angle

Displacement efficiency

Dislocation density

Projected ion range

Extinction distance

Ratio of the thermal vacancies at sink type $j$ to that in the bulk

Dislocation density

Atomic density of iron

Linear density of displaced atoms per unit length of ion range

Standard deviation

Interatomic distance where the interatomic potential is equal to zero

Angle between $\underline{b}$ and $\underline{n}$

Angle between $\underline{z}$ and $\underline{b}$

Angle between $\underline{z}$ and $\underline{n}$

Correction term for the hard sphere diameter of an atom

Atomic volume 
CHAPTER 1

\section{INTRODUCTION}

Selection of a material for the first wall and blanket structure of fusion power reactors will be a difficult task for designers. The blanket must convert the neutron kinetic energy to thermal energy and contain a coolant which conducts the heat to the power conversion system. A leak or rupture in the first wall would release fluids into the plasma chamber and quench the plasma reaction. Repair or replacement of the wall would then be required - a process expensive in terms of both money and lost generating capacity. Therefore, selection or development of sound first wall materials is a prerequisite for the commercialization of fusion electrical power systems.

The first wall will be subjected to the harsh plasma environment and the strenuous requirements of reactor operation. The combined effects of high temperatures, high thermal fluxes, possible pulsed operation, and neutron bombardment on the materials are of concern. Specifically, the effect of irradiation on physical and mechanical properties must be evaluated for various candidate structural materials in order to select the best possible ones for final use.

It is particularly difficult to evaluate radiation damage that will occur in the fusion environment as no prototype reactors currently exist. The basic reaction in the most probable plasma fuel is:

$$
\mathrm{D}+\mathrm{T} \rightarrow a(3.5 \mathrm{MeV})+\mathrm{n}(14.08 \mathrm{MeV})
$$

These plasma reaction products will cause the first wall and blanket structure to be bombarded by a large fast neutron flux with $20 \%$ of the neutrons above $10 \mathrm{MeV}$, with a maximum energy of $14.1 \mathrm{MeV}$. In addition, some of the energetic helium ions and plasma hydrogen ions will reach the surfaces facing the plasma. The light lons cause damage within a few micrometers of the surface. Eventually the surface may blister 
or exfoliate, which would contaminate the plasma. Also important are the effects of the neutron flux on the structural components. The neutrons cause atomic displacements and the formation of several transmutation products, the most important of which are believed to be hellum and hydrogen. The properties of a material can be dramatically affected by these alterations. Gabriel et al. have calculated the atomic displacement and gas generation rates for various candidate structural materials and their elemental constituents in the neutron spectrum expected for the first wall of a $D-T$ fusion reactor $[1,2]$.

Separate components of the surface radiation damage can be investigated utilizing irradiation of specimens with light ions from accelerators. The usual technique employed is scanning electron microscope (SEM) examination of irradiated surfaces. The dynamic deve1opment of the surface damage has been observed in an SEM-accelerator system which allows simultaneous ion bombardment and examination of the specimen [3]. Surface damage mechanisms have been studied on the microstructural level by several methods. The most common is transmission electron microscope (TEM) examination of specimens prepared by postirradiation back-thinning of bulk specimens. Dynamic development of the microstructure has been studied in a high voltage electron microscope (HVEM) accelerator system which allows simultaneous lon bombardment and microstructural observation of electron transparent specimens $[4-9]$.

Methods for investigating the effects of high energy neutrons on materials involve irradiation of specimens by (1) high energy neutrons from accelerator-based neutron sources, (2) fission reactor neutrons, (3) one, two, or three co-impinging ion beams, or (4) high energy electrons. A brief summary of each type of irradiation follows. A more detailed discussion of these irradiation techniques can be found in references [10-24]. A comparison of the microstructures which develop with the different methods of irradiation is found in references [10] and [11]. 
The accelerator-based neutron sources, such as the Rotating Target Neutron Source II (RTNS-II) and the cyclotron $B e(d, n)$ sources, provide the closest approximation available to the fusion environment. RTNS-II irradiations involve only $14 \mathrm{MeV}$ neutrons and, therefore, lack the lower energy neutrons found in the expected fusion neutron spectrum. The cyclotron source irradiations have a broad spectrum ( 1 to $30 \mathrm{MeV}$ ) of neutron energies. However, the higher energy neutrons ( $>14.1 \mathrm{MeV}$ ) are not found in the expected fusion neutron spectrum [10]. Due to the low fluxes, these facilities are primarily used to study the earliest stages $\left(\sim 10^{-4} \mathrm{dpa}\right)$ of radiation damage using, for example, TEM, field ion microscopy (FIM), resistivity measurements, x-ray diffuse scattering, or in situ internal friction measurements. They are not able to reach fluences representative of reactor service. These types of investigations are examined in more detail in the reviews of Goland [12] and Wiffen and Stiegler [15]. In addition, the small test volume available precludes the use of accelerator-based neutron sources for studies involving a large number of materials and limits their use in irradiations of the larger specimens required for mechanical property tests $[13,14]$.

Fission reactor neutron spectra lack the high energy component of neutrons in the fusion spectrum. It has been established by comparisons of fission reactor data to data from accelerator-based neutron source irradiations that fission reactors provide an adequate simulation of the displacement damage (see review by Wiffen and Stiegler [15] and Goland [12]). The major problem in using fission neutron spectra is the Inability to match the production of the transmutation products to those expected for fusion reactors. This difficulty results because most of the transmutation reactions of interest require threshold energles of $\sim 5$ to $10 \mathrm{MeV}$ or greater. Fusion neutron energies are above these threshold energies; fission neutrons are not. Fission reactor tests are often planned to include many different specimens and can be designed for both microstructural and mechanical property evaluations. 
In mixed spectrum fission reactors, such as the oak Ridge Research Reactor (ORR) and the High Flux Isotope Reactor (HFIR), both the fast neutron and thermal neutron fluxes exceed $3 \times 10^{18}$ neutrons $\mathrm{m}^{-2} \mathrm{~s}^{-1}$. The fast neutrons produce both displacement damage and, to a very limited extent, He ( $n, \alpha$ reactions) and $H$ ( $n, p$ reactions). The relatively low energy of the neutrons yields only quite low gas production rates. In alloys containing, for example, ${ }^{58} \mathrm{Ni}, 10_{\mathrm{B}}$, or ${ }^{6} \mathrm{Li}$, the thermal neutrons can produce He ( $n_{t h}, \alpha$ reactions). Gas and displacement production rates for ORR and HFIR neutron spectra have been compiled by Gabriel et al. for candidate structural materials and their constituent elements [1]. In ORR low fluxes limit displacement damage production rates to those equivalent to only about $20 \%$ of the projected fusion first wall damage rates. However, with spectrum tailoring, the He concentration/dpa (displacements per atom) ratios during tests on alloys containing sufficient quantities of nickel can be approximately matched to those expected for the first wa11. In HFIR, higher displacement damage can be attained, and for alloys containing 1 to 2 at. \% Ni the He/dpa ratio is also approximately equal to those in fusion reactors $[13,16]$.

In fast reactors, such as the Experimental Breeder Reactor II (EBR-II) and the Fast Flux Test Facility (FFTF), appropriate displacement damage rates can be obtained, but the He/dpa and H/dpa levels are much lower for all materials than the projected fusion values. (Displacement and gas production rates for EBR-II are tabulated in reference [1].) Irradiations in fast reactors are primar11y used to study the effects of near-1ifetime values of displacement damage [13,16].

Ion irradiations are primarily used to produce specimens for TEM evaluation of the microstructural effects of irradiation. The effects of helium and hydrogen on the microstructural evolution of radiation damage can also be evaluated. The most common arrangement used in fusion simulation studies is a dual-beam irradiation in which energetic heavy ions and helium ions bombard the specimen simultaneously. The role of the heavy ions is to create atomic displacements through Rutherford 
collisions. The helium is injected to simulate transmutation-produced helium. In dual-beam irradiations, the He/dpa ratios expected for the first wall can be easily matched. Other advantages compared to neutron irradiations are: (1) relatively low cost; (2) short times required to generate high displacement damage and helium content; (3) easily controlled specimen temperatures; (4) avallability of test facilities, and (5) the irradiated specimens are not radioactive. The disadvantages include: (1) the location of the damage is within a few micrometers of the specimen surface, allowing for possible complications due to surface effects; (2) the strong depth dependence of the damage; (3) the lack of the transmutation-produced hydrogen present in the fusion environment; (4) the difficulty in correlating data obtained to data from neutron irradiation experiments, and (5) the studies are restricted to microstructural evaluations.

"Triple-beam" irradiations overcome the problem of the lack of implanted hydrogen. Triple-beam irradiations add an energetic deuterium ion beam to the helium and heavy ion beams. The substitution of deuterium for hydrogen required for this technique is not expected to significantly affect the role of hydrogen in the development of the defect structure [17]. Since the mass of diatomic deuterium and atomic helium are the same, they can be accelerated and implanted simultaneously with a single accelerator. The diatomic deuterium dissociates upon impact with the target surfaces. The range of these deuterium atoms is approximately the same as the range of the helium. In this type of irradiation, fusion $\mathrm{H} / \mathrm{dpa}$ and $\mathrm{He} / \mathrm{dpa}$ ratios can be maintained throughout the irradiation [17-19].

Difficulties in data correlation between neutron and ion irradiations have been only partially overcome. For comparison to neutron data it is necessary to: (1) increase the ion irradiation temperature by large amounts (up to $\sim 200 \mathrm{~K}[20,21]$ ); (2) consider the effect of the injected interstitials and diffusional spreading in the ion irradiation [21]; and (3) consider possible differences in the rate dependencies of the components of radiation damage - i.e., radiation-induced phase 
instability differences [23]. For a more complete discussion of these requirements, see references [20-25].

Irradiations by high energy electrons are utilized for dynamic observation of microstructural damage development in an HVEM. Once again, correlations to the actual reactor situation are difficult [11].

While the above techniques have been used extensively in producing specimens for the characterization of the defect structures and mechanical properties in many candidate structural naterials, relatively little data are avaliable for defect structures in ferritic stainless steels. This is because the interest in ferritic steels for use in both fast breeder fission reactors and fusion power reactors is quite recent. An example that focuses this interest is the selection of HT-9 as the blanket material in the conceptual design of the Tandem Mirror Reactor, WITAMIR-I. The steel is required to operate at $600 \mathrm{~K}$ $\left(330^{\circ} \mathrm{C}\right)$ to $800 \mathrm{~K}\left(530^{\circ} \mathrm{C}\right)$ and to accumulate $\sim 340 \mathrm{dpa}, \sim 9600$ appm $\mathrm{H}$ and $\sim 2300$ appm He during service in WITAMIR-I [26].

The lack of avallable microstructural data may be partially explained by the difficulty of TEM examination of ferromagnetic materials. These difficulties are further enhanced by the complicated unirradiated microstructures of the ferritic steels.

The purpose of this investigation is to study the development of microstructures during the irradiation of pure iron and simple iron-chromiun alloys using TEM, HVEM, and analytical electron microscopy (AEM) techniques. These materials were chosen because they are the basis of the more complex commercial steels. Both ORR and "triplebeam" irradiations have been employed. The effects of irradiation temperature, Eluence (dpa), helfum Implantation, and chromium content have been investigated. The results from this study will provide a foundation for expanded analyses of radiation damage in ferritic steels. 
This dissertation is organized into seven main sections:

Chapter 2: A summary of the available literature concerning radiation damage in ferritic materials is presented. An emphasis is placed on the microstructural aspect of radiation damage.

Chapter 3: The damage observed in ORR neutron-irradiated iron as it depends on temperature is presented.

Chapter 4: The experimentally determined depth dependence of damage in "triple-beam" irradiated iron is presented and discussed.

Chapter 5: Defect structure development in "triple-beam" Fe-10\% $\mathrm{Cr}$ is presented as it depends on both temperature and fluence. The effects of helium are explored through comparisons of triple-beam irradiation microstructures to those obtained by heavy ion irradiation with no helium and heavy ion irradiation preceded by cold ( $300 \mathrm{~K}$ ) helium preinjection. The damage structures found in $\mathrm{Fe}, \mathrm{Fe}-5 \% \mathrm{Cr}$ and $\mathrm{Fe}-10 \%$ Cr triple-beam-irradiated under identical conditions are compared. The above observations are discussed and compared to the relevant. 1iterature.

Chapter 6: The neutron and "trlple-beam" irradiation results are discussed.

Chapter 7: A summary of the results of this investigation is presented. Also incorporated in this chapter is a discussion of experiments suggested by this study.

Appendices: Experimental irradiation details, specimen preparation and electron microscopy procedures and detailed calculations are presented in appendix form. 
v

-

- 
CHAPTER 2

\section{LITERATURE REVIEW}

Only a few investigations of radiation-induced microstructures in ferritic materials have been reported and unfortunately, the majority of these do not include detailed TEM characterization of the defect structures. In this chapter, a survey of these studies is presented. For the reader's convenience, the chapter is divided into two major sections: First, a summary of the experimental studies, emphasizing those with detalled microstructural analyses, and second, a discussion of the mechanisms for radiation damage, emphasizing swelling suppression mechanisms.

\subsection{Experimental Investigations}

\subsubsection{Neutron-Irradiation Experiments}

In 1962, Eyre [27] reported the first observation of "blackspot" damage in neutron-irradiated iron. The threshold fluence for the formation of observable damage in this study was between $5 \times 10^{22}$ and $1 \times 10^{23}$ neutrons $\mathrm{m}^{-2}(\mathrm{E}>1 \mathrm{MeV})$ for irradiation temperatures of $\sim 60^{\circ} \mathrm{C}$ $(333 \mathrm{~K})$. Similar damage and fluence dependence were also reported by Bryner [28]. A subsequent investigation by Eyre and Bartlett [29] demonstrated that the "black-spot" damage consisted of point defect clusters.

In 1969, Kulcinski et a1. [30] reported the first observation of volds in neutron-irradiated iron. The volds had a crystallographic shape. The reported void morphology was a truncated octahedron with $\{110\}$ planes as both the facets and truncations. The swelling in the iron of $\sim 0.12 \%$ was less than the swelling observed for similar purity 
fcc and bcc metals irradiated at approximately the same homologous temperature $\left(\sim 0.4 \quad \mathrm{~T}_{\mathrm{M}}\right)$ and more than an order of magnitude lower fluence. The void distribution was homogeneous within the grains with a wide $(0.8-1.0 \mu \mathrm{m})$ denuded region at grain boundaries. In contrast, Farre11 and Houston [31] observed a heterogeneous distribution of voids in similar purity iron. Although the irradiation conditions in the two studies were similar, the specimens used by Kulcinski et al. were annealed prior to irradiation while those used by Farrell and Houston were irradiated in a warm-worked condition. In the latter study, recrystallization occurred during the irradiation and the voids formed in "walls," suspected to be the sites of the original grain boundaries. It was further suggested that the voids preferentially nucleated at these sites due to the presence of impurities, originally segregated to the grain boundaries, which were left behind when recrystallization occurred.

After these initial investigations, unti1 the late 1970s, the majority of the reported studies of neutron-irradiation effects in iron [32-34], simple iron alloys [33-36], and ferritic steels [32,33,37-42] centered on mechanical property effects with only limited TEM examinations. In these studies, one common observation was that ferritic materials are resistant to void swelling. In the ferritic steels, some phases swelled more than others. In particular, the titanium dioxide $\left(\mathrm{TiO}_{2}\right)$ phase $[39,40]$ and $\sigma-p h a s e[42]$ have shown enhanced swelling compared to the ferrite matrix. other reported microstructural effects in the steels include coarsening and re-distribution of $\mathrm{M}_{23} \mathrm{C}_{6}$ precipitates and recovery of dislocation structures in tempered martensite regions [41]. In none of the studies was an analysis of void morphology presented. The dislocation microstructures were also not examined in any detail. The most detailed microstructural studies were of the irradiated binary alloys. In the investigations by Smidt and coworkers $[33,34]$, the microstructures in irradiated pure $\mathrm{Fe}, \mathrm{Fe}-0.3 \% \mathrm{Cu}$, $\mathrm{Fe}-0.3 \% \mathrm{Ni}, \mathrm{Fe}-0.3 \% \mathrm{~V}, \mathrm{Fe}-0.3 \% \mathrm{P}$, and $\mathrm{Fe}-0.1 \% \mathrm{C}$ were compared. In these studies, observations of the dislocation microstructures were 
reported together with the void characteristics. The effect of the solutes on the swelling and dislocation structures was dependent on the irradiation conditions. However, a higher density of voids than found in pure iron was consistently observed in $\mathrm{Fe}-0.3 \% \mathrm{NI}$ and $\mathrm{Fe}-0.1 \% \mathrm{C}$. Although the swelling could not always be measured in Fe-0.1\% $\mathrm{C}$ due to the presence of dendritic voids at some irradiation conditions, the swelling in $\mathrm{Fe}-0.3 \% \mathrm{Ni}$ was higher than in pure 1ron. The Fe-0.3\% V alloy showed no vold formation. Also, the dislocation microstructure In the Fe-0.3\% V alloy still consisted of dislocation loops when the structures of the other alloys and pure iron contained dislocation segments and a few large loops [34].

Recently, more detailed investigations have been published. Of particular interest are the investigations of little and Stow $[43,44]$ and Gelles [45]. In these studies a survey of radiation damage in high-purity iron-chromium alloys is presented. Little and co-workers have also included pure iron (both zone-refined and hydrogen-treated) as wel1 as several ferritic steels in their experiments $[44,46,47]$.

Little and Stow $[43,44]$ have examined specimens of iron (zonerefined), $\mathrm{Fe}-1 \% \mathrm{Cr}, \mathrm{Fe}-5 \% \mathrm{Cr}, \mathrm{Fe}-10 \% \mathrm{Cr}$, and $\mathrm{Fe}-15 \% \mathrm{Cr}$ which were irradiated at temperatures of $\sim 380,420,460$, and $615^{\circ} \mathrm{C}(653-888 \mathrm{~K})$ to a fluence of $1.14-1.3 \times 10^{26}$ neutrons $\mathrm{m}^{-2}(\sim 30 \mathrm{dpa})$. In addition, iron (zone-refined) specimens were irradiated at $\sim 440,510,525,575$, and $595^{\circ} \mathrm{C}(713-868 \mathrm{~K})$ to a fluence of $0.85-1.05 \times 10^{26}$ neutrons $\mathrm{m}^{-2}(\sim 23$ dpa). The emphasis of the TEM examinations was the characterization of the swelling, average cavity diameter, and cavity concentration. Their results for swelling as a function of irradiation temperature are presented in Figure 2.1. No swelling was observed for the $615^{\circ} \mathrm{C}(888 \mathrm{~K})$ irradiation in any of the specimens. The peak swelling temperature at $30 \mathrm{dpa}$ was $\sim 420^{\circ} \mathrm{C}(693 \mathrm{~K})$. For the $23 \mathrm{dpa}$ irradiation of iron, a second swelling peak at $\sim 510^{\circ} \mathrm{C}(783 \mathrm{~K})$ is apparent in Figure 2.1 . A similar peak at $\sim 510^{\circ} \mathrm{C}$ was observed in pure, hydrogen-treated, iron irradiated to $23 \mathrm{dpa}$ at $440-595^{\circ} \mathrm{C}[44]$. In Figure 2.2, the peak swelling at $420^{\circ} \mathrm{C}$ is plotted as a function of chromiun content. The 


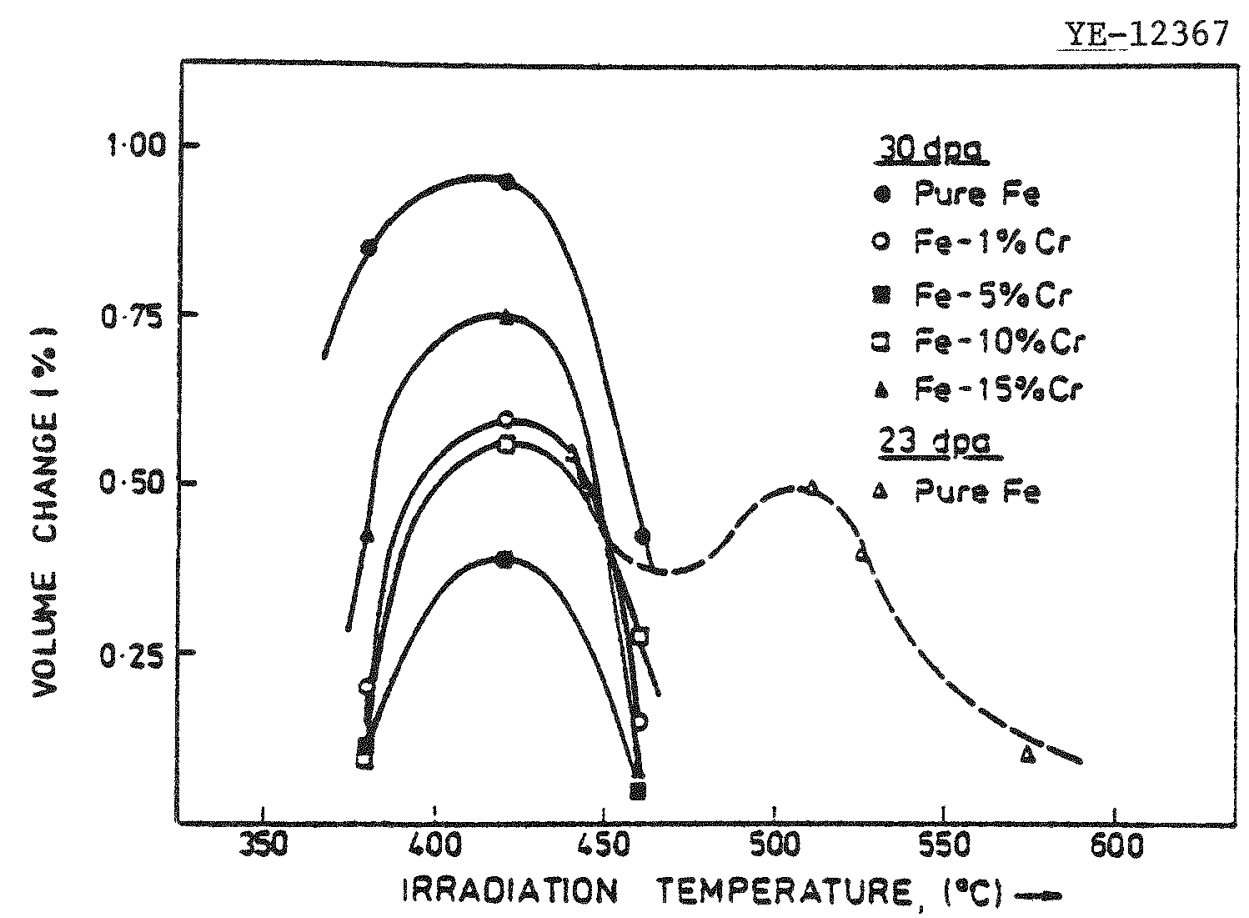

Figure 2.1. Plot of swelling as a function of irradiation temperature for neutron-irradiated iron and iron-chromium alloys. After ref. [43].

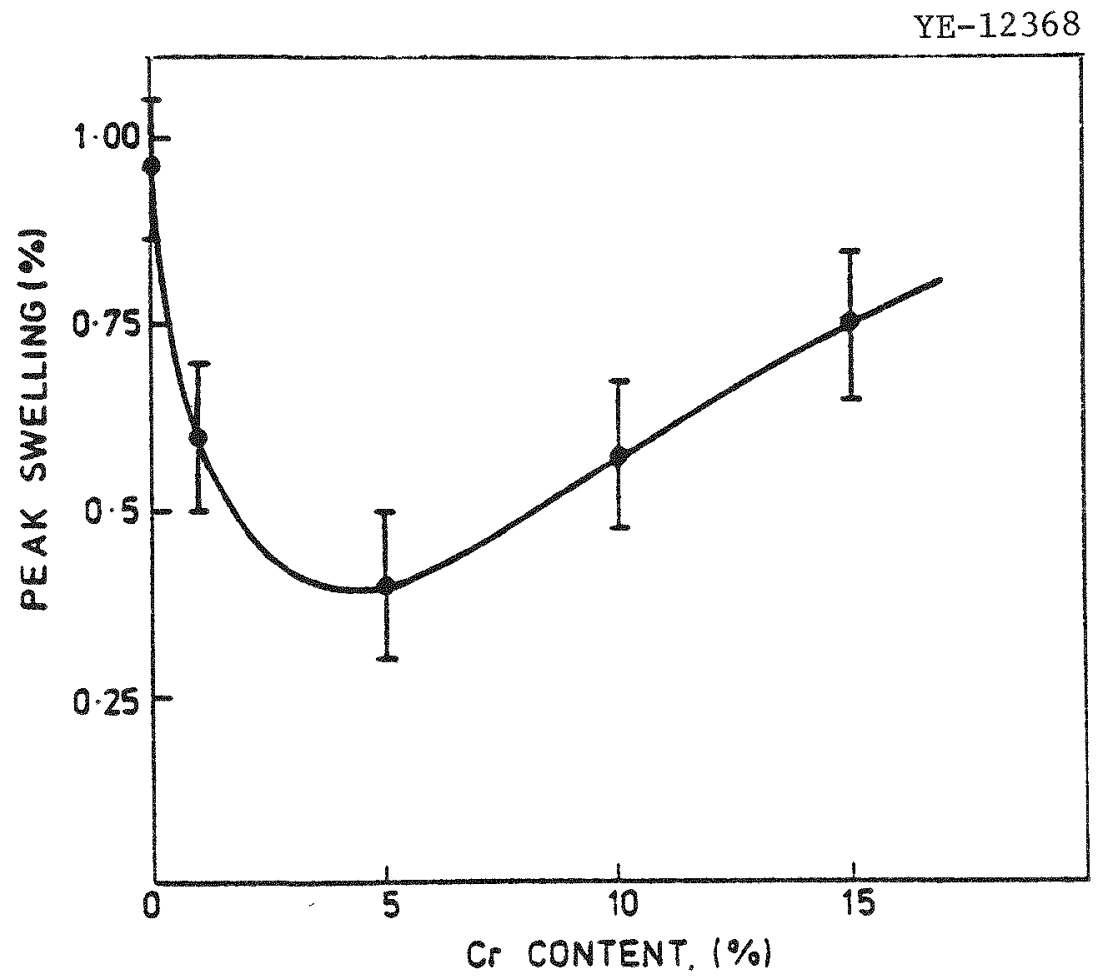

Figure 2.2. Plot of swelling as a function of alloy chromium content for iron-chromium alloys neutron-irradiated at $420^{\circ} \mathrm{C}$ to $30 \mathrm{dpa}$. After ref. [43]. 
lowest swelling was observed for $\mathrm{Fe}-5 \% \mathrm{Cr}$. In the $\mathrm{Fe}-10 \% \mathrm{Cr}$ and $\mathrm{Fe}-15 \%$

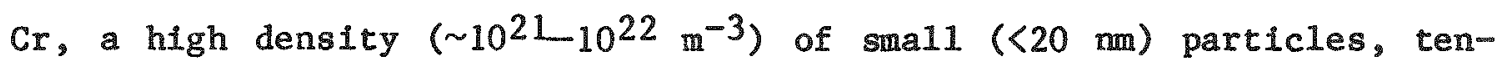
tatively identified as $\alpha^{-}$(chromium-rich ferrite) precipitates, were observed. Large zones around the cavities were denuded of these particles. It was suggested by the authors that the depletion of chromium in the matrix due to the $\alpha^{\prime}$ precipitation could be responsibie for the enhanced swelling in the $\mathrm{Fe}-10 \% \mathrm{Cr}$ and $\mathrm{Fe}-15 \% \mathrm{Cr}$ alloys. This explanation is based on the assumption that weak binding interactions between chromium atoms in solution and irradiation-produced vacancies can enhance point defect recombination and thus reduce swelling. With this assumption, removal of chromium atoms from solid solution, as in $a^{\text {- }}$ precipitation, would reduce the trapping-induced point defect recombination, making more vacancies available to contribute to swelling.

A Iimited characterization of the dislocation microstructures in the iron (zone-refined) irradiated to $30 \mathrm{dpa}$ at $420^{\circ} \mathrm{C}(693 \mathrm{~K})$ was presented by Little et al. [46]. The structure consisted of a homogeneous distribution of dislocation segments. Many segments had $\underline{b}=a\langle 100\rangle$ 1 ying along $\langle 100\rangle$ trace directions. Individual dislocation loops were reported to be only rarely discernible in the network.

Gelles [45] has examined specimens of $\mathrm{Fe}-3 \% \mathrm{Cr}, \mathrm{Fe}-6 \% \mathrm{Cr}, \mathrm{Fe}-9 \%$ $\mathrm{Cr}$, and $\mathrm{Fe}-12 \% \mathrm{Cr}$ which were irradiated at $400^{\circ} \mathrm{C}(673 \mathrm{~K})$ to $3.4 \times 10^{26}$ neutrons $\mathrm{m}^{-2}(\sim 15 \mathrm{dpa})$, at $425^{\circ} \mathrm{C}(698 \mathrm{~K})$ to $4.3 \times 10^{26}$ neutrons $\mathrm{m}^{-2}$ $(\sim 19 \mathrm{dpa})$, and at $450^{\circ} \mathrm{C}(723 \mathrm{~K})$ to $2.8 \times 10^{26}$ neutrons $\mathrm{m}^{-2}(\sim 13 \mathrm{dpa})$. Specimens of Fe-15\% $\mathrm{Cr}$ and Fe-18\% $\mathrm{Cr}$, irradiated to $\sim 13 \mathrm{dpa}$ at $450^{\circ} \mathrm{C}$, were also examined. In this study, no cavities were found in the Fe$3 \% \mathrm{Cr}$ and $\mathrm{Fe}-18 \% \mathrm{Cr}$ specimens irradiated at $450^{\circ} \mathrm{C}$. In specimens with cavities, grain boundaries had cavity-free zones. Linear arrays of cavities were often observed - interpreted as an indication of heterogeneous nucleation on dislocations. The swelling measurements for the alloys are summarized in Figure 2.3. For easy comparison, Little and Stow's data are also plotted in Figure 2.3. Both studies reported strong swelling suppression for the low chromium content alloys 


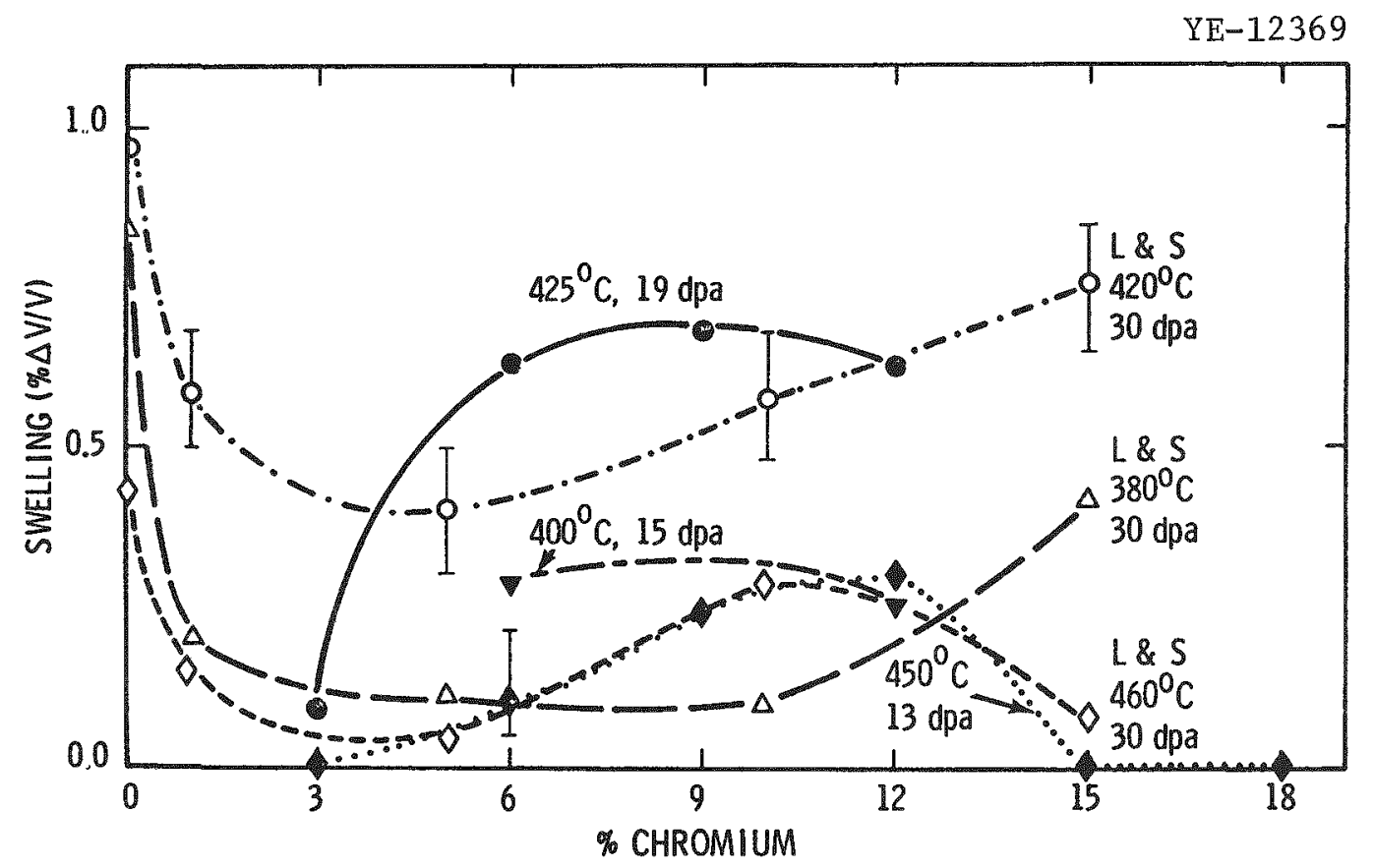

Figure 2.3. Comparison of swelling measurements as a function of alloy chromium content for the indicated irradiation conditions. The data of Gelles [45] and of Little and Stow [43,44] (labelled L \& S) are plotted. After ref. [45].

(Fe-3\% Cr or Fe-5\% Cr). Gelles also reported that the cavities usually had a truncated shape with $\{111\}$ faces.

In addition to the cavity data, Gelles also measured the dislocation densities and performed 11mited Burgers vector analyses for both loop and network dislocation structures. For the 425 and $450^{\circ} \mathrm{C}$ irradiations, the reported dislocation density increased with increasing chromium content from the Fe-3\% Cr alloy to the Fe-9\% $\mathrm{Cr}$ alloy. A slight decrease in the dislocation density was found for $\mathrm{Fe}-12 \% \mathrm{Cr}$ compared to $\mathrm{Fe}-9 \% \mathrm{Cr}$. In the $\mathrm{Fe}-3 \% \mathrm{Cr}$ alloy, the dislocation microstructure consisted primarily of 10ops with $\underline{b}=a\langle 100\rangle$. For Fe $-6 \%,-9 \%$, and $-12 \%$ Cr alloys, a network consisting of dislocations with both $\underline{b}=$ $a\langle 100\rangle$ and $b=a / 2\langle 111\rangle$ was reported. Loops were also observed in Fe$15 \% \mathrm{Cr}$ and $\mathrm{Fe}-18 \% \mathrm{Cr}\left(\mathrm{T}_{\mathrm{I}}=450^{\circ} \mathrm{C}\right)$ near grain boundaries and precipitates. Unlike the loops in the Fe-3\% Cr alloys, 10ops with $\underline{b}=a / 2\langle 111\rangle$ 
were predominant for Fe $-15 \%$ and $-18 \% \mathrm{Cr}$, although loops with $\underline{b}=a\langle 100\rangle$ were also reported.

In Gelles' study, precipitates, tentatively identified as $\alpha^{\star}$, were observed in all of the irradiated specimens. The number density of the precipitates was highest for the higher chromium alloys. Rodshaped precipitates, identified as $\mathrm{M}_{7} \mathrm{C}_{3}$, were also reported for the Fe-3\% Cr alloy.

Based on the above results, Gelles has correlated the swelling resistance of ferritic steels to the presence of dislocations with $b$ $=a\langle 100\rangle$. He suggested that the shift in the Burgers vector from

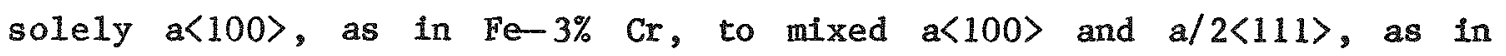
Fe-12\% $\mathrm{Cr}$, is primarily responsible for the higher swelling observed in Fe-12\% $\mathrm{Cr}$. Gelles further suggested that segregation of chromium and carbon and precipitates containing these elements contribute to swelling resistance by controlling swelling incubation and the swelling rate.

Little and co-workers [44,46] and Bullough et al. [47] have also presented damage analyses for neutron-irradiated ferritic steels. The swelling in all of the steels was suppressed compared to similarly irradiated pure iron. No voids were observed in commercial $12 \% \mathrm{Cr}$ martensitic stainless steels (FI, $C R-12$, and FV448) irradiated at 425$615^{\circ} \mathrm{C}$ (698 to $888 \mathrm{~K}$ ) to $30 \mathrm{dpa}$. A few voids were found in small isolated regions in the FI and FV448 steels irradiated at $380^{\circ} \mathrm{C}(653 \mathrm{~K}$, $30 \mathrm{dpa})$. A detalled description of the microstructures observed in FV448 has been presented by Little et al. [46] and Bullough et al. [47]. (These papers present results from the same experiment.) They report three distinct structural regions: loop colonies, void colonies, and lath martensite surrounding the loop colonies with a high network dislocation density (similar to the unirradiated structure). Void colonies were observed only for the $380^{\circ} \mathrm{C}$ irradiation. Loop colonies of homogeneously distributed, non-interacting, perfect loops on $\{100\}$ with $\underline{b}=a\langle 100\rangle$ were observed for irradiation temperatures from 380 to $460^{\circ} \mathrm{C}$ 
(653 to $733 \mathrm{~K})$. Only interstitial loops were found in the colonies, although the nature of all the loops could not be determined. No irregular loop shapes were reported. The measured loop concentration and dianeter as a function of irradiation temperature are summarized in Figure 2.4. No loops were observed for the $615^{\circ} \mathrm{C}$ irradiation. There Is some evidence of increasing recovery in the high dislocation density regions surrounding the loop colonies with increasing irradiation temperature.

Gelles [48,49] has reported microstructural observations for five commercial ferritic alloys: $21 / 4 \mathrm{Cr}-1 \mathrm{Mo}, \mathrm{H}-11, \mathrm{EM}-12,416$ and $430 \mathrm{~F}$, Irxadiated to a maximum fluence of $1.76 \times 10^{27}$ neutrons $\mathrm{m}^{-2}$ (E) 0.1 $\mathrm{MeV})$ at 400 to $650^{\circ} \mathrm{C}(673$ to $923 \mathrm{~K})$. Once again, low swelling was reported for all alloys. The major effect of the irradiation was second phase precipitation. The effects of the radiation varied with the pre-irradiation microstructures found in the steels. In EM-12, for example, the ferrite regions showed enhanced void formation compared to the martensite regions. Gelles' results are summarized in Table 2.1. The reader is referred to the aforementioned papers for a qualitative discussion of the dislocation structures. No dislocation densities or Burgers vector analyses are presented except for EM-12 irradiated at $425^{\circ} \mathrm{C}$. In this material, the majority of the dislocations had $b$ $=a\langle 100\rangle$ although dislocations with $\underline{b}=a / 2\langle 111\rangle$ were also observed.

Another current and particularly relevant investigation of low dose neutron damage in pure iron is that of Robertson, English, and Jenkins [50]. A final copy of their paper is not available, and will therefore not be discussed. However, the investigation includes detailed TEM examinations of the dislocation microstructures, including extensive Burgers vector and loop nature analyses. 


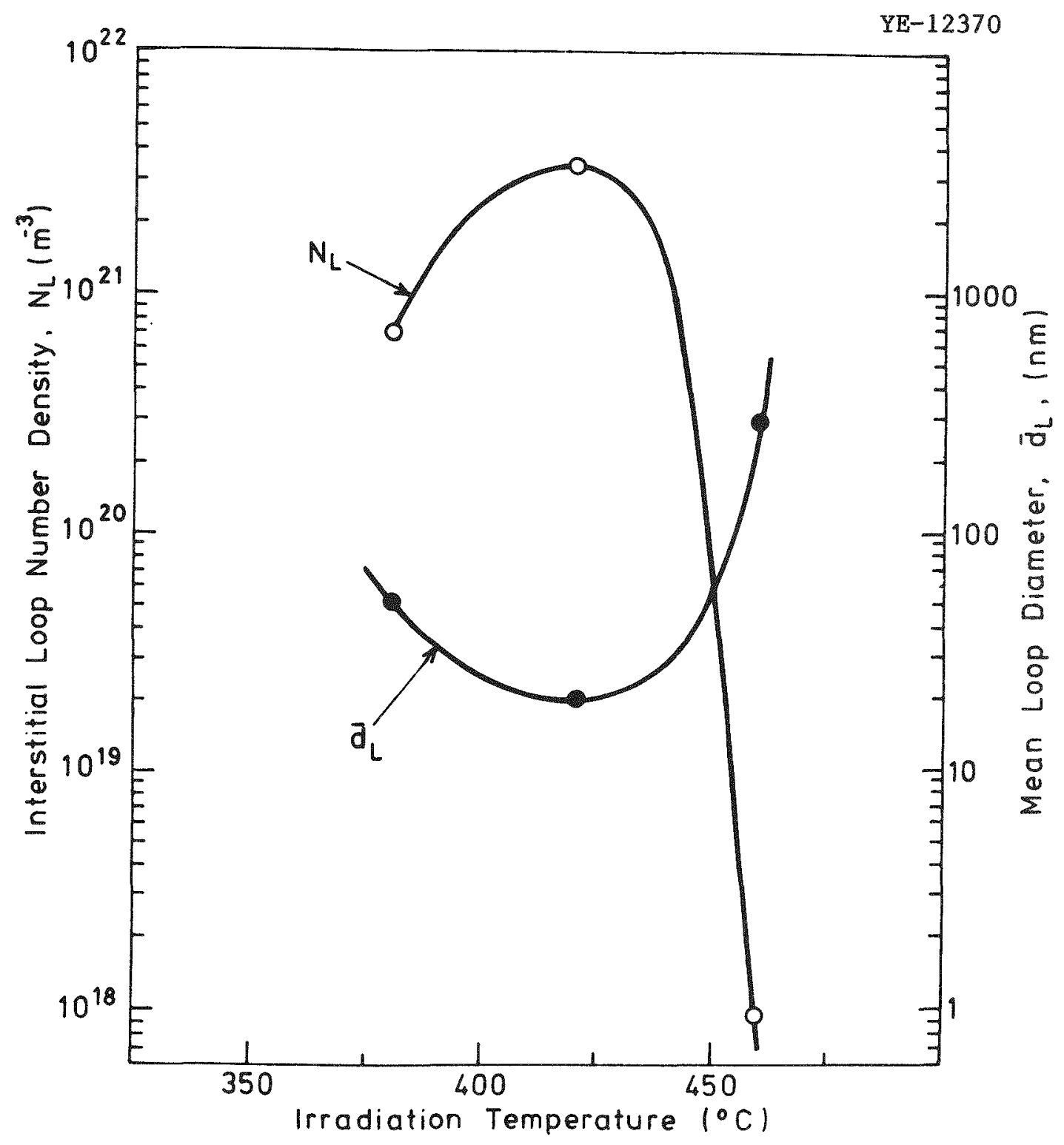

Figure 2.4. PInt of the interstitial loop concentration and mean loop diameter as a function of irradiation temperature for neutronirradiated FV448 ferritic steel, 30 dpa. After ref. [47]. 
Table 2.1. Summary of the microstructural results reported by Gelles for neutron-irradiated ferritic alloys. Parentheses indicate that a low number density was observed. * indicates unidentified precipitates After ref. [49].

\begin{tabular}{|c|c|c|c|c|c|}
\hline $\begin{array}{l}\text { Irradiation } \\
\text { Temperature } \\
\quad\left({ }^{\circ} \mathrm{C}\right)\end{array}$ & 400 & 425 & 425 & 510 & 650 \\
\hline $\begin{array}{l}\text { Fluence }\left(10^{26}\right. \\
\left.\text { neutrons } \mathrm{m}^{-2}\right)\end{array}$ & 14 & 5.1 & 15.8 & 17.2 & 17.6 \\
\hline $2 \frac{\text { Alloy }}{1 / 4 \mathrm{Cr}-1}$ Mo & & $\mathrm{M}_{6} \mathrm{C}, \mathrm{Mo}_{2} \mathrm{C}$ & $\begin{array}{l}\mathrm{M}_{6} \mathrm{C}, \mathrm{Mo}_{2} \mathrm{C} \\
\text { voids }\end{array}$ & $\mathrm{M}_{6} \mathrm{C}$ & $\left(M_{6} C\right)$ \\
\hline $\mathrm{H}-11$ & & $\mathrm{M}_{6} \mathrm{C}, \mathrm{Mo}_{2} \mathrm{C}$ & $\mathrm{M}_{6} \mathrm{C}, \quad \mathrm{Mo}_{2} \mathrm{C}$ & $\mathrm{M}_{6} \mathrm{C}, \mathrm{Mo}_{2} \mathrm{C}$ & \\
\hline$E M-12$ & $\begin{array}{l}\text { voids } \\
\text { ppts* }\end{array}$ & $x$, voids & $x$, voids & $x$ & (Laves) \\
\hline 416 & & $\begin{array}{l}\left(\mathrm{M}_{23} \mathrm{C}_{6}\right) \\
\text { (voids) } \\
\text { (ppts.*) }\end{array}$ & $\begin{array}{l}\left(\mathrm{M}_{23} \mathrm{C}_{6}\right) \\
(\text { voids) } \\
(\text { ppts. }\end{array}$ & $\left(M_{23} C_{6}\right)$ & \\
\hline 430 & & $a^{\prime}$ & $\alpha^{\prime}$ & $\left(\alpha^{\infty}, \mathbb{M}_{23} C_{6}\right)$ & \\
\hline
\end{tabular}

\subsubsection{Ion Irradiation Experiments}

In this section, investigations of damage microstructures in ferritic alloys resulting from heavy ion bombardment are summarized. The studies have utilized iron-ion [51-53], nickel-ion [34,54-56], carbon-ton [57], and other heavy ion ( $\left.\mathrm{Ge}^{+}, \mathrm{Kr}^{+}, \mathrm{Xe}^{+}, \mathrm{W}^{+}\right)$[52] irradiations. Only one study [58] has employed a dual beam of heavy ions (nickel) and helium ions to simulate the fusion environment.

The majority of the experiments have primarily studied the swelling characteristics of the materials and present only qualitative descriptions of the dislocation microstructures. Only two studies, Masters [51] and Jenkins et al. [52], report dislocation Burgers vector analyses. In 1964, Masters [51] reported the first observation of interstitial, edge dislocation loops with $\underline{b}=a\langle 100\rangle$ in irradiated iron. 
In this study, iron targets were bombarded with $150 \mathrm{keV} \mathrm{Fe}$ ions at $550^{\circ} \mathrm{C}$ to a fluence of $\sim 1.7 \times 10^{20}$ ions $\mathrm{m}^{-2}$. The loops were rectilinear with $\langle 100\rangle$ sides. Jenkins et al. observed vacancy loops with $\underline{b}=a\langle 100\rangle$ and $\underline{b}=a / 2\langle 111\rangle$ in iron irradiated to $\left\langle 5 \times 10^{16}\right.$ ions $\mathrm{m}^{-2}$ with $80 \mathrm{keV} \mathrm{w}^{+}$ ions. No damage was produced for self-ion irradiations to $\leqslant 5 \times 10^{16}$ ions $\mathrm{m}^{-2}$ for ion energies of 40 to $240 \mathrm{keV}$. The authors suggested that this observation implies that cascade collapse to vacancy loops does not occur for self-ion irradiated iron. They further suggested that cascade collapse will not occur in neutron-irradiated iron and therefore the dose for the onset of visible damage in neutron-irradiated iron will be determined by the growth of interstitial loops to visible sizes.

The only other iron-ion irradiation experiment was reported by smidt et al. [53]. They examined two commercial ferritic alloys, HT-9 and EM-12, which were first implanted with 1 at. ppm He and then bombarded with $2.8 \mathrm{MeV} \mathrm{Fe}^{+}$ions. They found that, for damage levels of $150 \mathrm{dpa}$, the peak swelling temperatures were $550^{\circ} \mathrm{C}$ for $\mathrm{EM}-12$ and $500^{\circ} \mathrm{C}$ for HT-9. Cavities were found in association with unidentified interand intra-granular precipitates. For 450 and $650^{\circ} \mathrm{C}$ irradiations, no cavities were observed in either material. The evolution of the damage microstructures with increasing damage between 40 and $250 \mathrm{dpa}$ at the peak swelling temperature was also studied. At $250 \mathrm{dpa}$, the swelling was quite low - $2.6 \%$ in EM-12 and $4.7 \%$ in HT-9. In EM-12, although large cavities $(\sim 95 \mathrm{~nm})$ were associated with intergranular precipitates at all damage levels, a second population of smaller cavities was present in the grain interiors for damage levels $\geqslant 150 \mathrm{dpa}$. A few extremely large cavities $(\sim 325 \mathrm{~nm})$ were found at large, blocky, intragranular precipitates. In HT-9, cavities were observed only at intergranular precipitates.

Smidt et al. [34] have also studied $2.8 \mathrm{MeV}$ nickel-ion irradiated $\mathrm{Fe}, \mathrm{Fe}-0.3 \% \mathrm{Cu}$, and $\mathrm{Fe}-0.3 \% \mathrm{~V}$ alloys. They reported the irradiation temperature dependence $\left(450-700^{\circ} \mathrm{C}\right)$ at 16 and $32 \mathrm{dpa}$. Cavities were observed only at $650^{\circ} \mathrm{C}$ in iron and at 450 and $550^{\circ} \mathrm{C}$ in $\mathrm{Fe}-0.3 \% \mathrm{Cu}$ specimens. No cavity formation was observed in $\mathrm{Fe}-0.3 \% \mathrm{~V}$. 
Nickel-ion damage in binary iron-chromium alloys has been investigated by Johnston et al. [54,58]. Prior to irradiation, the target materials were preinjected with 5 to 50 at. ppm helium. They reported the effect of chromium content (Fe- $7 \%,-15 \%$, and $-20 \% \mathrm{Cr}$, $\left.550^{\circ} \mathrm{C}, 140 \mathrm{dpa}\right)$, the effect of irradiation temperature $(\mathrm{Fe}-15 \% \mathrm{Cr}$, $\left.140 \mathrm{dpa}, 425-625^{\circ} \mathrm{C}\right)$, and the effect of fluence $\left(\mathrm{Fe}-15 \% \mathrm{Cr}, 550^{\circ} \mathrm{C}\right.$, 40-275 dpa) on the swelling. Their results are summarized in Figures $2.5,2.6$, and 2.7. Johnston et al. [54-56] also report low swelling $(<1 \%)$ for $21 / 4 \mathrm{Cr}-1$ Mo ferritic steel irradiated to $140 \mathrm{dpa}$ at $625^{\circ} \mathrm{C}$.

A single investigation has reported cavity morphology. Ohnuki et al. [57] have reported the observation of cubic cavities with $\{100\}$ faces in ixon irradiated with $200 \mathrm{keV} \mathrm{C}$ ions at $798 \mathrm{~K}\left(525^{\circ} \mathrm{C}\right)$. Radiation induced segregation and precipitation were also studied, although the effects of implanted carbon dominated these processes.

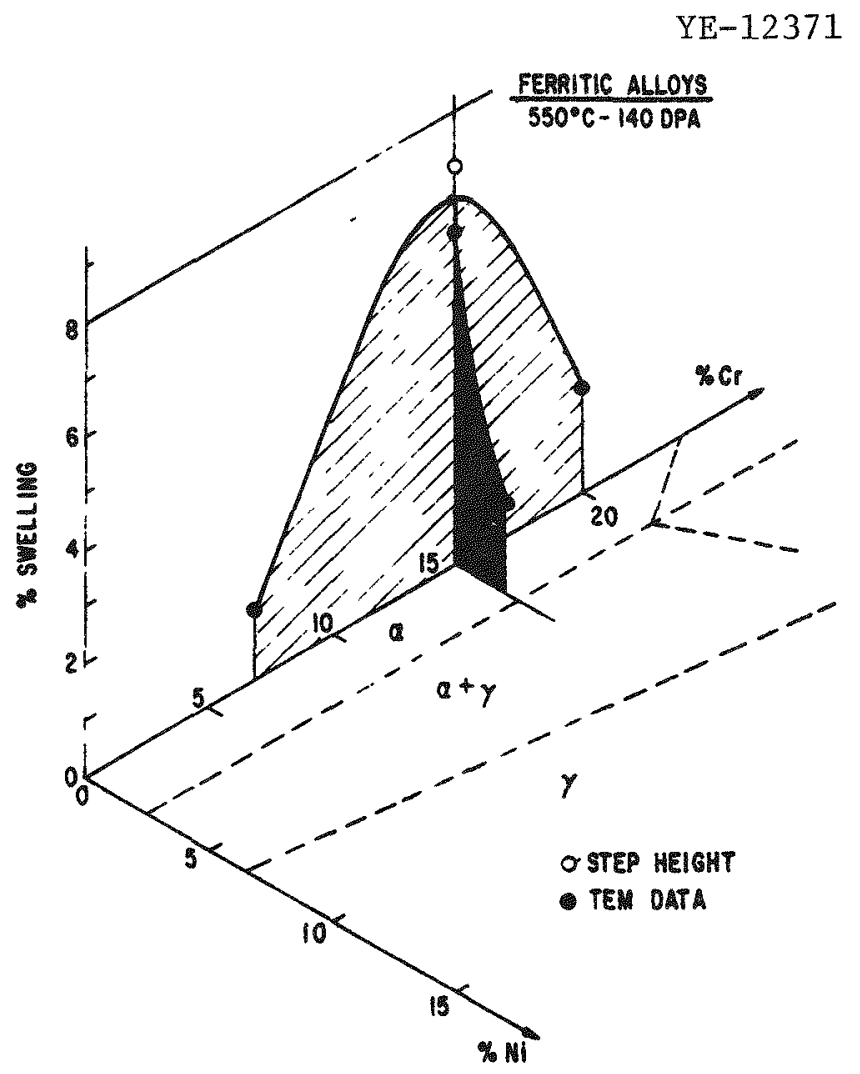

Figure 2.5. Plot of swelling as a function of chromium content for nickel-ion-irradiated iron-chromium alloys. $\mathrm{T}_{\mathrm{I}}=550^{\circ} \mathrm{C}, 140 \mathrm{dpa}$. After ref. [54]. 


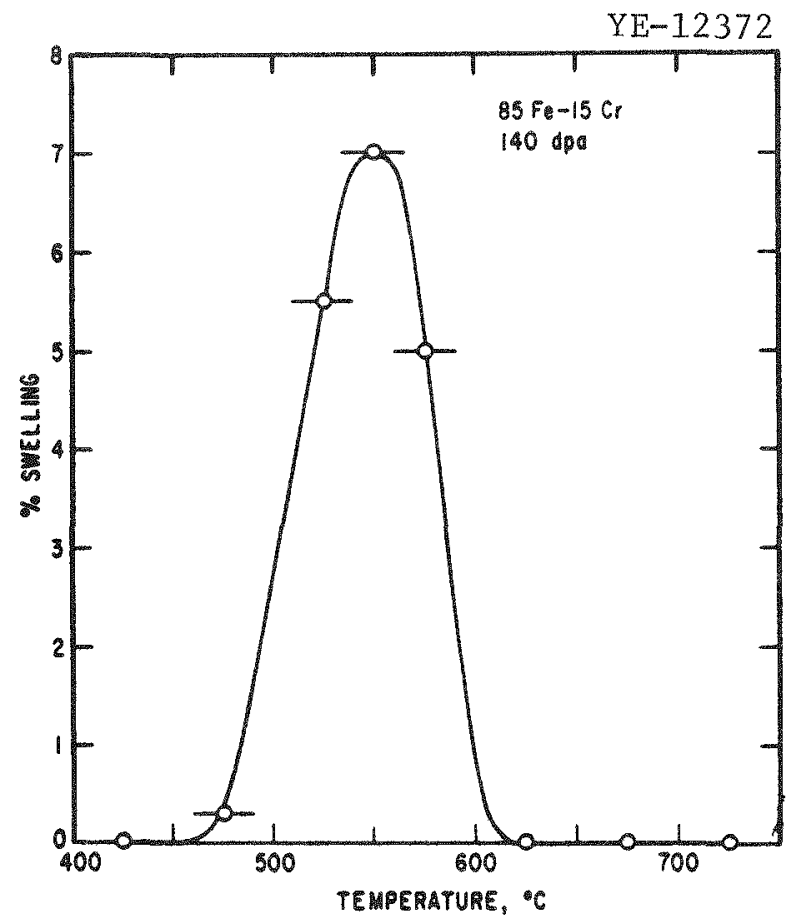

Figure 2.6. Plot of swelling as a function of irradiation temperature for nickel-ion-irradiated $\mathrm{Fe}-15 \% \mathrm{Cr}$, 140 dpa. After ref. [54].

YE-12373

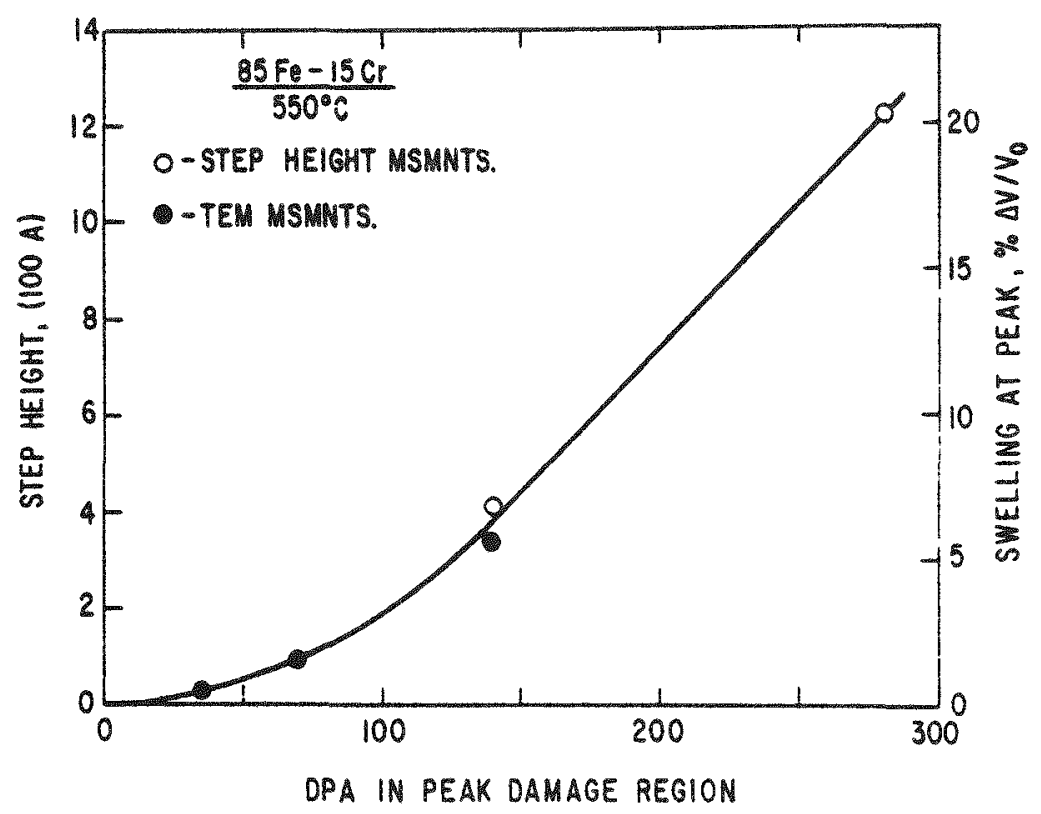

Figure 2.7. P1ot of the dose dependence of swelling for nickel-ion-irradiated $\mathrm{Fe}-1.5 \% \mathrm{Cr}, \mathrm{T}_{\mathrm{I}}=550^{\circ} \mathrm{C}$. After ref. [54]. 
Kuramoto et al. [58] have reported the only fusion environment simulation study. They irradiated pure iron with a dual beam of $4 \mathrm{MeV}$ nickel lons and 200-400 $\mathrm{keV}$ (the beam energy was sinusoidally ramped) helium ions $(\sim 10$ at. ppm helium/dpa). Single nickel lon irradiations were also performed. The targets were irradiated to fluences of 5,20 , and $70 \mathrm{dpa}$ (peak damage region) at temperatures of $350-500^{\circ} \mathrm{C}(623-773 \mathrm{~K})$. At $350^{\circ} \mathrm{C}$, small dislocation loops or dots, reported to be vacancy 10ops, were observed in single ion irradiations to $5 \mathrm{dpa}$. Cavities were observed for 450 and $500^{\circ} \mathrm{C}$ irradiations. Compared to the single ion irradiations, dual ion beam irradiation caused a decrease in mean cavity diameter, an increase in cavity concentration, and, at 20 and $70 \mathrm{dpa}$, a decrease in swelling. At $5 \mathrm{dpa}\left(500^{\circ} \mathrm{C}\right)$ the swelling was higher for the dual ion irradiation. It should be noted that the data for these experiments was not measured in the peak damage region. The data was measured from HVEM micrographs of backthinned specimens. Therefore, the region examined was from the surface to $0.7 \mu \mathrm{m}$. This is especially important in the dual ion irradiations as the calculated level portion of the helium profile, where the desired helium concentration to dpa ratios are attained, begins at $0.7 \mu \mathrm{m}$. Very little helium is deposited at depths less than $\sim 0.5 \mu \mathrm{m}$.

\subsubsection{Electron Irradiation Experiments}

The first reported study of electron damage in a ferritic material was published by Little and Eyre [59] in 1972. In alpha-iron irradiated at $550^{\circ} \mathrm{C}(823 \mathrm{~K})$, they observed rectilinear, pure edge, interstitial dislocation loops with $\underline{b}=a\langle 100\rangle$. The sides of the loops were parallel to $\langle 100\rangle$. Dislocation loops with similar geometry have also been reported by Little [60] for electron irradiation studies of mild steel $\left(\mathrm{T}_{\mathrm{I}}=550^{\circ} \mathrm{C}\right)$ and by Yoshida et al. [61] for pure iron $\left(\mathrm{T}_{\mathrm{I}}\right.$ $>350^{\circ} \mathrm{C}$ ). For irradiation temperatures $<300^{\circ} \mathrm{C}$, Yoshida et al have reported irregularly-shaped, pure edge, interstitial loops with $\underline{b}=$ $a\langle 100\rangle$. They referred to the loop shape as "flower-shaped." They believe that this shape is the result of enhanced growth of the corners 
of small rectilinear loops. Interstitials are preferentially attracted to the loop corners where there is a greater dilation in the strain field as compared to the straight loop segment. Another important result from this study is the determination of an activation energy of $0.26 \mathrm{eV}$ for interstitial migration in iron. Kiritani $[62,63]$ and Kiritani et al. [64] also discuss the experiment of Yoshida et al. in their reviews of electron radiation damage of metals and interactions of electron-irradiation-induced point defects and dislocations.

In a more recent investigation, Suganuma and Kayano [65] have studied the nucleation and growth of dislocation loops in $\mathrm{Fe}-15 \% \mathrm{Cr}$. For $200^{\circ} \mathrm{C}$ irradiations, clusters of black-spot damage were observed to form. Rectangular clusters of small loops were formed at 300 and $400^{\circ} \mathrm{C}$ (573 and 673 K). The majority of these clusters formed on $\{100\}$ and had $\langle 100\rangle$ sides. Less than $10 \%$ were on $\{111\}$ with $\langle 100\rangle$ sides. Above $450^{\circ} \mathrm{C}(723 \mathrm{~K})$, perfect "star-shaped" loops were formed. Loops formed on both $\{111\}$ and $\{100\}$. Some of the loops formed in pairs. These loops were on parallel planes and were almost identical in size and shape. Above $560^{\circ} \mathrm{C}(833 \mathrm{~K})$, no dislocations or other defects were observed. The authors suggested that the formation of clusters of small loops is due to the growth of $\mathrm{Cr}-(\mathrm{C}, \mathrm{N})$ atmospheres around small dislocation loops. The flow of interstitials towards the loops is hindered by these atmospheres, forming interstitial-rich areas near the loops and promoting further loop nucleation.

only four investigations have reported void formation in electron irradiated iron and ferritic steels. In 1972, Little [60] reported the first observation of voids in mild steel irradiated at $550^{\circ} \mathrm{C}$. The voids had an octahedral shape. However, the planes forming the facets of the voids were not reported. At $30 \mathrm{dpa}$, the void swelling was quite low - only 0.9 to $1.3 \%$. Void formation in $1.25 \mathrm{MeV}$ electron-irradiated high-purity iron has been reported to occur for irradiation temperatures of 325 to $387^{\circ} \mathrm{C}[66,67]$. This temperature range is narrow compared to that found for similarly irradiated fcc metals. Arkell and Williams [68] have reported void formation over a wider range of temperatures 
$\left(300-550^{\circ} \mathrm{C}\right.$, fluence $\left.\geqslant 3 \mathrm{dpa}\right)$ in $1 \mathrm{MeV}$ electron-irradiated FV 607 ferritic steel.

The effects of preinjected helium has also been studied. In pure iron, preinjection of 1 at. ppm He enhanced swelling but did not alter the peak swelling temperature of $\sim 350^{\circ} \mathrm{C}(625 \mathrm{~K})$. The helium also significantly reduced the incubation fluence for void formation. However, for specimens preinjected with 100 at. ppm He, swelling was suppressed and the peak swelling temperature was $50^{\circ}$ higher than for pure iron $[66,67]$. The effects of preinjection with 10 at. ppm He prior to irradiation of FV 607 steel were quite complex [68]. In general, the void concentration was higher and the average void diameter was smaller in the preinjected specimens. At $40 \mathrm{dpa}$, the peak swelling temperature was $450^{\circ} \mathrm{C}(723 \mathrm{~K})$ for the preinjected specimen and $400^{\circ} \mathrm{C}(673 \mathrm{~K})$ for the uninjected specimen. The magnitude of the swelling was $\sim 4 \%$ for both specimens. At $10 \mathrm{dpa}$, however, the results were the opposite the peak swelling temperature for the preinjected specimen was $400^{\circ} \mathrm{C}$ and for the uninjected specimen was $450^{\circ} \mathrm{C}$. The preinjected specimen had also swelled more than the uninjected specimen.

Radiation-induced precipitation and segregation have also been investigated for electron-irradiated iron [69] and iron binary alloys [70]. Of particular interest from these studies is the experimentally determined dissociation temperature for carbon-vacancy $(C-V)$ and nitrogen vacancy $(\mathrm{N}-\mathrm{V})$ pairs. Takeyama and Takahashi [69] determined the detrap temperature for $\mathrm{C}-\mathrm{V}$ pairs as $240^{\circ} \mathrm{C}(513 \mathrm{~K})$ and for $\mathrm{N}-\mathrm{V}$ pairs as $200^{\circ} \mathrm{C}(473 \mathrm{~K})$.

\subsubsection{Summary of Experimental Observations}

Several characteristics of the damage microstructures are common to all three types of irradiation experiments. In general, iron and ferritic alloys are resistant to void swelling as compared to other fcc and bcc metals and alloys. The reasons for the swelling suppression are not clear. Several mechanisms which have been proposed to explain this phenomenon are discussed in the last part of this chapter. 
Additional features of the cavity formation process are also of interest. The cavity morphologies reported vary considerably. Truncated octahedra with $\{110\}$ faces, cubes with $\{100\}$ faces and truncated octahedra with $\{111\}$ faces have all been reported. The peak swelling temperature varied considerably, depending on the alloy and irradiation experiment. These temperatures are summarized in Table 2.2. In experiments with helium preinjection, a shift in the peak swelling temperature compared to noninjected specimens was reported. Also, preinjection or co-implantation of helium generally increased the cavity concentration and decreased cavity diameter in irradiated iron and FV 607 ferritic steels.

Table 2.2. Summary of the reported peak swelling temperatures

\begin{tabular}{llll}
\hline Material & $\begin{array}{c}\text { Type of } \\
\text { Irradiation }\end{array}$ & $\begin{array}{c}\text { Peak } \\
\text { Swelling } \\
\text { Temperature }\end{array}$ & Reference \\
\hline $\mathrm{Fe}$ & Neutron & $420^{\circ} \mathrm{C}(693 \mathrm{~K})$ & $43,44,46$ \\
$\mathrm{Fe}$ & Neutron & $510^{\circ} \mathrm{C}(783 \mathrm{~K})$ & $43,44,46$ \\
$\mathrm{Fe}$ & Electron & $350^{\circ} \mathrm{C}(623 \mathrm{~K})$ & 66,67 \\
$\mathrm{Fe}{ }^{\mathrm{a}}$ & Electron & $350^{\circ} \mathrm{C}(623 \mathrm{~K})$ & 67 \\
$\mathrm{Fe}$ & Electron & $400^{\circ} \mathrm{C}(673 \mathrm{~K})$ & 66,67 \\
$\mathrm{Fe}-\mathrm{Cr} \mathrm{C}$ & Neutron & $420^{\circ} \mathrm{C}(693 \mathrm{~K})$ & 43,44 \\
$\mathrm{FV}-448$ & Neutron & $380^{\circ} \mathrm{C}(653 \mathrm{~K})$ & 46,47 \\
$\mathrm{HT}-9^{\mathrm{a}}$ & Iron Ion & $500^{\circ} \mathrm{C}(773 \mathrm{~K})$ & 53 \\
$\mathrm{EM}-12^{\mathrm{a}}$ & Iron Ion & $550^{\circ} \mathrm{C}(823 \mathrm{~K})$ & 53 \\
$\mathrm{FV}-607$ & Electron & $400, \mathrm{~d} 450^{\circ} \mathrm{C}$ & 68 \\
& & $(673,723 \mathrm{~K})$ & \\
$\mathrm{FV}-607^{\mathrm{E}}$ & Electron & $450, \mathrm{~d} 400^{\circ} \mathrm{C}$ & 68 \\
& & $(723,673 \mathrm{~K})$ &
\end{tabular}

$a_{1}$ at. ppm He preinjected.

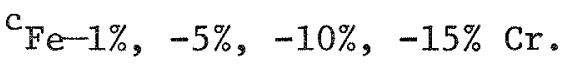
$\mathrm{e}_{10 \mathrm{dpa}}$

\footnotetext{
${ }^{b} 100$ at. ppm He preinjected.

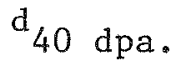

$\mathrm{f}_{10}$ at. ppm He preinjected.
} 
An unusual feature of the dislocation microstructure for all types of irradiation was the observation of interstitial dislocation loops with $a\langle 100\rangle$ Burgers vectors. Only rarely were loops with $\underline{b}$ $=a / 2\langle 111\rangle$ reported. In other bcc metals and alloys, loops with a/2<111> Burgers vectors are usual1y observed. Eyre and Bu11ough [71] have proposed a mechanism which accounts for the formation of interstitial loops with both Burgers vectors in bcc metals. They suggest that the loops form from common faulted loop nuclei on a $\{110\}$ plane. The stacking fault can be eliminated by a shear in either a $\langle 100\rangle$ or $\langle 110\rangle$ direction as described by the dislocation reactions

$$
\begin{aligned}
& a / 2[110]+a / 2[00 \overline{1}] \rightarrow a / 2[11 \bar{i}] \text { and } \\
& a / 2[110]+a / 2[\overline{1} 10]+a[010] \text {. }
\end{aligned}
$$

These reactions form perfect dislocation loops. It is suggested that, since the $\langle 110\rangle$ shear requires greater energy, loops with $\underline{b}=a\langle 100\rangle$ will form only in small numbers and only at elevated temperatures. The elastic energy of either loop can be lowered by rotation of the loop habit plane from $\{110\}$ to a pure edge configuration on $\{100\}$ or $\{111\}$. Recently, Bullough et al. [47] have evaluated the probability for a<100> $100 p$ formation for a number of bcc metals. Their calculations show a relative probability of $5.7 \times 10^{-9}$ for $\alpha$-iron. Higher relative probabilities were found only for niobium $\left(4.3 \times 10^{-5}\right)$ and vanadium $\left(5.5 \times 10^{-5}\right)$.

Another interesting aspect of the dislocation loops was the variation in loop shape. Irregularly-shaped loops have been reported for heavy ion irradiations (rectilinear loops) and for electron irradiations ("flower-shaped" and "star-shaped" loops). Regular loop shapes are reported for neutron irradiations.

Generally, vacancy loops are not observed in irradiated ferritic alloys. This observation is supported by early work of Jenkins et al. [52] which suggests that cascade collapse to vacancy loops does not occur in iron. 
Many of the investigations suggest that impurity and solute atoms have a strong influence on the damage microstructures. For example, in binary alloys, alloying with vanadium and chromium suppressed swelling while alloying with nickel and carbon enhanced it. Additions of 3-5\% chromium to pure iron suppressed swelling more than additions of 9-15\% chromium. Many secondary phases have shown enhanced swelling compared to the ferrite matrix. Also, cavity formation is enhanced at many types of precipitates. Radiation-induced precipitation is also frequently observed, especially in the ferritic steels.

\subsection{Swelling Suppression Mechanisms}

Currently, few mechanisms have been presented to explain the low swelling behavior exhibited by ferritic alloys. In this section, three of the more detailed models will be summarized.

Hayns and Williams [72] have presented a model based solely upon point defect trapping to explain the void swelling characteristics of electron-irradiated FV 607 ferritic steel. This is the only available model which utilizes rate theory calculations to provide semiquantitative support for the conclusions. By incorporating point defect trapping into a rate theory model, the qualitative features of the void swelling behavior in FV 607 steel could be consistently explained. The model yielded the low peak swelling temperature, the overall low swelling and the rapid drop in swelling at temperatures above the peak swelling temperature as observed in the experiments [68]. The authors suggest that, if their model is correct, there would be a weaker dose rate dependence for ferritic steels than that usually observed in austenitic alloys. Thus, the peak swelling temperature "shift" usually observed when comparing reactor and accelerator irradiations would be less for ferritic alloys than the 100 to $150^{\circ}$ difference often observed. A "shift" in the peak swelling temperature of $\sim 55^{\circ}$ is suggested for FV 607 by the rate theory calculations. 
Little [73] has suggested that both point defect trapping and solute-dislocation interactions play key roles in controlling the void swelling response of ferritic steels. Three mechanisms, all of which contribute to the suppression of void swelling, were considered to operate strongly in ferritic steels.

First, the effect of point defect trapping by solute atoms was considered. Little concluded that the trapping of radiation-induced vacancies, rather than interstitials, by the interstitial solutes carbon and nitrogen would be the most likely trapping scenario. Vacancy trapping by substitutional solute atoms could also occur. The vacancysolute complexes act as sites for preferred recombination for interstitials. As a result, growth of both volds and dislocation loops is reduced due to the reduced number of available point defects. In addition, the vacancy supersaturation would be reduced which should reduce vold nucleation. Void nucleation and growth could be further affected by nonequilibrium segregation of solutes to sinks as a result of the point defect trapping.

In the second mechanism, interactions of interstitial and substitutional solute atoms with dislocations are considered to be strong enough to reduce the dislocation bias for preferential self-interstitial capture. Enhanced recombination of point defects would result, reducing the number of vacancies available for vold growth. In the most extreme case, vold nucleation could be prevented by the failure to establish the necessary vacancy supersaturation. Calculations by Weertman and Green [74] have shown that void growth can be completely eliminated if the dislocations are surrounded by a condensed atmosphere of oversized substitutional atoms or interstitial atoms.

In Little's third swelling suppression mechanism, the effect of the interactions between solute atoms and dislocations on dislocation climb was considered. If the diffusivity of the solute atoms is of the correct magnitude, solutes are considered likely to be effective in impeding climb and glide of dislocations due to the restraining force 
of the impurity atmospheres on the dislocation. When climb cannot occur, a dislocation acts as a neutral, but saturable, sink for point defects - it cannot accept interstitials and vacancies separately, only simultaneously. Thus, the dislocations can act as recombination centers and reduce void growth.

In summary, Little has suggested that the above mechanisms can act together to produce the extremely low swelling or total lack of voids often observed in ferritic steels. In addition, the formation of precipitates, as in many $12 \%$ Cr steels, could further enhance point defect recombination and reduce swelling if the precipitates behave in a manner analogous to isolated solute atoms.

Little et al. [47] have proposed a model which considers the relationship between the swelling resistance and dislocation evolution. (This model is also presented by Bullough et al. [48]). Their model was developed to explain the observation of interstitial loops with exclusively $a\langle 100\rangle$ Burgers vectors and no cavities in regions of FV 448 ferritic steel specimens which contalned a high network density of $a / 2\langle 111\rangle$ dislocations prior to irradiation. Essentially, this mechanism considers that interstitial loops with $\underline{b}=a\langle 100\rangle$ and with $\underline{b}=a / 2\langle 111\rangle$ are formed prior to cavity nucleation due to the high

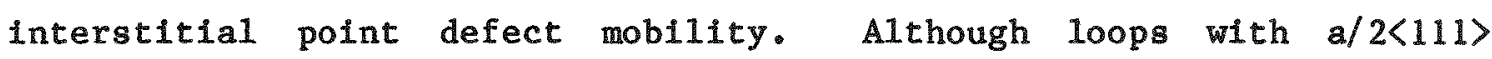

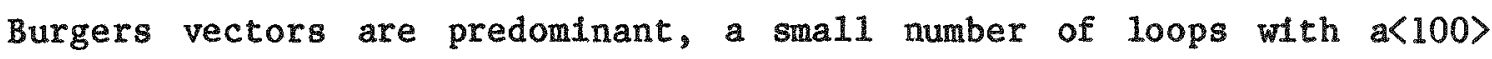
are also nucleated. The a<100> loops constitute biased sinks for preferential interstitial absorption, while the pre-existing $a / 2\langle 111\rangle$ dislocation network and the irradiation-induced $a / 2\langle 111\rangle$ loops are relatively neutral sinks. (The bias of a $\langle 100\rangle$ dislocations is higher than the bias of $a / 2\langle 111\rangle$ dislocations since the magnitude of the $a<100\rangle$ Burgers vector, $|b|=a$, is greater than the magnitude of the $a / 2\langle 111\rangle$ Burgers vector, $|\mathrm{b}|=\sqrt{3} \mathrm{a} / 2_{\bullet}$ ) The vacancy supersaturation resulting from the growth of the a<100> loops can be absorbed by the surrounding neutral a/2<111> dislocation sinks, causing vold nucleation

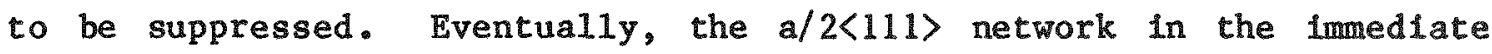


vicinity of the $a\langle 100\rangle$ loops disappears. Subsequent nucleation of a $\langle 100\rangle$ loops coupled with annihilation (by vacancy absorption) and renucleation of $a / 2<111\rangle$ loops establishes a steady-state condition in which vold nucleation is unlikely. The formation of an array of discrete domains which contain only a<100> loops is suggested by the above mechanism. Each domain grows around an initial, isolated a<100> 10op. As more a $\langle 100\rangle$ loops form around the initial loop, the growth of the Inner loop gradually ceases due to the shielding by the outer 10ops. This yields domains of approximately equal-sized loops. Between the domains, regions containing both the dislocation network and small a/2<111> loops remain. Voids can nucleate in these regions. However, if the network dislocation density is sufficiently high, both loop and vold nucleation will be suppressed by point defect recombination at the network.

The above model was formulated to provide a consistent explanation for the heterogeneous microstructures found in neutron-irradiated FV 448 martensitic steels. Little et al. expect that their mechanism would also apply to other ferritic and certain bcc metals - e.g., nioblum and vanadium, if the necessary preirradiation dislocation network is present.

In summary, none of the above mechanisms presents a clear, general picture of the radiation damage processes which occur in ferritic materials. In order to develop a complete understanding of the microstructural evolution, a more complete data base is necessary. 
CHAPTER 3

\section{NEUTRON-IRRADIATION EXPERIMENTS}

A TEM study of the defect structures produced in neutronirradiated iron is presented in this chapter. The specimens were $3 \mathrm{~mm}$ diameter disks fabricated from two-pass zone-refined Ferrovac-E Iron (30 wt ppm C). (See Appendix A for the complete chemical analysis.) The disks were irradiated in the Oak Ridge Research Reactor (ORR) at temperatures from $455 \mathrm{~K}\left(182^{\circ} \mathrm{C}\right)$ to $773 \mathrm{~K}\left(500^{\circ} \mathrm{C}\right)$ to $\sim 1$ dpa $(2.6$ at. ppm $\mathrm{He} / \mathrm{dpa}$ and 5.1 at. ppm H/dpa). Specimens were also irradiated at 923 $\mathrm{K}\left(650^{\circ} \mathrm{C}\right)$ and $1013 \mathrm{~K}\left(740^{\circ} \mathrm{C}\right)$ to $\sim 0.5 \mathrm{dpa}(3.9$ at. ppm He/dpa and 5.1 at. ppm H/dpa). The irradiated specimens were prepared for TEM examination using standard electropolishing techniques.

Experimental details, including specimen preparation, ORR test assembly and run detalls, irradiation parameters and electropolishing procedures can be found in Appendix A. TEM examinations were performed in a JEM $120 \mathrm{C}$ and a Hitach1 $1 \mathrm{MeV}$ HVEM. The JEM 120C was equipped with a special objective lens pole piece (AMG) for the observation of magnetic materials. Detalls concerning the microscopy and data analyses are in Appendix B.

TEM examinations of unirradiated specimens revealed a low dislocation density, $\rho$, of $10^{11} \mathrm{~m}^{-2}$. Obvious texture was apparent in the specimens. Many of the grains had a foil normal near [111]. As shown in Figure 3.1, the low-angle grain boundaries were resistant to electropolishing. This unusual effect was presumably due to impurity segregation at the boundaries.

Precipitates, believed to be $\mathrm{B}_{4} \mathrm{C}$, were also observed. These precipitates are quite important since $\sim 90 \%$ of the helium produced during the irradiation is from transmutation reactions involving boron (Appendix A). TEM studies have shown that damage halos or shells can form in association with boron-containing precipitates in neutronirradiated materials such as austenitic steels [75-79], nickel-base 


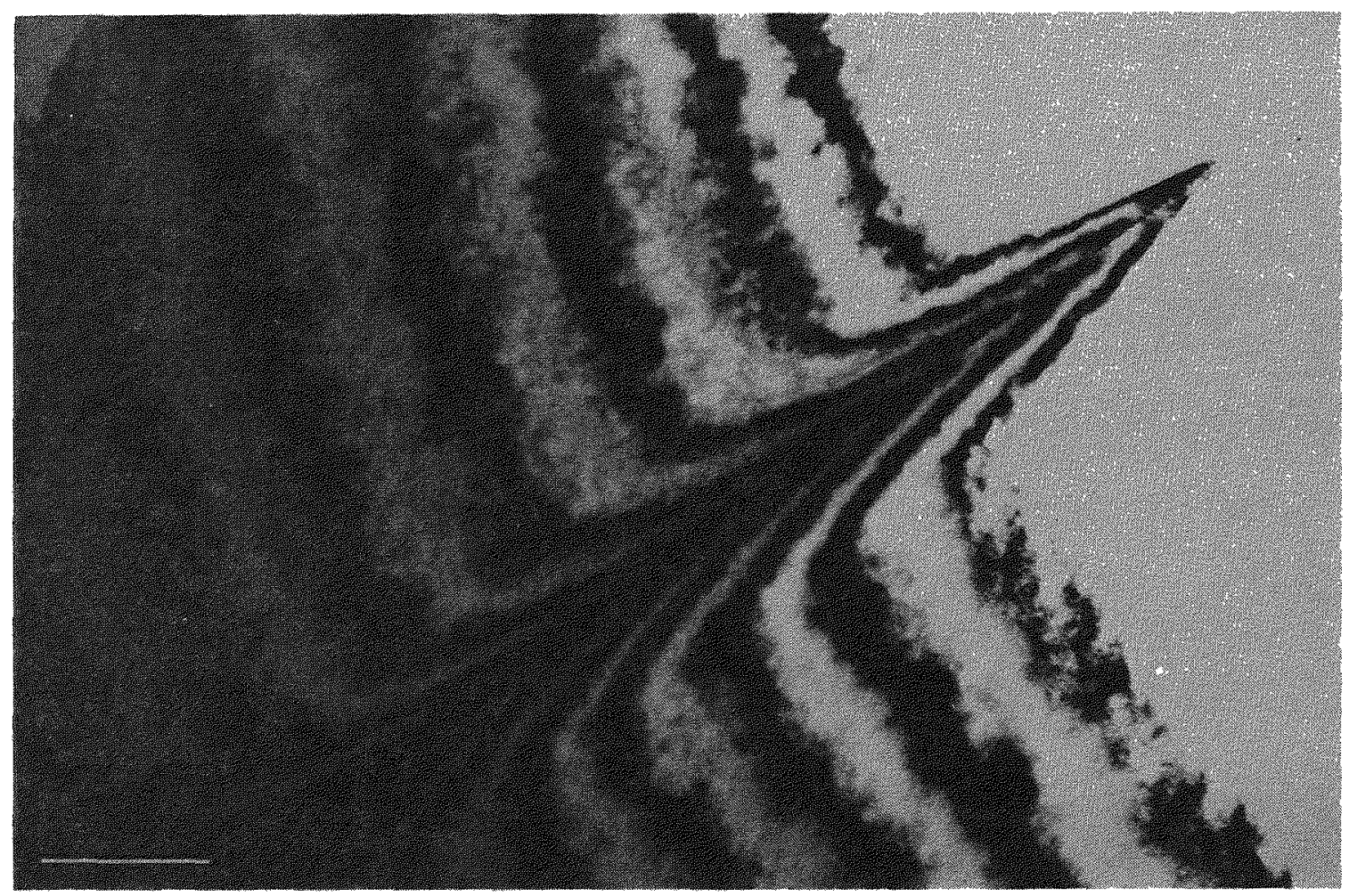

Figure 3.1. Micrograph of a low-angle grain boundary in an unirradiated iron specimen. Scale marker is $300 \mathrm{~mm}$.

alloys [78], copper [79], and vanadium [80-82]. Halos have also been found at inclusions of unknown composition in neutron-irradiated aluminum [83]. In all materials, these halos are duplex in nature, conslsting of two concentric rings of damage. In three dimensions, the halos are believed to be concentric spheres of damage surrounding the precipitate, except in vanadium. In vanadium, due to the morphology of the boron-containing precipitate, it has been suggested that the damage forms as concentric cylinders around elongated $V_{3} B_{2}$ precipitates [84]. Halos are believed to be the damage caused by the recoil products from elements, such as $10_{B}$, that undergo $(n, \alpha)$ transmutation reactions. For boron, this reaction is:

$$
{ }^{10}{ }_{B}+{ }^{1} n_{t h}+{ }^{4} \alpha(1.47 \mathrm{MeV})+{ }^{7} \mathrm{Li}(0.84 \mathrm{MeV}) .
$$

The range of $0.84 \mathrm{MeV}$ lithium in iron is $\sim 1.3 \mu \mathrm{m} \mathrm{[85].} \mathrm{The} \mathrm{range} \mathrm{of}$ $1.47 \mathrm{MeV} \alpha$ particles in iron is $\sim 2.4 \mu \mathrm{m}$ [86]. 
In the balance of this chapter, the results of the TEM study w111 be presented and discussed. Observations of the irradiation temperature dependence of the major defect structures - dislocations, cavities and halos - are presented separately.

\subsection{Irradiation-Induced Dislocation Microstructures}

The dislocation microstructures which resulted from neutron irradiation can be classified as belonging to one of four groups corresponding to four irradiation-temperature ranges. Within any one group, the structures were qualitatively similar. Each temperature range and its corresponding microstructures are presented separately.

Low Irradiation Temperatures: 455 to $523 \mathrm{~K}$ - At the three lowest irradiation temperatures of 455,493 , and $523 \mathrm{~K}$, the dislocation component of the microstructure was primarily limited to small defect clusters formed near preirradiation dislocation segments. A representative micrograph of the structure at each temperature is shown in Figure 3.2. The black-white contrast exhibited by the small defects can be seen in the dark-field micrograph in Figure 3.3.

At $455 \mathrm{~K}$, a fairly homogeneous background of "black-spot" defects less than $6 \mathrm{~nm}$ in diameter was also observed. The concentration of these defects was $\sim 10^{22} \mathrm{~m}^{-3}$. As shown in Figure 3.4, these defects also exhibited black-white contrast.

Based on the assumption that these small defects were small dislocation loops, a Burgers vector determination was attempted using the black-white image contrast of the defect. However, due to the presence of an unavoidable surface oxide and the usual problems associated with microscopy of magnetic specimens, high resolution images of the defects at the required diffraction conditions could not be obtained.

Preferential clustering of defects at dislocation lines has also been observed in neutron-irradiated copper $[87,88]$. In the study by Scheidler et al. [87], the degree of defect dislocation line interaction was found to be strongly dependent on the irradiation temperature in low fluence $\left(\sim 10^{22}\right.$ neutrons $\left.\mathrm{m}^{-2}\right)$, neutron-irradiated copper. At 4 and $77 \mathrm{~K}$, there was a fairly homogeneous distribution of small defects 
$455 \mathrm{~K}$

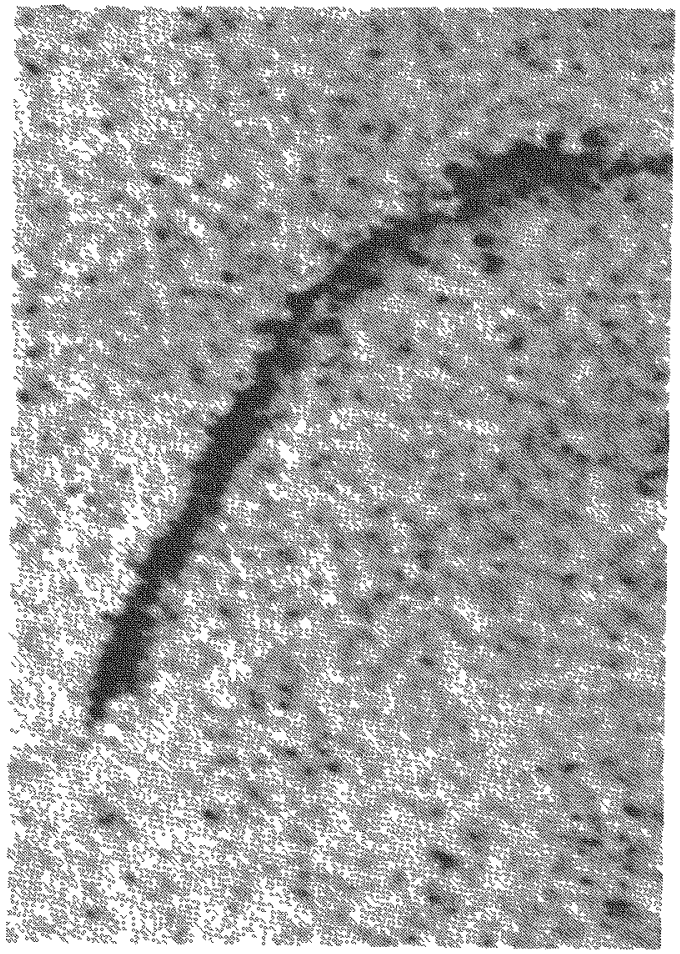

$493 \mathrm{~K}$

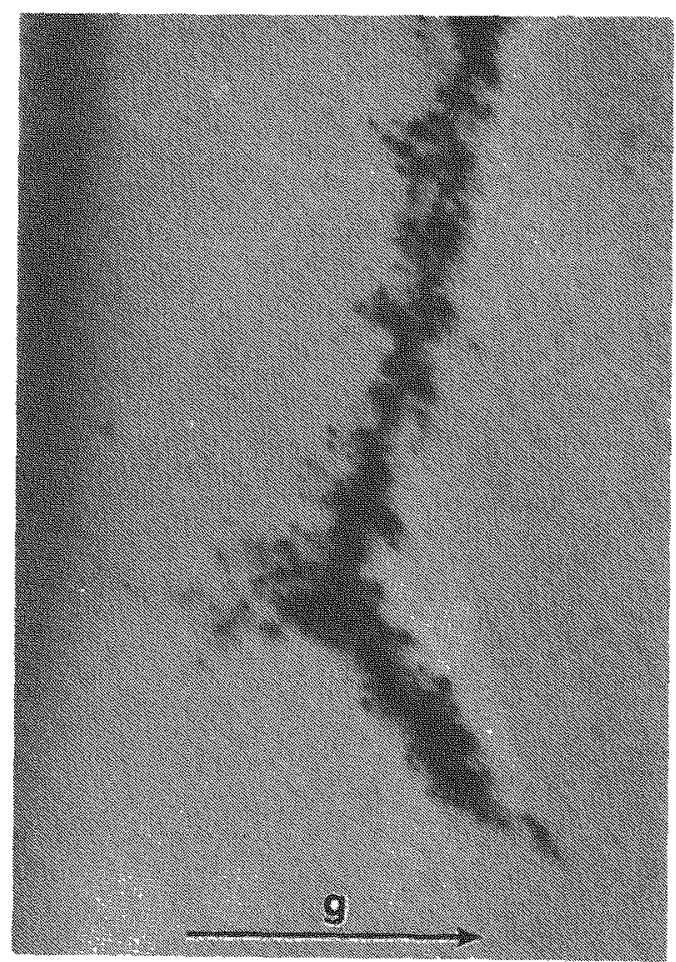

$523 \mathrm{~K}$

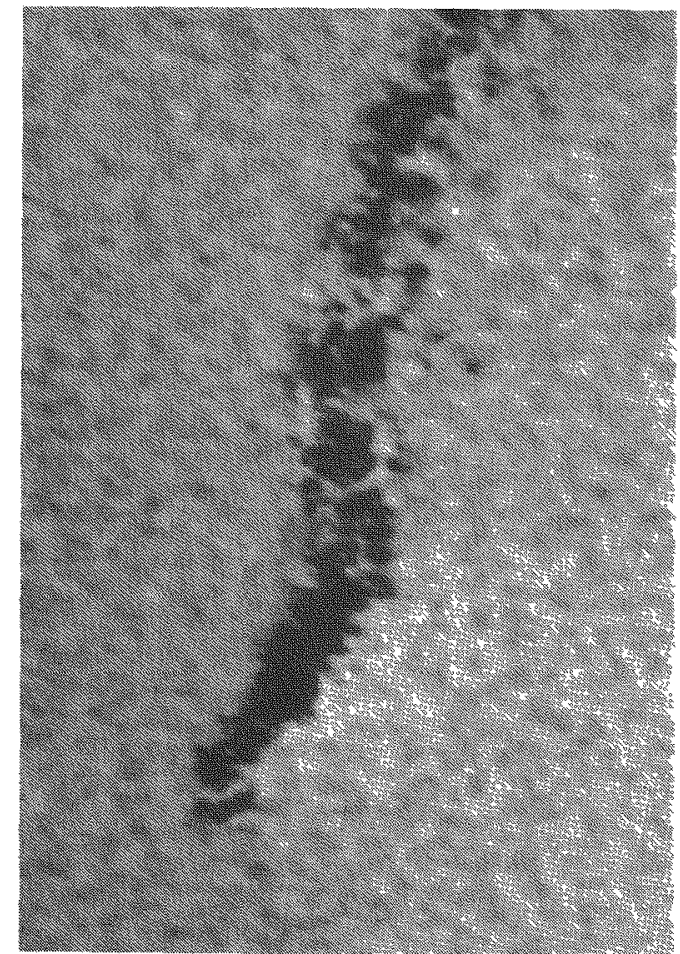

Figure 3.2. Representative micrographs of decorated dislocation segments found in specimens irradiated at 455,493 , and $523 \mathrm{~K}$. Length of arrow is $300 \mathrm{~nm} \cdot \mathrm{g}=\langle 110\rangle$. $z$ near [111]. 


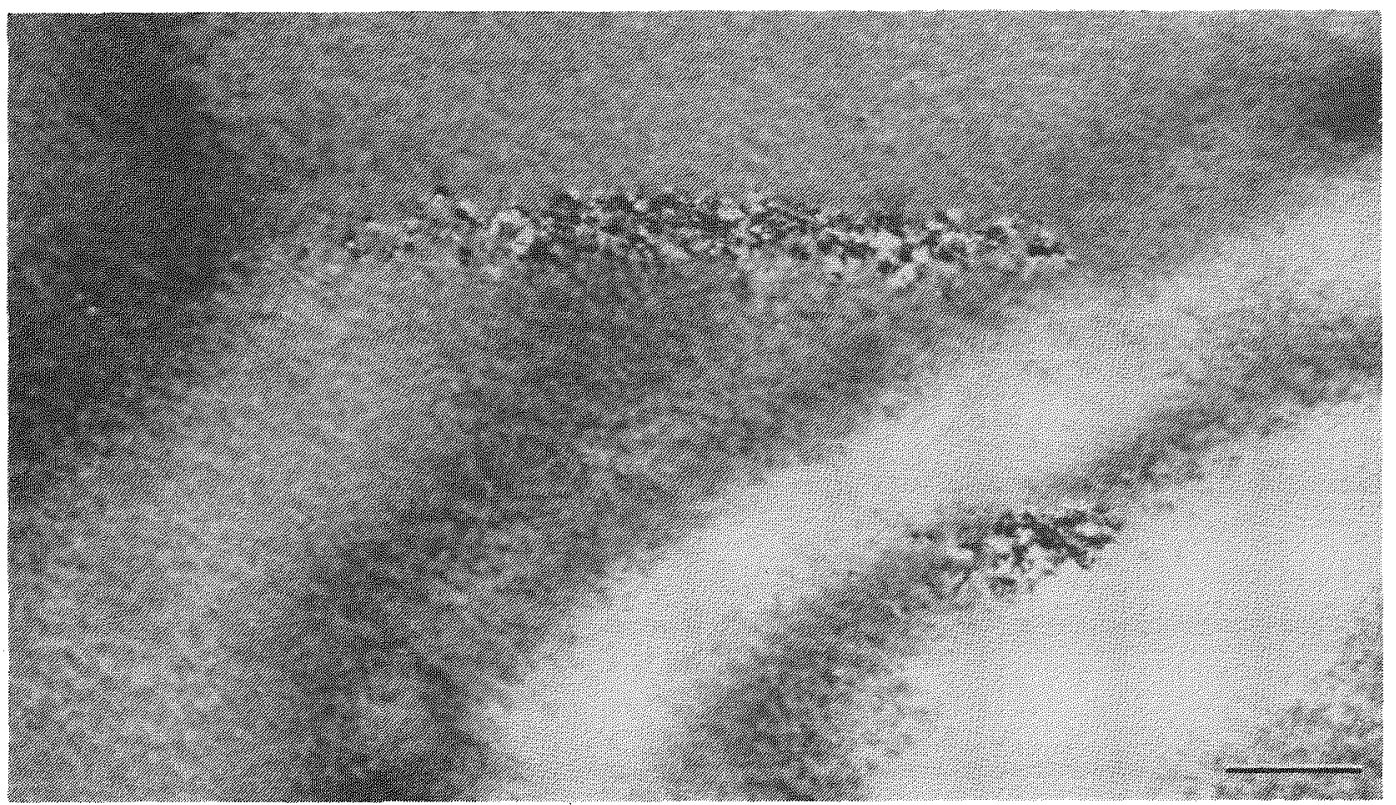

Figure 3.3. Dark field micrograph using a $\langle\overline{1} \overline{0}\rangle$ reflection of the "black-white" contrast exhibited by the small defects decorating the preirradiation dislocations. Scale marker is $200 \mathrm{~nm} \cdot \mathrm{T}_{\mathrm{I}}=523 \mathrm{~K} . \underline{z}$ near [111].

$\mathrm{YE}-12387$

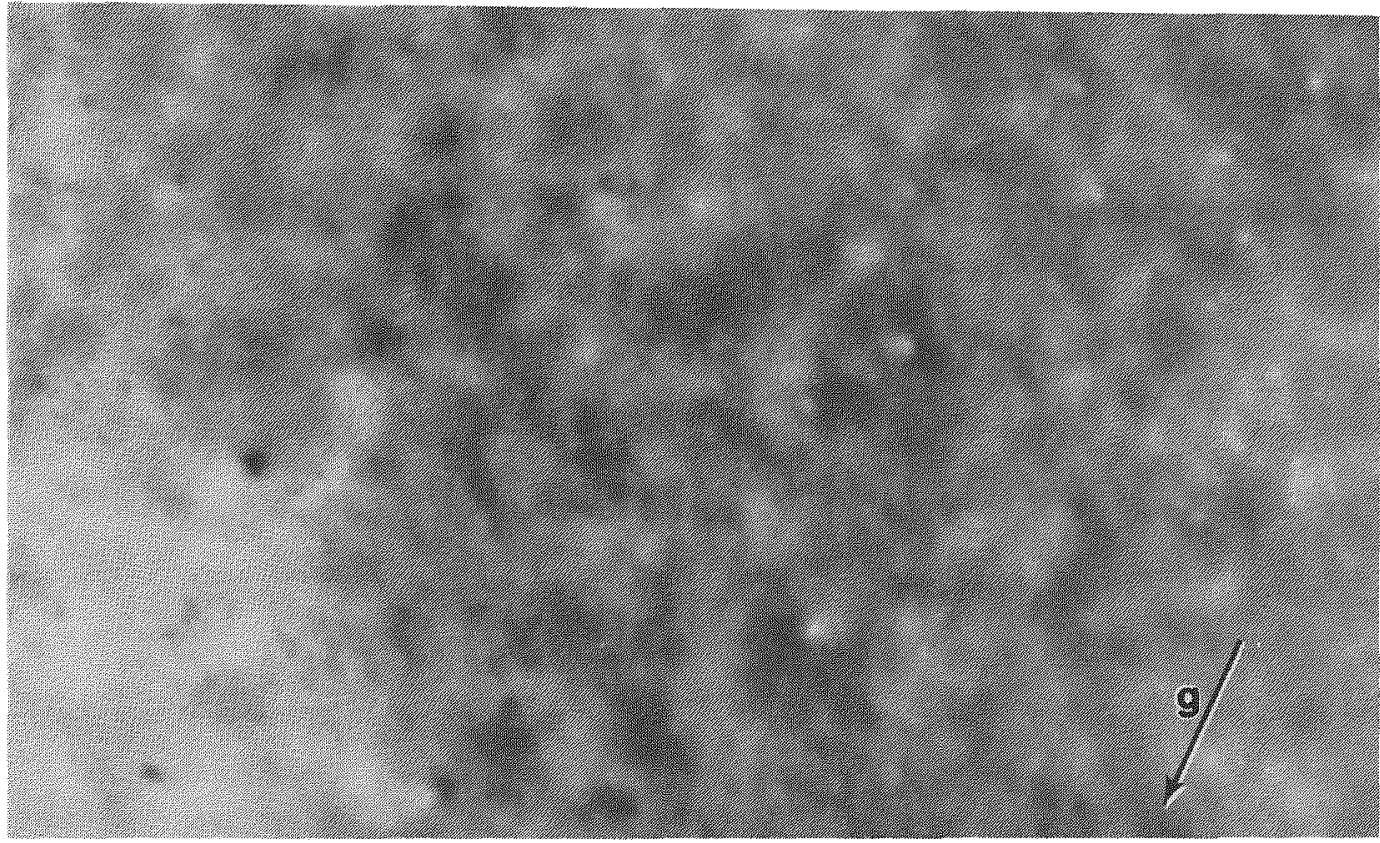

Figure 3.4. Micrograph of the "background" defects found in specimens irradiated at $455 \mathrm{~K}$. Note the "black-white" contrast exhibited by the defects. Length of arrow is $50 \mathrm{~nm}, \underline{g}=\langle 0 \overrightarrow{1} 1\rangle . \underline{z}$ near $[122] . w=0$. 
with little evidence of defect clustering, while at $293 \mathrm{~K}(0.21 \mathrm{~T})$ and $378 \mathrm{~K}(0.28 \mathrm{~T})$ decorated dislocation lines were a prominent microstructural feature. Hulett et al. [88] have also observed decorated dislocations in copper irradiated at $\sim 675 \mathrm{~K}(0.5 \mathrm{~T})$ to a low neutron fluence of $\sim 10^{22}$ neutrons $\mathrm{m}^{-2}$.

The nucleation of defect clusters at dislocations is believed to be promoted by the strain field of the dislocation [88]. The presence of Cottrell atmospheres at the dislocations could further enhance cluster formation by trapping interstitials and preventing their absorption by the dislocations. Once a cluster is formed, its strain field could promote further cluster formation nearby [88]. With increasing irradiation temperature, the interstitials can migrate more easily, enhancing cluster formation near the dislocations. Clustering at the dislocations would be significantly reduced when the irradiation temperature exceeded the temperature at which the Cottrel1 atmospheres are no longer efficient interstitial traps.

The above explanation for decorated dislocations is consistent with the current observations for iron. Cottrell atmospheres formation at dislocations is a well-known phenomenon in iron. The PortevinLechatelier effect often observed in stress-strain curves for iron is a result of successive aging and yielding caused by dislocation dragging by and escaping from the cottrell atmospheres.

The background of "black-spot" damage at $455 \mathrm{~K}\left(0.25 \mathrm{~T}_{\mathrm{M}}\right)$ could be small vacancy loops formed by cascade collapse. An analysis of the loop nature is required to determine if this proposal is correct. At irradiation temperatures of 493 and $523 \mathrm{~K}\left(0.27\right.$ and $0.29 \mathrm{~T}_{\mathrm{M}}$, respectively), these black-spot defects were not observed, possibly due to increased vacancy mobility at the higher temperatures. In neutronirradiated molybdenum, Bentley et al. [89] have observed that the concentration of vacancy loops decreased rapidly with increasing irradiation temperature for the temperature range of 0.16 to $0.26 \mathrm{~T}_{\mathrm{M}}$, with few vacancy loops observed at higher temperatures. The high stacking fault energies in bcc materials is a barxier to loop formation from cascade collapse. Therefore, vacancy migration from the cascade centers occurs 
at relatively low temperatures. Void nuclei can also be formed, especially if gas is present to stabilize the vacancy clusters [90].

Low-Intermediate Irradiation Temperatures: 548 and $573 \mathrm{~K}$ - At 548 and $573 \mathrm{~K}$, clusters of small 10ops were observed. Microstructures typical of those observed at these temperatures are shown in Figure 3.5. The Individual loops making up each cluster can be seen in the $2 \times$-enlarged inset area. Individual clusters did not appear to be associated with either precipitate or dislocation segments. A higher density of clusters and individual dislocation loops was observed at low angle grain boundarles, as shown in Figure 3.6. Earlier examination by K. Farrell using a $200 \mathrm{kV}$ Hitachi TEM of thick regions of specimens irradiated at $548 \mathrm{~K}$ indicated that a higher density of clusters was also found at preirradiation dislocation segments [91].

The quantitative data for the clusters is shown in Table 3.1. Cluster and dislocation loop parameters (average loop diameter, $\mathrm{d}_{\mathrm{L}}$; average number of loops per cluster, $\mathrm{N}_{\mathrm{L}}$ /cluster; average cluster diameter, dcluster; cluster concentration, $\mathrm{C}_{c l}$ luster; and dislocation density, $\Lambda$ ) were difficult to measure and are intended only as approximate values. In particular, the dislocation density was measured in two ways to minimize errors due to the difficulties of distinguishing individual loops in the clusters and those errors due to the anisotropic distribution of loops. First, the density was measured using the standard techniques as outlined in Appendix B. Then the density was calculated using the measured cluster parameters that appear in Table 3.1 according to the expression:

$$
\begin{aligned}
& \Lambda=\text { (10op circumference) } \times \text { (number of loops/cluster) } \\
& \times \text { (cluster concentration) } \\
& \Lambda=\left(\pi d_{L}\right)\left(N_{L} / \text { cluster }\right)\left(C_{\text {cluster }}\right)
\end{aligned}
$$

The two values obtained for $\Lambda$ were about a factor of 2 different - fairly good agreement for this type of distribution. The value for $\Lambda$ in Table 3.1 is the average of these two values. 
$548 \mathrm{~K}$

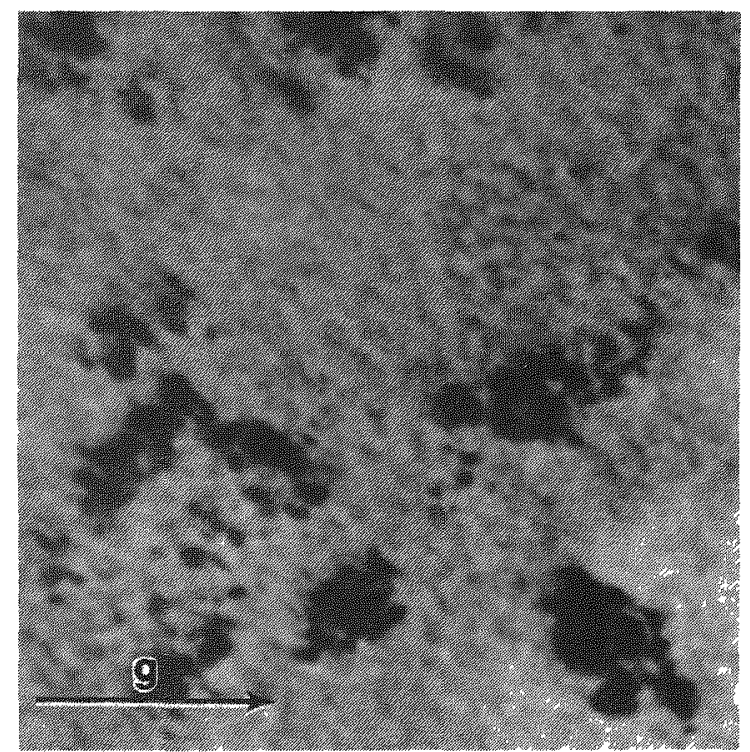

ORNL-Photo $3987-80$

$573 \mathrm{~K}$

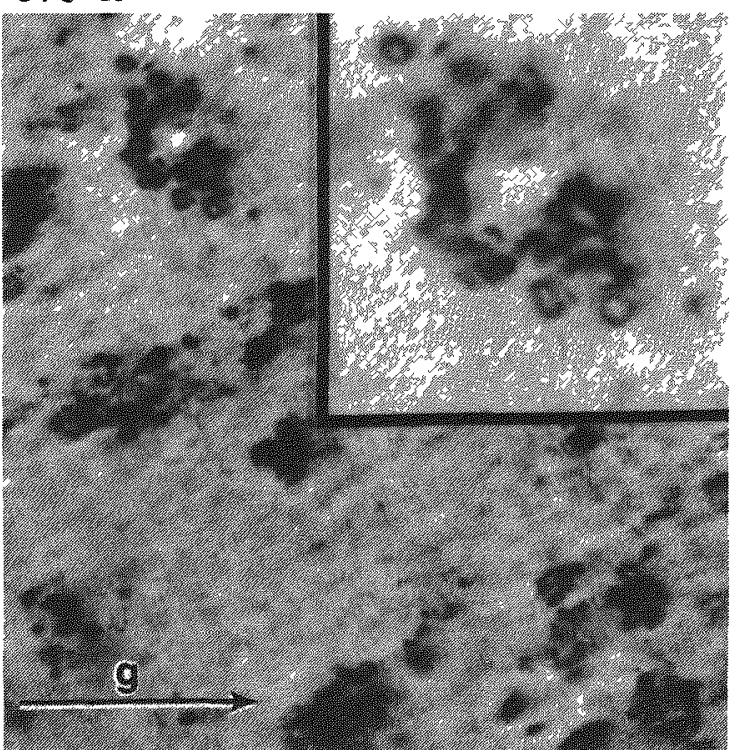

Figure 3.5. Representative micrographs of the raft-like clusters of small dislocation loops found in specimens irradiated at 548 and $573 \mathrm{~K}$. The inset enlargement $(2 x)$ of a single cluster shows the individual loops. Length of arrow is $200 \mathrm{~nm} \cdot \underline{\mathrm{g}}=\langle 1 \overline{1} 0\rangle, \underline{z}$ near [111].

$\mathrm{YE}-12388$

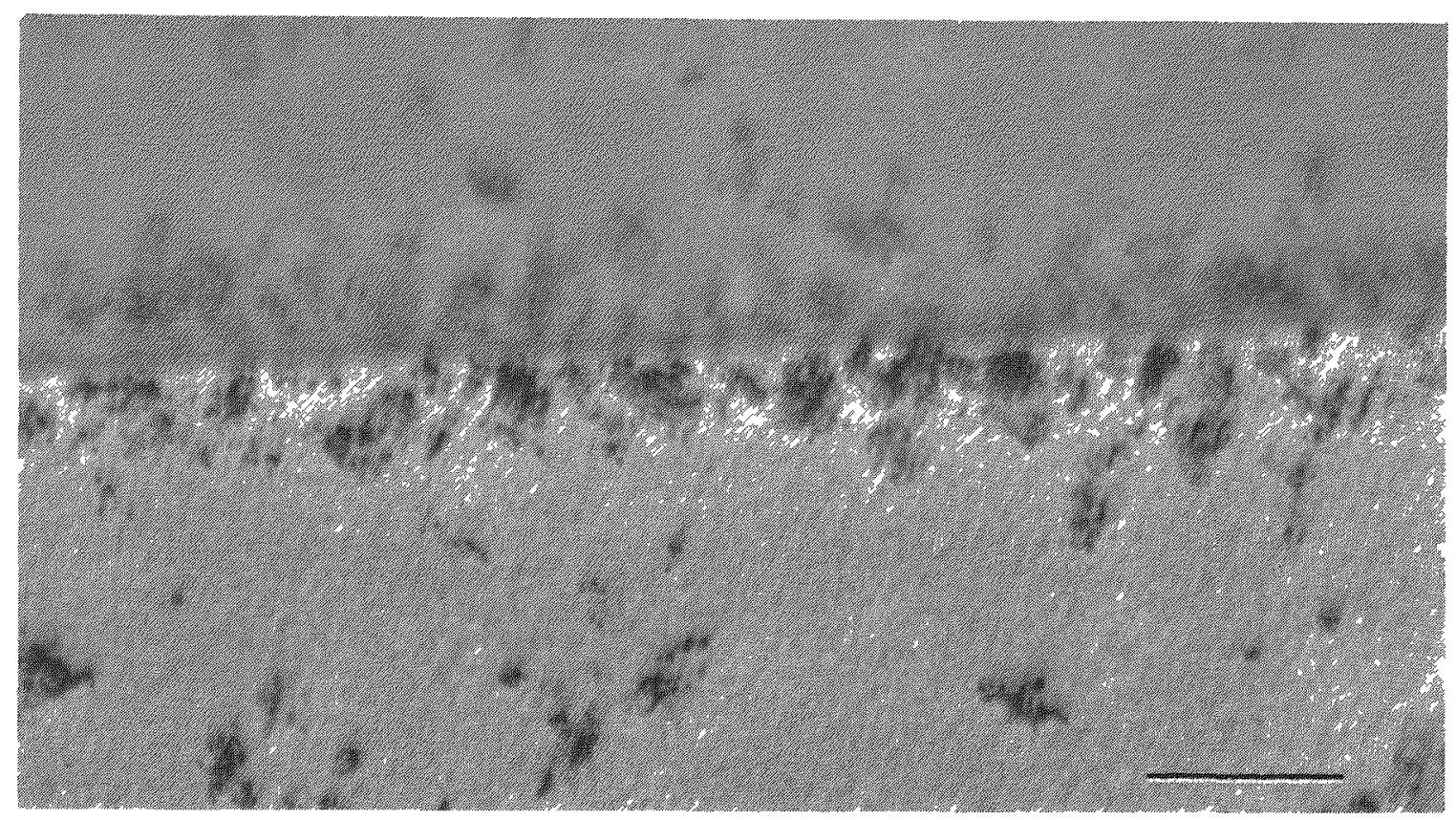

Figure 3.6. Micrograph of the enhanced cluster concentration found at the low angle grain boundaries. Scale marker is $300 \mathrm{~nm} \cdot \mathrm{T}_{\mathrm{I}}=548 \mathrm{~K}$. 
Table 3.1. Quantitative data for dislocation microstructures observed at 548 and $573 \mathrm{~K}$

\begin{tabular}{|c|c|c|c|c|c|c|}
\hline \multicolumn{2}{|c|}{ Temperature } & \multirow{2}{*}{$\underset{(n m)}{d_{L}}$} & \multirow{2}{*}{$\mathrm{N}_{L} /$ Cluster } & \multirow{2}{*}{$\begin{array}{c}\mathrm{d}_{\text {cluster }} \\
(\mathrm{nm})\end{array}$} & \multirow{2}{*}{$\begin{array}{c}C_{\text {cluster }} \\
\left(\mathrm{m}^{-3}\right)\end{array}$} & \multirow{2}{*}{$\begin{array}{c}\Lambda \\
\left(\mathrm{m}^{-2}\right)\end{array}$} \\
\hline$(\mathrm{K})$ & $\left({ }^{\circ} \mathrm{C}\right)$ & & & & & \\
\hline 548 & 275 & $\sim 10$ & $\sim 20$ & 150 & $9 \times 10^{19}$ & $9 \times 10^{13}$ \\
\hline 573 & 300 & $\sim 10$ & $\sim 15$ & 100 & $1.5 \times 10^{19}$ & $1.3 \times 10^{14}$ \\
\hline
\end{tabular}

The general appearance of these clusters is similar to the "rafts" of small dislocation loops and black-spot clusters observed in other neutron-irradiated bcc materials such as molybdenum $[89,92,93]$, TZM [89,92,94,95], vanadium [91], and tungsten [96]. For the studies of molybdenum, TZM, and tungsten, detailed analyses of raft geometry and loop characteristics have been reported. In these materials, rafts form on $\{111\}$ planes. The thicknesses of the rafts are $\sim 10-20 \mathrm{~nm}$. A11 of the loops within a raft have the same a/2<111> Burgers vector [92]. The loops are assumed to be interstitial in nature. The raft-like clusters in iron differ from these rafts in several aspects, as discussed below.

While a complete analysis of the loops in the clusters in iron was not possible due to the high loop concentration, a partial analysis, following the procedures outlined in Appendix $B$, was performed. The series of micrographs in Figure 3.7 is part of this analysis. Close inspection of the micrographs in Figure $3.7(a-c)$, with $\underline{g}=\langle 110\rangle$ and $\underline{z}$ near [111], shows that the individual clusters are made up of loops with different Burgers vectors. For a given $\langle 110\rangle$ diffracting vector, few clusters entirely exhibit a residual image. In Figure 3.7(d), with diffracting conditions of $\underline{g}=[110]$ and $\underline{z}$ near [001], many of the individual loops appear to be in a near edge-on orientation on (100) and (010) planes. Based on this observation, it can assumed that many of the loops are nearly edge dislocation loops with $\underline{b}=\langle 100\rangle$. In Figure $3.7(e, f)$ with diffracting conditions of $\underline{g}=[020]$ and $\underline{g}=[0 \overline{2} 0]$ with $z$ near [101], the character of a few of the loops with $\underline{b}=[010]$ can be determined. The circled loops exhibit "inside" contrast for $\underline{g}=[0 \overline{2} 0]$ and "outside" contrast for $\underline{g}=[020]$, indicating that these loops are interstitial in nature. Another conclusion, based on the observed 

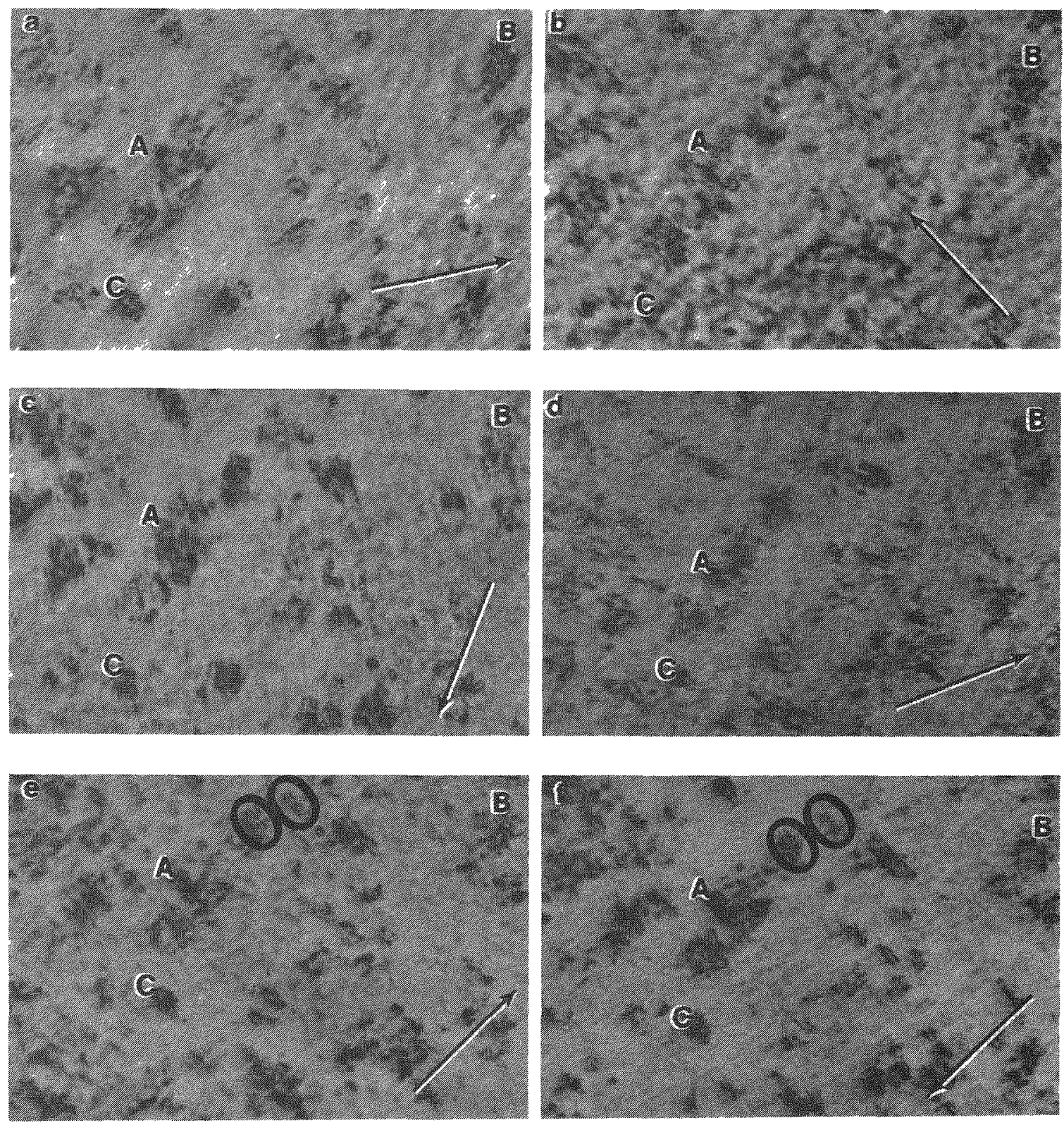

Figure 3.7. Partial analysis of the dislocation loops in the raftlike clusters. The same clusters are labeled in each micrograph. $\mathrm{T}_{\mathrm{I}}$ $=573 \mathrm{~K}$. Length of the arrows is $300 \mathrm{~nm}$. (a) $\mathrm{g}=[\overline{1} 10], \underline{z}$ near $[1 \overline{1} 1]$, (b) $\mathrm{g}=[10 I], \underline{z}$ near $[111],(\mathrm{c}) \mathrm{g}=[011], \underline{z}$ near $[111], \quad$ (d) $\underline{g}=$ [i10], $z$ near [001], (e) circled loops show "outside contrast," g $=[020], z$ near $[101]$, (f) circled loops show "Inside contrast," $\underline{\mathrm{g}}$ $=[0 \overline{2} 0], z$ near [101]. Arrows denote direction of $g$. 
variation in the cluster shape during high-angle tilting experiments, was that the clusters of loops in iron are approximately threedimensionally equiaxed and are certainly not planar arrays.

Rafts are believed to form by dislocation loops with the same Burgers vectors gliding together as a result of the elastlc interactions between the loops $[89,90,93]$. A similar interaction which is not limited to loops of the same Burgers vector could be responsible for the cluster formation in iron. However, loops with $\underline{b}=a\langle 100\rangle$, as were observed in the clusters in iron, are believed to require a higher glide stress for motion than loops with $\underline{b}=a / 2\langle 111\rangle$. Therefore, $a$ more complex mechanism may be responsible for the cluster formation in iron. An alternative explanation for cluster formation is outlined below.

At elevated temperatures, it is believed that both a 100$\rangle$ and a/2<111〉 interstitial loops are formed in irradiated bcc metals [71]. The probability for a $\langle 100\rangle$ loop nucleation is higher for iron than for many other bcc materials, such as molybdenum [46,47]. Possibly, during neutron-irradiation of iron, a/2<111> loops formed early in the irradiation glide together as proposed for raft formation. Loops with $\underline{b}$ $=a\langle 100\rangle$ which formed in the vicinity of these a/2<111> loop clusters could grow more quickly than those away from the clusters due to the greater influx of interstitials toward the clusters. Also, the $a\langle 100\rangle$ $(|b|=a)$ loops would grow faster than the $a / 2\langle 111\rangle(|b|=\sqrt{3} a / 2)$ loops since, due to the larger magnitude of the Burgers vector, the a<100> 10ops have a larger bias for interstitials [46]. By this process clusters of both $a\langle 100\rangle$ and $a / 2\langle 111\rangle$ loops would be formed. The majority of the observable, well-defined loops in the clusters would be the larger a<100>10ops. In addition, a few loops would also be expected to grow to observable dimensions between the clusters, as observed.

There is insufficient data available to more clearly define the exact mechanism of the cluster formation. Either of the above mechanisms seems plausible, although both are speculative in nature. Regardless of the formation mechanism, enhanced cluster concentrations at the grain boundaries and at preirradiation dislocation segments are presumably related to impurity segregation at these locations. 
Intermediate Irradiation Temperatures: $\underline{623}$ to $\underline{773} \mathrm{~K}-$ At $623-$ $773 \mathrm{~K}$, a fairly homogeneous distribution of loops and network segments is present. Representative areas from specimens irradiated at 623, 673,723 , and $773 \mathrm{~K}$ are shown in Figure 3.8. The extremely coarse distribution at $773 \mathrm{~K}$ required examination in an HVEM to obtain a true impression of the microstructure [Figure 3.8(d)]. As shown in this figure, many loops intersect the specimen surfaces. At 723 and $773 \mathrm{~K}$, unidentified precipitates were present in the center of most loops. This can be seen in the HVEM micrographs of the specimen irradiated at $773 \mathrm{~K}$, Figure 3.8(d) and, more clear1y, in Figure 3.9.

The measured quantitative data for the loop structures are found in Table 3.2 and Figure 3.10. The average loop diameter, $\mathrm{d}_{\mathrm{L}}$, increases and the loop concentration, $C_{L}$, decreases with increasing temperature. Also, the dislocation density (including loops) decreases by about an order of magnitude for each $50^{\circ}$ temperature increase. (There are two methods of measuring the dislocation density, indicated by $\rho$ and $\Lambda$, where $\Lambda=2 \rho$. For details, as well as a discussion of the error calculations, see Appendix B).

Loop analyses - The highest concentration of dislocation loops was present at the $623 \mathrm{~K}$ irradiation temperature. Analyses of the geometry and nature of the loops were performed following the techniques of Maher and Eyre [97] as outlined in Appendix B. A part of the analysis of the 1oop geometry is illustrated in Figure 3.11. In the micrographs in this figure, the loops that were analyzed are labeled alphabetically. In Figure $3.11(a),(b)$, and (c), micrographs taken with the three $\langle 110\rangle$ diffracting vectors near the [111] pole are shown. The invisibilities indicated by this sequence cannot distinguish $\underline{b}=a\langle 100\rangle$ and $\underline{b}=$ $a / 2\langle 111\rangle$. A survey of more diffracting vectors is required. Figure $3.11(\mathrm{~d})$ and (e) are micrographs taken with $\mathrm{g}=[\overline{1} 01]$ near the [010] pole and $g=[1 \overline{1} 0]$ near the [001] pole, respectively. These micrographs show the loops in a near edge-on loop orientation with the loop image aligned perpendicular to the $\langle 100\rangle$ directions. This observation indicates that these loops are nearly edge dislocation loops lying on $\langle 100\rangle$ planes and have predominantly a<100> Burgers vectors. This conclusion was substantiated by micrographs taken with $\underline{\mathrm{g}}=[200]$, 
$623 \mathrm{~K}$
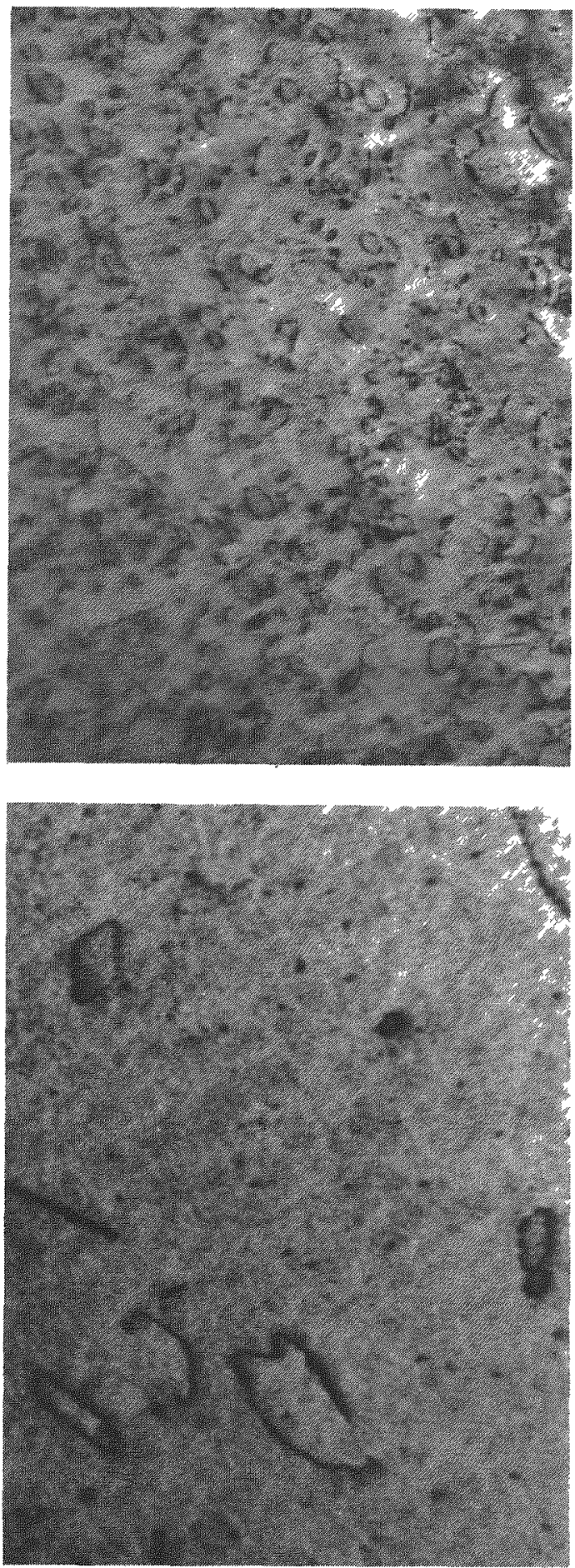

$723 \mathrm{~K}$
$673 \mathrm{~K}$

$\mathrm{Y}-185236$
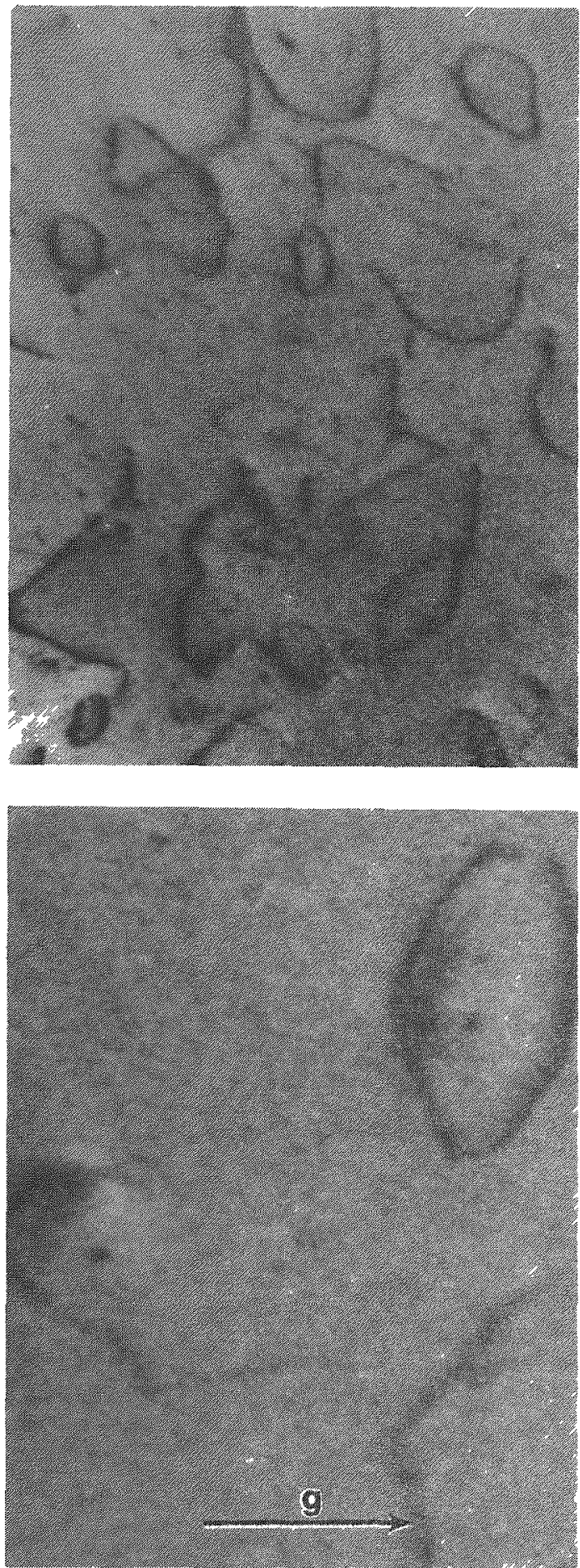

$773 \mathrm{~K}$

Figure 3.8. Representative micrographs of the dislocation loop structure observed in specimens irradiated at 623 to $773 \mathrm{~K}$. Length of arrow is $500 \mathrm{~nm} \cdot \underline{\mathrm{g}}=\langle 110\rangle$. $\underline{\mathrm{z}}$ near $\langle 111\rangle$. 
YE-12389

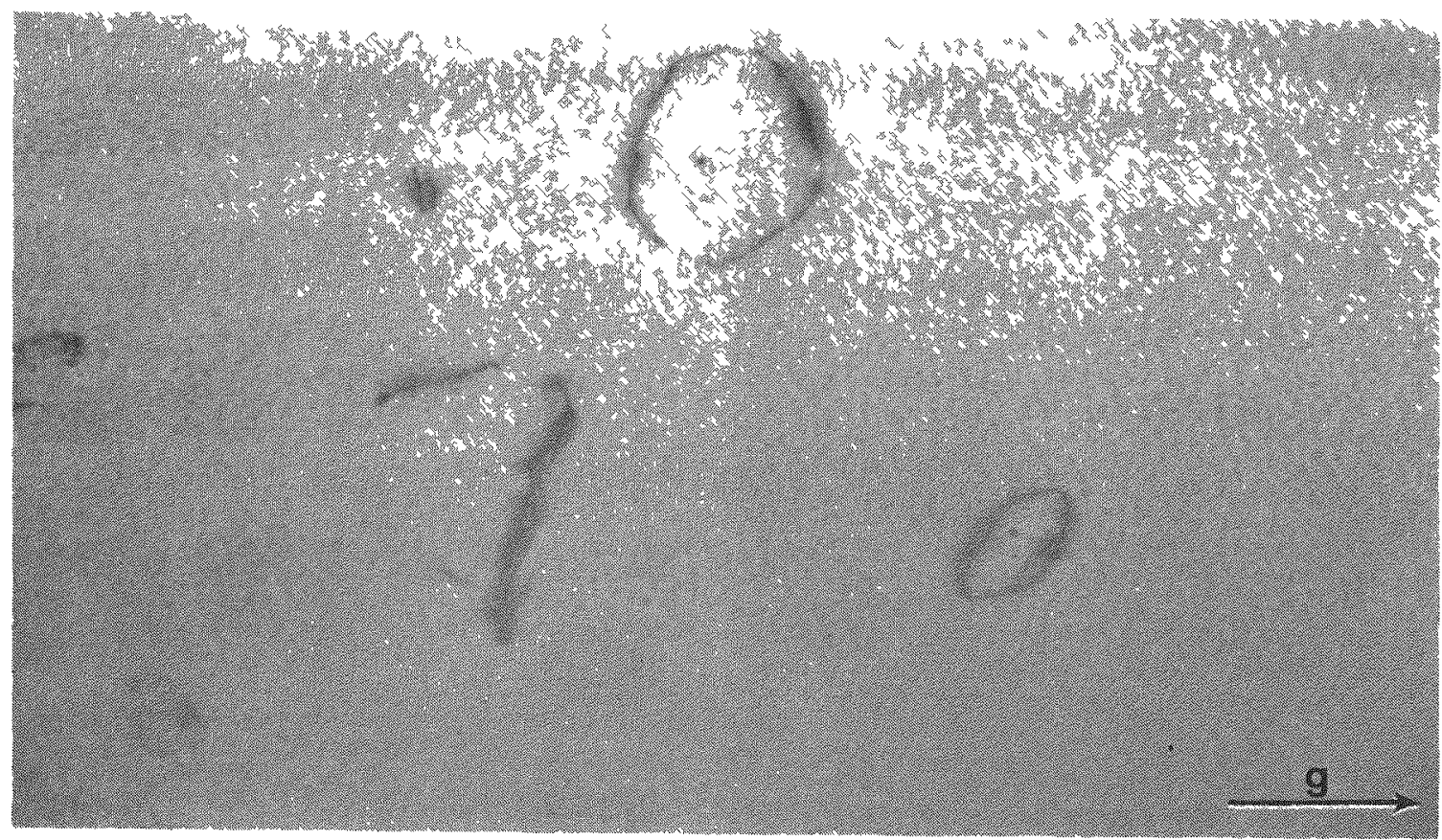

Figure 3.9. HVEM micrograph of precipitates associated with dislocation loops. $\mathrm{T}_{\mathrm{I}}=773 \mathrm{~K}$. Length of arrow is $1 \mu \mathrm{m} \cdot \mathrm{g}=\langle 110\rangle$.

[020], and [002]. In these micrographs, the dislocation 1oop images were resldual for diffracting conditions appropriate for a $\langle 100\rangle$ Burgers vector - e.g., for $\underline{g}=[200]$, loops with $\underline{b}=a[010]$ exhibited residual contrast.

The nature of the loops is determined using $g^{\circ} b= \pm 2$ diffracting conditions as shown by the micrographs of Figure 3.12. The loops are labeled as in Figure 3.11. Figures $3.12(\mathrm{a})$ and $3.12(\mathrm{~b})$, with $\mathrm{g}=$ [1̄i2] and [112], yield the "inside-outside" contrast for loops with $\underline{b}=$ a[001]. Similarly, Figures $3.12(\mathrm{c})$ and (d) determine the nature of loops with $\underline{b}=a[010]$ and $3.12(e)$ and $(f)$ for loops with $\underline{b}=a[100]$. Al1 loops were of the interstitial type.

A summary of these results is presented in Table 3.3 , where the letters refer to the loops labeled in Figures 3.11 and 3.12 . Only one 10op, that labeled "A," was determined not to have a Burgers vector of $a\langle 100\rangle$; it had $\underline{b}=a / 2[111]$ and was interstitial in nature (inside contrast for $g=[2 \overline{1} \overline{1}]$, outside contrast for $g=[\overline{2} 11])$. Since the $100 \mathrm{p}$ 


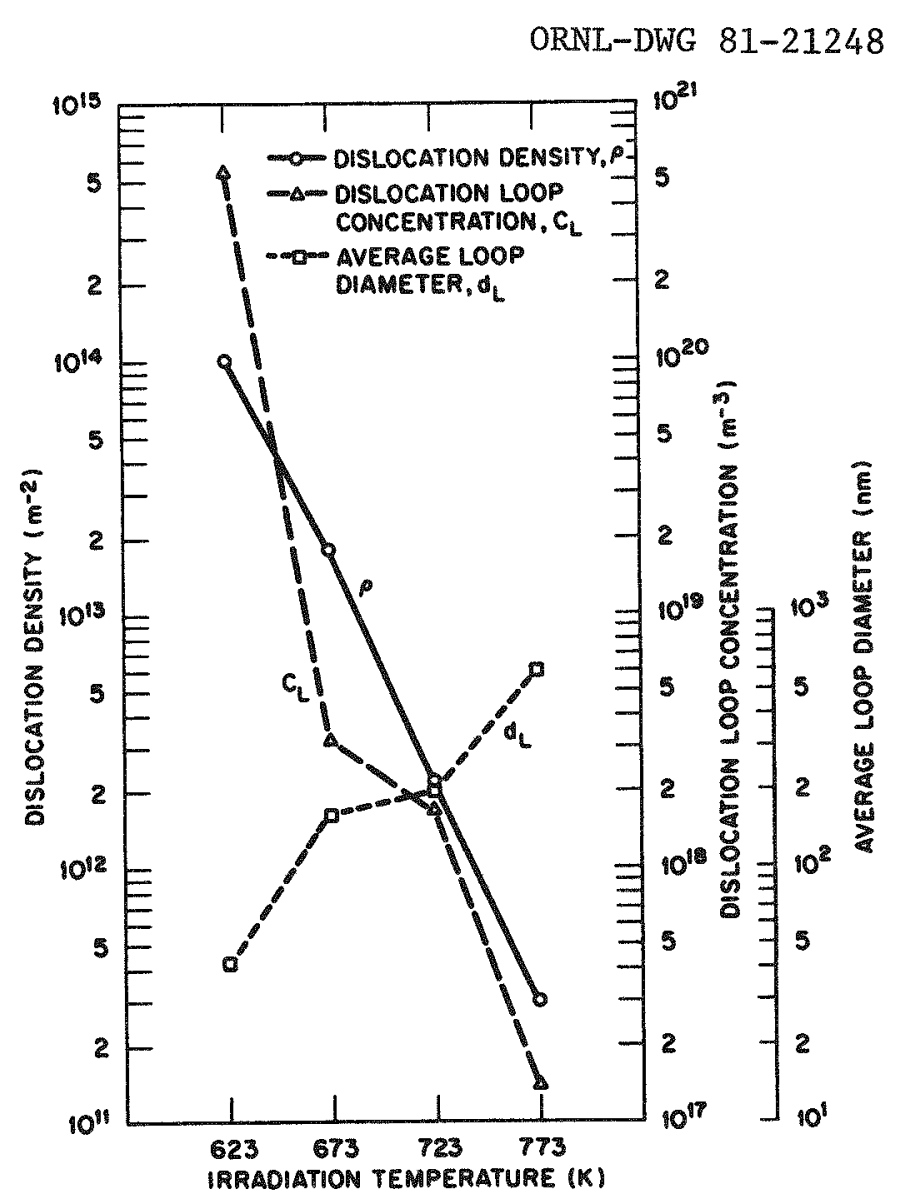

Figure 3.10. Dislocation loop parameters and total dislocation density for neutron-irradiated iron for intermediate irradiation temperatures.

Table 3.2. Dislocation density and loop parameters

\begin{tabular}{|c|c|c|c|c|c|c|c|c|c|}
\hline \multicolumn{2}{|c|}{ Temperature } & \multirow{2}{*}{$\begin{array}{c}C_{\mathrm{L}} \\
\left(\mathrm{m}^{-3}\right)\end{array}$} & \multirow{2}{*}{$\begin{array}{c}\mathrm{d}_{\mathrm{L}_{s}} \\
(\mathrm{~nm})\end{array}$} & \multicolumn{3}{|c|}{$2 p$} & \multicolumn{3}{|c|}{$\Lambda$} \\
\hline ( $\mathrm{K})$ & & & & & $\left.m^{-2}\right)$ & $\begin{array}{c}\text { Error } \\
(\%)\end{array}$ & & -2) & $\begin{array}{l}\text { eror } \\
\%)\end{array}$ \\
\hline 623 & 350 & $5.4 \times 10^{20}$ & 43 & & - & -- & $1 \times$ & $10^{14}$ & 7 \\
\hline 673 & 400 & $3.2 \times 10^{18}$ & 160 & 1.8 & $\times 10^{13}$ & 13 & 1.8 & $\times 10^{13}$ & 10 \\
\hline 723 & 450 & $1.7 \times 10^{18}$ & 200 & 2.2 & $\times 10^{12}$ & 10 & & - & -- \\
\hline 773 & 500 & $1.4 \times 10^{17}$ & 600 & 3.0 & $\times 10^{11}$ & 14 & & - & - \\
\hline
\end{tabular}



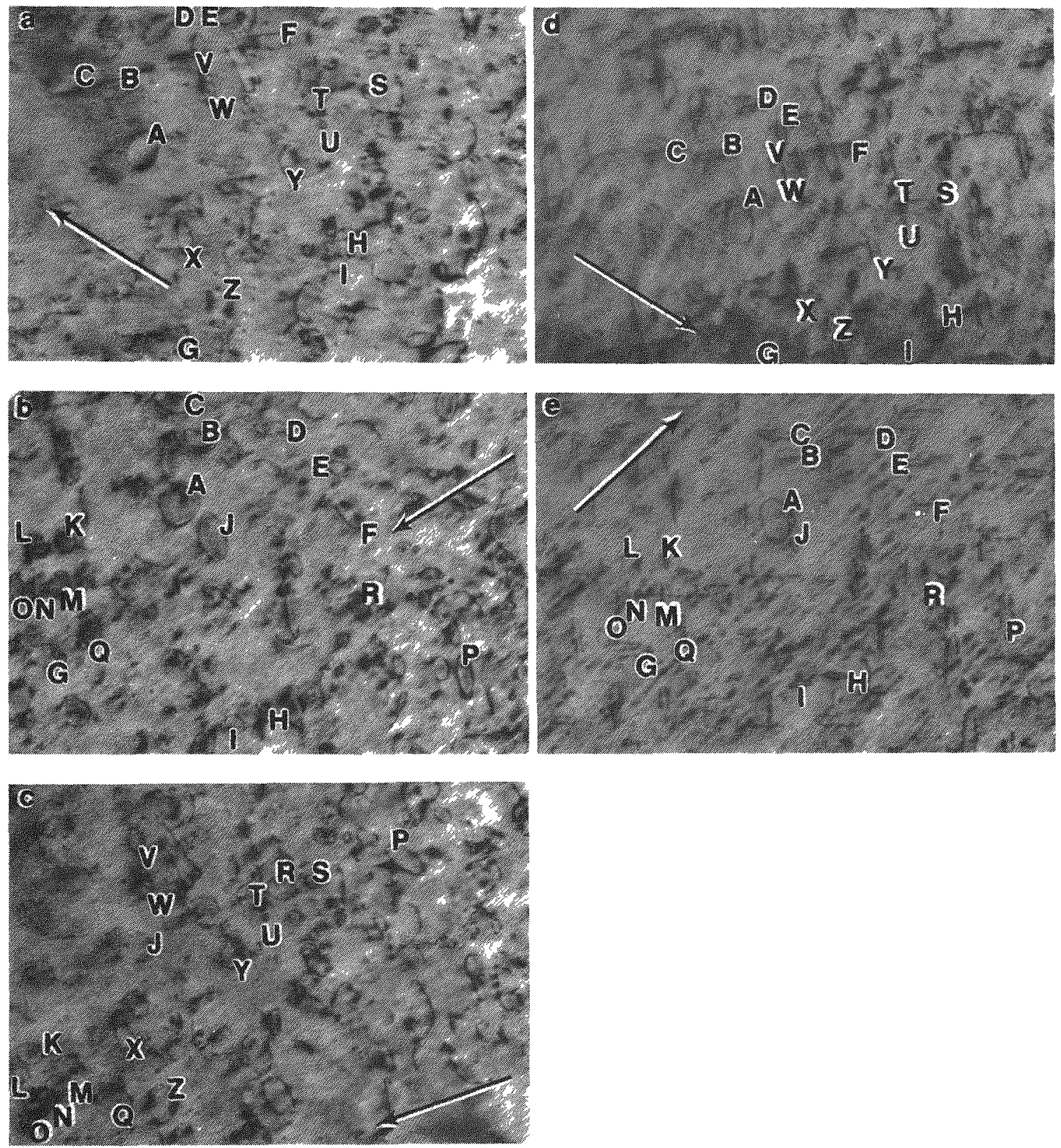

Figure 3.11. Micrographs comprising the Burgers vector determination aspect of dislocation loop analysis. Individual loops are labeled alphabetical1y. $T_{I}=623 \mathrm{~K}$. Length of arrows is $300 \mathrm{~mm}$.
(a) $\underline{g}=[101], \underline{z}$ near $[111]$,
(c) $\frac{g}{g}=[011], \frac{\bar{z}}{\mathrm{~g}}$ near $[111]$,
(e) $\frac{g}{g}=[1 \overline{10}, \underline{z}$ near $[001]$.
(b) $g=[110], z$ near [111]
(d) $\underline{g}=[\overline{101}], \underline{z}$ near [010]
Arrows denote direction of $\mathrm{g}$. 
$\mathrm{Y}-185238$
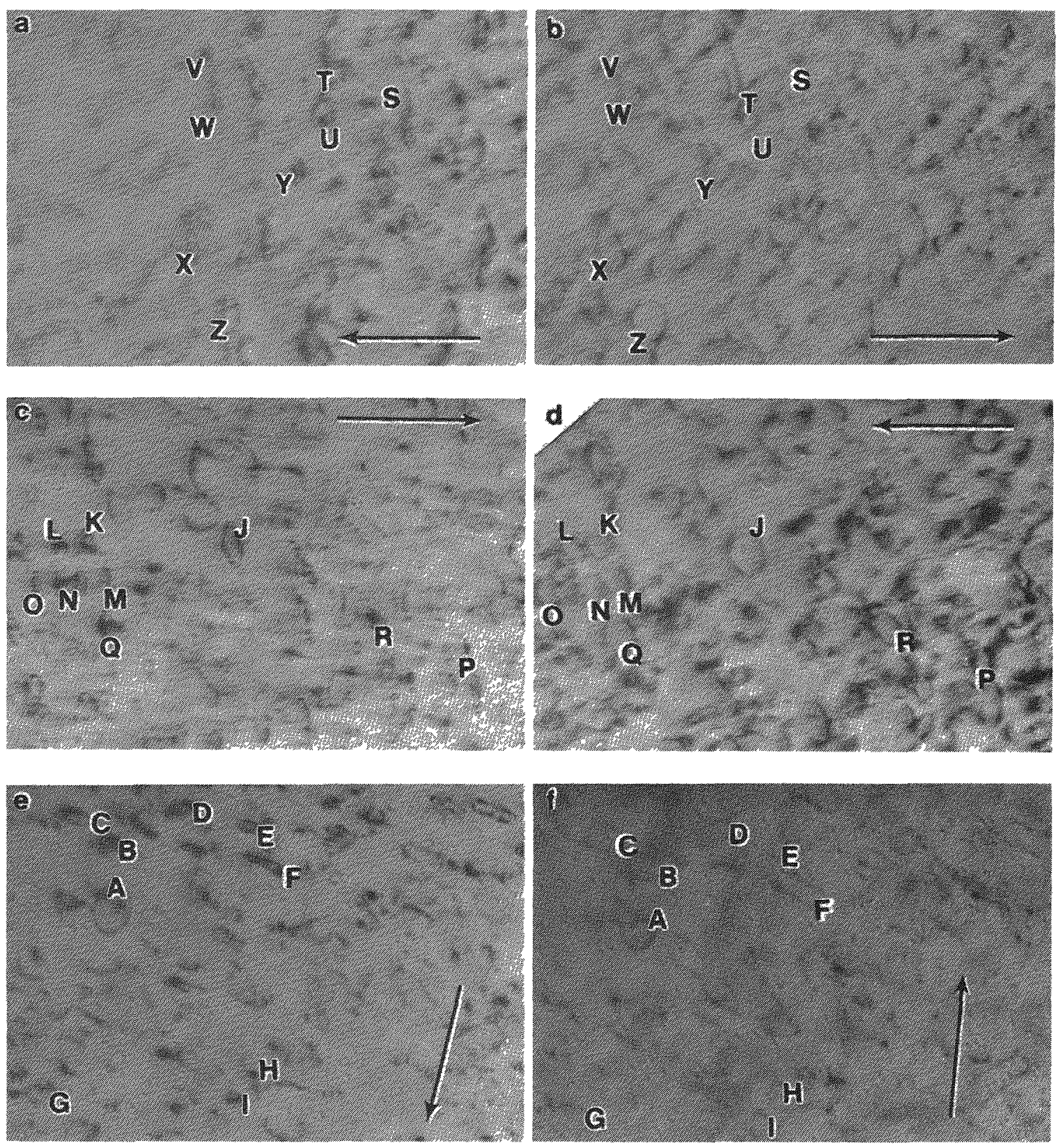

Figure 3.12. Nature determination for the same loops shown in Figure 3.10. $a, c, e$ show "Inside contrast." $b, d, f$ show "outside contrast." Length of arrows is $300 \mathrm{~nm}, z$ near [111].

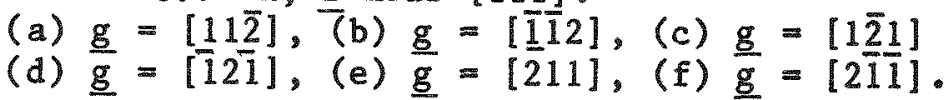
Arrows denote direction of $\mathrm{g}$. 
Table 3.3. Summary of the dislocation loop analysis shown in Figures 3.11 and 3.12

\begin{tabular}{|c|c|c|c|c|c|}
\hline \multirow[b]{2}{*}{ Loop } & \multirow[b]{2}{*}{$\begin{array}{l}\text { g Yielding } \\
\text { Invisibility }\end{array}$} & \multirow[b]{2}{*}{$\underline{b}$} & \multicolumn{2}{|c|}{ g Yielding } & \multirow[b]{2}{*}{ Nature } \\
\hline & & & $\begin{array}{l}\text { Inside } \\
\text { Contrast }\end{array}$ & $\begin{array}{l}\text { Outside } \\
\text { Contrast }\end{array}$ & \\
\hline $\mathbb{A}$ & $-[01 \overline{1}],[0 \overline{1} 1]$ & $a / 2[\overline{1} 11]$ & [2피] & {$[\overline{2} 11]$} & Interstitial \\
\hline$B-I$ & $\begin{array}{l}{[01 \overline{1}],[0 \overline{1} 1],[020]} \\
{[020],[002],[002]}\end{array}$ & $a[100]$ & {$[\overline{2} 11]$} & {$[2 \overline{1}]$} & Interstitial \\
\hline$J-R$ & {$\left[\begin{array}{l}\overline{10} 1],[10 \overline{1}],[200] \\
{[\overline{200}],[002],[002]}\end{array}\right.$} & $a[010]$ & {$[1 \overline{2} 1]$} & {$[\overline{1} 2 \overline{1}]$} & Interstitial \\
\hline$S-z$ & {$\left[\frac{110],[\overline{110}],[200]}{[200],[020],[020]}\right.$} & $\mathrm{a}[001]$ & {$[11 \overline{2}]$} & [112] & Interstitial \\
\hline
\end{tabular}

normal was not determined, it is uncertain whether a safe orientation was used for this analysis. However, the possible geometries suggest that the loop normal is less than $90^{\circ}$ from the beam direction, as required for a safe orientation. of 53 loops analyzed for this specimen, all were interstitial and $34 \%$ had $\underline{b}=\underline{a}[010] ; 25 \%$ had $\underline{b}=$ $a[100] ; 39 \%$ had $\underline{b}=a[001] ;$ and $2 \% \operatorname{had} \underline{b}=a / 2[111]$.

Similar loop analyses were performed for loops formed at irradiation temperatures of 673 and $723 \mathrm{~K}$. The majorlty of the loops at these temperatures were loop segments as shown in Figure 3.8. The nature of the loops was determined whenever a sufficient part of the loop remained to determine "inside-outside" contrast.

A portion of the loops that were analyzed for the specimen irradiated at $673 \mathrm{~K}$ is shown in Figure 3.13. The loops and loop segments are labeled $(A, B, C \ldots)$. The Burgers vectors and nature of these loops are listed in Table 3.4. All of the loops analyzed were of near edge character with $\underline{b}=a\langle 100\rangle$ and were interstitial in nature.

A portion of the loops that were analyzed in the $723 \mathrm{~K}$ specimen is shown in Figure 3.14. The loops and segments labeled are listed with the Burgers vectors and loop natures in Table 3.5. The 1oop nature determination with loop segments is 1llustrated for this analysis in Figure 3.15. Once again, all of the loops were near edge with $\underline{b}=a\langle 100\rangle$ and were interstitial in nature. 
$Y-185239$
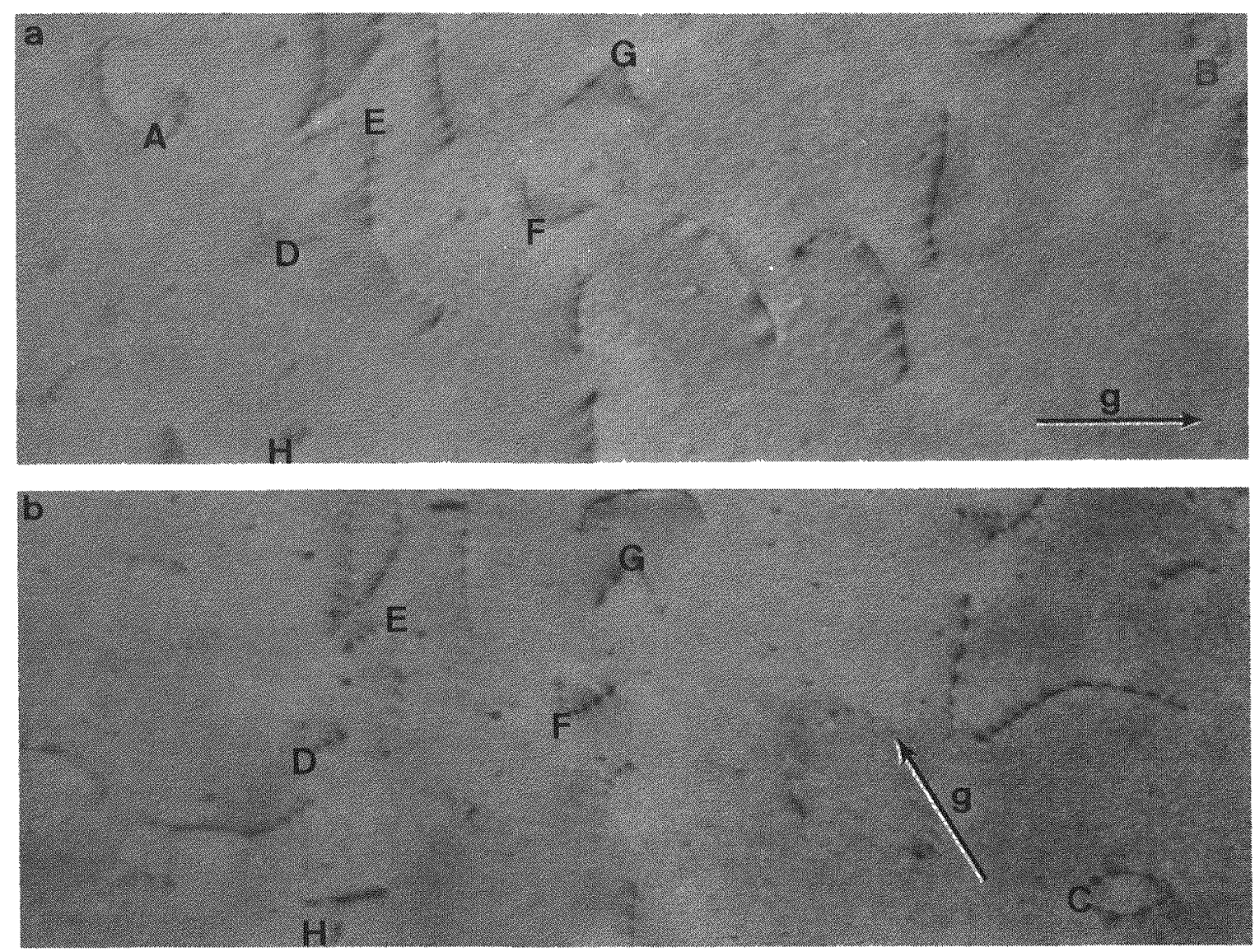

Figure 3.13. Dislocation loops and loop segments found in the specimen irradiated at $673 \mathrm{~K}$. A Burgers vector and loop nature determination was performed for the labeled dislocations. Length of arrows is $300 \mathrm{~mm}$. $\underline{z}$ near $[111]$. (a) $\underline{g}=[10 \overline{1}],(b) \underline{g}=[1 \overline{1} 0]$.

Table 3.4. Summary of the dislocation loop analysis for the loops and loop segments shown in Figure 3.13

\begin{tabular}{|c|c|c|c|c|c|}
\hline \multirow[b]{2}{*}{ Loop } & \multirow[b]{2}{*}{$\begin{array}{l}\text { g Yielding } \\
\text { Invisibility }\end{array}$} & \multirow[b]{2}{*}{$\underline{b}$} & \multicolumn{2}{|c|}{ g Yielding } & \multirow[b]{2}{*}{ Nature } \\
\hline & & & $\begin{array}{l}\text { Inside } \\
\text { Contrast }\end{array}$ & $\begin{array}{l}\text { Outside } \\
\text { Contrast }\end{array}$ & \\
\hline$A, B$ & $\pm[1 \overline{1} 0], \pm[200], \pm[020]$ & $a[001]$ & {$[11 \overline{2}]$} & [1̄12] & Interstitial \\
\hline $\mathrm{C}$ & $\pm[10 \overline{1}], \pm[200]$ & $a[010]$ & {$[1 \overline{2} 1]$} & {$[\overline{1} 2 \overline{1}]$} & Interstitial \\
\hline $\mathrm{D}-\mathrm{H}$ & $\pm[0 \overline{1} 1], \pm[020]$ & $a[100]$ & {$[\overline{2} 11]$} & {$[2 \overline{1} \overline{1}]$} & Interstitial \\
\hline
\end{tabular}



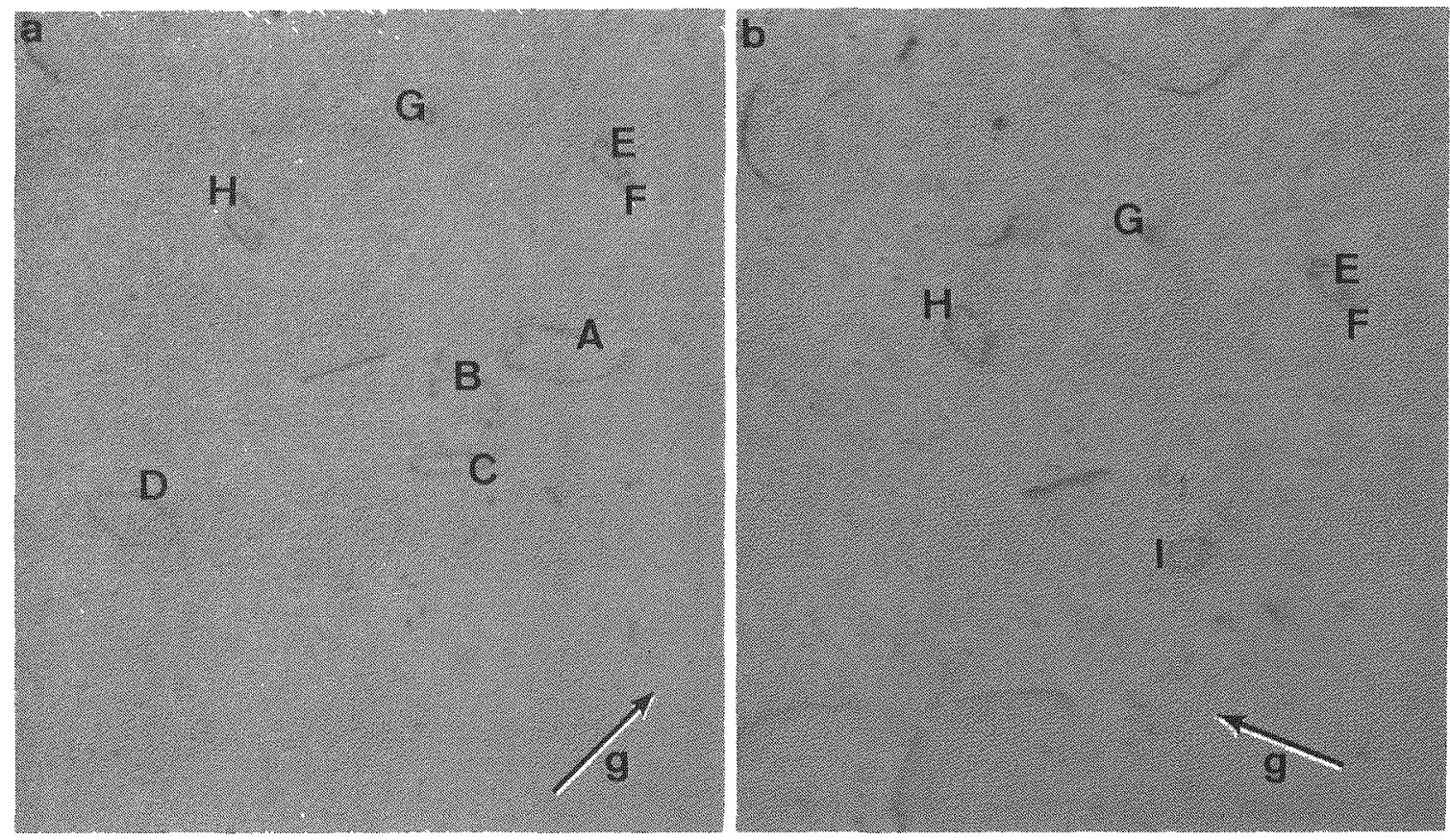

Flgure 3.14. Dislocation loops and loop segments found in the specimen irradiated at $723 \mathrm{~K}$. A Burgers vector and loop nature determination was performed for the labeled dislocations. Length of arrows is $500 \mathrm{~nm}$. $z$ near [111]. (a) $\mathrm{g}=[\overline{1} 10],(\mathrm{b}) \mathrm{g}=[0 \overline{1} 1]$.

Table 3.5. Summary of the dislocation loop analysis for the loop and 1oop segments shown in Figures 3.14 and 3.15

\begin{tabular}{|c|c|c|c|c|c|}
\hline \multirow[b]{2}{*}{ Loop } & \multirow{2}{*}{$\begin{array}{l}\text { g Yielding } \\
\text { Invisibility }\end{array}$} & \multirow[b]{2}{*}{$\underline{b}$} & \multicolumn{2}{|c|}{ g Yielding } & \multirow[b]{2}{*}{ Nature } \\
\hline & & & $\begin{array}{l}\text { Inside } \\
\text { Contrast }\end{array}$ & $\begin{array}{l}\text { Outside } \\
\text { Contrast }\end{array}$ & \\
\hline
\end{tabular}

\begin{tabular}{|c|c|c|c|c|c|}
\hline$A-D$ & $\begin{array}{l}{[0 \overline{1} 1],[01 \overline{1}],[020]} \\
{[0 \overline{2} 0]}\end{array}$ & $a[100]$ & {$[\overline{2} 11]$} & {$[2 \overline{1} \overline{1}]$} & Interstitial \\
\hline $\mathrm{E}-\mathrm{H}$ & $\begin{array}{l}{[\overline{1} 01],[10 \overline{1}],[200]} \\
{[\overline{2} 00]}\end{array}$ & $\mathrm{a}[010]$ & [121] & [12I] & Interstitial \\
\hline I & $\begin{array}{l}{[\overline{1} 10],[1 \overline{1} 0],[200]} \\
{[\overline{2} 00],[020],[0 \overline{2} 0]}\end{array}$ & $a[001]$ & {$[11 \overline{2}]$} & [i12] & Interstitial \\
\hline
\end{tabular}


$\mathrm{Y}-185241$
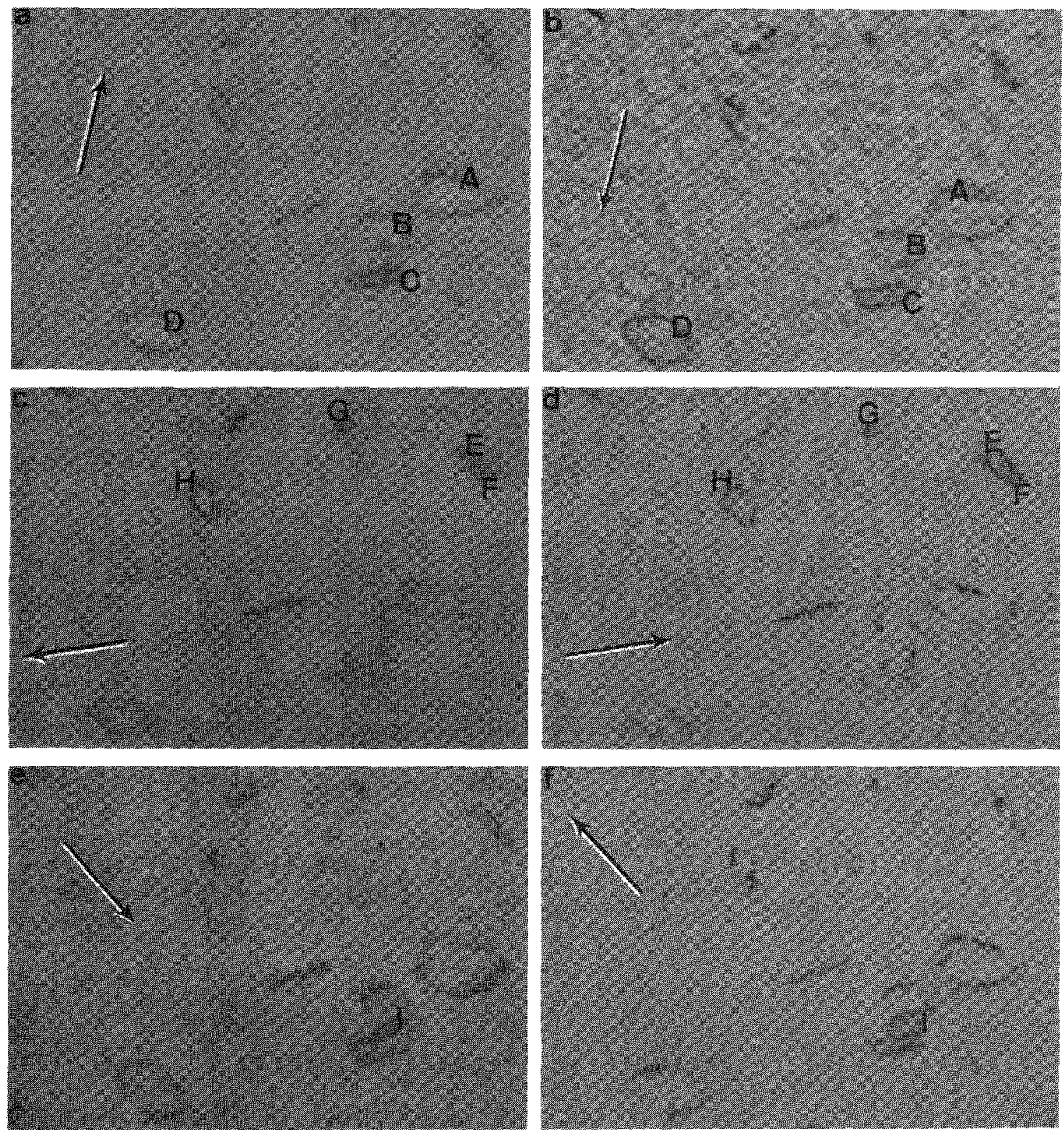

Figure 3.15. Analysis of the loop nature for the loops and loop segments shown in Figure 3.14. a,c,e - "inside contrast." b,d,f - "outside contrast." Length of arrows is $500 \mathrm{~nm}, z$ near [111].

(a) $g=[\overline{2} 11]$, (b) $g=[2 \bar{l} \overline{1}],(\bar{c}) g=[\underline{1} \underline{2} 1]$

(d) $\underline{g}=[\overline{1} 2 \overline{1}]$, (e) $\underline{g}=[112]$, (f) $\underline{g}=[\overline{1} 12]$.

Arrows denote direction of $\mathrm{g}$. 
The dislocation loops and network structures observed in neutronirradiated iron are similar to those observed in the ion irradiation studies (Chapters 4 and 5). Therefore, a detailed discussion of these dislocation microstructures will be deferred to Chapter 6 .

High Irradiation Temperatures: 923 and $1013 \mathrm{~K}$ - At irradiation temperatures of 923 and $1013 \mathrm{~K}$, there was no difference in the dislocation microstructure as compared to the residual, unirradiated microstructures. A typical micrograph illustrating this from a specimen irradiated at $923 \mathrm{~K}$ is shown in Figure 3.16.

YE-12390

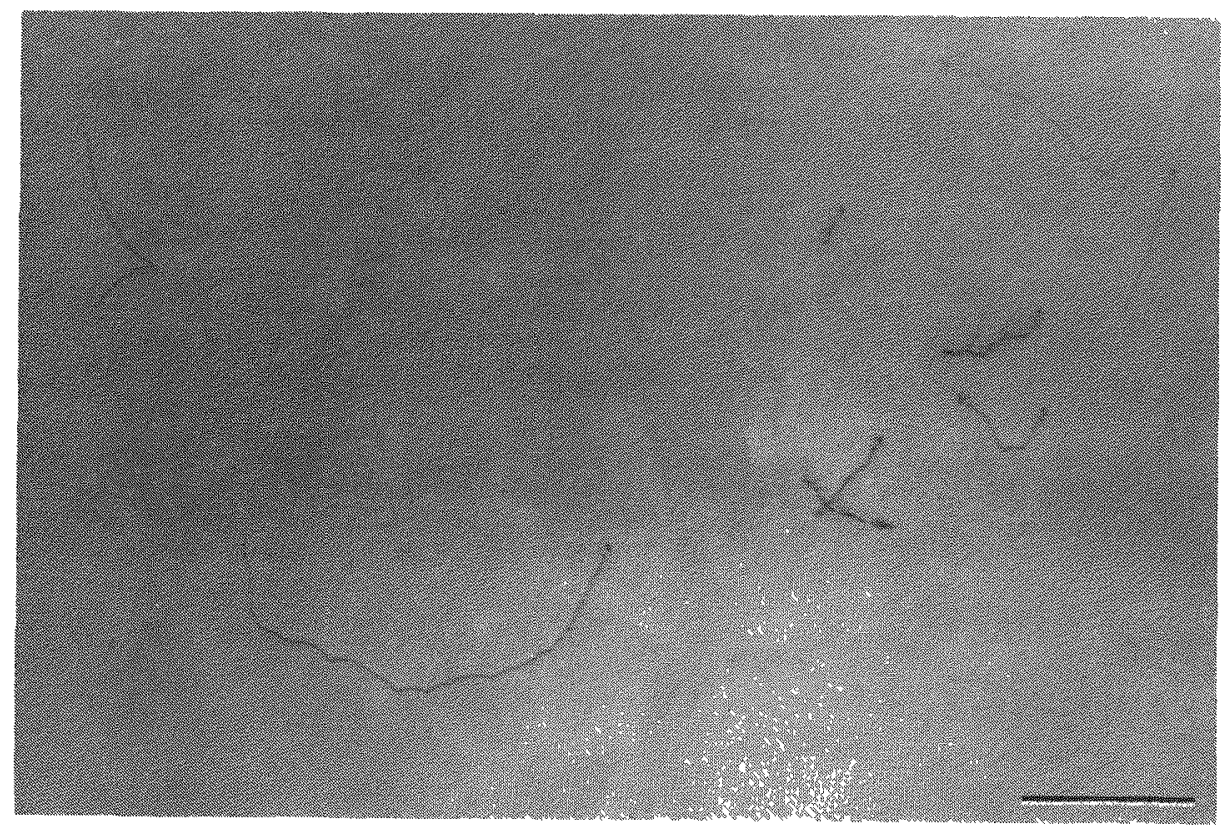

Figure 3.16. Typical micrograph of the dislocation structure found for high irradiation temperatures. $T_{I}=923 \mathrm{~K}$. Scale marker is $2 \mathrm{\mu m}$.

\subsection{Irradiation-Induced Cavity Microstructures}

Significant cavity formation occurred over a limited irradiation temperature range of 548 to $723 \mathrm{~K}$. The cavity microstructures present at these irradiation temperatures are shown in Figure 3.17. The cavity distribution is homogeneous only at 623 and $673 \mathrm{~K}$. At 548 and $573 \mathrm{~K}$, the cavities are found in association with the clusters of dislocation loops. This association is a clear example of the cooperative growth of cavities and interstitial loops. The association can be clearly 
$Y-185242$
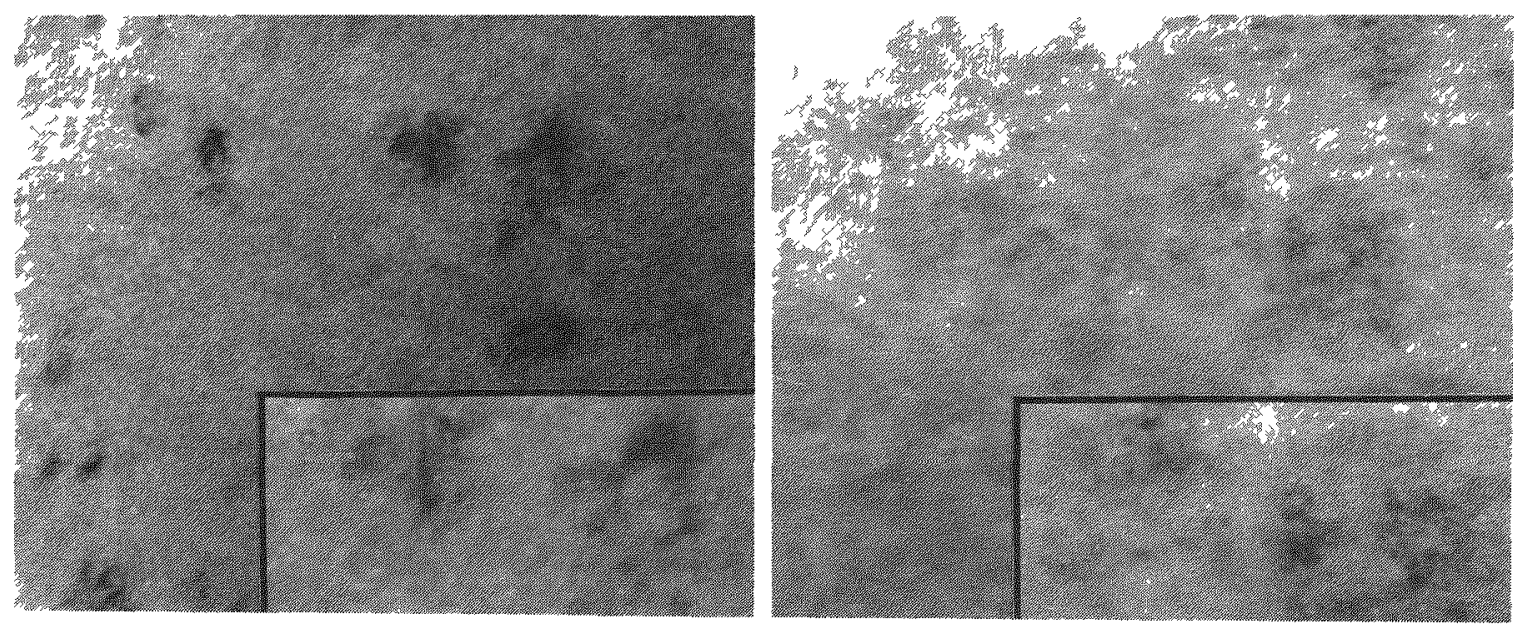

$548 \mathrm{~K}$

$573 \mathrm{~K}$

$623 \mathrm{~K}$

$673 \mathrm{~K}$
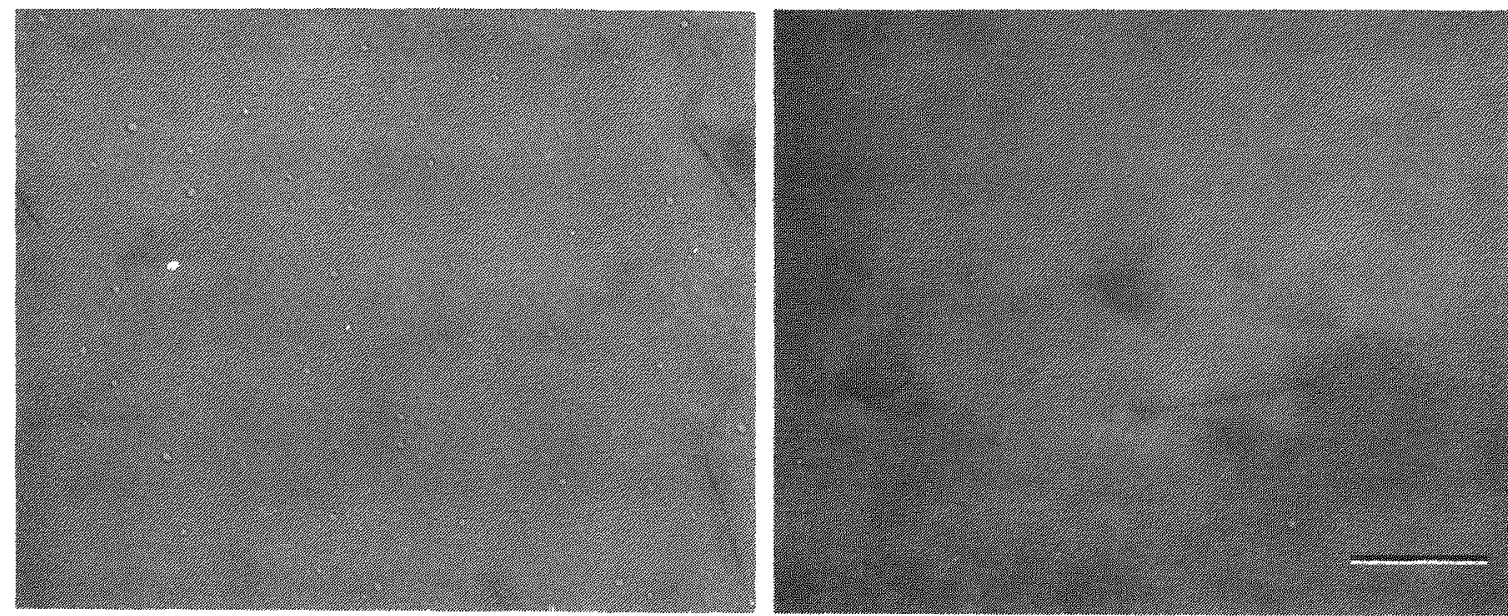

Figure 3.17. Representative micrographs of the cavity microstructure found in specimens irradiated at 548 to $723 \mathrm{~K}$. The insets ( 548 and $573 \mathrm{~K})$ are $2 \times$ enlargements. Scale marker is $200 \mathrm{~nm}$. $\underline{z}$ near [111], $\underline{g}=\langle 3 \overline{3} 0\rangle$. 
seen in the enlarged (2x) inset of Figure $3.17(a)$. No grain boundaries were observed in the thin regions of these specimens, so no relationship between grain boundaries and cavities could be determined.

At $773 \mathrm{~K}$, cavities were very sparse and were observed only in association with unidentified precipitates (probably carbides), as shown in Figure 3.18. Similar cavity-precipitate association was sometimes observed in the specimen irradiated at $723 \mathrm{~K}$. At $923 \mathrm{~K}$, excluding the damage halos, only a few cavities, located on grain boundaries, were observed.

Facets on the larger cavities were clearly visible, but for the small cavities no conclusions in regard to their shape could be reached. Surprising1y, detailed tilting experiments showed that the faceted cavities were $\{111\}$ octahedra with $\{100\}$ truncations. The cavity morphology is discussed in more detail in Chapter 6. The cavity diameters were measured assuming a circular projection with the same area as the observed crystallographic shape.

The quantitative cavity data are summarized in Table 3.6 and Figure 3.19. The volume-averaged cavity diameter, $d_{c}$, reaches a maximum of $\sim 12 \mathrm{~nm}$ at $673 \mathrm{~K}$. The cavity concentration, $C_{C}$, is a maximum of
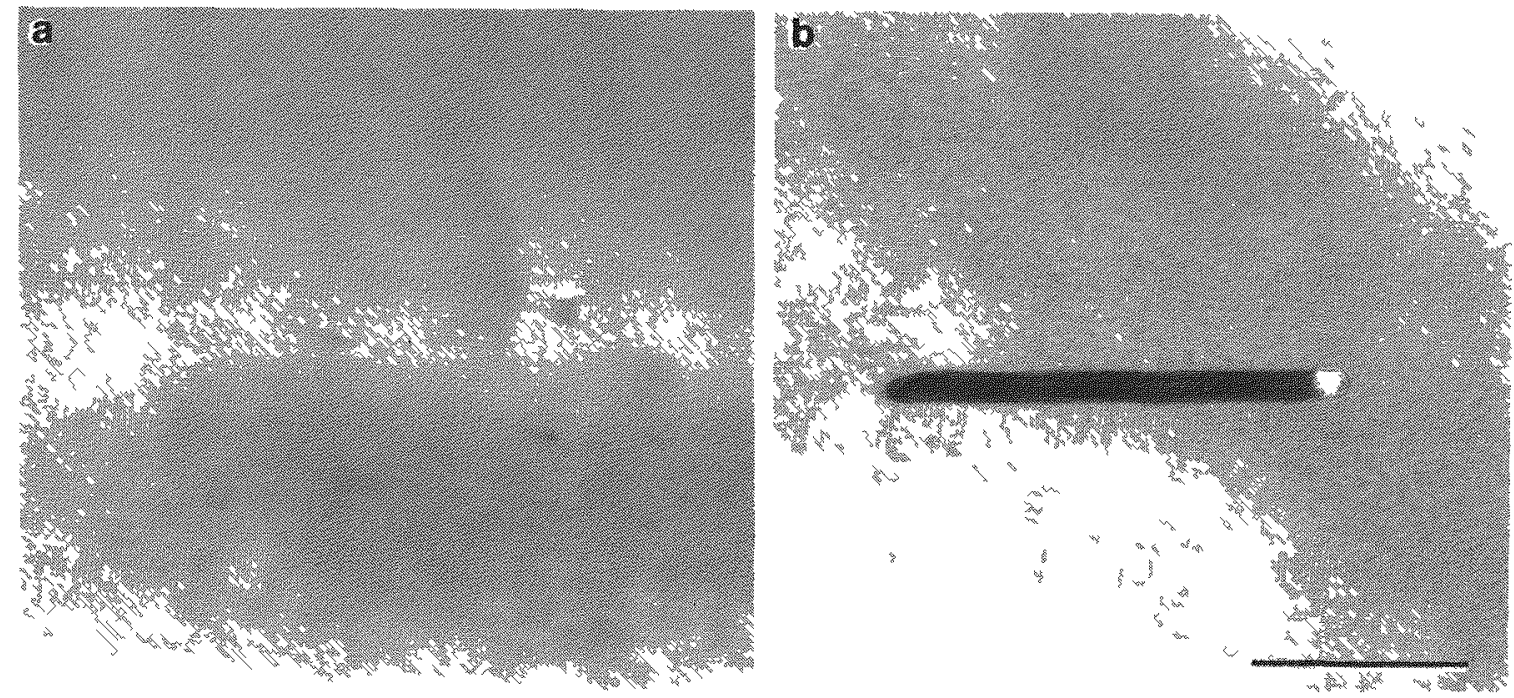

Figure 3.18. Micrograph of cavities found in association with precipitates. Scale marker is $500 \mathrm{~nm}$. (a) $\mathrm{T}_{\mathrm{I}}=723 \mathrm{~K}$. (b) $\mathrm{T}_{\mathrm{I}}=773 \mathrm{~K}$. 
Table 3.6. Quantitative Cavity Data

\begin{tabular}{|c|c|c|c|c|}
\hline \multicolumn{2}{|c|}{ Temperature ${ }^{a}$} & \multirow{2}{*}{$\begin{array}{c}\mathrm{d}_{\mathrm{c}} \\
(\mathrm{nm})\end{array}$} & \multirow{2}{*}{$\underset{\left(m^{-3}\right)}{C_{c}}$} & \multirow{2}{*}{$\begin{array}{c}\text { Swelling } \\
(\%)\end{array}$} \\
\hline$(\mathrm{K})$ & $\left({ }^{\circ} \mathrm{C}\right)$ & & & \\
\hline 548 & 275 & 5.7 & $6.4 \times 10^{20}$ & 0.006 \\
\hline 573 & 300 & 8.5 & $9.8 \times 10^{20}$ & 0.032 \\
\hline 623 & 350 & 10.5 & $1.1 \times 10^{21}$ & 0.067 \\
\hline 673 & 400 & 12.0 & $8.2 \times 10^{20}$ & 0.073 \\
\hline 723 & 450 & 10.2 & $7.7 \times 10^{19}$ & 0.004 \\
\hline
\end{tabular}

${ }^{a}$ No cavity data were obtained for the 773 and $923 \mathrm{~K}$ irradiation temperatures.

$b_{\text {Typical error } ~} 34 \%$.

ORNL-DWG 81-21249

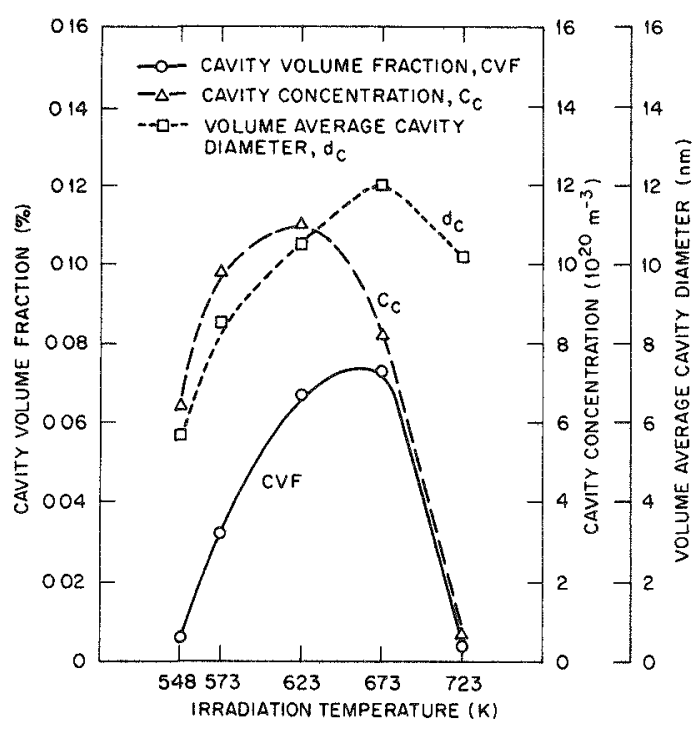

Figure 3.19. Quantitative data for cavities in neutron-irradiated iron.

$\sim 10^{21} \mathrm{~m}^{-3}$ at irradiation temperatures of 573 to $673 \mathrm{~K}$. The calculated maximum swelling or cavity volume fraction (CVF) of $\sim 0.07 \%$ occurs at 623 and $673 \mathrm{~K}$.

\subsection{Irradiation-Induced Halo Microstructures}

Halos centered on $B_{4} C$ precipitates were observed in specimens irradiated at $493,523,723$, and $923 \mathrm{~K}$. The low concentration of halos $\left(<10^{11} \mathrm{~m}^{-3}\right)$ allowed them to be considered as a separate feature of the microstructure. 
A schematic diagram of a planar section through a damage halo in iron is shown in Figure 3.20. As discussed previously, the damage halo actually consists of two individual halos - one caused by energetic lithium lons and one caused by energetic helium ions. In three dimensions, these halos are concentric spheres of damage. Halos are believed to result largely from the impurity effect (e.g., helium and lithium) on the bulk neutron displacement damage. However, the displacement damage and damage rates will be higher within the halo region.

The appearance of a halo in a thinned specimen depends on many factors. The halo diameter and width are dependent on the precipitate size as well as the foil sectioning. For example, the larger the precipitate, the larger the halo diameter and the greater the width. For sufficiently large precipitates, the individual halos could merge. If the foll intersects both damage spheres and the precipitate, halos similar to that depicted in Figure 3.20 would be observed. If the intersection occurs above or below the precipltate, the observed halo diameters would be smaller than the actual value for the halo diameter. When the intersection occurs at the lithium halo, the planar section would consist of a small inner damage field (1ithium halo), surrounded

YE-12391

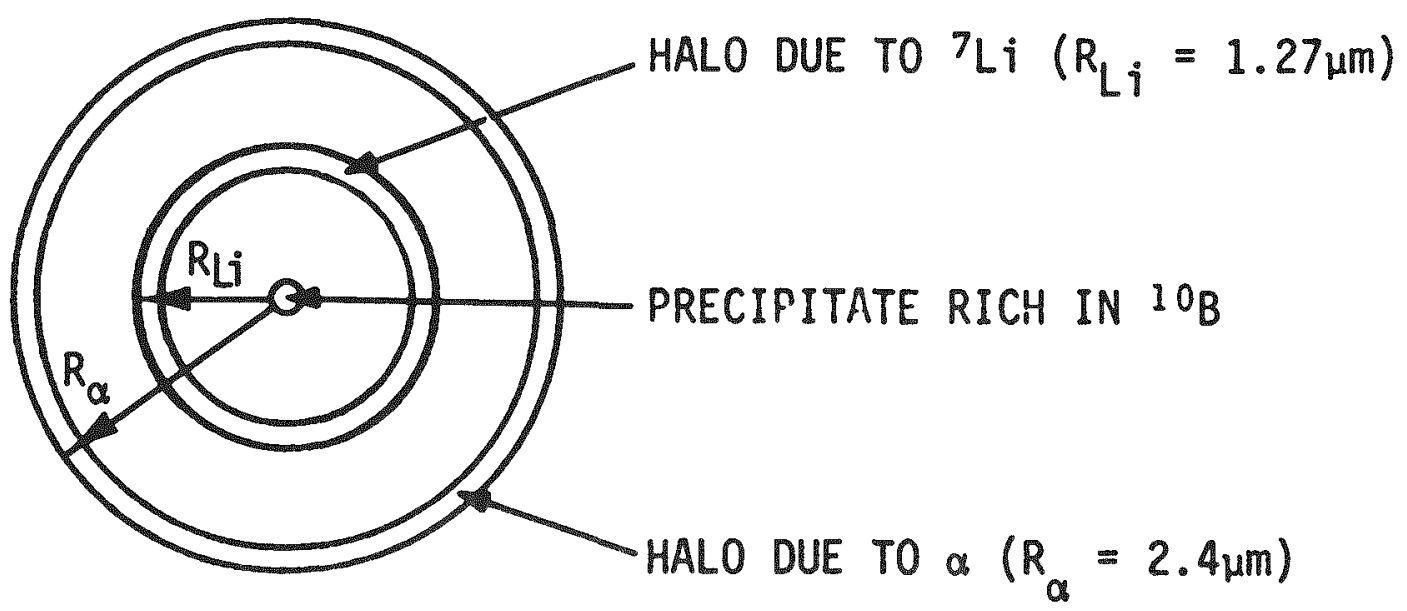

Figure 3.20. Schematic diagram of a planar section through a damage halo in iron. 
by a ring of damage (helium halo). Finally, in the extreme case - i.e., intersection at the helium halo - only a small, circular damage field would be observed.

The type of damage clusters in the halos depends on the irradiation temperature. The actual density of the clusters within a halo further depends on the boron content of the prectpitate - the larger the boron content, the higher the displacement damage and the larger the effect of the helium and lithium ions on the damage.

For irradiation temperatures of 493 and $523 \mathrm{~K}$, double halos of small dislocation loops were observed. At $723 \mathrm{k}$, the two halos were not distinct in any of the observed halos. The halo microstructure consisted of larger dislocation loops. At $923 \mathrm{~K}$, the halo microstructure consists of a high concentration of small cavities. For comparison, each of these halo structures is pictured in Figure 3.21. Each halo structure is discussed in more detail below.

Low-Irradiation-Temperature Halo Microstructures - An array of the damage halos observed at 493 and $523 \mathrm{~K}$ is shown in Figures 3.22 and 3.23. In these micrographs, it can be seen, as was discussed above, that the width, diameter, and defect density vary from halo to halo. The diameters of the lithium and helfum halos were measured on micrographs of foils irradiated at 493 and $523 \mathrm{~K}$ in which both the halos and the precipitate that formed the halos were observed. The diameters measured were those defined by the center of the halo damage region. The average diameter of the 1fthium halo was $\sim 2.5 \mu \mathrm{m}$ and the diameter of the helium halo was $\sim 5.2 \mu \mathrm{m}$. These average measurements correlate well with the calculated values.

A partial analysis of the dislocation loops was performed for the small loops within the indicated region of the halo shown in Figure 3.24. Note that this halo in a specimen irradiated at $523 \mathrm{~K}$ intersected the electropolishing hole. Higher magnification micrographs were taken of the marked region of Figure 3.24 with $g=[1 \overline{1} 0]$, [ $\overline{200]}$, and [020] near the [001] pole. These micrographs are shown in Figure 3.25. Many of the loops seen in this series of micrographs are not in the edge, $\underline{b}=a\langle 100\rangle$, configuration. Also, as indicated by the lettered loops, many loops appear in contrast in all of the micrographs 
ORNL-Photo 3992-80
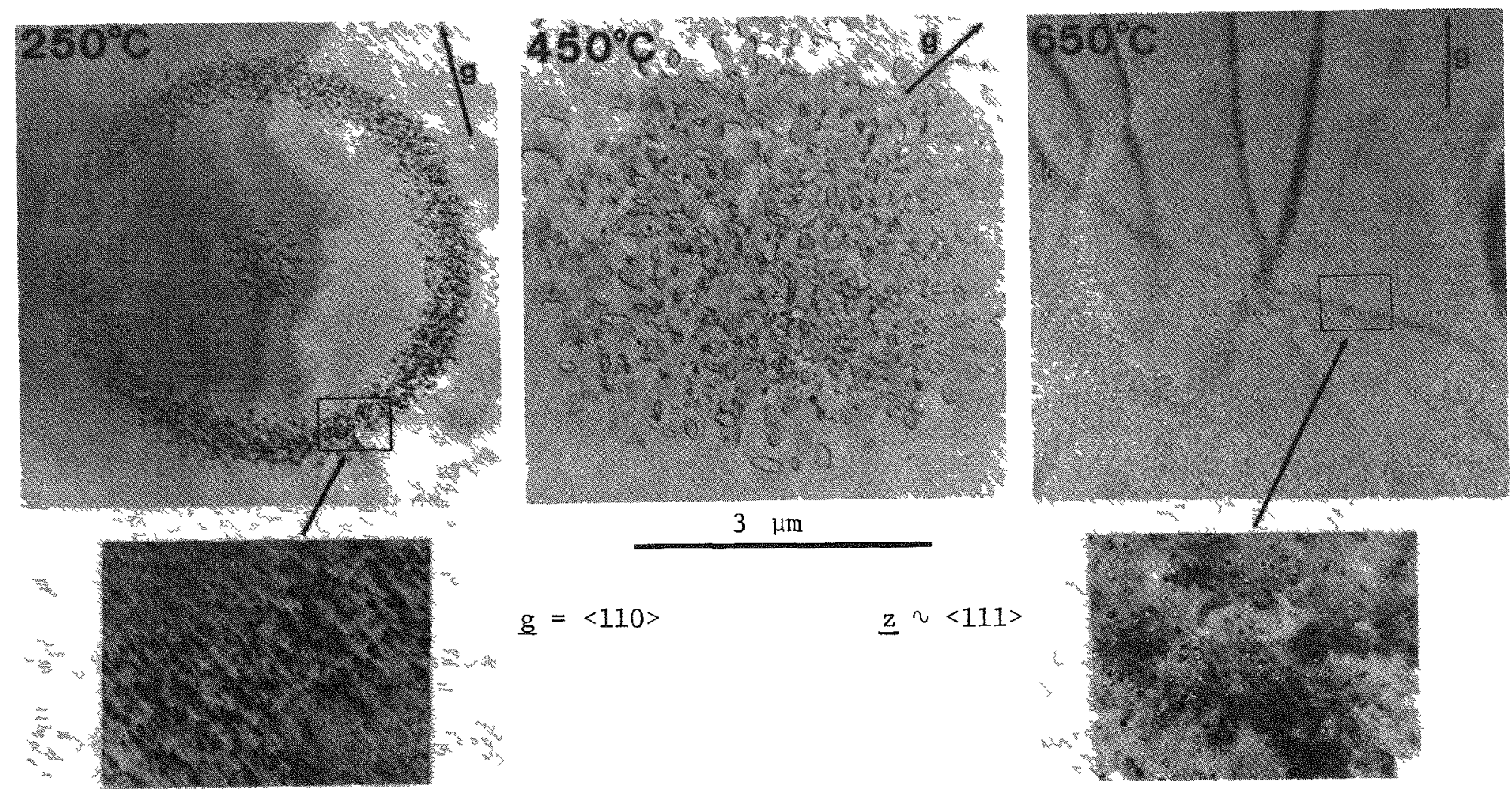

$\underline{z} \sim\langle 111\rangle$

$\underline{\mathrm{g}}=\langle 110\rangle$

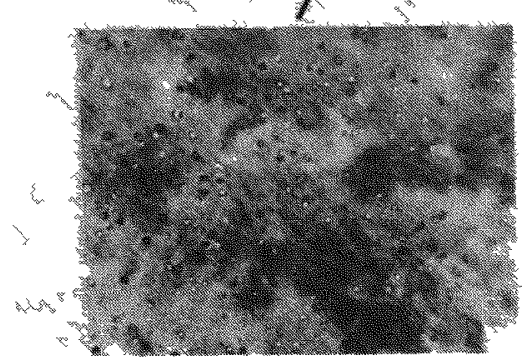

Figure 3.21. Representative micrographs of the halo microstructures found at $523 \mathrm{~K}$ $\left(250^{\circ} \mathrm{C}\right), 723 \mathrm{~K}\left(450^{\circ} \mathrm{C}\right)$, and $923 \mathrm{~K}\left(650^{\circ} \mathrm{C}\right)$. Inset areas are $2 \times$ enlargements. 
$523 \mathrm{~K}$

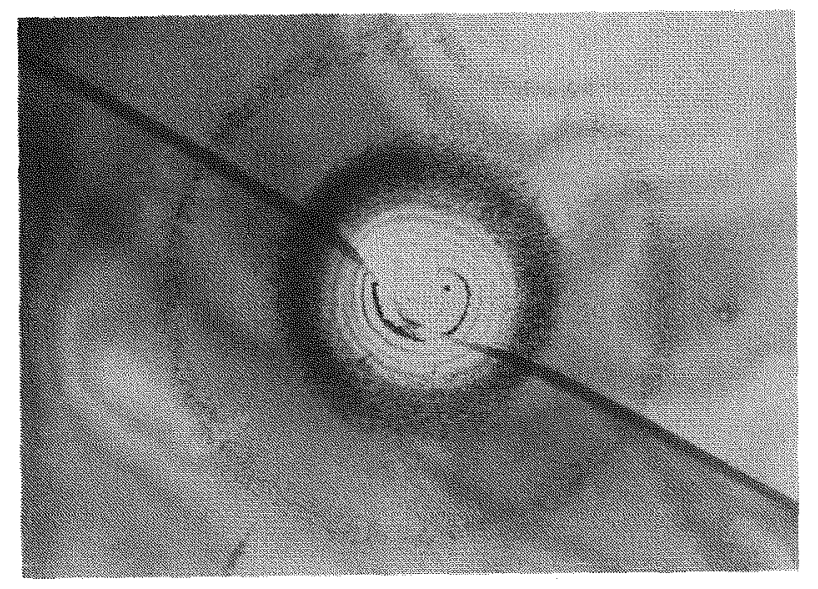

$523 \mathrm{~K}$

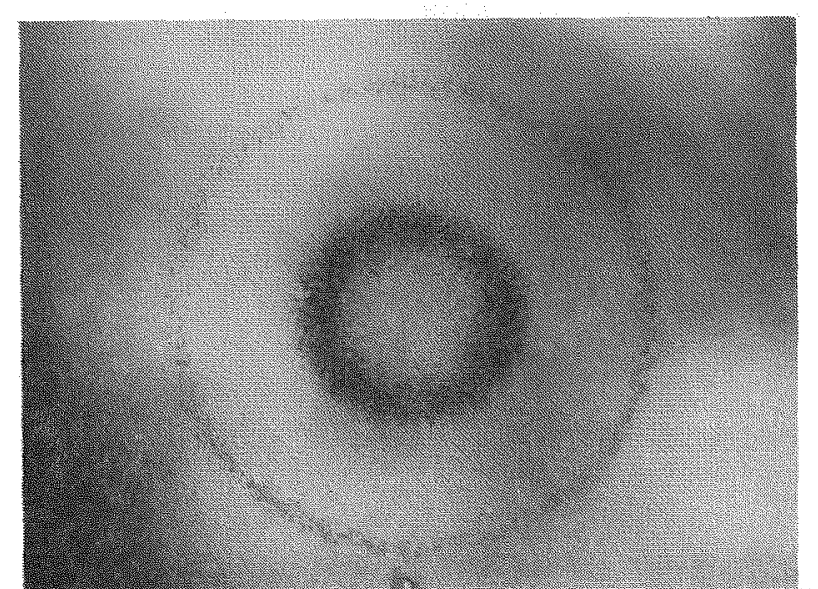

Figure 3.22. Micrographs of the halos found in specimens irradiated at 493 and $523 \mathrm{~K}$. Scale marker is $2 \mu \mathrm{m}$.
$523 \mathrm{~K}$

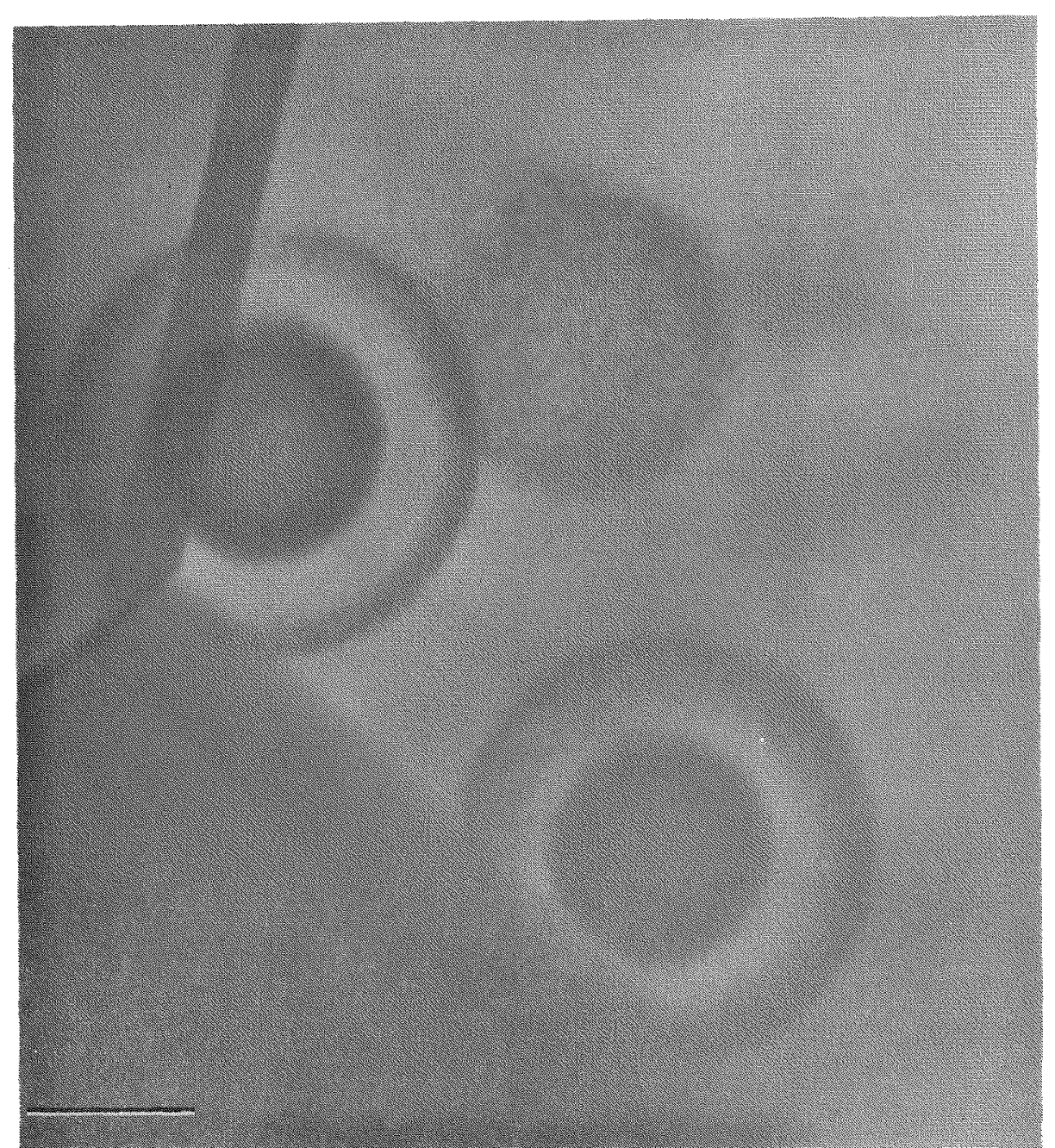

\section{G}


$Y-185245$

$493 \mathrm{~K}$

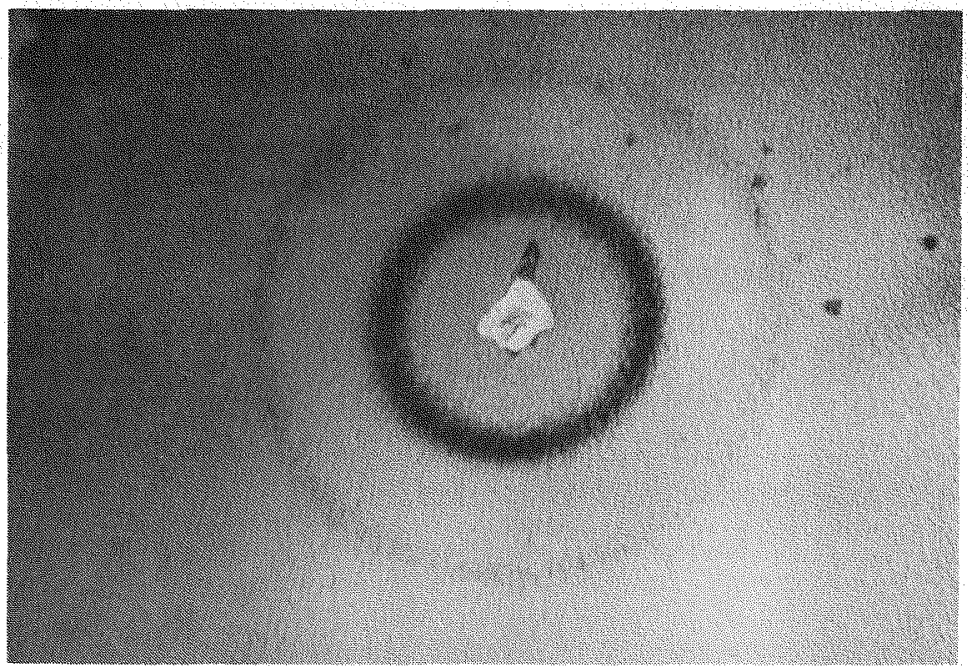

$523 \mathrm{~K}$

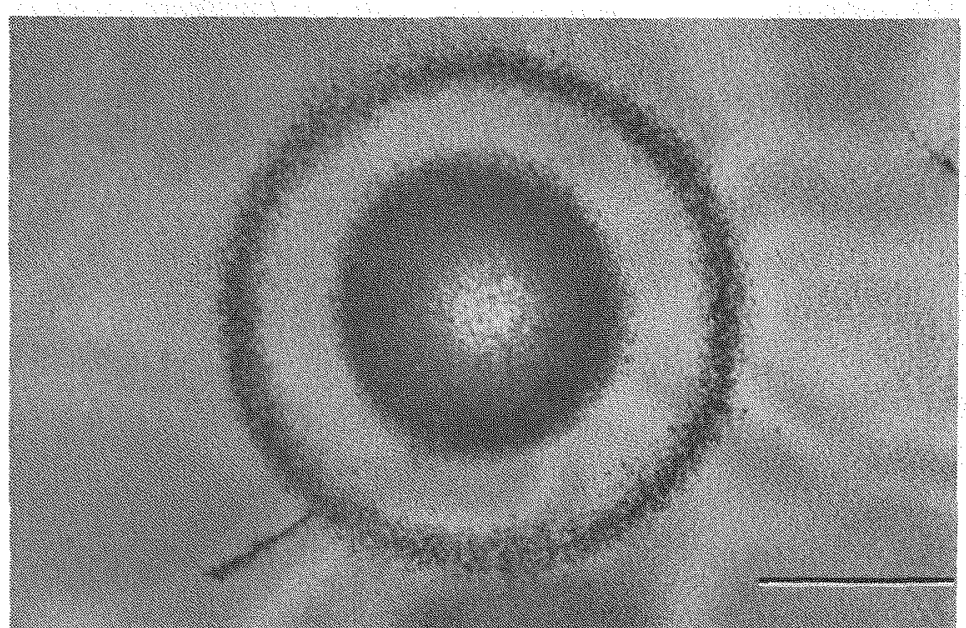

Figure 3.23. Micrographs of the halos found in specimens irradiated at 493 and $523 \mathrm{~K}$. Scale marker is $2 \mu \mathrm{m}$.
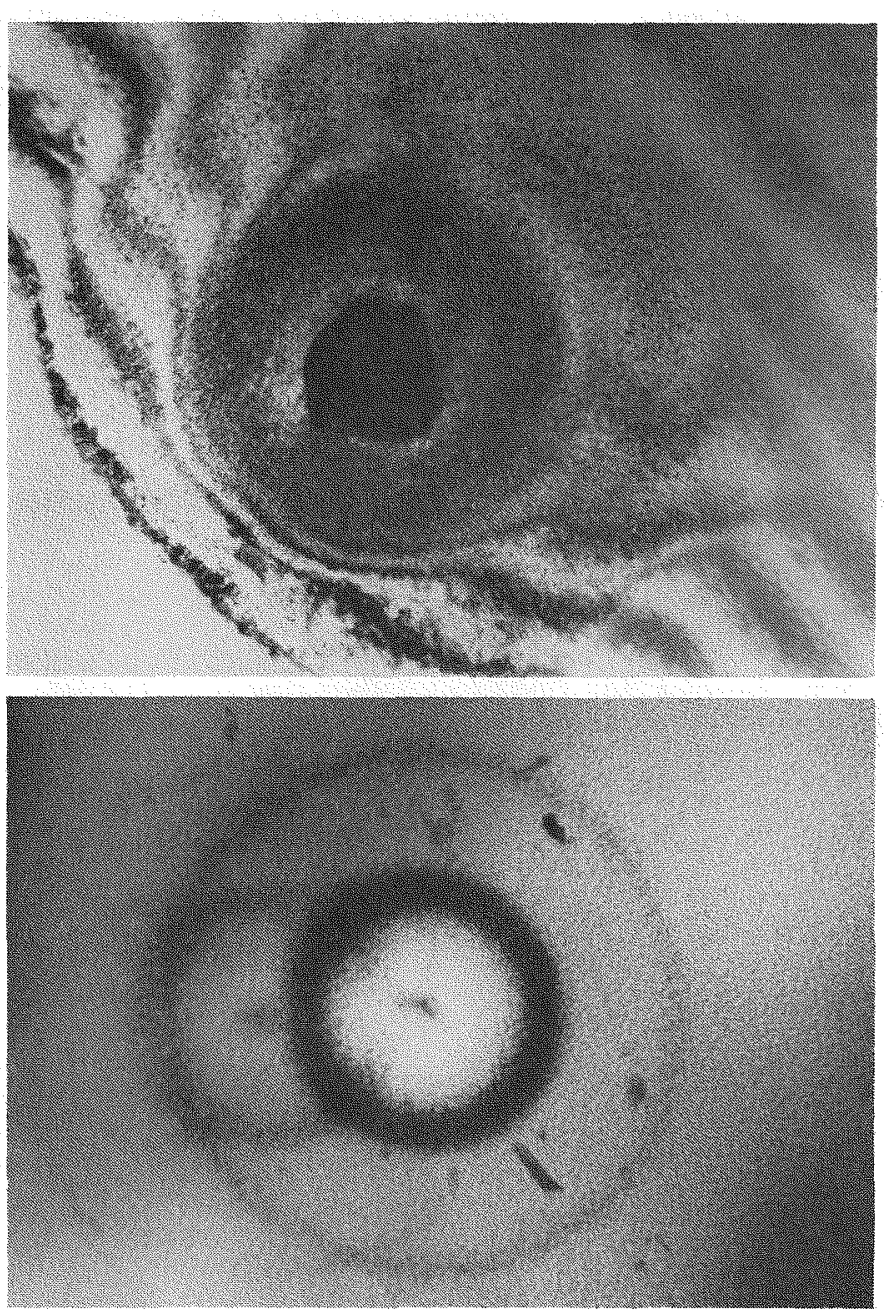

$493 \mathrm{~K}$ 


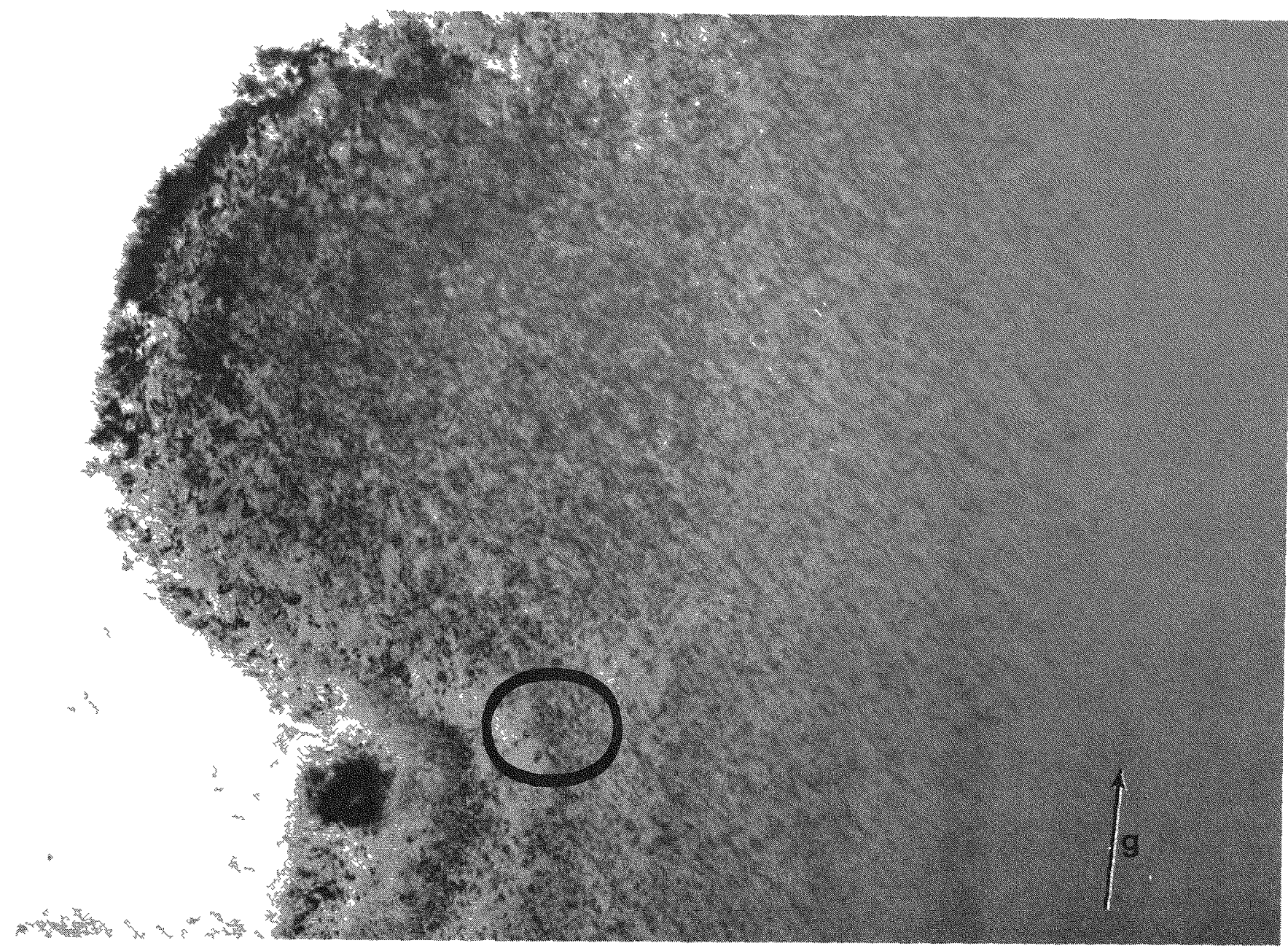

Figure 3.24. Micrograph of a halo intersecting the electropolishing hole. Circled area is shown in the enlarged micrographs in Figure $3.23 . T_{I}=523 \mathrm{~K}$. Length of arrow is $500 \mathrm{~nm} . \mathrm{g}=[\overline{1} 10] . \underline{z}$ between [113] and [112]. 

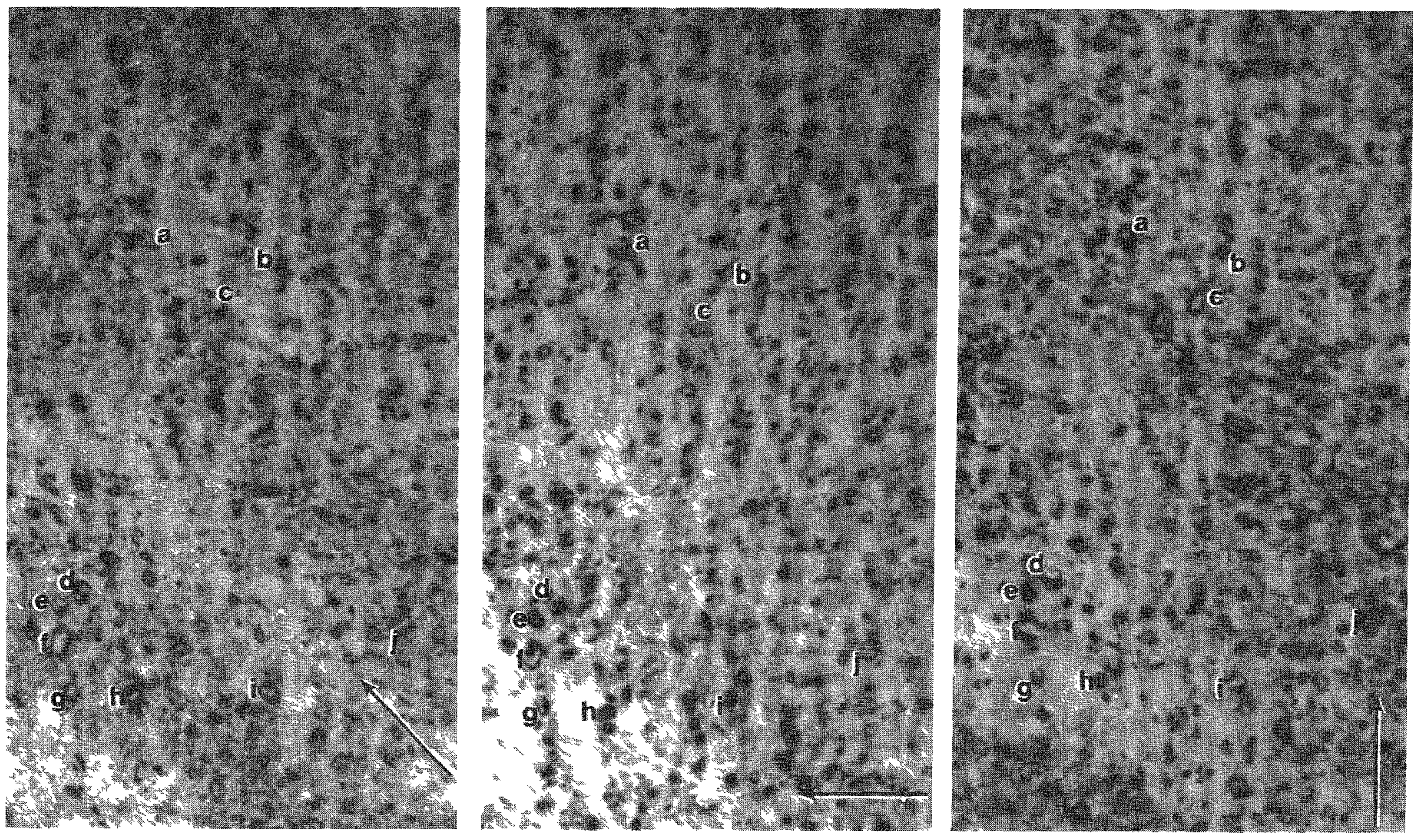

Figure 3.25. Higher magnification micrographs of the circled region in Figure 3.22. The 1etters mark the same loops in each micrograph. $\mathrm{T}_{\mathrm{T}}=523 \mathrm{~K}$. Length of arrows is $200 \mathrm{~nm}$. $z$ near [001]. (a) $\underline{g}=[\overline{1} 10],(b) g=[\overline{2} 00]$, (c) $\underline{g}=[020]$. Arrows denote direction of $g$. 
- an indication that their Burgers vectors are $a / 2[1 \overline{1} 1]$ or $a / 2[\overline{1} 11]$. The loop density over the halo region was higher for $\underline{g}=\langle 200\rangle$ than for $\underline{g}=[1 \overline{1} 0]-$ as would be expected if loops of $\underline{b}=a / 2[111]$ and $a / 2[11 I]$ were present. In conclusion, it appears that $a / 2\langle 111\rangle$ loops as well as a $\langle 100\rangle$ loops are formed at $523 \mathrm{~K}$.

Intermediate Temperature Halo Microstructures - At $723 \mathrm{~K}$, the larger loop structure in the halo microstructure permitted a complete loop analysis. A part of this analysis, for loops with $b=a[001]$, is shown in Figure 3.26. As in the non-halo dislocation microstructure, all loops were near edge character, with $\underline{b}=a\langle 100\rangle$ and were interstitial in nature. The quantitative parameters for the halo and non-halo structures are shown in Table 3.7. The loops in the halos are smaller and have a factor of 30 higher concentration than the loops not found in the halos. The dislocation density was an order of magnitude higher for the halo dislocations.

High-Temperature Halo Microstructures - At $923 \mathrm{~K}$, two diffuse cavity halos are observed. These halos are shown in Figure 3.21. An enlargement of an area across the two halos is shown in Figure 3.27 . The cavities in the inner halo are larger and have a lower concentration than those in the outer halo. The width of the inner halo is $\sim 1.2$ $\mu \mathrm{m}$ and the gap between halos is $\sim 0.3 \mu \mathrm{m}$. The width of the outer halo, measured in a region away from the grain boundary, is also $\sim 1.2 \mu \mathrm{m}$, with a few cavities in the regions surrounding the halo. Near the halos, cavities form on and near grain boundaries, as is shown in Figure 3.28. The cavities on the boundary are about a factor of 2 larger than those within the halo. As described earlier, a few cavities were also found on grain boundaries in reglons away from the halos.

Discussion: At all of the irradiation temperatures, the defect clusters in the halos varied significantly from those in non-halo regions. These differences may be caused by the higher displacement damage, damage rates, helium concentration and lithium concentration in the halo regions. While it is impossible to assign a single cause to the microstructural features observed in the halos, it is belleved that the higher helium and lithium concentrations have the strongest effect. 
ORNL-Photo $3989-80$
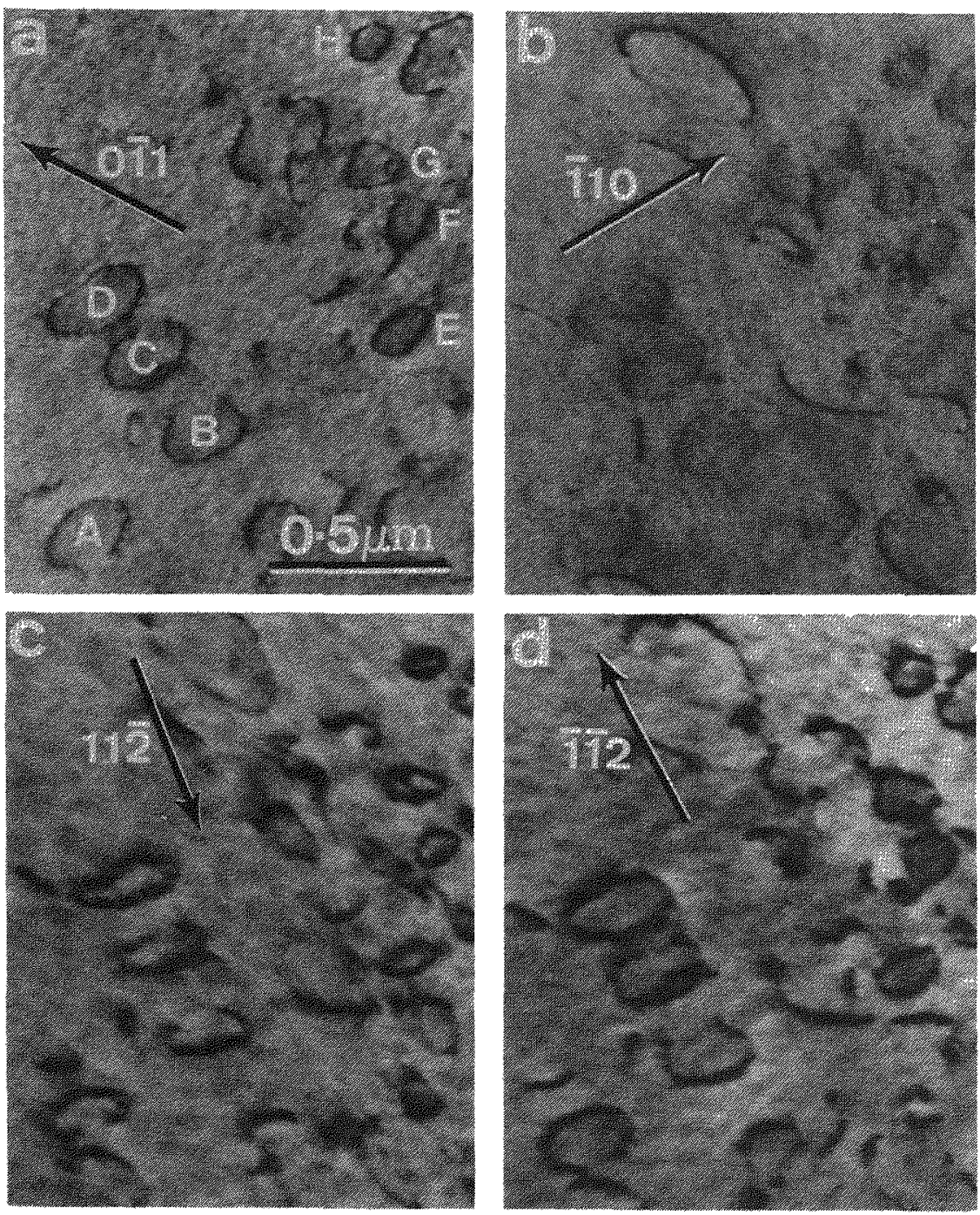

Figure 3.26. Analysis of the geometry and nature of dislocation loops found in a damage halo. The labeled loops have a Burgers vector of $a[001]$ and are interstitial in nature. $\mathrm{T}_{\mathrm{I}}$ $=723 \mathrm{~K}$. $\underline{z}$ near [111]. $\mathrm{g}$ as indicated on the individual micrographs.

Table 3.7. Dislocation density and 1oop parameters for halo and non-halo areas of a specimen irradiated at $723 \mathrm{~K}$

\begin{tabular}{lccccc}
\hline & $\mathrm{C}_{\mathrm{L}}$ & $\mathrm{d}_{\mathrm{L}}$ & \multicolumn{2}{c}{$2 p$} \\
\cline { 5 - 6 }$\left(\mathrm{m}^{-3}\right)$ & $(\mathrm{nm})$ & \multicolumn{1}{c}{$\left(\mathrm{m}^{-2}\right)$} & $\begin{array}{c}\text { Error } \\
(\%)\end{array}$ \\
\hline Non-halo & $1.7 \times 10^{18}$ & 200 & $2.2 \times 10^{12}$ & 10 \\
Halo & $5.8 \times 10^{19}$ & 140 & 2 & $\times 10^{13}$ & 7 \\
\hline
\end{tabular}


YE-12393

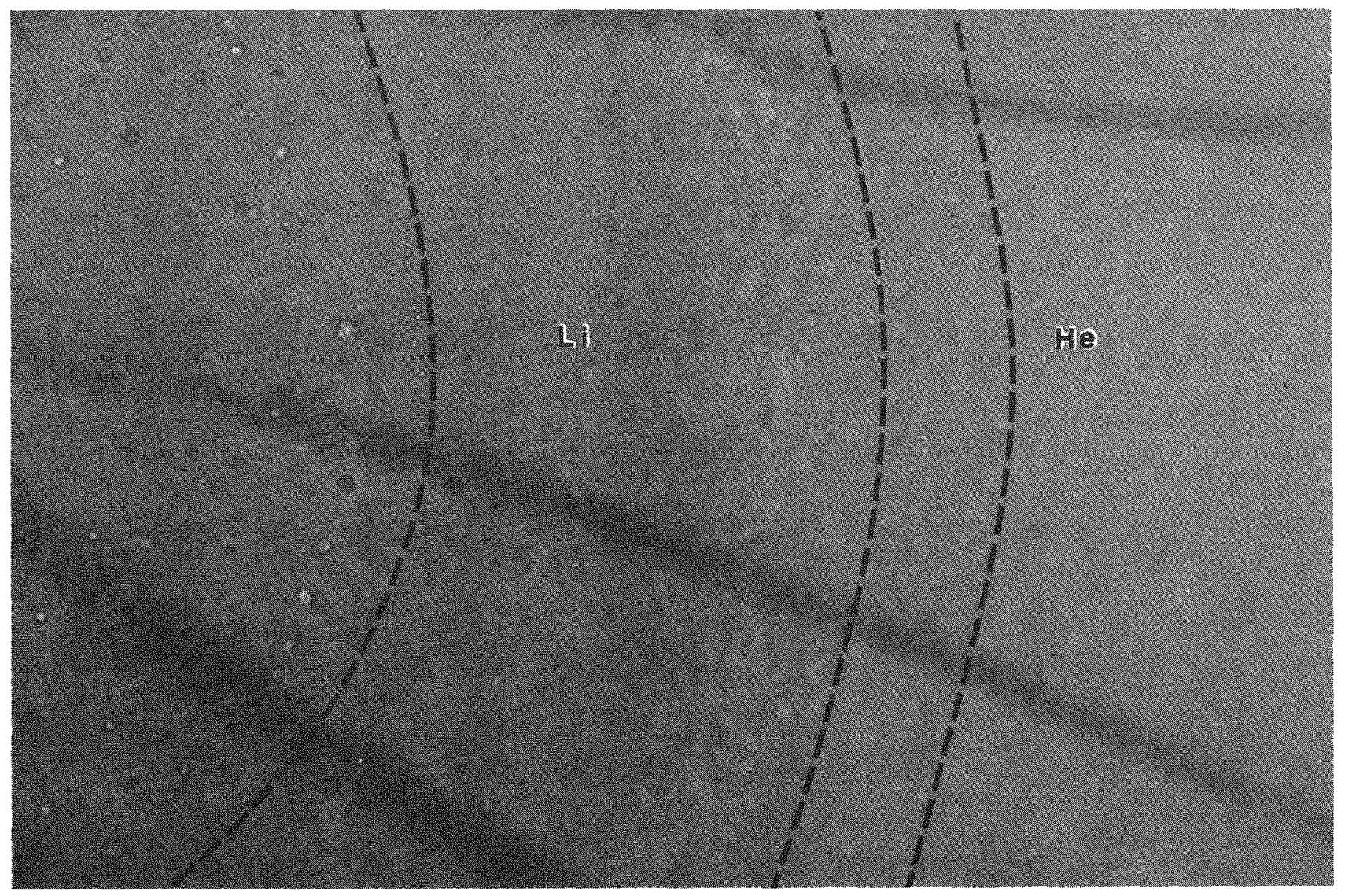

Figure 3.27. Enlargement of a cavity damage halo. The lithium and helium halos are indicated. $T_{\mathrm{I}}=923 \mathrm{~K}$. Scale marker is $300 \mathrm{~nm}$. 
YE-12394

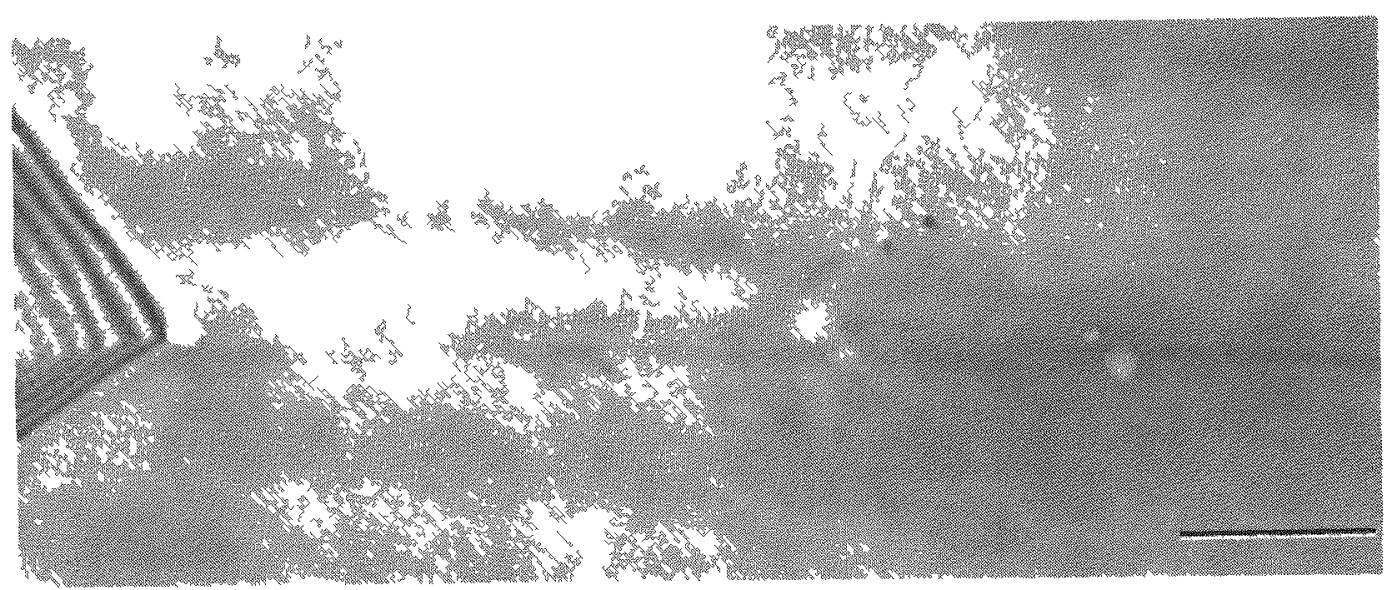

Figure 3.28. Micrograph of cavities on and near a grain boundary which is close to a damage halo. $T_{I}=923 \mathrm{~K}$. Scale marker is $300 \mathrm{~nm}$.

Ion irradiation studies of austenitic materials have shown that preimplanted and co-implanted helium can enhance cavity and interstitial dislocation loop formation [25]. The characteristics of the hightemperature halos are probably most influenced by the presence of helium. At these temperatures, except for a few cavities at grain boundarles, cavities were observed only within or near the halos. While the higher displacement damage in the halos is undoubtedly a contributing factor, the helium is probably more important in aiding cavity formation. Cavity formation in low damage regions outside the main halo regions is probably due to the diffusion of helium away from the halo. This "spread" of cavities away from the main damage regions is responsible for the rather diffuse appearance of the halos.

A similar temperature dependence of the halo microstructures has been observed by $K$. Farrel1 [91] in neutron-irradiated iron specimens that were doped with $B_{4} C$ precipitates. These specimens were also irradiated in ORR-228 in the same capsules as the iron used for this study. In the $\mathrm{Fe}_{-} \mathrm{B}_{4} \mathrm{C}$ specimens, due to the higher precipitate concentration, halos were found at all irradiation temperatures. Presumably halos were also present in all of the iron specimens, but, due to the low precipitate concentration, the halos were not found in the electron transparent regions of all of the specimens. 
CHAPTER 4

DAMAGE DEPTH PROFILES IN TRIPLE-BEAM IRRADIATED IRON

In ion-irradiated specimens, the damaged region is within a few micrometers of the irradiated surface. The defect structures vary significantly with distance from the irradiated surface. Simple "backthinning" from the unirradiated surface of the specimen would limit 120 $\mathrm{kV}$ TEM examinations to the region within about $250 \mathrm{~nm}$ of the irradiated surface. In this region, surface effects would dominate the observations. Therefore, controlled removal or "sectioning" of the damaged region to some predetermined depth from the irradiated surface is necessary prior to back-thinning for preparation of TEM specimens. Usually, the sectioning depth is selected by studying the computer-calculated deposited energy profiles. The sectioning depth can best be selected, however, by experinentally determining the depth distribution of the defect structures.

Three experimental methods are commonly used to determine defect profiles. In the first, the Ion-irradiated specimen is back-thinned and examined in an HVEM. The defect distribution is then determined from stereo pairs. This method is suitable on1y for microstructures with low defect concentrations because of image overlap problems and relies heavily on the experimenter's accuracy in stereo measurements. Also, $1 \mathrm{MeV}$ electrons allow examination of medium atomic number materials at foil thicknesses of only up to $\sim 1.5 \mu \mathrm{m}$, which may be less than the calculated range of high energy ions in these materials. Further, at these high electron energies, electron displacement damage becomes a difficulty. The final limitation is that there are relatively few $1 \mathrm{MV}$ HVEMs available as compared to 100-120 kV TEMs. The HVEM technique has been used to characterize qualitatively the depth dependence of the damage structures in Ni-ion-irradiated 316 stainless steel $[98,99]$ and nicke1 alloys $[99,100]$. 
The second technique for determining defect profiles utilizes sectioning of many specimens to various depths followed by backthinning and TEM examination. In order to reduce the number of specimens required, this technique has been modified by a number of investigators. Evans [101] used a single specimen which was first back-thinned and examined. Then the irradiated surface was electropolished to remove a known depth of material and the specimen was reexamined. This process was repeated several times. The depth of material removed was measured by weight loss. F1at, circular specimen surfaces were assumed and polishing of the spectmen edges was neglected. Johnston et al. [102] used a technique similar to that of Evans. Instead of electropolishing the irradiated surface, however, a commerclal ion milling machine was used. An estimated accuracy of $\pm 10 \%$ for the amount of material removed during each ion miling operation was quoted. Yet another approach to this technique was used by Lee and Rowcliffe [103]. In their procedure, the bombarded surface was initially sectioned by covering a large portion of the surface with microstop lacquer and sectioning the exposed surface. By repeating the microstop-sectioning procedure for different areas on the irradiated surface, varying depths of removed material were obtained. The specimen was then back-thinned to perforation in one of these sectioned areas. After TEM examination, the first hole was covered with opaque lacquer and the specimen was back-thinned to perforation in another sectioned area. Once again, the major drawback of these various techniques is the difficulty in accurately determining the distance of the damage from the original irradiated surface.

The final method for determining the defect profiles is TEM examination of a cross-sectional area of a bombarded foil. In order to bring the damaged region to the center of the TEM cross-sectional specimens, it is first necessary to increase the cross-sectional area of the foil.

Many investigators have used electroplating of the base metal or an alloy constituent to increase the cross-sectional area of the foil. The plated specimen is then sliced normal to the irradiated surface. The resulting wafers are cut into $3 \mathrm{~mm}$ diameter disk specimens. These 
disks are electropolished from both sides to perforation at the interface of the electroplate and the irradiated surface. This technique was first used by Spurifing and Rhodes [104] in 1972. Since then, this basic technique has been used by a number of investigators to study damage profiles in stainless steel [104,105], nickel [106-113], copper [114-116], and iron [58]. Details of the electroplating vary in each investigation, but the major differences are (1) whether the surface was cleaned prior to electroplating and (2) how the wafers were sliced from electroplated specimens.

Henager et a1. [117] have used high rate sputtering to increase the cross-sectional area of irradiated molybdenum. Sputter etching was performed to clean the surface prior to sputter deposition of molybdenum on the specimen. Fabrication of TEM disks from the enlarged specimen was the same as for electroplated specimens.

The electroplating, cross-sectional area technique that does not utilize surface cleaning prior to plating probably provides more accurate damage-depth profiles than the other techniques. Since the irradiated surface remains intact, the depth measurements are more accurate than those obtained after sectioning. The largest drawback, as with all electroplating methods, is the difficulty in preparing the specimens.

For the present study, the depth distribution of the defect structures in $10 \mathrm{dpa}$, "triple-beam" ion irradiated iron (100 at. ppm He 410 at. ppm D) was determined using the electroplating, cross-sectional area technique. Detalls of the specimen preparation and triple-beam Ion irradiation technique and its application in the current investigation are found in Appendices $C$ and $D$. The sectioning depth for the iron and iron-chromium alloys was selected based on the defect profile for iron. The defect profiles for the iron-chromium alloys were not determined because of the difficulties in electroplating specimens with a chromium oxide surface layer.

\subsection{Electroplating Procedure}

The apparatus used for electroplating the fron specimens is pictured in Figure 4.1. The plating solution was ferrous chloride with 




Figure 4.1. Electroplating apparatus used for plating triple-beam irradiated iron. 
calcium chloride added at a ratio of 1 part $\mathrm{CaCl}_{2}$ to 2.6 parts $\mathrm{FeCl}_{2}$. For each liter of plating solution, $370 \mathrm{~g} \mathrm{FeCl}_{2}$ and $145 \mathrm{~g} \mathrm{CaCl}_{2}$ were used. The plating bath was held in a 1 liter Pyrex beaker. The plating was performed at $370-372 \mathrm{~K}\left(97-99^{\circ} \mathrm{C}\right)$, as measured by a standard Celsius thermometer. Heating was provided by a Briskeat high-temperature heating tape wrapped around the beaker. The temperature was controlled by varying the heat tape voltage with a Varlac autotransformer voltage regulator. The $\mathrm{pH}$ of the plating solution was maintained between 0.15 and 1.5 with additions of small amounts of hydrochloric acid. The pH was determined with a Beckman Zeromatic SS-3 pH meter.

To reduce evaporation during the plating, the beaker was covered with a Plexiglas lid. This lid was designed to fit securely in the top of the beaker without making contact with the plating bath. Holes were drilled in the lid for the thermometer, anode, and specimens. The spout of the beaker was plugged with Saran wrap. The spout provided easy access to the bath for removal of solution to check the $\mathrm{pH}$ and for additions of hot distilled water to counter losses due to evaporation and hydrochloric acid to correct the $\mathrm{pH}$.

The bath was constantly agitated during the plating by a Corning PC-351 hot plate magnetic stirrer. In the initial stages of determining the best plating procedure, a non-heating magnetic stirrer was used. This stirrer did not function very well for extended times at the temperatures resulting from contact with the hot plating bath container.

The anode used for the plating was a $3 \mathrm{~mm}(\sim 1 / 8 \mathrm{in}$.$) thick sheet$ of Ferrovac-E iron. This sheet was welded to a $3 \mathrm{~mm}$ diameter rod of low carbon steel. The rod fit tighty into a hole in the plexiglas cover descrlbed above. Use of an iron anode in a magnetically stirred bath presented some problems. If the anode was too long, the stircing bar was strongly attracted to the iron. This resulted in uneven stirring or, in the worst case, a halt in the stirring if the stirring bar attached itself to the anode.

The current density used for the plating was 1.0 to $10 \mathrm{kA} / \mathrm{m}^{2}$. The power supply used was a Universal Power Source (Mode1 6050A). The current was conducted to the specimens via a $0.18 \mathrm{~mm}(0.007 \mathrm{in.})$ 
diameter iron wire spot-welded to the edge of the specimen, as sketched in Figure 4.2(a). As shown in Figure 4.2(b) this wire was encased in a glass tube to prevent plating of the wire. The apparatus was designed to allow electroplating of up to three specimens simultaneously, with each specimen at the same distance from the anode.

In the actual plating of the specimens, the first layers of the plate were the most important. To facilitate the development of a good specimen-plate interface, the following procedure was used:

ORNL-Photo $0986-81$

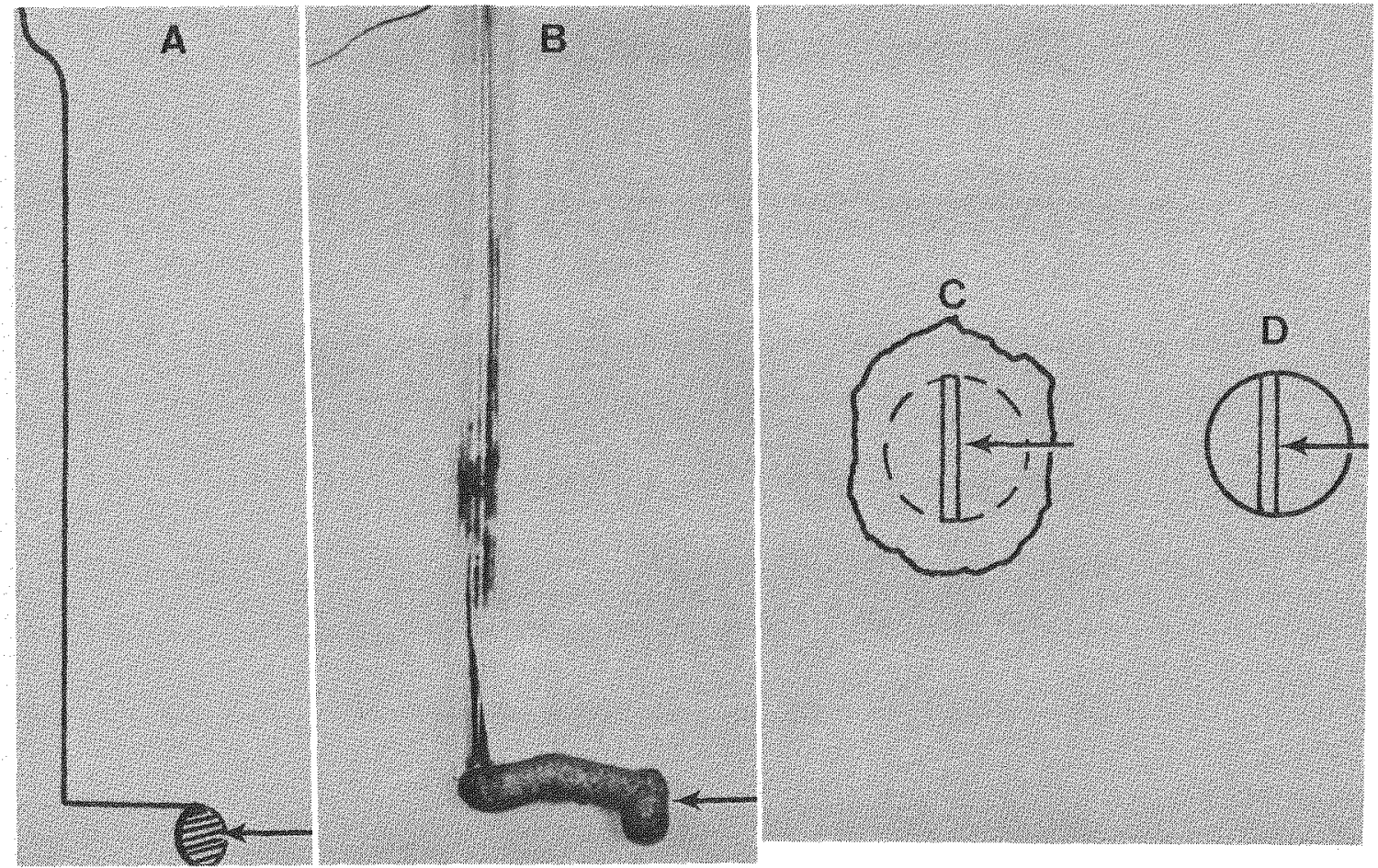

Figure 4.2. Steps in preparing a disk specimen using the electroplating technique. The irradiated surface is indicated by an arrow. (a) Sketch of the irradiated specimen spot-welded to iron wire. (b) Photograph of the plated specimen. Note that the iron wire was encased in a glass tube. (c) Sketch of a wafer cut from a plated specimen. (d) Sketch of a TEM disk electrodischarge machined from the wafer. Note that the original irradiated surface-electroplate interface is in the center of the disk. 
1. A fresh plating bath was used for each plating session. The bath was mixed, brought to the desired plating temperature, and held at temperature under agitation for at least 2 hours prior to starting the plating. If necessary, the bath pH was adjusted.

2 . During the 2 hour equilibrating period, the irradiated specimens were removed from the desiccator and wires were spot welded to the edge of each specimen with the irradiated surface forward (away from the wire). [See Figure 4.2(a)].

3. Before the specimens were inserted into the bath, they were cleaned with alcohol and acetone.

4. Al1 specimens to be plated were lowered into the bath simultaneously with the plating current turned on just before the specimens entered the bath.

In order to avold problems associated with variations in the level of the bath due to evaporation, the specimens were lowered 50 to $80 \mathrm{~mm}$ below the surface of the plating bath. Plating of 4 to $5 \mathrm{~mm}$ of iron required two to three days. The plating procedure was under constant observation for the first 2 hours and then checked every half hour for the next 10 hours. For the balance of the required plating time, the bath was checked about once per hour, except for the two overnight periods ( 8 hours). During these periods, the bath was not checked.

\subsection{TEM Specimen Preparation}

Wafers were cut with a high-speed silicon carbide slitting wheel from the electroplated specimens with slices normal to the original, irradiated surface. The wafers were cut with a thickness of $0.3 \mathrm{~mm}$. Three or four wafers were obtained from each electroplated specimen. In the balance of the specimen preparation, great care was taken to distinguish the irradiated surface interface from the unirradiated surface interface.

As shown in Figure 4.2(c) and 4.2(d), $3 \mathrm{~mm}$ diameter disks were cut from the wafers. The wafers were sanded and given a light electropolish to expose the irradiated surface-electroplate interface. The 
wafers were then glued to a copper block with a mixture of graphite and Du Pont DUCO cement. The copper block was clamped in a Hansvedt SM 120 a electrodischarge machine (EDM) equipped with a hollow tube graphite tool for cutting $3 \mathrm{~mm}$ diameter disks. This tool was specifically designed for precision fabrication of $3 \mathrm{~mm}$ diameter disks by L. Turner of ORNL. The tool was machined from POCO EDM-3 Graphite, a high conductivity, fine grain and wear resistant graphite manufactured by PocoGraphite, Inc., a subsidiary of Union 0il Company [118]. The wafer was aligned carefully in the EDM so that the irradiated surfaceelectroplate interface would be in the center of the $3 \mathrm{~mm}$ diameter disk [Figure 4.2(d)]. Only one disk specimen was obtained from each wafer.

Final electropolishing was performed in a Tenupole jet electropolishing apparatus. An A-3 polishing solution $[600 \mathrm{~m} 1$ methyl alcohol, $360 \mathrm{ml}$ ethylene g1ycol monobutyl ether (butylcellusolve), $60 \mathrm{ml}$ perchloric acid and $2 \mathrm{ml}$ Solvent $\mathrm{X}]$ cooled to $255 \mathrm{~K}\left(-18^{\circ} \mathrm{C}\right)$ was used with a polishing current of $90 \mathrm{~mA}$. The two-side electropolishing removed any deformation introduced by the high-speed slitting wheel.

The disk specimen was placed in a standard Tenupole holder with the irradiated surface-electroplate interface in the center of the polishing area. If perforation did not occur at the interface, the hole was covered with MOBILE Mudge, an opaque lacquer, and the specimen was repolished. After electropolishing, the specimen was rinsed in acetone and 200 proof ethyl alcohol. The electropolished specimens were stored in a vacuum desiccator continuously evacuated by a mechanical pump.

The TEM specimen preparation procedure had about a $30 \%$ success rate. Specimens with irradiation temperatures of 770,850 , and $900 \mathrm{~K}$ were plated. Of these, only TEM specimens from the plated $850 \mathrm{~K}$ specimen have been successfully prepared and examined. (For details of the irradiation parameters for the $850 \mathrm{~K}$ specimen, see Appendix D, run 534 , specimen 32. )

\subsection{TEM Observations}

The TEM specimens were examined in a JEM 120C TEM equipped with a special objective lens pole piece (AMG) for the observation of magnetic 
materials. Details of the microscopy are outlined in Appendix B. Two disks from the iron specimen irradiated at $850 \mathrm{~K}$ were examined in detail. The results described below were obtained from several areas with different orlentations in these specimens.

In genera1, the damage microstructures consisted of both dislocations and cavities. As shown in the TEM micrograph in Figure 4.3, the microstructural distributions varied significantly with distance from the irradiated surface-electroplate interface. As shown in Figure 4.4, the cavities had a crystallographic shape. There was a zone denuded of cavities from the interface to $0.1 \mu \mathrm{m}$ from the interface, $a$ typical observation in ion damage profiles. The major form of the dislocation microstructure was a coarse network. A few dislocation loops appear with the network at 1.6 to $2.2 \mu \mathrm{m}$ from the interface. A higher concentration of loops is present within a discrete band extending from 2.4 to $3.4 \mu \mathrm{m}$ from the interface. The loops in both regions were ana1yzed using the method of Maher and Eyre [97] (Appendix B). The 1oops were determined to be interstitial in nature with predominantly a<100> Burgers vectors. A few $2 / 2\langle 111\rangle$ Burgers vectors were also observed in each of the two loop regions.

The quantitative data for the dislocation density and the cavity parameter (volume-averaged diameter and cavity concentration) profiles were measured for each of seventeen $0.2 \mu \mathrm{m}$ wide regions covering the entire width of the damaged region. The values obtained for each region are plotted at the midpoint of the region in Figure $4.5(\mathrm{a})$ for the cavity parameters and in Figure $4.5(\mathrm{c})$ for the dislocation density. The dislocation density, $\Lambda$, was measured using the line intersection method (see Appendix B). The dislocation loops were included in the dislocation density measurement. The diameter of the individual cav1ties was measured assuming a circular projection of approximately the same area as the projected crystallographic shape. The cavity volume fraction (CVF) or swelling was calculated from the volume average diameter, $d_{c}$, and the cavity concentration, $C_{c}$, according to the relationship:

$$
\operatorname{CVF}(\%)=\left(\frac{C_{c} \pi d_{c}}{6}\right) \times 100
$$




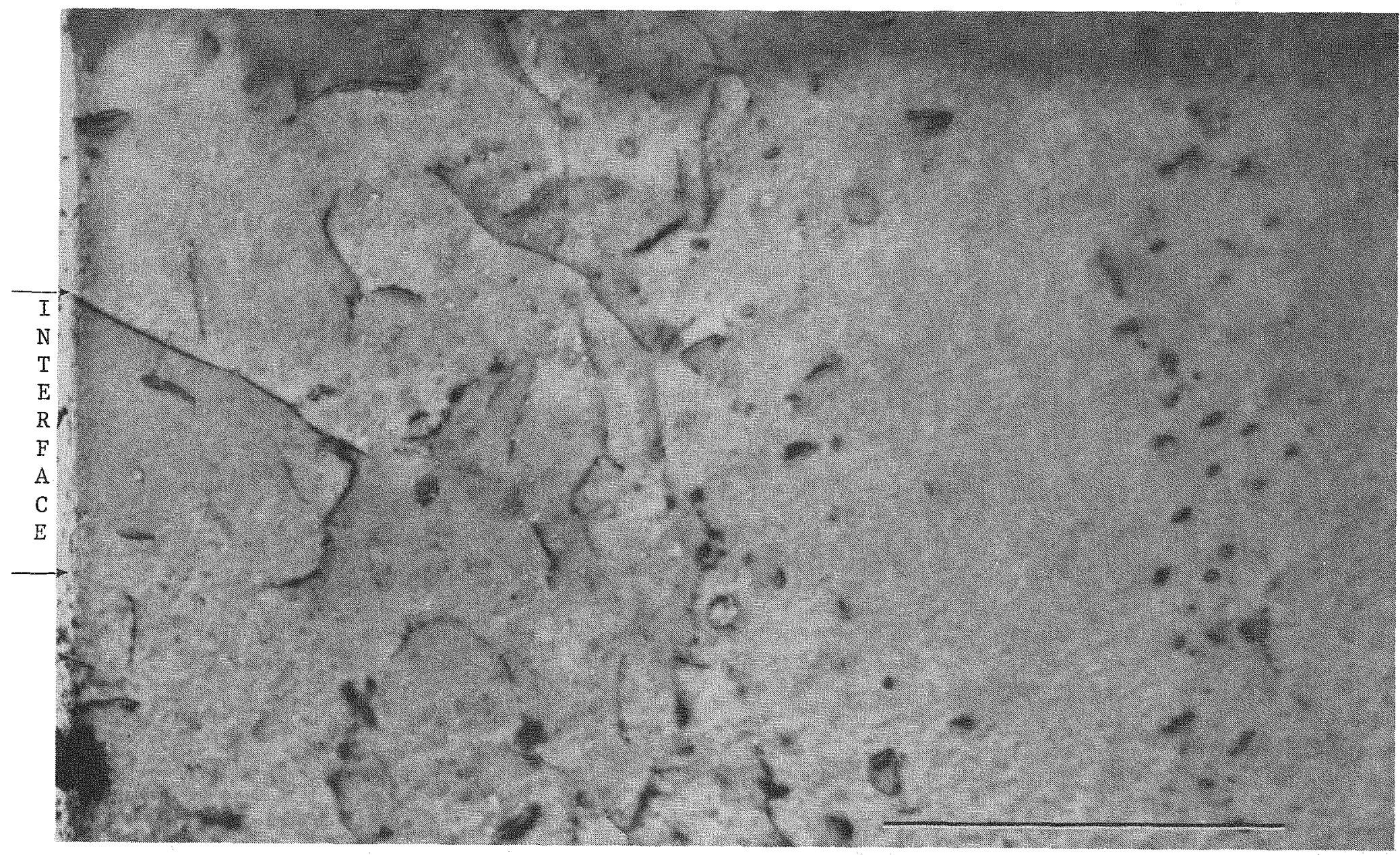

Figure 4.3. TEM micrograph of the damage profile in iron, "triple-beam" ion-irradiated at $850 \mathrm{~K}$. Scale marker is $1 \mu \mathrm{m}$. 


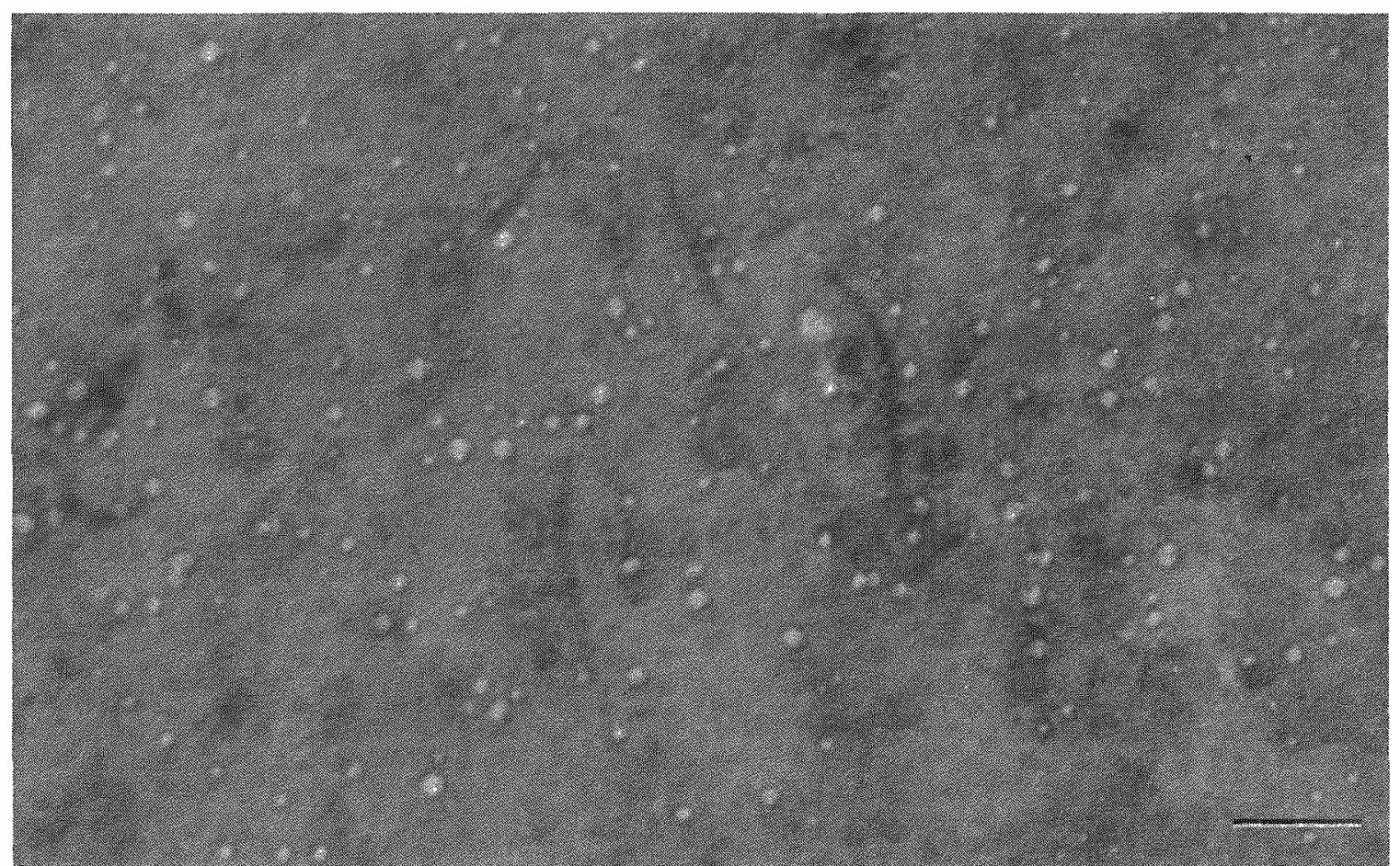

Figure 4.4. TEM micrograph showing the crystallographic shape of cavities found in the damage profile for iron. Scale marker is $100 \mathrm{~nm}$.

The swelling for each region is plotted with the cavity parameters in Figure $4.5(\mathrm{a})$. The swelling was a maximum of about $0.22 \%$ over a range of depths from 0.9 to $1.1 \mu \mathrm{m}$ from the interface. The detalls of the cavity parameter measurement and error calculations are found in Appendix B.

To allow direct comparison of the damage distribution with the quantitative damage profiles, the micrograph of Figure 4.3 is included in Figure 4.5 .

\subsection{Discussion}

No damage profiles for a triple-beam irradiated material are reported in the 1iterature. Damage profiles are reported for only three three dual Ion beam (heavy Ion + gas fon) irradiations. Henager et al. [117] have reported a damage profile for molybdenum irradiated simultaneously with $5 \mathrm{MeV} \mathrm{Ni}^{++}$and $0.2 \mathrm{MeV} \mathrm{He}^{+}$1ons. Farrel1 et al. [111] and 
ORNL-Photo $5116-81$
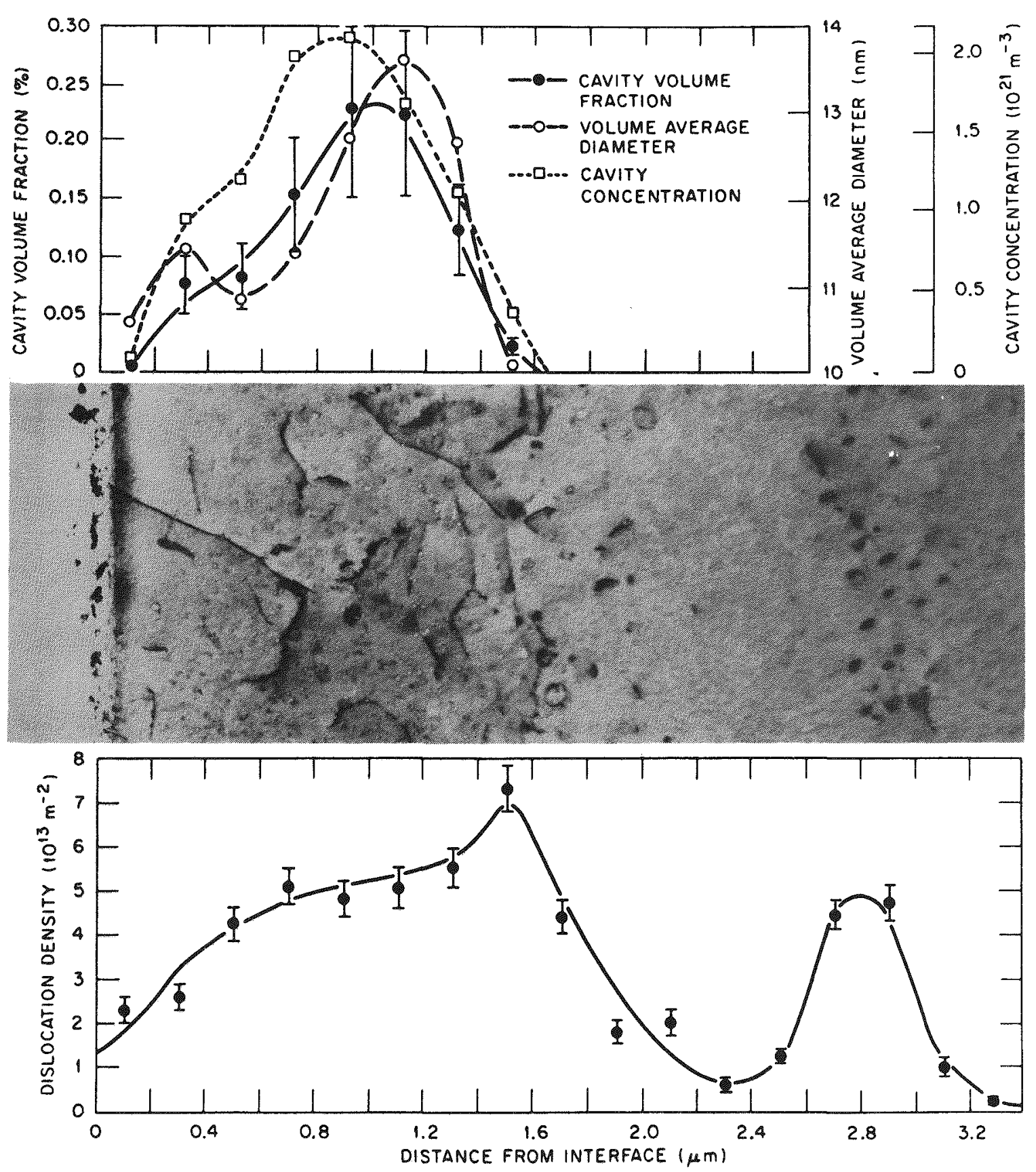

Figure 4.5. The damage profiles for triple-beam 1on-irradiated iron. The distances from the interface indicated on the horizontal axis at the bottom of the figure apply to the micrograph, the dislocation density profile, and the cavity parameter profiles. 
Packan et al. [110] have reported damage profiles for nickel simultaneously irradiated in the ORNL facility with a dual beam of $4 \mathrm{MeV} \mathrm{Ni}$ ions and $\mathrm{He}^{+}$ions whose energies were ramped from 0.2 to $0.4 \mathrm{MeV}$. The only damage profiles available for iron are the currently unpublished results of Kuramoto et al. [58]. These profiles for iron are for single $4 \mathrm{MeV} \mathrm{Ni}{ }^{++}$ion bombardments.

The qualitative appearance of the damage profiles observed in the current investigation agrees with those reported in the literature for dual ion irradiations of other materials with one exception. The band of dislocation loops centered at $\sim 2.8 \mu \mathrm{m}$ from the interface is a unique observation. The possible origins of this band will be discussed later in this section.

\subsubsection{Comparison of Calculated and Experimental Damage Profiles}

In order to select a sectioning depth, the profiles were compared to the calculated deposited energy and ion depth profiles for $4 \mathrm{MeV} \mathrm{Fe}^{+H}$ ion bombardment of iron shown in Figure 4.6. These profiles were plotted from the deposited energy and ion profiles calculated by the E-DEP-1 computer code of Manning and Mueller [119]. The E-DEP-1 output is tabulated with the calculated dpa profiles in Appendix $E$. The dpa(x) $=C S_{D}(x)$, where $C$ is a constant and $S_{D}(x)$ is the deposited energy. Therefore, the normalized deposited energy profile in arbitrary units (i.e., normalized to 1 ) is equivalent to the dpa profile. Figure 4.6(a) contains the profiles obtained for an electronic stopping power, $k$, of 0.156 , the LSS (Lindhard, Schraff, and Schiott) value for $k$. Figure $4.6(b)$ and $4.6(c)$ contains the profiles for $k=0.121$ and 0.108 , respectively.

A comparison of the experimentally obtained profiles in Figure 4.5 and the calculated profiles in Figure 4.6(a) indicates that the peak swelling corresponds to the peak of the deposited lons rather than the expected correspondence to the peak in the deposited energy (dpa). As shown in Figure $4.6(\mathrm{~b})$, if the value for $k$ is reduced by $22 \%$ to 0.121 , the maximum in the deposited energy calculated using E-DEP-1 is 


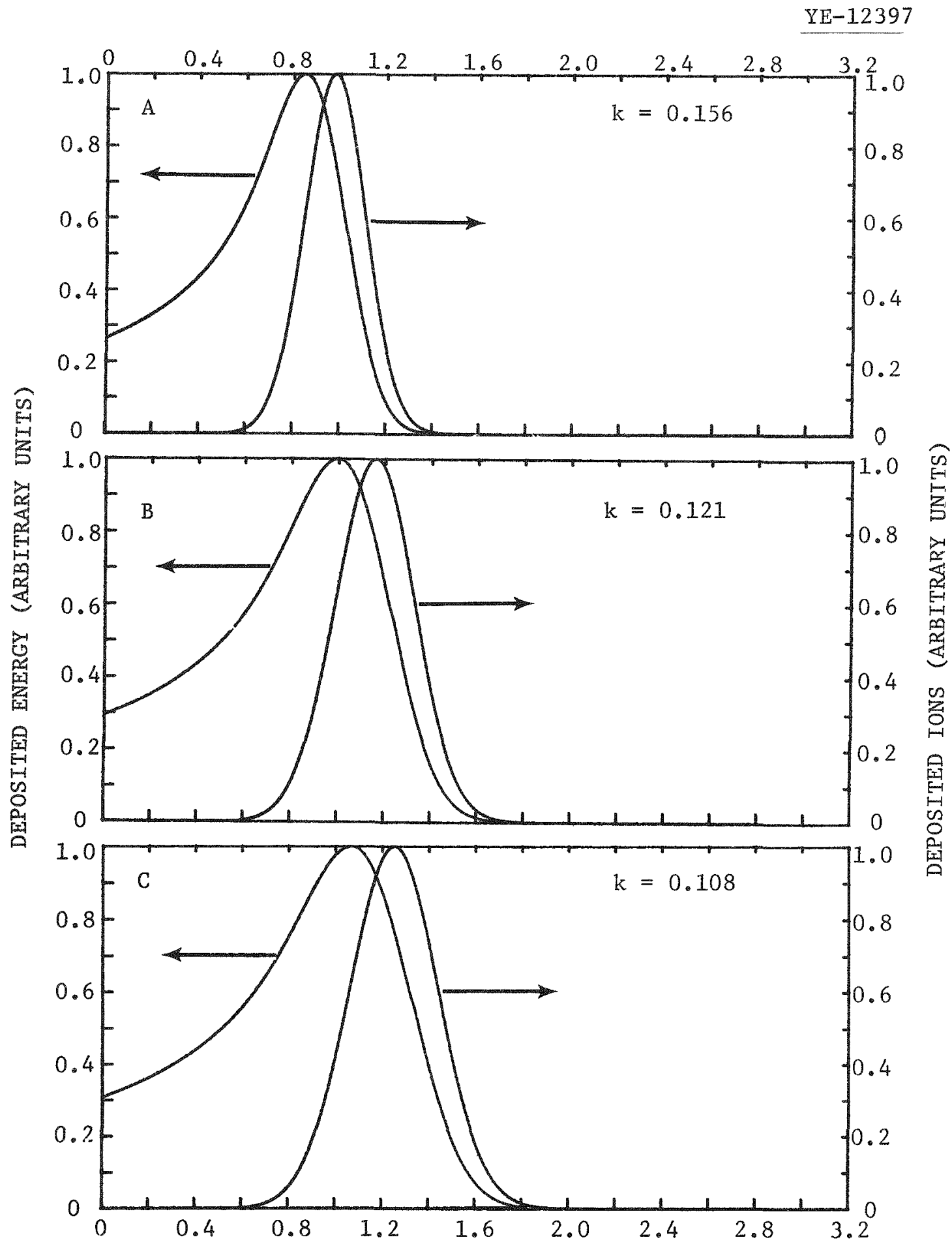

Figure 4.6. Deposited energy and deposited ion profiles calculated with $\mathrm{E}-\mathrm{DEP}-1$ computer code. $\mathrm{k}=$ electronic stopping power. 
at $1.0 \mu \mathrm{m}$ from the irradiated surface. This is the approximate location of the observed swelling peak.

The possibility of an overestimate in the LSS theoretical value for $k$ has been suggested by other investigators. Narayan and Oen [106] observed that the maximum in the point defect density profile was $18 \%$ deeper in nickel irradiated with $4 \mathrm{MeV} \mathrm{Ni}^{++}$Ions than the location of the maximum of the deposited energy profile predicted by E-DEP-1. Similar observations were reported by Narayan et al. for copper irradiated by $4 \mathrm{MeV} \mathrm{Ni}{ }^{++}$ions [114] and by 5, 16, 27, and $38 \mathrm{MeV}$ copper lons [116]. For the nickel ion irradiations, the calculated depths agreed with the experimental observation if $k$ was reduced by $22 \%$ for nicke1 [106] and $28 \%$ for copper [114]. For copper ion irradiations of nickel, the amount of reduction in $k$ required for the calculated profile to agree with the experimental profile varied with the ion energy. A reduction in $k$ of $28 \%$ was required for $5 \mathrm{MeV}$ ions, $16 \%$ for $16 \mathrm{MeV}$ ions, and $9 \%$ for $27 \mathrm{MeV}$ ions. No reduction was required for $38 \mathrm{MeV}$ copper lons [116].

Narayan and co-workers believe that the lower values of $k$ required to achieve agreement between calculated and experimental profiles are related to the oscillations of the electronic stopping when plotted as a function of the target atomic number, $z_{2}$, and the bombarding ion atomic number, $z_{1}$. The " $z_{2}$ oscillations" have a local minimum at $z_{2}=29$ (copper) $[120,121]$. For $4 \mathrm{MeV}$ ion energies, the curve is approximately level between $z_{2}=26$ (nickel) and $z_{2}=28$ (iron). The experimental results for copper and nickel, indicating a larger overestimate of $k$ for copper, are in good qualitative agreement with the $z_{2}$ oscillation curve. With increasing ion energy, the $z_{2}$ oscillations are reduced, as indicated by the lower reductions in $k$ required for higher ion energies.

The " $z_{1}$ oscillations" have a local minimum at $z_{1}=31$ (gallium) [122]. Both nickel and copper lons lie near to the minimum and could contribute to a lower stopping power requirement. However, in the energy range of these and the current studies, the $z_{2}$ effect is believed to be more important than the $z_{1}$ effect [123]. If the difference in the bombarding ion species is neglected, the level region from $z_{2}=26$ to 28 on the $z_{2}$ oscillation curve indicates that the percentage 
of overestimation of $k$ should be about the same for $4 \mathrm{MeV}$ ion bombardment of iron and nickel targets. This supports the current observation that a $22 \%$ reduction in $\mathrm{k}$ for iron leads to agreement between the calculated deposited energy and experimental profiles.

However, the damage profiles for triple-beam irradiated iron are more complex than the profile for low dose single lon irradiations such as those used by Narayan and co-workers. The cavity profile is affected by many factors other than the deposited energy from the heavy ions. Deposited interstitials, diffusional spreading, and gas concentration influence the observed cavity profiles [124].

The deposited interstitials are believed to have the most impact on the location of the swelling peak. It has been suggested by Mansur and Yoo [124] that, in a low swelling material such as iron, the deposited interstitials will cause the swelling peak to be closer to the irradiated surface than the deposited energy peak. In considering the current swelling profile for iron, this effect of the deposited interstitials implies that the maximum in the deposited energy profile is even further from the irradiated surface than that calculated previous$1 y$ for $k=0.121$. Calculations by Beeler and Beeler [125] for fcc iron indicate that a value of $k=0.108$ is more accurate than the E-DEP-1 value. Use of this value for $k$ as input for E-DEP-1 (with the density of bcc Iron as an input parameter) gave the maximum in the deposited energy at $1.06 \mu \mathrm{m}$ and a deposited ion peak at $1.25 \mu \mathrm{m}(\sigma=0.19 \mu \mathrm{m})$ from the Irradiated surface as is shown in Figure 4.6(c). (Details of the $E-D E P-1$ results for $k=0.108$ are found in Appendix $E_{0}$ ) The maximum effect of the deposited interstitials would be within $\pm \sigma$ of the ion peak, 1.06 to $1.44 \mu \mathrm{m}$, where $\sim 68 \%$ of the deposited interstitials are found. A lesser effect would be seen from $\sigma$ to $2 \sigma, 0.87$ to $1.06 \mu \mathrm{m}$ and 1.44 to $1.63 \mu \mathrm{m}$, where $\sim 27 \%$ of the deposited interstitials are located. In considering the region from 0.9 to $1.1 \mu \mathrm{m}$, the swelling would be least affected by the deposited interstitials at $0.9 \mu \mathrm{m}$, with an increasing effect up to $1.1 \mu \mathrm{m}$, where the peak in the deposited energy is found. This could lead to only slight variations in the swelling profile from 0.9 to $1.1 \mu \mathrm{m}$, as is observed. The combination of the decreasing deposited energy and the strong interstitial effect could 
cause the observed rapid decrease in the swelling at depths greater than $1.1 \mu \mathrm{m}$.

Diffusional spreading refers to the migration of vacancies and interstitials to the free surface and to depths beyond the deposited energy profile. The usual manifestation of diffusional spreading of vacancies is a broadening of the cavity profiles and reduced cavity growth near to the surface. In the current profile, due to the low swelling and strong effect of the deposited interstitials, it is unlikely that diffusional spreading would lead to cavity growth beyond the range of the deposited energy from the heavy ions. The effect of the surface on the profile is indicated by the lack of cavities within 0.1 $\mu \mathrm{m}$ of the surface.

The effect of the deposited gas is more difficult to ascertain. From comparisons of $\mathrm{Fe}-10 \% \mathrm{Cr}$ specimens bombarded with a triple-beam of ions and specimens irradiated only with $4 \mathrm{MeV} \mathrm{Fe}^{++}$ions, the effect of the gas has been observed to be increased cavity density, decreased cavity diameters and increased swelling (see Chapter 5). The slight decrease in the cavity diameter shown in Figure 4.6 at $\sim 0.5 \mu \mathrm{m}$ could be attributed to the effect of the implanted gas. There is, however, no obvious indication in the profile of the effect of the gas at the deeper regions. In fact, the cavity profiles are smooth, suggesting that either the gas is having little effect on the profiles or that any indication of the decreasing gas concentration is masked by the effect of the deposited interstitials and the decreasing damage rate. The latter suggestion implies that the gas concentration levels do not change significantly over the region from 0.9 to $1.1 \mu \mathrm{m}$. Further support of this conclusion is provided by the location of the peak cavity concentration within this region. As shown in Figure 4.7, the calculated depths for the region of uniform helium and deuterium deposition are $\sim 0.65$ to $0.9 \mu \mathrm{m}$. (Details concerning the deposited helium and deuterium profiles can be found in Appendix $D_{0}$ ) However, the location of the deposited ions is calculated from the stopping powers for hellum and deuterium in iron, which in the energy range for these irradiations, is not well known. In fact, the few experimental values for the stopping powers reported have differences of up to $20 \%$ for helium and 


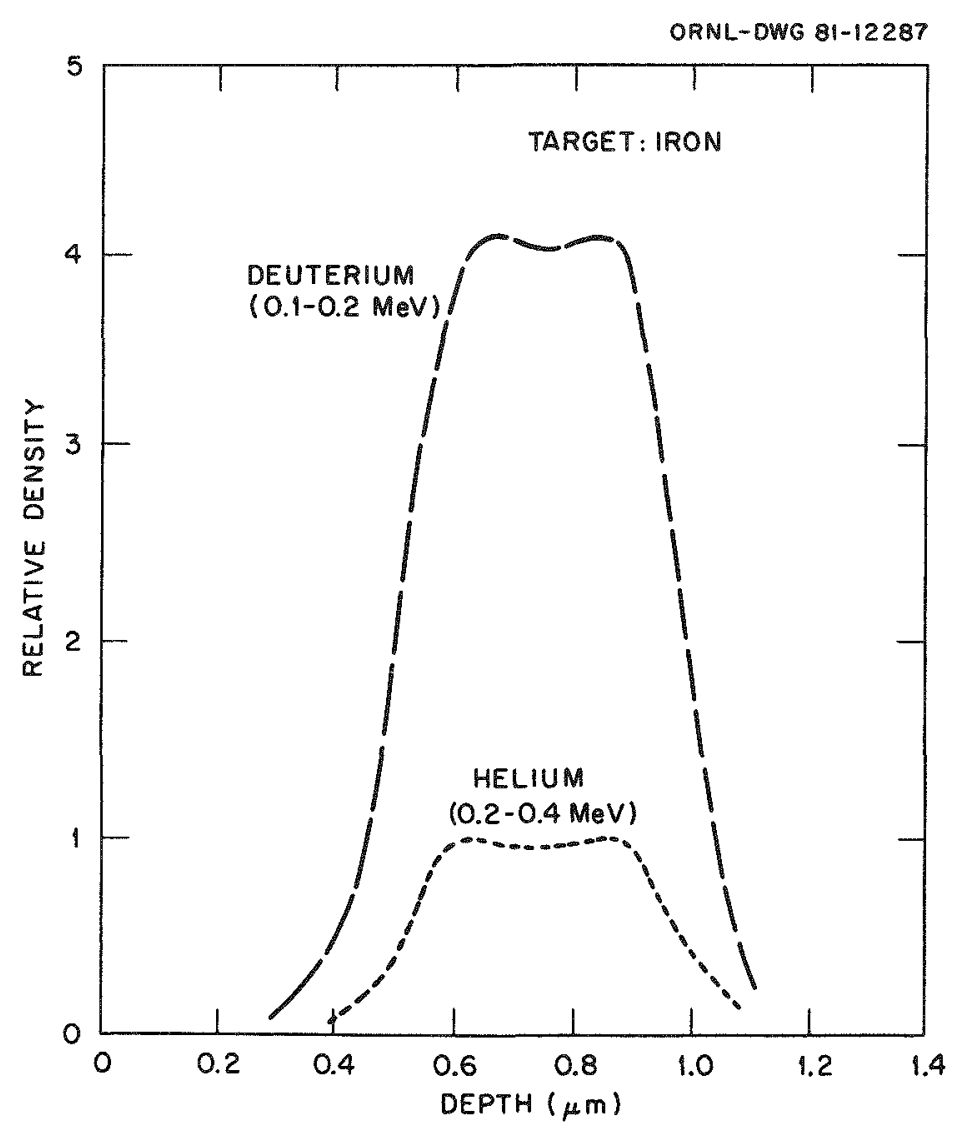

Figure 4.7. Calculated helium and deuterium profiles for an iron target with an ion beam consisting of $\mathrm{He}^{+}$and $\mathrm{D}_{2}^{+}$with sinusoidally ramped energies $\left(0.2\right.$ to $\left.0.4 \mathrm{MeV}, 2.5 \times 10^{-2} \mathrm{~Hz}\right)$. The $\mathrm{D}_{2}^{+}$splits to atomic species ( 0.1 to $0.2 \mathrm{MeV}$ energies) upon impact with target.

$10 \%$ for deuterium $[86,126]$. Therefore, a significant error in the light ion ranges is possible. A $20 \%$ error in the ranges would increase the depth of the region of uniform helium and deuterium deposition from $\sim 0.8$ to $1.1 \mu \mathrm{m}$, as suggested above.

The calculated profile which leads to the deepest damage profile is that for $\mathrm{k}=0.108$ [Figure $4.6(\mathrm{c})$ ]. Even in this case, the deposited energy is less than $0.0001 \mathrm{MeV} / \mu \mathrm{m}-10 \mathrm{n}$ for depths greater than $\sim 2.0 \mu \mathrm{m}$. The damage is less than $0.01 \mathrm{dpa}(10 \mathrm{dpa}$ at the peak) for depths greater than $\sim 1.9 \mu \mathrm{m}$. In the current profile, irradiationinduced dislocation microstructures were observed in the low damage region at depths up to $2.2 \mu \mathrm{m}$ (excluding the discrete band of dislocation loops at 2.4 to $3.4 \mu \mathrm{m}$ ). 
The presence of dislocations in the low damage regions in dualion damage profiles is not unusual. Henager et al. [117] have observed dislocation loops at 2 to $3 \mu \mathrm{m}$ from the irradiated surface in molybdenum bombarded with $5 \mathrm{MeV}$ nickel and $0.2 \mathrm{MeV}$ helium ions. The peak in the deposited energy profile for $5 \mathrm{MeV}$ nickel ions in molybdenum was calculated by E-DEP-1 (LSS value of $k$ ) to be $0.9 \mu \mathrm{m}$ from the irradiated surface. In the experimental damage profile, the largest dislocation loops and the coarsest distribution was found at $2 \mu \mathrm{m}$, with the 1oop diameter decreasing with increasing depth. This behavior can be explained as an effect of the decreasing dose and dose rate within the damage tail coupled with diffusional spreading of the interstitials. At the higher displacement levels, i.e., closer to the surface, the loops appeared to have agglomerated to form a network with a consequent reduction in the dislocation density. In the current profile for iron, this type of dislocation microstructure variation was observed in the major portion of the damage profile from the surface to $2.2 \mathrm{\mu m}$. From the surface to $1.6 \mu \mathrm{m}$, a coarse dislocation network was observed. From 1.6 to $2.2 \mu \mathrm{m}$, dislocation loops were observed in the distribution analogous to those observed by Henager et al. in molybdenum.

The discrete band of dislocation loops observed in iron at 2.4 to $3.4 \mu \mathrm{m}$ from the irradiated surface is not easily explained. This band cannot be confused with the loops observed by Henager et al. [117]. Although both groups of dislocation loops are located at about the same distance from the surface, the band of loops in iron does not have the distinctive size distribution observed for the loops in molybdenam. Also, as discussed above, the dislocation distribution in the major portion of the damage profile for iron correlates well with observations of Henager et al.

Farre11 et al. [111] have observed a discrete band of dislocations resembling a creep cell wall at $2.0 \mu \mathrm{m}$ from the irradiated surface in damage profiles for nickel bombarded with $4 \mathrm{MeV}$ nickel ions and energetically ramped $(0.2$ to $0.4 \mathrm{MeV}$ ) helium ions (ORNL facility). They believe that this band is caused by the movement and accumulation of dislocations caused by swelling-induced stresses. In the dislocation band observed in iron, the majority of the loops had Burgers vectors 
of $a\langle 100\rangle$. There was no evidence of network dislocations in this region. This observation implies that the stress caused by the swelling in iron was not sufficient to cause the migration of network segments from the major part of the damaged zone to deeper regions. It therefore seems unlikely that the stress would be sufficient to cause dislocation loops nucleated within the major damage region to move to the depth of the dislocation band. In fact, it has been suggested that a<100> loops are essentially sessile $[51,60]$.

Channeling has also been considered as a possible explanation for the dislocation band. However, the same features have been observed in differently oriented grains. Yet another possible cause could have been an external stress. This explanation can be rejected due to the lack of similar dislocation loop observations in the damage profiles of other investigators using the same irradiation facility and specimen loading procedures. Another suggestion considered was that the loops were vacancy loops resulting from diffusion of vacancies to this region. Analyses of the nature of the loops indicated, however, that the loops were interstitial in nature. Finally, although the profile of the band was not consistent with the usual damage profile, the possibility of an extraneous energetic ion causing the loop formation was considered. Referring to the tabulated ion ranges found in the Handbook of Range Distributions for Energetic Ions in AII Elements [85], no ions were found to have the correct range within the energy range and mass selection range of the accelerators. The remote possibility of a $0.4 \mathrm{MeV} \mathrm{D}_{2}$ splitting and giving all of its energy to a single deuteron, rather than creating two $0.2 \mathrm{MeV}$ deuterons, was considered. However, the range of $0.4 \mathrm{MeV}$ deuterons is only $\sim 1.8 \mu \mathrm{m}$. Even with a $20 \%$ range error, this depth is increased only to $2.1 \mu \mathrm{m}$ - not deep enough to account for the dislocation loop band.

In conclusion, no likely explanation was found for this discrete band of loops. Further experiments (e.g. - fluence and temperature dependence), which were deemed beyond the scope of this dissertation, are required to more completely characterize the band of dislocation 1oops. Hopefully, with this additional information, a satisfactory explanation of the origin of the band can be derived. 


\subsubsection{Sectioning Depth}

Based on the above discussion, a sectioning depth of $0.9 \mu \mathrm{m}$ was selected for the triple-beam ion-irradiated iron, $\mathrm{Fe}-10 \% \mathrm{Cr}$, and $\mathrm{Fe}-5 \%$ Cr specimens. This sectioning depth allowed TEM (120 kV) examination of the damage from 0.9 to $1.1 \mathrm{\mu m}$ from the irradiated surface. In the region extending from 0.8 to $1.20 \mu \mathrm{m}$, both the swelling and the dislocation density have only small variations in their respective values in the damage profiles. Therefore, an error of $\pm 0.05 \mu \mathrm{m}$ in the sectioning depth would not adversely affect the quantitative swelling and dislocation density data. This section depth error would also result in less than a $10 \%$ error in the cavity concentration and diameter data. Therefore, the quantitative data obtained from a sectioned specimen would be reasonably accurate if the sectioning depth was between 0.85 and 0.95 $\mu \mathrm{m}$, allowing for small experimental measurement and sectioning errors. 
$x$

-

- 
CHAPTER 5

TRIPLE-BEAM ION IRRADIATION EXPERIMENTS

A TEM study of the defect structures produced in "triple-beam" ion-irradiated $\mathrm{Fe}-10 \% \mathrm{Cr}, \mathrm{Fe}-5 \% \mathrm{Cr}$, and $\mathrm{Fe}$ specimens is presented in this chapter. The alloy fabrication, specimen preparation, irradiation procedure, and irradiation parameters are discussed in detail in Appendices $C$ and D. The irradiations utilized a "triple-beam" of $4 \mathrm{MeV}$ iron ions and energetically ramped $(0.2$ to $0.4 \mathrm{MeV})$ helium and deuterium 1ons. The gas concentration levels were 10 at. $\mathrm{ppm} \mathrm{He} / \mathrm{dpa}$ and 41 at. $\mathrm{ppm} \mathrm{D} / \mathrm{dpa}$. These are the concentration-to-dpa ratios expected for ferritic stainless steels in a fusion reactor first wal1 [2,127]. In the first set of irradiations (Runs 532-537), specimens of all the materials were bombarded at temperatures of $725,775,800,850,900$, and $950 \mathrm{~K}$ to $10 \mathrm{dpa}$, the approximate displacement damage expected for 1 megawatt year $/ \mathrm{m}^{2}$ of reactor service [127]. The goal of this experiment was to determine the irradiation temperature at which the maximum swelling occurred, to study the effect of chromium content on the damage microstructure, and to select a single material for the balance of this study. In the second set of irradiations (Runs 592, 593,596, and 601), specimens of the selected material, Fe-10\% Cr, were irradiated at the peak swelling temperature $(850 \mathrm{~K})$ to displacement damage levels of $0.3,1,3,10,30$, and $100 \mathrm{dpa}$. In this experiment, the evolution of damage with increasing dpa was studied. A third experiment (Runs 594 and 602) was conducted without utilizing the triple-beam irradiation technique in order to study the effect of helium and deuterium implantation on the damage in $\mathrm{Fe}-10 \% \mathrm{Cr}$. In Run 594, the light ions were pre-injected at room temperature to $100 \mathrm{at}$. ppm He and 410 at. ppm $D$, followed by irradiation to $10 \mathrm{dpa}$ with $4 \mathrm{MeV}$ iron ions at $850 \mathrm{~K}$. Run 602 consisted of a single, $4 \mathrm{MeV}$ iron ion irradiation to 10 and 30 dpa at $850 \mathrm{~K}$. 
The specimen preparation for TEM examination consisted of sectioning to $0.9 \mu \mathrm{m}$, followed by back-thinning. The exact procedures used are presented in Appendix D. A JEM 120C TEM, equipped with an AMG objective lens pole piece for the observation of magnetic specimens, was used for the majority of the examinations. A JEM 120CX and Philips 400T-FEG (Field Emission Gun) were used for analytical and high magnification microscopy. When required, a Hitachi $1 \mathrm{MeV}$ HVEM was employed for examinations of specimens. Details concerning the microscopy procedures and measurement of the quantitative microstructural data are located in Appendix B. In this chapter, the microstructural observations will be presented in the following sequence:

1. the microstructure of unirradiated specimens,

2. the temperature dependence of the damage microstructures,

3. the evolution of the microstructure with increasing damage levels,

4. the effect of chromium content on the damage microstructure, and

5. the effect of the gas on the damage microstructures.

\subsection{Unirradiated Microstructures}

The dislocation density in the as-prepared, unirradiated specimens of $\mathrm{Fe}, \mathrm{Fe}-5 \% \mathrm{Cr}$, and $\mathrm{Fe}-10 \% \mathrm{Cr}$ was $\sim 10^{12} \mathrm{~m}^{-2}$. Precipitates were found at the grain boundaries in the $\mathrm{Fe}-5 \% \mathrm{Cr}$ and $\mathrm{Fe}-10 \% \mathrm{Cr}$ specimens. Various techniques were employed to characterize these precipitates. Preliminary analyses of in-foll precipitates using standard $S A D$ (Selected Area Diffraction) techniques were not successful. The only conclusion from these studies was that the lattice parameter of the precipitate was probably not as large as that of many carbides such as $M_{23} C_{6}\left(a_{0}=10.621 \AA\right)$ and $M_{6} C\left(a_{0}=11.082 \AA\right)$. Energy Dispersive $X$-Ray Spectroscopy (EDS) studies of in-foil precipitates indicated that the precipitates were rich in chromium. A comparison of the spectrum obtained for a precipitate and the matrix is shown in Figure 5.1. Electron Energy Loss Spectroscopy (ELS) studies of thin, in-foil, precipitates also yielded inconclusive results. Few precipitates were thin enough to be analyzed. Additional difficulties were encountered due to a temporary electronics problem in the ELS system. The position 


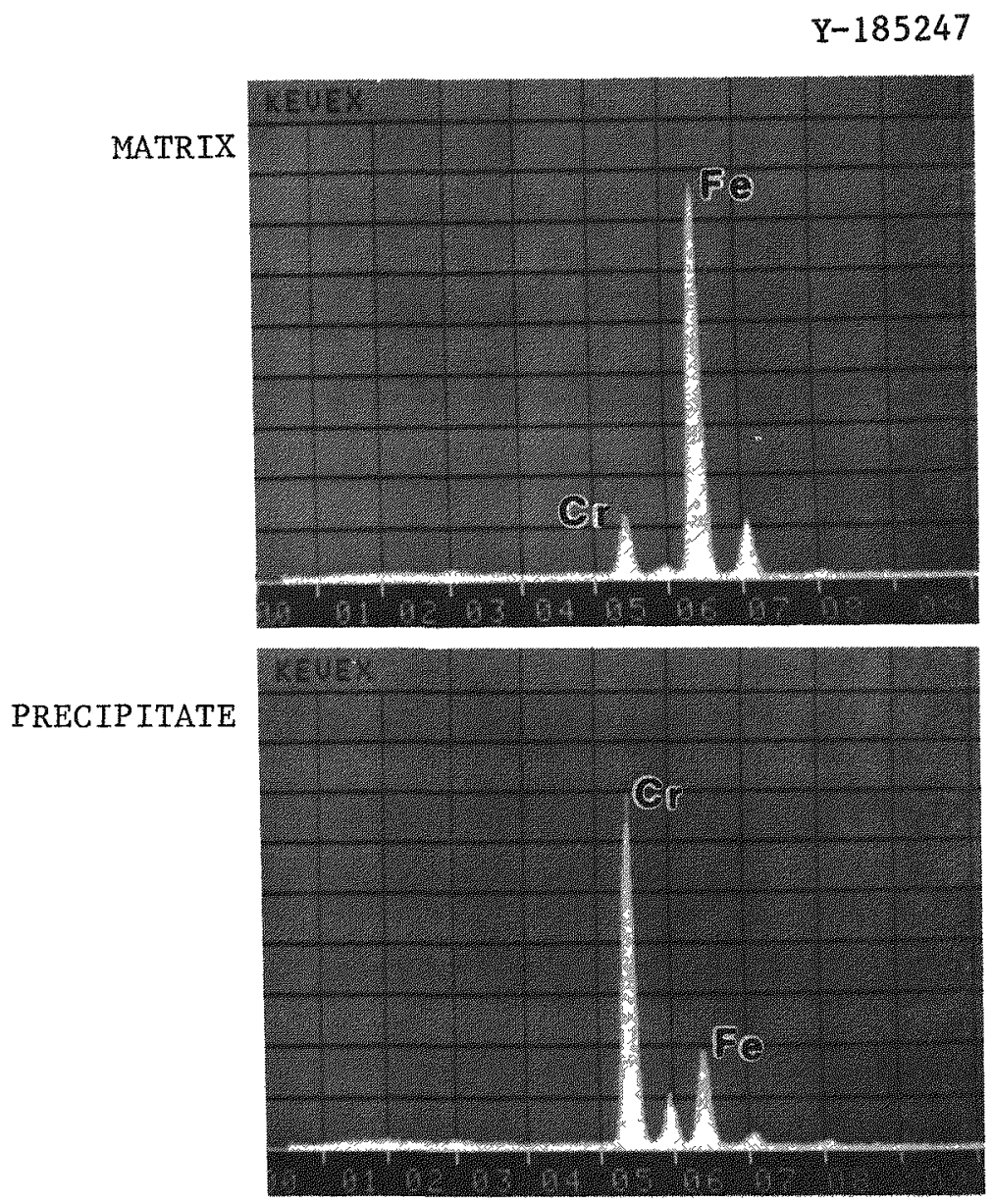

Figure 5.1. EDS spectra obtained from the matrix and an in-foil precipitate. $\mathrm{Fe}-10 \% \mathrm{Cr}$.

of the carbon edge was masked by an electronically generated edge eliminating any possibility of identifying small amounts of carbon in the spectrum. This electronic artifact also prevented any quantitative analysis of the edges that were observed since the background fitting routines could not accommodate 1t. The only additional information obtained from this analysis was that nitrogen was present in at least one of the precipitates. A comparison of the ELS spectrum obtalned for this precipitate and the matrix is shown in Figure 5.2.

In a final effort to analyze the precipitates, a carbon extraction replica of an $\mathrm{Fe}-10 \% \mathrm{Cr}$ specimen was made. Standard techniques were employed for replica fabrication. First, an Fe-10\% Cr specimen was 1ightly etched in a $10 \% \mathrm{HC}-90 \% \mathrm{H}_{2} \mathrm{O}$ (distilled) water solution and then carbon was evaporated onto the etched specimen. The carbon 
$\mathrm{Y}-185248$
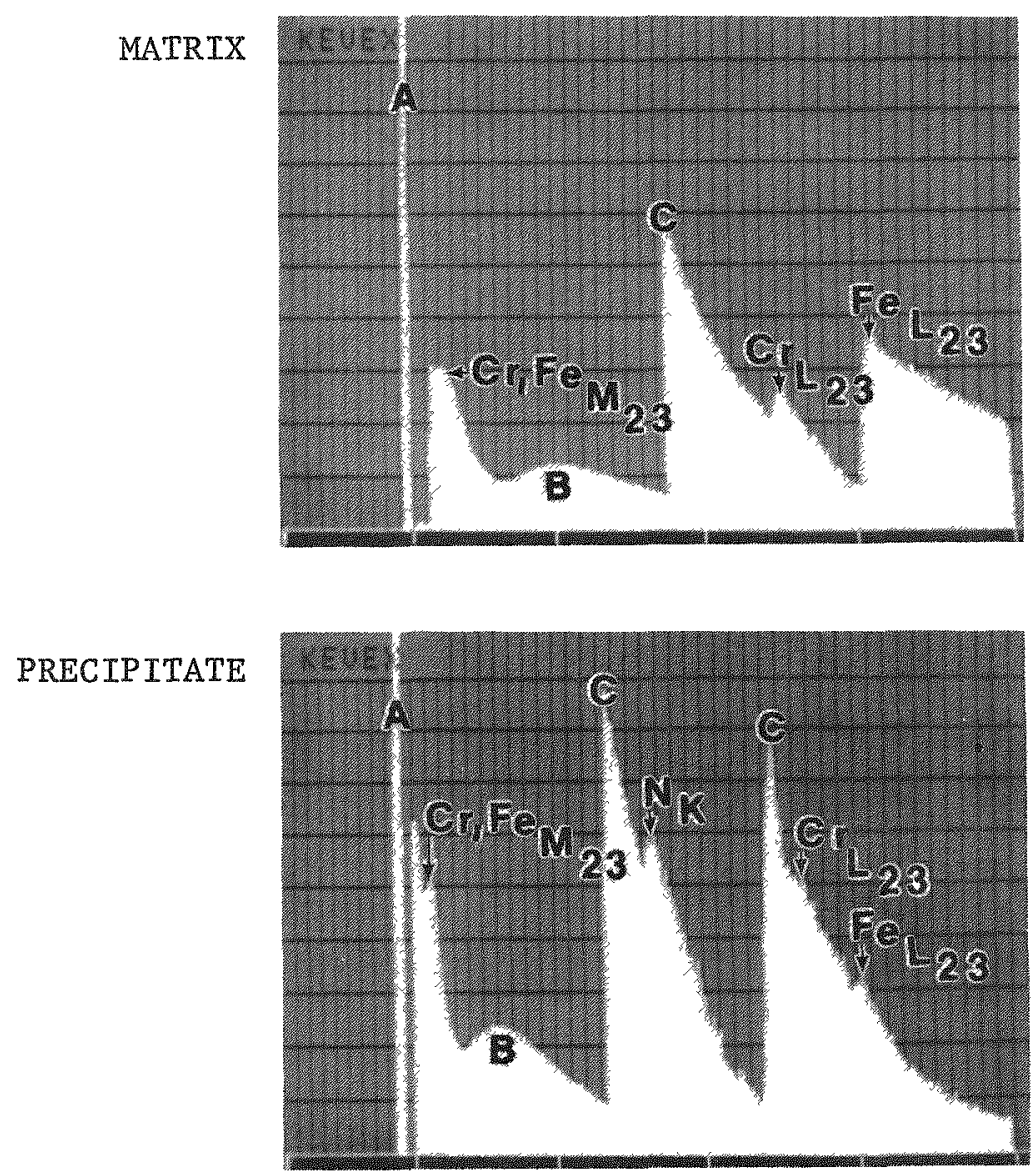

Figure 5.2. ELS spectra for the matrix and an in-foil precipitate. Fe$10 \% \mathrm{Cr}$. Notation: (A) Zero-1oss peak, (B) electronic artifact, (C) gain change.

replica was removed from the specimen by submering it in the etching solution (more hydrochloric acid was added to the solution if necessary). A beryllium grid was used to support the replica for TEM studies.

The precipitates extracted on the replica were analyzed using the EDS system. TEM micrographs of the replica and the precipitates studied are shown in Figure 5.3. The precipitates are labeled alphabetically with the letters corresponding to the labels on the EDS spectra in Figure 5.4. Only one precipitate, that labeled "d," contained any elements $(z>11)$ other than chromium and iron; sulfur was also indicated in the EDS spectrum. 

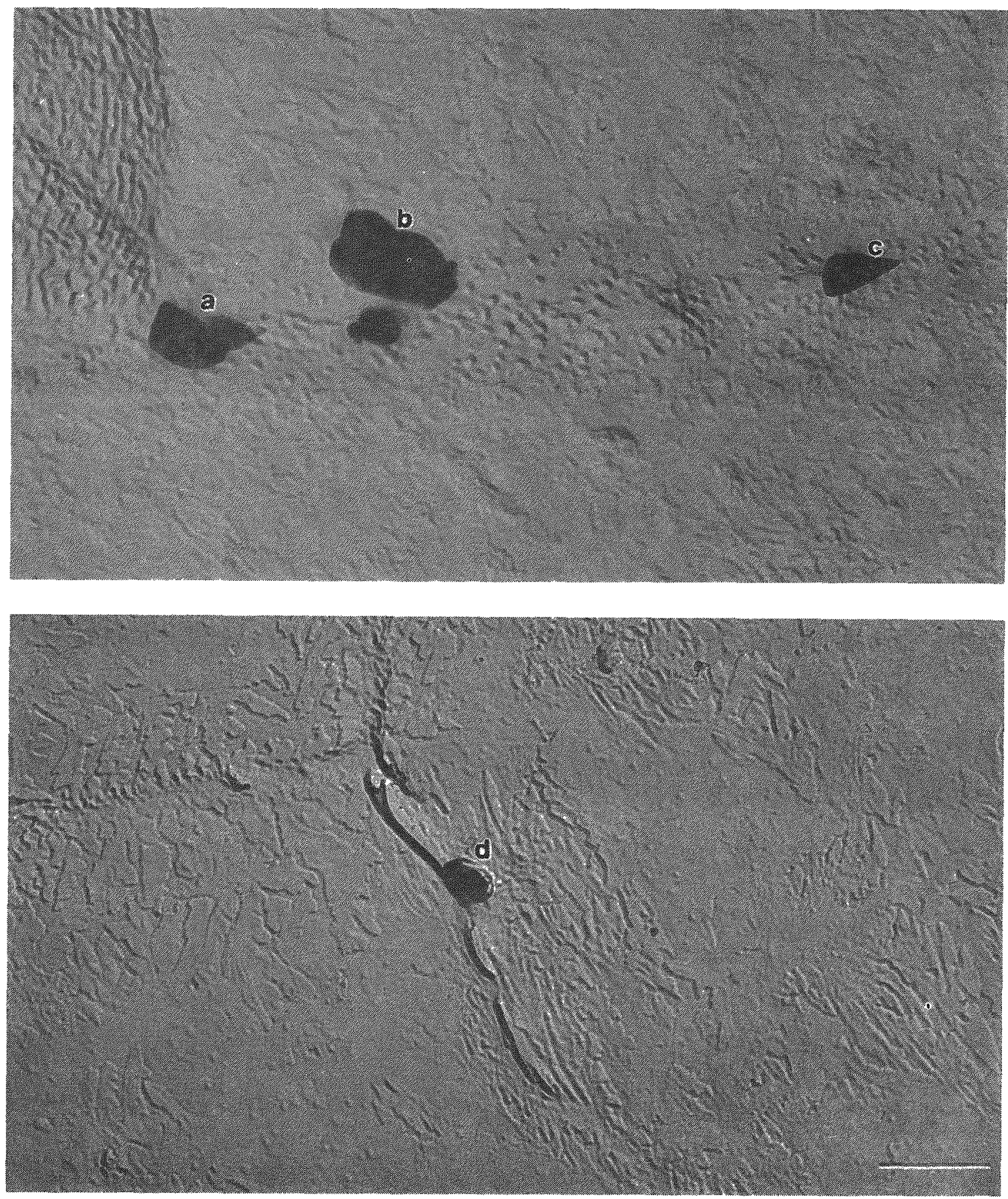

Figure 5.3. TEM micrographs of a carbon replica from a Fe-10\% Cr spectmen with grain boundary precipltates. The letters labeling the preclpitates correspond to the labels on the EDS spectra in Figure 5.4. Scale marker is $500 \mathrm{~nm}$. 

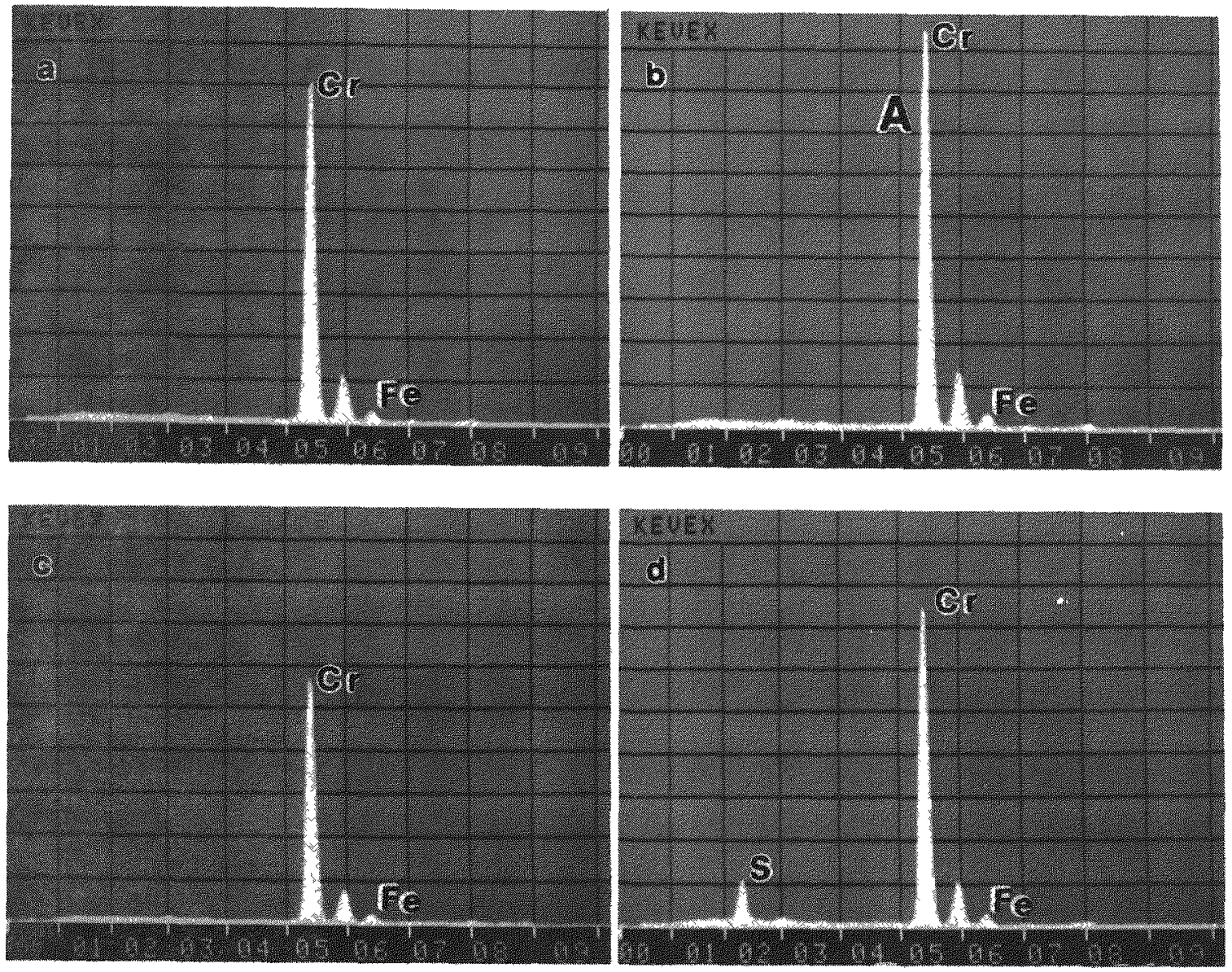

Figure 5.4. EDS spectra from the precipitates shown in Figure 5.3.

Extended study of the extracted precipitates using $S A D$ and Convergent Beam Electron Diffraction (CBED) techniques, as well as reevaluation of the in-foll precipitates with the ELS system, would probably identify the precipitate unambiguously. However, for the purposes of this Investigation, exact identification was not essential. It seems 1ikely that the preclpitates are simply the result of impurities in the alloys migrating to the grain boundaries during the annealing process. This implies that the matrix away from the grain boundaries is of higher purity than indicated by the chemical analyses In Appendix C. 


\subsection{Temperature Dependence Studies}

The $\mathrm{Fe}-10 \% \mathrm{Cr}$ specimens were selected for extensive examination to determine the temperature dependence of the radiation-induced microstructures. These specimens were selected for two major reasons. The TEM specimen preparation has a higher success rate for Fe-10\% Cr specimens than for $\mathrm{Fe}-5 \% \mathrm{Cr}$ and $\mathrm{Fe}$ specimens. Also, studies of the defect structures in $\mathrm{Fe}-10 \% \mathrm{Cr}$ were considered to be the most relevant to the fusion materials research effort since the chromium content of many of the ferritic stainless steels being consldered for fusion applications is $\sim 10 \%$.

In general, ion bombardment of $\mathrm{Fe}-10 \% \mathrm{Cr}$ resulted in the development of both dislocation and cavity microstructures. No radiationinduced changes in the precipitation were observed. The series of micrographs in Figures 5.5 and 5.6 show the development of the dislocation and cavity microstructures with increasing irradiation temperature. The quantitative data, as measured by the techniques discussed in Appendix B, are summarized in Table 5.1. Also reproduced in this table are the irradiation conditions for each specimen examined. Listed in the first column of the table are the run number and array location for the specimen as described in Appendix $D$.

A comparison of the dislocation densities in Table 5.1 shows a general coarsening of the structure with increasing temperature. This feature is demonstrated by a plot of the dislocation density as a function of temperature in Figure 5.7. As shown in the micrographs in Figure 5.5, the dislocation microstructure for irradiation temperatures between 723 and $803 \mathrm{~K}$ consisted primarily of 1oops. As indicated in Table 5.1, except for the specimen irradiated at $765 \mathrm{~K}$, the loop diameter, $d_{L}$, increased and the loop concentration, $C_{L}$, decreased with increasing irradiation temperature. (Within the experimental error, the data obtained for the specimens irradiated at 723 and $729 \mathrm{~K}$ are equivalent.) The smaller than expected loop diameter found in the specimen irradiated at $765 \mathrm{~K}$ is probably related to the lower damage in this specimen $(8.3 \mathrm{dpa})$ as compared to the other specimens. At all temperatures, loops were observed to intersect the foll surfaces 

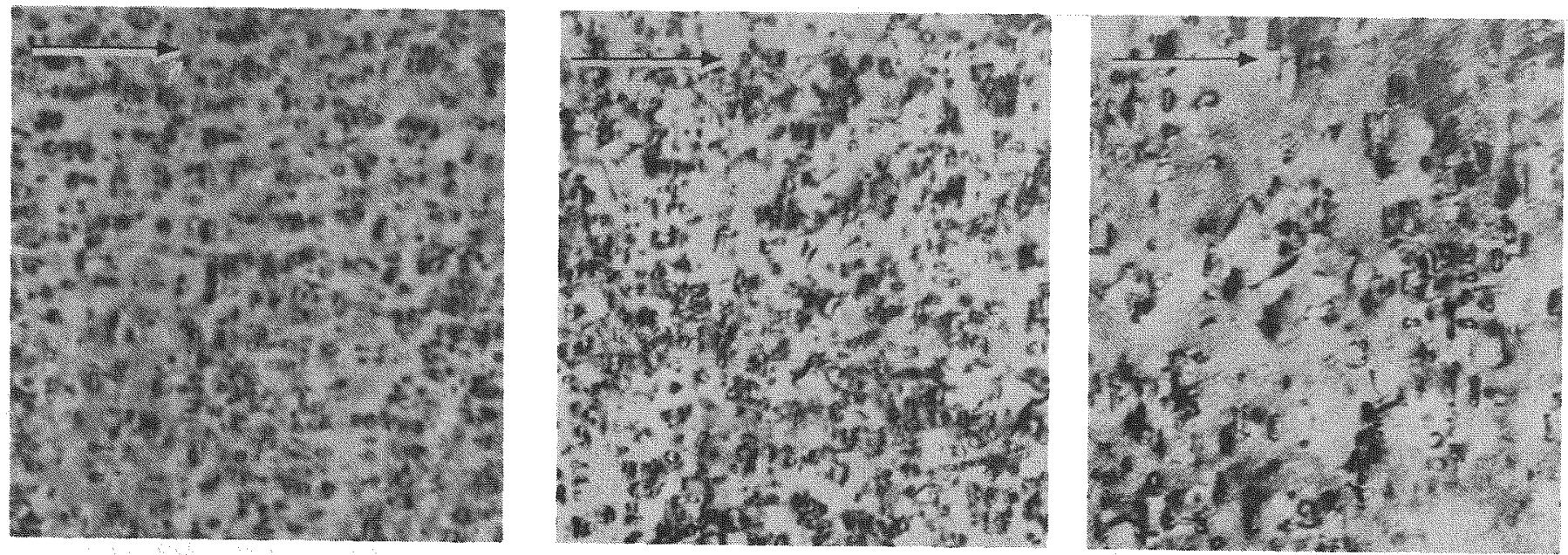

$773 \mathrm{~K} ; \mathrm{g}=<0 \overline{1} 1>; \mathrm{z} \sim[011]$
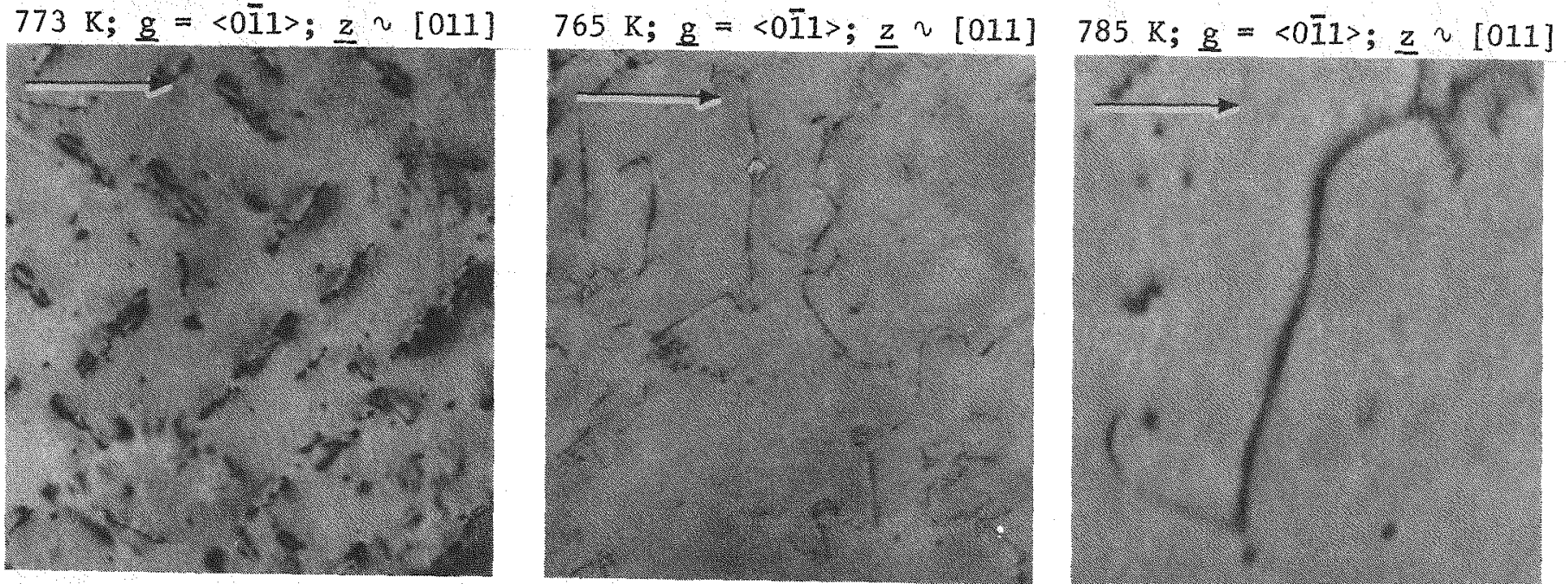

$849 \mathrm{~K} ; \mathrm{g}=\langle 0 \overline{1} 1\rangle ; \mathrm{z} \sim[133]$

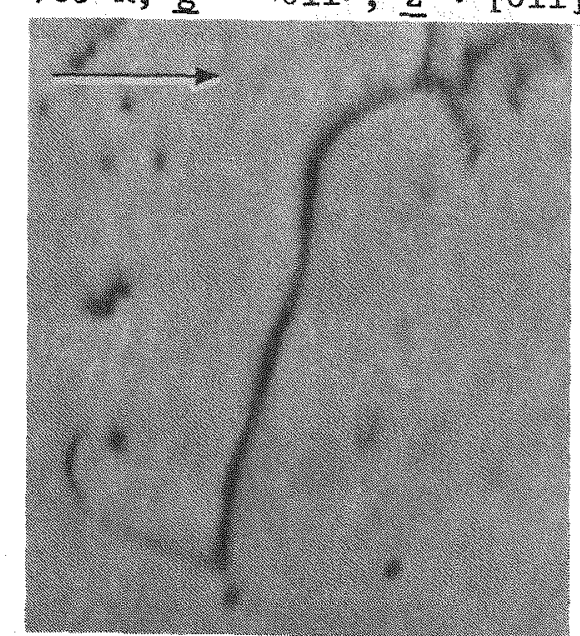

$803 \mathrm{~K} ; \underline{g}=\langle 1 \overline{1} 0\rangle ; \underline{z} \sim[115]$

Figure 5.5. Series of micrographs of the dislocation microstructures observed in $\mathrm{Cr}$. Arrows denote direction of $\mathrm{g}$, length of arrows equals $200 \mathrm{~nm}$. $10 \mathrm{dpa}$. indicated. 

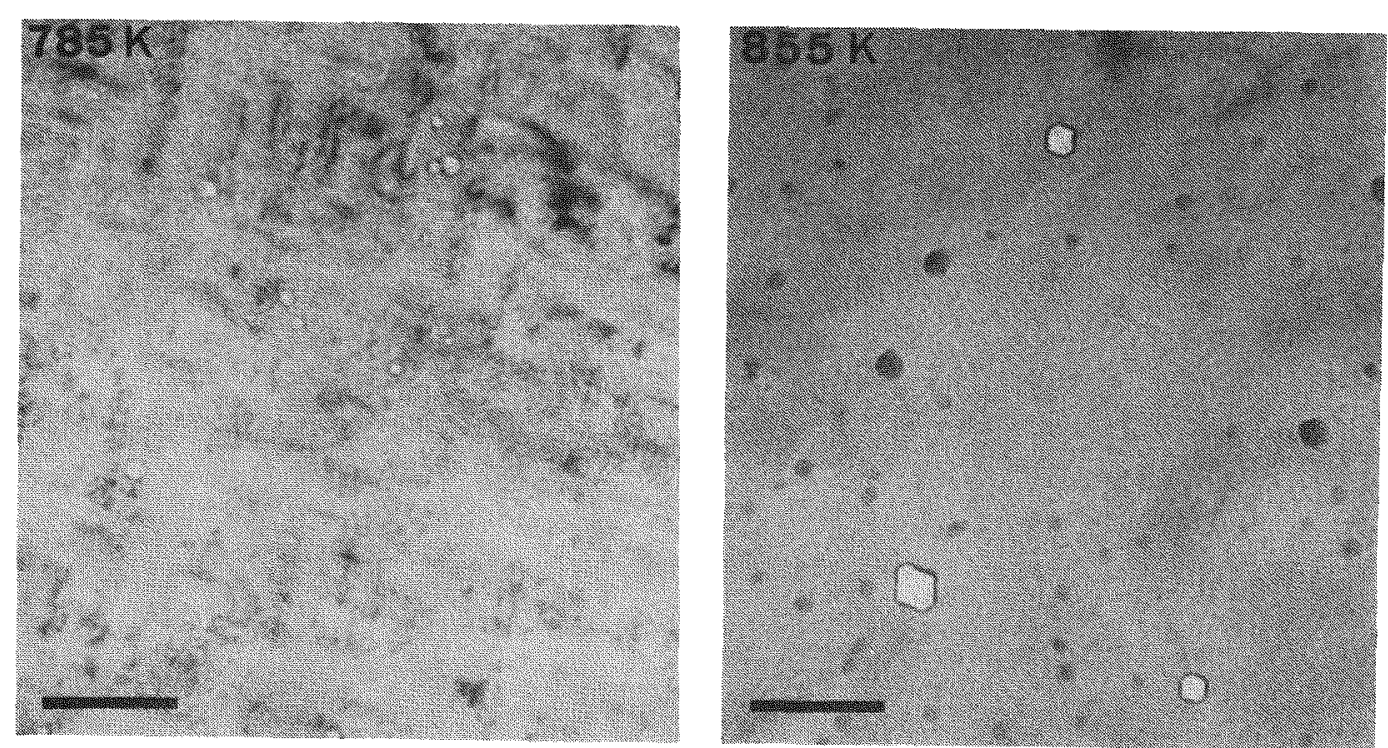

YE-12399

Figure 5.6. Series of micrographs of the cavity microstructures observed in Fe-10\% Cr. Arrows indicate sma11 cavities. Scale markers equal $100 \mathrm{~nm} .10 \mathrm{dpa} . \mathrm{T}_{\mathrm{T}}$ as indicated. 
Table 5.1. Quantitative data and irradiation parameters for triple-beam ion irradiation, temperature dependent experiment

Fe-10\% Cr, nominal dpa $=10,100$ at. ppm He, 41 at. ppm $\mathrm{D}^{\mathrm{a}}$

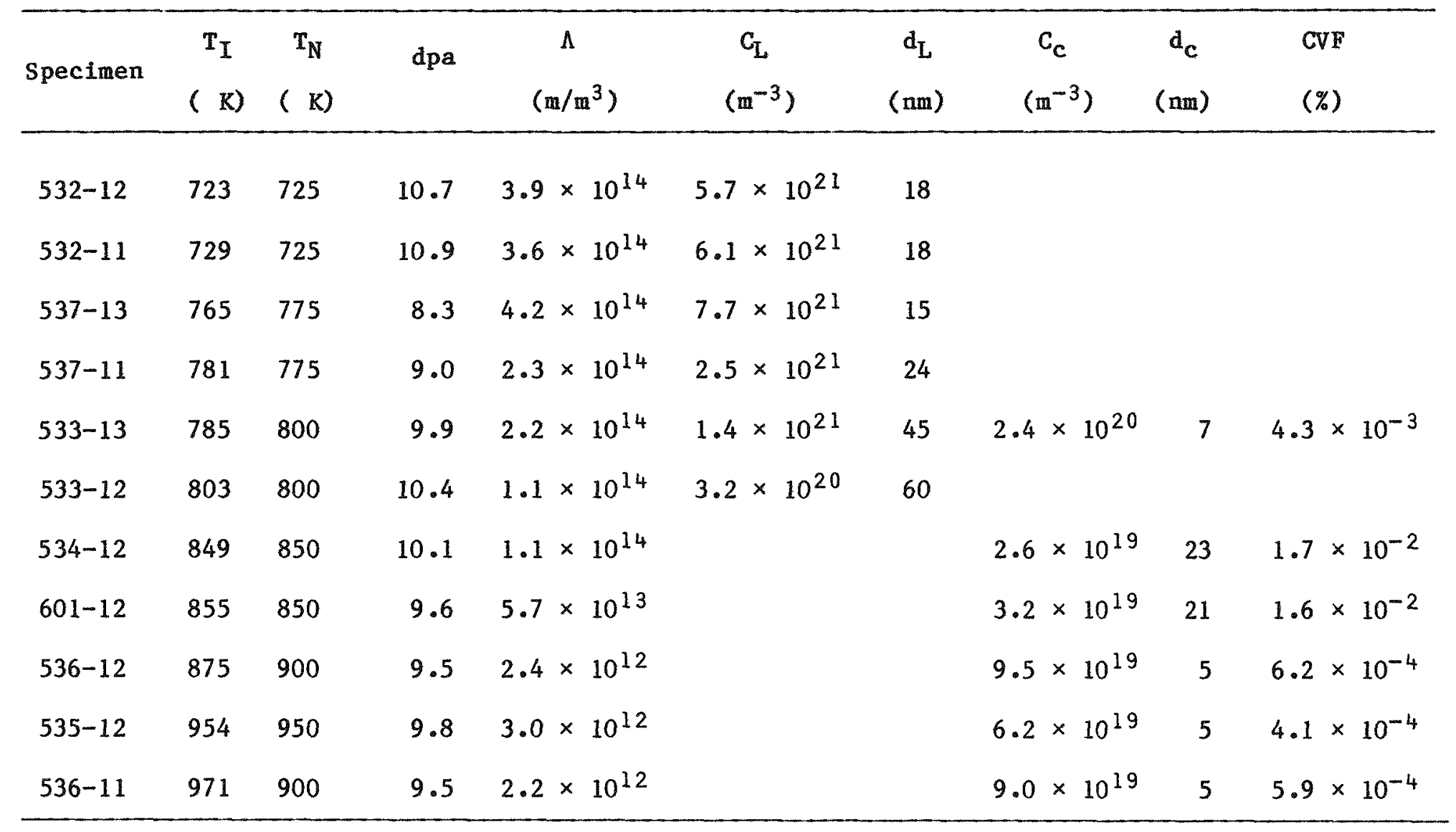

${ }^{\text {Notation: }} \mathrm{T}_{\mathrm{I}}$ actual irradiation temperature; $\mathrm{T}_{\mathrm{N}}$, nominal irradiation temperature (see Appendix $\mathrm{D}), \Lambda$, dislocation density (including loops); $\mathrm{C}_{\mathrm{L}}$, loop concentration; $\mathrm{d}_{\mathrm{L}}$, loop diameter; $C_{c}$, cavity concentration; $d_{c}$, volume average cavity diameter; and $C V F$, cavity volume fraction or swelling. 


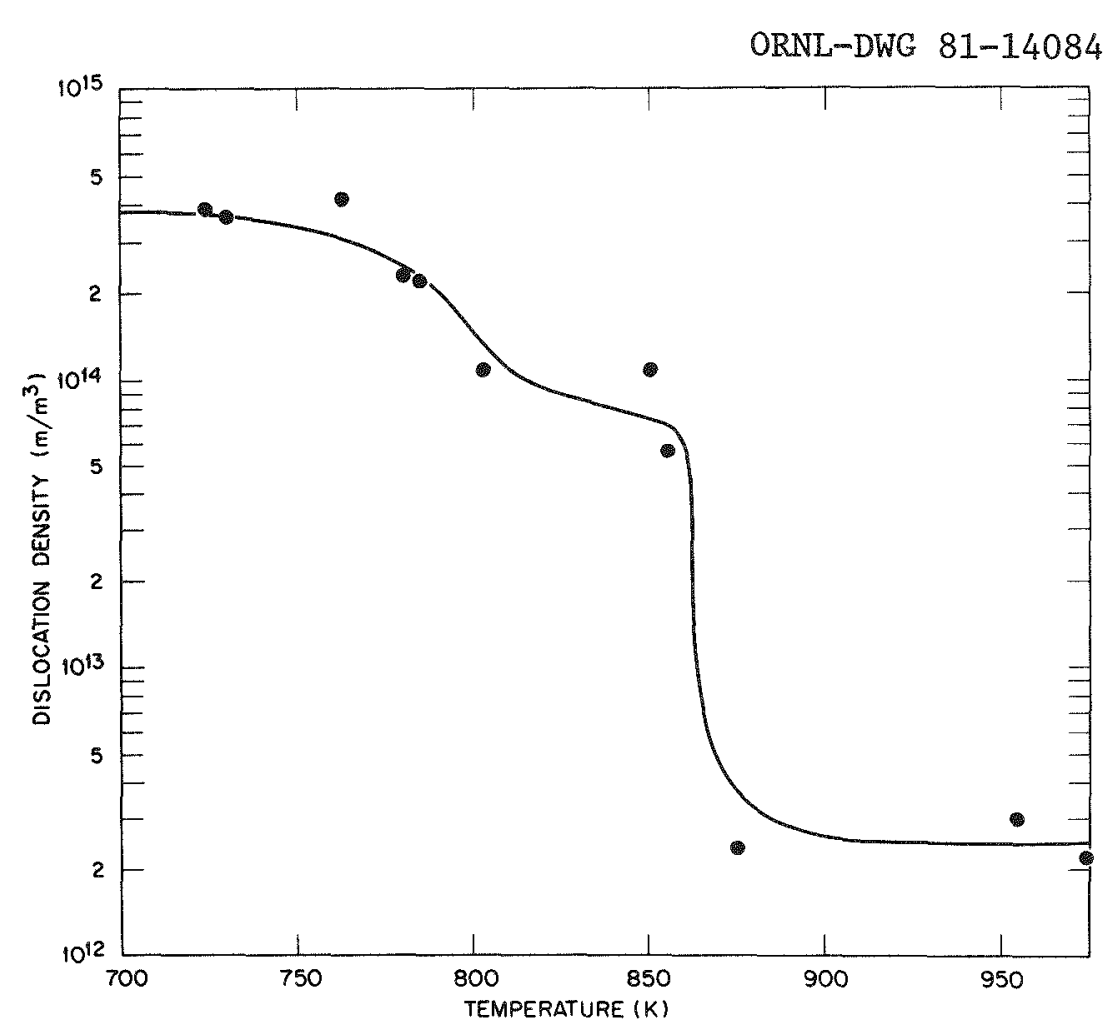

Figure 5.7. Graph of the irradiation temperature dependence of the dislocation density in $\mathrm{Fe}-10 \%$ Cr. $10 \mathrm{dpa}$.

without gliding out of the foil. For beam directions near $\langle 001\rangle$ the majority of the loops were observed to have a near-edge character and lie on $\{100\}$ planes. This type of loop alignment can also be observed for foil orientations near $\langle 011\rangle$ for loops with $\underline{b}=\langle 100\rangle$, as shown in Figure 5.5. In the micrograph (Figure 5.5) of the specimen irradiated at $803 \mathrm{~K}$, taken with a beam direction near $\langle 115\rangle$, the loops are nearly edge-on, at $\sim 45^{\circ}$ angle to the $\langle 1 \overline{1} 0\rangle$ diffracting vector, as expected for near-edge loops with $\underline{b}=\langle 100\rangle$ and $\langle 010\rangle$. A complete analysis of the loops was not performed. However, the complete analyses of the geometry and nature of the loops in both neutron- (Chapter 3) and ion- (Chapter 4 ) irradiated iron, as well as in the fluence-dependent studies of ionirradiated $\mathrm{Fe}-10 \% \mathrm{Cr}$ (see Section 5.3), indicate that the loops are probably interstitial, of near-edge character, and have $\underline{b}=a\langle 100\rangle$. The few loops that did not show the characteristic edge-on orientation for beam directions near $\langle 001\rangle$ were probably a/2<111> interstitial loops since loops of this type were also found in the complete analyses mentioned above. 
Smal1 loops $(\underline{b}=a\langle 100\rangle)$ varied in shape from round to rectilinear, with the sides aligned along $\langle 100\rangle$. For irradiation temperatures above $765 \mathrm{~K}$, many of the loops had a convoluted shape. One such loop, with $\underline{b}=a[001]$, is shown in Figure 5.8, a higher magnification micrograph of the specimen irradiated at $803 \mathrm{~K}$. This micrograph was taken with multiple-beam diffracting conditions at the [012] pole in order to view the a[010] loops and edge-on profiles of a[100] loops as well as the full perimeter profile of the al001] 1oops. The 1oops appear to have grown preferential1y along $\langle 110\rangle$ directions.

At irradiation temperatures of $\sim 850 \mathrm{~K}$, a coarse distribution of network segments was observed. At higher irradiation temperatures ( $875 \mathrm{~K})$, the dislocation structures appeared to be little changed from those existing in unirradiated specimens. Between 850 and $875 \mathrm{~K}$, more than an order of magnitude decrease in the dislocation density is observed (Table 5.1 and Figure 5.7).

Cavities were observed in specimens irradiated at temperatures of 785 and above, except at $803 \mathrm{~K}$. This result may suggest that there are actually two swelling peaks, one near $780 \mathrm{~K}$ and another at $\sim 850 \mathrm{~K}$ with lower swelling at $\sim 800 \mathrm{~K}$. However, at $780-800 \mathrm{~K}, 10 \mathrm{dpa}$ is apparently near the threshold damage level required for visible cavity formation. Therefore, a higher dose experiment is required to determine whether or not there are actually two swelling peaks. The larger ORNL-Photo $4542-81$

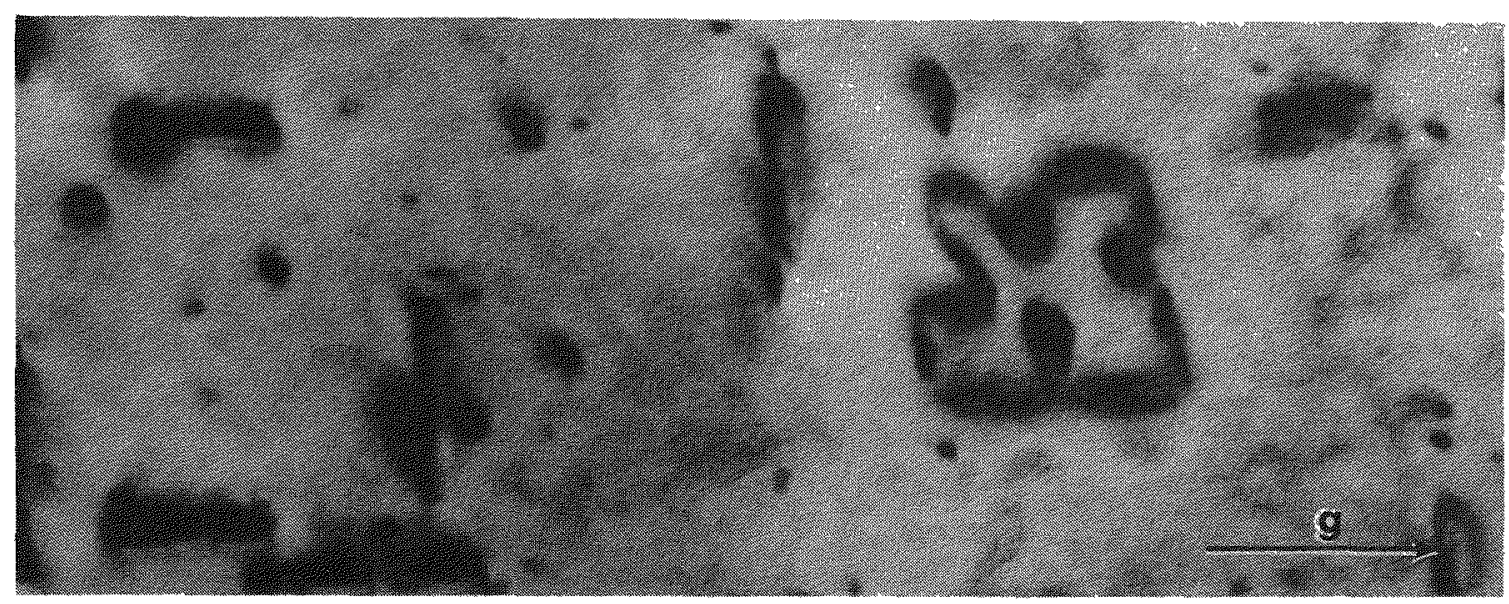

Figure 5.8. TEM micrograph of a "convoluted" dislocation loop with b $=a[001]$. Arrow shows [200] direction; length of arrow equals $100 \mathrm{~nm}$. Fe-10\% $\mathrm{Cr}, \mathrm{T}_{\mathrm{I}}=803 \mathrm{~K}, 10 \mathrm{dpa}, \underline{z} \sim[012]$. Multiple beam diffracting conditions. 
cavities had a definite crystallographic shape, as can be seen in Figure 5.6. The cavity morphology was consistent with a truncated octahedron with $\{111\}$ facets and $\{100\}$ truncations. As discussed previously, the cavity diameter was measured from a circular projection judged to have the same area as the observed crystallographic shape. The maximum cavity volume fraction (CVF) or swelling of $0.02 \%$ occurred for irradiation temperatures of $\sim 850 \mathrm{~K}$ (see Table 5.1). Compared with the cavities which developed at $\sim 805 \mathrm{~K}$, the cavities observed at both higher and lower temperatures were smaller and more numerous. However, for irradiation temperatures above $850 \mathrm{~K}$, the cavity distribution was less homogeneous than at the lower temperature. As illustrated in Figure 5.9, zones denuded of cavities were observed near grain boundaries. While the grain boundaries were also free of cavities at lower temperatures, cavities were found on the boundaries in specimens irradiated at $\sim 950 \mathrm{~K}$. At this temperature, as shown in Figure 5.10, cavities were also observed at the intersection of grain boundaries and chromium-rich precipitates. For irradiation temperatures of 785 and $850 \mathrm{~K}$, cavities were observed along dislocations.

ORNL-Photo 4543-81

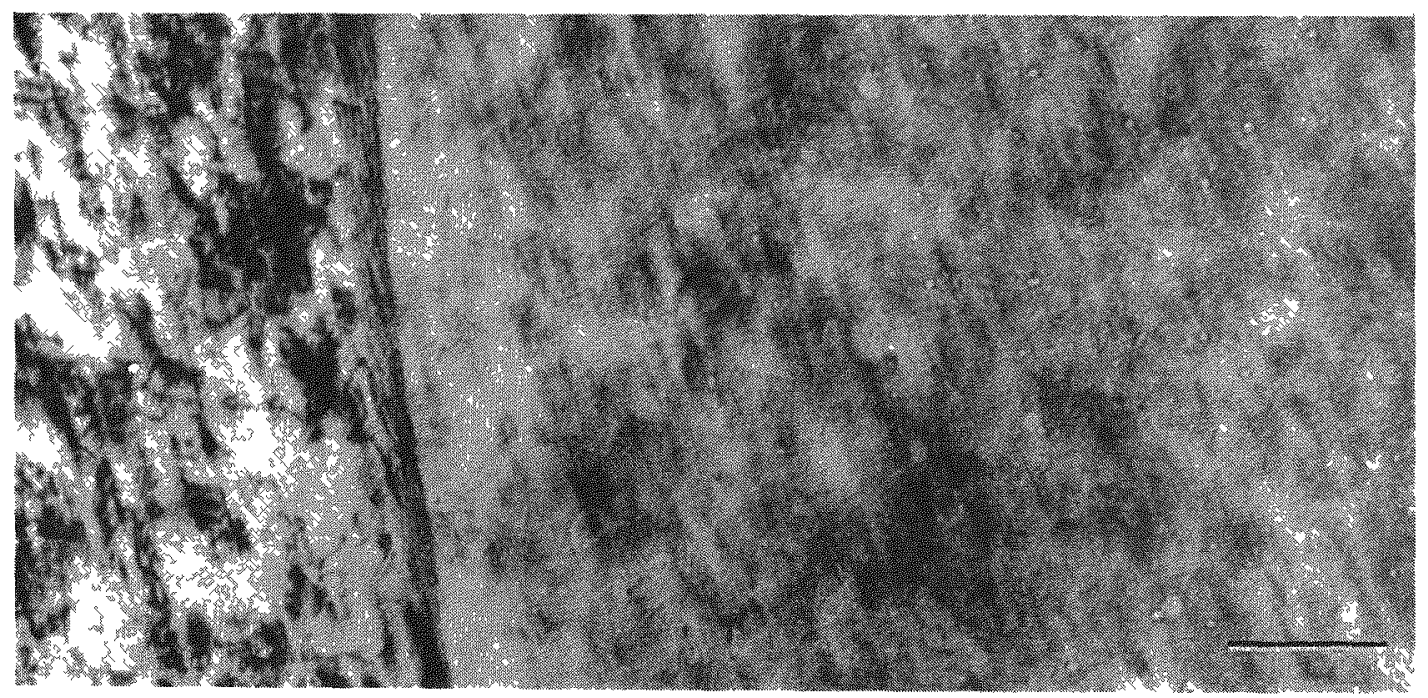

Figure 5.9. TEM micrograph of a cavity denuded zone typical of those found at the grain boundaries in $\mathrm{Fe}-10 \% \mathrm{Cr}$. Length of marker equals $200 \mathrm{~mm}$. $10 \mathrm{dpa}, \mathrm{T}_{\mathrm{I}}=785 \mathrm{~K}$. 
ORNL-Photo 0982-81

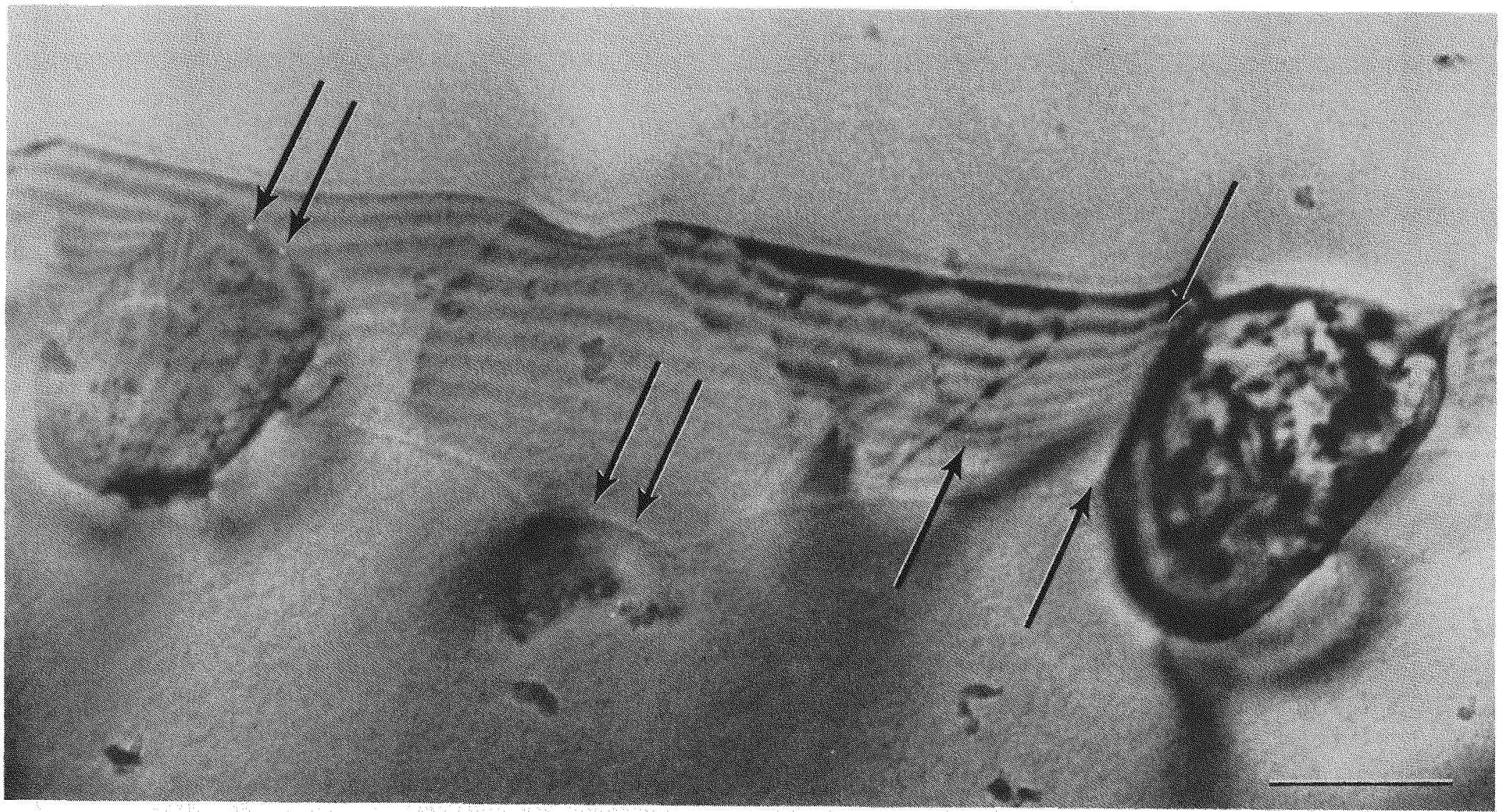

Figure 5.10. TEM micrograph of cavities along grain boundaries and at the intersection of grain boundaries and chromium-rich precipitates. Arrows indicate small cavities. Scale marker equals $200 \mathrm{~nm}$. Fe-10\% Cr, $10 \mathrm{dpa}, \mathrm{T}_{\mathrm{I}} \sim 950 \mathrm{~K}$. 
The cavity-dislocation association is clearly seen in the micrograph of the specimen irradiated at $849 \mathrm{~K}$ in Figure 5.5.

Based on the above observations, the peak swelling temperature of $850 \mathrm{~K}$ was selected as the irradiation temperature for the dose dependent experiment. Again, the Fe-10\% $\mathrm{Cr}$ alloy was selected because of the high success rate of TEM specimen preparation and because of its relevance to commercial ferritic steels.

\subsection{Dose Dependence Studies}

As in the previous experiment, the damage microstructures observed in ion-bombarded Fe-10\% Cr consisted of dislocations and cavities. Once again, no radiation-induced precipitation was observed. The series of micrographs in Figures $5.11,5.12$ and 5.13 summarize the dislocation and cavity microstructures observed with increasing displacement damage. The quantitative data, including the irradiation conditions for each specimen examined, are summarized in Table 5.2. Listed in the first column of Table 5.2 are the run number and array location for the specimen as described in Appendix D.

As shown in Figure 5.11, at low damage levels $(0.3$ to $3 \mathrm{dpa})$, dislocation loops were observed as the primary form of the dislocation microstructure. Inspection of the quantitative data in Table 5.2 shows that the loop concentration decreases and loop diameter increases with increasing displacement damage. At 1 and $3 \mathrm{dpa}$, many of the dislocation loops had a convoluted shape, similar to that discussed in Section 5.2 . As before, preferential growth appears to have occurred in $\langle 110\rangle$ directions.

A complete analysis of the nature and geometry of the dislocation loops was performed for the 0.3 dpa specimen following the techniques of Maher and Eyre [97]. (See Appendix B for details of the application of this procedure.) A portion of this analysis is shown in Figure 5.14. In the first micrograph in the sequence, $\underline{g}=[1 \overline{1} 0]$ and $\underline{z}$ $\sim$ [001], al1 loops are in contrast and in a near edge-on orientation. Invisibilities for the loops labeled A-F are obtained for $\underline{g}=[200]$ and 
ORNL-Photo $4544-81$

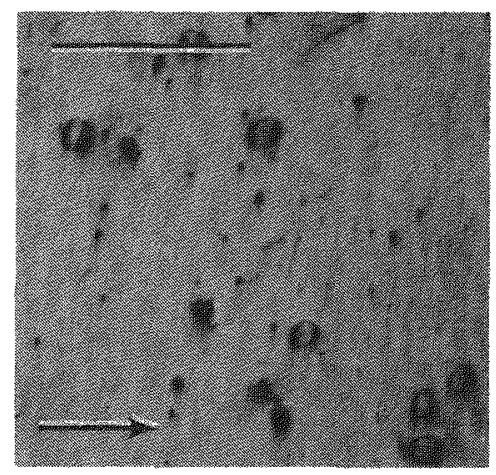

$0.3 \mathrm{dpa}$

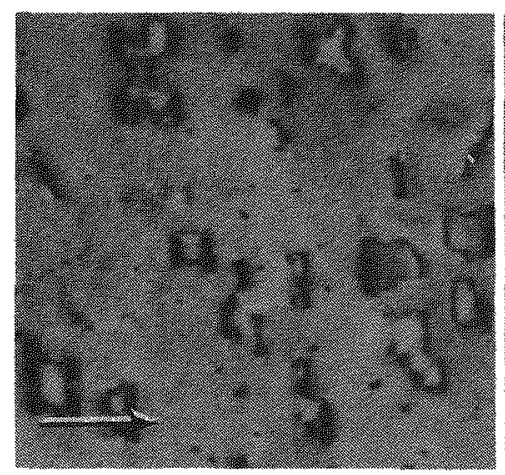

$1 \mathrm{dpa}$

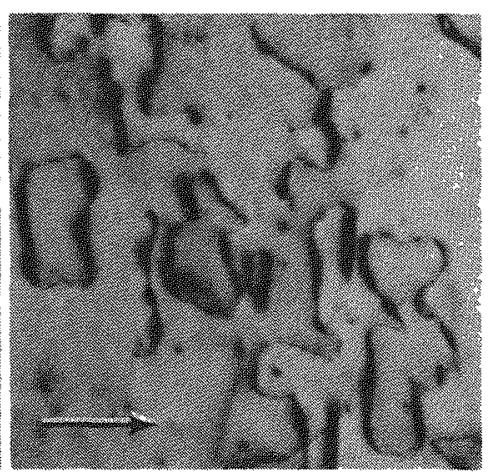

3 dpa

$$
\underline{g}=\langle 01 \bar{I}\rangle \quad \underline{z} \sim[111] \quad \underline{g}=\langle 01 \bar{I}\rangle \quad \underline{z} \sim[011] \quad \underline{g}=\langle 01 \overline{1}\rangle \quad \underline{z} \sim[011]
$$

Figure 5.11. TEM micrographs of the dislocation microstructure observed in $\mathrm{Fe}-10 \% \mathrm{Cr}$ irradiated to high levels of displacement damage. $\mathrm{T}_{\mathrm{I}} \sim$ $850 \mathrm{~K}$. Scale marker equals $300 \mathrm{~mm}$. Arrows indicate direction of $\mathrm{g}$.

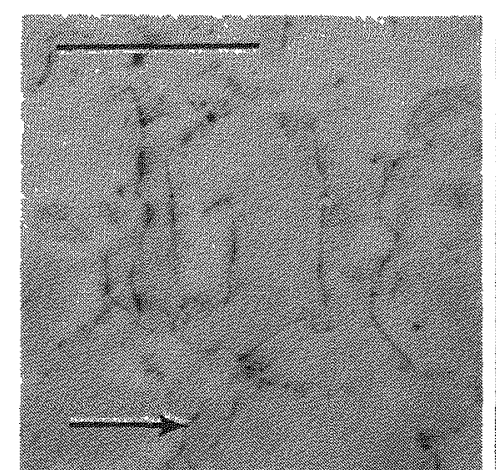

$10 \mathrm{dpa}$

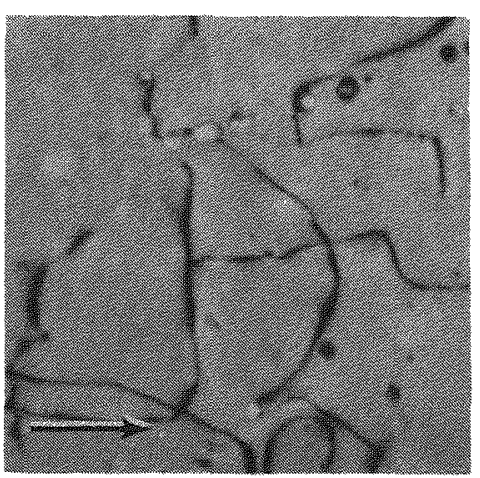

$30 \mathrm{dpa}$

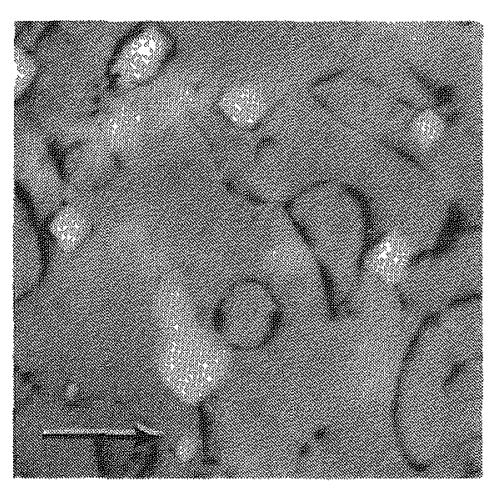

$100 \mathrm{dpa}$

$$
\underline{g}=\langle 0 \overline{1} 1\rangle \quad \underline{z} \sim[133] \quad g=\langle 0 \overline{1} 1\rangle \quad \underline{z} \sim[011] \quad g=\langle 200\rangle \underline{z} \sim[011]
$$

Figure 5.12. TEM micrographs of the dislocation microstructures observed in $\mathrm{Fe}-10 \% \mathrm{Cr}$ irradiated to high levels of displacement damage. $\mathrm{T}_{\mathrm{I}} \sim 850 \mathrm{~K}$. Scale marker equals $300 \mathrm{~nm}$. Arrows indicate direction of g. 
ORNL-Photo $4547-81 R$

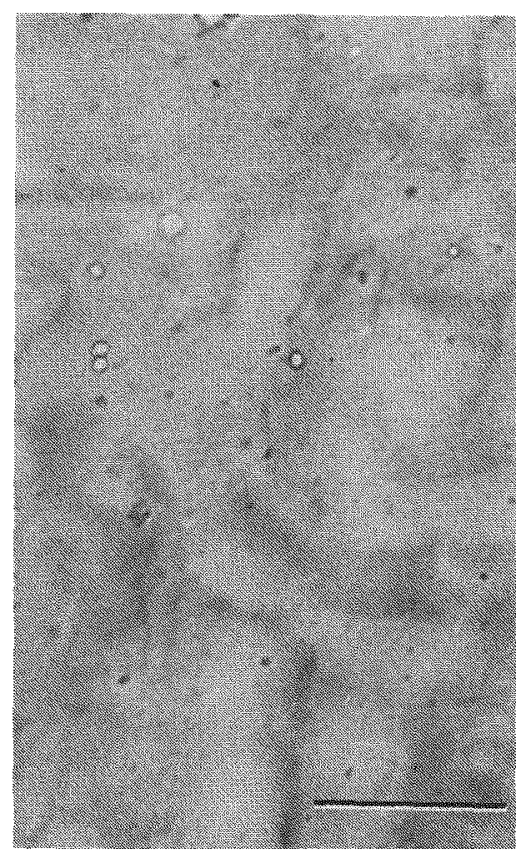

$3 \mathrm{dpa}$

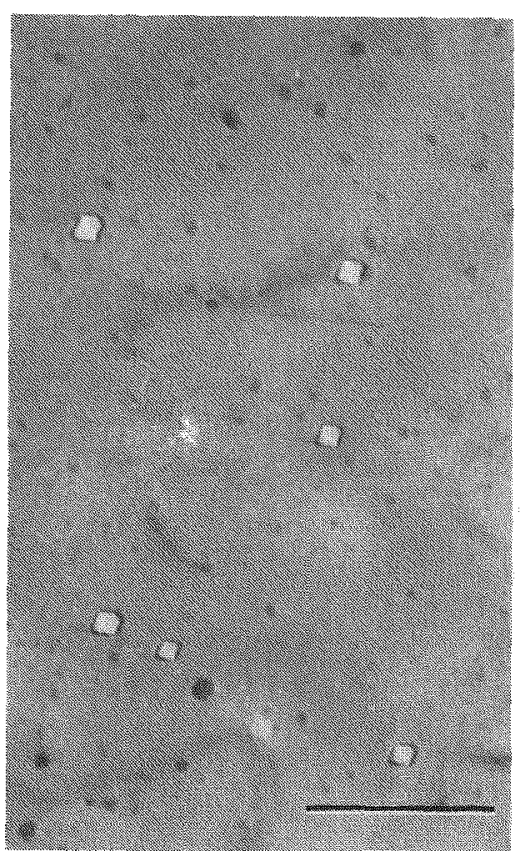

$10 \mathrm{dpa}$

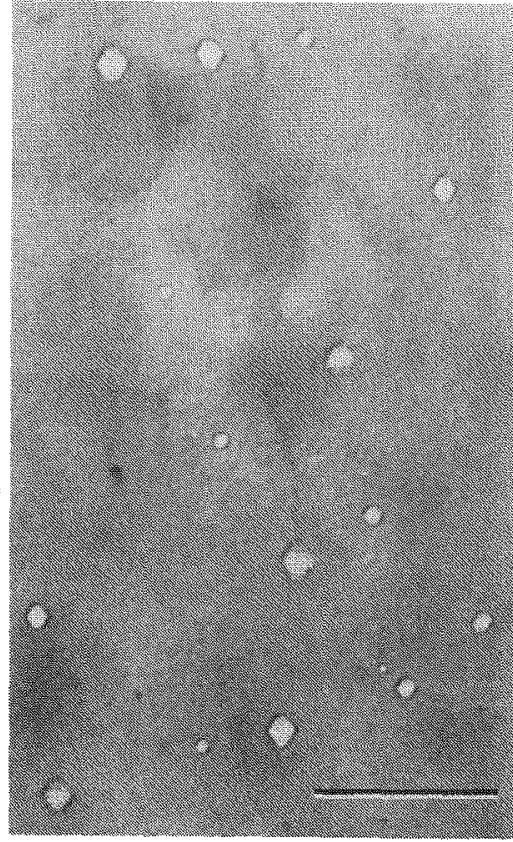

$30 \mathrm{dpa}$

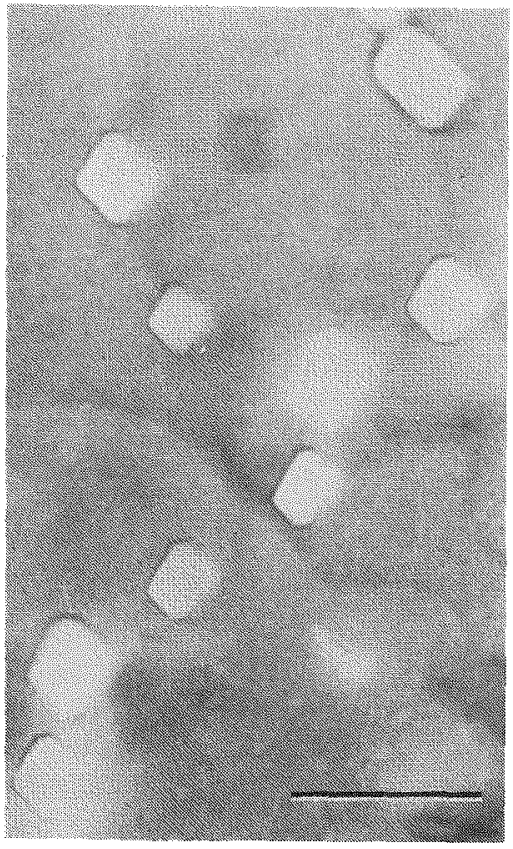

$100 \mathrm{dpa}$

Figure 5.13. TEM micrographs of the cavity microstructures observed in $\mathrm{Fe}-10 \% \mathrm{Cr}$. Scale markers are $200 \mathrm{~nm}$. Fluence as indicated. $\mathrm{T}_{\mathrm{T}} \sim 850 \mathrm{~K}$. 
Table 5.2. Quantitative data for triple-beam ion irradiation dose dependent experiment Fe-10\% Cr, $850 \mathrm{~K}, 10$ at. ppm He/dpa, 41 at. ppm $\mathrm{D}^{\mathrm{a}}$

\begin{tabular}{lccccccccc}
\hline Specimen & $\begin{array}{c}\mathrm{T}_{\mathrm{I}} \\
(\mathrm{K})\end{array}$ & $\mathrm{dpa}$ & $\mathrm{dpa}_{\mathrm{N}}$ & $\begin{array}{c}\Lambda \\
\left(\mathrm{m} / \mathrm{m}^{3}\right)\end{array}$ & $\begin{array}{c}\mathrm{C}_{\mathrm{L}} \\
\left(\mathrm{m}^{-3}\right)\end{array}$ & $\begin{array}{c}\mathrm{d}_{\mathrm{L}} \\
(\mathrm{nm})\end{array}$ & $\begin{array}{c}\mathrm{C}_{\mathrm{C}} \\
\left(\mathrm{m}^{-3}\right)\end{array}$ & $\begin{array}{c}\mathrm{d}_{\mathrm{c}} \\
(\mathrm{nm})\end{array}$ & $\begin{array}{c}\mathrm{CVF} \\
(\%)\end{array}$ \\
\hline $593-12$ & 848 & 0.3 & 0.3 & $3.7 \times 10^{13}$ & $1.8 \times 10^{20}$ & 37 & & & \\
$592-21$ & 848 & 1.0 & 1.0 & $3.9 \times 10^{13}$ & $8.1 \times 10^{19}$ & 94 & & 0.001 \\
$593-22$ & 853 & 3.0 & 3.0 & $6.8 \times 10^{13}$ & $6.4 \times 10^{19}$ & 150 & $3.5 \times 10^{19}$ & 9 & \\
$593-21$ & 847 & 3.1 & 3.0 & $4.4 \times 10^{13}$ & $4.1 \times 10^{19}$ & 150 & $\mathrm{~b}$ & & \\
$534-12$ & 849 & 10.1 & 10.0 & $1.1 \times 10^{14}$ & $\mathrm{c}$ & & $2.6 \times 10^{19}$ & 23 & 0.02 \\
$601-12$ & 855 & 9.6 & 10.0 & $5.7 \times 10^{13}$ & $<10^{18}$ & $\sim 200$ & $3.2 \times 10^{19}$ & 21 & 0.02 \\
$601-23$ & 844 & 25.9 & 30.0 & $3.2 \times 10^{13}$ & $\sim 10^{18}$ & $\sim 150$ & $2.0 \times 10^{20}$ & 20 & 0.09 \\
$596-22$ & 867 & 101.4 & 100.0 & $4.8 \times 10^{13}$ & $7 \times 10^{18}$ & $\sim 110$ & $9.9 \times 10^{19}$ & 77 & 2.45 \\
\hline
\end{tabular}

a Notation: $\mathrm{T}_{\mathrm{I}}$, actual irradiation temperature; $\mathrm{dpa} \mathrm{N}_{\mathbb{N}}$, nominal dpa; $\Lambda$, dislocation density (including loops); $C_{L}$, loop concentration; $d_{L}$, loop diameter; $C_{c}$, cavity concentration; $d_{c}$, volume average cavity diameter; and CVF, cavity volume fraction or swelling.

$b_{\text {Not measured. }}$

$c_{\text {None observed. }}$ 
ORNL-Photo 4545-81
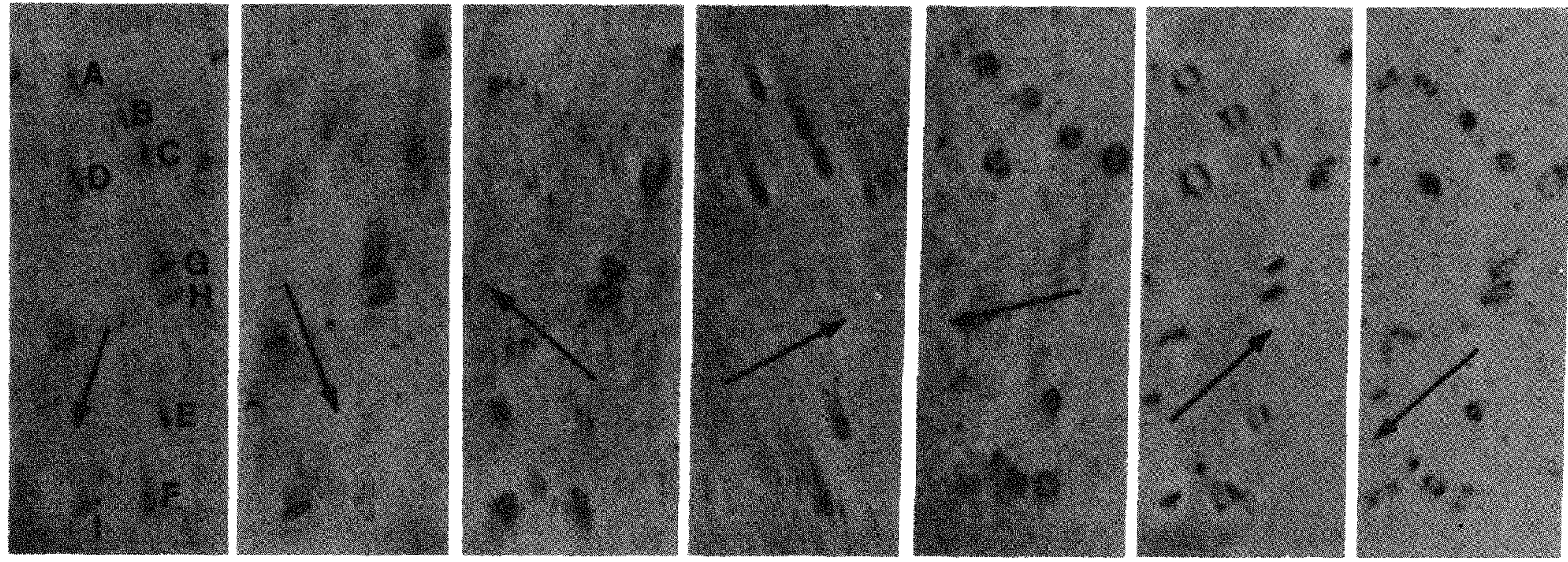

$\mathrm{g}=[1 \overline{1} 0]$

$\mathrm{g}=[200]$

$\underline{g}=[\overline{1} 01]$

$\mathrm{g}=[020]$

$\mathrm{g}=[0 \overline{1} 1]$

$\mathrm{g}=[\overline{1} 2 \overline{1}]$

$\underline{g}=[1 \overline{2} 1]$

$\underline{z} \sim[001]$

$\underline{z} \sim[001]$

$\underline{z} \sim[111]$

$\underline{z} \sim[001]$

$\underline{z} \sim[111]$

$\underline{z} \sim[111]$

$\underline{z} \sim[111]$

\section{$300 \mathrm{~nm}$}

Figure 5.14. Analysis of the geometry and nature of dislocation loops in $\mathrm{Fe}-10 \% \mathrm{Cr} .0 .3 \mathrm{dpa}, \mathrm{T}_{\mathrm{I}} \sim 850 \mathrm{~K}$. Loops $A-F, \underline{b}=a[010]$, interstitial. Loops $G-I, \underline{b}=a[100]$, interstitial. Arrows indicate direction of $\mathrm{g}$. 
[101], indicating that these loops have $\underline{b} \pm \mathrm{a}[010]$. Similarly, invisibilities for the loops labeled $G-I$ are obtained for $\underline{g}=[020]$ and [0I1], Indicating that these loops have $\underline{b}= \pm a[100]$. The last two micrographs of the sequence in Figure 5.14, $g=[\overline{1} 2 \overline{1}]$ and [1리 yield the nature of both sets of loops. Loops $A-F$ show "outside" contrast for $g=[\overline{1} 2 \overline{1}]$ $(\underline{g} \cdot \underline{b}=+2)$ and "inside" contrast for $\underline{g}=[1 \overline{2} 1](\underline{g} \cdot \underline{b}=-2)$, demonstrating that the loops are interstitial in nature. Conversely, loops $G-I$ show "outside" contrast for $\underline{g}=[\overline{1} 1]\left(\underline{g^{\circ}} \underline{b}=+1\right)$ and "inside" contrast for $\underline{g}=[\overline{1} 2 \overline{1}](\underline{g} \cdot \underline{b}=-1)$, demonstrating that these loops are also interstitial in nature. This conclusion was confirmed by micrographs taken with the appropriate $(\underline{g} \cdot \underline{b}= \pm 2)$ diffracting conditions. Al1 loops ( 75 ) analyzed for this specimen were near-edge dislocation loop character having an interstitial nature with $\underline{b}=a\langle 100\rangle$.

While a complete analysis of the loops was not performed for the 1 dpa specimen, inspection of stereo TEM micrographs showed that all of the loops were on $\{100\}$ planes. For all foll orientations used for the examination, all loops were observed to be consistent with the expected configuration for loops with $\underline{b}=a\langle 100\rangle$.

At $3 \mathrm{dpa}$, the initial stages of the formation of a dislocation network from the loop structure are observed. Part of the analysis to determine the Burgers vectors for the dislocation loops and segments found in this specimen is shown in Figure 5.15. The results for this analysis of the labeled dislocations in figure 5.15 are listed in Table 5.3. As indicated, several of the loops had $\underline{b}=a / 2\langle 111\rangle$. The shape of these loops differed significantly from the shape of the loops with $\underline{b}=a\langle 100\rangle$. The $a / 2\langle 111\rangle$ loops, as shown in Figure 5.15 , were round while the $a\langle 100\rangle$ loops had a convoluted shape. When the nature of the loops could be safely determined, the loops were found to be interstitial dislocation loops. Two groups of dislocation loops and segments (marked $A, B, C$, and $D, E, F$ ) show the merging of two loops to form a dislocation segment. In the first group, loop $A\left(b_{A}=a / 2[\overline{1} 11]\right.$ and loop $B$ $\left(b_{B}=a[001]\right.$ have merged to form segment $C$. Analysis of the Burgers vector of segment $c$ indicated that $\underline{b}_{c}= \pm a / 2[1 \overline{1} 1]$. In the second group, loop $D\left(\underline{b}_{D}=a / 2[1 \overline{1} 1]\right)$ and loop segment $E\left(\underline{b}_{E}=a[001]\right.$ have joined to form segment $F\left(\underline{b}_{F}= \pm a / 2[\overline{1} 11]\right)$. 

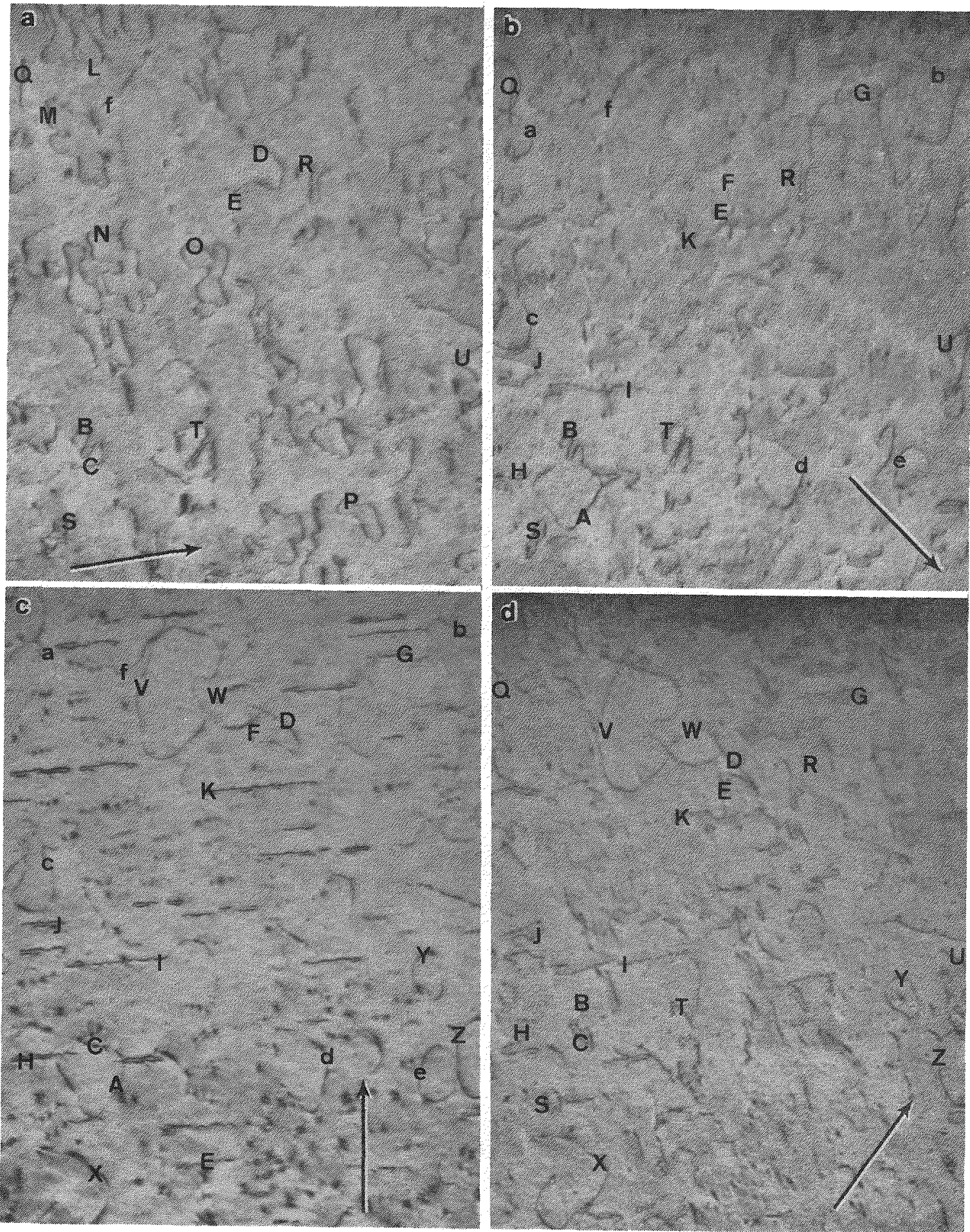

Figure 5.15. Series of micrographs comprising part of a dislocation loop analysis in $\mathrm{Fe}-10 \% \mathrm{Cr}$. Arrows denote direction of $\mathrm{g}$, length of arrows equals $500 \mathrm{~nm} .3 .0 \mathrm{dpa}, \mathrm{T}_{\mathrm{I}} \sim 850 \mathrm{~K} . \mathrm{g}=[01 \overline{1}], \underline{z} \sim[111]$; (b) $\underline{g}=[10 \overline{1}], \underline{z} \sim[111] ;$ (c) $\underline{g}=[\overline{2} 00], \underline{z} \sim[011] ; \quad$ (d) $\underline{\mathrm{g}}=[\overline{1} 0 \overline{1}]$, $z \sim$ [II1]. 
Table 5.3. Results of the analyses of the dislocation loops and segments in Figure 5.15. All loops are interstitial, unless otherwise noted, the Burgers vectors of the dislocation segments were not determined exactly and are plus or minus the indicated vector

\begin{tabular}{cccc}
$\begin{array}{c}\text { Loop } \\
\text { or } \\
\text { Segment }\end{array}$ & $\begin{array}{c}\text { Burgers } \\
\text { Vector }\end{array}$ & $\begin{array}{c}\text { Loop } \\
\text { or } \\
\text { Segment }\end{array}$ & $\begin{array}{c}\text { Burgers } \\
\text { Vector }\end{array}$ \\
\hline A & $a / 2[\overline{1} 11]$ & $G-K$ & $a[100]$ \\
B & $a[001]$ & L-D & $a[010]$ \\
$C^{a}$ & $\pm a / 2[1 \overline{1} 1]$ & $Q-U$ & $a[001]$ \\
$D$ & $a / 2[1 \overline{1} 1]$ & $V-Z$ & $a / 2[111]$ \\
E & $a[001]$ & $a-e$ & $a / 2[\overline{1} 11]$ \\
$F^{a}$ & $\pm a / 2[\overline{1} 11]$ & $f$ & $a / 2[11 \overline{1}]$ \\
\hline
\end{tabular}

${ }^{2}$ Segment Burgers vector was determined.

From $10 \mathrm{dpa}$ to $100 \mathrm{dpa}$, as shown in Figure 5.12, the dislocation microstructure consisted primar11y of a coarse distribution of network segments. The graph of the dislocation density (including loops) as a function of displacement damage in Figure 5.16 clearly shows that the maximum dislocation density occurs at $10 \mathrm{dpa}$. A sharp drop in the dislocation density occurs between 10 and $30 \mathrm{dpa}$. Surprising1y, the loop concentration increases and the average diameter decreases from 10 to $100 \mathrm{dpa}$ (see Table 5.2). At $10 \mathrm{dpa}$, very few, large $(\sim 200 \mathrm{~mm})$, round dislocation loops remained in the structure $\left(C_{L}<10^{18} \mathrm{~m}^{-3}\right)$. The loops observed at 30 and $100 \mathrm{dpa}$ are compared in Figure 5.17. As in the 10

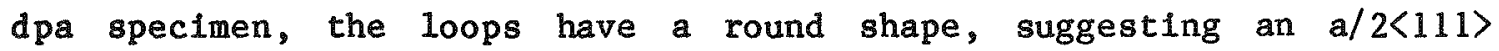
Burgers vector. If the loop character was near edge with $\underline{b}=a\langle 100\rangle$, the loops would exhibit an edge-on profile for beam directions near [001]. Instead, as shown in Figure 5.17, a full perimeter profile is observed for $z \sim[001]$, supporting the conclusion that the loops have $\underline{b}$ $=a / 2\langle 111\rangle$. Analysis of the loops in the 100 dpa specimen showed that $\sim 90 \%$ of the analyzable 10ops had $\underline{b}=a / 2\langle 111\rangle$ and $\sim 10 \%$ of the loops had 


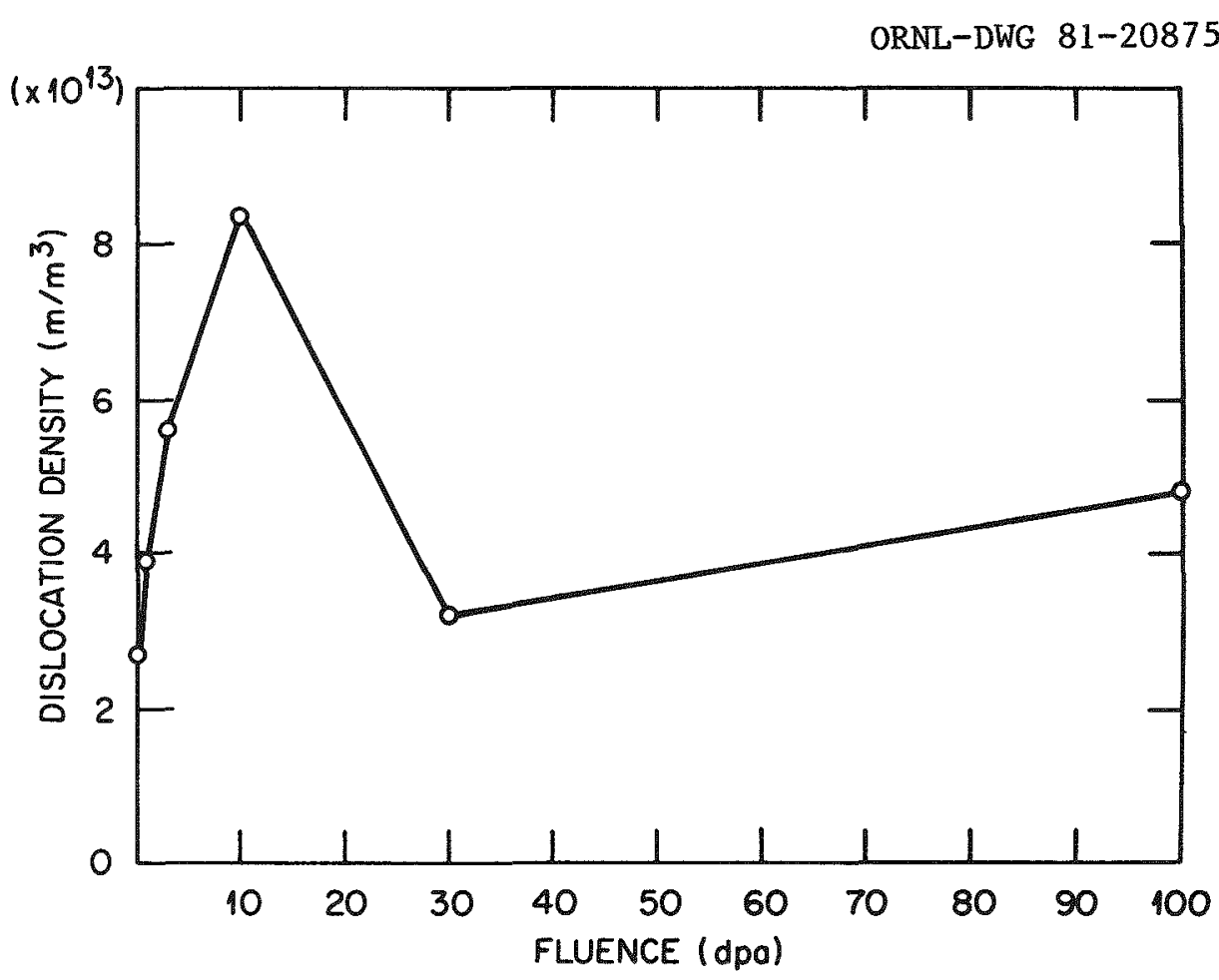

Figure 5.16. Graph of the fluence dependence of the dislocation density (including loops) for $\mathrm{Fe}-10 \% \mathrm{Cr} . \mathrm{T}_{\mathrm{I}} \sim 850 \mathrm{~K}$.
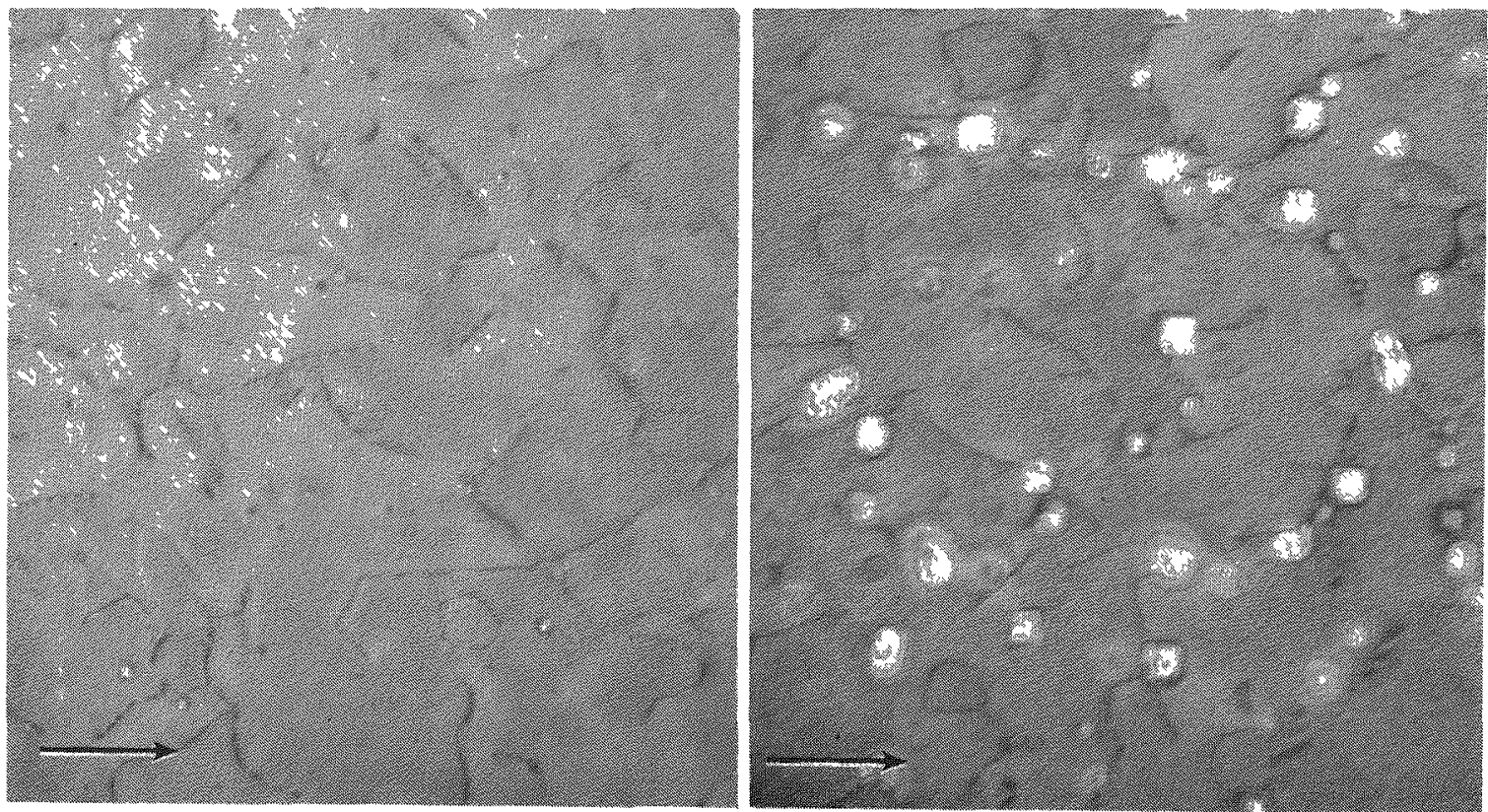

Figure 5.17. TEM micrographs of the dislocation loops observed at 30 and $100 \mathrm{dpa}$ in $\mathrm{Fe}-10 \% \mathrm{Cr}$. Length of arrows equals $300 \mathrm{~mm}$. $\mathrm{T}_{\mathrm{I}} \sim 850 \mathrm{~K}$. $\underline{g}=[1 \overline{1} 0], \underline{z} \sim[001]$. Arrows indicate direction of $g$. 
$\underline{b}=a\langle 100\rangle$. The nature of the loops was not determined. However, it Is likely that they are interstitial loops. A higher concentration of these loops $(\underline{b}=a / 2\langle 111\rangle)$ was observed near grain boundaries than in the grain interior.

Cavity microstructures were observed in specimens irradiated to 3 dpa and above, as shown in Figure 5.13. The cavity distribution was fairly homogeneous in all specimens. Bimodal size distributions were found for the cavity microstructures at 30 and $100 \mathrm{dpa}$. Zones with no cavities were found near the grain boundaries in the specimens irradiated to 10-100 dpa. These grain boundary denuded zones are shown in Figure 5.18 for the 30 and 100 dpa specimens. No grain boundaries were observed in the thin areas of the $3 \mathrm{dpa}$ specimen. Cavities were also observed in association with dislocations. Lines of cavities were found as well (Figure 5.19), another possible indication of cavity growth on dislocations.

The observed cavities had a crystallographic shape. Figure 5.19 shows micrographs of the cavities in a specimen irradiated to $30 \mathrm{dpa}$ taken near each of the three major poles $(\langle 100\rangle,\langle 110\rangle,\langle 111\rangle)$ with $\mathrm{g}$ $=\langle 330\rangle$. Detalled study of the cavity shape shown in these micrographs Indicated that the cavities have a truncated octahedral shape. The cavity facets are on $\{111\}$ with truncations on $\{100\}$. Comparisons of the micrographs taken near the $\langle 100\rangle$ and $\langle 110\rangle$ poles with the computed images of truncated octahedral cavities ( $\{111\}$ facets, $\{100\}$ truncations) for these orientations [128] supported this conclusion. The cavity shape is the one expected for fcc materials. The expected shape for cavities in bcc materials is a truncated twelve-sided polyhedron with $\{110\}$ faces and $\{100\}$ truncations.

According to Wulff's theorem, the ratio of the surface energy, $\gamma_{\text {hkl }}$, to the perpendicular distance, $d_{h k 1}$, between diametrically opposite (hk1) planes which serve as faces of an equilibrium bubble is a constant $[129,130]$. This relationship has been used to measure the ratio of the surface energies of different low index planes for cavities in copper, aluminum, and molybdenum [131]. For the cavities in iron, 
ORNL-Photo 4551-81

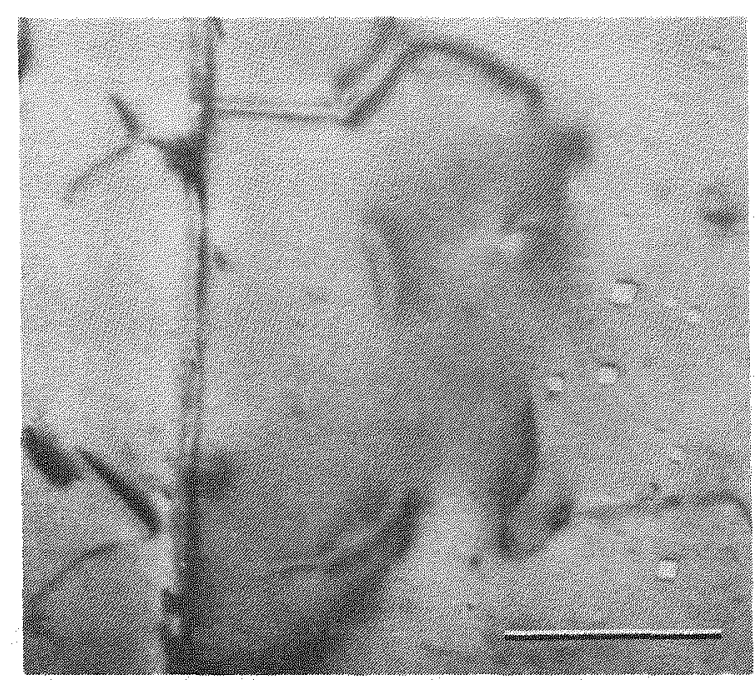

$30 \mathrm{dpa}$

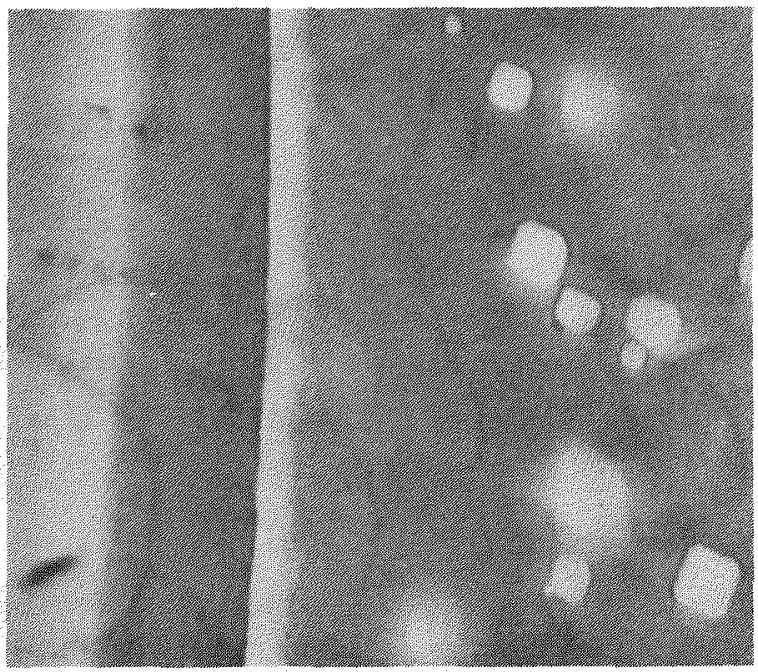

$100 \mathrm{dpa}$

Figure 5.18. TEM micrographs of the zones free of cavities found near grain boundaries in Fe-10\% Cr. $T_{I} \sim 850 \mathrm{~K}$. Scale marker is $300 \mathrm{~nm}$.

ORNL-Photo $4548-81$

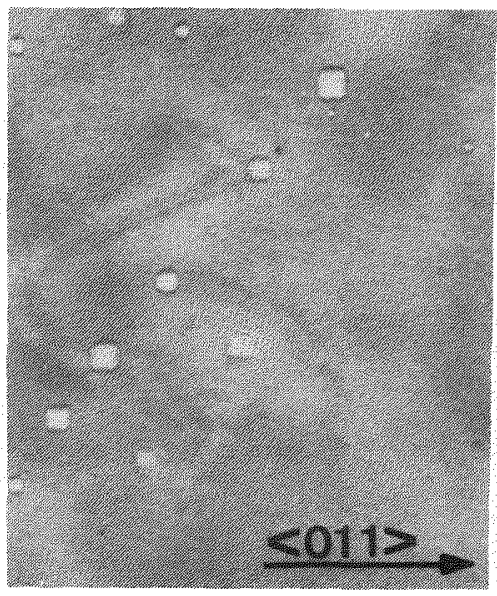

$\underline{z} \sim\langle 100\rangle$

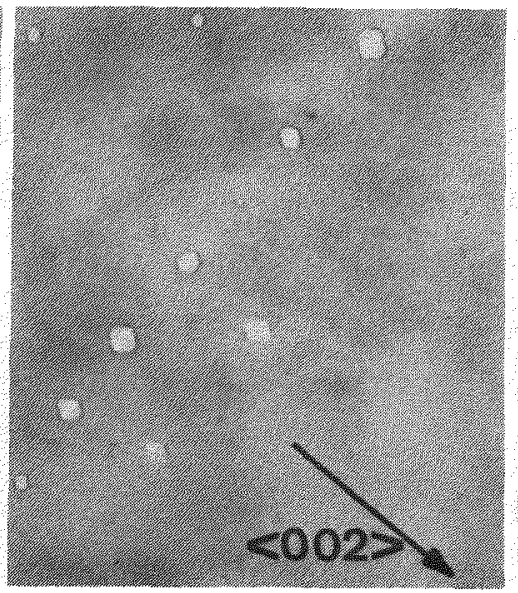

$\underline{z}^{2}\langle 110\rangle$

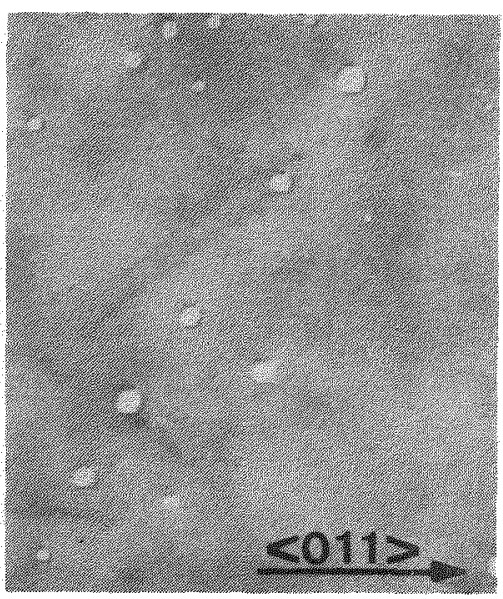

$\underline{z} \sim<111\rangle$

Figure 5.19. TEM micrographs showing the analysis of the cavity shape for triple-beam ion-irradiated $\mathrm{Fe}-10 \% \mathrm{Cr} .30 \mathrm{dpa}, \mathrm{T}_{\mathrm{I}} \sim 850 \mathrm{~K}$. Length of arrows is $200 \mathrm{~nm}$. 


$$
\frac{r_{111}}{d_{111}}=\frac{r_{100}}{d_{100}}=a \text { constant }
$$

or

$$
\frac{r_{111}}{r_{100}}=\frac{d_{111}}{d_{100}}
$$

Equation (5.1) was used to determine the surface energy ratio for the cavities found in the $30 \mathrm{dpa}$ specimen. Both $d_{111}$ and $d_{100}$ were measured for a number of cavities from the cavity projection for a $\langle 110\rangle$ beam direction. Additional measurements of $d_{100}$ were made for each cavity from a projection for a $\langle 100\rangle$ beam direction. The average value for the $d_{111} / d_{100}$ ratios calculated from these measurements was 0.77 with a standard deviation of 0.06 . Therefore, the surface energy ratio is given by

$$
\frac{r_{111}}{r_{100}}=0.77 \pm 0.06
$$

The quantitative data for the measured cavity concentration and average cavity diameter are plotted with the calculated cavity volume fraction (swelling) in Figure 5.20. (The values for these data are also 1isted in Table 5.2.) At 30 and $100 \mathrm{dpa}$, the cavities had a bimodal distribution for the measured cavity diameters. In the histogram of the measured cavity diameters for the 30 dpa specimens, about $25 \%$ (a concentration of $\sim 4.4 \times 10^{19} \mathrm{~m}^{-3}$ ) of the cavities were found in a peak centered at $\sim 7 \mathrm{~nm}$ while the remaining $75 \%$ (a concentration of $\sim 1.6 \times$ $10^{20} \mathrm{~m}^{-3}$ ) were in a peak centered at $\sim 20 \mathrm{~mm}$. The volume-averaged cavity diameters for these peaks were 7.8 and $22.1 \mathrm{~nm}$. At $100 \mathrm{dpa}$, the two peaks were 1 ess distinct. The lower peak ( $20 \%$ of the cavities) had a volume-averaged diameter of $\sim 40 \mathrm{~nm}$ and the upper peak $(\sim 80 \%$ of the cavities) had a volume-averaged diameter of $\sim 84 \mathrm{~nm}$. The values listed in Table 5.2 and Figure 5.20 for the volume-averaged cavity diameter and the cavity concentration are the values for the entire distribution. 
ORNL-DWG 81-20876

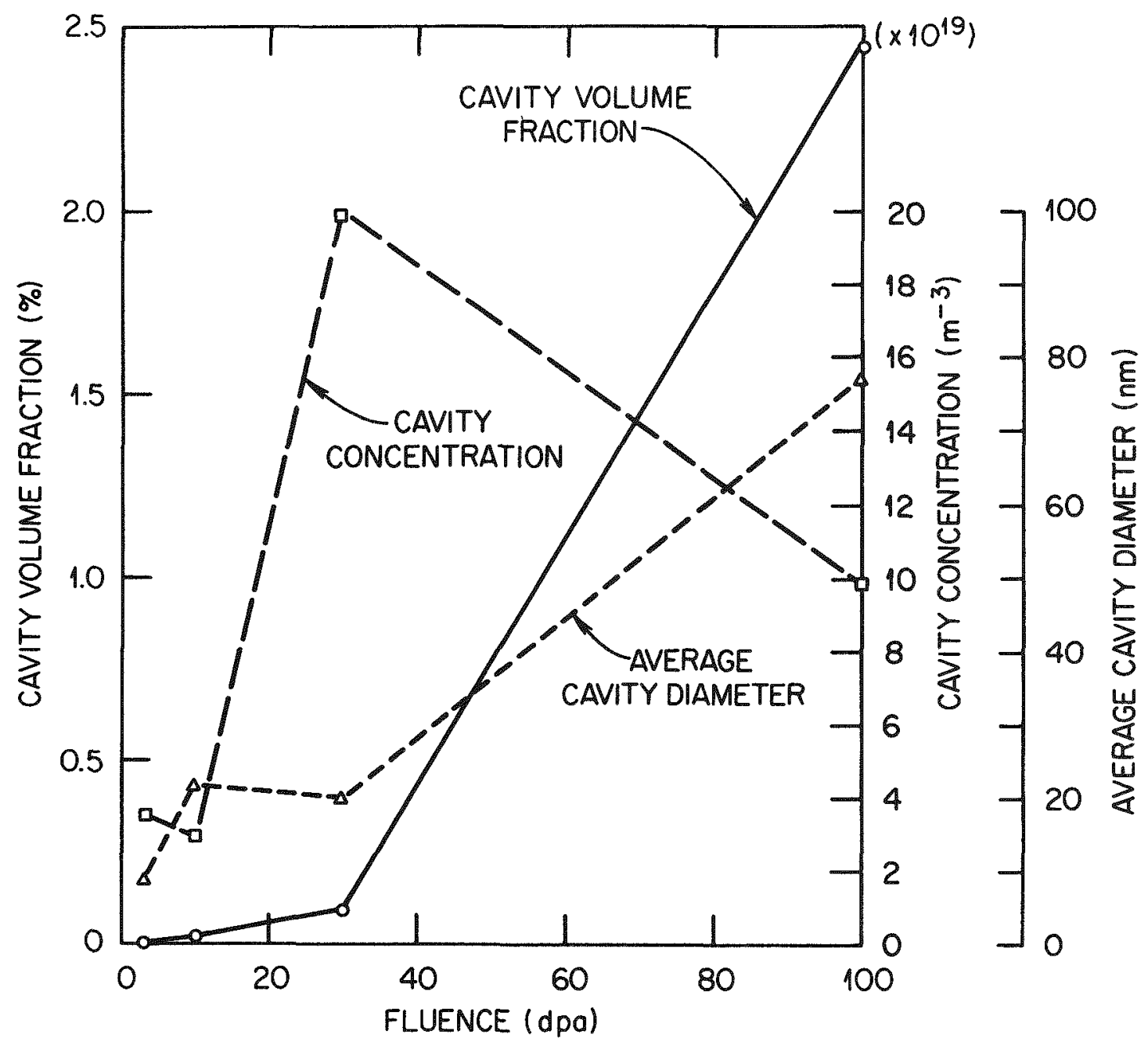

Figure 5.20. Graph of the fluence dependence of the cavity concentration, average cavity diameter, and cavity volume fraction (swelling) for $\mathrm{Fe}-10 \% \mathrm{Cr}$. $\mathrm{T}_{\mathrm{I}} \sim 850 \mathrm{~K}$.

In studying the quantitative cavity data, it is interesting to note that the average cavity diameter and the swelling increase aramatically between 30 and $100 \mathrm{dpa}$. Possible causes of this observation will be discussed in Chapter 6 .

5.4 Effect of Alloy Chromium Content

The microstructures found in specimens of $\mathrm{Fe}, \mathrm{Fe}-5 \% \mathrm{Cr}$, and Fe-10\% Cr triple-beam Ion-irradiated to $10 \mathrm{dpa}$ at $850 \mathrm{~K}$ (Runs 534 and 601 ) are shown in Figure 5.21. The quantitative data are summarized in 


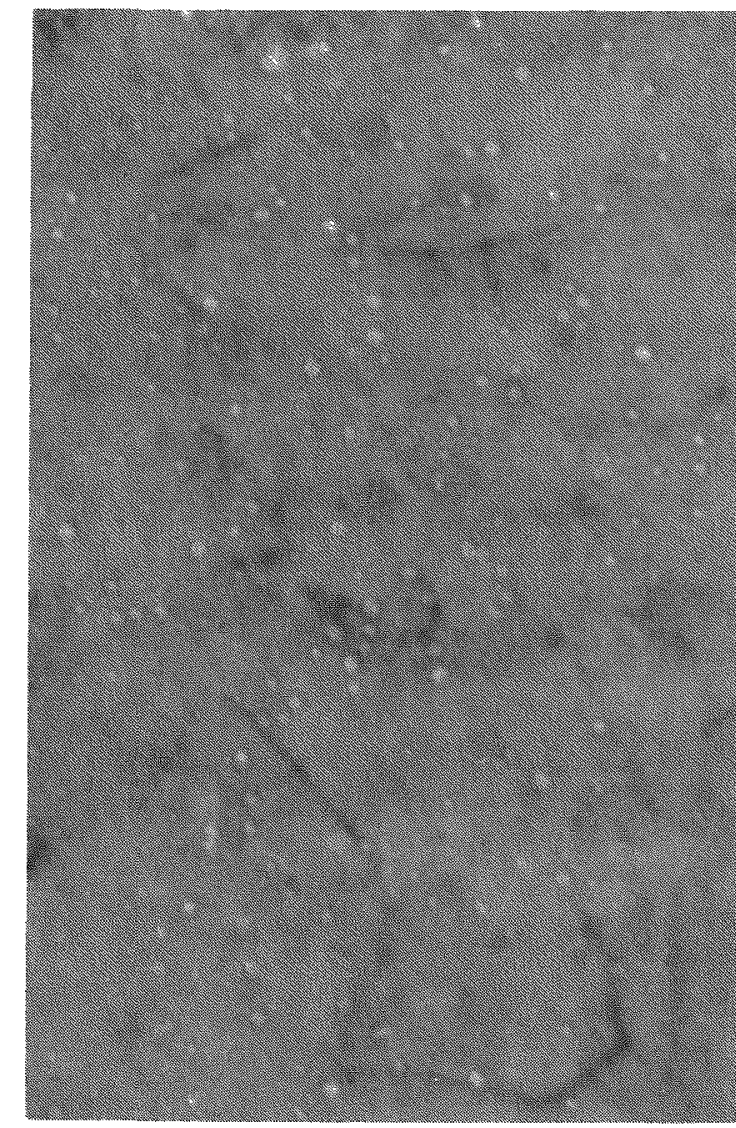

$\mathrm{Fe}$

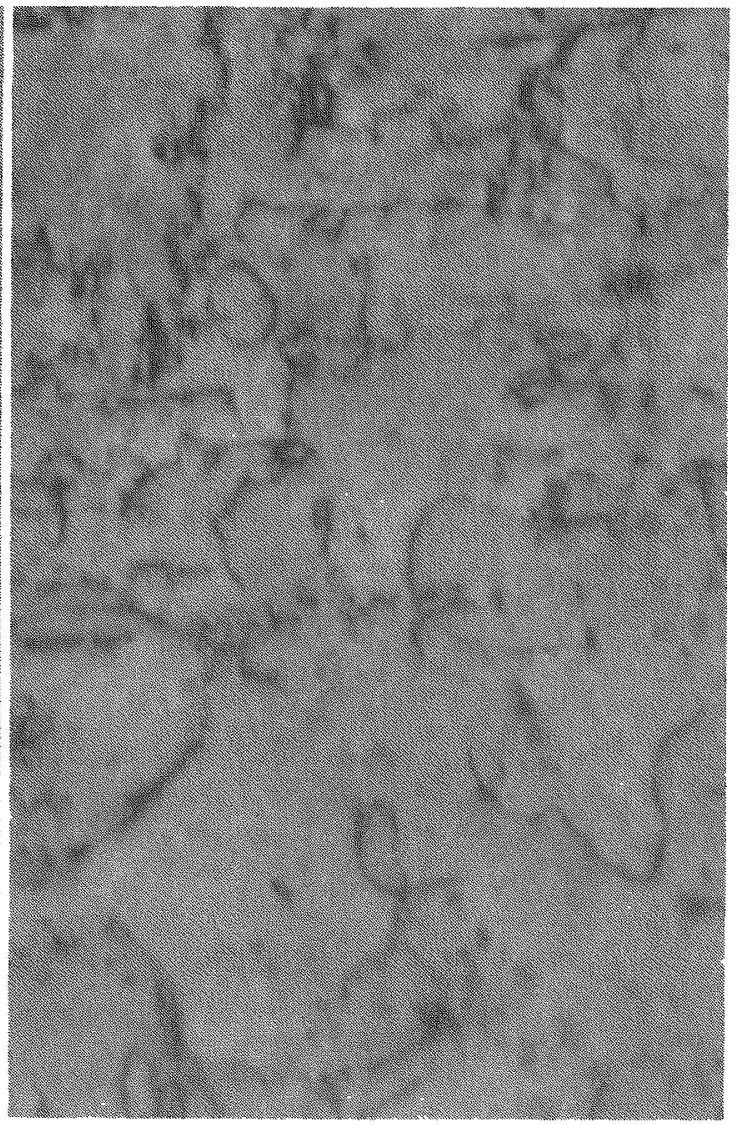

$\mathrm{Fe}-5 \% \mathrm{Cr}$

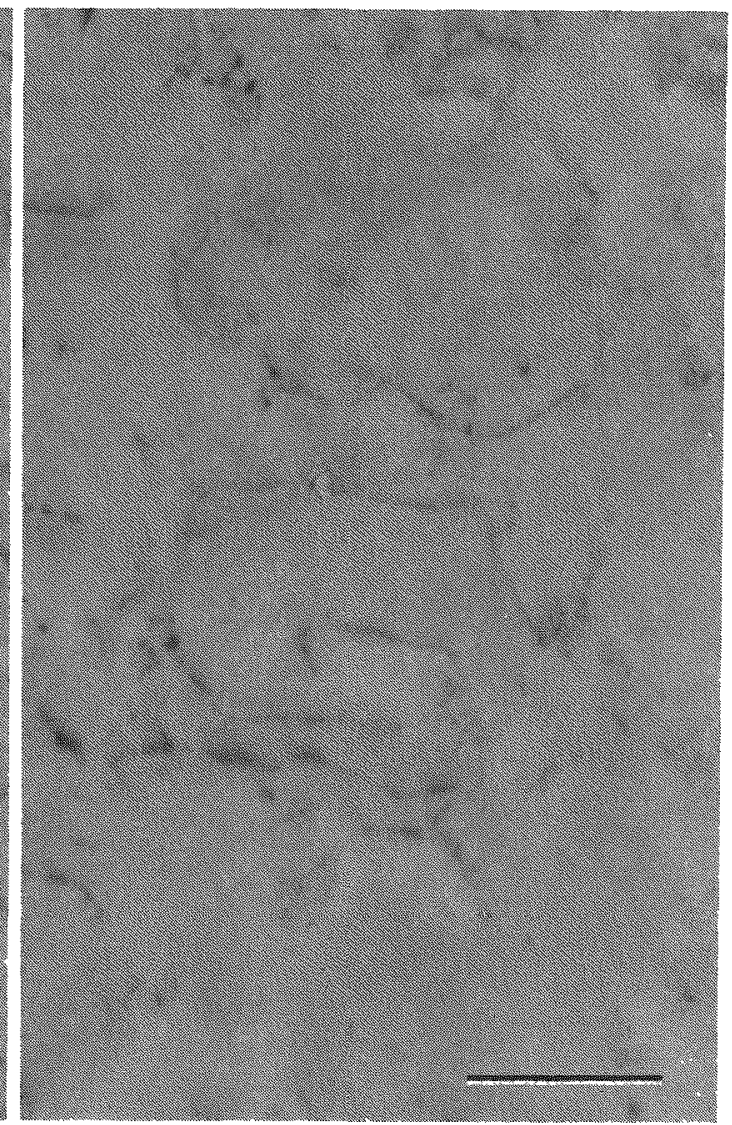

$\mathrm{Fe}-10 \% \mathrm{Cr}$

Figure 5.21. TEM micrographs showing a comparison of the microstructures observed in triple-beam ionirradiated $\mathrm{Fe}, \mathrm{Fe}-5 \% \mathrm{Cr}$, and $\mathrm{Fe}-10 \% \mathrm{Cr} .10 \mathrm{dpa}, 100$ at. ppm He, 410 at. ppm $\mathrm{D}, \mathrm{T}_{\mathrm{I}} \sim 850 \mathrm{~K}$. Scale marker is $200 \mathrm{~nm}$. 
Table 5.4 and the graph in Figure 5.22. The dislocation microstructures in all of the alloys consisted primarily of a coarse network of dislocation segments. The dislocation density was highest for the Fe-5\% Cr specimen and lowest for the iron specimen. Cavities were found only in the iron and $\mathrm{Fe}-10 \% \mathrm{Cr}$ specimens. The cavities in the Fe-10\% Cr specimens were about a factor of 2 larger in diameter and two orders of magnitude lower in concentration than the cavities in the iron specimen. The swelling (cavity volume fraction) was, therefore, about an order of magnitude lower in $\mathrm{Fe}-10 \% \mathrm{Cr}$ than in iron.

In neutron-irradiation experiments, Little and Stow [44] have observed a relationship between chromium content and swelling similar to that reported here $\left[\mathrm{T}_{\mathrm{I}} \sim 700 \mathrm{~K}\left(420^{\circ} \mathrm{C}\right)\right]$. They stated that the increase in the swelling in $\mathrm{Fe}-10 \% \mathrm{Cr}$ as compared to the swelling in Fe-5\% Cr was related to $\alpha^{-}$precipitation and the corresponding nonequilibrium segregation of chromium in Fe-10\% $\mathrm{Cr}$. However, no $\alpha^{\prime}$ precipitates were observed in the current investigation, which suggests that the increase in swelling is based on a different mechanism.

Table 5.4. Summary of the quantitative microstructural data for specimens examined to determine the effect of chromium content on the irradiation-induced microstructure. The nominal irradiation temperature, $\mathrm{T}_{\mathrm{N}}$, is $850 \mathrm{~K}$; the nominal damage level, $\mathrm{dpa}_{\mathrm{N}}$ is $10 \mathrm{dpa}^{a}$

\begin{tabular}{lcccccccc}
\hline Material & Specimen & $\begin{array}{c}\mathrm{T}_{\mathrm{I}} \\
(\mathrm{K})\end{array}$ & $\mathrm{dpa}$ & $\begin{array}{c}\Lambda \\
\left(\mathrm{m} / \mathrm{m}^{3}\right)\end{array}$ & $\begin{array}{c}\mathrm{C}_{\mathrm{C}_{3}} \\
\left(\mathrm{~m}^{-3}\right)\end{array}$ & $\begin{array}{c}\mathrm{d}_{\mathrm{c}} \\
(\mathrm{nm})\end{array}$ & $\begin{array}{c}\mathrm{CVF} \\
(\%)\end{array}$ \\
Iron & $534-32$ & 850 & 10.6 & $5 \times 10^{13}$ & $2 \times 10^{21}$ & 13 & 0.22 \\
Fe-5\% Cr & $534-22$ & 852 & 10.6 & $1.3 \times 10^{14}$ & $\mathrm{~b}$ & & \\
Fe-10\% Cr & $534-12$ & 848 & 10.1 & $1.1 \times 10^{14}$ & $2.6 \times 10^{19}$ & 23 & 0.02 \\
Fe-10\% Cr & $601-12$ & 855 & 9.6 & $5.7 \times 10^{13}$ & $3.2 \times 10^{19}$ & 21 & 0.02
\end{tabular}

\footnotetext{
a Notation: $T_{I}=$ actual irradiation temperature; $\Lambda=$ dislocation density; $\mathrm{C}_{\mathrm{c}}=$ cavity concentration, $\mathrm{d}_{\mathrm{c}}=$ volume-average cavity diameter; and $\mathrm{CVF}=$ cavity volume fraction or $b_{\text {None observed. }}$ swelling.
} 


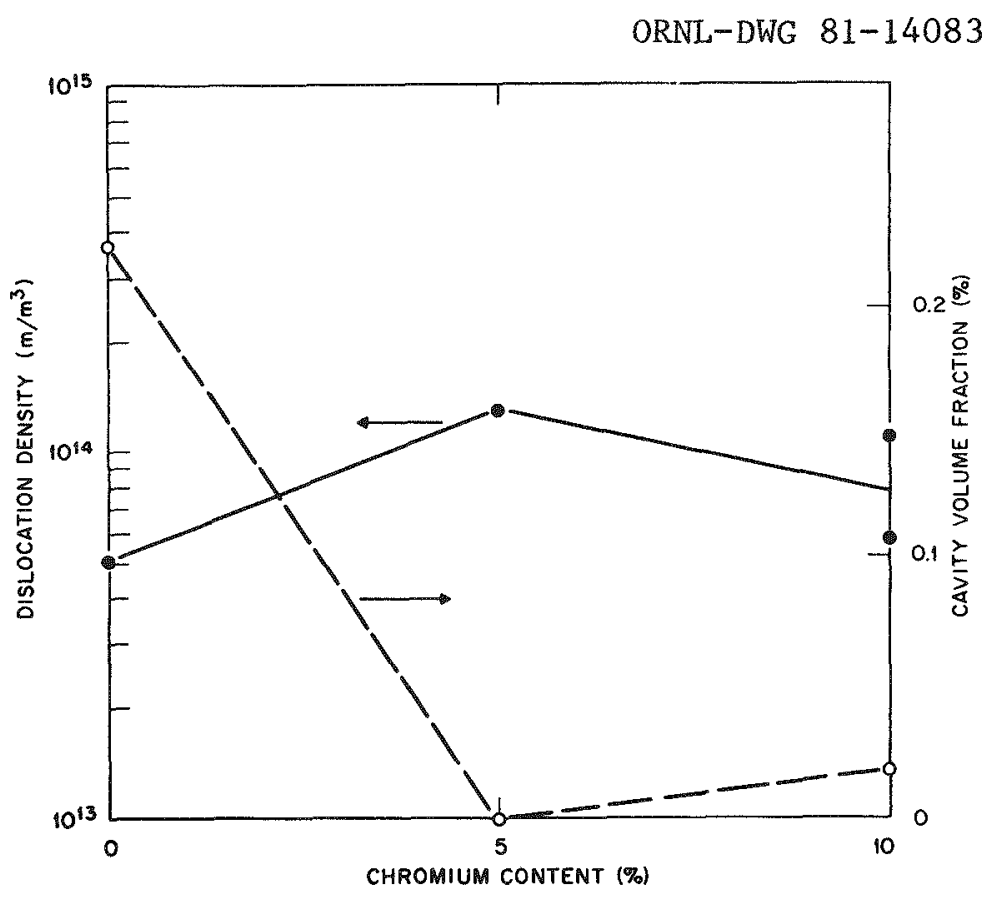

Figure 5.22. Graph showing the dependence of the dislocation density and cavity volume fraction on the alloy chromium content. $10 \mathrm{dpa}, \mathrm{T}_{\mathrm{I}} \sim 850 \mathrm{~K}$.

Gelles [45] has observed that neutron-irradiated Fe-3\% Cr specimens swell less than specimens of $\mathrm{Fe}-6 \% \mathrm{Cr}, \mathrm{Fe}-9 \% \mathrm{Cr}$, and $\mathrm{Fe}-12 \% \mathrm{Cr}$ for irradiation temperatures of $700 \mathrm{~K}\left(425^{\circ} \mathrm{C}\right)$. In his study, a higher concentration of $a\langle 100\rangle$ dislocations was present in the irradiated $\mathrm{Fe}-3 \% \mathrm{Cr}$ specimens than in the higher chromium content alloys. Also, the fraction of $a / 2\langle 111\rangle$ dislocations found in the specimens increased with increasing chromium content. Gelles suggests that there is a strong correlation between the presence of a<100> dislocations and swelling resistance. In the present study, Burgers vector analyses for the dislocation networks were not performed. However, primarily based on the higher density of dislocations in the $\mathrm{Fe}-5 \% \mathrm{Cr}$ specimen than in the $\mathrm{Fe}$ and $\mathrm{Fe}-10 \% \mathrm{Cr}$ specimens, it seems likely that more a $\langle 100\rangle$ dislocations would be present in the $\mathrm{Fe}-5 \% \mathrm{Cr}$ specimen.

\subsection{Effect of Helium and Deuterium}

Comparisons of the microstructures found in Fe-10\% $\mathrm{Cr}$ triplebeam ion-irradiated to $30 \mathrm{dpa}$ ( 300 at. ppm He, 1230 at. ppm D, Run 601) and those found in $\mathrm{Fe}-10 \% \mathrm{Cr}$ irradiated with a single beam of $4 \mathrm{MeV}$ 
iron ions to $30 \mathrm{dpa}$ (Run 602) are shown in Figures 5.23 and 5.24. The quantitative data for this comparison are listed in Table 5.5.

The dislocation microstructures found in both the triple-beam and iron ion-irradiated specimens consist primarily of a coarse network of dislocation segments (Figure 5.23). A few large, round dislocation loops were found for both types of irradiated specimens. The dislocation density for the triple-beam irradiation was $~ 50 \%$ higher than for the single ion irradiation.

The cavities found in both types of irradiated specimens had a crystallographic shape. However, the cavities in the triple-beam ionirradiated specimens had a bimodal distribution, as discussed previousIy (Section 5.3), while those in the single-ion irradiated specimen did not. The cavities in the triple-beam ion-irradiated specimen were smaller in diameter by $\sim 50 \%$ but were present at a concentration larger by a factor of $\sim 30$ than the cavities in the iron ion-irradiated specimens. The swelling in the specimen irradiated by the triple-beam technique was higher by a factor of $\sim 4.5$ than the swelling in the specimen irradiated only by iron lons.

The above results are consistent with the helium effects observed for other materials. A higher concentration of smaller cavities has been observed in austenitic stainless steel irradiated with a dual beam of nickel and helium ions as compared to the cavity distribution found in specimens irradiated with only nickel lons [132]. Although the dislocation density is not as sensistive to helium implantation, coimplantation with helium and nickel ions produced a higher dislocation density than irradiation with only nickel ions [132]. In general, for triple-beam ion (nickel, helium, deuterium) irradiation of austenitic stainless steel, especially in the temperature range from 800 to $950 \mathrm{~K}$, these effects are further enhanced - e.g., the cavities are smaller and have a higher concentration and the dislocation density is higher than for dual ion (nickel and helium) irradiations [133].

Specimens of $\mathrm{Fe}-10 \% \mathrm{Cr}$ were also preinjected at room temperature with 100 at. ppm He and 410 at. ppm D followed by bombardment with $4 \mathrm{MeV}$ $\mathrm{Fe}^{++}$ions at $850 \mathrm{~K}$ to $10 \mathrm{dpa}$. No cavities were observed in these specimens. The dislocation structure consisted of $100 \mathrm{p}$ and network segments, 


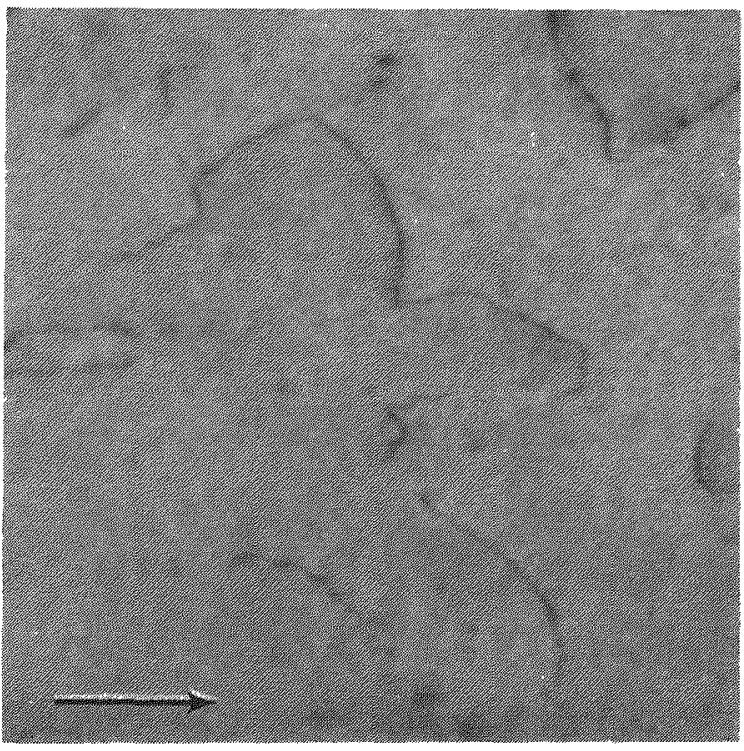

triple-beam

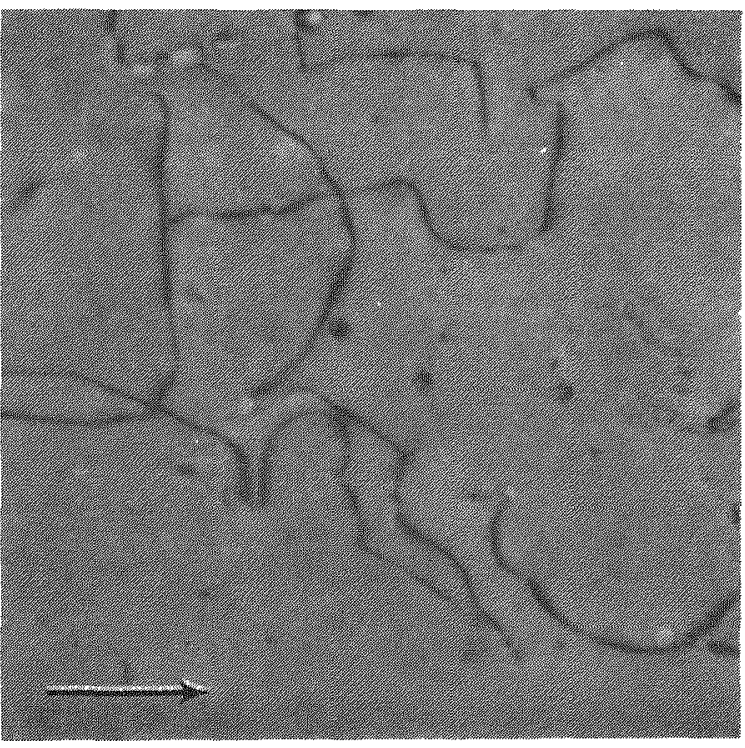

single beam

Figure 5.23. TEM micrographs of the dislocation microstructures found in Fe-10\% Cr irradiated with a triple-beam ( $\mathrm{Fe}, \mathrm{He}, \mathrm{D})$ and a single beam ( $\mathrm{Fe}$ ) of ions. $30 \mathrm{dpa}, \mathrm{T}_{\mathrm{I}} \sim 850 \mathrm{k}, \underline{\mathrm{g}}=[0 \overline{\mathrm{I}} 1], z \sim[011]$. Length of arrows is $200 \mathrm{~nm}$. Arrows indicate direction of $\mathrm{g}$.

ORNL-Photo $4549-81$
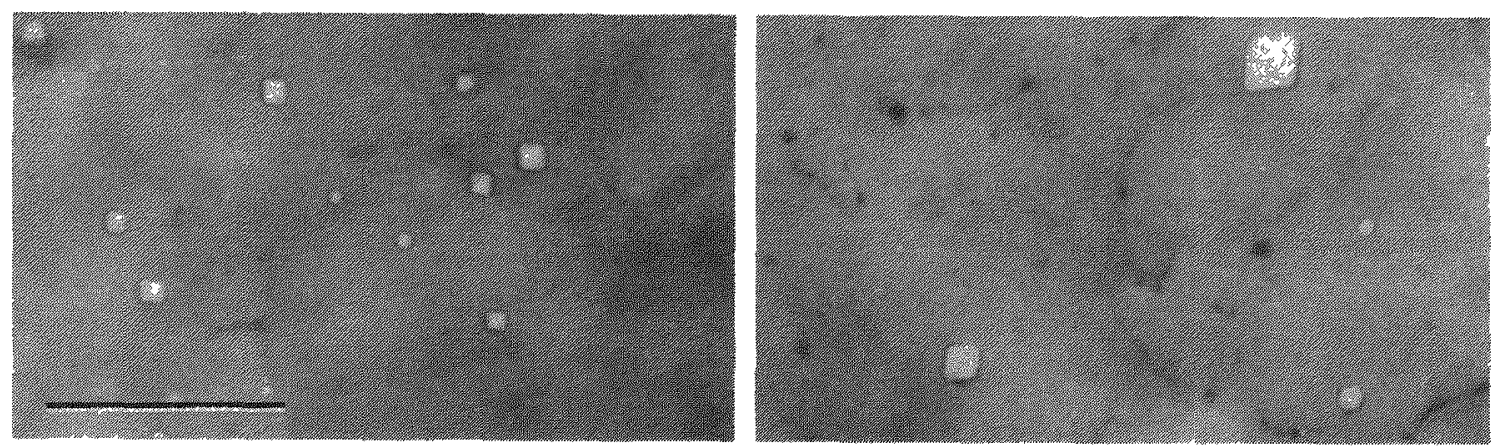

Figure 5.24. TEM micrographs of the cavity microstructures found in $\mathrm{Fe}-$ $10 \% \mathrm{Cr}$ irradiated with a triple beam ( $\mathrm{Fe}, \mathrm{H}, \mathrm{D}$ ) and a single beam ( $\mathrm{Fe}$ ) of ions. $30 \mathrm{dpa}, \mathrm{T}_{\mathrm{I}} \sim 850 \mathrm{~K}$. Scale marker is $300 \mathrm{~nm}$. 
Table 5.5. Quantitative data for triple-beam and 4 MeV iron ion-irradiated $\mathrm{Fe}-10 \% \mathrm{Cr} .30 \mathrm{dpa}^{\mathrm{a}}$

\begin{tabular}{llccccc}
\hline Specimen & Irradiation & $\begin{array}{c}\Lambda \\
\left(\mathrm{m} / \mathrm{m}^{3}\right)\end{array}$ & $\begin{array}{c}\mathrm{C}_{\mathrm{c}_{3}} \\
\left(\mathrm{~m}^{-3}\right)\end{array}$ & $\begin{array}{l}\mathrm{d}_{\mathrm{c}} \\
(\mathrm{nm})\end{array}$ & $\begin{array}{l}\mathrm{CVF} \\
(\%)\end{array}$ \\
\hline $601-23$ & Triple bean & $3.2 \times 10^{13}$ & $2.0 \times 10^{20}$ & 20 & 0.09 \\
$602-21$ & 4 MeV Fet+ & $2.1 \times 10^{13}$ & $7 \times 10^{18}$ & 37 & 0.02 \\
\hline
\end{tabular}

a Notation: $\Lambda=$ dislocation density (including loops); $C_{c}=$ cavity concentration, $d_{c}=$ volume-average cavity diameter; and $C V F=$ cavity volume fraction or swelling.

as shown in Figure 5.25, with a density of $\Lambda=4.96 \times 10^{13} \mathrm{~m}^{-2}$ (2p $=4.96 \times 10^{13} \mathrm{~m}^{-2}$ as well). For Fe-10\% Cr specimens irradiated at 850 $\mathrm{K}$ to $10 \mathrm{dpa}$ with a triple beam of ions, the dislocation density was somewhat higher than in the specimens preinjected with helium and deuterium. Cavities were also observed in the triple-beam ion-irradiated specimen, as discussed earlier.

As in the current study, Packan and Farrel1 [132] have found that, for irradiations of an austenitic alloy similar to 316 stainless stee1, room-temperature pre-injection of helium followed by $4 \mathrm{MeV}$ $\mathrm{Ni}^{+H}$ bombardment to $10 \mathrm{dpa}$ at $898 \mathrm{~K}$ caused a reduction in the dislocation density compared to triple-beam ion-irradiation (10 dpa, 200 at. ppm He, 500 at. ppm D, $898 \mathrm{~K}$ ). They also found that the cavity concentration was about two orders of magnitude higher and the volumeaveraged cavity diameter was about an order of magnitude smaller in the specimens pre-injected with helium than in the triple-beam ionirradiated specimens. If a similar decrease in the cavity diameter occurred in the $\mathrm{Fe}-10 \% \mathrm{Cr}$ specimens, the cavity diameter for the preinjected specimens would be $\sim 2 \mathrm{~nm}$. It is possible that these small cavities were present in the specimen, but that they were too small to be resolved with the JEM 120C TEM using the AMG pole-piece. Further examination with the JEM $120 \mathrm{CX}$ did not reveal cavities, suggesting that, if they are present, the cavity diameter is less than $2 \mathrm{~nm}$. Further experiments using higher damage and gas concentration are required to clarify the effects of gas pre-injection on the microstructura1 damage. 
YE- 12400

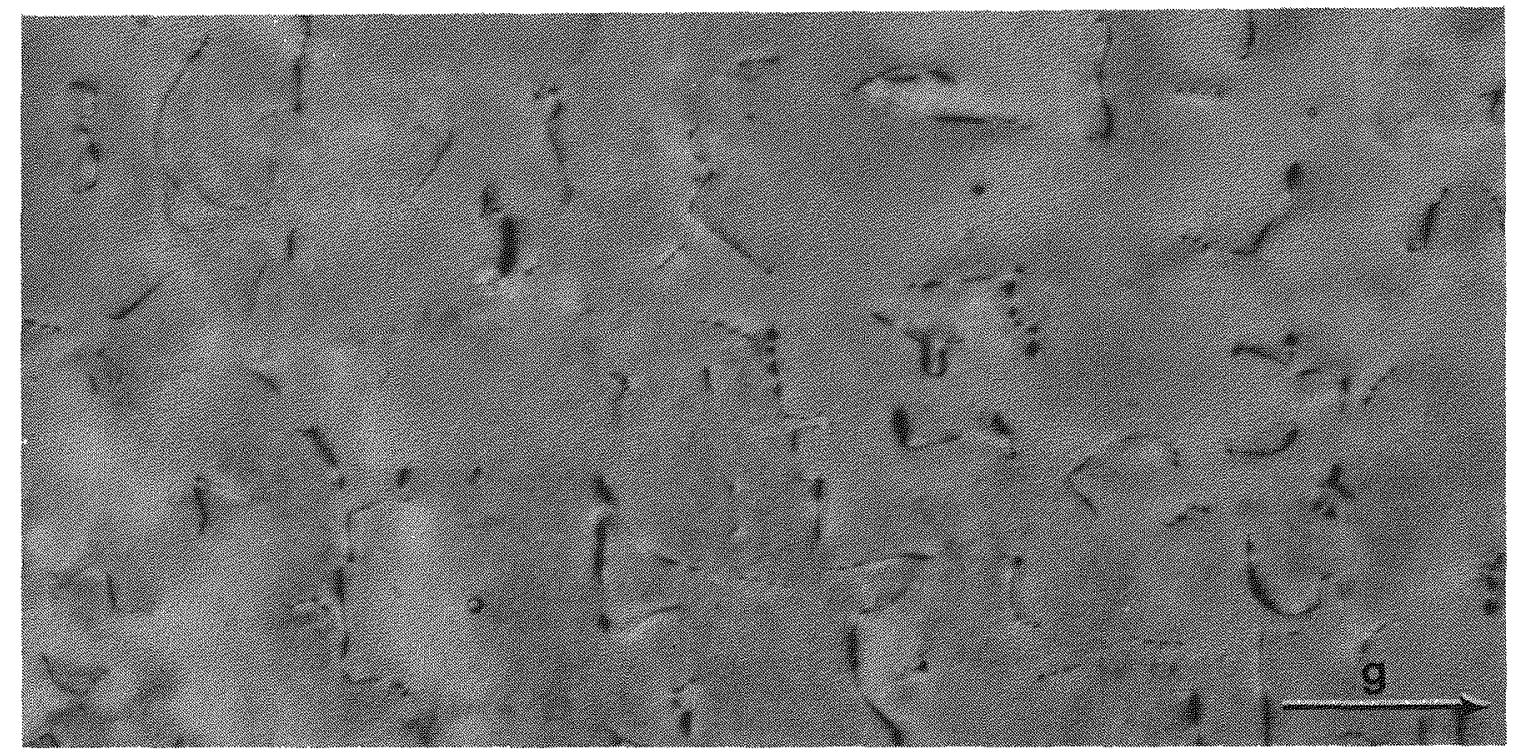

Figure 5.25. TEM micrograph showing the dislocation microstructures found in $\mathrm{Fe}-10 \% \mathrm{Cr}$ which was pre-injected with 100 at. ppm He and 410 at. ppm D followed by bombardment with $4 \mathrm{MeV} \mathrm{Fe}{ }^{++}$ions at $850 \mathrm{~K}$ to 10 $\mathrm{dpa}$. The arrow indicates the direction of $\underline{\mathrm{g}}=[0 \overline{1} 1]$. The length of the arrow is $300 \mathrm{~nm}$. 
In the first section of this chapter, several aspects of the characteristics of the damage microstructures common to both the neutron and the ion irradiation experiments will be discussed (section 6.1). Then the results will be related to the currently proposed mechanisms for swelling suppression in ferritic materials (Section 6.2). A possible mechanism for the evolution of the damage microstructure with increasing fluence, based on the $\mathrm{Fe}-10 \% \mathrm{Cr}$ ion irradiation results, will be considered in the final section (6.3) of this chapter.

\subsection{Damage Microstructures}

\subsubsection{Dislocations}

One microstructural feature common to both the neutronirradiated iron and the ion-irradiated iron and iron-chromium alloys was the observation of near-edge, interstitial loops with predominantly $a\langle 100\rangle$ Burgers vectors. No vacancy loops were observed for either type of irradiation. As presented in Chapter 2, these observations are consistent with the reports of other investigators. A large percentage of loops with $\underline{b}=a / 2\langle 111\rangle$ were found only in the halos in iron specimens neutron-irradiated at the relatively low irradiation temperature of 523 $\mathrm{K}$, supporting the idea that formation of loops with $\underline{b}=a\langle 100\rangle$ requires elevated irradiation temperatures. In the $\mathrm{Fe}-10 \% \mathrm{Cr}$ ion-irradiated at $850 \mathrm{~K}$ to 3,30 , and $100 \mathrm{dpa}$, a few a/2<111〉 interstitial loops were also observed. Especially interesting is the apparent continuing nucleation of a/2<111> loops between 30 and $100 \mathrm{dpa}$. This observation will be discussed in more detail in Section 6.3 .

In the ion-irradiated specimens the dislocation loops with $b$ $=a\langle 100\rangle$ had a convoluted shape, except at low fluences $(0.3 \mathrm{dpa})$. The shape of the loops suggested preferential growth in the $\langle 110\rangle$ directions. 
The dislocation loops in the neutron-irradiated specimens were not convoluted. Irregularly shaped loops have also been observed in electron-irradiated iron [61], Fe-15\% Cr [65], aluminum [62], and austenitic stainless steel $[134,135]$, as we11 as in neutron-irradiated austenitic stainless steels $[134,135]$. The exact cause of irregularly shaped loops is not clear. For a $\langle 100\rangle$ loops in the ferritic materials, it has been suggested that interstitials are preferentially attracted to the corners of small, rectilinear loops with $\langle 100\rangle$ sides [61]. In electron and ion irradiations, the interstitial generation rate is much higher than in neutron irradiations. During neutron irradiations, the arrival rate of interstitials at the loop corners could be slow enough to allow diffusion along the loop, resulting in regularly shaped loops, as are observed. During electron and iron irradiation, however, the higher arrival rate for interstitials at the loop corners could cause enhanced growth at the corners as compared to the straight segments of the loops. This would lead to enhanced growth in the $\langle 110\rangle$ directions, as observed in the current investigation. The formation of small precipitates on the loops and segregation of impurity atoms, including chromium, to the vicinity of the loops could also contribute to the growth of irregularly shaped loops. It has been suggested that preclpitate intersection events cause the irregular loop shapes in the austenitic stainless steels $[134,135]$. The rectilinear shape of small a $\langle 100\rangle$ loops could force preferential segregation on the $\langle 100\rangle$ sides leading to enhanced growth in only the $\langle 110\rangle$ directions, as observed. In addition, the $a / 2\langle 111\rangle$ interstitial loops found with the convoluted a $\langle 100\rangle$ loops in ion-irradiated $\mathrm{Fe}-10 \% \mathrm{Cr}(850 \mathrm{~K} ; 3,30$, and $100 \mathrm{dpa})$ did not have an irregular shape. This could be the result of preferential segregation to the $\{100\}$, as suggested by reports of some precipitates [e.g., carbides and nitrides] forming on $\{100\}$ in bcc iron alloys [136].

Many of the a $\langle 100\rangle$ dislocation 1oops were observed to intersect the foil surfaces without gliding out of the foil. This could be the result of dislocation pinning by impurity atmospheres. However, this observation could also be interpreted as an indication of the nearly sessile nature of $a\langle 100\rangle$ dislocation loops $[51,60]$. The image forces 
may not be strong enough to initiate loop glide. In addition, the surface oxide present in both the iron and the iron-chromium alloys could restrict the gliding of dislocations from the foll.

In the irradiation-temperature-dependence studies for both neutron-irradiated iron and ion-irradiated $\mathrm{Fe}-10 \% \mathrm{Cr}$, a rapld decrease to near preirradiation dislocation densities was observed. In the neutron-irradiated iron, this drop occurred for an increase in the irradiation temperature from 773 to $923 \mathrm{~K}$. No specimens were irradiated over the interval of $773 \mathrm{~K}\left(\rho=3 \times 10^{11} \mathrm{~m}^{-2}\right)$ to $923 \mathrm{~K}\left(\rho<10^{11} \mathrm{~m}^{-2}\right)$. In the ion-irradiated Fe-10\% $\mathrm{Cr}$, the drop to residual dislocation density levels was more dramatic. It occurred for an increase in the irradiation temperature of $25^{\circ}(850$ to $875 \mathrm{~K})$. In the Ion irradiations, the drop in the dislocation density may be emphasized due to enhanced loss of point defects to the surface at the higher irradiation temperature [124]. These observations suggest a strong temperature dependence of the dislocation microstructures, especially the interstitial dislocation loop nucleation rate.

\subsubsection{Cavities}

The low swelling reported in this investigation is consistent with previously reported results for ferritic materials, as outined in Chapter 2. As expected from the literature, the observed swelling was much lower than that found in many similarly irradiated austenitic refractory bcc materials.

Cavity Growth Modes - of particular interest was the dramatic increase in the swelling found when comparing the swelling for Fe-10\% Cr irradiated at $850 \mathrm{~K}$ to 30 and $100 \mathrm{dpa}$. The increase in the swelling was primarily caused by an increase in the volume-averaged cavity diameter from $\sim 20 \mathrm{~nm}$ at $30 \mathrm{dpa}$ to $\sim 75 \mathrm{~nm}$ at $100 \mathrm{dpa}$. Rate theory calculations were performed to determine if this rapid cavity growth resulted from the cavity radius exceeding the critical cavity radius, $r_{\text {crits }}$ required for bias-driven cavity growth. These calculations are shown in Appendix $F$. Values of $r_{\text {crit }}$ were calculated for an irradiation temperature of $850 \mathrm{~K}$, the dislocation density and cavity concentration 
measured for the $30 \mathrm{dpa}$ specimen, and the assumed values for the other variables as presented in Appendix $F$. The value for $r_{c r i t}$ varied from $\sim 0.2 \mathrm{~nm}$ (hellum pressure in the bubble, $P$, equal to $90 \%$ of the pressure required for an equilibrium bubble, $P_{\text {eq }} ; i_{\text {.e. }}, P=0.9 P_{\text {eq }}$ ) to $\sim 0.9 \mathrm{~nm}$ $\left(P=0.5 P_{e q}\right)$ to $\sim 1.5 \mathrm{~nm}\left(P=0.1 \quad P_{e q}\right)$. Since the average cavity diameter is $20 \mathrm{~nm}$ at $30 \mathrm{dpa}$, it is therefore quite unlikely that a shift to bias-driven growth is the cause of the large increase in the cavity diameter.

As an alternative explanation, the possibility that all of the cavity growth, from 3 to $100 \mathrm{dpa}$, occurred as equilibrium bubble growth was considered. In order to establish whether the observed cavities could be equilibrium bubbles, the number of helium atoms, $n_{e q}$, required to stabilize the cavities as equilibrium bubbles was calculated. The high-density-equation-of-state (HDEOS) proposed by Wolfer $[137,138]$ was used to calculate $\mathrm{n}_{\mathrm{eq}}$. This calculation is presented in detail and compared to similar calculations using the ideal gas law and a modified Van der Waals equation of state in Appendix $G$. The values of $\mathrm{n}_{\text {eq }}$ calculated for an irradiation temperature of $850 \mathrm{~K}$, a surface energy of $2 \mathrm{~J} \mathrm{~m}^{-2}$, and the average cavity diameters observed at each fluence are listed in Table 6.1. For each fluence, the average number of helium atoms, $n$, in each cavity (assuming that all of the helium is in the resolvable cavities) is given by the expression

$$
\mathrm{n}=\frac{\mathrm{C}_{\mathrm{He}}}{\mathrm{C}_{\mathrm{c}}},
$$

where $C_{H e}$ is the concentration of implanted helium atoms and $C_{C}$ is the concentration of cavities. In order to calculate $\mathrm{C}_{\mathrm{He}}$ in units of $\mathrm{m}^{-3}$, the concentration in at. ppm must be multiplied by the atomic density of Iron, $\rho_{\mathrm{Fe}}, 8.5 \times 10^{28}$ atoms $\mathrm{m}^{-3}$. For example, for specimens implanted with 10 at. ppm He ( $1 \mathrm{dpa})$, the helium concentration in units of $\mathrm{m}^{-3}$ is given by:

$$
\left.\mathrm{C}_{\mathrm{He}}\right)_{1 \mathrm{dpa}}=\frac{10 \text { atoms } \mathrm{He}}{10^{6} \text { atoms } \mathrm{Fe}} \rho_{\mathrm{Fe}}=8.5 \times 10^{23} \text { atoms } \mathrm{He}-\mathrm{m}^{-3} \text {. }
$$


Table 6.1. Values of the average number of helium atoms per cavity, n; the calculated number of helium atoms in an equilibrium bubble (with a diameter equal to the average cavity diameter), $\mathrm{n}_{\mathrm{eg}}$; and the calculated sink strengths for $\mathrm{Fe}-10 \% \mathrm{Cr}$, triple-beam ionirradiated at $850 \mathrm{~K}$ to fluences of $3,10,30$, and 100 dpa. Experimental values for the cavity concentration, $c_{c}$, and the volume-averaged cavity diameter, $d_{c}$, are also tabulated

\begin{tabular}{ccccccccccc}
\hline $\begin{array}{c}\text { Fluence } \\
(\mathrm{dpa})\end{array}$ & $\begin{array}{c}\mathrm{d}_{\mathrm{c}} \\
(\mathrm{nm})\end{array}$ & $\begin{array}{c}\mathrm{C}_{\mathrm{c}} \\
\left(\mathrm{m}^{-3}\right)\end{array}$ & $\begin{array}{c}\mathrm{n}_{\mathrm{eq}} \\
\text { (He atoms) }\end{array}$ & $\begin{array}{c}\mathrm{n} \\
\text { (He atoms) }\end{array}$ & $\mathrm{n} / \mathrm{n}$ eq & Dislocations Cavities & $\begin{array}{c}\text { Ratio: } \\
\text { Disl/Cav }\end{array}$ \\
\hline 3 & 9 & $3.5 \times 10^{19}$ & $1.4 \times 10^{4}$ & $7.3 \times 10^{4}$ & 5.2 & $5.6 \times 10^{13}$ & $2.0 \times 10^{12}$ & 28 \\
10 & 22 & $2.9 \times 10^{19}$ & $1.2 \times 10^{5}$ & $2.9 \times 10^{5}$ & 2.4 & $8.4 \times 10^{13}$ & $4.0 \times 10^{12}$ & 21 \\
30 & 20 & $2.0 \times 10^{20}$ & $9.5 \times 10^{4}$ & $1.3 \times 10^{5}$ & 1.4 & $3.2 \times 10^{13}$ & $2.5 \times 10^{13}$ & 1.3 \\
100 & 77 & $9.9 \times 10^{19}$ & $1.9 \times 10^{6}$ & $8.5 \times 10^{5}$ & 0.5 & $4.8 \times 10^{13}$ & $4.8 \times 10^{13}$ & 1 \\
\hline
\end{tabular}


The values calculated for $\mathrm{n}$ for each fluence are also listed in Table 6.1. Comparison of $\mathrm{n}$ and $\mathrm{n}_{\mathrm{eq}}$ for each fluence indicates that the apparent "bubble-character" of the cavities decreases with increasing fluence. At $3 \mathrm{dpa}$, a factor of 5 more helium atoms are available than are required for an equilibrium bubble with a diameter equal to the volume-averaged cavity diameter. However, at $100 \mathrm{dpa}$ only $50 \%$ of the helium atoms required for equilibrium bubbles was implanted. This suggests that the cavities are not growing as equilibrium bubbles at $100 \mathrm{dpa}$.

If partitioning of mobile helium to the dislocations is considered, the suggestion that the cavity growth occurred as equilibrium bubble growth is further discredited. According to the rate theory model of Yoo and Mansur [139], hellum will be particioned to internal sinks according to their sink strengths. The sink strength of the dislocations for helium is given by $z_{\alpha} L$, where $z_{\alpha}$ is the dislocation capture efficiency for helium and $L$ is the dislocation density. The value for $z_{\alpha}$ is not known. For the purpose of this discussion, $z_{\alpha}$ is assumed to be about 1. The sink strength of the cavities is given by $2 \pi d_{c} C_{c}$. The calculated sink strengths are listed in Table 6.1 for each fluence. of course, the amount of helium accumulated at the microstructural features as a result of partitioning is not accurately reflected by the instantaneous sink strengths. In order to perform exact calculations, an integral approach incorporating the continually changing microstructural parameters would be required. However, for the semiquantitative approach used in this discussion, simple calculations assuming the instantaneous sink strengths have been employed. For fluences of 3 and $10 \mathrm{dpa}$, as indicated by the ratio of the dislocation sink strength to the cavity sink strength, less than $5 \%$ of the implanted helium atoms would go directly to the cavities. At 30 and $100 \mathrm{dpa}$, about $50 \%$ of the hellum would go directly to the cavities. Since many of the cavities are observed to be associated with dislocations, part of the helium partitioned to the dislocations would diffuse along the dislocations to the cavities. It seems likely, however, that a large percentage of the helium atoms would be trapped in small clusters along the dislocations, especially at the lower fluences. At $3 \mathrm{dpa}$, if more than $80 \%$ of the 
implanted helium were trapped in these clusters, the number of hellum atoms in the average cavity would be less than $1.4 \times 10^{4}$, the number of helium atoms required for an equilibrium bubble with the average cavity diameter. Similarly, at $10 \mathrm{dpa}$, trapping of $\geqslant 60 \%$ of the hellum atoms would reduce the number of hellum atoms in the cavities to less than $\mathrm{n}_{\mathrm{eq}}$. And, at $30 \mathrm{dpa}$, if only $30 \%$ of the implanted helium were in small clusters, the cavities could not be equilibrium bubbles. Thus, with a reasonable degree of partitioning of the helium to dislocations, the average cavity would not be an equilibrium bubble - even at $3 \mathrm{dpa}$.

In evaluating the above discussion of the possibility of equiIibrium bubble growth, it is important to note that the value for the surface energy is not accurately known for a helium-containing cavity in Fe-10\% Cr. The value for the surface energy used, $2 \mathrm{~J} \mathrm{~m}^{-2}$, is the crystal-vapor surface energy for iron [140]. If the surface energy were actually $\sim 1 \mathrm{~J} \mathrm{~m}^{-2}$, the values for $\mathrm{n}_{\mathrm{eq}}$ given in Table 6.1 would be reduced by a factor of 2 . With this reduced value for $n_{e q}$, the cavities at $100 \mathrm{dpa}$ would nearly be equilibrium bubbles. However, for all of the fluences, a reasonable degree of partitioning of helium to clusters at the dislocations could reduce the number of hellum atoms in the cavities to less than $50 \%$ of $\mathrm{n}_{\mathrm{eq}}$. In addition, if the dislocation capture efficiency for helium were actually $\sim 2$, rather than 1 , as assumed previously, the dislocation sink strength would double - enhancing the likelihood of partitioning to the dislocations. In conclusion, if partitioning of the implanted helium to the dislocations is considered, it seems likely that the cavities did not grow as equilibrium bubbles.

In the temperature-dependent ion irradiation study of $\mathrm{Fe}-10 \% \mathrm{Cr}$ $(10 \mathrm{dpa})$, another cavity-growth phenomenon is observed. Here, the volume-averaged cavity diameter decreased from $\sim 22$ nn for an irradiation temperature of $850 \mathrm{~K}$ to $\sim 5 \mathrm{~nm}$ for an irradiation temperature of $970 \mathrm{~K}$. Along with the decrease in cavity diameter, the dislocation density is two orders of magnitude lower at the higher temperature. If the dislocations are assumed to be biased sinks for interstitials, the cavities at $850 \mathrm{~K}$ would be more void-like due to the presence of excess vacancies (bias-driven growth). At $970 \mathrm{~K}$, more defect recombination would occur due to the lower dislocation density, constraining the 
cavities to grow as bubbles (gas-driven growth). Further experimental support for gas bubbles being present at the higher temperature is provided by the observation of cavities along the grain boundaries.

The above conclusions are also supported by two calculations. First, at $850 \mathrm{~K}$, the average cavity radius, $11 \mathrm{~nm}$, is much greater than any of the calculated values for the critical radius for bias-driven growth, as calculated using rate theory equations (see Appendix F). According to these calculations, the critical radius varied from $\sim 0.2$ $\mathrm{nm}\left(\mathrm{P}=0.9 \mathrm{P}_{\mathrm{eq}}\right)$ to $\sim 1 \mathrm{~nm}\left(\mathrm{P}=0.5 \mathrm{P}_{\mathrm{eq}}\right)$ to $\sim 1.8 \mathrm{~nm}\left(\mathrm{P}=0.1 \mathrm{P}_{\mathrm{eq}}\right)$. Therefore, the cavities are probably growing by a bias-driven growth mechanism. At $970 \mathrm{~K}$, however, the calculated values for the critical radius varied from $\sim 2.0 \mathrm{~nm}\left(\mathrm{P}=0.9 \mathrm{P}_{\mathrm{eq}}\right)$ to $\sim 7.0 \mathrm{~nm}\left(\mathrm{P}=0.5 \mathrm{P}_{\mathrm{eq}}\right)$ to $\sim 15$. $\mathrm{mm}\left(\mathrm{P}=0.1 \mathrm{P}_{\mathrm{eq}}\right)$. (The lower dislocation density at the higher temperature is primarily responsible for the differences in $r_{c r i t}$ for the two irradiation temperatures.) Based on these calculated values, the observed cavity radius of $2.5 \mathrm{~nm}$ could easily be less than $x_{\text {crit }}$. If this is true, the cavities would be growing by a gas-driven growth mechanism.

In addition, these conclusions also gain support from comparisons of the average number of helium atoms, $n$, in each cavity and the calculated number of helium atoms, $n_{e q}$, required to stabilize the observed cavities as equilibrium bubbles at the two irradiation temperatures. The calculations involved in this comparison were discussed previously in this section.

According to calculations using the HDEOS, in order for the cavities at $850 \mathrm{~K}$ to be equilibrium bubbles, assuming the average cavity diameter, $\sim 1.1 \times 10^{5}$ helfum atoms must be contained in each cavity. For the cavities at $970 \mathrm{~K}$ to be equilibrium bubbles, $\sim 3.4 \times 10^{3}$ helium atoms must be contained in each cavity. At $850 \mathrm{~K}, \mathrm{C}_{\mathrm{c}} \simeq 2.9 \times 10^{19} \mathrm{~m}^{-3}$, yielding, according to $\mathrm{Eq} .(6.1), \mathrm{n}=2.9 \times 10^{5}$ helium atoms/bubble. At $970 \mathrm{~K}, \mathrm{C}_{\mathrm{c}} \simeq 6 \times 10^{19} \mathrm{~m}^{-3}$, yielding $\mathrm{n}=1.4 \times 10^{5}$ helium atoms/bubble. Comparing these values to those calculated by the HDEOS, less than $10 \%$ of the implanted helium must be in the cavities observed at $970 \mathrm{~K}$ in order for the cavities to be equilibrium bubbles. At $850 \mathrm{~K}, \sim 50 \%$ of the implanted helium is required for equilibrium bubbles. Thus, it is 
more likely that the cavities are growing as bubbles at $970 \mathrm{~K}$ rather than at $850 \mathrm{~K}$.

Extending the above calculations to 785 and $875 \mathrm{~K}$, it has also been shown that the cavities at $785 \mathrm{~K}$ are likely to be growing by a bias-driven mechanism, while those at $875 \mathrm{~K}$ are likely to be gas bubbles. This once again demonstrates the exaggerated effect that the surface has on the developing microstructures at high irradiation temperatures for ion bombardments.

Peak Swelling Temperatures - The peak swelling temperature of $673 \mathrm{~K}$ observed for the $1 \mathrm{dpa}$ neutron-irradiated iron in this study agrees well with the peak swelling temperature of $693 \mathrm{~K}$ reported by Little and co-workers $[43,44,46]$ for $30 \mathrm{dpa}$, neutron-irradiated iron. Little and co-workers also report a second swelling peak at $783 \mathrm{~K}$ (23 dpa). However, it should be noted that the $30 \mathrm{dpa}$ irradiation was not performed for irradiation temperatures above $\sim 730 \mathrm{~K}$, and the $23 \mathrm{dpa}$ irradiation was not performed for irradiation temperatures below $\sim 710 \mathrm{~K}$. The approximate location of the swelling peak reported for the 23 dpa irradiation was $773 \mathrm{k}$, but in the current study the swelling at this temperature was too small to be accurately measured. It is difficult to draw any firm conclusions based on comparisons of these rather limited data. A more detailed, high fluence study is required to determine if two peak swe11ing temperatures actually occur in neutronirradiated iron.

In neutron-irradiated $\mathrm{Fe}-10 \% \mathrm{Cr}$, a peak swelling temperature of $693 \mathrm{~K}$ has been reported $[43,44,46]$. This is $\sim 150^{\circ}$ lower than the peak swelling temperature reported by this study for ion-irradiated $\mathrm{Fe}-10 \%$ $\mathrm{Cr}$. While this shift may be partially due to the higher concentration of helium in the ion irradiation [141], it is probably primarily due to the difference in the damage rates in the two types of irradiation. This "reactor-accelerator" shift in the peak swelling temperature is usually observed in comparisons of neutron and ion-irradiation results.

Cavity Morphology - The same crystallographic cavity shape was found in the neutron and the ion-irradiated specimens containing cavities. The observed cavity morphology, a truncated octahedron with \{111\} facets and $\{100\}$ truncations, is the morphology usually found in 
fcc materials. Gelles [45] has reported a similar morphology for neutron-irradiated iron-chromium alloys.

The expected morphology of bubbles is that which produces the minimum surface energy, $\gamma$, for a specific volume. This shape is derived from a Wolff construction. In bcc materials, the expected surface energy relationship for the low-Index planes is $\gamma_{\{110\}}<\gamma_{\{100\}}$ $<\gamma\{111\}$. This surface energy relationship has been found, for example, in molybdenum [131]. The expected and commonly observed morphology in bcc materials is a truncated 12-faced polyhedron with $\{110\}$ facets and $\{100\}$ truncations. It is not considered unusual, however, to observe cubic cavities with $\{100\}$ sides. Expected cavity morphologies have been reported twice for irradiated ferritic materials. Kulcinski et a1. [30] have reported "truncated octahedral" cavities with $\{110\}$ faces and $\{110\}$ truncations (presumably this reported shape could also be referred to as a 12-sided polyhedron with $\{110\}$ faces) in neutronirradiated iron. Ohnuki et al. [57] have reported cubic cavities with $\{100\}$ faces in $C^{+}$irradiated iron.

The formation of truncated octahedral cavities with $\{111\}$ faces could be due to surface energy variations caused by impurities preferentially segregating to a specific set of planes. Impurities have been shown to affect cavity shape. For example, in fcc copper, bubbles with $\{100\}$ and $\{110\}$ facets are reported for "clean" foils while bubbles with $\{111\}$ and $\{100\}$ facets develop in regions exposed to oxygen [131]. This is consistent with preferential absorption of oxygen on the $\{111\}$ and $\{100\}$ planes in copper. In the current study, measurements of the surface energy ratio, $\gamma_{\{111\}} / \gamma_{\{100\}}$, from the cavity geometry in the Fe-10\% Cr specimens, indicated that $\gamma_{\{111\}}=0.77 \gamma_{\{100\}}$. This observation of no $\{110\}$ facets suggests that $\gamma\{110\}$ is greater than both $\gamma_{\{111\}}$ and $\gamma_{\{100\}}$. Thus $\gamma_{\{111\}}<\gamma_{\{100\}}<\gamma_{\{110\}}$ - exact1y the opposite of the expected surface energy relationship.

The unexpected shape could also be a result of non-equilibrium conditions during cavity growth. Further experiments - e.g., postirradiation annealing - would show if the cavities have a nonequilibrium shape, as suggested by this explanation. 


\subsection{Swelling Suppression Mechanisms}

In this section, the implications of the results of the current study to proposed swelling mechanisms will be presented. The reader is referred to Chapter 2 for a detailed summary of these mechanisms.

One of the predictions of the model proposed by Hayns and Williams [72] is that the "reactor-accelerator" shift in the peak swelling temperature would be less for ferritic materials than the usual 100 to $150^{\circ}$ shift often observed. However, as discussed earlier, a comparison of the peak swelling temperatures reported for ionirradiated (this study) and neutron-irradiated (Little and Stow $[43,44]$ ) Fe-10\% $\mathrm{Cr}$ indicates a $150^{\circ}$ shift. Therefore, on the basis of the mode1 of Hayns and Williams, this large temperature shift would suggest that the lower swelling observed in ferritic materials is not entirely due to point defect trapping at solute atoms.

Little [73] has considered the effects of the interactions of impurity solutes with both the point defects and the dislocations in his model. Many of the features of the damage microstructures observed in this study suggest that impurity or Cottrell atmospheres at dislocations influence the damage evolution. In the neutron-irradiated iron, the effects of impurities on the microstructural development by trapping of point defects and segregation to defect clusters is particularIy evident - probably due to the longer irradiation times at elevated temperatures required for the neutron irradiations as compared to the ion irradiations. Since the concentration of metallic impurities is low, the observed effects are considered most likely to be due to carbon. Clustering of defects at pre-irradiation dislocation segments observed for irradiation temperatures of 455 to $523 \mathrm{~K}$ is probably related to the presence of Cottrell atmospheres at the dislocations. At 548 and $573 \mathrm{~K}$, the higher concentration of $100 \mathrm{p}$ clusters found at dislocations is probably also related to these impurity clouds. The observation of dislocation loops intersecting the foil surfaces, also observed in the Ion-1rradiation experiments, could be the result of pinning by Cottre11 atmospheres, although, as discussed earlier, other explanations are possible. In addition, for irradiation temperatures 
of $723 \mathrm{~K}$ or higher, small precipitates were found in the center of the dislocation loops and on voids. In the ion-irradiation experiments, impurity segregation could also be partially responsible for the growth of convoluted dislocation loops. Finally, as also discussed earlier, variations in the surface energies due to segregation could be the reason for the observed cavity shape.

The last mode1, that of little et al. [47] (also presented by Bullough et al. [48]), requires a high pre-irradiation dislocation network and is, therefore, not directly applicable to the current investigation. Some features of this model have, however, been incorporated in the damage evolution model presented in the next section of this chapter.

\subsection{Damage Evolution}

The evolution of the damage microstructures with increasing fluence in ion-irradiated $\mathrm{Fe}-10 \% \mathrm{Cr}$ can be summarized briefly as follows:

Interstitial loops with $\mathrm{b}=\mathrm{a}\langle 100\rangle$ were dominant in the dislocation microstructure from 0.3 to $3 \mathrm{dpa}$. The $100 \mathrm{p}$ size increased and the concentration decreased with increasing fluence. At 1 and $3 \mathrm{dpa}$, the loops had a convoluted shape. At $3 \mathrm{dpa}$, round, interstitial loops with b $=a / 2\langle 111\rangle$ loops were also observed. The a/ $2\langle 111\rangle$ loops

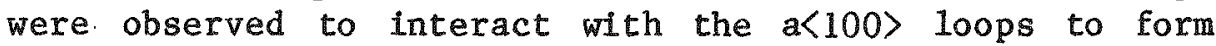
network segments with $\underline{b}=a / 2\langle 111\rangle$. Cavities were first observed at $3 \mathrm{dpa}$. At 10 to $100 \mathrm{dpa}$, a coarse distribution of network segments was found along with an increasing concentration of round loops with $b=a / 2\langle 111\rangle$ (assumed to be interstitial). The loop diameter decreased and the concentration increased with increasing fluence. The cavity diameter increased and the concentration decreased slight1y from 3 to $10 \mathrm{dpa}$. From 10 to $30 \mathrm{dpa}$, the cavity concentration increased and the average cavity diameter decreased. A bimodal cavity size distribution was observed at 30 and $100 \mathrm{dpa}$. Between 30 and $100 \mathrm{dpa}$, the cavity concentration decreased, but the average cavity diameter increased by more than a factor of 3 , resulting in a dramatic increase in the swelling. 
In order to explain this behavior, the following sequence of events for the damage evolution is suggested. In the early stages of damage, both types of interstitial loops, those with $b=a\langle 100\rangle$ and those with $\underline{b}=a / 2\langle 111\rangle$, are nucleated prior to cavity formation. As proposed by Eyre and Bullough [71], many more a/2<111 $\rangle$ loops are nucleated than a<100> 10ops. However, as suggested by Little and co-workers $[47,48]$, the a<100> loops act as a biased sink for interstitials and the $a / 2\langle 111\rangle$ loops act as relatively neutral sinks and absorb the excess vacancies early in the irradiation. The a $<100\rangle$ loops grow with

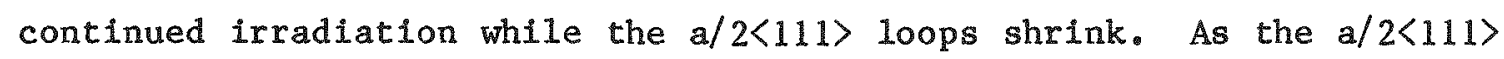
loops are annihilated, reducing the number of vacancy sinks, a vacancy supersaturation develops. Eventually, small cavities are nucleated, providing a new sink for the vacancies. Then the remaining a/2<111> loops start to grow from interstitial absorption. Thus, cavities and a/2<111 interstitial loops are observed at approximately the same time during the irradiation. In addition, more $a\langle 100\rangle$ loops are observed than $a / 2<111\rangle$ loops since the majority of the $a / 2<111\rangle$ loops disappear due to vacancy absorption. Most of the helium injected during this phase of the irradiation would be partitioned to the loops and form small clusters of helium atoms.

When the loops are large enough, dislocation network segments with $\underline{b}=a / 2\langle 111\rangle$ develop from the interaction of $a\langle 100\rangle$ loops and the appropriate $a / 2\langle 111\rangle$ loops. Segments with $\underline{b}=a\langle 100\rangle$, remnants of the a $\langle 100\rangle$ loops and from the interactions of a<100> 10ops, would also be found in the network. In addition, a few loops would probably be retained in the structure. It is difficult to conceive of a reasonable dislocation interaction involving loops with only $\underline{b}=a\langle 100\rangle$ which yields segments with $\underline{b}=a / 2\langle 111\rangle$. In many of the reported investigations of irradiated iron, only a<100> interstitial loops have been observed. The current study suggests that, in order to form a network from these loops, either a reasonable pre-irradiation dislocation ( $\underline{b}=$ $a\langle 111\rangle$ ) density would have to exist or, $a / 2<111\rangle 100 p s$ would have to be present (and not observed) or develop at higher fluences.

While the dislocation network is evolving, the cavities grow quickly, probably by a bias-driven growth mechanism. After the 
dislocation network is formed, however, the segments continue to interact with many of the segments being annihilated, causing a decrease in the dislocation density. During the early part of the irradiation, small clusters of helium atoms formed along the dislocations due to partitioning of helium. When the dislocation segments are annihilated, these clusters become active cavity nuclei. As these small nuclei grow, a bimodal cavity size distribution develops. However, since the lower density of dislocations increases the number of interstitials available for recombination with vacancies, the cavity growth is slower than the cavity growth with the higher dislocation densities present in the earlier stages of dislocation development. Impurity clouds at the dislocations could also reduce their ability to absorb interstitials. As the interstitials continue to flow toward the dislocations without being removed at the dislocations, a high density of interstitials could develop - encouraging the renucleation of interstitial dislocation 1oops. Impurity segregation at grain boundaries could similarly result in nucleation of loops near the boundaries. Once again, many

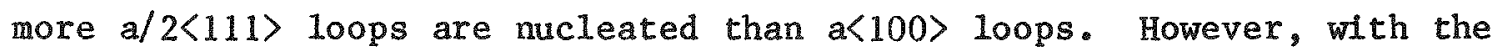
cavities available to absorb vacancies, both types of 100 ps will grow at this point in the irradiation. Thus, with increasing fluence, an

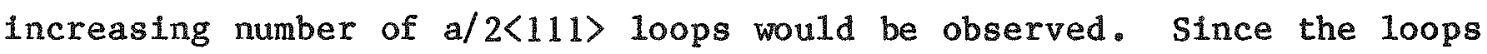
would not nucleate simultaneously throughout the specimen, the number of dislocation loops would increase with fluence (as expected), but the average loop diameter could decrease with increasing fluence until the loop nucleation ends. With the introduction of these interstitial sinks, the cavity growth rate would increase. As the cavities grow, their concentration could decrease if cavities coalesce through impingement during their growth. The swelling rate could also be enhanced with increasing fluence if the mode of cavity growth were surface controlled rather than diffusion controlled. With dislocations as the dominant sink, for surface-controlled cavity growth the swelling is porportional to (fluence) ${ }^{3}$ while for diffusion-controlled cavity growth the swelling is proportional to (fluence) $3 / 2$ [142].

The above mechanism presents one possible explanation for the damage evolution sequence observed in this investigation. As in the 
swelling suppression model proposed by Little and co-workers $[47,48]$, it depends on the dislocation loops with $\underline{b} a / 2\langle 111\rangle$ acting as vacancy sinks early in the irradiation. In addition, it proposes that renucleation of interstitial loops and/or surface-controlled cavity growth are responsible for the dramatic increase in the swelling observed between 30 and $100 \mathrm{dpa}$. Both of these proposals require further study to determine if they are valid. 
$13 \varepsilon$

○

- 


\section{CHAPTER 7}

\section{SUMMARY}

This investigation of the microstructural aspects of radiation damage in neutron-irradiated (ORR) iron and triple-beam ( $\mathrm{Fe}^{++}, \mathrm{He}^{+}, \mathrm{D}_{2}^{+}$) ion-irradiated iron, $\mathrm{Fe}-5 \% \mathrm{Cr}$, and $\mathrm{Fe}-10 \% \mathrm{Cr}$ has shown:

1. A comparison of the experimental damage-depth profile for ion-irradiated iron to the deposited energy and deposited ion profiles calculated by the E-DEP-1 code indicated a possible overestimate of the LSS stopping power of at least $22 \%$.

2. Based on this experimental damage-depth profile, a sectioning depth of $0.9 \mu \mathrm{m}$ was selected for studies of 1ron and bcc iron alloys irradiated with a triple beam of energetic $\mathrm{Fe}^{++}$(4 MeV), $\mathrm{He}^{+}$, and $\mathrm{D}_{2}^{+}$.

3. The dislocation microstructures coarsen with increasing irradiation temperature. In neutron-irradiated $(\sim 1 \mathrm{dpa})$ iron, the structure developed from decorated dislocations $\left(\mathrm{T}_{\mathrm{I}}=455\right.$ to $\left.523 \mathrm{k}\right)$, through clusters of loops $\left(T_{I}=548\right.$ and $\left.573 \mathrm{~K}\right)$, to loops and network segments $\left(\mathrm{T}_{\mathrm{I}}=623\right.$ to $\left.773 \mathrm{~K}\right)$. In triple-beam ion-1rradiated (10 dpa) Fe-10\% Cr, the structure developed from sma11, regularly shaped loops $\left(\mathrm{T}_{\mathrm{I}} \sim 725 \mathrm{~K}\right)$, through convoluted loops $\left(\mathrm{T}_{\mathrm{I}}=765\right.$ to $\left.800 \mathrm{~K}\right)$, to a coarse distribution of network segments $\left(T_{I} \sim 850 \mathrm{~K}\right)$. At higher irradiation temperatures, a rapid decrease in the dislocation density to preirradiation levels was observed for both types of irradiation. The predominant type of dislocation loop observed was interstitial with $\underline{b}=a\langle 100\rangle$ and near-edge character.

4. Extensive cavity formation was observed for irradiation temperatures of 548 to $723 \mathrm{~K}$ in neutron-1rradiated tron with homogeneous distributions only at 623 and $673 \mathrm{~K}$. At 548 and $573 \mathrm{~K}$, the cavities were found only in association with clusters of dislocation loops. The maximum swelling of $\sim 0.07 \%$ occurred at 623 and $673 \mathrm{~K}$. Only one swelling peak was found. 
5. In triple-beam ion-irradiated $\mathrm{Fe}-10 \% \mathrm{Cr}$, cavities were observed for Irradiation temperatures of $785 \mathrm{~K}$ and above, except at 803 $\mathrm{K}$. The peak swelling of $\sim 0.02 \%$ occurred at $850 \mathrm{~K}-150^{\circ}$ higher than the reported peak swelling temperature for neutron-irradiated $\mathrm{Fe}-10 \% \mathrm{Cr}$. The cavities observed at $850 \mathrm{~K}$ were about a factor of 4 larger than those observed at irradiation temperatures of $875 \mathrm{~K}$ and higher. It appears that the cavities at $850 \mathrm{~K}$ were growing by a bias-driven growth mechanism while those at the higher irradiation temperatures were growing by a gas-driven growth mechanism. This conclusion is supported by calculations of the critical cavity radius for bias-driven growth using a rate theory approach and by calculations of the number of helium atoms required for an equilibrium gas bubble using a high density equation of state (a bubble radius equal to the observed average cavity radius at the corresponding irradiation temperatures was assumed).

6. Damage halos centered at $\mathrm{B}_{4} \mathrm{C}$ precipitates were observed in neutron-irradiated iron. These halos result from ${ }^{10} B(n, \alpha){ }^{7} \mathrm{~L}$ reactions.

7. In the study of the evolution of the damage microstructure In triple-beam ion-irradiated Fe-10\% Cr $(850 \mathrm{~K})$, the dislocation microatructures developed from regularly shaped, small, interstitial, nearedge loops with $\underline{b}=a\langle 100\rangle(0.3 \mathrm{dpa})$, through convoluted, larger interstitial 10ops with $\underline{b}=a\langle 100\rangle(1 \mathrm{dpa})$, to dislocation segments $(\underline{b}=a\langle 100\rangle$ and $\underline{b}=a / 2\langle 111\rangle$ together with convoluted loops $(\underline{b}=a\langle 100\rangle)$ and round, large interstitial loops with $\underline{b}=a / 2<111\rangle$ ( 3 dpa). Cavities were first observed at $3 \mathrm{dpa}$. At 10 to $100 \mathrm{dpa}$, a coarse network of dislocation segments was observed together with round, interstitial loops with $\underline{b}=a / 2\langle 111\rangle$. The concentration of $a / 2\langle 111\rangle$ loops increased and the average loop diameter decreased with increasing fluence from 10 to $100 \mathrm{dpa}$. Between 30 and $100 \mathrm{dpa}$, the average cavity diameter and the swelling increased dramatically. Calculations of the critical radius for bias-driven growth indicated that a shift to biasdriven growth is probably not responsible for this rapid cavity growth. A possible mechanism was proposed to account for the observed damage evolution. 
8. The damage microstructures observed in this study differ significantly from the structures observed in other bcc materials. For example:

- While similar to the "rafts" of small dislocations loops observed in other neutron-irradiated bcc materlals, the clusters of loops in neutron-irradiated iron were unlike the "rafts" in that they contained loops with dissimilar Burgers vectors, were not planar, and contained interstitial loops with $\underline{b}=a\langle 100\rangle$.

- Interstitial dislocation loops in other irradiated bec materials have predominant 1 y a/ $2\langle 111\rangle$ Burgers vectors while those in iron, $\mathrm{Fe}-5 \% \mathrm{Cr}$, and $\mathrm{Fe}-10 \% \mathrm{Cr}$ have predominantly a $\langle 100\rangle$ Burgers vectors.

- The cavity shape observed in irradiated iron and Fe$10 \% \mathrm{Cr}$, a truncated octahedron with $\{111\}$ facets and $\{100\}$ truncations, is not observed in other irradiated bcc materials.

9. The observed swelling in both the neutron and fon irradiation experiments was much lower than that found in many equivalently irradiated fcc and refractory bcc materials.

10. A higher concentration of smaller cavities was observed in triple-beam ( $\mathrm{Fe}^{++}$, $\mathrm{He}^{+}$, and $\mathrm{D}_{2}^{+}$) ion-irradiated $\mathrm{Fe}-10 \% \mathrm{Cr}$ than in single ion ( $\mathrm{Fe}^{++}$) irradiated $\mathrm{Fe}-10 \%$ Cr $(850 \mathrm{~K}, 30 \mathrm{dpa})$.

11. At $850 \mathrm{~K}$ and $10 \mathrm{dpa}$, the swelling was an order of magnitude lower in triple-beam ion-irradiated $\mathrm{Fe}-10 \% \mathrm{Cr}$ than in iron. No cavity formation was observed in simllarly irradiated Fe-5\% $\mathrm{Cr}$.

12. The observations suggest a strong effect of impurities upon the microstructural development due to trapping and segregation.

Further study is required before a comprehensive mechanism for radiation damage in ferritic alloys can be developed. Many questions remain unanswered as a result of this investigation. of particular interest is the damage evolution between 30 and 100 dpa in Ionirradiated $\mathrm{Fe}-10 \% \mathrm{Cr}$. A series of irradiations between 30 and 100 dpa 
would provide a clearer picture of the reasons for the observed rapid cavity growth and the dislocation evolution. With the observed swelling of $\sim 2.5 \%$ at $100 \mathrm{dpa}$, the question arises as to the possibility that extremely high swelling could be obtalned at higher damage levels.

Analyses of the geometry of the small dislocation loops in low temperature, neutron-irradiated iron would also facilitate understanding of the dislocation evolution processes in iron.

Additional experiments to determine why the observed cavity morphology is a truncated octahedron with $\{111\}$ facets and $\{100\}$ truncations are necessary. Annealing of cavity-containing specimens would be expected to yleld the equilibrium cavity shape. If the cavity morphology did not change upon annealing, solute segregation effects on the surface energies would need to be investigated.

Further studies of the effects of chromium on the damage microstructures are also of interest. The reasons for swelling suppression in ion-irradiated Fe-5\% $\mathrm{Cr}$ at the peak swelling temperature for Fe-10\% $\mathrm{Cr}, 850 \mathrm{~K}$, are not known. A survey of specimens of $\mathrm{Fe}-5 \% \mathrm{Cr}$ for a range of irradiation temperatures would determine if the cavity formation in $\mathrm{Fe}-5 \% \mathrm{Cr}$ has a different temperature dependence than $\mathrm{Fe}-10 \%$ $\mathrm{Cr}$ - 1.e., if Fe-5\% $\mathrm{Cr}$ swells appreciably at temperatures other than $850 \mathrm{~K}$.

From the damage-depth profile for ion-irradiated iron, several unanswered questions arise. Foremost is the cause of the dislocation loop band at $2.8 \mu \mathrm{m}$ from the interface. Study of low temperature and low dose, single-ion ( $4 \mathrm{MeV} \mathrm{Fe}{ }^{+t}$ ) damage profiles should provide some insight into this phenomenon.

In conclusion, this study has investigated several of the fundamental aspects of radiation damage in ferritic materials. A damage evolution sequence was proposed which accounts for the observed damage development in $\mathrm{Fe}-10 \% \mathrm{Cr}$. The reported microstructural features should provide direction and aid in the microstructural analyses for future studies of the damage in irradiated ferritic alloys. 
APPENDIX A

EXPERIMENTAL DETAILS - ORR NEUTRON IRRADIATION OF IRON

The information presented in this appendix was primarily provided by Dr. K. Farre11 of Oak Ridge National Laboratory. Dr. Farre11, J. W. Woods, and W. W. Davis, all of ORNL, were responsible for the planning and assembly of $\mathrm{ORR}-228$, the experiment in which the fron specimens examined in the current investigation were irradiated. $J$. Houston was responsible for both the final specimen preparation for the experiment and the electropolishing of the irradiated specimens for TEM examinations.

\section{A.1 Specimen Preparation}

The starting material for the specimens used in this experiment was commercially obtained Ferrovac-E iron rod (Lot 167). Prior to rolling into a $0.5 \mathrm{~mm}$ thick sheet, the iron was two-pass zone refined in a $1.3 \mu \mathrm{Pa}\left(10^{-8}\right.$ torr) vacuum by the staff of the solid state Division at ORNL. Chemical analysis of two-pass zone refined Ferrovac E iron in wt $\mathrm{ppm}(99.94 \% \mathrm{Fe})$ is shown as follows:

$\begin{array}{lrrrlrll}\mathrm{C} & 30 & \mathrm{BI} & 1 & \mathrm{~K} & <1 & \mathrm{~S} & 15 \\ \mathrm{H} & 7 & \mathrm{Br} & 1 & \mathrm{Mg} & <5 & \mathrm{SI} & 90 \\ \mathrm{~N} & 4 & \mathrm{Ca} & 20 & \mathrm{Mn} & 3 & \mathrm{Ta} & 1 \\ 0 & 8 & \mathrm{Cl} & 60 & \mathrm{Na} & 10 & \mathrm{TI} & 0.3 \\ \mathrm{~A} 1 & 75 & \mathrm{Cr} & 3 & \mathrm{Nb} & <1 & \mathrm{~V} & 1 \\ \mathrm{As} & 0.4 & \mathrm{Cu} & 5 & \mathrm{NI} & 180 & \mathrm{~W} & 3 \\ \mathrm{~B} & 2 & & & \mathrm{P} & 5 & \mathrm{Zn} & <1\end{array}$

Disk specimens, $3 \mathrm{~mm}$ diameter, were mechanically punched from the sheet, deburred, given a rough mechanical polish and ultrasonically cleaned prior to annealing for one hour at $1023 \mathrm{~K}\left(750^{\circ} \mathrm{C}\right)$ in a $<1 \mathrm{mPa}$ (< $10^{-5}$ torr) vacuum. 
A.2 ORR - Specimen Loading and Assembly Details

The iron disks together with disks of other materials to be irradiated in ORR Run 228 were loaded into thin-walled stainless steel tubes with a thick plug welded to one end. Fifty to one hundred twenty specimens were stacked into each of a total of 39 of these capsules. Prior to final sealing with a thick plug of stainless steel, the capsules were evacuated and filled with helium. The plugs at both ends of the capsule had a deep indentation for thermocouple attachment. previous experiments had determined that the temperature over the length of the capsule varled by only a few degrees.

The stainless steel capsules were placed into aluminum sleeves which were wound with a heating coil of electrical resistance heating wire (nickel-chromium alloy). There was one stainless steel capsule per aluminum sleeve except where high temperatures were desired. Then, up to three capsules were placed into one large-diameter aluminum sleeve to take maximum advantage of the $\gamma$-heating.

The partially completed test assembly is shown in Figure A.1. Referring to this figure, it can be seen that the aluminum sleeves (1abeled A) were welded at six levels to a large central aluminum tube (labeled $B$ ) in a radial configuration by aluminum bars of various sizes (labeled C). In ORR 228, level 1 contained 8 capsules; levels 2,3 , and 4 contalned 7 capsules/1evel; level 5 contalned 3 capsules; and level 6 contained 2 aluminum sleeves with 3 capsules per sleeve.

The central aluminum tube was filled with flowing cold water for removing heat generated by $\gamma$-heating. Sufficient cooling was provided to reduce the temperature to below that desired for each capsules. The amount of $\gamma$-heating removed depended on the size and length of the aluminum connecting bars and the location of the capsule relative to the inlet water in the central aluminum tube. For example, the lowest temperature capsules were in level 1 , on the water inlet end of the central tube, connected to the central tube with a short, thick aluminum bar. Electrical heat was supplied by the heating colls to maintain each capsule at the desired irradiation temperature. The temperature of each capsule was monitored and controlled via thermocouples attached to the capsules. 


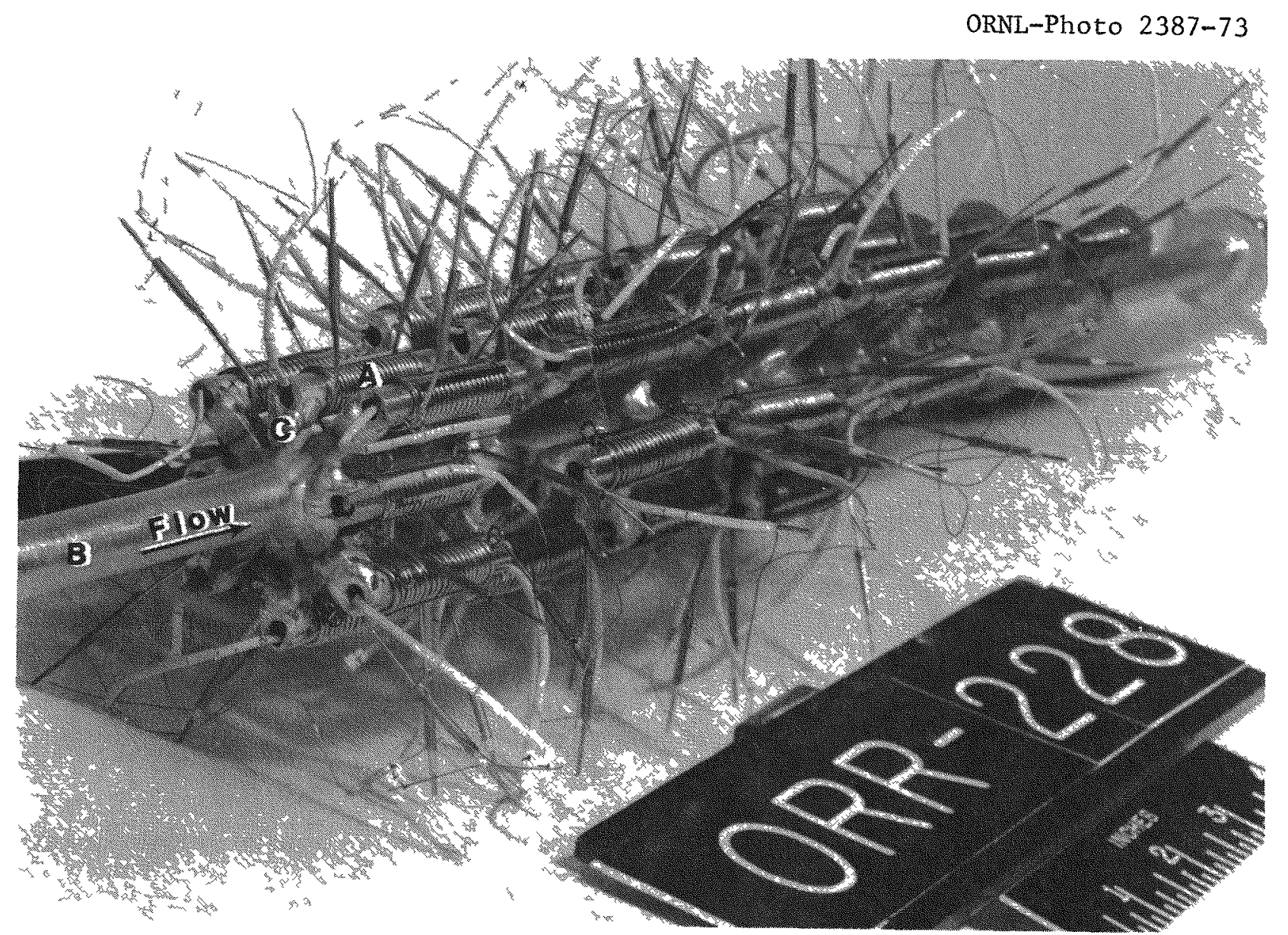

Figure A.1. Partially completed test assemb1y for ORR 228. (A) Aluminum sleeves wound with electrical resistance heating wire (nickel-chromium alloy), (B) central aluminum tube containing the cooling water (flow direction indicated), (C) aluminum connecting bars. 
In order to monitor the neutron flux during the irradiation, flux monitors were placed at strategic points on the assembly outside the heating coils. In Figure A.2, which shows the completed test assembly, the visible flux monitors are marked by arrows. The flux monitors were stainless steel envelopes containing preweighed dosimeters of $\mathrm{Al}-0.1 \% \mathrm{CO}, \mathrm{Al}-0.15 \% \mathrm{Ag}$, and natural iron. Fourteen flux monitors were used in ORR-228. Seven were in various locations along the length of the assembly in the SE (southeast) position. The reference for the directions used in the ORR reactor experiments is shown in Figure A.3. Two monitors were just above (water inlet side) level 1 in the $\mathrm{NE}-\mathrm{N}$ and $\mathrm{SW}-\mathrm{W}$ positions. The same positions were occupied by monitors just below (water exit side) level 6. The remaining monitors were in the $N-N E, E-N E$, and $S W-W$ positions between levels 3 and 4.

Dimensionally, the final assembly was $\sim 400 \mathrm{~mm}$ long and $\sim 110 \mathrm{~mm}$ across the diagonal of a roughly rectangular cross section.

A.3 Irradiation Details Including Calculations of dpa and Concentrations of Helium and Hydrogen

The assembly shown in Figure A.2 was loaded into ORR position $B-8$ on October 24,1973 . The core configuration during the irradiation is shown in Figure A.3. The experiment ran in ORR from cycle 115-B through cycle 117-A for a total exposure of 3089.1 megawatt days. The total fast $(>0.1 \mathrm{MeV})$ and thermal $(2200 \mathrm{~m} / \mathrm{s})$ fluences for each capsule position were derived graphically by Dr. Farrell from the fluences measured at the 14 flux monitor sites.

Table A.1 contains the irradiation temperatures, fluences, dpa, and helium and hydrogen concentrations for the ORR-228 capsules which contained iron specimens. The dpa and concentrations of helium and hydrogen were calculated using the individual capsule fluxes and the information available in the tables from Gabriel, Bishop and Wiffen [1]. In these tables, the dpa, helium, and hydrogen production per unft total fluence for various materials are presented as a function of position relative to the horizontal assembly midplane for ORR position C-3. In order to apply these data to the iron irradiated in position $\mathrm{B}-8$, the following procedure was used: 
ORNL-Photo $2535-73$

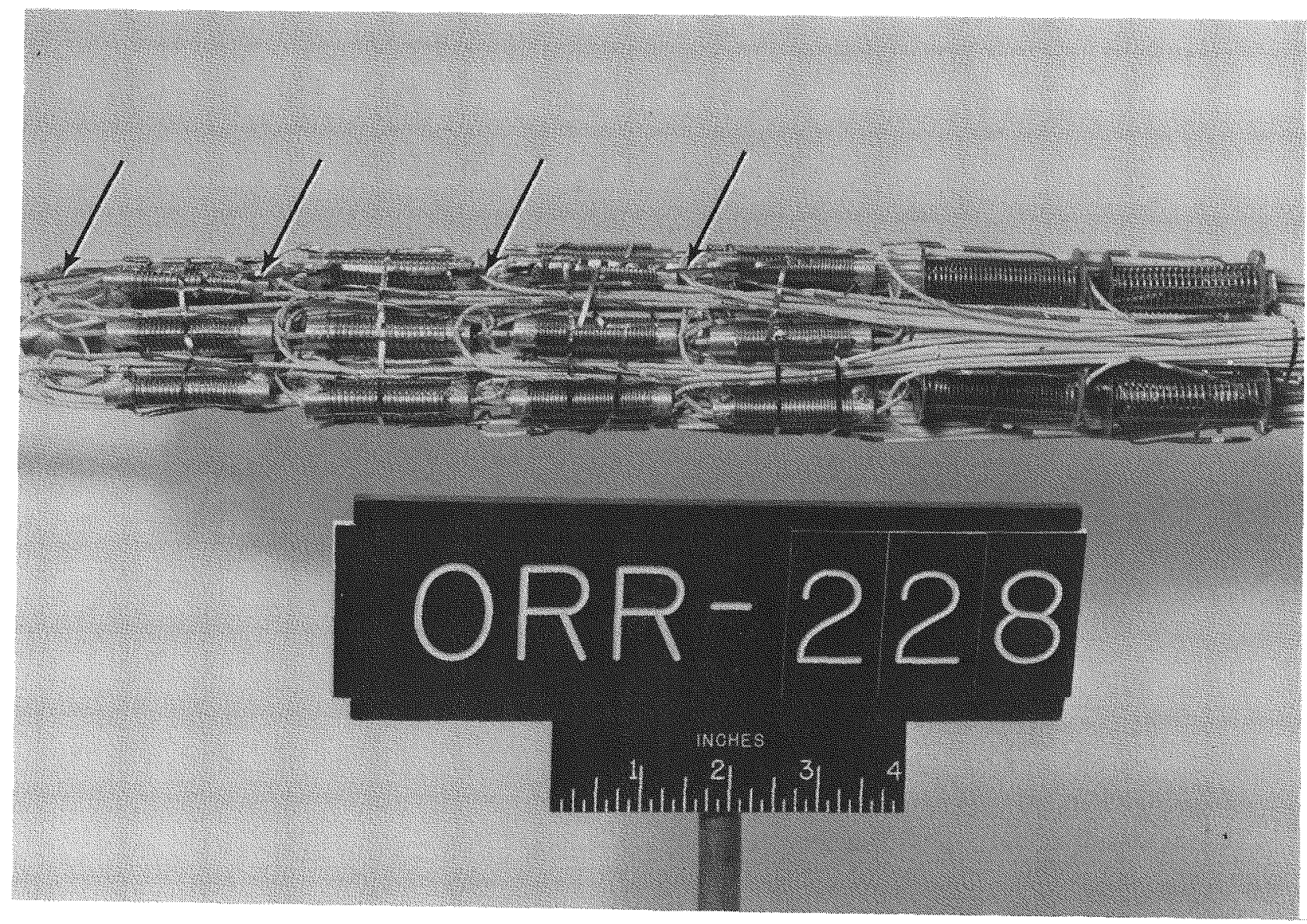

Figure A.2. Completed ORR 228 test assembly. Several of the flux monitors are indicated with arrows. 


\begin{tabular}{|c|c|c|c|c|c|c|c|c|}
\hline \multicolumn{7}{|c|}{ W } & \multicolumn{2}{|c|}{$Y E-12360$} \\
\hline A.l & $A-2$ & $A=3$ & $A-4$ & A-5 & $A-6$ & $A=7$ & $A-8$ & $A=9$ \\
\hline $\mathrm{Be}$ & $\mathrm{Be}$ & $F$ & $F$ & $F$ & $\mathrm{~F}$ & $\mathrm{Be}$ & $\mathrm{Be}$ & $\mathrm{Be}$ \\
\hline 8.1 & $B-2$ & B-3 & $\mathrm{B}-4$ & $3-5$ & $8-\infty$ & $B-7$ & B-8 & B.9 \\
\hline $\mathrm{Be}$ & $F$ & $F$ & $F$ & $F$ & $F$ & $F$ & $(228)$ & $\mathrm{Be}$ \\
\hline C. 1 & $C-2$ & $C-3$ & Cod & C-5 & $c-6$ & $C-7$ & $C-8$ & C.9 \\
\hline $\mathrm{Be}$ & $F$ & Expt & $\mathrm{F}$ & $F$ & $F$ & $\mathrm{~F}$ & $\mathrm{Be}$ or & $\mathrm{Be}$ \\
\hline$D-8$ & 0.2 & $D-3$ & $D-4$ & $0-5$ & D-6 & 0.7 & $D .8$ & 0.9 \\
\hline $\mathrm{Be}$ & $F$ & $F$ & $F$ & $F$ & $\mathrm{~F}$ & $F$ & $\mathrm{HT}$ & $\mathrm{Be}$ \\
\hline E-1 & $E-2$ & $E-3$ & $E-4$ & $E-5$ & $E=6$ & $E-7$ & $E-8$ & $E-9$ \\
\hline $\mathrm{Be}$ & $\Gamma$ & Expt & $F$ & F & $F$ & $F$ & $\mathrm{Be}$ & $\mathrm{Be}$ \\
\hline$F-1$ & $F-2$ & $F-3$ & $F-4$ & $F \cdot 5$ & $F-6$ & $F-7$ & $F=8$ & $F=9$ \\
\hline $\mathrm{Be}$ & $\mathrm{F}$ & $F$ & $F$ & $F$ & $F$ & Be & $\mathrm{Be}$ & $\mathrm{Be}$ \\
\hline $0-1$ & $0-2$ & $G-3$ & $G-4$ & G.5 & G-6́ & 6.7 & G.8 & G-9 \\
\hline $\mathrm{Be}$ & $\mathrm{Be}$ & $\mathrm{Be}$ & $\mathrm{Be}$ & $\mathrm{Be}$ & $\mathrm{Be}$ & $\mathrm{Be}$ & $\mathrm{Be}$ & $\mathrm{Be}$ \\
\hline
\end{tabular}

E

Figure A.3. ORR core configuration and reference directions for ORR228. The test assembly is located at position $B-8 . F=$ fuel element; $\mathrm{Be}=$ beryllium reflector; Expt = experimental capsule; $\mathrm{HT}=$ hydraulic tube.

Table A.1. Irradiation parameters for the capsules containing iron specimens in ORR-228

\begin{tabular}{|c|c|c|c|c|c|c|c|c|c|}
\hline \multirow[t]{2}{*}{ Capsule } & \multirow{2}{*}{$\begin{array}{l}\text { Location } \\
\text { Position/ } \\
\text { Level }\end{array}$} & \multicolumn{2}{|c|}{ Temperature } & \multicolumn{3}{|c|}{$\begin{array}{l}\text { Fluence, } 10^{25} \\
\text { neutrons } / \mathrm{m}^{2}\end{array}$} & \multirow[t]{2}{*}{$\mathrm{dpa}$} & \multicolumn{2}{|c|}{$\begin{array}{l}\text { Concen- } \\
\text { tration } \\
\text { (at. ppm) } \\
\end{array}$} \\
\hline & & $(\mathrm{K})$ & $\left({ }^{\circ} \mathrm{C}\right)$ & Total & Fast & Thermal & & $\mathrm{He}$ & $\mathrm{H}$ \\
\hline 1 & N1 & 455 & $(182)$ & 3.65 & 1.13 & 1.16 & 0.84 & 2.3 & 4.3 \\
\hline 7 & W1 & 493 & $(220)$ & 3.65 & 1.13 & 1.16 & 0.84 & 2.3 & 4.3 \\
\hline 6 & SW1 & 523 & (250) & 4.26 & 1.17 & 1.21 & 0.87 & 2.3 & 4.4 \\
\hline 3 & E1 & 548 & (275) & 4.00 & 1.29 & 1.19 & 0.96 & 2.3 & 4.9 \\
\hline 8 & NW1 & 573 & $(300)$ & 3.65 & 1.05 & 1.14 & 0.78 & $2 \cdot 3$ & 4.0 \\
\hline 15 & W2 & 623 & $(350)$ & 3.70 & 1.18 & 1.17 & 0.88 & 2.3 & 4.5 \\
\hline 10 & NE2 & 673 & $(400)$ & 3.70 & 1.32 & 1.20 & 0.98 & 2.3 & 5.0 \\
\hline 17 & NE3 & 723 & $(450)$ & 3.50 & 1.26 & 1.15 & 0.94 & 2.3 & 4.8 \\
\hline 29 & W4 & 773 & $(500)$ & 3.27 & 1.02 & 1.03 & 0.74 & 2.3 & 3.9 \\
\hline 35 & NE6 & 923 & $(650)$ & 2.25 & 0.69 & 0.75 & 0.51 & 2.2 & 2.6 \\
\hline 32 & SW5 & 1013 & $(740)$ & 3.35 & 0.87 & 0.95 & 0.64 & 2.2 & 3.3 \\
\hline
\end{tabular}


1. In calculating the dpa, the fast neutrons are of primary interest. Therefore, the value for the dpa/unit total fluence for iron found in the tables [1] was multiplied by 2.8 , the ratio of the total fluence to the fast fluence for position $\mathrm{C}-3$. (Note: all fluences have dimensions of neutron $\mathrm{m}^{-2}$.) As these values for the dpa/unit fast fluence varied by less than $10 \%$ over the length of the assembly, a single average value of $7.45 \times 10^{-26} \mathrm{dpa} /$ unit fast fluence was used in the dpa calculations. Multiplying this average value by the fast fluence for a capsule in the $B-8$ position yields the dpa for the $B-8$ capsule. In equation form this can be expressed as:

$$
\begin{aligned}
\mathrm{dpa} & =\left(\frac{\mathrm{dpa}}{\text { unit total fluence }}\right)_{\mathrm{C}-3} \times\left(\frac{\text { total fluence }}{\text { fast fluence }}\right)_{\mathrm{C}-3} \times \text { (fast fluence) }_{\mathrm{B}-8} \\
& =7.45 \times 10^{-26} \mathrm{dpa} / \text { unit fast fluence } \times\left(\text { fast fluence) }{ }_{\mathrm{B}-8}\right.
\end{aligned}
$$

Variations in the dpa due to the impurities in the iron were assumed to be negligible.

2. The impurities found in the iron specimens which would contribute significantly to the helium production through thermal neutrons are ${ }^{58} \mathrm{Ni}$ and ${ }^{10} \mathrm{~B}$. Production due to the other impurities was assumed to be negligible for both helium and hydrogen.

The helium and hydrogen production from the iron was calculated with an equation similar to equation A.1. The helium at. ppm/unit total fluence and hydrogen at. $\mathrm{ppm} /$ unit total fluence from the tables were substituted for the dpa/unit total fluence in calculating the helium and hydrogen concentrations, respectively. Since the nickel and boron impurities make up approximately 182 wt ppm of the specimen, the resulting concentrations of helium and hydrogen $\left(\mathrm{C}_{\mathrm{He}}\right.$ and $\mathrm{C}_{\mathrm{H}}$ ) from the iron calculation should be multiplied by $\sim 0.9998$ - a negligible correction. The final equations were:

$\mathrm{C}_{\mathrm{He}}$ (at. ppm)

$=\left(2.20 \times 10^{-26} \text { at. } \mathrm{ppm} \text { He/unit fast fluence) } \times \text { (fast fluence) }\right)_{\mathrm{B}-8}$; 
$\mathrm{C}_{\mathrm{H}}$ (at. ppm)

$=\left(3.78 \times 10^{-25}\right.$ at. $\mathrm{ppm} \mathrm{H} /$ unit fast fluence) $\times$ (fast fluence) $\mathrm{B}-8$.

The contribution of nickel to the hellum production is due to the ${ }^{58} \mathrm{Ni}$ which makes up $67.88 \%$ of naturally occurring nickel. The concentration of ${ }^{58} \mathrm{Ni}\left(\mathrm{C}_{58}{ }_{\mathrm{Ni}}\right)$, assuming the total atomic weight of the specimen is approximately that of iron, is given by:

$C_{58 \mathrm{Ni}}(\mathrm{at} \cdot \mathrm{ppm})=\frac{(0.6788)(\mathrm{wt} \mathrm{ppm} \mathrm{Ni})(\mathrm{at} \cdot \mathrm{wt} \mathrm{Fe})}{a t \cdot \text { wt of } \mathrm{Ni}}$

$$
=\frac{(0.6788)(180)(55.84)}{58.70}=116
$$

The transmutation reaction occurs as a two-step process:

$$
\begin{gathered}
{ }^{58} \mathrm{Ni}+{ }^{1} \mathrm{n} \rightarrow{ }^{59} \mathrm{Ni} \\
{ }^{59} \mathrm{Ni}+{ }^{1} \mathrm{n} \rightarrow{ }^{56} \mathrm{Fe}+{ }^{4} \mathrm{He}
\end{gathered}
$$

The helium produced by ${ }^{58} \mathrm{Ni}$ for the ORR-228 thermal fluence of $\sim 1$ $\times 10^{25}$ neutrons $\left./ \mathrm{m}^{2}\right) 2200 \mathrm{~m} / \mathrm{s}$ is $2.8 \times 10^{-5}$ atoms $\mathrm{He} /$ initial atom of ${ }^{58} \mathrm{Ni}$ [143]. Or, for 116 at. ppm ${ }^{58} \mathrm{Ni}$, a contribution of $<0.004$ at. ppm - a negligible amount.

Due to its large cross section for thermal neutron capture, essentially all of the ${ }^{10_{B}}$ in the specimen is converted to helium within the ORR-228 thermal fluences [144] . ${ }^{10} \mathrm{~B}$ makes up $19.78 \%$ of naturally occurring boron. The contribution of the boron to the helium concentration $\left(\mathrm{C}_{\mathrm{He}}\right)$ is given by:

$\mathrm{C}_{\mathrm{He}}$ (at. ppm) $=\mathrm{C}_{10}$ (at. ppm)

$=\frac{(0.1978)(\text { wt ppm B })(\text { at. wt Fe) }}{\text { at. wt B }}=\frac{(0.1978)(2)(55.84)}{(10.81)}=2.04$.

The majority of the helium produced during the irradiation is from the boron, only about $10 \%$ is from the iron. 


\section{A.4 TEM Specimen Preparation}

The neutron-irradiated iron specimens were electropolished using the two-step semiautomatic method developed by DuBose and Stiegler $[145,146]$.

In the first step, the specimen was "dimpled" on both sides using an electrolytic jet technique. The electrolyte was A-2, a solution consisting of $700 \mathrm{ml}$ ethyl alcohol, $100 \mathrm{~m} \ell$ butylcellusolve, $125 \mathrm{~m} \ell$ distilled water and 78 me perchloric acid. The polishing was performed at room temperature with a current of $\sim 180 \mathrm{~mA}$.

Final polishing was performed in a standard electrolytic polishing cell equipped with a fiber-optics light source and sensitive photocell to detect perforation of the dimpled region of the specimen. Activation of the photocell stopped the polishing action. The electro1yte was $\mathrm{A}-2$, as described above. The polishing was performed at $\sim 268$ $\mathrm{K}\left(-5^{\circ} \mathrm{C}\right)$ with a current of $200-300 \mathrm{~mA}$. 
1.52

- 


\section{APPENDIX B}

\section{ELECTRON MICROSCOPY PROCEDURES}

The majority of the electron microscopy for this investigation was performed with a JEM $120 \mathrm{C}$ transmission electron microscope (TEM) equipped with a special objective lens pole-piece for the observation of magnetic materials, referred to as the AMG. With this pole-piece, as shown in Figure $B .1$, the magnetic field at the specimen position is less than 3.4 Gauss $[147,148]$. With the AMG installed in a JEM 120C TEM, the minimum selected area diffraction (SAD) camera length ( $c_{0}$. $_{0}$ ) is $620 \mathrm{~mm}$, the maximum selected area magnification (SAM) is $22,000 \times$, and the maximum magnification (MAG mode) is $80,000 \times$. The point resolution is better than $1 \mathrm{~nm}$ [147]. With the AMG, extensive tilting experiments, such as Burgers vector analyses, could be performed utilizing a double-tilt specimen stage.

Limited examinations using an Hitachi $1 \mathrm{MV}$ high-voltage electron microscope (HVEM) and a JEM 120C TEM or JEM 120CX analytical electron microscope (AEM) were also performed when required. The HVEM was used for observations of coarse defect distributions. Due to the design of the Gatan stages for the HVEM, insertion of the double-tilt stage loaded with a magnetic specimen into the objective lens field caused the gimbal to rotate such that the specimen surface was parallel to the electron beam. Tilting of the specimen could not compensate for this rotation, eliminating the possibility of doing double-tilt experiments. For defect distributions requiring high resolution of magnifications greater than $80,000 \times$, the JEM $120 \mathrm{C}$ or $120 \mathrm{CX}$ was used with the standard objective lens pole-piece installed. Only small angles of specimen tilt were possible with this pole-piece. The amount of tilt possible before good images could no longer be obtained (due to uncorrectable beam deflection or beam tilt, and/or image astigmatism) varied from specimen to specimen.

In addition to the standard TEM examinations, some analytical microscopy was also performed. The JEM 120CX AEM and a Philips 400T AEM (120 kV, Field Emission Gun) were used for these studies. Both 
YE-12361

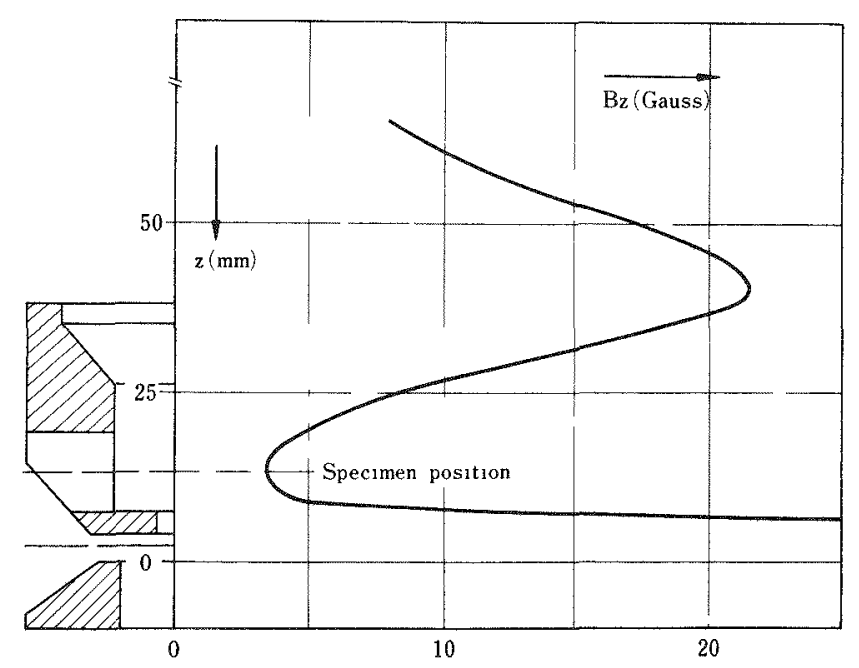

Figure B.1. The magnetic field distribution curve with a cross section of the AMG objective lens pole-piece. After ref. [148].

microscopes are equipped for scanning transmission electron microscopy (STEM), energy dispersive $x$-ray spectroscopy (EDS), and electron energy loss spectroscopy (EELS). The JEM 120CX AEM is equipped with a Kevex EDS detector/cryostat and the Philips 400T/FEG AEM is equipped with an Edax EDS detector/cryostat. Both systems are interfaced through a Kevex 5100 multichannel analyzer (MCA) to a PDP-11/34 computer and peripherals. Both microscopes were optimized for $x$-ray microanalysis according to the procedures outlined by Bentley et al. [149]. Bery1lium specimen stages were used. Standard operating procedures were followed (see, for example, ref. [150]). Large specimen tilt angles are required to obtain EDS spectra with these systems, e.g., for the $120 \mathrm{cX}$ the specimen is tilted to $45^{\circ}$ to both the electron beam and the detector. Therefore, the quality of the image was usually poor for the magnetic specimens. Because of the poor images, it was difficult to identify the areas of the specimens for which EDS analysis was desired after the specimen was tilted to the required angle.

Energy loss spectra were obtained using a symmetrical, doublefocusing $90^{\circ}$ magnetic sector spectrometer, designed by N. J. Zaluzec [151] and built at Oak Ridge National Laboratory. In this study, the 
EELS system interfaced to the JEM 120CX AEM was employed. Intensities were recorded by pulse counting from a photomultiplier tube detector and data were stored in a Kevex $5100 \mathrm{MCA}$ interfaced to a PDP-11/34 computer and peripherals. The microscope was operated in the STEM mode with specimen areas selected using shadow images in the disks of the convergent beam electron diffraction pattern. The incident beam divergence was $\sim 3 \mathrm{mrad}$ and the collection angle at the specimen was $\sim 7 \mathrm{mrad}$. Specimens were cooled to $<220 \mathrm{~K}$ in a single-tilt cooling holder in order to minimize hydrocarbon contamination. Typically, spectra were recorded at 30 to $100 \mathrm{~ms} / \mathrm{eV}$ with a resolution of $\sim 6 \mathrm{eV}$.

\section{B.1 Calibration of the JEM $120 \mathrm{C}$ - AMG}

Magnification and rotation calibration experiments were performed to characterize the AMG pole-plece. For the magnification cal1bration, a standard calibration grid with 2160 1ines/mm $(0.463 \mu \mathrm{m}$ spacing) was used. The actual magnifications, as calculated from the measured line spacing, are listed with the indicated magnifications and the objective lens current in Table B.1 for the MAG, SAM, and SCAN modes.

For the rotation calibration, an $\mathrm{Al}-3.8 \% \mathrm{Cu}$ foll containing $\theta^{-}$ precipitates was used. In order to determine the diffraction rotation relative to the image, the image and the SAD pattern were superimposed on a single negative. Diffracting conditions were selected such that the diffracting vector, $\underline{g}$, was [200] and the beam direction, $\underline{z}$, was not near a low index pole. Since the $\theta^{\prime}$ preclpitates lie on $\{100\}$, for these diffracting conditions, [100] is perpendicular to the precipitates with an edge-on orientation. The SAD/image rotation is the angle between the [200] direction on the SAD pattern and the perpendicular to these precipitates. Table B.2 lists the rotation angles required to move the [200] direction on the SAD to the direction of the precipitate normal on the image for magnifications within the SAM mode. The angles in Table B.2 marked with an asterlsk $(*)$ were calculated from the relative diffraction rotations listed in Table B.3. In this table, the angles 1isted are those required to rotate the [200] on the lower camera length SAD pattern to the [200] on the higher camera length SAD 
Table B.1. Magnification calibration for the JEM $120 \mathrm{C}$ with the AMG objective lens pole-piece

\begin{tabular}{|c|c|c|}
\hline $\begin{array}{l}\text { Listed } \\
\text { Magnification } \\
\left(10^{3} x\right)\end{array}$ & $\begin{array}{l}\text { Actual } \\
\text { Magnification } \\
\left(10^{3} \times\right)\end{array}$ & $\begin{array}{c}\text { Objective } \\
\text { Lens } \\
\text { Current } \\
\text { (amperes) }\end{array}$ \\
\hline \multicolumn{3}{|l|}{ SAM } \\
\hline 22 & 27.6 & 0.7396 \\
\hline 17 & 20.9 & 0.7390 \\
\hline 12 & 15.5 & 0.7389 \\
\hline 9.4 & 12.0 & 0.7389 \\
\hline 7.2 & 9.88 & 0.7389 \\
\hline 6.0 & 8.47 & 0.7389 \\
\hline 5.0 & 6.81 & 0.7389 \\
\hline 3.6 & 5.53 & 0.7389 \\
\hline 2.6 & 2.59 & 0.7389 \\
\hline 2.0 & 1.85 & 0.7389 \\
\hline 1.3 & 1.77 & 0.7389 \\
\hline \multicolumn{3}{|l|}{ SCAN } \\
\hline 0.63 & 0.63 & 0.6444 \\
\hline \multicolumn{3}{|l|}{ MAG } \\
\hline 86 & 99.6 & 0.7258 \\
\hline 54 & 69.6 & 0.7289 \\
\hline 44 & 57.1 & 0.7313 \\
\hline 33 & 39.5 & 0.7096 \\
\hline 22 & 27.4 & 0.7100 \\
\hline 17 & 19.6 & 0.7104 \\
\hline 12 & 14.2 & 0.7244 \\
\hline 9.4 & 11.5 & 0.7267 \\
\hline 7.2 & 8.76 & 0.7287 \\
\hline 6.0 & 7.42 & 0.7309 \\
\hline 5.0 & 5.99 & 0.7329 \\
\hline 3.6 & 4.57 & 0.7370 \\
\hline 3.0 & 3.90 & 0.7370 \\
\hline 2.6 & 2.66 & 0.6875 \\
\hline 2.0 & 2.02 & 0.6733 \\
\hline 1.3 & 1.31 & 0.6500 \\
\hline 1.0 & 1.01 & 0.6442 \\
\hline 0.76 & 0.79 & 0.6442 \\
\hline 0.63 & 0.67 & 0.6445 \\
\hline 0.51 & 0.51 & 0.6445 \\
\hline
\end{tabular}


Table B.2. SAD/image rotation callbration for the JEM $120 \mathrm{C}$ with the AMG objective lens pole-piece

Angles listed are the rotations of the $S A D$ required to make it colinear with the image

$$
+=\mathrm{cw} ;-=\mathrm{ccw}
$$

Measured on enlargements printed emulsion "up"

* indicates calculated rotations

\begin{tabular}{ccccc}
\hline Camera Length & 62 & 135 & 185 & 295 \\
\hline $\begin{array}{c}\text { Indicated } \\
\text { Magnification } \\
\left(10^{3} \times\right)\end{array}$ & & & & \\
\hline SAM & & & & \\
22 & +16.3 & +39.9 & -62.6 & -56.0 \\
17 & +32.5 & $+56.1^{*}$ & $-46.5^{*}$ & -40.1 \\
12 & +44.9 & $+68.5^{*}$ & $-34.1^{*}$ & $-27.7^{*}$ \\
9.4 & +53.0 & +76.2 & -25.4 & -19.4 \\
7.2 & +56.8 & $+80.4^{*}$ & $-22.2^{*}$ & $-15.8^{*}$ \\
6.0 & +58.9 & $+82.5^{*}$ & $-20.1^{*}$ & $-13.7^{*}$ \\
5 & +62.5 & +86.5 & -17.1 & -10.5 \\
3.6 & +64.5 & $+88.5^{*}$ & $-15.1^{*}$ & $-8.5^{*}$ \\
2.6 & -48.9 & $-25.3^{*}$ & $-127.9^{*}$ & $-121.5^{*}$ \\
2.0 & -41.9 & $-18.3^{*}$ & $-120.9^{*}$ & $-114.5^{*}$ \\
1.3 & -28.6 & $-50^{*}$ & $-107.6^{*}$ & $-101.2^{*}$ \\
\hline
\end{tabular}

Table B.3. SAD rotation calibration for the JEM $120 \mathrm{C}$ with the AMG objective lens pole-piece

Angles listed are the rotations of the lower camera length SAD required to make it colinear with the higher camera length $S A D$ $+=\mathrm{cw} ;-=\mathrm{ccw}$

Measured on enlargements printed emulsion "up"

\begin{tabular}{crrrr}
\hline $\begin{array}{c}\text { Camera Length } \\
(\mathrm{cm})\end{array}$ & 62 & 135 & 185 & 295 \\
\hline 62 & $-\overline{3.6}$ & - & & \\
135 & +23.0 & +102.6 & - & \\
185 & +72.6 & +96.2 & -6.4 & - \\
295 & & & & \\
\hline
\end{tabular}


pattern. Similarly, Table B.4 lists the relative image rotation angles. In this table, the angles listed are the angles required to rotate the direction of the precipitate normal on the lower magnification image to the direction of the precipitate normal on the higher magnification 1mage. In Tables B.2-B.4, "+" indicates a clockwise rotation and "-" indicates a counterclockwise rotation. It is important to note that these angles were measured from photographic enlargements printed with the negative emulsion "up" - i.e., prints same as the lmage on the microscope viewing screen. If these rotation calibrations were used for micrographs printed with the negative emulsion "down," the direction of the rotations would be the opposite of that listed.

Table B.4. Image rotation calibration for the JEM $120 \mathrm{C}$ with the AMG objective lens pole-piece

Angles listed are the rotations of the lower magnification image required to make it colinear with the higher magnification image $+=\mathrm{cw} ;-=\mathrm{ccw}$

Measured on enlargements printed emulsion "up"

\begin{tabular}{|c|c|c|c|c|c|c|c|c|c|}
\hline $\begin{array}{l}\text { Indicated } \\
\text { Magnif1- } \\
\text { cation } \\
\left(10^{3} \times,\right. \\
\text { SAM) }\end{array}$ & d & 17 & 12 & 9.4 & 7.2 & 6.0 & 5.0 & 3.6 & 2.6 \\
\hline $\begin{array}{l}22 \\
17 \\
12 \\
9.4 \\
7.2 \\
6.0 \\
5.0 \\
3.6 \\
2.6\end{array}$ & $\begin{array}{c}-- \\
-16.2 \\
-28.6 \\
-36.7 \\
-40.5 \\
-42.6 \\
-46.2 \\
-48.2 \\
+65.2\end{array}$ & $\begin{array}{l}-\overline{-} \\
-12.4 \\
-20.5 \\
-24.3 \\
-26.4 \\
-30.0 \\
-32.0 \\
+81.4\end{array}$ & $\begin{array}{r}-- \\
-8.1 \\
-11.9 \\
-14.0 \\
-17.6 \\
-19.6 \\
+93.8\end{array}$ & $\begin{array}{c}-- \\
-3.8 \\
-5.9 \\
-9.3 \\
-115 \\
+101.9\end{array}$ & $\begin{array}{r}- \\
-2.1 \\
-5.7 \\
-7.7 \\
+105.7\end{array}$ & $\begin{array}{r}- \\
-3.6 \\
-5.6 \\
+107.8\end{array}$ & $\begin{array}{c}- \\
-2.0 \\
+111.4\end{array}$ & $+\overline{113.4}$ & -- \\
\hline
\end{tabular}

\section{B.2 Microscopy Procedures for Magnetic Specimens}

In this section, adaptations of the standard microscopy procedures required for examination of magnetic specimens with TEM w11 be presented. No special procedures were required for the HVEM examinatons. Emphasis is placed on the use of the JEM 120C/AMG TEM. 


\section{B.2.1 Microscope alignment}

The standard alignment procedures for the microscopes were followed using non-magnetic specimens. It was especially important to align the beam deflector coils with the condenser alignment : wobbler controls. In addition, the objective lens current center was aligned with the "dark-field" condenser alignment controls.

After the AMG pole-piece was installed in the JEM 120C, the alignment of the image forming system required large mechanical adjustments of the intermediate lens and the projector lens. It was often necessary to repeat the alignment of the image forming system after the objective lens current center was aligned.

\section{B.2.2 Specimen loading and stage insertion}

Great care in specimen loading was essential in order to prevent the loss of magnetic specimens within the microscope. With the regular objective lens pole-piece, silver paint was applied over the spring clip to help hold the specimen in the gimbal of the double-tilt stage. (No gimbals with screw-in caps were available.) Silver paint was applied around the specimen edges when the single-tilt stage was used. With the AMG, no silver paint was necessary.

While inserting or removing the specimen stages, it was necessary to have the objective lens off. This precaution also applled when the AMG pole-piece was installed. For the JEM $120 \mathrm{C}$ and $120 \mathrm{cX}$ microscopes, the objective lens is off in the LOW MAG mode.

\section{B.2.3 Eucentric height : magnetic specimens}

Because of the lmage sweep assoclated with tilting a magnetic specimen, it is difficult to set the eucentric height. Traditionally, for ferromagnetic specimens, the eucentric height is set at the position that the direction of the image sweep changes. However, when using this method, it is quite easy for the spectmens to be pulled from the specimen stage due to the abrupt tilting required. To avold this problem, the procedure described below was used for this study. 
Before a magnetic specimen was inserted into the microscope, the eucentric height was set using a non-magnetic specimen. After setting the eucentric height, the specimen was focused at SAM 22 and the current alignment checked using the "dark-fleld" condenser allgnment controls. Now, the magnetic specimen was inserted into the microscope (using a LOW MAG mode, as discussed previously). The magnification was gradually increased to SAM 22 - with no attempts to focus the specimen. In order to set the eucentric height, the specimen focus was then corrected with the eucentric helght control knob.

\section{B.2.4 Specimen t1lting procedures : magnetic specimens}

In order to correct the current center after tilting even a few degrees, a11 "bright-field" microscopy of magnetic specimens was performed using the "dark-fleld" condenser alignment controls. For the JEM $120 \mathrm{C}$ and $120 \mathrm{CX}$ microscopes, the "dark-field" controls allow $6^{\circ}$ of beam tilt while the "bright-field" controls allow less than $1^{\circ}$ of tilt [152]. While operating the microscope in this manner, centered darkfield microscopy was very difficult. This problem was eliminated with the installation of a second set of "dark-field" controls on the JEM $120 \mathrm{C}$.

When tilting a magnetic specimen, the electron beam could move a significant amount. This movement was minimized with the AMG poleplece. With each specimen tilt, the current center would need to be realigned and the objective astigmatism would have to be corrected. Usually, the illumination would also require recentering. At large specimen tilt angles, with the current center set, the transmitted or forward-scattered beam in the SAD mode was often at a different location on the microscope viewing screen than the location of the transmitted beam for no specimen tilt. If the transmitted beam was too far from this original location, the image obtained was poor - probably due to the off-center path of the electron beam through the lenses. When this occurred, the best image was often obtained with the beam tilted to the original location of the transmitted beam, although some image rotation occurred when focusing. In some situations, however, the best 
image was obtained with the beam tilted to some position between the location of the transmitted beam with the current center set at its original location.

In order to set up a specific set of diffracting conditions, an involved process was required. First, the specimen was slowly tilted toward the desired beam direction, $\underline{z}$, and diffracting vector, $\underline{g}$. While tilting the specimen, the electron beam was tilted to maintain the location of the transmitted beam spot close to its location prior to tilting the specimen (this kept the current center roughly aligned). After tilting to the desired diffracting conditions, the current center was aligned. However, since movement of the current center changes the diffracting conditions, the specimen would again be tilted to the desired diffracting conditions. Now, if the current center was still aligned, the astigmatism was corrected. (If the current center was not still aligned, the above steps were repeated until both the current center and the diffracting conditions were satisfactory.) If the image was poor, corrections to the diffracting conditions were made in an effort to improve the image (including resetting the location of the transmitted beam to the location obtained with no specimen tilt, as discussed previously).

\section{B.2.5 Additional precautions}

In order to avold "losing" the specimen within the microscope, in addition to the procedures already presented, the following precautions were taken. Abrupt specimen tilts were always avoided. In addition, when changing the operating modes on the JEM microscopes, it was important not to allow all of the buttons, which were used to select the modes, to be "out," i.e., off, at the same time. In this condition, the objective lens voltage increases to its maximum value, increasing the field around the specimen and possibly causing the specimen to be lost within the microscope. 


\section{B.3 Thickness Measurements}

In this study foil thicknesses were determined either from the thickness fringes or from stereo pairs. In utilizing the thickness fringes, micrographs taken at the Bragg angle with $\underline{g}=\langle 110\rangle$ were usually used. The extinction distance for a $\langle 110\rangle$ reflection, $\xi_{110}$, for $120 \mathrm{keV}$ electrons was calculated from the value of $\xi_{110}$ for 100 $\mathrm{keV}$ electrons $(27 \mathrm{~nm})$ using the procedures outlined by Hirsch et al. [153]. The value obtained from this calculation $(29 \mathrm{~nm})$ was reduced for many beam effects [154] to $26 \mathrm{~nm}$. This corrected value for $\xi_{110}$ was used for the thickness measurements.

The stereo pairs were usually taken with the same $\langle 110\rangle$ diffracting vector with $\sim 10^{\circ}$ tilt between the micrographs. Stereo measurements were made with a Hilger and Watts Folding Mirror Stereoscope (Mode1 SB180). The parallax, p, was measured from three or more areas of each stereo pair with at least two sets of readings per area. Unless there was a large deviation between the areas $(>10 \%)$, an average thickness was calculated for the area represented by the micrographs. The thickness, $t$, was calculated with the expression

$$
t=\frac{p}{2 M \sin (\theta / 2)}
$$

where $M$ is the magnification of the micrographs and $\theta$ is the tilt angle of the stereo pair.

\section{B.4 Dislocation Density Measurements}

Two methods were used to determine the dislocation densities. For a more complete discussion of these techniques, see, for example, Hirsch et al. [153]. Generally, the dislocation density was measured on micrographs in which all dislocations, including the dislocations with "residual" images, had sufficlent contrast to be included in the measurement.

With the first method, the number of dislocation ends, $N$, on a micrograph are counted. This ylelds the total number of dislocation 
intersections with the top and bottom foll surfaces. The dislocation density, $\rho$, is given by

$$
\rho=\frac{\mathrm{N}}{2 \mathrm{~A}}
$$

where $A$ is the surface area of the specimen included in the micrograph. It is difficult to use this method to measure densities greater than $\sim 10^{13} \mathrm{~m}^{-2}[153]$.

With the second measurement technique, the foil thickness must be measured, as discussed in section B.2. The dislocation density, $\Lambda$, is calculated from the number of intersections that the dislocations make with a set of random lines of length $L$. In equation form

$$
\Lambda=\frac{2 \mathrm{~N}}{\mathrm{Lt}}
$$

where $t$ is the measured foil thickness in the region that the dislocation line intersections were counted. For this study, a series of concentric circles was used for the "random 1ines." This method can be used for dislocation densities up to $10^{15}$ to $10^{16} \mathrm{~m}^{-2}$ [153]

It has been shown by schoeck [155] that the relationship between the dislocation density measured by the surface intersection technique $(\rho)$ and that measured by the line length per unit volume method $(\Lambda)$ is given by

$$
\Lambda=2 \rho
$$

for an isotropic distribution.

\section{B.5 Dislocation Loop Analyses}

For this study, the Burgers vector and nature (vacancy or interstitial) of dislocation loops were of interest. The methods described by Maher and Eyre [97] were utilized in these analyses. 
The direction of the Burgers vector was determined from the standard "invisibility" criterion. In the analyses, the dislocations were imaged with $s_{g}$, the deviation from the Bragg angle, positive. For dislocation loops, the residual image was confirmed by comparisons of the images obtained for $+\underline{g}$ and $-\underline{g}$, with the same value of $s_{g}$ used for both diffracting vectors. For the loops in residual contrast, all features of the image should be the same in both micrographs. Residual images were obtained for at least two different diffracting vectors for each loop analyzed.

For these analyses, the Burgers vector was defined by taking the positive direction around the loop as clockwise when the loop was viewed from above the foil and then applying the FS/RH (finish-start) right-handed) perfect crystal convention. According to this convention:

$$
\begin{aligned}
& (\underline{g} \cdot \underline{b}) s_{g}>0 \text { gives "outside" contrast; and } \\
& \left(g^{\circ} \underline{b}\right) s_{g}<0 \text { gives "inside" contrast. }
\end{aligned}
$$

These relationships were used to determine the sense (t or $\rightarrow$ ) of the Burgers vectors. For the "inside-outside" image shifts, the diffracting vectors were selected such that $\underline{g} \cdot \underline{b} \geqslant \pm 2$.

Another important consequence of this Burgers vector definition is that, for interstitial loops, $\underline{n^{*}} \underline{b}>0$ and, for vacancy loops, $\underline{n}^{\bullet} \underline{b}$ $<0$, where $\underline{n}$ is the upwards loop normal. Thus, the nature of the loop can be determined from the sense of $\underline{b}$ if sufficient information is known concerning $n$. According to Maher and Eyre, the nature of the loop can be determined from the direction and sense of $b$ if the location of $\underline{n}$ is known relative to the two "domains" bounded by $\underline{n}^{*} \underline{z}=0$ (i.e., edge-on loops) and $\underline{n} \cdot \underline{b}=0$ (i.e., shear loops) shown in the stereographic projection in Figure B.2. Within the unshaded portion of the stereographic projection, the angle between $\underline{b}$ and $\underline{n}, \phi_{\mathrm{bn}}$, is $\leqslant 90^{\circ}$. If the analysis is performed with "safe" orientations, i.e., with b sufficiently close to $\underline{z}$ such that $\underline{n}$ falls within the unshaded region of the stereographic projection, then the loop exhibits the same contrast behavior as the corresponding edge loop configuration with the same Burgers vector irrespective of the loop inclination, $i_{\bullet} e_{\bullet}$, for $\underline{b} \underline{z}$ $>0$, the loop has an interstitial nature, and for $\underline{b} \underline{z}<0$, the loop has a vacancy nature. However, if the analysis is performed with foil 


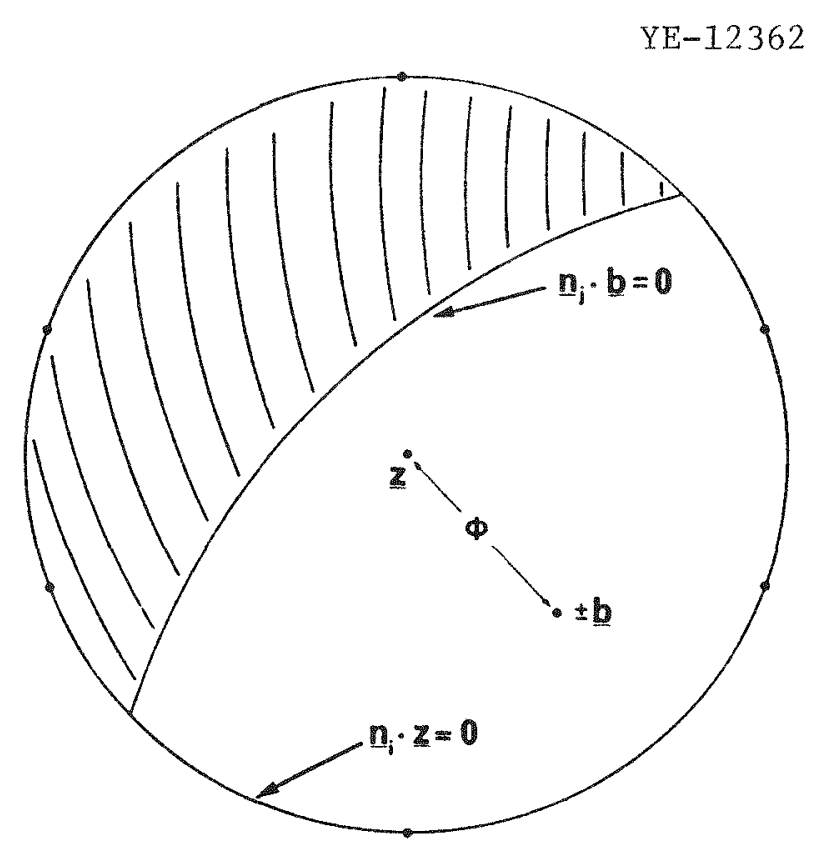

Figure B.2. Stereographic projection which defines the two domains for $\underline{n}$ for non-edge dislocation loops. After ref. [97].

orientations such that $\underline{\mathrm{n}}$ falls in the unshaded region of the stereographic projection, referred to as the "region of reverse contrast," then the loop exhibits contrast opposite to that of an edge 1oop, i.e., for $\underline{b} \underline{z}>0$, the loop has a vacancy nature, and for $a \underline{b} \underline{z}<0$ the loop has an interstitial nature.

If $\underline{n}$ is not determined experimentally, the nature of the loop can still be obtained if limits can be placed on $\mathbf{n}$ from a consideration of the geometry of loop formation. Assuming that the model for interstitial loop nucleation in bcc materials proposed by Eyre and Bullough [71] is correct, loops in iron nucleate on $\{110\}$ and shear to $a / 2\langle 111\rangle /$ $\{110\}$ or $a\langle 100\rangle /\{110\}$, then rotate towards a pure edge orientation. Therefore, the loop normal for loops with $\underline{b}=a / 2<111\rangle$ would lie between $\langle 110\rangle$ and $\langle 111\rangle$ and the loop normal for loops with $\underline{b}=a\langle 100\rangle$ would lie between $\langle 110\rangle$ and $\langle 100\rangle$. If the loops normal is not determined, the maximum possible $\phi_{\mathrm{bn}}$ must be assumed in calculating "safe" orientations. For bcc materials, the maximum $\phi_{\mathrm{bn}}$ occurs for $\underline{\mathrm{n}}=\langle 110\rangle$, e.g., for $\underline{b}=a / 2\langle 111\rangle, \phi_{b n}$ is $\sim 36^{\circ}$ and for $\underline{b}=a\langle 100\rangle, \phi_{b n}$ is $\sim 45^{\circ}$. 
As stated above, by definition $\phi_{z n}$ must be $\leqslant 90^{\circ}$. The most restrictive scenario is one in which $\underline{b}, \underline{z}$, and $\underline{n}$ lie on a single great circle on the stereographic projection. In this situation, in order for $\underline{n}$ to remain in the unshaded or "safe" region of the stereographic projection (Figure B.2), the angle between $\underline{z}$ and $\underline{b}, \phi_{\mathrm{zb}}$, must be $\leqslant 54^{\circ}$ for $\phi_{\mathrm{bn}} \sim 36^{\circ}$ $(\underline{b}=a / 2\langle 111\rangle)$. Likewise, for $\phi_{b n} \sim 45^{\circ}(\underline{b}=a\langle 100\rangle), \phi_{z b}$ must be $\leqslant 45^{\circ}$ for "safe" orientations.

For the loop analyses in the present study, the loops with $\underline{b}=$ $a\langle 100\rangle$ were found to be nearly edge dislocation loops. Therefore, $\phi_{\text {bn }}$ was close to $0^{\circ}$, allowing for correct analyses with values of $\phi_{Z \mathrm{~b}}$ approaching $90^{\circ}$. For example, for loops with $\underline{\mathrm{b}}=[100]$, the loop nature analysis was performed with beam directions near [111], i.e., $\phi_{\mathrm{zb}} \sim 55^{\circ}$. In this analysis, an interstitial loop yielded "outside" contrast for $\underline{g}=[2 \overline{1} \overline{1}]$ and "inside" contrast for $\underline{g}=[\overline{2} 11]$.

\section{B.6 Cavity Analyses}

Micrographs of the cavities were taken with diffracting vectors that were positive of $3 \mathrm{~g}$. Usually, diffracting vectors of $\langle 330\rangle$ with a positive deviation from the Bragg angle were used.

Usually, the cavities were measured on micrographs with underfocused images. With this focusing condition, a cavity appears as a white dot surrounded by a dark, Fresnel-1ike ring. The cavity diameter was measured across only the white portion of the image. For crystallographic cavities, the cavity diameter was assumed to be the same as the diameter of a circular projection with approximately the same area as the crystallographic projection.

The diameter measurements were made elther with a Zeiss Particle Size Analyzer (Mode1 TGZ-3) or, for coarse distributions, with a handheld Polaron 10x Magnifier. From these measurements, the volumeaveraged cavity diameter, $d_{c}$, was calculated according to the equation

$$
d_{c}=\left(\frac{\sum N_{i} d_{i}}{\sum N_{i}}\right)^{I / 3},
$$


where $\mathrm{N}_{1}$ is the number of cavities within the cavity diameter interval centered on a diameter of $d_{i}$.

The concentration of cavities, $C_{C}$, was calculated from the total number of cavities, $\mathrm{N}_{C}$, found in a specimen volume, $\mathrm{V}$. The thickness of the specimen projected along the beam direction, $t$, was measured using the techniques presented in section B.3. Thus,

$$
C_{c}=\frac{N_{c}}{t A} \text {, }
$$

where $A$ is the area of the specimen included in the micrograph used for the cavity concentration measurement.

The cavity volume fraction (CVF) was calculated from the volumeaveraged cavity diameter, $d_{c}$, and cavity concentration, $c_{c}$, according to the expression

$$
\begin{gathered}
\mathrm{CVF}=\frac{\pi}{6} \mathrm{~d}_{\mathrm{c}}^{3} \mathrm{C}_{\mathrm{c}} . \\
\text { Swelling }=\frac{\mathrm{CVF}}{1-\mathrm{CVF}}
\end{gathered}
$$

was used to calculate the swelling in this investigation. Since the CVF in this investigation was sma11, the swelling $\simeq$ CVF.

\section{B.7 Error Analysis}

The calculus approach as presented by, for example, Topping [156] was used for error analysis in this investigation. This method is based on the specification of the uncertainties in experimental measurements such as the micrograph magnification, Mag; the specimen thickness, $t$; and individual cavity diameter measurements. According to this type of error analysis, if a quantity $Q$ is a function of several measured quantities $x, y, z, \ldots$. , the error in $Q, \delta Q$, due to errors $\delta x, \delta y, \delta z, \ldots$. in $x, y, z, \ldots .$, respectively, is given by 


$$
\delta Q=\left[\left(\frac{\partial Q}{\partial x} \delta x\right)^{2}+\left(\frac{\partial Q}{\partial y} \delta y\right)^{2}+\left(\frac{\partial Q}{\partial z} \delta z\right)^{2}+\ldots\right]^{1 / 2}
$$

As an example, the error calculation for the dislocation density, $\Lambda$, given by Eq. (B.3), will be considered in detail. First, the length of the lines, $L$, measured on the micrograph must be corrected for the magnification, yielding a modified version of $\mathrm{Eq} \cdot(\mathrm{B} .3)$

$$
\Lambda=\frac{2 \mathrm{~N}(\mathrm{Mag})}{\mathrm{Lt}} \text {. }
$$

The error in $\Lambda, \delta \Lambda$, is given by, according to Eq. (B.9),

$$
\delta \Lambda=\left[\left(\frac{\partial \Lambda}{\partial N} \delta N\right)^{2}+\left(\frac{\partial \Lambda}{\partial(\mathrm{Mag})} \delta(\mathrm{Mag})\right)^{2}+\left(\frac{\partial \Lambda}{\partial L} \delta \mathrm{L}\right)^{2}+\left(\frac{\partial \Lambda}{\partial t} \delta t\right)^{2}\right]^{1 / 2}
$$

The partial derivatives with respect to each of the variables are

$$
\begin{aligned}
& \frac{\partial \Lambda}{\partial N}=\frac{2(\mathrm{Mag})}{L t}, \frac{\partial \Lambda}{\partial(\mathrm{Mag})}=\frac{2 \mathrm{~N}}{\mathrm{Lt}} \\
& \frac{\partial \Lambda}{\partial \mathrm{L}}-\frac{2 \mathrm{~N}(\mathrm{Mag})}{\mathrm{L}^{2} t} \text { and } \frac{\partial \Lambda}{\partial t}=-\frac{2 N(\mathrm{Mag})}{L^{2}}
\end{aligned}
$$

The parameter errors were assumed to be:

$$
\begin{aligned}
& \delta \mathrm{N}=(1 / \sqrt{\mathrm{N}}) \mathrm{N}, \quad \delta(\mathrm{Mag})=2 \%(\mathrm{Mag}), \\
& \delta L=1 \% L, \quad \text { and } \quad \delta t=10 \% t \text {. }
\end{aligned}
$$

Substituting Eqs. (B.12) and (B.13) into Eq. (B.11) yields

$$
\begin{aligned}
\delta \Lambda=\left[\left(\frac{2(\mathrm{Mag})}{\mathrm{Lt}}\right)^{2}\left(\frac{\mathrm{N}}{\sqrt{\mathrm{N}}}\right)^{2}+\left(\frac{2 \mathrm{~N}}{\mathrm{Lt}}\right)^{2}(0.02)^{2}(\mathrm{Mag})^{2}\right. & +\left(-\frac{2 \mathrm{~N}(\mathrm{Mag})}{\mathrm{L}^{2} \mathrm{t}}\right)^{2}(0.01)^{2} \mathrm{~L}^{2} \\
& \left.+\left(-\frac{2 \mathrm{~N}(\mathrm{Mag})}{\mathrm{L} \mathrm{t}^{2}}\right)^{2}(0.10)^{2} \mathrm{t}^{2}\right]^{1 / 2}
\end{aligned}
$$

or

$$
\delta \Lambda=\Lambda\left[\left(\frac{1}{\sqrt{N}}\right)^{2}+(0.02)^{2}+(0.01)^{2}+(0.10)^{2}\right]^{1 / 2}
$$

Similarly, the error in the dislocation density, $\rho$, given by Eq. (B.2) is found to be 


$$
\delta \rho=\rho\left[\left(\frac{1}{\sqrt{N}}\right)^{2}+4(0.02)^{2}+2(0.01)^{2}\right]^{1 / 2},
$$

where the parameter errors for the length and width measurements (used to calculate the area, A) were assumed to be $1 \%$.

For the cavity analyses, the error in the volume-averaged cavity diameter, $d_{c}$, was assumed to be $10 \%$. Since, for this investigation, the volume of the cavities was much less than the specimen volume, the errors in the swelling were the same as the errors in the CVF. With $C_{c}=N_{c} /(t A)$ and incorporating the print magnification correction, Eq. (B.7) becomes

$$
\mathrm{CVF}=\frac{\frac{\pi}{6} \mathrm{~d}_{\mathrm{c}}^{3} \mathrm{~N}_{\mathrm{c}}}{\text { tA (Mag) }}
$$

Implementing the calculus approach for the error in the swelling, $\delta(C V F)$, yields

$\delta(\mathrm{CVF})=\mathrm{CVF}\left[\left(\frac{1}{\sqrt{\mathrm{N}_{\mathrm{C}}}}\right]^{2}+9(0.10)^{2}+2(0.01)^{2}+(0.10)^{2}+(0.02)^{2}\right]^{1 / 2}$

The second term of the bracketed expression in Eq. (B.17), the contribution due to the error in the measurement of $d_{c}$, dominates the error in the swelling measurement. However, while the actual error in $d_{c}$ is probably about the $10 \%$ exror assumed, this error is largely systematic and does not prohibit good comparisons of the swelling values found in this study.

In addition to the above expressions for error analysis, the error in $C_{c}, \delta C_{c}$, was derived using the same techniques. This error is given by

$$
\delta C_{c}=C_{c}\left[\left(\frac{1}{\sqrt{N_{c}}}\right)^{2}+4(0.02)^{2}+2(0.01)^{2}+(0.10)^{2}\right]^{1 / 2} .
$$


$17 i$

- 
APPENDIX C

PREPARATION OF IRON-CHROMIUM ALLOYS

The iron and chromium used for the fabrication of $\mathrm{Fe}-5 \% \mathrm{Cr}$ and Fe $10 \% \mathrm{Cr}$ alloys was obtained from Materials Research Corporation (MRC). The iron was MARZ grade $(99.99+\%)$ and the chromium was IOCHROME $(99.996+\%)$. Chemical analyses as obtained from the manufacturer are given in Table C.1.

Table C.1. Chemical Analysis Provided by MRC of MARZ Grade Iron and IOCHROME Used in this Study (in wt $\mathrm{ppm}$ )

\begin{tabular}{|c|c|c|c|c|c|c|c|c|}
\hline & $\mathrm{Fe}$ & $\mathrm{Cr}$ & & $\mathrm{Fe}$ & $\mathrm{Cr}$ & & $\mathrm{Fe}$ & $\mathrm{Cr}$ \\
\hline C & 12 & 0.001 & $\mathrm{Ga}$ & $<0.1$ & & Pt & $<0.1$ & \\
\hline $\mathrm{H}$ & $<1$ & $0.2\left(\mathrm{H}_{2}\right)$ & In & $<0.1$ & & $S$ & 2.6 & \\
\hline$N$ & 10 & $0.9\left(\mathrm{~N}_{2}\right)$ & $\mathrm{K}$ & 1.8 & & $\mathrm{Sb}$ & $<0.1$ & \\
\hline 0 & 60 & $12.0\left(\mathrm{O}_{2}\right)$ & $\mathrm{Mg}$ & 0.87 & 0.1 & Si & $<0.1$ & 10.0 \\
\hline $\mathrm{Ag}$ & $<0.1$ & & Mn & & 0.1 & $\mathrm{Sn}$ & $<0.1$ & \\
\hline $\mathrm{A} 1$ & $<0.1$ & 0.3 & Mo & $<0.1$ & & $\mathrm{Ta}$ & $<0.1$ & \\
\hline $\mathrm{Au}$ & $<0.1$ & & $\mathrm{Na}$ & 1.6 & & $T \mathbf{I}$ & 1.4 & \\
\hline $\mathrm{Ca}$ & 0.8 & 0.3 & $\mathrm{Nb}$ & $<0.1$ & & $\nabla$ & & 0.3 \\
\hline $\mathrm{Cl}$ & 0.8 & & $\mathrm{Ni}$ & $<0.1$ & 0.3 & $w$ & $<0.1$ & \\
\hline $\mathrm{Cr}$ & 1.6 & Major & $\mathrm{P}$ & 0.7 & & $\mathrm{Zn}$ & 1.9 & \\
\hline $\mathrm{Cu}$ & 0.6 & & $\mathrm{~Pb}$ & $<0.1$ & & $\mathrm{Zr}$ & $<0.1$ & \\
\hline $\mathrm{Fe}$ & Major & 12 & $\mathrm{Pd}$ & $<0.1$ & & & & \\
\hline
\end{tabular}

The as-received $12.7-\mathrm{mm}(0.5 \mathrm{in.})$ diameter iron rod was rolled to $3.2 \mathrm{~m}(0.125 \mathrm{in.})$ thick slabs. To assure that the material would not be contaminated from the rollers, it was carefully cleaned and placed between a sandwich of clean, Ferrovac-E iron for the rolling process. These slabs were cut into pieces about 25 long. After careful cleaning in dilute hydrochloric acid, the pleces of iron were placed in a horizontal furnace arranged for wet/dry hydrogen annealing. This apparatus was operated with the help of G. Petersen of the Metals and Ceramics Division of ORNL. 
Previous treatment of similar materials with this annealing process indicated that the carbon and oxygen impurities would be reduced to less than 10 wt ppm with a six-day (three days per stage) anneal in flowing, high-purity hydrogen at $1200 \mathrm{~K}$. A wet/dry hydrogen anneal of the iron was performed as described above.

The iron-chromium alloys were fabricated with the assistance of H. Harmon and Dr. Y. Chang of the Solid State Physics Division of ORNL. The required weights of iron and chromium for each alloy were arc melted together under clean conditions in an argon atmosphere containing less than $0.3 \mathrm{ppm}$ of oxygen and water. The alloys were remelted several times to assure homogeneity of the alloy. For control material, a third melt of the pure iron was performed under the same conditions as those during the alloy fabrication.

The bars of $\mathrm{Fe}, \mathrm{Fe}-5 \% \mathrm{Cr}$, and $\mathrm{Fe}-10 \% \mathrm{Cr}$ were swaged to $\sim 3.2-\mathrm{mm}-$ dia rods. The swaging dies were carefully cleaned to reduce the possibility of contamination of the material.

At this stage, a carbon analysis of each material was performed by the Analytical Chemistry Division of ORNL. A Leako Carbon Analyzer was used for the analysis. The results were: $\mathrm{Fe}, 36$ wt ppm $\mathrm{C}$; $\mathrm{Fe}-5 \%$ $\mathrm{Cr}, 35$ wt ppm $\mathrm{C}$; and $\mathrm{Fe}-10 \% \mathrm{Cr}, 32$ wt ppm C.

In an effort to reduce the carbon impurities, a second hydrogen anneal was performed. Only the dry hydrogen part of the anneal was ut1lized. The conditions were chosen to optimize both carbon removal and post-anneal grain size, thereby avoiding a second anneal. The anneal was performed within a high-purity alumina tube to reduce possible silicon contamination. The rods were annealed for 24 hours at $1400 \mathrm{~K}$, furnace cooled for approximately 2 hours to $1000 \mathrm{~K}$, held at this temperature for 2 hours, and furnace cooled to room temperature. AgIng at $1000 \mathrm{~K}$ was chosen as this temperature was in the ferrite transformation temperature region and was believed to be above the temperature at which carbide precipitation would occur. After the anneal, the rods were centerless ground to $3 \mathrm{~mm}$ diameter. 
The final chemical analyses of the iron and iron-chromium alloys are shown in Table C.2. Metallography of specimens chemically etched with dilute hydrochloric acid indicated that the grain size was about $200 \mu \mathrm{m}$ for the $\mathrm{Fe}-10 \% \mathrm{Cr}$ alloy and about $400 \mu \mathrm{m}$ for the Fe-5\% $\mathrm{Cr}$ alloy.

\begin{tabular}{|c|c|c|c|}
\hline & $\mathrm{Fe}$ & $\mathrm{Fe}-5 \% \mathrm{Cr}$ & $\mathrm{Fe}-10 \% \mathrm{Cr}$ \\
\hline$c^{b}$ & 34 & 24 & 34 \\
\hline $\mathrm{H}^{\mathrm{C}}$ & 2 & 8 & 2 \\
\hline $\mathrm{NC}^{\mathrm{C}}$ & $<1$ & 17 & 4 \\
\hline $0^{c}$ & 6 & $<1$ & 6 \\
\hline $\mathrm{Cr}$ & 20 & $4.3 \%$ & $6.98 \%$ \\
\hline $\mathrm{Ag}$ & 5 & 5 & 2 \\
\hline As & 20 & 20 & 20 \\
\hline B & 2 & 0.5 & 2 \\
\hline $\mathrm{Ba}$ & $<1$ & 100 & 4 \\
\hline $\mathrm{Br}$ & 0.7 & $\leqslant 3$ & 5 \\
\hline $\mathrm{Cl}$ & 70 & - & 40 \\
\hline $\mathrm{Ca}$ & 1 & 40 & 4 \\
\hline Co & 40 & 3 & 4 \\
\hline $\mathrm{Cu}$ & 50 & 50 & 30 \\
\hline $\mathrm{Ga}$ & 50 & 30 & 50 \\
\hline $\mathrm{Ge}$ & 10 & 20 & 20 \\
\hline $\mathrm{K}$ & 0.2 & 70 & 7 \\
\hline $\mathrm{Mg}$ & $\leqslant 1$ & $\leqslant 2$ & $\leqslant 3$ \\
\hline $\mathrm{Mn}$ & 40 & 50 & 10 \\
\hline Mo & $\leqslant 1$ & 6 & $\leqslant 2$ \\
\hline $\mathrm{Na}$ & 1 & 100 & 4 \\
\hline $\mathrm{Nb}$ & 5 & - & 2 \\
\hline $\mathrm{NI}$ & 10 & 40 & 20 \\
\hline $\mathrm{P}$ & 10 & 7 & 10 \\
\hline $\mathbf{S}$ & 7 & 20 & 20 \\
\hline $\mathrm{Sb}$ & 3 & 2 & 2 \\
\hline $\mathrm{Sn}$ & 60 & 70 & 70 \\
\hline $\mathrm{Ta}$ & $\leqslant 100$ & $\leqslant 300$ & $\leqslant 3$ \\
\hline V & $\leqslant 0.5$ & 1 & 1 \\
\hline$W$ & $\leqslant 10$ & $\leqslant 10$ & $\leqslant 10$ \\
\hline $\mathrm{Zn}$ & $\leqslant 0.2$ & 70 & 10 \\
\hline
\end{tabular}

analysis by mass spectroscopy unless otherwise indicated.

bAnalysis by the Leako Carbon Method.

cAnalysis by the Vacuum Fusion Method. 
$1-7$

- 
APPENDIX D

\section{PROCEDURE - TRIPLE-BEAM IRRADIATION}

The triple-beam irradiation technique allows the simultaneous bombardment of specimens with energetic heavy ions, helium and hydrogen. The facility used to perform the triple-beam irradiations for the present study was the dual Van de Graaff accelerator system at ORNL. This facility is operated by M. Lewis, R. Buhl, and S. Cook. The ORNL facility and the details of the triple-beam irradiation technique are discussed below. Following this discussion, the details of the triplebeam irradiation of the iron and iron-chromium alloys are presented. A more in-depth presentation of the triple-beam technique and the ORNL. facility is found in refs. [17-19] and ref. [157].

D.1 Description of the Triple-Beam Technique and the ORNL Irradiation Facility

Accelerator System - The facility used for triple-beam irradiation at ORNL, as shown in Figure D.1, consists of two Van de Graaff accelerators. The beam lines of both accelerators terminate at a single target chamber.

The heavy tons are accelerated to $4 \mathrm{MeV}$ by a vertical CN Van de Graaff accelerator. This accelerator is equipped with a Model 910 Physicon ion source [18]. Incorporated in the beam line is a specially designed Johnson split-field lens [158]. The purpose of this lens is to reduce the beam divergence to obtain a $1 \mathrm{~cm}^{2}$ uniform beam area.

A $400 \mathrm{kV}$ horizontal AN Van de Graaff is used to accelerate ions of helium and deuterium simultaneously. Deuterium is used instead of hydrogen because molecular deuterlum has the same mass as hellum. Thus, the helium and deuterium gases can be mixed, fonized to the same charge, and accelerated together by a single accelerator. Another advantage is that the deuterons are loosely bound in the fonized molecular deuterium and separate upon impact with the target surface. Each deuteron has half of the incident energy. Since the stopping power of a deuteron in a target is about half that of helium, the deuterons and the helium come to rest at about the same depth from the target surface $[17,19]$. 
YE-12363

(a) CN VANDEGRAFF ACCELERATOR (4 MV)

(b) AN ACCELERATOR (400 keV)

(c) STEERER

(d) DIFFUSION PUMP

(e) GAS STRIPPER

(†) $90^{\circ}$ MAGNET

(9) BEAM STOP AND CONTROL SLITS

(b) BEAM SCANNER

(i) JOHNSON LENS

(1) CRYO PUMP

(B) EXPERIMENT CHAMBER

(1) QUADRAPOLE, SINGLET LENS (1)

(ii) DTFC

(i) $30^{\circ}$ MAGNET

(D) FARADAY CUPS

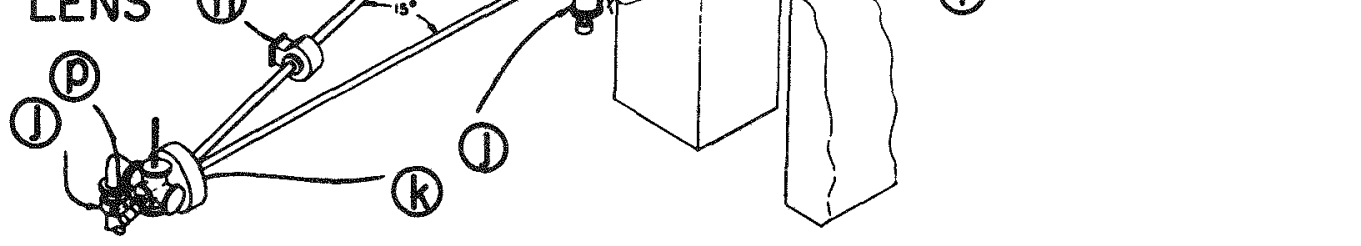

Figure D.1. Oak Ridge National Laboratory Van de Graaff Accelerator System. After ref. [157]. 
The terminal voltage of the light-ion accelerator can be varied continuously while maintaining a uniform intensity at the target. By selecting the proper energy range, the implanted helium and deuterium ions can be superimposed over the heavy ion damage region. Typically, the energy of the gas beam is ramped sinusoidally at $2.5 \times 10^{-2} \mathrm{~Hz}$ between 200 and $400 \mathrm{keV}$.

Target Chamber - At the target chamber, the heavy ion beam strikes the specimens normal to the surface. The 1ight-ion beam impinges at a $75^{\circ}$ angle $[17,19]$. Initial pumpdown is provided by two liquid nitrogen sorption pumps. The ultrahigh vacuum in the target chamber is provided by a cryopump. During bombardments, pressures of 1 to $\sim 0.1 \mu \mathrm{Pa}\left(10^{-7}\right.$ to $10^{-8}$ torr $)$ are reached $[18,157]$.

Target Assembly - The target assembly consists of six specimen holder-heater stations. Each station is bombarded separately, allowing up to six "runs" with different irradiation conditions without reloading specimens. Specimen heating is provided by dispenser cathodetriode type electron gun assemblies at each station [18].

Specimen Holders - The specimen holders consist of two parts - a thermalizer block and a face plate. The specimens are clamped between these two parts. Both parts are fabricated from Kulite-112 machineable tungsten alloy to reduce bonding to the specimens. The thermallizer blocks are ground flat to assure good thermal contact with the specimens [18]. In the specimen holder usually used, the face plate has a $3 \times 3$ array of $\sim 2 \mathrm{~mm}$ diameter holes. On the back of the face plates, the area around each hole is recessed to position a $3 \mathrm{~mm}$ diameter specimen over the hole. A $3 \mathrm{~mm}$ diameter crushable, annealed platinum wire ring, 0.1$0.2 \mathrm{~mm}$ thick and a $0.05 \mathrm{~mm}$ thick oxidized stainless steel spacer (optional) are placed between the specimen and the face plate. The platinum ring accommodates small specimen-to-specimen thickness variations so that none of the specimens are loose in the holder. The stainless steel spacer is placed between the platinum ring and the specimen to prevent welding of the platinum to the specimen during high-temperature irradiations. A clamping force of $\sim 60 \mathrm{~kg}(\sim 125 \mathrm{lb})$ is applied to the faceplate-specimen-thermalizer block assembly to compress the platinum rings 
[18]. Four screws hold the specimen holder together and maintain this pressure.

Temperature Control and Monitoring - The specimen temperature is controlled during the irradiation by any one of three thermocouples. Two thermocouples fit into recessed holes on the edge of the thermalizer block. The surface temperature is monitored by a third thermocouple spot welded to one of the nine specimens in the specimen holder array.

The temperature readout from the surface thermocouple is used to callbrate an infrared pyrometer which can be sighted on the surface of each of the specimens. The Individual specimen temperatures are measured during the irradiation with this pyrometer. The movement of the pyrometer to each spectmen location is directed by a pre-set electronic positioning system. This system is designed to allow fast, accurate movement of the pyrometer to each specimen location. The temperature of all nine specimens can be read and recorded in $<2 \mathrm{~min}$.

Current Monitoring and Specimen Masking - An array of nine miniature Faraday cups is used to monitor the ion beam current. Each Faraday cup corresponds to the exact location of a specimen in the specimen holder array. Beam current readings are taken and recorded automatically during the irradiation at pre-set time intervals.

Each row of the specimen array can be irradiated to a different dose. This is accomplished with a mask which covers one or two rows of the array during the initial part of the irradiation. The mask moves to uncover the rows at an appropriate time during the irradiation to enable the specimens to be bombarded to the required dose. The mask movement is computer-controlled.

Computer Control - As indicated above, many aspects of the ion Irradiation utilize some form of computerized automation. A TP-50 (Tennecomp Products) minicomputer is programmed for control of the valves In the beam line (for starting and stopping the run), beam current monitoring, mask movement, etc. A printout is provided during each run with details such as the dpa/specimen, the ion current/specimen, and the projected time at the end of the run. Only the irradiation temperature must be recorded manual1y. 
D.2 Specimen Preparation of $\mathrm{Fe}, \mathrm{Fe}-5 \% \mathrm{Cr}$, and $\mathrm{Fe}-10 \% \mathrm{Cr}$ for Triple-Beam Irradiation

The specimens were prepared for Van de Graaff irradiation with the assistance of $\mathrm{J}$. Houston. The procedure used is outlined below.

Specimens with $\sim 0.5 \mathrm{~mm}$ thickness were sliced with a silicon carbide slitting wheel from the $3 \mathrm{~mm}$ diameter rods of annealed $\mathrm{Fe}, \mathrm{Fe}-5 \%$ $\mathrm{Cr}$, and $\mathrm{Fe}-10 \% \mathrm{Cr}$ (alloy preparation is discussed in Appendix $\mathrm{C}$ ). These specimens were deburred and mounted in groups on a lapping block with epoxy. The lapping block was the standard, cylindrical, stainless steel block used for syntron polishing. The epoxy covering the surfaces of the specimens was ground away with 600 grit paper on a rotating wheel. The exposed surfaces were lightly polished on a Syntron lapping wheel with Linde $C$ abrasive, a powder of 1 um diameter particles of alpha alumina, until each specimen surface was flat and shiny. An alloy identification mark was then scratched on each specimen.

The next steps were removal of the specimens from the block, remounting them with the marked surface toward the block, and polishing the new surface for bombardment. The specimens were removed from the block by immersing it in $\mathrm{N}, \mathrm{N}-\mathrm{Dimethyl}$ Formamide heated to $90-100^{\circ} \mathrm{C}$. Any remnants of epoxy remaining adhered to the specimens were carefully removed with sharpened, softwood sticks while immersed in acetone.

The specimens were remounted on the block in the same manner as described previously. As before, the epoxy was removed from the specimen surface with 600 grit paper. The specimens were ground with the $600 \mathrm{grit}$ paper until they were within $\sim 100 \mu \mathrm{m}$ of the final desired specimen thickness of $0.3-0.36 \mathrm{~mm}$. The specimens were then syntron polished with the abrasives listed below. The abrasives are listed in the order of polishing sequence:

1. Linde $\mathrm{C}-1 \mathrm{\mu m}$ diameter alpha alumina particles,

2. Diamond paste $-0.5 \mu \mathrm{m}$ diameter particles,

3. Diamond paste $-0.1 \mu \mathrm{m}$ diameter particles.

The final Syntron polish was of sufficient duration to produce a scratch-free surface. The specimens were removed from the block in the 
same manner as discussed previously. Specimens were stored in a mechanically pumped desiccator until just before bombardment.

Final polishing deformation was removed just before loading the specimens for the bombardment by electropolishing the surface to be irradiated. A vertical jet electropolishing apparatus developed by E. Lee [103] was used for the electropolishing with an $80 \%$ ethyl alcohol- 20\% perchloric acid polishing solution. The vertical jet apparatus removes the same amount of material over the entire specimen surface. A polishing current of $275 \mathrm{~mA}$ removed $\sim 5 \mathrm{~m}$ in $5 \mathrm{~s}$.

The electropolished specimens were loaded into a $3 \times 3$ array specimen holder in the manner described previously. Both platinum rings and stainless steel washers were used in the loading procedure.

\section{D.3 Specimen Arrays}

Specimens positions within the specimen holder array were identified using matrix notation. The first row contalned specimens 11 , 12, and 13; the second row, specimens 21,22 , and 23; and the third row, specimens 31,32 , and 33 . Specimen 33 had a Chrome1-Alumel thermocouple welded to the surface near the edge of the disk.

\section{D.4 Irradiation Parameters}

For this study, the triple-beam irradiations utilized $\mathrm{He}^{+}, \mathrm{D}_{2}^{+}$, and $4 \mathrm{MeV} \mathrm{Fe}$ ions. The energy of the $\mathrm{He}^{+}$and $\mathrm{D}_{2}^{+}$beam was ramped sinusoldally at $2.5 \times 10^{-2} \mathrm{~Hz}$ between 0.2 and $0.4 \mathrm{MeV}$. Ratios of 10 at. ppm He/dpa and 41 at. ppm D/dpa were attained. The estimated depth profiles for the helium and deuterium in iron are shown in Figure D.2. The helium profile was experimentally determined for a nickel target and the deuterium profile was determined for a stainless steel target [19]. These profile shapes were assumed to also apply to an iron target. The depth of the profiles was determined from the tabulated values for the helium and deuterium ion ranges $[126,86]$. These values are calculated from the stopping powers of helium and deuterium in iron, which, in the energy range of interest, are not well known. The 


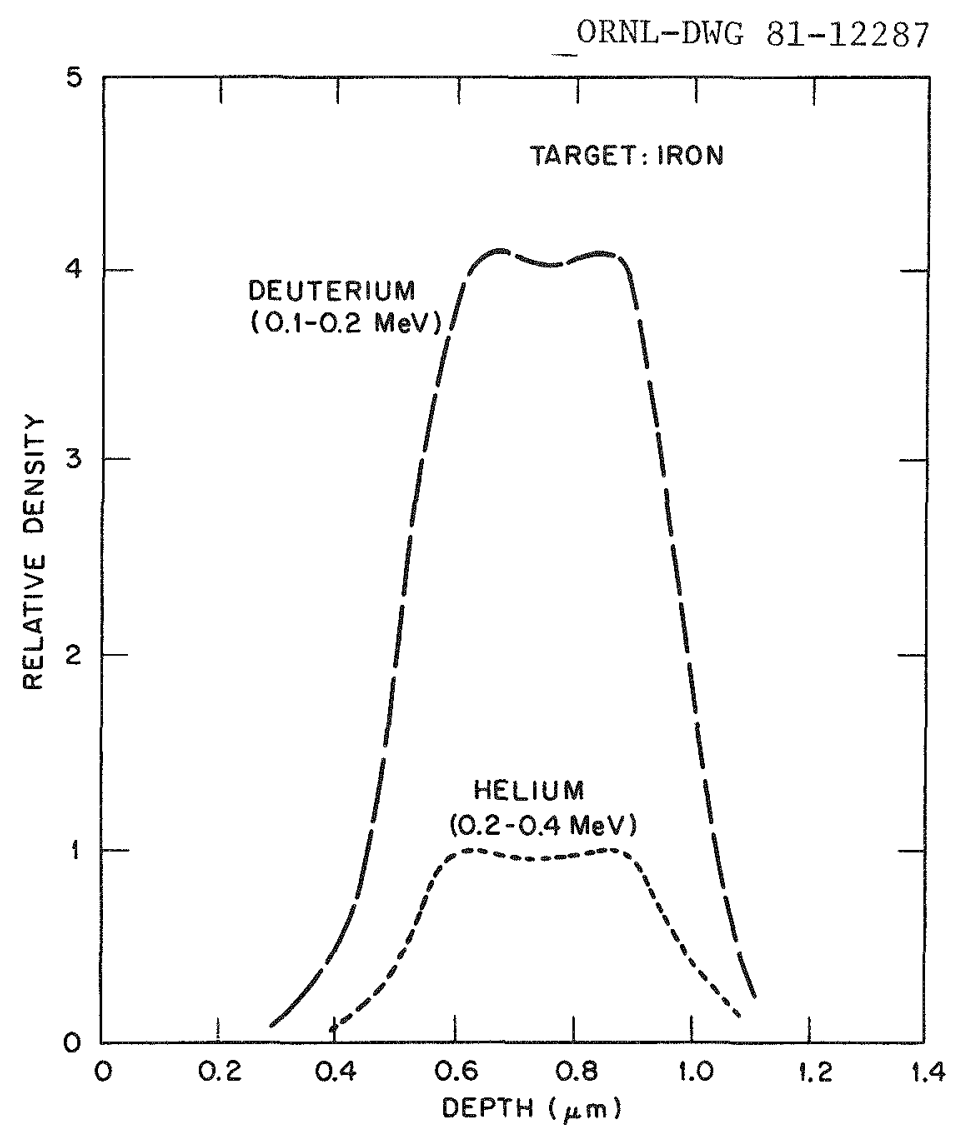

Figure D.2. Calculated helium and deuterium profiles for an iron target with an ion beam consisting of $\mathrm{He}^{+}$and $\mathrm{D}_{2}^{+}$with sinusoidally ramped energies $\left(0.2\right.$ to $\left.0.4 \mathrm{MeV}, 2.5 \times 10^{-2} \mathrm{~Hz}\right)$. The $\mathrm{D}_{2}$ splits to atomic species ( 0.1 to $0.2 \mathrm{MeV}$ energies) upon impact with targets.

few experimental values reported have differences of up to $20 \%$ for helium and $10 \%$ for deuterium, indicating that a significant error in the ion range is possible.

The irradiation parameters and specimen materials used in each irradiation run are found in Table D.1. Columns $1-3$ of the table contain the run number, nominal irradiation temperature $\left(\mathrm{T}_{\mathrm{I}}\right.$ ) and nominal dpa. Columns $4-7$ contain the relevant data for each specimen in the specimen holdex array. The actual dpa for each specimen was calculated from the iron ion current measured by the Faraday cups. The value for the dpa actually varies significantly over the ion range. The dpa values as a function of distance from the surface of the specimen are tabulated in Appendix $E_{\text {. }}$. The listed value in Table $D .1$ is the maximum dpa which was calculated with the expression: 
Table D.1. Irradiation Parameters and Specimen Material for Each Specimen in Ion Irradiation Runs ${ }^{a}$

\begin{tabular}{|c|c|c|c|c|c|c|c|}
\hline $\begin{array}{l}\text { Run } \\
\text { Number }\end{array}$ & $\begin{array}{l}\text { Nomina1 } \\
\mathrm{T}_{\mathrm{I}} \\
(\mathrm{K})\end{array}$ & $\begin{array}{l}\text { Nominal } \\
\mathrm{dpa}\end{array}$ & $\begin{array}{l}\text { Specimen } \\
\text { Position }\end{array}$ & Alloy & $\begin{array}{l}\mathrm{T}_{\mathrm{I}} \\
(\mathrm{K})\end{array}$ & dpa & $\begin{array}{c}\text { Sectioning } \\
\text { Depth } \\
(\mu \mathrm{m})\end{array}$ \\
\hline \multirow{9}{*}{532} & \multirow{9}{*}{725} & 10 & 11 & $\mathrm{Fe}-10 \% \mathrm{Cr}$ & 719 & 10.90 & 0.85 \\
\hline & & 10 & 12 & $\mathrm{Fe}-10 \% \mathrm{Cr}$ & 722 & 10.65 & 0.85 \\
\hline & & 10 & 13 & $\mathrm{Fe}-10 \% \mathrm{Cr}$ & 720 & 9.57 & \\
\hline & & 10 & 21 & $\mathrm{Fe}-5 \% \mathrm{Cr}$ & 728 & 11.59 & 0.90 \\
\hline & & 10 & 22 & $\mathrm{Fe}-5 \% \mathrm{Cr}$ & 730 & 13.32 & 1.00 \\
\hline & & 10 & 23 & $\mathrm{Fe}-5 \% \mathrm{Cr}$ & 735 & 9.00 & \\
\hline & & 10 & 31 & $\mathrm{Fe}$ & 731 & 9.26 & \\
\hline & & 10 & 32 & $\mathrm{Fe}$ & 730 & 9.06 & 1.2 \\
\hline & & 10 & 33 & $\mathrm{Fe}-5 \% \mathrm{Cr}$ & 744 & 8.66 & \\
\hline \multirow{9}{*}{533} & \multirow{9}{*}{800} & 10 & 11 & $\mathrm{Fe}-10 \% \mathrm{Cr}$ & 802 & 10.43 & 0.95 \\
\hline & & 10 & 12 & $\mathrm{Fe}-10 \% \mathrm{Cr}$ & 803 & 10.43 & 0.90 \\
\hline & & 10 & 13 & $\mathrm{Fe}-10 \% \mathrm{Cr}$ & 785 & 9.90 & 0.90 \\
\hline & & 10 & 21 & $\mathrm{Fe}-5 \% \mathrm{Cr}$ & 803 & 11.14 & 0.85 \\
\hline & & 10 & 22 & $\mathrm{Fe}-5 \% \mathrm{Cr}$ & 803 & 10.63 & 0.95 \\
\hline & & 10 & 23 & $\mathrm{Fe}-5 \% \mathrm{Cr}$ & 798 & 9.99 & \\
\hline & & 10 & 31 & $\mathrm{Fe}$ & 803 & 7.98 & \\
\hline & & 10 & 32 & $\mathrm{Fe}$ & 800 & 10.94 & 0.85 \\
\hline & & 10 & 33 & $\mathrm{Fe}-5 \% \mathrm{Cr}$ & 788 & 10.96 & \\
\hline \multirow{9}{*}{534} & \multirow{9}{*}{850} & 10 & 11 & $\mathrm{Fe}-10 \% \mathrm{Cr}$ & 848 & 10.06 & \\
\hline & & 10 & 12 & $\mathrm{Fe}-10 \% \mathrm{Cr}$ & 846 & 10.06 & 0.95 \\
\hline & & 10 & 13 & $\mathrm{Fe}-10 \% \mathrm{Cr}$ & 842 & 9.24 & \\
\hline & & 10 & 21 & $\mathrm{Fe}-5 \% \mathrm{Cr}$ & 851 & 10.72 & 0.95 \\
\hline & & 10 & 22 & $\mathrm{Fe}-5 \% \mathrm{Cr}$ & 852 & 10.58 & 0.95 \\
\hline & & 10 & 23 & $\mathrm{Fe}-5 \% \mathrm{Cr}$ & 848 & 9.91 & 0.85 \\
\hline & & 10 & 31 & $\mathrm{Fe}$ & 839 & 8.54 & 1.2 \\
\hline & & 10 & 32 & $\mathrm{Fe}$ & 850 & 10.60 & Plated \\
\hline & & 10 & 33 & $\mathrm{Fe}-5 \% \mathrm{Cr}$ & 871 & 10.87 & \\
\hline \multirow{9}{*}{535} & \multirow{9}{*}{950} & 10 & 11 & $\mathrm{Fe}-10 \% \mathrm{Cr}$ & 950 & 8.98 & \multirow{3}{*}{1.0} \\
\hline & & 10 & 12 & $\mathrm{Fe}-10 \% \mathrm{Cr}$ & 954 & 9.80 & \\
\hline & & 10 & 13 & $\mathrm{Fe}-10 \% \mathrm{Cr}$ & 948 & 9.40 & \\
\hline & & 10 & 21 & $\mathrm{Fe}-5 \% \mathrm{Cr}$ & 963 & 11.31 & 0.95 \\
\hline & & 10 & 22 & $\mathrm{Fe}-5 \% \mathrm{Cr}$ & 958 & 11.62 & 0.90 \\
\hline & & 10 & 23 & $\mathrm{Fe}-5 \% \mathrm{Cr}$ & 962 & 10.47 & \\
\hline & & 10 & 31 & $\mathrm{Fe}$ & 959 & 8.68 & \\
\hline & & 10 & 32 & $\mathrm{Fe}$ & 965 & $10 \cdot 13$ & 0.95 \\
\hline & & 10 & 33 & $\mathrm{Fe}-5 \% \mathrm{Cr}$ & 945 & 8.83 & \\
\hline
\end{tabular}


Table D.1 (Continued)

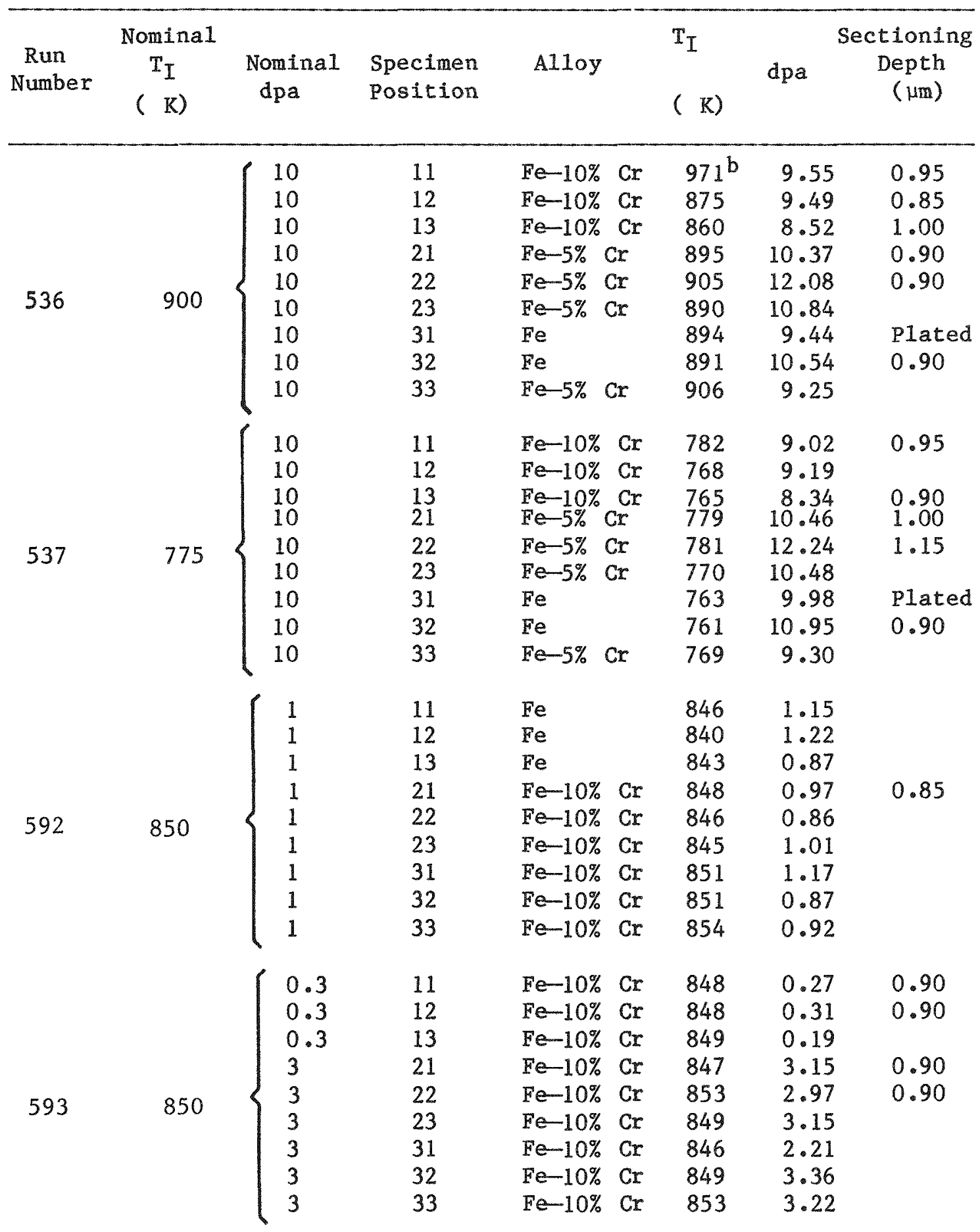


Table D.1 (Continued)

\begin{tabular}{|c|c|c|c|c|c|c|c|c|}
\hline $\begin{array}{l}\text { Run } \\
\text { Number }\end{array}$ & $\begin{array}{l}\text { Nominal } \\
\qquad \mathrm{T}_{\mathrm{I}} \\
(\mathrm{K})\end{array}$ & $\begin{array}{l}\text { Nominal } \\
\text { dpa }\end{array}$ & $\begin{array}{l}\text { Specimen } \\
\text { Position }\end{array}$ & Alloy & & $\begin{array}{l}\mathrm{T}_{\mathrm{I}} \\
(\mathrm{K})\end{array}$ & $\mathrm{dpa}$ & $\begin{array}{c}\text { Sectioning } \\
\text { Depth } \\
(\mu \mathrm{m})\end{array}$ \\
\hline $594^{\mathrm{c}, \mathrm{d}}$ & 850 & $\begin{array}{l}10 \\
10 \\
10 \\
10 \\
10 \\
10 \\
10 \\
10 \\
10\end{array}$ & $\begin{array}{l}11 \\
12 \\
13 \\
21 \\
22 \\
23 \\
31 \\
32 \\
33\end{array}$ & $\begin{array}{l}\text { Fe-10\% } \\
\text { Fe-10\% } \\
\text { Fe-10\% } \\
\text { Fe-10\% } \\
\text { Fe-10\% } \\
\text { Fe-10\% } \\
\text { Fe-10\% } \\
\text { Fe-10\% } \\
\text { Fe-10\% }\end{array}$ & $\begin{array}{l}\mathrm{Cr} \\
\mathrm{Cr} \\
\mathrm{Cr} \\
\mathrm{Cr} \\
\mathrm{Cr} \\
\mathrm{Cr} \\
\mathrm{Cr} \\
\mathrm{Cr} \\
\mathrm{Cr}\end{array}$ & $\begin{array}{l}853 \\
842 \\
847 \\
850 \\
898^{b} \\
845 \\
850 \\
850 \\
860\end{array}$ & $\begin{array}{r}10.51 \\
10.27 \\
7.12 \\
10.63 \\
12.10 \\
10.28 \\
9.18 \\
8.93 \\
7.55\end{array}$ & $\begin{array}{l}0.85 \\
0.90 \\
0.95 \\
0.70\end{array}$ \\
\hline 596 & 850 & $\begin{array}{l}100 \\
100 \\
100 \\
100 \\
100 \\
100 \\
100 \\
100 \\
100\end{array}$ & $\begin{array}{l}11 \\
12 \\
13 \\
21 \\
22 \\
23 \\
31 \\
32 \\
33\end{array}$ & $\begin{array}{l}\mathrm{Fe} \\
\mathrm{Fe} \\
\mathrm{Fe} \\
\mathrm{Fe}-10 \% \\
\mathrm{Fe}-10 \% \\
\mathrm{Fe}-10 \% \\
\mathrm{Fe}-10 \% \\
\mathrm{Fe}-10 \% \\
\mathrm{Fe}-10 \%\end{array}$ & $\begin{array}{l}\mathrm{Cr} \\
\mathrm{Cr} \\
\mathrm{Cr} \\
\mathrm{Cr} \\
\mathrm{Cr} \\
\mathrm{Cr}\end{array}$ & $\begin{array}{l}850 \\
859 \\
836 \\
866 \\
867 \\
846 \\
846 \\
851 \\
840\end{array}$ & $\begin{array}{r}108.74 \\
98.91 \\
71.41 \\
120.92 \\
101.42 \\
98.14 \\
107.47 \\
104.52 \\
88.54\end{array}$ & 0.90 \\
\hline 601 & 850 & $\begin{array}{l}10 \\
10 \\
10 \\
30 \\
30 \\
30 \\
30 \\
30 \\
30\end{array}$ & $\begin{array}{l}11 \\
12 \\
13 \\
21 \\
22 \\
23 \\
31 \\
32 \\
33\end{array}$ & $\begin{array}{l}\text { Fe-10\% } \\
\text { Fe }-10 \% \\
\text { Fe }-10 \% \\
\text { Fe-10\% } \\
\text { Fe-10\% } \\
\text { Fe-10\% } \\
\text { Fe-10\% } \\
\text { Fe-10\% } \\
\text { Fe-10\% }\end{array}$ & $\begin{array}{l}\mathrm{Cr} \\
\mathrm{Cr} \\
\mathrm{Cr} \\
\mathrm{Cr} \\
\mathrm{Cr} \\
\mathrm{Cr} \\
\mathrm{Cr} \\
\mathrm{Cr} \\
\mathrm{Cr}\end{array}$ & $\begin{array}{l}850 \\
855 \\
845 \\
853 \\
850 \\
844 \\
853 \\
851 \\
826\end{array}$ & $\begin{array}{r}9.24 \\
9.64 \\
6.12 \\
34.30 \\
34.53 \\
25.85 \\
32.78 \\
29.64 \\
27.63\end{array}$ & $\begin{array}{l}0.90 \\
0.90 \\
0.90 \\
0.85 \\
0.85\end{array}$ \\
\hline $602^{c}$ & 850 & $\begin{array}{l}10 \\
10 \\
10 \\
30 \\
30 \\
30 \\
30 \\
30 \\
30\end{array}$ & $\begin{array}{l}11 \\
12 \\
13 \\
21 \\
22 \\
23 \\
31 \\
32 \\
33\end{array}$ & $\begin{array}{l}\mathrm{Fe}-10 \% \\
\mathrm{Fe}-10 \% \\
\mathrm{Fe}-10 \% \\
\mathrm{Fe}-10 \% \\
\mathrm{Fe}-10 \% \\
\mathrm{Fe}-10 \% \\
\mathrm{Fe}-10 \% \\
\mathrm{Fe}-10 \% \\
\mathrm{Fe}-10 \%\end{array}$ & $\begin{array}{l}\mathrm{Cr} \\
\mathrm{Cr} \\
\mathrm{Cr} \\
\mathrm{Cr} \\
\mathrm{Cr} \\
\mathrm{Cr} \\
\mathrm{Cr} \\
\mathrm{Cr} \\
\mathrm{Cr}\end{array}$ & $\begin{array}{l}837 \\
839 \\
853 \\
841 \\
850 \\
853 \\
839 \\
851 \\
885\end{array}$ & $\begin{array}{r}10.36 \\
10.03 \\
7.00 \\
34.81 \\
24.40 \\
29.64 \\
30.39 \\
32.97 \\
29.97\end{array}$ & 0.85 \\
\hline
\end{tabular}

aunless otherwise noted the run was a triple-beam irradiation (4 MeV Fe+ 10 at. ppm He/dpa, 41 at. ppm D/dpa).

${ }^{b}$ Specimen loose in holder. $c_{4} \mathrm{MeV} \mathrm{Fe}+$ only.

dCold preinjection with 100 at. ppm He, 410 at. ppm $D$. 


$$
\mathrm{dpa}=\mathrm{dpa}(\mathrm{x})_{\max }=\frac{\rho(\mathrm{x})_{\max } \Omega}{\mathrm{A}_{\text {beam }}} \mathrm{C}_{\mathrm{T}}
$$

where $C_{T}=$ the total ion beam charge in Coulombs,

$\Omega=$ the target atomic volume,

$A_{\text {beam }}=$ the area of the ion beam, $1.25 \mathrm{~cm}^{2}$, and

$\rho(x)_{\max }=$ the maximum value of the linear density of the displaced atoms per unit length of range.

For small ranges, the value for $\rho(x)_{\max }$ can be calculated using the expression $[119,159]$ :

$$
\rho(x)_{\max } \simeq \frac{\kappa}{2 E_{d}} S_{D}(x)_{\max }
$$

where $\quad k=$ the displacement efficiency,

$E_{\mathrm{d}}=$ the average energy required to produce one atomic displacement, and

$S_{D}(x)_{\max }=$ the maximum value for the energy deposited per unit length of range.

For iron, $E_{d}$ is $\sim 40 \mathrm{eV} /$ displacement [160] and $K$ is $\sim 0.8$ [159]. $\mathrm{S}_{\mathrm{D}}(\mathrm{x})_{\max }$ was assumed to be $0.9720 \mathrm{MeV} / \mu \mathrm{m}-1$ ion $(\mathrm{x}=1.00 \mu \mathrm{m})$ as calculated by the E-DEP-1 computer code for an electronic stopping power, $k$ of 0.121 . This code and the output for $4 \mathrm{MeV} \mathrm{Fe}$ t+ ion bombardment of iron are discussed in Appendix $E$. Since the ions are doubly charged, each ion is equivalent to $3.204 \times 10^{-19} \mathrm{C}$ (i.e., $2 \times 1.602 \times 10^{-19} \mathrm{C}$ ). Substituting the above values in Eq. $(D .2)$ yields: $\rho(x)_{\max }=3.034$ $\times 10^{20}$ displacement/ $/ \mathrm{C}-\mathrm{cm}$. Substituting this value for $\rho(x)_{\max }$ and the atomic volume of iron of $1.178 \times 10^{-23} \mathrm{~cm}^{3} /$ atom [161] into Eq. (D.1) yields:

$$
\mathrm{dpa}=\frac{2.86 \times 10^{-3} \text { displacement }}{\mu \mathrm{C} \text {-atom }} \mathrm{C}_{\mathrm{T}} .
$$

All runs used the triple-beam irradiation technique as described above except for runs 594 and 602. The irradiation for run 594 consisted of room-temperature preinjection of 100 at. ppm He and 410 at. ppm $D$ followed by an $850 \mathrm{~K}$ irradiation with $10 \mathrm{dpa}$ of $4 \mathrm{MeV} \mathrm{Fe}$ ions. 
In run 602 , a single beam of $4 \mathrm{MeV} \mathrm{Fe}$ ions was used for the irradiation. To prevent oxidation after irradiation, the specimens were stored in a vacuum desiccator evacuated by a mechanical pump.

\section{D.5 TEM Specimen Preparation}

One problem associated with ion irradiations is the presence of the damaged region of the specimen within a few micrometers of the irradiated surface. The usual method for preparing these specimens for TEM examination involves the controlled removal or "sectioning" of the damaged region to some predetermined depth from the irradiated surface, followed by electropolishing from the unirradiated surface ("backthinning") to perforation. The sectioning depth is important as the characteristics of the defect structures vary significantly with distance from the irradiated surface. The sectioning depth can best be selected by experimentally determining the depth distribution of the defect structures. The procedure for determining this depth distribution and selecting a sectioning depth is outlined in Chapter 4. From the depth distribution of the defects in triple-beam-irradiated iron, a sectioning depth of $0.9 \mu \mathrm{m}$ was selected. Assistance in the preparation of TEM specimens was provided by C. G. McKamey of ORNL.

The specimens were sectioned electrolytically using the vertical jetting apparatus developed by E. H. Lee [103]. An $80 \%$ ethyl alcohol$20 \%$ perchloric acid electrolyte cooled to $223 \mathrm{~K}\left(-50^{\circ} \mathrm{C}\right)$ was used with a polishing current of $150 \mathrm{~mA}$.

The polishing times required to remove $0.9 \mu \mathrm{m}$ were: $\sim 12.5 \mathrm{~s}$ for $\mathrm{Fe}-10 \% \mathrm{Cr}, \sim 8 \mathrm{~s}$ for $\mathrm{Fe}-5 \% \mathrm{Cr}$, and $\sim 6.6 \mathrm{~s}$ for iron. The amount of material removed was measured from a small area that was covered with a layer of microstop lacquer during the removal. The measurements were made with a DEKTAK profilometer manufactured by Sloan Corporation. Multiple measurements were made around the microstopped area. These readings were averaged to obtain the value listed in the last column of Table D.1. The spread in the measurements was usually less than $0.1 \mu \mathrm{m}$.

Back-thinning was performed in a Tenupol electropolishing apparatus using an electrolyte of $900 \mathrm{ml}$ acetic acid, $100 \mathrm{ml}$ methyl alcohol, 
and $100 \mathrm{ml}$ perchloric acid. The electrolyte temperature was $286 \mathrm{~K}$ $\left(13^{\circ} \mathrm{C}\right)$. A slow jet speed was used with $\sim 90 \mathrm{~mA}$ polishing current. The irradiated, sectioned surface of the specimen was protected during the polishing by a heavy coat of microstop lacquer. The standard Tenupol specimen holder was used.

After perforation, the specimens were carefully rinsed in acetone and methyl ethyl ketone to remove the microstop. Final rinsing was in 200-proof ethyl alcohol. The electropolished specimens were stored in a vacuum desiccator evacuated by a mechanical pump.

Many difficulties were encountered in preparing TEM specimens from the lon-irradiated iron and iron-chromium alloys. The above procedure is the end result of many months of experimentation with various techniques and electrolytes. The results are still not completely satisfactory. Sectioning at low temperatures presents some difficulties. It was difficult to maintain the electrolyte temperature. This led to a certain amount of non-repeatability in the amount of material removed for a given polishing time. Great care was required in applying the microstop layer on the irradiated surface for back-thinning. Leakage was a common problem; it resulted in etched specimen surfaces, especially in the electron thin regions. The Tenupol jet speed was very important. If the speed was too fast, the folls were extremely bent. If the speed was too slow, the polishing was uneven and etching often occurred. Dirty specimens often resulted from insufficient rinsing to remove the remnants of the microstop. In general, specimen preparation difficulties increased as the chromium content decreased. 
178

- 


\section{APPENDIX E \\ E-DEP-1 COMPUTER CODE: DEPOSITED ENERGY, DEPOSITED \\ ION AND DPA PROFILES}

The E-DEP-1 computer code written by Manning and Muel1er [119] calculates the depth distribution of deposited energy and the projected ion range for ion irradiations. The target material can contain up to six atomic components and is assumed to be amorphous. This calculation is based on the assumption of Kulcinski et al. [162] that the energy distribution can be estimated by relating it to the range straggling. This calculation is refined to take into account the inelastic collisions by using the Lindhard Partition Theory [163]. The mean Lon range and range straggling are obtained from the LSS (Lindhard, Schraff, and Schiott) theory [164]. The LSS value for $k$, the electronic stopping power, can be overruled by an input value.

At Oak Ridge National Laboratory, the E-DEP-1 computer code is run on the IBM 3033 Computer System. The orfginal E-DEP-1 program was modified as per Manning and Mueller by O. Oens in 1976 [165]. For the current investigation, the program was used to calculate the deposited energy and ion distribution for $4 \mathrm{MeV}$ iron ion bombardment of iron. The LSS value of $k, 0.156$, as well as two lower values for $k, 0.121$ and 0.108 , were utilized. The calculated values for the projected ion range, $x_{m}$, the standard deviation (RMS) for the projected range, $\sigma_{p}$, the standard deviation (RMS) for the total range, $\sigma_{r}$, the distance from the target surface that the maximum value for the deposited energy is found, $x_{\max }$, and the maximum value for the deposited energy, $S_{D}(x)_{\max }$, are tabulated in Table $E_{.1}$ for each value of $k$ used.

The dpa distribution as a function of distance from the irradiated surface of the target was calculated following the procedures simi1ar to that outlined in Appendix D. The peak in the dpa distribution, $\mathrm{dpa}(\mathrm{x})$, which corresponds to the peak in the deposited energy, was assumed to be $10 \mathrm{dpa}$. The $\mathrm{dpa}(\mathrm{x})$ can be calculated using the expression: 
Table E.1. E-DEP-1 calculated values for $k=0.156,0.121$, and 0.108

\begin{tabular}{ccccccc}
\hline $\mathrm{k}$ & $\begin{array}{c}\mathrm{x}_{\mathrm{m}} \\
(\mu \mathrm{m})\end{array}$ & $\begin{array}{c}\sigma_{\mathrm{p}} \\
(\mu \mathrm{m})\end{array}$ & $\begin{array}{c}\sigma_{\mathrm{r}} \\
(\mu \mathrm{m})\end{array}$ & $\begin{array}{c}\mathrm{x}_{\max } \\
(\mu \mathrm{m})\end{array}$ & $\begin{array}{c}\mathrm{s}_{\mathrm{D}}(\mathrm{x})_{\max } \\
(\mathrm{MeV} / \\
\mu \mathrm{m}-\text { ion })\end{array}$ & $\begin{array}{c}\mathrm{C} \\
(\mathrm{dpa}-\mathrm{MeV} / \\
\mu \mathrm{m}-\text { ion })\end{array}$ \\
\hline 0.156 & 0.980 & 0.125 & 0.131 & 0.85 & 0.9772 & 10.23 \\
0.121 & 1.164 & 0.160 & 0.173 & 1.00 & 0.9720 & 10.29 \\
0.108 & 1.252 & 0.178 & 0.194 & 1.06 & 0.9669 & 10.34 \\
\hline
\end{tabular}

$$
\mathrm{dpa}(\mathrm{x})=\frac{\mathrm{C}_{\mathrm{T}} \Omega \rho(\mathrm{x})}{\mathrm{A}_{\text {beam }}}
$$

where the atomic volume, $\Omega,=1.178 \times 10^{-23} \mathrm{~cm}^{3} /$ atom [161], Abeam the beam area, $=1.25 \mathrm{~cm}^{2}, C_{T}$ is the total ion beam charge in coulombs required to produce $10 \mathrm{dpa}$ at the damage peak $[119,159]$, and

$$
\rho(x)=\frac{K}{2 E_{d}} S_{D}(x),
$$

with the constant, $\kappa_{2}=0.8$ [119], the displacement threshold energy, $E_{\mathrm{d}}=40 \mathrm{eV} / \mathrm{displacement}[160]$, and $\mathrm{S}_{\mathrm{D}}(\mathrm{x})$, the deposited energy per unit length of range, calculated by the E-DEP-1 code. Substituting Eq. (E.2) into Eq. (E.1) yields:

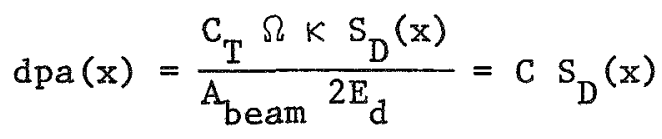

Here $C$ is a constant equal to $C_{T} \Omega k /\left(A_{\text {beam }} 2 E_{d}\right)$. At the peak damage depth,

$$
\mathrm{C}=\frac{\mathrm{dpa}(\mathrm{x})_{\max }}{\mathrm{S}_{\mathrm{D}}(\mathrm{x})_{\max }}=\frac{10 \mathrm{dpa}}{\mathrm{S}_{\mathrm{D}}(\mathrm{x})_{\max }} .
$$

Using Eq. (E.4), C can be calculated for each of the energy distributlons using the values for $S_{D}(x)_{\max }$ found in Table $E .1$. The values for $C$ are tabulated in the final column of Table E.1. Then, using Eq. (E.3) with the appropriate value of $C$ and the E-DEP-1 values for the deposited energy at various depths from the irradiated surface, $S_{D}(x)$, the 
dpa-depth profile can be calculated. The depth, $x, s_{D}(x)$, and dpa( $\left.x\right)$ are tabulated in Table E.2 for $k=0.156$, Table $E .3$ for $k=0.121$, and in Table $\mathrm{E} .4$ for $k=0.108$.

The iron ions are assumed to be deposited in a Gausian distribution centered on the projected ion range. For the E-DEP-1 code, the standard deviation, $\sigma$, of the ion distribution is assumed to be equal to the larger of the two distribution deviations, $\sigma_{\mathrm{p}}$ or $\sigma_{\mathrm{r}}$. For the distributions listed in Table E.1, $\sigma=\sigma_{r}$. The deposited ion-depth profile can be calculated using the equation for a normal distribution [119]:

$$
f(x)=\frac{1}{\sqrt{2 \pi} \sigma} e^{\frac{(x-\mu)^{2}}{2 \sigma^{2}}}
$$

where $f(x)=$ the deposited ion distribution,

$x=$ the depth from the target surface,

$\mu=x_{\mathrm{m}}$, the projected ion range, and

$\sigma=\sigma_{r}$, the standard deviation of the total range. 
Table E.2. Values for the deposited energy, $S_{\mathrm{p}}(\mathrm{x})$, and the displacements per atom, dpa(x), as a function of the distance from the target surface, $x, k=0.156$

\begin{tabular}{|c|c|c|c|c|c|}
\hline $\begin{array}{c}\mathrm{x} \\
(\mu \mathrm{m})\end{array}$ & $\mathrm{dpa}(\mathrm{x})$ & $\begin{array}{c}S_{D}(x) \\
(\mathrm{MeV} / \\
\mu m-i o n)\end{array}$ & $\stackrel{\underset{x}{x}}{(\mu \mathrm{m})}$ & $\operatorname{dpa}(\mathrm{x})$ & $\begin{array}{c}\mathrm{S}_{\mathrm{D}}(\mathrm{x}) \\
(\mathrm{MeV} / \\
\mu \mathrm{m}-\mathrm{ion}) \\
\end{array}$ \\
\hline 9.50 & 2.05 & .2594 & $.7 E$ & 9.15 & .8949 \\
\hline $.6 z$ & $\therefore-1$ & $=049$ & .78 & 9.45 & .3245 \\
\hline .64 & $\therefore \rightarrow$ & .705 & .80 & 9.71 & .9491 \\
\hline .05 & $E .5$ & .2765 & 8 & 9.89 & .9068 \\
\hline .08 & $E .99$ & .2820 & .84 & 4.99 & .9700 \\
\hline .16 & $\therefore 90$ & .2890 & .80 & 9.95 & .9756 \\
\hline $.1 \equiv$ & $3.0=$ & .2950 & .89 & $\exists .80$ & .9635 \\
\hline .14 & 3.10 & .3920 & .90 & 9.51 & .9395 \\
\hline .16 & 3.17 & .3090 & .95 & 9.5 & .9638 \\
\hline .18 & $3=25$ & .3173 & .94 & 8. 10 & .8563 \\
\hline .20 & 3.33 & .3252 & .96 & 8.19 & .8060 \\
\hline .22 & 3.41 & .3334 & .96 & $\vec{T}: 5=$ & .7349 \\
\hline .24 & 3.50 & .3420 & 1.616 & $E .79$ & $=0034$ \\
\hline$=26$ & 3.5 & .3511 & 1.95 & 8.63 & .5890 \\
\hline .8 & 3.03 & .3060 & 1.04 & 5.30 & .5260 \\
\hline .30 & 3.79 & .3796 & 1.50 & 4.60 & .4495 \\
\hline 32 & 3.90 & .3811 & 1.08 & 3.80 & .375 \\
\hline .34 & 4.01 & .3922 & 1.10 & 3.18 & .3169 \\
\hline .35 & 4.13 & .4939 & $1.1=$ & $\because 5 E$ & .2506 \\
\hline .38 & 4.26 & .4163 & 1.14 & $\Xi .05$ & .1980 \\
\hline .40 & 4.40 & .4205 & 1.18 & 1.57 & .1533 \\
\hline .42 & 4.54 & .4435 & 1.18 & 1.19 & $=11 E$ \\
\hline .44 & 4.69 & .4584 & $1 . \pm \overline{0}$ & .89 & .0854 \\
\hline $.4 E$ & 4.65 & .4744 & 1.23 & .64 & .0530 \\
\hline 48 & 5.03 & .4918 & 1.24 & .48 & .0449 \\
\hline .56 & 5.5 & .5106 & 1. $2 E$ & $.3 z$ & .9314 \\
\hline $.5 E$ & $5.4=$ & .5290 & 1.28 & Z & $.5 \geq 14$ \\
\hline .54 & 5.64 & .5514 & 1.30 & .15 & .0143 \\
\hline .56 & 5.85 & .574 & 1.32 & .10 & .0094 \\
\hline .58 & E.1. & .5997 & 1.34 & .00 & .0000 \\
\hline .85 & $E .4 Z$ & .8269 & 1.36 & .014 & .0538 \\
\hline .62 & $E .71$ & .5560 & 1.38 & 62 & .0023 \\
\hline .84 & 7.03 & .6872 & 1.40 & .01 & .0517 \\
\hline .65 & 1.37 & .7203 & 1.42 & .01 & .0000 \\
\hline .86 & 7.33 & .7550 & 1.44 & .91 & .0909 \\
\hline .70 & 8.09 & .7906 & 1.45 & .00 & .0053 \\
\hline .72 & 8.46 & .8265 & 1.48 & .06 & .0602 \\
\hline .74 & 8.82 & .8617 & 1.50 & .61 & .0061 \\
\hline
\end{tabular}


Table E.3. Values for the deposited energy, $S_{D}(x)$, and the displacements per atom, dpa(x), as a function of the distance from the target surface, $x . k=0.121$

\begin{tabular}{|c|c|c|c|c|c|}
\hline $\begin{array}{c}\mathbf{x} \\
(\mu \mathrm{m})\end{array}$ & $\operatorname{dpa}(\mathrm{x})$ & $\begin{array}{c}\mathrm{S}_{\mathrm{D}}(\mathrm{x}) \\
(\mathrm{MeV} / \\
\mu \mathrm{m} \text {-ion) }\end{array}$ & $\begin{array}{c}\mathrm{x} \\
(\mu \mathrm{m})\end{array}$ & $\operatorname{dpa}(\mathrm{x})$ & $\begin{array}{l}\mathrm{S}_{\mathrm{D}}(\mathrm{x}) \\
(\mathrm{MeV} / \\
\mu \mathrm{m} \text {-ion) }\end{array}$ \\
\hline 0.00 & $\therefore \quad \exists+$ & ニ $5 E \Xi$ & .94 & 9.7 & .9512 \\
\hline $.0=$ & $\therefore .98$ & $=911$ & .95 & 9.91 & .9533 \\
\hline .64 & $\because .65$ & a $=$ ニ & .98 & 9.98 & .9705 \\
\hline $.0 E$ & 3.16 & .3013 & 1.00 & 10.00 & $.97 \Xi 0$ \\
\hline .69 & $3.1 E$ & .3057 & 1. $19=$ & 9.95 & .9544 \\
\hline .15 & 2.1 & $.31 \Xi$ & 1.94 & 9.83 & .9559 \\
\hline $.1 E$ & 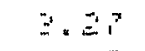 & .3179 & 1.96 & 9.5 .4 & $.937=$ \\
\hline .17 & $\because: 23$ & .3239 & 1.08 & 9.38 & .9120 \\
\hline $.1 E$ & 3.40 & .300 & 1.10 & 9.65 & .8794 \\
\hline .18 & $=.45$ & .335 & 1.12 & 5.65 & .8412 \\
\hline .20 & 3.53 & $.24=9$ & 1.14 & 5.50 & .7974 \\
\hline$\Xi \Xi$ & $3 . E 0$ & $.345-$ & 1.15 & $\Gamma \cdot \Gamma 1$ & .7492 \\
\hline$= \pm 4$ & $\therefore B$ & .3589 & 1.18 & $\because .14$ & 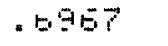 \\
\hline$\therefore E$ & 3.75 & .3541 & 1.20 & 5.59 &.$E 400$ \\
\hline .28 & $\therefore 8 E$ & $.371 \%$ & 1. $=$ & $E .61$ & .5844 \\
\hline .30 & 3.91 & . 3797 & 1. $=4$ & 5.55 & .5396 \\
\hline $.3=$ & 3.90 & $.59-9$ & 1. 20 & 4.95 & .4814 \\
\hline .34 & 4.08 & .3955 & 1. 28 & 4.37 & .4245 \\
\hline .35 & $4.1 \%$ & .4055 & 1.30 & 3.61 & .5703 \\
\hline .35 & $4.3-1$ & .4149 & $1.3=$ & 3.29 & .3195 \\
\hline .40 & $4.2 \pi$ & .4248 & 1.34 & $\therefore 80$ & $\therefore=4$ \\
\hline .45 & 4.48 & .4351 & 1.30 & $\therefore 35$ & .2297 \\
\hline .44 & 4.59 & .4459 & 1.38 & 1.97 & .1914 \\
\hline $.4 E$ & 4.70 & $.457 \Xi$ & 1.49 & 1.E & $.157 \mathrm{E}$ \\
\hline .48 & 4.55 & 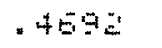 & 1.45 & 1. IE & .1285 \\
\hline .50 & 4.46 & .4819 & 1.44 & $1.0 E$ & .1030 \\
\hline $.5=$ & 5.49 & .4951 & 1.45 & .84 & .5617 \\
\hline .54 & 5.54 & .5092 & 1.49 & $=E$ & $.0 E+1$ \\
\hline $.5 E$ & 5.39 & $.5 \Xi 4 \Xi$ & 1.59 & .51 & .6490 \\
\hline .58 & 5.58 & .5405 & $1.5=$ & .39 & .0385 \\
\hline .60 & $5.7 z$ & $.55 E 8$ & 1.54 & .31 & $.529 \vec{~}$ \\
\hline .52 & 5.91 & .5747 & 1.58 & $\therefore \Xi$ & .9214 \\
\hline .84 & $E .11$ & .5937 & 1.58 & $.1 E$ & .9158 \\
\hline . EE & $E . \equiv 1$ & .5139 & 1.50 & .12 & .0115 \\
\hline $.6 E$ & $5.5=$ & .852 & $1 . E=$ & .69 & .0083 \\
\hline .75 & $E . \vec{B}$ & 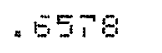 & 1.54 & .51 & .695 \\
\hline .72 & 7.01 & .8215 & 1.EE & .54 & $.851+1$ \\
\hline .74 & $\vec{i}=\overrightarrow{3}$ & .7064 & 1.68 & .03 & .6029 \\
\hline.$T B$ & 7.53 & $.73=3$ & 1.70 & $.9=$ & .9020 \\
\hline .73 & T.E1 & .7590 & 1. & .01 & .01913 \\
\hline .50 & 0.09 & $-8 \overrightarrow{2}$ & 1.74 &.$\sqrt{11}$ & .0609 \\
\hline .8 & 5.37 & .8135 & 1. TE & .01 & .51960 \\
\hline .84 & $5 \times 5$ & .3407 & $1 . ; \mathrm{G}$ & .09 & .0504 \\
\hline $.8 E$ & 5.92 & .8570 & 1.80 & .001 & .0062 \\
\hline .88 & 9.18 & .0919 & 1.92 & .95 & .0002 \\
\hline .96 & $\because .41$ & $. \quad=14 \pi$ & 1.94 & .60 & .0001 \\
\hline .92 & G. & .9347 & $1 . E 5$ & .06 & .0001 \\
\hline
\end{tabular}


Table E.4. Values for the deposited energy, $S_{D}(x)$, and the displacements per atom, dpa $(x)$, as a function of the distance from the target surface, $x . k=0.108$

\begin{tabular}{|c|c|c|c|c|c|}
\hline $\begin{array}{c}x \\
(\mu \mathrm{m})\end{array}$ & $\operatorname{dpa}(\mathrm{x})$ & $\begin{array}{c}\mathrm{S}_{\mathrm{D}}(\mathrm{x}) \\
(\mathrm{MeV} / \\
\mu \mathrm{m} \text {-ion) }\end{array}$ & $\begin{array}{c}x \\
(\mu \mathrm{m})\end{array}$ & $\mathrm{dpa}(\mathrm{x})$ & $\begin{array}{c}\mathrm{S}_{\mathrm{D}}(\mathrm{x}) \\
(\mathrm{MeV} / \\
\mu \mathrm{m} \text {-ion) }\end{array}$ \\
\hline 0.05 & 3.69 & × 290 & .94 & $\because \leq 4$ & .9930 \\
\hline .02 & 3.13 & $.36=7$ & $=98$ & 4.44 & .9128 \\
\hline .64 & 3.18 & .3075 & .98 & $\exists=E$ & .9503 \\
\hline $.9 E$ & 3.2 & .3124 & 1.65 & 9.7 & .9449 \\
\hline .09 & 3.20 & .3174 & $1.0=$ & 9.99 & ×5E \\
\hline .10 & 3.34 & ה & 1.04 & 9.95 & .953 \\
\hline .12 & 3.39 & .3280 & $1.0 E$ & 10.610 & .9559 \\
\hline .14 & 3.45 & .3356 & 1.08 & 7.45 & .9554 \\
\hline .16 & 3.51 & .3433 & 1.10 & 9.95 & .3590 \\
\hline .18 & 3.57 & $.345=$ & 1.12 & 9.79 & .9470 \\
\hline .20 & $3 . E$ & .3513 & 1.14 & $9 . \pm 1$ & . \\
\hline$\therefore=$ & 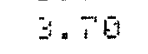 & .3575 & $1.1 E$ & 9.3 & .9654 \\
\hline .24 & $3.7 \overrightarrow{1}$ & $.58+1$ & 1.18 & 9.69 & $.877 E$ \\
\hline .20 & 3.83 & .3018 & $1 .=0$ & 6.75 & .8445 \\
\hline .28 & 3.91 & 4728 & 1. $\Xi 2$ & 5.34 & .9059 \\
\hline .30 & 3.96 & .3550 & 1. $=4$ & 7.41 & $\because 553$ \\
\hline .32 & $4.0 E$ & .3925 & 1. $2 \bar{E}$ & $i=45$ & .7207 \\
\hline .34 & 4.14 & .4003 & 1.26 & $E .9 E$ & . \\
\hline $.3 E$ & $4.2=$ & .4084 & 1.30 & 5.44 & $B=20$ \\
\hline .38 & 4.31 & .4188 & $1.3 z$ & 5.95 & $.5-28$ \\
\hline .40 & $4 \times 45$ & $.425 E$ & 1.34 & $5.5=$ & .5340 \\
\hline$=4 E$ & 4.56 & .4348 & 1. $3 E$ & 4.40 & $48=7$ \\
\hline .44 & 4. E. & $.4+43$ & 1.38 & 4.47 & .4355 \\
\hline $.4 E$ & 4.70 & $.45+3$ & 1.40 & 3.97 & .3939 \\
\hline .45 & 4.91 & .4548 & $1.4=$ & 2.45 & .3375 \\
\hline .50 & 4.92 & .4757 & 1.44 & 3.95 & $=2945$ \\
\hline .52 & 5.04 & $.487 \equiv$ & $1.4 E$ & $E . E Z$ & $.25+5$ \\
\hline .54 & $5.1 E$ & .4993 & 1.48 & $\Xi .55$ & .179 \\
\hline $.5 E$ & 5.30 & .5150 & 1.50 & 1.91 & .1845 \\
\hline .58 & 5.45 & .5254 & 1.52 & $1 . E 1$ & .1553 \\
\hline.$E 0$ & 5.50 & .5395 & 1.54 & 1.54 & .1293 \\
\hline . Ë & 5.73 & .5544 & $1.5 E$ & 1.10 & $.15 \mathrm{~T}$ \\
\hline .54 & 5.70 & .5701 & 1.59 & .70 & $.59 \cdot 1$ \\
\hline$E E$ & $E . \overline{6}$ & .585. & 1.50 & .73 & .6794 \\
\hline .56 & $E= \pm 5$ & $\cdot 654=$ & 1.52 & .58 & .9564 \\
\hline .70 & $0 .+4$ & $.5 \pm 27$ & 1.5 .4 & $.4 E$ & $.94+7$ \\
\hline .72 & $E . E 4$ & $.64 \geq 1$ & 1.56 & $.3 E$ & .0351 \\
\hline. $\overrightarrow{1} 4$ & $E .35$ & $\cdot E E 24$ & 1.5 & .78 & . \\
\hline $.7 E$ & $\therefore .95$ & $.583 T$ & 1.70 & $\Xi E$ & $.9 \Xi 19$ \\
\hline $.7 \xi$ & $\overrightarrow{7} .30$ & .7059 & $1 . \overrightarrow{1} 4$ & .13 & $.01=1$ \\
\hline .86 & ‥54 & .7280 & 1.78 & .97 & .9057 \\
\hline $.9=$ & 7.75 & $.75 \pm 4$ & $1.8=$ & .84 & $.515=5$ \\
\hline .84 & 6.03 & ・・E4 & 1.56 & .52 & .9018 \\
\hline.$E E$ & 8.28 & .5607 & 1.95 & .011 & .0609 \\
\hline .80 & 2.53 & .8248 & 1.94 & .05 & .0004 \\
\hline .95 & 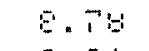 & .8485 & 1.95 & .051 & $.650=$ \\
\hline . $9 コ$ & 9.01 & .914 & & & \\
\hline
\end{tabular}




\section{APPENDIX F}

\section{CALCULATION OF THE CRITICAL CAVITY RADIUS}

The growth rate of a cavity of radius $r_{c}$ is determined by the net flux of vacancies to the cavity. Schematically, the equation for the growth rate, $\mathrm{dr}_{\mathrm{c}} / \mathrm{dt}$, is given by:

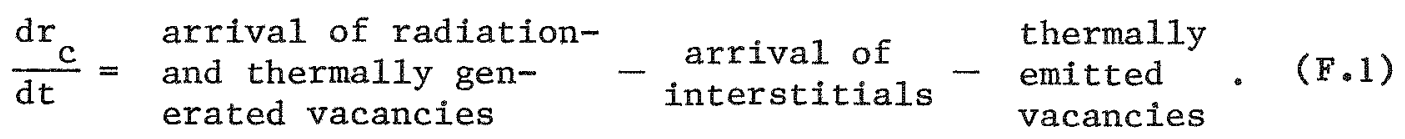

For a cavity radius above the "critical" cavity radius, $r_{\text {crit }}$, the cavity will grow by vacancy absorption, i.e., bias-driven growth. Below $r_{\text {crit, }}$ a cavity will shrink if only bias-driven growth is considered. Gas-driven growth could still occur if the cavities are considered to be equilibrium bubbles. At $r_{\text {crit, }}$ the cavities would neither grow nor shrink, i.e.,

$$
\left.\frac{\mathrm{dr}}{\mathrm{dt}}\right)_{r_{\text {crit }}}=0
$$

In order to evaluate $\mathrm{r}_{\text {crit, }}$ a rate theory approach has been employed. The relationships used to describe the point defect kinetics have been developed by others (see, for example, the review by Mansur [142]). The equations necessary to evaluate $r_{\text {crit }}$ are outlined below. Papers of Mansur [21,142] and Hayns and Mansur [141] were the major references used for this section.

\section{F.1 Continuity Equations and Point Defect Sink Strengths}

After an initial transient and away from the free specimen surfaces, point defect conservation can be described by the equations

$$
G_{V}-R C_{V} C_{1}-R_{v} C_{v}=0,
$$

and 


$$
G_{1}-R C_{v} C_{1}-K_{1} C_{1}=0
$$

where the subscripts denote vacancies and interstitials, the $G^{\prime} s$ are the point defect generation rates per unit volume, $R$ is the coefficient of recombination, the $C^{\prime} s$ are the concentrations of point defects per unit volume, and the $K^{\prime} s$ are the loss rates of point defects to distributed sinks.

In equation form,

$$
\begin{gathered}
G_{i}=\operatorname{Gf}\left(1+\varepsilon_{i}\right), \\
G_{v}=\operatorname{Gf}\left(1-\varepsilon_{v}\right)+G_{T}, \\
R=4 \pi r_{r}\left(D_{i}+D_{v}\right), \\
K_{v}=D_{v} S_{v}, \\
K_{i}=D_{i} S_{i},
\end{gathered}
$$

where $G=$ atomic generation rate per unit volume,

$f=$ ratio of the generation rate of defects surviving the cascade to the displacement rate,

$\varepsilon_{i}=$ the additional interstitial generation rate due to the selfions injected by the bombarding ion beam,

$\varepsilon_{\mathrm{v}}=$ the fraction of the vacancies retained in vacancy loops,

$\mathrm{G}_{\mathrm{T}}=$ the vacancy generation rate by thermal emissions from sinks, Eq. (F.10),

$I_{r}=$ the radius of recombination,

$\mathrm{D}_{\mathrm{i}}=$ the interstitial diffusion coefficient, Eq. (F.14),

$D_{v}=$ the vacancy diffusion coefficient, Eq. (F.13),

$S_{\mathrm{V}}=$ the total sink strength for vacancies, Eq. (F.19),

$S_{i}=$ the total sink strength for interstitials, Eq. (F.20).

$G_{T}$ is further defined by the equation

$$
G_{T}=D_{v} C_{v}^{e} \sum_{j} \xi^{j} S_{v}^{j},
$$


where $C_{v}^{e}=$ the bulk thermal vacancy concentration per unit volume,

$$
=\Omega^{-1} \exp \left(S_{V}^{f} / k_{B}\right) \exp \left(-E_{V}^{f} / k_{B} T\right) \text {, }
$$

$\Omega=$ the atomic volume,

$\mathrm{s}_{\mathrm{v}}^{f}=$ entropy of vacancy formation,

$\mathrm{E}_{\mathrm{V}}^{\mathrm{f}}=$ energy of vacancy formation,

$\mathrm{k}_{\mathrm{B}}=$ Boltzmann's constant,

$\mathrm{T}=$ the absolute temperature,

$\mathrm{S}_{\mathrm{v}}^{j}=$ the sink strength of sink type $j$ for vacancies, and

$\xi^{j}=$ the ratio of thermal vacancies at sink type $j$ to that in the bulk.

For cavities with radius $r_{c}$,

$$
\xi^{c}=\exp \left[\left(\frac{2 \gamma}{r_{c}}-\mathrm{P}\right) \Omega / k_{B} T\right] .
$$

where $\gamma=$ the surface tension at the cavity-matrix interface, and

$P=$ the pressure of the gas within the cavity.

For dislocations,

$$
\xi^{\mathrm{d}}=1
$$

The vacancy and interstitial diffusion coefficients are given by the expressions:

$$
D_{\mathrm{v}}=D_{\mathrm{v}}^{0} \exp \left(-\mathrm{E}_{\mathrm{v}}^{\mathrm{m}} / k_{\mathrm{B}} \mathrm{T}\right)
$$

and

$$
D_{i}=D_{i}^{0} \exp \left(-E_{i}^{m} / k_{B} T\right)
$$

where $D_{v}^{0}$ and $D_{i}^{0}$ are constants, $E_{v}^{m}=$ the vacancy migration energy, and $E_{i}^{\mathrm{m}}=$ the interstitial migration energy. 
Finally, the sink strengths of cavities for vacancies, $\mathrm{S}_{\mathrm{v}}^{\mathrm{c}}$, and for interstitials, $S_{i}^{c}$, can be expressed as

$$
S_{v}^{c}=4 \pi r_{c} N_{c} Z_{v}^{c}
$$

and

$$
s_{i}^{c}=4 \pi r_{c} N_{c} z_{i}^{c}
$$

where $\mathrm{N}_{\mathrm{c}}=$ the concentration of cavities and $\mathrm{Z}_{i, c}^{c}=$ the cavity capture efficiencies.

The sink strengths of dislocations for vacancies, $\mathrm{S}_{\mathrm{V}}^{\mathrm{d}}$, and for interstitials $\mathrm{S}_{i}^{\mathrm{d}}$, can be expressed as

$$
\begin{aligned}
& S_{v}^{d}=Z_{v}^{d} L, \quad \text { and } \\
& S_{i}^{d}=Z_{i}^{d} L,
\end{aligned}
$$

where $\mathrm{L}=$ the dislocation density, and $\mathrm{z}_{\mathrm{i}, \mathrm{v}}^{\mathrm{d}}=$ the dislocation capture efficiencies. Therefore, for a damage distribution containing only cavities and dislocations,

$$
\begin{aligned}
& S_{v}=S_{v}^{c}+S_{v}^{d}=4 \pi r_{c} N_{c} Z_{v}^{c}+z_{v}^{d} L \text {, and } \\
& S_{i}=S_{i}^{c}+S_{i}^{d}=4 \pi r_{c} N_{c} Z_{i}^{c}+z_{i}^{d} L \text {. }
\end{aligned}
$$

\section{F.2 Cavity Growth Equations}

The growth rate of a cavity, expressed previously in schematic form (Eq. F.1), can be written in analytic form as

$$
\frac{d r c}{d t}=\frac{\Omega}{r_{c}}\left\{z_{v}^{c} D_{v} c_{v}-z_{i}^{c} D_{i} c_{i}-z_{v}^{c} D_{v} c_{v}^{e}\left(r_{c}\right)\right\}
$$


where

$C_{v}^{e}\left(r_{C}\right)=C_{v}^{e} \exp \left[-\left(P-\frac{2 \gamma}{r_{c}}\right) \Omega / k_{B} T\right]$

= the thermal vacancy concentration near a cavity of radius $r_{c}$.

If Eq. (F.3) and Eq. $(F .4)$ are solved simultaneously, an expression for $\mathrm{C}_{\mathrm{V}}$ which is independent of $\mathrm{C}_{i}$ and an expression for $\mathrm{C}_{\mathrm{i}}$ which is independent of $\mathrm{C}_{\mathrm{v}}$ can be obtained. If these expressions are substituted into Eq. (F.21), the following equation can be obtained:

$$
\begin{aligned}
\frac{d r}{d t}=\frac{\Omega_{l}}{r_{c}}\left\{\frac{Z_{v}^{C} D_{v}}{2 R K_{v}}\left[K_{i} K_{v}+R\left(G_{i}-G_{v}\right)\right] \times\left[\left(1+\frac{4 R G_{v} K_{i} K_{v}}{\left[K_{i} K_{v}+R\left(G_{i}-G_{v}\right)^{2}\right]}\right)^{1 / 2}-1\right]\right. \\
-\frac{Z_{i}^{C} D_{i}}{2 R K_{i}}\left[K_{i} K_{v}+R\left(G_{v}-G_{i}\right)\right] \times\left[\left(1+\frac{4 R G_{i} K_{i} K_{v}}{\left[K_{i} K_{v}+R\left(G_{v}-G_{i}\right)\right]^{2}}\right)^{1 / 2}-1\right] \\
-Z_{v}^{C} D_{v} C_{v}\left(r_{c}\right)
\end{aligned}
$$

At $r_{c}=r_{c r i t}, E q .(F .23)$ equals zero as discussed earlier. To solve for an exact value for $r_{c r i t}$, an iterative approach is required. However, $r_{c r i t}$ lies between the values for $r_{c}$ which yield $\mathrm{dr}_{c} / \mathrm{dt}<0$ and those which yield $\mathrm{dr}_{\mathrm{c}} / \mathrm{dt}>0$. Therefore, an approximate value for $\mathbf{r}_{\text {crit }}$ can be obtained by solving $\mathrm{Eq} .(\mathbf{F} .23)$ for a series of values for $r_{c}$ with a small interval between each $r_{c}$. The value for $r_{c r i t}$ would lie between the last value for $r_{c}$ for which $d r_{c} / d t$ was less than zero and the first value for $r_{c}$ for which $\mathrm{dr}_{c} / \mathrm{dt}$ was greater than zero. This approach was used in the current investigation. By selecting a small interval between the values for $r_{c}(e . g ., 0.1$ to $1 \mathrm{~nm}$ ), a close approximation to the value for $r_{\text {crit }}$ was obtained. Considering the inaccuracy of many of the values used for the parameters (e.g., the capture efficiencies, the interstitial diffusion constant, etc. are not well known for iron - and these were used for $\mathrm{Fe}-10 \% \mathrm{Cr}$ ), this approximate value of $r_{\text {crit }}$ was sufficient for the purpose of this study. 
The BASIC computer program shown in Figure F.l (written for the Hewlett Packard 9845A Desk Top Computer) calculates $\mathrm{dr} / \mathrm{dt}$ for the ionirradiation experiment using $\mathrm{Fe}-10 \% \mathrm{Cr}$. The following values were used for the required parameters:

$$
\begin{aligned}
& \Omega=1.18 \times 10^{-29} \mathrm{~m}^{3} \text { atom }^{-1}, \\
& \mathrm{k}_{\mathrm{B}}=8.61 \times 10^{-5} \mathrm{eV}-\mathrm{K}^{-1} \text {, } \\
& \gamma=2 \mathrm{~J}-\mathrm{m}^{-2} \text {, } \\
& \mathrm{G}=6.8 \times 10^{26} \mathrm{~m}^{-3}-\mathrm{s}^{-1}\left(8 \times 10^{-3}{\left.\mathrm{dpa}-\mathrm{s}^{-1}\right)}^{-1}\right. \\
& f=0.2 \text {, } \\
& \varepsilon_{i}=0 \text {, } \\
& \varepsilon_{\mathrm{v}}=0 \text {, } \\
& r_{\mathrm{r}}=0.344 \mathrm{~nm} \text {, } \\
& D_{\mathrm{v}}^{0}=1.9 \times 10^{-4} \mathrm{~m}^{2}-\mathrm{s}^{-1} \text {, } \\
& D_{i}^{0} \sim 10^{-6} \mathrm{~m}^{2}-\mathrm{s}^{-1} \text {, } \\
& \mathrm{S}_{\mathrm{v}}^{\mathrm{f}}=0 \text {, } \\
& \mathrm{E}_{\mathrm{V}}^{\mathrm{m}}=1 \mathrm{eV} \text {, } \\
& \mathrm{E}_{\mathrm{V}}^{\mathrm{f}}=1.6 \mathrm{eV} \text {, } \\
& \mathrm{E}_{\mathrm{i}}^{\mathrm{m}}=0.26 \mathrm{eV} \text {, } \\
& z_{i}^{d}=1.03 \text {, } \\
& z_{i}^{c}=1 \text {, } \\
& z_{v}^{c}=1 \text {, } \\
& \mathrm{z}_{\mathrm{v}}^{\mathrm{d}}=1 \text {. }
\end{aligned}
$$

The pressure within the cavity was set equal to a fraction of the equilibrium bubble pressure, i.e., $P=P^{*} P_{e q}$, where $P_{e q}=2 \gamma / r_{c}$. For example, for $P^{\prime}=0.9$, the pressure within the cavity was $90 \%$ of $P_{\text {eq }}$. Values for $P^{\prime}$ of $0.1,0.5$, and 0.9 were used in these calculations. Three series of calculations were performed. First, the dislocation density and cavity concentration observed for the specimen irradiated to $30 \mathrm{dpa}$ at $850 \mathrm{~K}$,

$$
L=3.2 \times 10^{13} \mathrm{~m}^{-2},
$$

and

$$
\mathrm{N}_{\mathrm{c}}=2.0 \times 10^{20} \mathrm{~m}^{-3}
$$




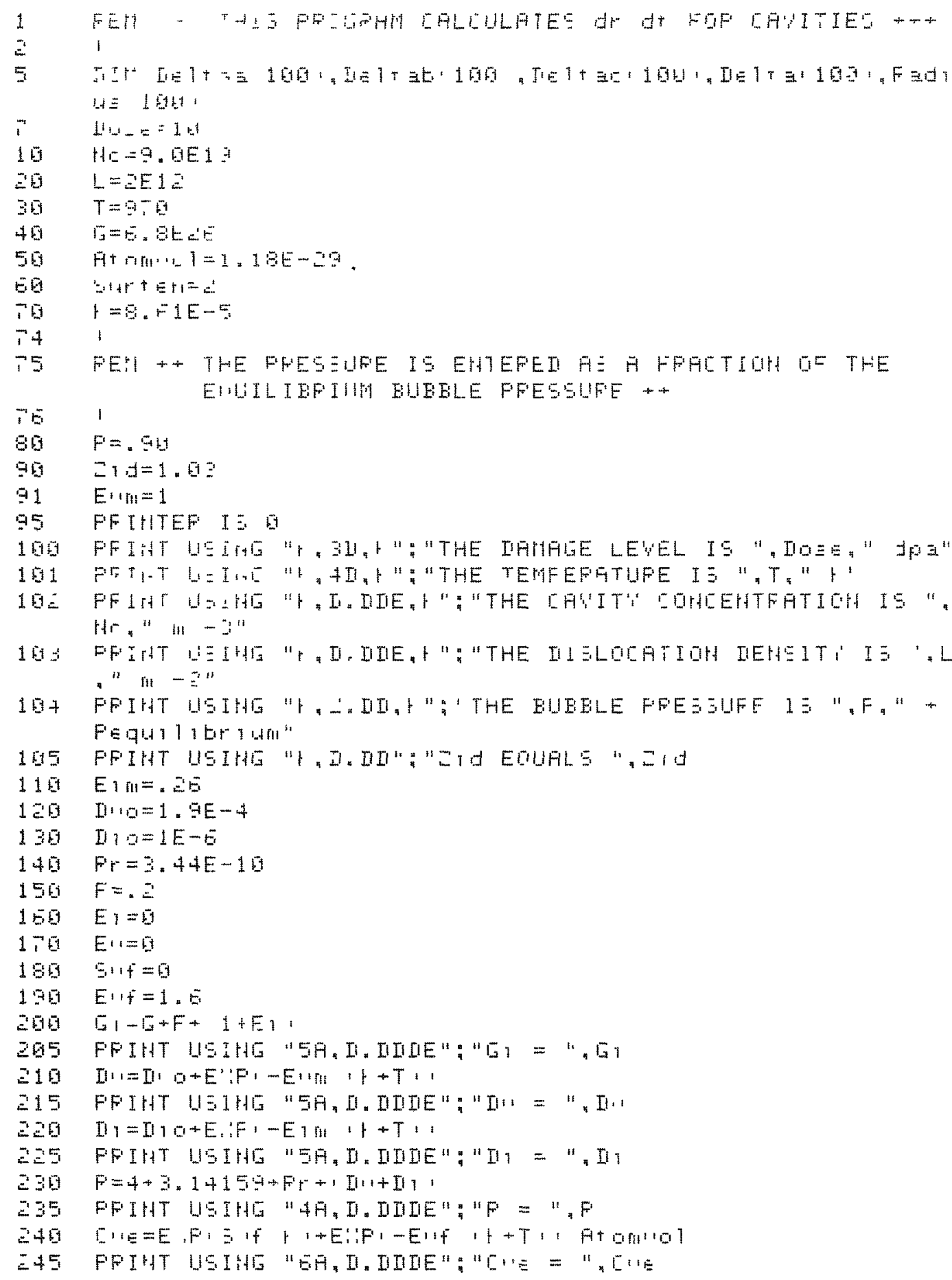

Figure F.1. BASIC computer program (HP 9845A) which calculates dr/dt for cavities. 


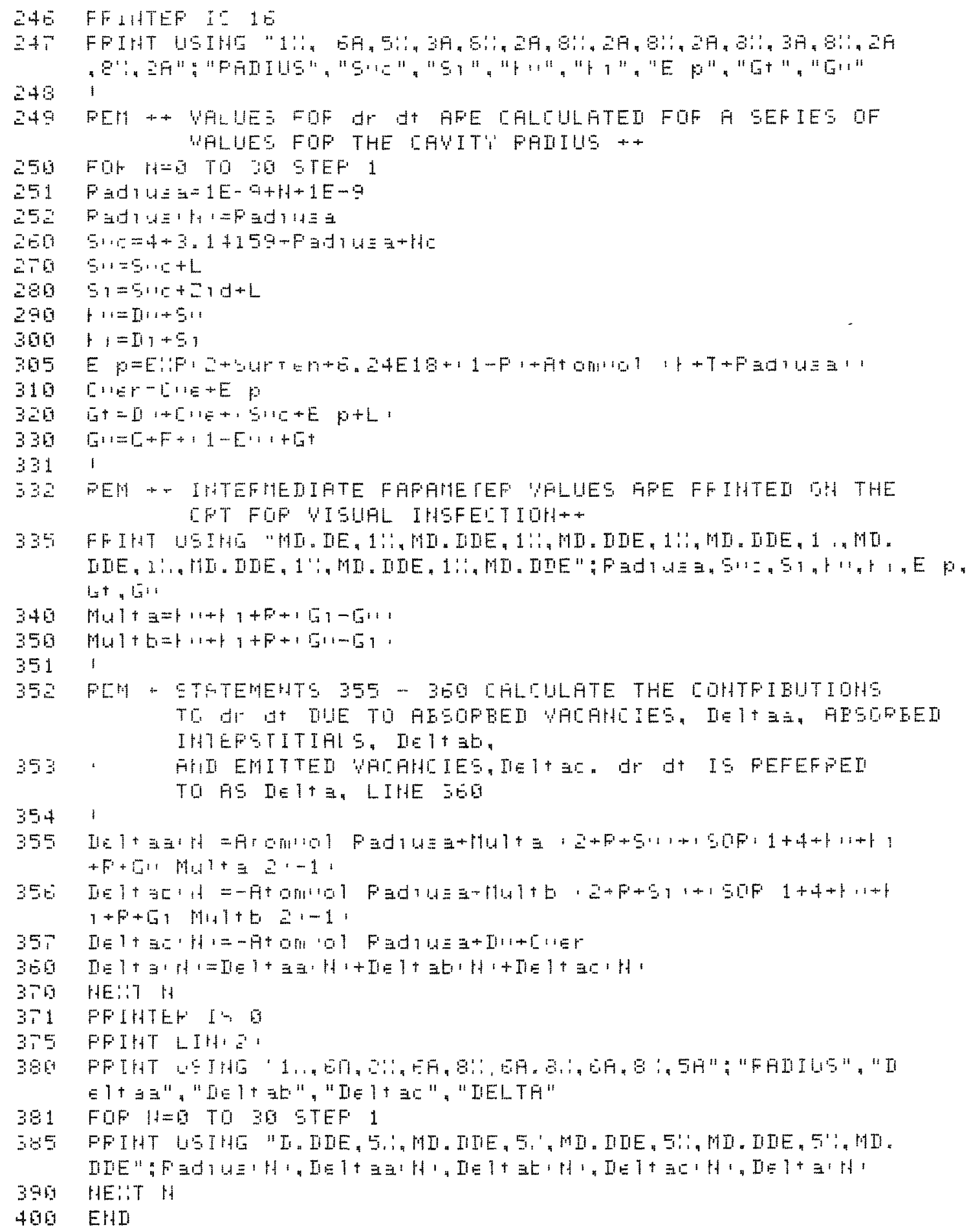


were used. The calculated values of $\mathrm{dr}_{\mathrm{c}} / \mathrm{dt}$ are compiled in Table $\mathrm{F} \cdot 1$ $\left(P^{\prime}=0.1\right)$, Table F.2 $\left(P^{\prime}=0.5\right)$, and Table F.3 $\left(P^{\prime}=0.9\right)$. In these tab1es,

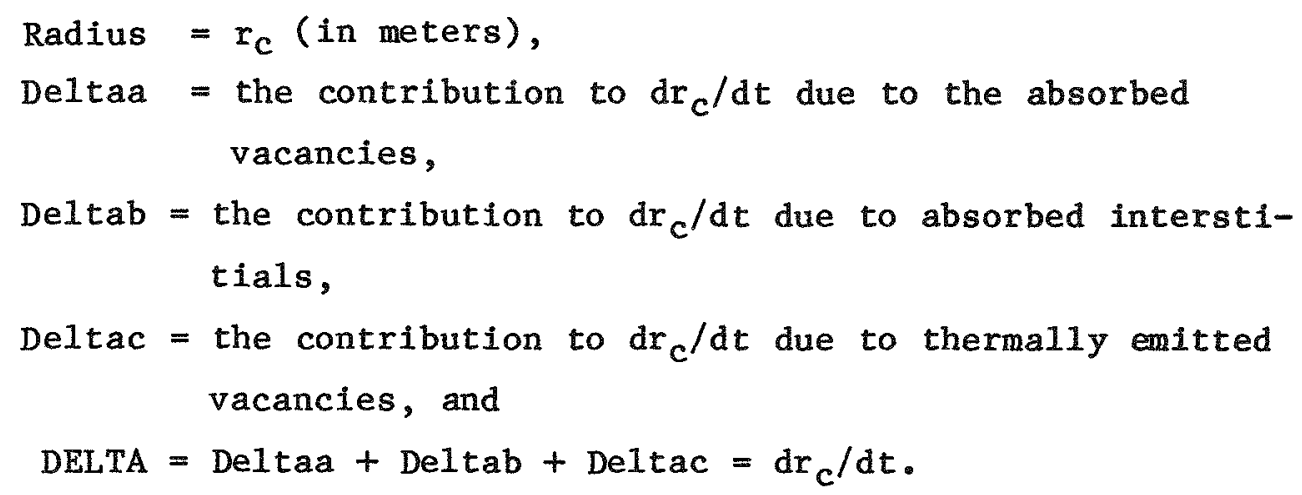

In the second set of calculations, the dislocation density and cavity concentration observed for the specimen irradiated to $10 \mathrm{dpa}$ at $850 \mathrm{~K}$,

$$
\mathrm{L}=8 \times 10^{13} \mathrm{~m}^{-2}
$$

and

$$
\mathrm{N}_{\mathrm{C}}=2.9 \times 10^{19} \mathrm{~m}^{-3}
$$

were used. The calculated values of $\mathrm{dr}_{\mathrm{c}} / \mathrm{dt}$ are compiled in Table $\mathrm{F}_{0} 4$ $\left(P^{\prime}=0.1\right)$, Table F.5 $\left(P^{\prime}=0.5\right)$, and Table F.6 $\left(P^{\prime}=0.9\right)$. Finally, in the third set of calculations, the dislocation density and cavity concentration observed for the specimen irradiated to $10 \mathrm{dpa}$ at $970 \mathrm{~K}$,

$$
L=2 \times 10^{12} \mathrm{~m}^{-2},
$$

and

$$
\mathrm{N}_{c}=9 \times 10^{19} \mathrm{~m}^{-3},
$$

were used. The calculated values of $\mathrm{dr}_{\mathrm{c}} / \mathrm{dt}$ are compiled in Table F.7, $\left(P^{\prime}=0.1\right)$, Table F.8 $\left(P^{-}=0.5\right)$, and Table F.9 $\left(P^{-}=0.9\right)$. The values for $r_{\text {crit }}$ obtained for these calculations are summarized in Table F.10. The value listed for $r_{c r i t}$ is the value for $r_{c}$ for which $d r_{c} / d t$ was closest to zero. 
Table F.1. Calculated values for $\mathrm{dr}_{\mathrm{c}} / \mathrm{dt}$ (DELTA) for cavity radii of 0.1 to $3.1 \mathrm{~mm}$. $30 \mathrm{dpa}, 850 \mathrm{~K}, \mathrm{P}=0.1 \mathrm{P}$ eq, $\mathrm{r}_{\text {crit }}=1.6 \mathrm{~nm}$

THE DAMATE LEVEL IS 30 GHE

THE TENFEFHTUFE IS $55 \mathrm{~S}$

THE IHWIT: EOHEEHTFATIOH IS E. BSE+EG M -3

THE DISLOCATIOH IIEHSITi IS $3.20 E+13$ ri -

THE EUEELE FFESEUPE IS 0.10 - FEqL1116R1um

ZI EDUALS 1.03

$E_{1}=1.3 E 0 E+E$

In: = $2.211 E-10$

$D_{1}=\Xi .5 \pm 5 E-65$

$F=1.245 E-1 E$

$C U=E . T 13 E+19$

FHIIUS

1. $010 E-10$

$2.60 E-10$

$3.00 E-10$

4. $00 E-10$

5. $90 E-10$

E. $00 E-10$

7. $50 E-10$

9. $19 E-10$

$9.90 E-19$

1. $965-69$

1. $10 E-99$

1. $=0 E-19$

1. $39 E-99$

1. $\$ 6 E-65$

1. $56 E-69$

1. $6.6 E-99$

1. $.9 E-59$

1. $90 E-99$

1. $30 E-09$

a. $06 E-99$

$2.10 E-69$

‥

$\therefore 30 E-69$

2. $40 E-69$

$2.50 E-99$

2. $50 E-69$

2. $76 E-99$

$\therefore$. $8 \mathrm{GE}-69$

2. 90E- 99

3. $96 E-59$

$3.10 E-69$
IIE

0. $00 E+50$

$-1.00 E-07$

$-\neg .7 D E-B D$

․ $10 E-9 a$

5. $09 E-09$

$3.95 E-138$

$3.3 \mathrm{E}-199$

$\because 9 B-58$

$\therefore 5+E-09$

I. IE $-\mathrm{G}$

$\therefore$. $15 \mathrm{E}-\mathrm{Bg}$

1. $83 E-18$

1. $73 E-09$

1. $E G E-6 \theta$

1. $49 E-69$

1. $39 E-69$

1. $31 E-6 G$

1. $23 E-59$

1. $1 E E-E S$

1. $19 E-09$

1. $95 E-19$

$9.99 E-69$

- $53 E-69$

‥ $11 E-99$

Q. $\div E-69$

9. $37 E-99$

6. $04 E-09$

$\therefore=73 E-69$

$\vec{P} .45 E-09$

ᄀ. $18 E-59$

E. $95-09$
IIE $1+\equiv \mathrm{E}$

0. $0 \mathrm{GE}+0 \mathrm{~g}$

$-5.6+5-11$

$-1.01 E-98$

$-4.23 E-6 \theta$

$-4.18 E-05$

$-3.51 E-05$

$-3.12 E-99$

$-2.73 E-98$

$-\because .4 E-09$

$-2.16 E-6 E$

$-1.96 E-59$

$-1.91 E-98$

$-1.5 E-58$

$-1.55 E-09$

$-1.4+E-68$

$-1.35 E-68$

-1.

$-1.19 E-19$

$-1.13 E-68$

$-1.07 E-68$

$-1.9 E E-08$

$-9.59 E-09$

$-9.5+E-69$

$-8.84 E-95$

$-9.47 E-69$

$-8.1 \pm E-59$

$-7.81 E-09$

$-7.51 E-69$

$-\vec{P} .3 E-99$

- $E$. 9E- 09

$-B .7 E-69$
$I=1+\equiv 5$

$-3.90 E+B E$

$-2.5 E-19$

$-4.13 E-55$

$-1.51 E-9 E$

$-1.98 E-67$

$-4.94 E-18$

$-1.79 E-G 9$

$-9.18 E-99$

$-4 .+1 E-69$

$-5.5 E-59$

$-1.7 \equiv E-69$

$-1.21 E-19$

$-3.63 E-10$

$-E . T E E-10$

$-5.29 E-19$

$-4.25 E-10$

$-3.55 E-10$

$-5.94 E-10$

$-2.51 E-10$

$-=1 E E-10$

$-1.89 E-19$

$-1.5 \cdot E-10$

$-1.49 E-10$

$-1.35 E-10$

$-1 .=1 E-10$

$-1.10 E-10$

$-1.00 E-10$

$-9.21 E-11$

$-8.51 E-11$

$-7.95-11$

$-7.34 E-11$
IEE TH $-3.35+3 E$ $-5.5 E-B=$ $-4.1+E-65$

$-1.4 .7 E-6 E$

$-1.8 .5-61$

$-4.55 E-09$

$-1.5=-09$

$-5.5+E-19$

$-3.35 E-19$

$-1.7 B E-09$

$-9.85 E-10$

$-5.55 E-10$

$-2.9 E E-10$

$-1.41 E-10$

$-4,2, E-11$

2. $09 E-11$

E. $27 E-11$

9. $9+E-11$

1. $99 E-10$

1. $=1 E-10$

1. $E E E-10$

1. $: E E-1 \mathrm{E}$

1. $35 E-15$

1. $35 E-18$

1. $35 E-10$

1. $3 E-10$

1. $3 E-10$

1. $Z \exists E-10$

1. $27 E-10$

1. $24 E-10$

1. $: 1 E-10$ 
Table F.2. Calculated values for $\mathrm{dr}_{\mathrm{c}} / \mathrm{dt}$ (DELTA) for cavity radii of

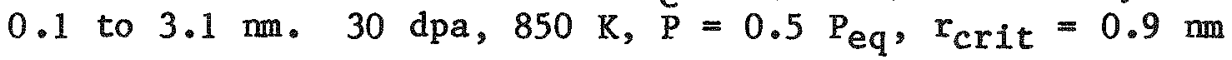

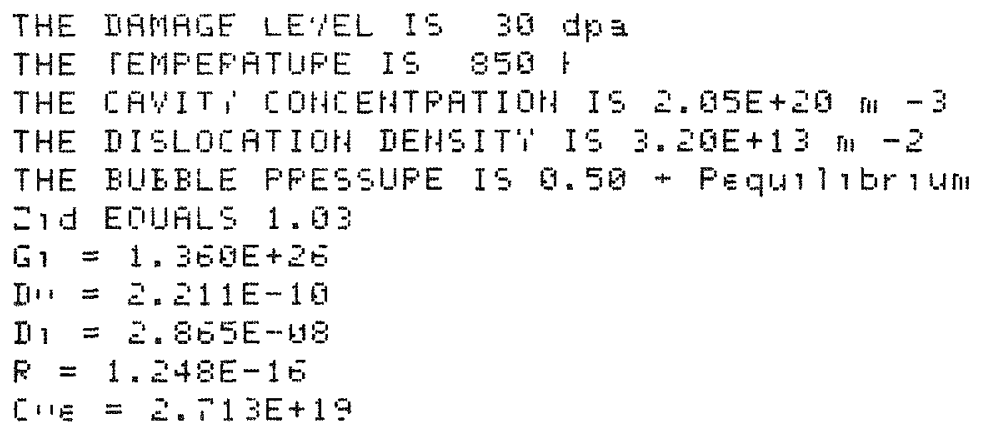

\begin{tabular}{|c|c|c|c|c|}
\hline FFIIUS & $I E I+\Xi \equiv$ & $D \equiv 1+\equiv \mathrm{L}$ & IE $1+ \pm 0$ & IELTA \\
\hline 1. $.5 B E-19$ & $-1.99 E-67$ & $-3.10 E-11$ & $-3.8 \mathrm{E} E-01$ & $-3.85 E-61$ \\
\hline$\therefore 80 E-19$ & $-1.90 E-07$ & $-7.7 \geq E-09$ & $-9.25 E-05$ & $-9.54 E-60$ \\
\hline $3.60 E-15$ & ᄀ. $9 E E-08$ & $\neg ロ コ E-68$ & $-1.93 E-67$ & $-1.8 E E-9 \vec{r}$ \\
\hline $4.50 E-10$ & $5.79 E-95$ & $-5.52 E-68$ & $-2.71 E-58$ & $-\therefore+4+E-68$ \\
\hline $5.60 E-10$ & $4.59 E-59$ & $-4.4 \Sigma E-98$ & $-7.9 \Omega E-09$ & $-5 .=9 E-99$ \\
\hline $5.00 E-10$ & $3.81 E-98$ & $-3.68 E-98$ & $-3.3-6-69$ & $-\Xi .95 E-69$ \\
\hline $7.60 E-10$ & $\exists=\Xi E E-69$ & $-3.15 E-69$ & $-1.79 E-09$ & $-7.65 E-16$ \\
\hline B. DOE-1D & $\because 84 E-18$ & $-2.75 E-98$ & $-1.09 E-69$ & $-1.76 E-10$ \\
\hline $9.50 E-15$ & $=52 E-19$ & $-a \cdot 4+E-65$ & $-7.35 E-10$ & $E \cdot G E E-11$ \\
\hline 1. $99 E-99$ & $\therefore \Xi E E-99$ & $-2.19 E-08$ & $-5.29 E-19$ & $1 . E+E-18$ \\
\hline 1.10E- 19 & $=.05 E-98$ & $-1.99 E-08$ & $-4.01 E-10$ & $\because .3 B E-19$ \\
\hline 1. $: 0 E-09$ & 1. 3TE- 58 & $-1.8 \Xi E-03$ & $-3.15 E-10$ & $\Xi .5 E-10$ \\
\hline 1. $39 E-99$ & 1. $\rightarrow 3 E-19$ & $-1 . E, E-5 \theta$ & $-2.5 B E-10$ & $\Xi: \vec{B} E-1 B$ \\
\hline $1.40 E-89$ & 1. $56 E-6 \theta$ & $-1.55 E-68$ & $-\therefore 13 E-10$ & $2 . T E E-10$ \\
\hline $1.56 E-93$ & $1.49 E-98$ & $-1.4+4 E-08$ & $-1.80 E-10$ & $\because .8 E-10$ \\
\hline 1. $56 E-09$ & $1.39 \mathrm{E}-19 \mathrm{E}$ & $-1.35 E-69$ & $-1.5 \bar{E} E-10$ & $E . E 0 E-10$ \\
\hline 1. $\neg \overline{D E}-\overline{9}$ & $1.31 E-5 G$ & $-1.27 E-68$ & $-1.35 E-10$ & $=5 \pm E-16$ \\
\hline 1. $90 E-69$ & 1. $=3 E-19$ & $-1 .=9 E-98$ & $-1 . \Xi 0 E-10$ & $2.4 E-10$ \\
\hline $1.50 E-0 \%$ & $1.1 E E-15 E$ & $-1.13 E-68$ & $-1.9 F E-10$ & $2.35 E-19$ \\
\hline $2.09 E-99$ & 1. $15 E-99$ & $-1.0, E-00$ & $-9.58 E-11$ & $\Xi E+E-10$ \\
\hline$\Xi 10 E-09$ & $1.05 E-05$ & $-1.62 E-59$ & $-9.79 E-11$ & $\Xi 15 E-10$ \\
\hline$\therefore 2 \mathrm{GE}-0 \mathrm{G}$ & $9.98 E-09$ & $-9.69 E-09$ & $-8.03 E-11$ & $\therefore . B E E-10$ \\
\hline $2.30 E-09$ & $9.52 E-69$ & $-9.25 E-69$ & $-\vec{\neg} .39 E-11$ & $1.95 E-10$ \\
\hline$\because 4 B E-89$ & $9.16 E-09$ & $-8.84 E-99$ & $-E \cdot \theta \Xi E-11$ & 1.95E-19 \\
\hline $5.55 E-09$ & $9 . \vec{P} E-19$ & $-8.47 E-09$ & $-E .33 E-11$ & 1. $5 E E-15$ \\
\hline$=50 E-59$ & 3.36E-195 & $-6.13 E-69$ & $-5,95 E-11$ & 1.75E-10 \\
\hline$\because 70 E-09$ & $9.63 E-99$ & $-7.91 E-69$ & $-5.52 E-11$ & 1.5. $5 E-15$ \\
\hline$\therefore .90 E-99$ & $\neg .73 E-59$ & $-7.51 E-69$ & $-5.19 E-11$ & 1. $E 2 E-18$ \\
\hline $2.95 E-99$ & $\because .44 E-99$ & $-7.24 E-59$ & $-4.88 E-11$ & $1.5 E E-10$ \\
\hline $3.50 E-09$ & $7.18 E-69$ & $-8.95 E-09$ & $-4 . E 1 E-11$ & $1.51 E-10$ \\
\hline $3.10 E-00$ & $5.93 E-69$ & $-E . T+E-\overline{0}$ & $-4.5-E-11$ & 1. $45 E-18$ \\
\hline
\end{tabular}


Table F.3. Calculated values for $d r_{c} / d t$ (DELTA) for cavity radil of 0.1 to $3.1 \mathrm{~nm} .30 \mathrm{dpa}, 850 \mathrm{~K}, \mathrm{P}=0.9 \mathrm{P}_{\text {eq }}, \mathrm{r}_{\text {crit }}=0.2 \mathrm{~nm}$

THE IAMATE LEVEL IS 30 APa

THE TEMFEFHTUFE 19 S50

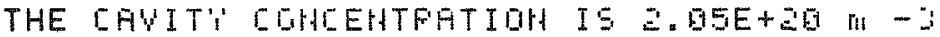

THE DISLDLATIOH IEHSIT' IS 3 EDE+13 $\mathrm{m}-\mathrm{z}$

THE BUEELE FFESEUFE IS 9.90 + FEqU1116"14m

ZIO EDUALS 1.93

$G_{1}=1.360 E+2 E$

Du $= \pm .211 E-10$

$n_{1}=2.8 E 5 E-193$

$R=1.248 E-1 E$

$C \cdot E=E .713 E+13$

\begin{tabular}{|c|c|}
\hline PHIIUS & $I E I+\equiv \equiv$ \\
\hline 1. $00 E-10$ & $\because 31 E-07$ \\
\hline $2.96 E-10$ & $1.15 E-9$ \\
\hline $3.60 E-10$ & $\because E E E-B$ \\
\hline $4.90 E-19$ & $5.7 \Xi E-0$ \\
\hline $5.06 E-10$ & $4.5, E-0$ \\
\hline 6. $90 E-10$ & $3.80 E-0$ \\
\hline $\overrightarrow{70.9 E-10}$ & $3.35 E-E$ \\
\hline $5.69 E-19$ & $2.94 E-5$ \\
\hline $9.90 E-19$ & $\therefore 5 \equiv E-E$ \\
\hline 1. $.0 E-69$ & $\therefore \Xi E E-G$ \\
\hline 1. $19 E-69$ & $2.65 E-5$ \\
\hline 1. $20 E-09$ & 1. BTE-E \\
\hline $1.36 E-69$ & $1.72 E-B$ \\
\hline $1.49 E-199$ & $1.69 E-6$ \\
\hline $1.56 E-19$ & $1.4 \% E-6$ \\
\hline 1. $661 E-69$ & $1.39 E-6$ \\
\hline $1.79 E-99$ & $1.31 E-D$ \\
\hline $1.56 E-69$ & $1 . \Xi 3 E-1$ \\
\hline $1.96 E-69$ & $1.1 E E-B$ \\
\hline $2.00 E-99$ & $1.10 E-E$ \\
\hline $2.10 E-69$ & $1.05 E-B$ \\
\hline $2.20 E-69$ & $9.97 E-1$ \\
\hline $2.30 E-99$ & $9.52 E-1$ \\
\hline $2.40 E-69$ & $19 E$ \\
\hline $2.59 E-69$ & $9 . \vec{r} 1 \mathrm{E}-$ \\
\hline $2.69 E-09$ & $8.36 E-1$ \\
\hline $2.75 E-59$ & $8.03 E-1$ \\
\hline $2.89 E-99$ & $\vec{i} \cdot \vec{i} 2 E-$ \\
\hline $2.96 E-69$ & $\vec{i} .44 E-1$ \\
\hline $3.06 E-199$ & $\vec{i}$ \\
\hline $3.10 E-51$ & \\
\hline
\end{tabular}

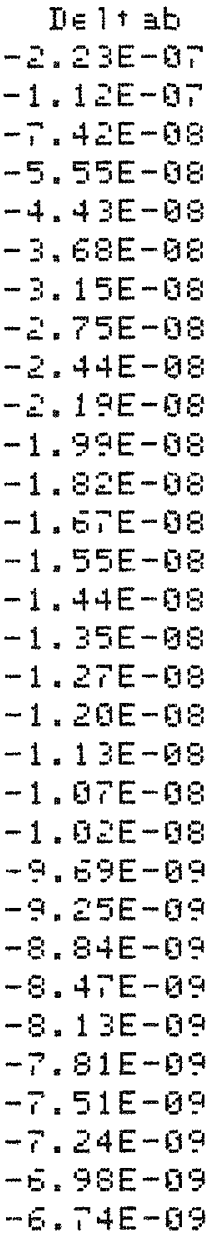

IlE $1+\equiv E$

$-3.9 E E-05$

$-5.5 E-69$

$-9.02 E-10$

$-4.94 E-10$

$-3.17 E-10$

$-\because 31 E-10$

$-1.56 E-10$

$-1.4 E E-10$

$-1.23 E-10$

$-1.9 E E-10$

$-9.2 E E-11$

$-8.5 E-11$

$-7.42 E-11$

-E. $\rightarrow 4 E-11$

-E. $17 E-11$

$-5.89 E-11$

-5. :TE-11

$-4.95 E-11$

$-4.6 \overline{G E}-11$

$-4.3 \exists E-11$

$-4.9 B E-11$

- 3. 8EE-11

- 3. E-E-11

$-3.49 E-11$

$-3.53 E-11$

$-3.1 \mathrm{E}-11$

$-3.5+E-11$

-

$-2.86 E-11$

$-E .79 E-11$

$-2 \cdot \overline{B g E}-11$
IIELTH

$-3.19 E-08$

1. $04 E-59$

1. $5: 2 E-89$

1. $\Xi E E-09$

1. $11 E-60$

$9.4 F-19$

E. $2 \mathrm{DE}-1 \mathrm{~B}$

$\because .1 E-19$

$E .41 E-10$

5. $T E E-10$

5. $22 E-19$

4. $T E E-10$

4. $I T E-10$

4. $3 E-10$

$3.7+E-16$

$3.4 \mathrm{EE}-10$

Z. $5 E-10$

$3.05 E-10$

$\therefore=8 E E-10$

$\therefore \overrightarrow{1} \mathrm{WE}-1 \mathrm{G}$

$\therefore 5 E-10$

$\therefore 4 E E-10$

$\therefore .9 E-10$

$\therefore 1 \mathrm{EE}-1 \mathrm{~g}$

$\therefore$. $97 E-15$

1. $9 E E-19$

1. $89 E-19$

$1.91 E-10$

1. $\vec{\prime} E-10$

1.EEE-10

1. $5 E-10$ 
Table F.4. Calculated values for $d_{c} / d t$ (DELTA) for cavity radil of 0.1 to $3.1 \mathrm{~mm} .10 \mathrm{dpa}, 850 \mathrm{~K}, \mathrm{P}=0.1 \mathrm{P}_{\text {eq }}, \mathrm{r}_{\text {crit }}=1.8 \mathrm{~mm}$

THE DAMALE LEUEL IS 10 AP

THE TEMFEFATUFE IS 859 .

THE CAUT'i COHCEHTFATIOH IS $2.96 E+19 \mathrm{~m}-3$

THE IISLOLATIOH IIEHSIT'i IS $8.09 E+13 \mathrm{~m}$ -

THE EUEELE FFESSUFE I' 0.10 + Fequilibrium

210 EOUHLS 1.03

$\square_{1}=1.3 E D E+2 E$

$I_{1} \cdot=211 E-10$

$D_{1}=2.8 E E-6 \theta$

$F=1.243 E-1 E$

LUE = $2 \cdot \vec{B} 13 E+1 \overrightarrow{0}$

\begin{tabular}{|c|c|c|c|c|}
\hline FAIIUS & $\mathrm{Il} \equiv 1+\Xi \Xi$ & $I E I+\equiv E$ & IE $1+\equiv 0$ & IIELTA \\
\hline 1. GDE-10 & $-5.11 E-6 ?$ & $0.96 E+00$ & $-3.90 E+0 E$ & $-3.9 \mathrm{GE}+\mathrm{BE}$ \\
\hline$\approx .90 E-10$ & $-\Xi .45 E-07$ & $-1.01 E-09$ & $-2.59 E-0=$ & $-\Xi .59 E-9 \Omega$ \\
\hline $9.95 E-1 D$ & $9 . E+E-69$ & $-4.65 E-65$ & $-4.13 E-65$ & $-4.13 E-05$ \\
\hline 4. $5 E-10$ & $4.05 E-195$ & $-3.5 E E-09$ & $-1.51 E-5 E$ & $-1.51 E-9 E$ \\
\hline $5.59 E-10$ & $=11 E-9 E$ & $-\Xi .9 B E-05$ & $-1.98 E-07$ & $-1.97 E-07$ \\
\hline E. BGE-ID & $=5 \cdot E-D B$ & $-2.47 E-09$ & $-4.94 E-69$ & $-4.84 E-58$ \\
\hline $7.00 E-10$ & $\therefore=0 \mathrm{E}-0 \mathrm{0}$ & $-\Xi \cdot 1 \Xi E-9 g$ & $-1 ., 9 E-09$ & $-1 . P 1 E-5 B$ \\
\hline 8. $85 E-10$ & $1.9=E-69$ & $-1.85 E-08$ & $-8.18 E-99$ & $-7.51 E-89$ \\
\hline $9.56 E-10$ & $1.7 \mathrm{GE}-\mathrm{Bg}$ & $-1.65 E-05$ & $-4.40 E-89$ & $-3.61 E-09$ \\
\hline 1. $50 E-09$ & 1. $53 \mathrm{E}-9 \mathrm{~g}$ & $-1.48 E-09$ & $-\Xi .5 E-99$ & $-\Xi 1 \Xi E-09$ \\
\hline $1.10 E-5$. & 1. $39 E-09$ & $-1.35 E-08$ & $-1.73 E-99$ & $-1.2 E E-199$ \\
\hline 1. $20 E-69$ & 1. $28 E-69$ & $-1 .=3 E-09$ & $-1.21 E-69$ & $-7.73 E-10$ \\
\hline 1.30E-9 & $1.15 E-05$ & $-1.14 E-08$ & $-6.93 E-10$ & $-4.84 E-10$ \\
\hline $1.46 E-69$ & 1. $19 \mathrm{E}-\mathrm{g9}$ & $-1.9 E E-09$ & $-6 . \neg 2 E-10$ & $-3.8 Z E-10$ \\
\hline $1.50 E-09$ & 1. $02 E-09$ & $-9.9 E E-59$ & $-5.28 E-10$ & $-1.95 E-15$ \\
\hline 1. $50 E-05$ & $\because 5 E E-9 \div$ & $-9.24 E-09$ & $-4.25 E-10$ & $-1.03 E-10$ \\
\hline $1.76 E-69$ & $9.00 E-99$ & $-8.70 E-09$ & $-3.56 E-10$ & $-4 . F E E-11$ \\
\hline 1. $8 \mathrm{BE}-0 \mathrm{G}$ & $9.56 E-09$ & $-8.21 E-09$ & $-2.94 E-10$ & $-8.38 E-12$ \\
\hline $1.96 E-09$ & $8.05 E-99$ & $-7.78 E-99$ & $-2.51 E-10$ & 1.9BE-11 \\
\hline $2.00 E-09$ & $7.5+E-19=$ & $-7.39 E-09$ & $-2.16 E-10$ & $4.01 E-11$ \\
\hline $2.10 E-95$ & $7.28 E-09$ & $-7.03 E-59$ & $-1.89 E-10$ & $5.55 E-11$ \\
\hline$\therefore=\Xi E E-59$ & $E \cdot 94 E-09$ & $-6.71 E-09$ & $-1 . E \cdot E-10$ & $E .59 E-11$ \\
\hline$=30 E-3=$ & $E=E 4 E-93$ & $-6.4 \Xi E-99$ & $-1.49 E-10$ & $7.39 E-11$ \\
\hline$\therefore .40 E-09$ & $E .3 E E-69$ & $-E .15 E-09$ & $-1.33 E-10$ & $\vec{i} .9 F-11$ \\
\hline $2.56 E-69$ & $E .10 E-19$ & $-5.96 E-69$ & $-1.21 E-15$ & $8.38 E-11$ \\
\hline$\therefore .59 E-09$ & $5.5 \cdot E-67$ & $-5.57 E-69$ & $-1.10 E-10$ & B. $67 E-11$ \\
\hline$\therefore=70 E-09$ & $5.55-95$ & $-5.46 E-09$ & $-1.50 E-10$ & 5. $87 E-11$ \\
\hline$\therefore .96 E-59$ & $5.45 E-95$ & $-5.25 E-89$ & $-9.21 E-11$ & 6. $9 E-11$ \\
\hline$=96 E-99$ & $5.25 E-99$ & $-5.68 E-69$ & $-8.51 E-11$ & $9.95 E-11$ \\
\hline $3.50 E-193$ & $5.85 E-69$ & $-4.91 E-09$ & $-7.39 E-11$ & $9.98 E-11$ \\
\hline $3.15 E-69$ & $4.91 E-09$ & $-4.75 E-69$ & $-7.34 E-11$ & $9.9 E E-11$ \\
\hline
\end{tabular}


Table F.5. Calculated values for $\mathrm{dr}_{\mathrm{c}} / \mathrm{dt}$ (DELTA) for cavity radii of 0.1 to $3.1 \mathrm{~nm} .10 \mathrm{dpa}, 850 \mathrm{~K}, \mathrm{P}=0.5 \mathrm{P}_{\text {eq }}, \mathrm{r}_{\text {crit }}=1.0 \mathrm{~nm}$

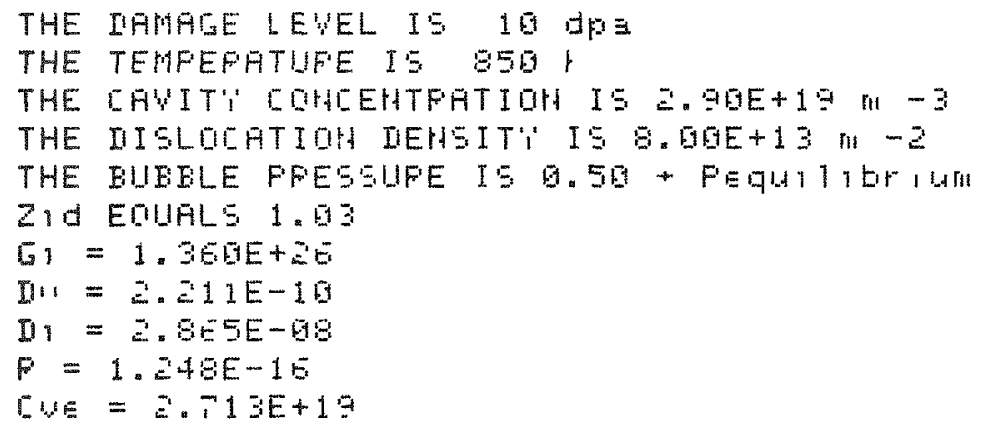

\begin{tabular}{|c|c|c|c|c|}
\hline FHIIIIS & $\Pi E 1+3 \equiv$ & $D \in 1+a t$ & $\Pi E 1+3 E$ & IIELTA \\
\hline 1.89E-19 & $-4.94 E-67$ & $-5.43 E-19$ & $-3.88 E-01$ & $-3.8 \geq E-61$ \\
\hline$=06 E-19$ & 9. $=9 E-09$ & $-\overrightarrow{1} .8 E-6 \theta$ & $-8.28 E-6 E$ & $-6 .=7 E-6 E$ \\
\hline $3.96 E-10$ & $5.14 E-15$ & $-4.94 E-98$ & $-1.93 E-57$ & $-1.91 E-97$ \\
\hline $4.00 E-10$ & $3.94 E-19$ & $-3.71 E-09$ & $-2.71 E-09$ & $-2.57 E-68$ \\
\hline 5. $95 E-15$ & $3 \times 97 E-60$ & $-\Xi .9 F E-198$ & $-7.9 \Xi E-19$ & $-E .87 E-99$ \\
\hline $6.00 E-15$ & $\therefore 56 E-09$ & $-2.47 E-108$ & $-3.37 E-59$ & $-2.51 E-09$ \\
\hline $7.05 E-10$ & $\therefore 19 E-19$ & $-\Xi .12 E-5 B$ & $-1.79 E-69$ & $-1.05 E-09$ \\
\hline $8.69 E-16$ & $1.9 a E-19$ & $-1.85 E-58$ & $-1.09 E-09$ & $-4.48 E-16$ \\
\hline $9.65 E-19$ & 1. $79 E-195$ & $-1.65 E-09$ & $-7.35 E-10$ & $-1 . E E E-10$ \\
\hline 1. $69 E-69$ & $1.53 E-69$ & $-1.48 E-85$ & $-5.29 E-19$ & $-1.4 \Sigma E-11$ \\
\hline $1.19 E-99$ & 1. $39 E-19$ & $-1.35 E-68$ & $-4.01 E-10$ & $E .70 E-11$ \\
\hline 1. $20 E-69$ & 1. $28 E-0 B$ & $-1.23 E-19$ & $-3.15 E-10$ & $1.13 E-19$ \\
\hline 1. $39 E-99$ & $1.18 E-98$ & $-1.14 E-58$ & $-2.5 E E-10$ & $1.39 E-10$ \\
\hline $1.40 E-69$ & 1. $95 E-98$ & $-1.56 E-08$ & $-\Xi .13 E-10$ & $1.54 E-1 E$ \\
\hline 1.5.5E- 59 & 1. $02 E-63$ & $-9.55 E-09$ & $-1.80 E-10$ & 1. $51 E-10$ \\
\hline 1.58E-69 & $9.56 E-59$ & $-9.24 E-99$ & $-1.5 E E-10$ & 1. $E 5 E-10$ \\
\hline $1.75 E-69$ & $9.65 E-59$ & $-9.7 D E-B 9$ & $-1.3 E E-10$ & $1.55 E-19$ \\
\hline 1.89E-69 & $9.49 E-99$ & $-5.51 E-69$ & $-1.20 E-10$ & 1. $54 E-10$ \\
\hline 1.95E-69 & 8. $155-69$ & $-\overrightarrow{7}, \overrightarrow{B E}-59$ & $-1.9, E-10$ & 1. $5 E E-16$ \\
\hline $2.00 E-09$ & $F=E+E-95$ & $-\vec{r} .39 E-99$ & $-9.8 B E-11$ & 1. $59 E-19$ \\
\hline $2.10 E-09$ & $7.27 \mathrm{E}-99$ & $-\overrightarrow{1} .03 E-59$ & $-9.79 E-11$ & $1.55 E-10$ \\
\hline $2.20 E-69$ & $E .9+E-99$ & $-E . T 1 E-69$ & $-9.03 E-11$ & 1. $5 E E-10$ \\
\hline $2.30 E-69$ & $E .54 E-05$ & $-E .4 E E-193$ & $-7.38 E-11$ & $1.48 E-19$ \\
\hline $2.40 E-90$ & $E .3 E E-99$ & $-E .15 E-199$ & $-E . E z E-11$ & $1.44 E-10$ \\
\hline $2.55 E-09$ & $E .10 E-99$ & $-5.90 E-193$ & $-E .3 J E-11$ & 1. $40 E-15$ \\
\hline $2.60 E-09$ & $5.87 E-09$ & $-5.5-5-09$ & $-5.90 E-11$ & $1.3, E-10$ \\
\hline$=78 E-99$ & $5.65 E-09$ & $-5.46 E-09$ & $-5.5 \mathrm{EE}-11$ & $1.35 E-10$ \\
\hline $2.86 E-69$ & $5: 45 E-99$ & $-5.26 E-139$ & $-5.19 E-11$ & $1.3 G E-16$ \\
\hline $2.99 E-99$ & $5.25 E-69$ & $-5.85 E-59$ & $-4.85 E-11$ & 1. $E E E-15$ \\
\hline $3.50 E-59$ & $5.08 E-99$ & $-4.91 E-99$ & $-4.51 E-11$ & 1. $=3 E-10$ \\
\hline $3.10 E-09$ & $4.91 E-199$ & $-4.75 E-09$ & $-4.3 E-11$ & 1. $: D E-19$ \\
\hline
\end{tabular}


Table F.6. Calculated values for $\mathrm{dr} / \mathrm{dt}$ (DELTA) for cavity radii of 0.1 to $3.1 \mathrm{~mm} .10 \mathrm{dpa}, 850 \mathrm{~K}, \mathrm{P}=0.9 \mathrm{P}$ eq, $\mathrm{r}_{\text {crit }}=0.2 \mathrm{~mm}$

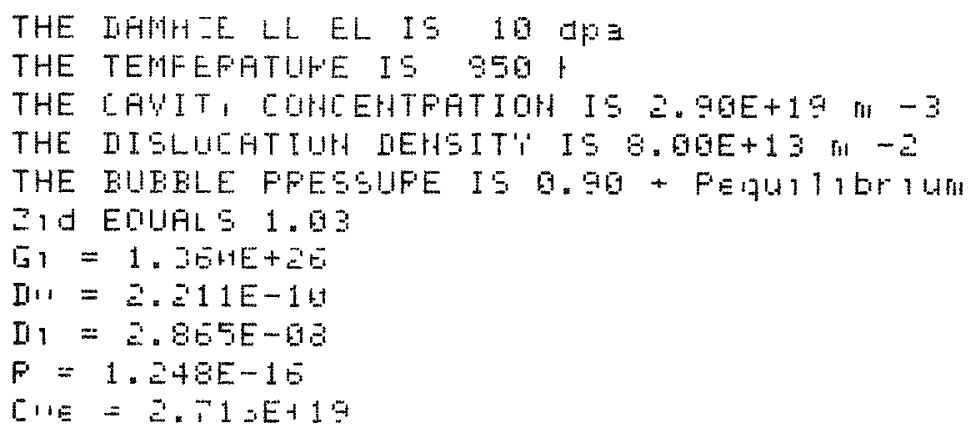

FAIIUE

1. $00 E-10$

$=.9 G E-10$

3. $90 \mathrm{E}-1 \mathrm{H}$

4. $00 E-10$

5. $00 E-10$

6. $90 E-10$

T. GUE-10

9. $60 E-10$

9. $90 E-16$

1. $00 E-69$

1. $10 E-6 \overline{1}$

1. $=\mathrm{DE}-\mathrm{G9}$

1. $30 E-69$

1. $+9 E-99$

1. $50 E-69$

1. EDE-69

1. $79 E-93$

1. $30 E-59$

1. $90 E-9 \%$

2. $05 E-51$

$\therefore 10 E-09$

$\therefore=D E-9=$

Z.30E- 19

$\Xi .49 E-69$

․ $50 E-09$

$\therefore 5 B E-619$

ㄷ $7 \mathrm{QE}-\mathrm{G}$

$\therefore .90 E-90$

2.9BE- 99

3. 00E- 19

$3.10 E-93$

\section{IIE $1+\equiv$}

$1.54 E-57$

ᄀ. EE- ES

5.1EE- 19

$3.84 E-6 E$

3. $9 T E-65$

$\therefore 5 E E-6 \theta$

ב. $19 E-08$

1. $: 2 E-08$

1. $P E E-69$

1. $5 E-69$

1. $3: 9 E-9 \theta$

1. $=B E-68$

1. $18 E-188$

1. $99 E-98$

1. $05 E-0 E$

$9.5 E E-09$

9. GEE-G

$0.49 E-99$

E. $94 E-09$

$\because E 4 E-99$

$7.2 T E-09$

E. 9HE-

E. $54 E-09$

B. $3 E E-B G$

E. 1EE-EG

5. 97E-93

$5.55 E-95$

$5.45 E-05$

5. $2 E E-59$

5. $05 E-95$

$4.91 E-59$
$I E I+$ It

$-1.48 E-01$

$-7.42 E-68$

$-4.95 E-6 \theta$

$-\Xi .1 E-B \theta$

- E. 97 $E-05$

$-2.47 E-68$

$-2.1 \Xi E-05$

$-1.85 E-18$

$-1 . E 5 E-108$

$-1.48 E-59$

$-1.35 E-58$

$-1 . \Xi \pm E-0 S$

$-1.14 E-19$

$-1.9 E E-9 B$

$-9.9 E E-99$

$-9.24 E-59$

$-5 .-9 E-69$

$-E .1 E-B S$

-T. $T E E-59$

$-7.39 E-199$

$-7.03 E-19$

-E. TIE- BI

$-E .4 E-G$ O

$-E .15 E-99$

$-5.9 E E-69$

$-5.67 E-19$

$-5.4 E E-19$

$-5.5 E E-69$

$-5.6 B E-D 9$

$-4.91 E-69$

$-4.75 E-69$
IIE]

$-3.95 E-68$

$-2.65 E-69$

$-9.0 E E-10$

$-4.9+E-15$

$-3.17 E-19$

$-\approx .31 E-16$

$-1.95 E-19$

$-1.4 E E-10$

$-1 .=3 E-1 D$

$-1.95 E-10$

- $5.5 E-11$

$-9 .-5 E-11$

$-7.4 E E-11$

$-E . T+4-11$

-E. 1TE-11

$-5.59 E-11$

$-5.27 E-11$

$-4.92 E-11$

$-4.59 E-11$

$-4.3 E-11$

$-4.59 E-11$

$-3.9 E E-11$

- 3. ETE-11

$-3.4 E E-11$

$-3.33 E-11$

$-3.1 \mathrm{BE}-11$

$-3.5+E-11$

$-2.92 E-11$

$-\mathrm{g} \theta E-11$

$-2 . \neg G E-11$

$-\Xi .5 E-11$
IELTTA

$-3.44 E-60$

$-E$. $E \bar{B}-11$

8. $1 E E-10$

9. $94 E-19$

$7.13 E-10$

E. ล. $E-10$

$5.55 E-10$

4. $=E E-10$

$4.48 E-10$

4. $97 E-19$

3. $T+4 E-10$

$3.45 E-10$

$3.90 E-10$

$\therefore 9 E-10$

$\therefore \rightarrow 9 E-10$

$\therefore E z E-16$

$=4 E-10$

ت. $35 \mathrm{E}-19$

$\because=3 E-10$

$\therefore 1 E E-10$

a. $9 E-10$

1. $93 E-10$

1. $85 E-10$

1. $7.7 E-19$

1. $70 E-15$

1. $E+E-15$

1. $58 E-10$

1. $5 E E-10$

1. $4, E-16$

1. $4 \Xi E-10$

1. $3 E-10$ 
Table F.7. Calculated values for $\mathrm{dr}_{\mathrm{c}} / \mathrm{dt}$ (DELTA) for cavity radii of 1.0 to $31.0 \mathrm{~mm}$. $10 \mathrm{dpa}, 970 \mathrm{~K}, \mathrm{P}=0.1 \mathrm{P}_{\text {eq }}, \mathrm{r}_{\text {crit }}=15.0 \mathrm{~nm}$

THE IARAGE LEUEL IS 19 dPa

THE TEMFEFHTUFE IS 9 TS 1 .

THE CAUIT: COHCEHTFATIOH IS 9. DUE+19 $\mathrm{m}-3$

THE IISLOCATIOH TIEHSIT; IS $2000 \mathrm{G}+1 \mathrm{a}-\mathrm{m}$

THE BUEFLE FFESSUFE IS 0.10 - Fequ111brim

Z1日 EDUALS $1.0 \mathrm{~S}$

$\square 1=1.3 E 0 E+2 E$

Iiv $=1.199 \mathrm{E}-0 \mathrm{~g}$

$I_{1}=4.445 E-08$

$R=1.97+4 E-1 E$

EVE $=4.655 E+20$

\begin{tabular}{|c|c|c|c|c|}
\hline FADIUS & IIE 1十 $\equiv \equiv$ & $\Pi E T+a t$ & $I H E 1+3 E$ & IIELTH \\
\hline 1. $90 E-09$ & $-5.73 E-5 E$ & $-4 . E 3 E-108$ & $-1.3 E-9$ & $-\Xi .41 E-9$ \\
\hline $2.60 E-09$ & $-3.59 E-09$ & $-\therefore .8 E E-0 B$ & $-1.40 E-60$ & $-\overrightarrow{1} .5 E-69$ \\
\hline $3.00 E-09$ & $\Xi 3 \neg E-0 E$ & $-1.93 E-6 z$ & $-5.51 E-09$ & $-1.1=E-60$ \\
\hline $4.60 E-09$ & $1.71 E-5 \theta$ & $-1.43 E-90$ & $-3.17 E-99$ & $-4.51 E-15$ \\
\hline $5.00 E-09$ & $1.32 E-09$ & $-1.13 E-08$ & $-\approx .1 E E-09$ & $-1 . T A E-10$ \\
\hline $6.00 E-09$ & $1.07 E-00$ & $-9.26 \mathrm{E}-09$ & $-1.5 E E-60$ & $-8.8 \Xi E-11$ \\
\hline $7.96 E-09$ & 8.97E-69 & $-7 .-3 E-09$ & $-1.29 E-99$ & $-4.81 E-11$ \\
\hline $0.06 E-69$ & ᄀ. ESE- 19 & $-E . E 1 E-09$ & $-1.0-E-09$ & $-a .-3 E E-11$ \\
\hline $9.60 E-69$ & E. E4E-69 & $-5.75 E-59$ & $-9.0 R E-10$ & $-1.59 E-11$ \\
\hline $1.00 E-09$ & $5.84 E-69$ & $-5.9 E E-09$ & $-\neg .88 E-10$ & $-9.37 E-1=$ \\
\hline $1.10 E-09$ & $5.19 E-69$ & $-4.50 E-09$ & $-E .9 E E-10$ & $-5.4 E E-1=$ \\
\hline 1. $20 \mathrm{E}-\mathrm{6g}$ & $4.65 \mathrm{E}-09$ & $-4.03 E-09$ & $-E .=3 E-10$ & $-3.05 E-12$ \\
\hline 1. $20 \mathrm{E}-0 \mathrm{\theta}$ & 4. $30 E-69$ & $-3 . E 4 E-69$ & $-5.53 E-10$ & $-1.5 \pm E-12$ \\
\hline $1.46 E-05$ & $3.82 E-09$ & $-3.31 E-09$ & $-5.1+E-10$ & $-5.69 E-13$ \\
\hline 1. $59 E-08$ & $3.49 E-09$ & $-3.02 E-09$ & $-4.72 E-10$ & $4.45 E-14$ \\
\hline 1. $-0 E-0 E$ & $3.20 E-09$ & 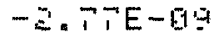 & $-4.3 \neg E-10$ & $4.34 E-13$ \\
\hline $1.70 E-69$ & $2.95 E-619$ & $-2.55 E-60$ & $-4.07 E-10$ & В. . $E E-13$ \\
\hline $1.80 E-00$ & $\therefore .74 E-09$ & $-2 \times 35 E-69$ & $-3.80 E-10$ & 8. $2=E-13$ \\
\hline $1.90 E-09$ & $\therefore 54 E-013$ & $-z=1 \mathrm{EE}-0 \mathrm{~g}$ & $-3.5 \cap E-10$ & $9.9 E E-13$ \\
\hline $2.60 E-018$ & $\therefore 3-E-09$ & $-2.03 E-00$ & $-3.36 E-10$ & $\because 46 E-13$ \\
\hline $2.19 E-98$ & $\therefore .21 E-619$ & $-1.89 E-09$ & $-3.18 E-10$ & $9 .+8 E-13$ \\
\hline $2.20 E-90$ & $\therefore 0.7 E-09$ & $-1.7 P E-09$ & $-3.01 E-10$ & $9.3 E-13$ \\
\hline $2.30 E-00$ & $1.94 E-09$ & $-1.56 E-0.9$ & $-2.8 E E-10$ & $9.15 E-13$ \\
\hline $2.40 E-698$ & $1.63 \mathrm{E}-0 \mathrm{~g}$ & $-1.5 E E-09$ & $-2.73 E-10$ & $9.94 E-13$ \\
\hline$\therefore .50 E-60$ & 1.72E-09 & $-1.4 E E-99$ & $-2.50 E-10$ & $8.49 E-13$ \\
\hline $2.60 E-08$ & $1.53 E-09$ & $-1.38 E-60$ & $-2.49 E-10$ & $8.11 E-13$ \\
\hline$=.70 E-0 S$ & $1.5+E-09$ & $-1.36 E-59$ & $-2.39 E-10$ & $\therefore \cdot a E-13$ \\
\hline $2.80 E-08$ & $1.46 E-69$ & $-1.23 E-09$ & $-Z .29 E-10$ & $\therefore .33 E-12$ \\
\hline $2.99 E-69$ & $1.39 E-69$ & $-1.1 \vec{F} E-9 \bar{C}$ & $-\Xi= \pm 1 E-10$ & $5.95 E-13$ \\
\hline $3.96 E-68$ & $1.3=E-09$ & $-1.11 E-09$ & $-\Xi .13 E-10$ & E. $58 \mathrm{E}-13$ \\
\hline $3.10 E-56$ & $1.25 E-09$ & $-1.65 E-09$ & $-E .05 E-10$ & $5.23 E-13$ \\
\hline
\end{tabular}


Table F.8. Calculated values for $\mathrm{dr}_{\mathrm{c}} / \mathrm{dt}$ (DELTA) for cavity radii of 1.0 to $31.0 \mathrm{~nm}$. $10 \mathrm{dpa}, 970 \mathrm{~K}, \mathrm{P}=0.5 \mathrm{P}_{\text {eq }}, \mathrm{r}_{\text {crit }}=7.0 \mathrm{~nm}$

THE DHMAGE LE' $E L$ IS 10 dP:

THE TENFEFATUFE IS 9,0

THE CAUIT COH. EHTFATIOH IS G. BEE+19 his -3

THE IISLOCATIUH DEHEIT'i IS $\mathrm{Bg}$.

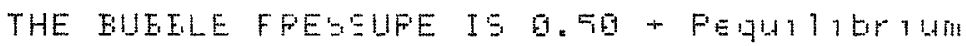

21d EOUALS 1.43

$G_{1}=1.3 E \mathrm{EH}+\mathrm{E}$

$\mathrm{IIn}_{11}=1.1 \mathrm{GGE}-\mathrm{GA}$

$I_{1}=4.47 E E-68$

$F=1.97+1 E-1 E$

$C_{1: E}=4.055 E+2 E$

\begin{tabular}{|c|c|c|}
\hline FAIIUS & I比1十三 & $I \equiv I+$ at \\
\hline 1. $015 E-19$ & $-7.6 E E-65$ & $-5.9 \pm E-95$ \\
\hline$=.09 E-99$ & $3.5 E E-69$ & $-3.01 E-50$ \\
\hline $9.5 E E-199$ & $\Xi . \Xi 9 E-59$ & $-1.98 E-58$ \\
\hline $4.50 E-69$ & $1.5 .7 E-B Q$ & $-1.4 E E-610$ \\
\hline $5.00 E-19$ & $1.30 E-65$ & $-1.14 E-1$ \\
\hline$E .65 E-69$ & 1. $5 E E-10$ & $-9.3 B E-9$ \\
\hline $7.60 E-09$ & 6. $95 E-69$ & $-7.79 E-\square$ \\
\hline $6.60 \mathrm{E}-\mathrm{Gg}$ & $\because .5 E E-09$ & $-E . E E E-g$ \\
\hline $9.6 \mathrm{GE}-19 \mathrm{G}$ & $E .5, E-99$ & $-5.79 E-1$ \\
\hline 1. $.9 E-9 G$ & $5.79 E-09$ & $-5.09 E-5$ \\
\hline $1.1 G E-6 \square$ & $5=14 E-109$ & $-4.52 E-9$ \\
\hline 1. $=9 E-99$ & $4.61 E-19$ & $-4.95 E-9$ \\
\hline 1. $39 E-195$ & $4.17 E-19$ & $-3, E E E-9$ \\
\hline $1.45 E-195$ & $3.79 E-59$ & $-3,3 z E-G$ \\
\hline 1. $55 E-199$ & $\because .4 E E-D 9$ & $-3,63 E-5$ \\
\hline 1. EDE-1. & $3.15 E-19$ & $- \pm . \neg E-E$ \\
\hline 1. $70 E-6 \theta$ & $\Xi 9 J E-99$ & $-\Xi: 5 E E-E$ \\
\hline $1.80 E-50$ & $\Xi \cdot \vec{E} E-B 9$ & $-Z=3 E E-G$ \\
\hline 1. $9 \mathrm{GE}-89$ & $\Xi .5 \Xi E-19 a$ & $-\Xi .19 E-g$ \\
\hline$\Xi \cdot 0 \bar{E}-103$ & $\Rightarrow 35 E-19$ & $-\Xi \cdot \theta \Xi-g$ \\
\hline$z .10 E-119$ & $\therefore \Xi \bar{E}-19$ & $-1.96 E-E$ \\
\hline$\therefore . \Xi 5 E-69$ & $\therefore=0 E E-19$ & $-1, \rightarrow F E-0$ \\
\hline$\therefore .30 E-60$ & 1. $93 E-19$ & $-1 \cdot \overrightarrow{E E} E-\mathrm{D}$ \\
\hline$E=4 G E-B \theta$ & 1. $B=E-19$ & $-1.5 E E-\theta$ \\
\hline$\Xi .50 E-65$ & $1 . \overrightarrow{1} 1 E-\overrightarrow{1}$ & $-1.47 E-0$ \\
\hline$\therefore 50 E-69$ & 1.E $\Xi E-199$ & $-1.3 E E-b$ \\
\hline$\therefore 76 E-8 B$ & $1.53 E-94$ & $-1.39 E-0$ \\
\hline$\therefore .9 \mathrm{GE}-\mathrm{ES}$ & $1.45 E-19=$ & $-1 . \Xi Z E-G$ \\
\hline$\Xi 961 E-60$ & $1.38 E-19$ & $-1 \cdot 1 \cdot E-1$ \\
\hline $3.95 E-59$ & 1. $31 E-19$ & $-1.11 E-G$ \\
\hline $3.15 E-55$ & 1. $=5 E-99$ & $-1.05 E-5$ \\
\hline
\end{tabular}

$\Gamma \in I+\equiv$

$-3: \exists+E-E B$

-

$-3.4+E-B=$

$-2.5 E-6=$

$-1 . B 3 E-E$

$-1 . E B E-95$

$-1.55 E-69$

$-9.9+4 E-10$

$-7.75 E-13$

$-E \times E+E-19$

-E. $1: E E-10$

$-5.54 E-19$

$-5.05 E-10$

$-4.55 E-15$

$-4.30 E-10$

$-4.65 E-19$

$-3.7+16-19$

$-3.51 E-10$

$-3.31 E-10$

$-3.13 E-15$

$-\because .9 E-19$

$-Z .8 E-10$

- Z. EGE-10

$-\because 5-5 E-10$

$-24 E E-10$

$-2.3 E-10$

- $A T E-1 B$

$-2.1 B E-19$

- E.1GE-10

$-2.03 E-10$

-1. GEE-1G
IELTH

- 1.E:EE-0T

$-1.4 E-69$

$-3.4-E-10$

$-1.11 .14$

$-3,-\because E-11$

- 1. 05E-11

-1. $. \pm E-1 \exists$

$4.19 E-12$

5. $2 T E-1=$

$E .3 E-1=$

E. $\Xi 4 E-1=$

5. 9QE-1

$5.4 E E-12$

5. $00 E-12$

$4.55-12$

4. $15 E-1 \Xi$

3. $T 5 E-1=$

$3.40 E-1 E$

3. $0+E-1 \Xi$

$\therefore 81 E-1 \Xi$

$=5 E E-1=$

$\therefore 3+E-1=$

$\therefore \quad 1 \neq E-1=$

1. $9 E E-1=$

1. $20 E-1 E$

1. $5 E-12$

1.5 $: E-1=$

1. $41 E-1=$

1. $30 E-1 z$

1. $=4 E-1 E$

1. $1=E-1=$ 
Table F.9. Calculated values for $\mathrm{dr}_{\mathrm{c}} / \mathrm{dt}$ (DELTA) for cavity radii of 1.0 to $31.0 \mathrm{~nm} .10 \mathrm{dpa}, 970 \mathrm{~K}, \mathrm{P}=0.9 \mathrm{P}_{\text {eq }}, \mathrm{r}_{\text {crit }}=2.0 \mathrm{~nm}$

THE DATIACE LE.EL IS 10 dF

THE TENFEFHTUFE IS STO

THE EAUITI EOHEEHTFATIOH IS $\triangle$ DOE+1:

THE DISLCTATIOH IEHSITI IS

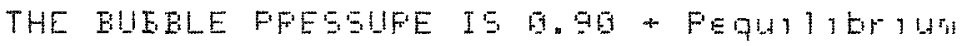

II EUUALS 1, U⿺

$G_{1}=1.3 E D E+E$

III $=1.195 E-57$

II $1=4.4 E E-5 E$

$F^{\prime}=1.974 E-1 E$

$[1 E=4.05 E+5$

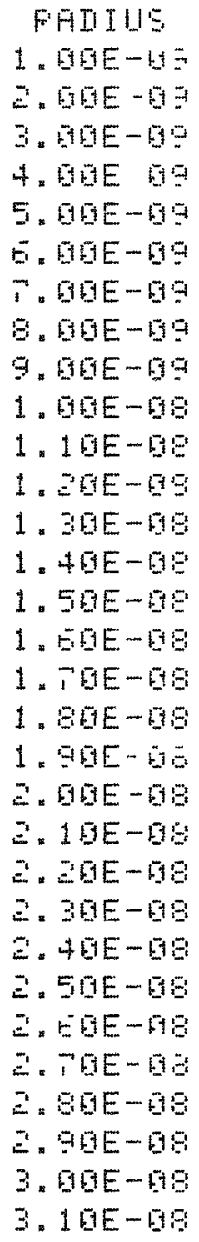

FAIIUS

$=50 E-63$

3. $\mathrm{GOE}-6 \mathrm{0}$

$4.00 \mathrm{E} \quad 0 \mathrm{C}$

$5.00 E-69$

6. $60 \mathrm{E}-69$

8. GOEE-10

$9.6 B E-09$

1. $00 \mathrm{E}-\mathrm{gg}$

1. $10 E-98$

1. $30 E-59$

$1.46 E-60$

1. $50 E-0 E$

1. $E G E-E \theta$

1. $8 G E-69$

1. $900-60$

a. $90 \mathrm{E}-0 \mathrm{~g}$

$\therefore 10 E-90$

$20 E-50$

E. $+6 E-6 E$

$\therefore 50 E-60$

$\therefore=6 E-79$

. TDE-6a

$\therefore 90 E-09$

3. $10 E-69$
$D=1+\exists \equiv$

‥ DQE-DE

$\therefore 45 E-63$

$Z= \pm 4 E-6 E$

1. $54 E-7 E$

$1.2 \mathrm{BE}-\mathrm{Ba}$

1. $94 \mathrm{E}-6 \mathrm{C}$

2. $: 5 E-69$

‥ $49 E-09$

E. 51E- 99

5. $73 E-59$

5. 10E-0:

$4.58 E-69$

$4 \times 14 E-69$

$\because A B E-69$

$\because 44 E-9$ ?

Z) $1 E E-09$

․ GIE-69

$\therefore-70 \mathrm{E}-69$

$=51 E-09$

$\because 34 E-65$

$\because 18 E-79$

$\therefore .95 E-69$

1. $9 E E-69$

1.91E- 09

1. TUE- 19

1. $51 E-09$

1. $52 E-69$

1. $44 E-69$

1. $\triangle T E-09$

1. $30 \mathrm{E}-0 \mathrm{9}$

1. $24 E-69$
IE $1+$ 프

$-E .51 E-8 E$

$-3.09 E-60$

$-\therefore .01 E-60$

-1. 4 EE- 98

$-1.15 E-08$

$-9.38 E-09$

$-T .85 E-09$

$-E . T 1 E-0.9$

$-5.92 E-09$

$-5.12 E-69$

$-4.54 E-90$

$-4.07 E-09$

$-3, E T E-E O$

$-3.33 E-69$

$-3.94 E-9 ?$

$-\geq .7 E E-9.9$

$-2.5 E E-60$

$-2.3 T E-09$

- $.19 E-09$

- $2014 E-65$

$-1.9 E E-99$

-1. TEE- G9

$-1.5 E E-0.9$

$-1.5 E E-69$

$-1.47 E-09$

$-1.38 \mathrm{E}-0 \mathrm{9}$

$-1.51 E-99$

$-1.25 E-69$

-1. ITE- -99

$-1.11 E-95$

$-1.05 E-60$
$0=1+3$

$-8.1 E E-09$

- $24.4 E-69$

$-\because 15 E-69$

$-1.5 T E-09$

$-1.25 E-09$

$-1.01 E-09$

- E. EE-10

$-T .49 E-10$

-E. E $3 E-10$

$-5.9+E-19$

$-5,3 E E-10$

$-4.9 E E-10$

$-4.53 E-10$

$-4.20 E-10$

$-3.91 E-10$

$-3.6 E E-15$

$-3.44 E-10$

$-3.25 E-10$

- 3. $5 \mathrm{BE}-1 \mathrm{~g}$

- $-9 E-10$

- $.7 \mathrm{TE}-15$

$-3.5 E-19$

$-\therefore .5 E-10$

- $243 E-1 E$

$-2.33 E-10$

- $20+E-10$

$-2.15 E-10$

$-\therefore .9 E-10$

$-\therefore$. $5 E-10$

$-1.33 E-10$

$-1.9 T E-10$
IELTH

$-3.4 E-10$

‥ $P 5 E-10$

1. $3 E-10$

9.5 $5 E-11$

E. $04 E-11$

5. $0.9 E-11$

$3.96 E-11$

$\because$ GEE-11

ב. $45 E-11$

2. $60 \mathrm{E}-11$

1. $E 5 E-11$

1. $3 E E-11$

1. $1, E-11$

1. $90 E-11$

$\therefore \quad 5 E-1=$

T. $5 E-1=$

E. $58 \mathrm{E}-1 \mathrm{E}$

5. $7=E-1 E$

5. $1 \mathrm{E} E-1 \mathrm{Z}$

4. 5EE-1

$4.97 E-1=$

$3.5 E-1=$

3. $2=E-1 a$

$\therefore$. $97 E-12$

$\therefore T E-12$

$\therefore 45 E-12$

$\therefore=4 E-12$

$2.65 E-12$

1. $98 \mathrm{E}-1 \mathrm{Z}$

1. $T E-1=$

1. $59 E-12$ 
Table F.10. Summary of the results for the calculations of $\mathbf{r}_{\text {crit }}$

\begin{tabular}{|c|c|c|c|}
\hline $\begin{array}{l}\text { Fluence } \\
\text { (dpa) }\end{array}$ & $\begin{array}{c}\text { Temperature } \\
\text { ( k) }\end{array}$ & $\begin{array}{l}\text { Pressure } \\
\text { (fraction } \\
\quad \text { of } \mathrm{P}_{\mathrm{eq}} \text { ) }\end{array}$ & $\begin{array}{l}r_{\text {crit }} \\
(\mathrm{nm})\end{array}$ \\
\hline 30 & 850 & $\begin{array}{l}0.1 \\
0.5 \\
0.9\end{array}$ & $\begin{array}{l}1.6 \\
0.9 \\
0.2\end{array}$ \\
\hline 10 & 850 & $\begin{array}{l}0.1 \\
0.5 \\
0.9\end{array}$ & $\begin{array}{l}1.8 \\
1.0 \\
0.2\end{array}$ \\
\hline 10 & 970 & $\begin{array}{l}0.1 \\
0.5 \\
0.9\end{array}$ & $\begin{array}{r}15.0 \\
7.0 \\
2.0\end{array}$ \\
\hline
\end{tabular}

F.3 Direct Calculation of $\mathbf{r}_{\text {crit: }}$ Dislocation Dominant Case

Hishinuma and Mansur [166] have derived a formula for $r_{c r i t}$ that applies when the sink strength of the dislocations is larger than that of the cavities, i.e.,

$$
L \gg 4 \pi r_{c} N_{c}
$$

For this condition

$$
r_{\text {crit }}=\frac{2 \gamma}{P+\frac{k_{B} T}{\Omega} \ln \left[\left(1-\frac{z_{i}^{c} z_{v}^{d}}{z_{v}^{c} z_{i}^{d}}\right) \frac{C_{v}}{c_{v}^{e}}+\frac{z_{i}^{c} z_{v}^{d}}{z_{v}^{c} z_{i}^{d}}\right]}
$$

where

$C_{V}=\left[\frac{K_{i} K_{v}+R\left(G_{i}-G_{v}\right)}{2 R K V}\right] \times\left[\left(1+\frac{4 R K_{v} K_{i} G_{v}}{\left[K_{i} K_{v}+R\left(G_{i}-G_{v}\right)\right]^{2}}\right]^{1 / 2}-1\right]$.

If $L \gg 4 \pi r{ }_{c} N_{c}$, then

$$
\mathrm{S}_{\mathrm{v}} \sim \mathrm{Z}_{\mathrm{v}}^{\mathrm{d}} \mathrm{L}
$$




$$
S_{i} \sim z_{i}^{d} L
$$

and

$$
G_{T} \sim D_{V} C_{V}^{e} L
$$

If $P$ is used in terms of $P_{e q}$ as described earlier,

$$
r_{\text {crit }}=\frac{2 \gamma\left(1-P^{\prime}\right)}{\frac{k_{B}^{T}}{\Omega} \ln \left[\left(1-\frac{z_{i}^{c} z_{v}^{d}}{z_{v}^{c} z_{i}^{d}}\right] \frac{c_{v}}{C_{v}^{e}}+\frac{z_{j}^{c} z_{v}^{d}}{z_{v}^{c} z_{i}^{d}}\right]}
$$

An additional advantage of Eq. (F.30) is that only the ratio of the capture efficiences, $z_{i}^{c} z_{v}^{d} / Z_{v}^{c} z_{i}^{d}$, is required. In the present investigation, Eq. (F.30) applies to the $30 \mathrm{dpa}, 850 \mathrm{~K}$ irradiation and to the $10 \mathrm{dpa}, 850 \mathrm{~K}$ irradiation. Table $\mathrm{F} .11$ summarizes the values of $r_{\text {crit }}$ calculated with Eq. (F.30) using the previously listed values for the required parameters. A comparison of Tables F.10 and F.11 demonstrates that both calculations yielded approximately the same values for $\mathrm{r}_{\text {crit. }}$

Table F.11. Summary of the values for $r_{\text {crit }}$ calculated with the assumption that

$L \gg 4 \pi r_{c} N_{c}$

\begin{tabular}{cccc}
$\begin{array}{c}\text { Fluence } \\
(\mathrm{dpa})\end{array}$ & $\begin{array}{c}\text { Temperature } \\
(\mathrm{K})\end{array}$ & $\begin{array}{c}\text { Pressure } \\
(\text { fraction } \\
\left.\text { of } \mathrm{P}_{\mathrm{eq}}\right)\end{array}$ & $\begin{array}{c}\mathrm{r}_{\text {crit }} \\
(\mathrm{nm})\end{array}$ \\
\hline \multirow{2}{*}{30} & \multirow{2}{*}{850} & 0.1 & 1.5 \\
& & 0.5 & 0.85 \\
& & 0.9 & 0.17 \\
10 & 850 & 0.1 & 1.8 \\
& & 0.5 & 1.0 \\
& & 0.9 & 0.2 \\
\hline
\end{tabular}




\section{APPENDIX G}

CALCULATIONS OF THE NUMBER OF HELIUM ATOMS IN AN EQUILIBRIUM BUBBLE

In this appendix, three calculations of the number of helium atoms in an equilibrium bubble are presented. These calculations utilize the ideal gas equation, a modified Van der Waals equation of state and a high density equation of state. The pressure, $\mathrm{P}_{\mathrm{eq}}$, in an equilibrium bubble of radius $x$ was assumed to be given by

$$
P_{\text {eq }}=\frac{2 \gamma}{r}
$$

where $\gamma$ is the surface tension between the bubble and the matrix.

In the simplest calculation, the ideal gas law was assumed to apply to helium bubbles in metals. The pressure given by the ideal gas law is

$$
\mathrm{P}=\frac{\mathrm{nk}_{\mathrm{B}} \mathrm{T}}{\mathrm{V}}
$$

where

$$
\begin{aligned}
\mathrm{k}_{\mathrm{B}} & =\text { Boltzmann's constant, } \\
\mathrm{n} & =\text { the number of helium atoms, } \\
\mathrm{T} & =\text { the absolute temperature, and } \\
\mathrm{V} & =\text { the volume of the bubble. }
\end{aligned}
$$

Assuming a spherical bubble, the volume of the bubble is given by the expression

$$
V=\frac{4}{3} \pi r^{3}
$$

Substituting Eqs. (G.1) and (G.3) into Eq. (G.2) yields the following expression for the number of helium atoms in an equilibrium bubble, $n_{e q}$ :

$$
\left.\mathrm{n}_{\text {eq }}\right|_{\text {ideal gas }}=\frac{8 \gamma \pi r^{2}}{3 k_{B} \mathrm{~T}} .
$$


A somewhat more complex approach used a modified Van der Waals equation of state. This calculation yields more accurate results than the ideal gas equation, especially for small bubbles. The usual form of the Van der Waals equation of state is given by:

$$
\left(\mathrm{p}+\frac{a \mathrm{n}^{2}}{\mathrm{v}^{2}}\right) \times(\mathrm{v}-\mathrm{n} b)=\mathrm{nk}_{\mathrm{B}} \mathrm{T}
$$

where $a$ is a measure of the attractive force between the atoms and $b$ is the effective volume of the atoms in a mole of gas [161]. For helium in the interatomic forces can be disregarded [167,168], yielding the modified form of the Van der Waals equation

$$
P(V-n b)=n k_{B} T
$$

Substituting Eqs. (G.1) and (G.3) into the above equation (with $P$ $=\mathrm{p}_{\mathrm{eq}}$ ) and solving for $\mathrm{n}_{\mathrm{eq}}$ gives

$$
\left.\mathrm{n}_{\text {eq }}\right|_{\substack{\text { Van der } \\ \text { Waa1s }}}=\frac{8 \pi r^{3} \gamma}{3\left(k_{B} \operatorname{Tr}+2 \gamma b\right)} .
$$

Equation (G.7) is the expression most frequently used to calculate $\mathrm{n}_{\mathrm{eq}}$ for helium bubbles. In using this equation, the selection of $b$ is quite important as $b$ is a function of temperature. As discussed by Cost and Chen [167], $b$ is equal to or nearly equal to the second virial coefficient, B, obtained from conventional compressibility studies. Tsederberg et al. [169] have derived an analytical expression for the temperature dependence of $B$. This expression is given by

$B(T)=\left[0.45 \times 10^{-3}+\frac{5.42}{(1890+T)}\right] \times 6.65 \times 10^{-27} \mathrm{~m}^{3}-$ atom $^{-1}$ (G.8)

In calculation of the number of helium atoms using the modified van der Waals equation $[\mathrm{Eq} .(\mathrm{G.7})], b$ was set equal to $B(T), E d .(G .8)$.

The third and most complex calculation used the high density equation of state (HDEOS) for gaseous helium presented by Wolfer [137, 138]. This calculation is applicable to bubbles with helium densities 
as high as the liquid density of helium. In deriving the HDEOS, Wolfer has utilized the interatomic potential for helium as given by Beck [170], the equation of state for a hard sphere system given by Carnahan and Starling [171], and the liquid state theory (applicable to dense gases) developed by Barker and Henderson [172,173], Anderson, Chandler, and Weeks [174], and Verlet and Weiss [175]. The HDEOS actually consists of several implicit equations which must be solved numerically. These equations are summarized below:

I) The modified form of the Beck Potential (Eq. 1, ref. [137]), with corrections as per Wolfer and Glasgow [176] is given by

$$
\frac{V(x)}{k_{B}}=A_{0} \exp \left[-a_{1} x-a_{2} x^{6}\right]-\left[\frac{D_{0}}{\left(x^{2}+x_{0}^{2}\right)^{3}} \times\left(1+\frac{x_{2}^{2}+3 x_{0}^{2}}{x^{2}+x_{0}^{2}}\right)\right]
$$

where

$$
\begin{aligned}
V(x)= & \text { interatomic potential of helium, } \\
A_{0}= & 4.63 \times 10^{6} \mathrm{~K}, \\
D_{0}= & 29.9950 \mathrm{~K}, \\
\mathrm{a}_{1}= & 11.5764, \\
\mathrm{a}_{2}= & 0.12596, \\
\mathrm{x}_{0}= & 0.25597, \\
\mathrm{x}_{2}= & 0.62416, \\
\mathrm{x}= & \mathrm{R} / \sigma, \\
\mathrm{R}= & \text { the interatomic distance, and } \\
\sigma= & 0.2637 \mathrm{~nm}, \text { the interatomic distance where the potential } \\
& \text { is equal to zero. }
\end{aligned}
$$

The minimum potential is at $R_{m}=0.2969 \mathrm{~nm}$, where

$$
\mathrm{V}\left(\mathrm{R}_{\mathrm{m}}\right) / \mathrm{k}_{\mathrm{B}}=-10.37 \mathrm{~K}
$$

The symbol $\varepsilon$ is also used for $v\left(R_{m}\right)$. 
II) The Weeks, Chandler, and Anderson values for $V_{0}(x)$, the repulsive part of the potential (Eq. G.10, ref. [137]) are given by

$$
V_{0}(x) \begin{cases}=v(x)+|\varepsilon| & \text { for } x \leqslant R_{m} / \sigma \\ 0 & \text { for } x>R_{m} / \sigma\end{cases}
$$

III) The Carnahan-Starling equation of state (Eq. 7, ref. [137] is given by

$$
z \int_{\substack{\text { hard } \\ \text { sphere }}}=\frac{1+y+y^{2}-y^{3}}{(1-y)^{3}} \text {, }
$$

where

$$
\begin{aligned}
z= & \text { the compressibility, } \\
y= & \text { the packing fraction }=(\pi / 6) \rho d^{3}, \\
\rho= & \text { the number density of gas atoms, and } \\
d= & \text { the effective hard sphere diameter for individual helium } \\
& \text { atoms. }
\end{aligned}
$$

IV) The Barker and Henderson equation for $d_{B}$, the effective hard sphere diameter without correction terms (Eq. 9, ref. [137]) is given by

$$
d_{B}=\sigma \int_{0}^{\infty}\left\{I-\exp \left[-V_{0}(x) / k_{B} T\right]\right\} d x
$$

V) The Verlet and Weiss equation for d (Eqs. 11-17, ref. [137], with corrections as per Wolfer and Glasgow [176]) is given by

$$
\mathrm{d}=\mathrm{d}_{\mathrm{B}}(1+\psi \delta)
$$

where

$$
\begin{gathered}
\delta=-1+2\left(\frac{\sigma}{d_{B}}\right)^{2} \int_{0}^{\infty}\left\{1-\exp \left[-v_{0}(x) / k_{B} T\right]\right\} x d x, \\
\Psi=\frac{(1+11 y) \sigma_{1}-(1-y) \sigma_{2}+3 y f(1-y)^{-3}}{\left.2[2+7 y) \sigma_{0}-(1-y) \sigma_{1}-1.5 y(1-y)^{-2}\right]}, \\
f=-7.5+y-17.3595 y^{2}-6.04 y^{3},
\end{gathered}
$$




$$
\begin{gathered}
\sigma_{0}=(1-y / 2) \times(1-y)^{3} \\
\sigma_{1}=\left(2-7.5 y+0.5 y^{2}-5.7865 y^{3}-1.51 y^{4}\right) \times(1-y)^{-4}
\end{gathered}
$$

and

$\sigma_{2}=\left(2-20 y+30 y^{2}+0.17 y^{3}-26.796 y^{4}+11.2241 y^{5}\right) \times(1-y)^{-5}$

The final form of the HDEOS is given by Eq. 19, ref. [137],

$$
\left.z=\frac{P}{\rho k_{B} T}=z\right) \underbrace{}_{\substack{\text { Hard } \\ \text { phere }}}-4 y \delta\left[\psi\left(\sigma_{0}+\sigma_{1}\right)-\sigma_{1}-\frac{1}{2} \sigma_{2}\right]
$$

The computer program (written for the Hewlett Packard 9845B Desktop Computer) shown in Figure G.1 calculates 2 as a function of $y_{0}$ (called Eta in the program) for several irradiation temperatures. $Y_{0}$ is a measure of the density, referred to as the hard sphere packing factor, which, in terms of $\sigma$, is given by the expression

$$
y_{0}=\frac{\pi}{6} \rho \sigma^{3}
$$

The output of the program is shown at the end of the progran 11sting. This program was provided by Wolfer and Glasgow [176]. Although not incorporated in this program, Wolfer and Glasgow recomended, based on recent re-evaluation of their calculations, the use of a correction factor of 1.9 in the calculation of $z$ [176]. This correction modifies Eq. (G.23) as shown below:

$$
\left.z=\frac{P}{\rho k_{B} T}=z\right)_{\substack{\text { Hard } \\ \text { Sphere }}}-4 y \delta\left[\psi\left(\sigma_{0}+\sigma_{1}\right)-\sigma_{1}-\frac{1}{2} \sigma_{2}\right] / 1.9 .
$$

In order to calculate the number of hellum atoms in an equilibrium bubble using the HDEOS, an indirect approach must be used. First, in terms of $y_{0}$, the number density of helium atoms is Ire-arranging Eq. (G.24)], 
YE-12365

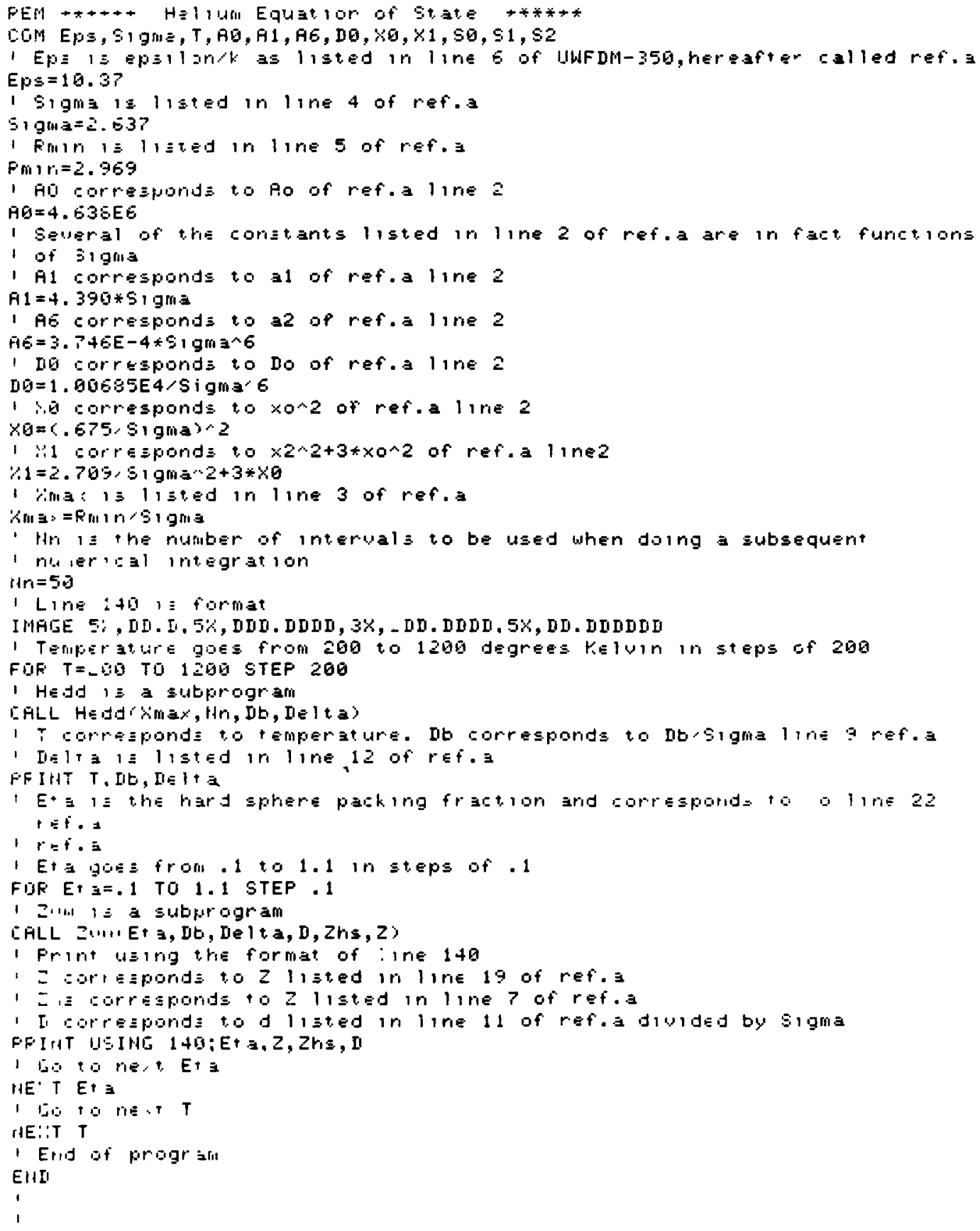

Figure G.1. BASIC computer program (HP 9845B) which calculates the compressibility, $z$, from the hard sphere packing factor, $y_{0}$ or eta, using the HDEOS for Irradiation temperatures of 200 to $1200 \mathrm{~K}$. Program supplied by Wolfer and Glasgow [176]. Reference a in the program is ref. [137] in this dissertation. The output is included at the end of the program. 


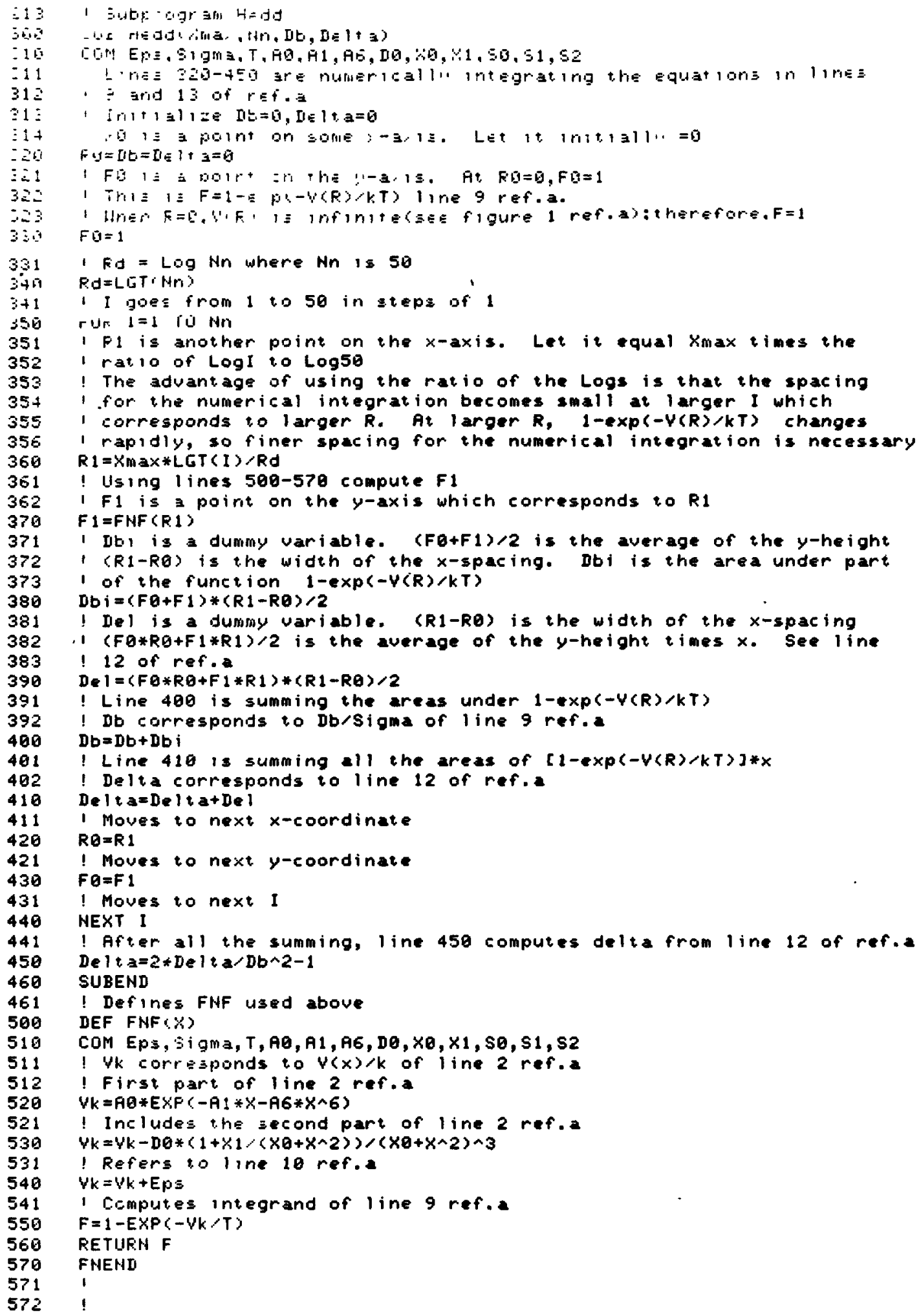

\section{Figure G.1. (continued)}




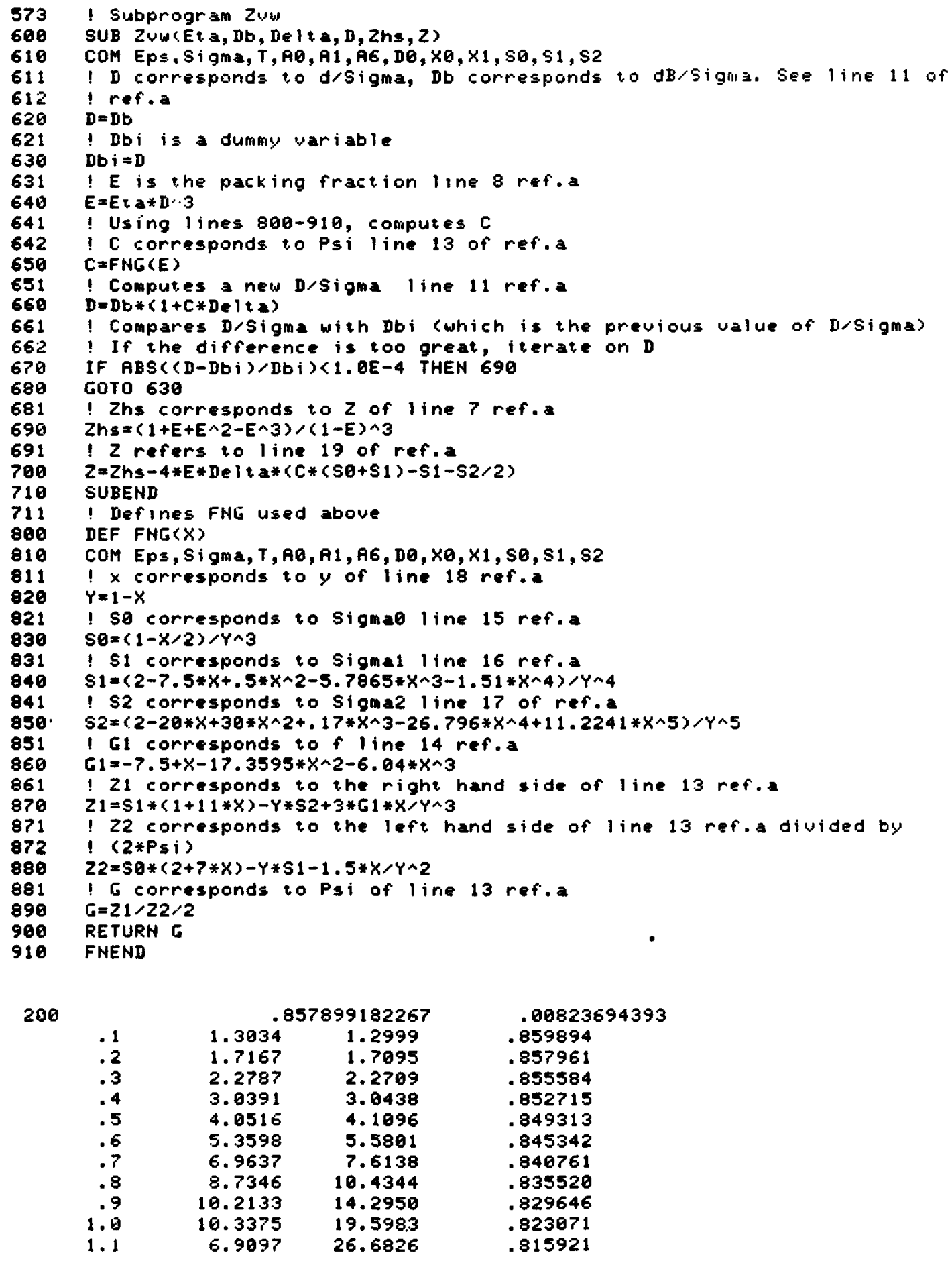

Figure G.1. (continued) 


\begin{tabular}{|c|c|c|c|}
\hline 450 & \multicolumn{2}{|c|}{.81035110 .495} & .01084964300 \\
\hline .1 & 1.2507 & 1.5471 & .813151 \\
\hline.$\Sigma$ & 1.5 .54 & 1.5574 & .81123 \\
\hline .3 & 1.9931 & 1.9823 & .808947 \\
\hline .4 & $\therefore 5: 51$ & 2.5200 & .8018201 \\
\hline.$\equiv$ & 3.564 & 3.2178 & .80315 \\
\hline . E & 4.5411 & 4.1134 & . \\
\hline .7 & 5.5451 & 5.2904 & .795007 \\
\hline. & 0.1353 & E.า. 80 & $.79+23$ \\
\hline .3 & 7.3050 & 0.5455 & . TEEลa \\
\hline 1.0 & 3.4395 & 11.1179 & .71191 \\
\hline 1.1 & $\therefore .133$ & 14.1900 & .775300 \\
\hline$=02+$ & & 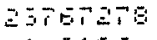 & - 112040925 ค \\
\hline 1 & 1. こここE & 1.2150 & $.734+86$ \\
\hline.$\Sigma$ & 1. 5.524 & 1.494 .1 & .785599 \\
\hline$\therefore=$ & $1.85=2$ & 1.0402 & .780450 \\
\hline .7 & $\therefore .5: 57$ & $\because .755$ & .7TBE4 \\
\hline .5 & $\Xi .65 \pm 5$ & $\Xi .913$ & .75497. \\
\hline .8 & $\Xi 4+8$ & 3.5035 & .771730 \\
\hline . & 4.2355 & 4.3542 & .700133 \\
\hline .5 & 5.1090 & 5.4097 & $.75+174$ \\
\hline.$\Xi$ & 0.0015 & 5.7213 & .759840 \\
\hline 1.9 & $\therefore$ G010 & 8.3230 & .755192 \\
\hline 1.1 & 7.7345 & 10.2883 & .750199 \\
\hline$=51$ & & 24094314 & .51404754012 \\
\hline .1 & 1. 2001 & 1.1990 & $.7 E 3597$ \\
\hline. & 1.4548 & 1.4452 & .751 .62 \\
\hline .3 & 1. TE: & 1.7502 & .759542 \\
\hline .4 & $\therefore 1384$ & 2.1243 & .757224 \\
\hline .5 & $\therefore 5923$ & 2.5839 & .754498 \\
\hline .5 & 3.1330 & 3.14 .00 & $.751+58$ \\
\hline T & 3.7647 & 3.8325 & .748110 \\
\hline .5 & $4.48+1$ & 4.60 .51 & .744454 \\
\hline .9 & 5. & 5.6700 & .746498 \\
\hline 1.6 & 5.0945 & 5.8924 & .736244 \\
\hline 1.1 & 0.8529 & 8.33 .4 & .731752 \\
\hline 1060 & & 06033225 & $.015=2500051$ \\
\hline .1 & $1.157^{-}$ & 1.1250 & $. \mp 1-14 a$ \\
\hline .2 & $1 .+2 \omega=$ & 1.4116 & .745 .5 \\
\hline .3 & 1. $69.8=$ & 1. $\operatorname{css} 0$ & .74375 \\
\hline .4 & $=013.45$ & $\approx .0187$ & .740953 \\
\hline .5 & $=.4335$ & 2.4215 & .738355 \\
\hline. & $\therefore 94+4$ & $\therefore .9005$ &. .354 .7 \\
\hline . & 3.4439 & 3.4881 & .732330 \\
\hline.$\Xi$ & 4.00 .68 & 4.1844 & .726906 \\
\hline .9 & $4.7+90$ & 5.6183 & . $\because 55012$ \\
\hline 1.5 & 5.4502 & 0.0020 & $.7212 E 4$ \\
\hline 1.1 & 5.1750 & ‥157 & .717100 \\
\hline $1=010$ & & 83555759 & .01625020508 \\
\hline .1 & 1.17 .99 & $1.17+E$ & $.73+55$ \\
\hline .2 & 1. 395 & 1. $38+8$ & .731731 \\
\hline$\therefore$ & 1.0551 & 1.63 .0 & .729738 \\
\hline .4 & $1.954=$ & 1.9388 & .75501 \\
\hline .5 & $\because 3153$ & 2.3003 & .725060 \\
\hline .5 & $\therefore-735$ & 2.7307 &.-22259 \\
\hline .7 & $3.21 \Xi E$ & 3.2405 &. .19268 \\
\hline$\therefore$ & $5 . T E 4 Z$ & 3.5435 & .715029 \\
\hline$\cdot$ & 4.3079 & $4.555=$ &. .12544 \\
\hline$\cdot$ & 50050 & 5.3867 & .708835 \\
\hline 1. 1 & 5.6514 & 0.3580 & .704920 \\
\hline
\end{tabular}




$$
\rho=\frac{6 y_{0}}{\pi \sigma^{3}}
$$

Substituting this equation for $\rho$ into $z=P / \rho k_{B} T$ yields

$$
z=\frac{P \pi \sigma^{3}}{6 y_{0} k_{B} T}
$$

Or, for an equilibrium bubble where $\mathrm{P}_{\mathrm{eq}}=\mathrm{P}_{\mathrm{eq}}=2 \gamma / \mathrm{r}$,

$$
z=\frac{\gamma \pi \sigma^{3}}{3 r y_{0} k_{B} T} \text {. }
$$

Re-arranging the above equation, the equilibrium bubble radius, $r$ eq, is given by

$$
r_{e q}=\frac{\gamma \pi \sigma^{3}}{3 z y_{0} k_{B} T} .
$$

Therefore, the radius of an equilibrium bubble can be calculated for a given $y_{0}$ and $T$. Since $z$ is a function of $y_{0}$ and $T, z$ must be calculated using the HDEOS.

The number of helium atoms, $n$, in a bubble can be derived from the definition

$$
\rho=\frac{n}{V}=\frac{3 n}{4 \pi r^{3}}
$$

Setting the expressions for $\rho$ given by Eqs. (G.26) and (G.27) equal yields

$$
\frac{3 \mathrm{n}}{4 \pi r^{3}}=\frac{6 \mathrm{y}_{0}}{\pi \sigma^{3}}
$$

or

$$
\text { n } \int_{H E D O S}=\frac{8 y_{0} r^{3}}{\sigma^{3}}
$$

Unlike calculations using the ideal gas and Van der Waals equations, the number of helium atoms required for an equilibrium bubble cannot be directly calculated for a given bubble radius using the HDEOS. Instead, as outlined above, $z$ must be calculated for a given $y_{0}$ and temperature. Then, $r_{\text {eq }}$ is calculated using Eq. (G.29). Finally, the number 
of helium atoms in the bubble is calculated using Eq. (G.31) with $r=r_{\text {eq }}$ i.e.,

$$
\mathrm{n} \text { eq } \int_{\mathrm{HDEOS}}=\frac{8 \mathrm{y}_{0} \mathrm{r}_{\mathrm{eq}}^{3}}{\sigma^{3}}
$$

Therefore, in order to best utilize the HDEOS, a serles of calculations of $r_{e q}$ and $n_{e q}$ using different values for $y_{0}$ and a single irradiation temperature must be performed. For small enough intervals between the yo values, a useful graph of $r_{e q}$ vs $n_{e q}$ can be obtained. A computer program to calculate and plot these data for $r_{e q}$ ( $R$ in the program) and $n_{e q}$ ( $M$ in the program) is shown in Figure G.2. This program also plots $z$ vs $y_{0}$. The symbols used in this program are the same as those in the program shown in Figure G.1. The output for the program shown in Figure G.2, for an irradiation temperature of $850 \mathrm{~K}$ and a surface energy of $2 \mathrm{~J}^{-\mathrm{m}^{-2}}$, is shown in Table G.1 (numerical data), Figure G.3 ( $z$ vs $y_{0}$ ), and Figure $G .4$ ( $r_{\text {eq }}$ vs $n_{e q}$ ). Figure $G .5$ shows a comparison of the $r_{e q} v s n_{e q}$ curves obtained for values of the surface energy of 1,2 , and $3 \mathrm{~J}-\mathrm{m}^{-2}$. A factor of 3 increase in the surface energy resulted in a factor of 3 increase in the number of helium atoms in an equilibrium bubble of a given radius. Likewise, Figure G.6 demonstrates the effect of the irradiation temperature $(300,750,850$, and $950 \mathrm{~K}$ ) on this curve. For irradiation temperatures of $750-950 \mathrm{~K}$, the irradiation temperature has only a small effect $(<20 \%)$ on the number of helium atoms in an equilibrium bubble of a given radius.

Calculations of $\mathrm{n}_{\mathrm{eq}}$ using the ideal gas equation, the modified Van der Waals EOS, and the HDEOS are compared in Figure G.7 (a plot of $r_{\text {eq }}$ vs $n_{e q}$ for each calculation) and Table $G 2$ (the numerical data) for an irradiation temperature of $850 \mathrm{~K}$ and a surface energy of $2 \mathrm{~J}-\mathrm{m}^{-2}$. The HDEOS predicts fewer hellum atoms for an equilibrium bubble than the modified Van der Waals and more hellum atoms than the Ideal gas equation for a given bubble radius. However, the differences in $n_{e q}$ calculated by each of the equations decrease with increasing bubble radius. In fact, for a bubble radius greater than $\sim 5 \mathrm{~nm}$, there is less than a $10 \%$ difference between the number of helium atoms predicted by the modified Van der Waals EOS and the HDEOS. 
YE-12366

10 REM **** CRLCULATIOH OF EQUILIBRIUM BUBBLE VARIAELES

15 USIHL THE HDEDS $* * * *$
20 IIM $Y(150)$, Matz(150), R(150),M(150)

30 COM Eps, Sigma, $T, A \theta, A 1, A 6, D Q, K \theta, X 1, S 9,51,52$

$40 \quad E p \equiv=10.37$

$50 \quad F^{\prime} i=3.14159$

$60 \quad K=1.38 E-16$

$70 \quad$ Sigma $=2.637$

$88 \quad$ Rmin $=2.969$

$90 \quad \mathrm{AQ}=4.638 \mathrm{E} 6$

$100 \quad A 1=4.399 * 5 i g m a$

$110 \mathrm{~A} E=3.746 \mathrm{E}-4 * \mathrm{Sigma \wedge}$

$120 \mathrm{DG}=1.00685 \mathrm{E} 4 / \mathrm{sigma} 6$

$130 \mathrm{XQ}=(.675 / \mathrm{sigma}) \times 2$

$140 \quad x_{1}=2.709 / \operatorname{sigma\wedge } 2+3 * x \theta$

150 Xmax=Rminssigma

$160 \quad \mathrm{~N} r=50$

170 IMAGE 5\%,D.DD, 5\%, DDD. DDDD, 5 , DDD.DD, 5,$D . D I D D E$

189 PRIHT "IHFUT THE TEMPERATURE IN KELVIN AND THE SLIRFACE

ENERGY IN Ergs/cm^2"

190 INPUT T, Gamma

209 FRIHTER IS 0

210

220

CALL HEdd ( $X_{\max }, \mathrm{Hn}, \mathrm{Dt}, \mathrm{D} \in \mathrm{lt}$ a)

PRIHT USING "K,4D K, 3D.2D K, D.4DE K , SD"; "THE TEMFERFTUR

E IN KELYIN IS ", T, "Db IS ", Db, "Delta IS ", Delta, "THE SURFAC

E EHERGY IN Ergs $\mathrm{cm}^{\wedge} 2$ I $\mathrm{S} "$, Gamma

225 PRINT LIN(2);SFA(7); "Y"; SFA(10); "Z"; SPA(9); "r(non)"; SFA (6); "He ATOMS"; LIN(1)

230 Courit $=9$

240 FOR Et $z=.01$ TO 1.3 STEP . 11

250 CALL Zuw(Eta, Ib, DE Tta, D,Zhs, Z)

260 Y(Count) $=$ Et.a

270 Matz(Count) $=2$

271

$272 !$

275 REM * * STATEMENTS 280 AHD 290 CALCULATE THE EQUILIERIUM FUBBLE RADIUS ANI THE HUMBER OF HELIUM FTOMS IN THE EQUILIBRIUM BIJEBLE**

276

$!$

R(Count) $)=$ anma*Fi $i$ S i gma^ $3 * 1 E-17 /(3 * K * T * 2 * E+a)$

M(Count) $=8 * R($ Count) $\wedge * E t a /(S i g m a \wedge 3 * 1 E-3)$

IF R(Count) 1 THEN GOTO 310

FRINT USING 179; Y(Count), Matz(Count), R(Count), MCCount)

IF Y(count $)=.38$ THEN PRINT PAGE

Count $=$ Count +1

NEXT Eta

Figure G.2. BASIC computer program (HP 9845A) written to calculate the number of helium atoms in an equilibrium bubble using the HDEOS. 


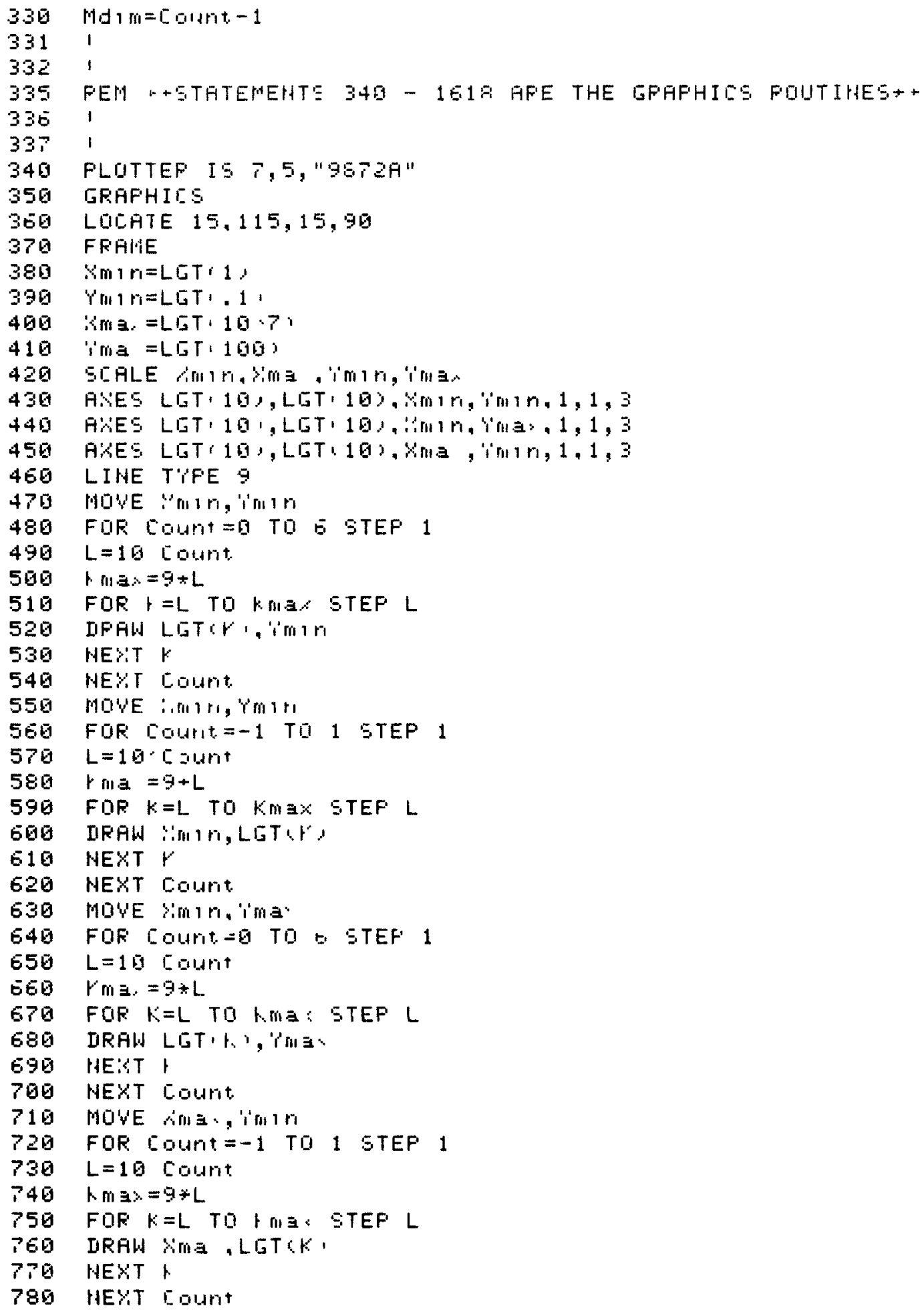

Figure G.2. (continued) 


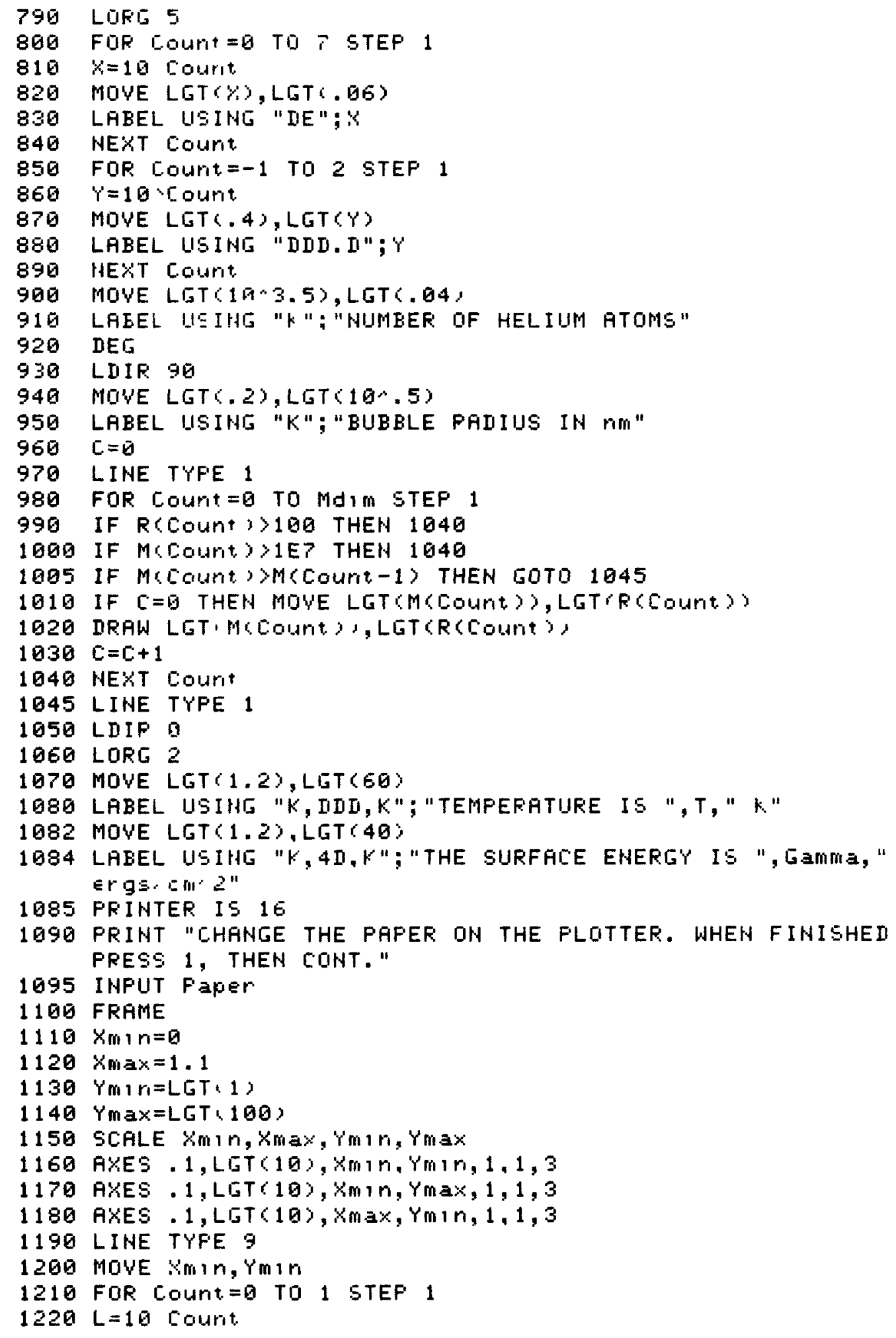

Figure G.2. (continued) 


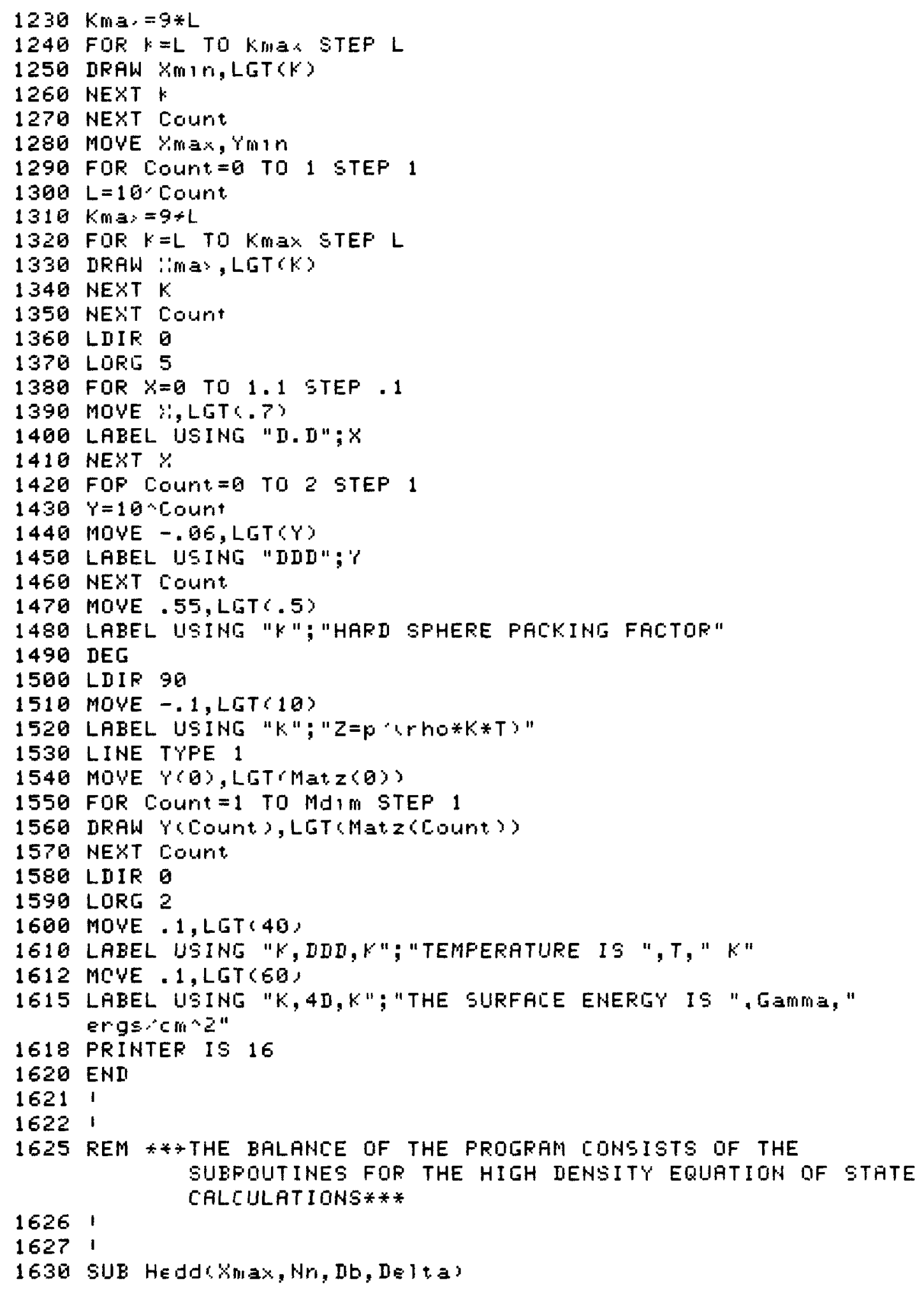

Figure G.2. (continued) 


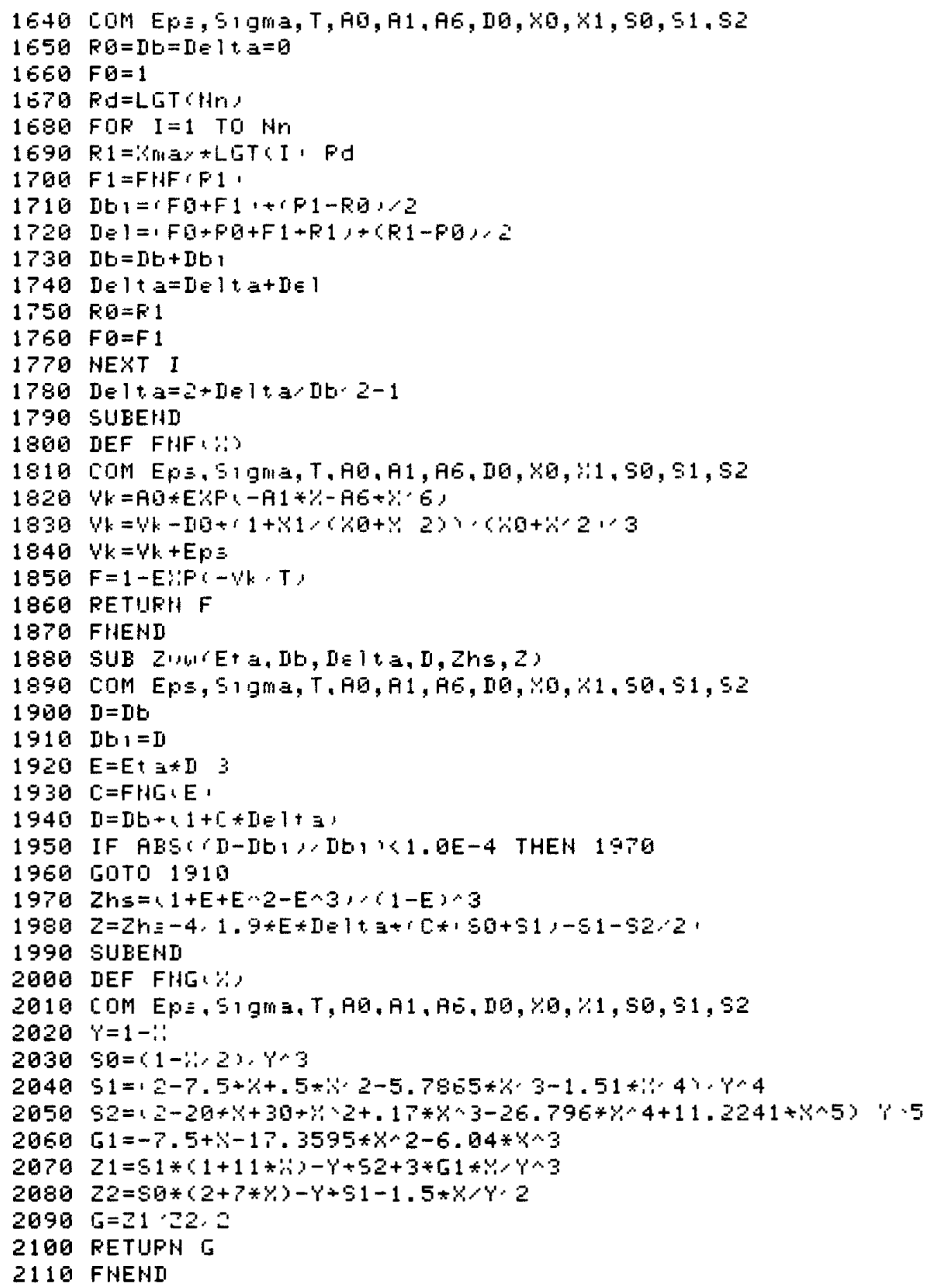

Figure G.2. (continued) 
Table G.1. Numerical output from the computer program shown in Figure G.2. Graphs of the output are found in Figure G.3 $\left(\mathrm{z}\right.$ vs $\left.\mathrm{y}_{0}\right)$ and Figure $\mathrm{G.4}$ ( $\mathrm{r}_{\mathrm{eq}}$ vs $\mathrm{n}_{\mathrm{eq}}$ )

THE TEMFEFHTUWE IH IELVIH IS 850

ID IS . TE

Ie 1 t. IS $1.43 E G E-62$

THE SUFFHEE EHEFG'i IH ERg

\begin{tabular}{|c|c|c|c|}
\hline 40 & $z$ & $r(n$ in) & HE ATON三 \\
\hline .01 & 1.9180 & $321.5 \Xi$ & $1.4514 \mathrm{E}+0 \mathrm{E}$ \\
\hline .92 & 1.6354 & 157.96 & $3.4390 \mathrm{E}+07$ \\
\hline $.9 \Xi$ & 1.0551 & 103.43 & 1. $4484 E+07$ \\
\hline .04 & 1.0743 & 70.19 & $7.7189 E+0 E$ \\
\hline .05 & 1.0938 & 59.86 & $4.6799 E+06$ \\
\hline .00 & 1.1138 & 48.99 & $3.0783 E+9 E$ \\
\hline .67 & 1.1342 & 41.24 & 2. $1+18 E+5 E$ \\
\hline .09 & 1.1550 & 35.43 & $1.5529 E+06$ \\
\hline .09 & $1.17 E 2$ & 30.93 & 1. $1616 E+0 E$ \\
\hline .10 & 1.1979 & 27.33 & $8.9071 E+95$ \\
\hline .11 & 1. 2201 & 24.49 & $6.9677 E+95$ \\
\hline .12 & 1. ה & 21.96 & $5.5412 E+05$ \\
\hline .13 & 1.2657 & 19.90 & $4.4680 E+95$ \\
\hline .14 & 1.2893 & 18.14 & $3.645 \Xi E+015$ \\
\hline .15 & 1.3133 & $16 . E \Xi$ & $3.9042 E+95$ \\
\hline $.1 E$ & 1.3379 & 15.30 & $2.4978 E+05$ \\
\hline .17 & 1.3629 & 14.13 & $2.0929 E+05$ \\
\hline .18 & 1.3885 & 13.10 & $1.7056 \mathrm{E}+05$ \\
\hline .19 & 1.4145 & 12.18 & $1.498 E E+05$ \\
\hline .25 & 1.4412 & 11.36 & 1.2P89E+05 \\
\hline .21 & 1.4683 & 10.62 & $1.0968 E+05$ \\
\hline $2=2$ & 1.4950 & 9.95 & $9.4486 E+04$ \\
\hline . & 1.5243 & 9.94 & $8.1725 E+194$ \\
\hline .24 & 1.5532 & 8.78 & ᄀ. 0950E+04 \\
\hline .25 & 1.5626 & 8. . 8 & $6.1896 E+04$ \\
\hline . $2 E$ & 1.E12E & 7.81 & $5.4010 E+04$ \\
\hline .27 & 1.6433 & 7.38 & $4.7333 E+04$ \\
\hline .28 & 1.5745 & E.98 & $4.1594 E+04$ \\
\hline .29 & 1.705 .4 & E.EZ & $3.6641 E+04$ \\
\hline .30 & 1.7392 & 6.28 & $3.2349 E+64$ \\
\hline .31 & 1.7.a1 & 5.96 & $2.9630 E+94$ \\
\hline .32 & 1.8059 & $5.6 ?$ & $2.538 T E+04$ \\
\hline$=33$ & 1.8404 & 5.39 & 2. $: 554 E+04$ \\
\hline .34 & 1.8756 & 5.13 & 2. $9674 E+54$ \\
\hline .35 & 1.9115 & 4.89 & 1. . $897 E+94$ \\
\hline . $3 t$ & 1.9461 & 4.E. & $1.5961 E+04$ \\
\hline $.3 i$ & 1.9853 & 4.45 & $1.4295 E+94$ \\
\hline .38 & 2.0230 & $4.2 E$ & 1. $279 \Omega E+04$ \\
\hline
\end{tabular}


Table G.1. (continued)

\begin{tabular}{|c|c|c|c|}
\hline 10 & $z$ & $r(n i n)$ & $H E$ ATUMS \\
\hline .39 & 2.0627 & 4.07 & 1. $1471 E+54$ \\
\hline .49 & 2.1023 & 3.89 & 1.0300E+04 \\
\hline .41 & 2.1427 & 3.73 & $9.2596 E+03$ \\
\hline .42 & 2.1838 & 3.57 & $8.3343 E+03$ \\
\hline .43 & 2.2258 & 3.42 & ㄱ. $5098 E+03$ \\
\hline .44 & 2.2686 & 3.28 & $6.7>42 E+03$ \\
\hline .45 & 2.3122 & 3.15 & $6.117 \overline{b E}+03$ \\
\hline .46 & 2.3566 & 3.02 & $5.5290 E+03$ \\
\hline .47 & 2.4919 & 2.90 & $5.0023 E+03$ \\
\hline .48 & 2.4480 & 2.79 & $4.5300 E+03$ \\
\hline .49 & 2.4951 & 2.68 & $4.1058 E+03$ \\
\hline .50 & 2.5430 & 2.53 & $3.7246 E+03$ \\
\hline .51 & $\therefore .5918$ & 2.48 & $3.3815 E+03$ \\
\hline .52 & 2.6415 & 2.38 & $3.0725 E+03$ \\
\hline .53 & 2.6921 & 2.29 & $2.7939 E+03$ \\
\hline .54 & 2.7437 & 2.21 & $2.5424 E+53$ \\
\hline .55 & 2.7962 & 2.13 & $2.3153 E+93$ \\
\hline .56 & 2.8497 & 2.55 & $2.1100 E+03$ \\
\hline 57 & 2.9041 & 1.98 & $1.9242 E+0.3$ \\
\hline .58 & 2.9595 & 1.91 & $1.7559 E+03$ \\
\hline .59 & 3.0160 & 1.84 & $1.6034 E+03$ \\
\hline .00 & 3.9734 & 1.78 & $1.4651 E+03$ \\
\hline.$E 1$ & 3.1319 & 1.71 & $1.3395 E+03$ \\
\hline.$G \Omega$ & 3.1914 & 1.65 & $1.2255 E+03$ \\
\hline .63 & 3.2506 & 1.60 & 1. $1232 E+03$ \\
\hline .64 & 3.3120 & 1.54 & $1.0290 E+03$ \\
\hline .65 & 3.3745 & 1.49 & $9.4316 E+02$ \\
\hline.$F E$ & 3.4380 & 1.44 & $8.6502 E+02$ \\
\hline .67 & 3.5026 & 1.40 & $7.9381 E+02$ \\
\hline .68 & 3.5682 & 1.35 & $7.2887 \mathrm{E}+02$ \\
\hline .69 & 3.6350 & 1.31 & $6.6962 E+02$ \\
\hline .70 & 3.7028 & 1.20 & $6.1553 E+02$ \\
\hline .1 & 3.771. & 1.22 & $5.6611 E+02$ \\
\hline .72 & 3.8417 & 1.18 & $5.2094 E+02$ \\
\hline ב & 3.9128 & 1.15 & $4.7963 E+02$ \\
\hline .74 & 3.9851 & 1.11 & $4.4184 E+02$ \\
\hline .75 & 4.0584 & 1.08 & $4.0724 E+02$ \\
\hline $.7 E$ & 4.1328 & 1.094 & $3.7554 E+02$ \\
\hline & 4.2084 & 1.01 & $3.4649 E+02$ \\
\hline
\end{tabular}




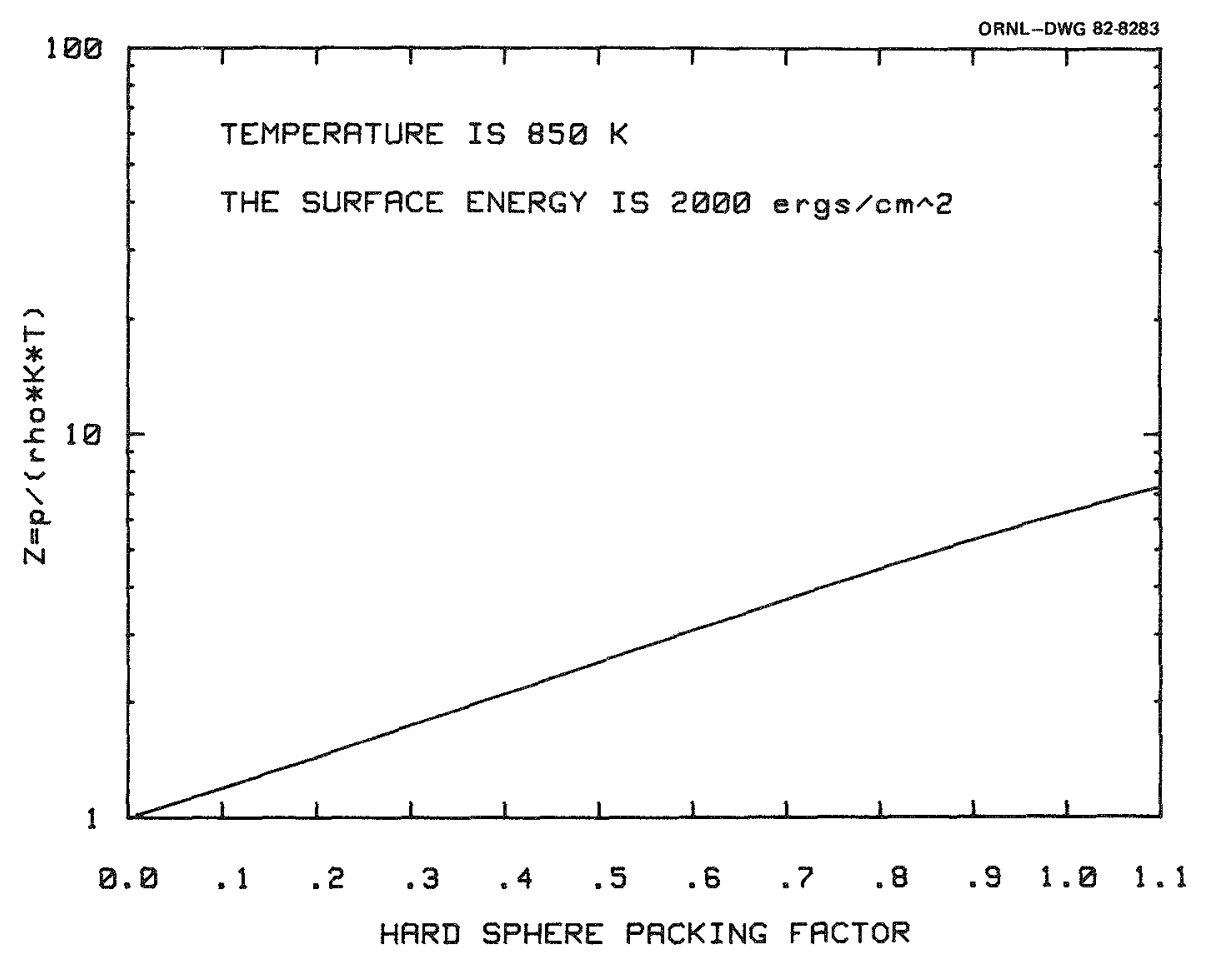

Figure G.3. Plot of the compressibility, $z$, vs the hard sphere packing factor, $\mathrm{y}_{0}$, as calculated by the HDEOS.

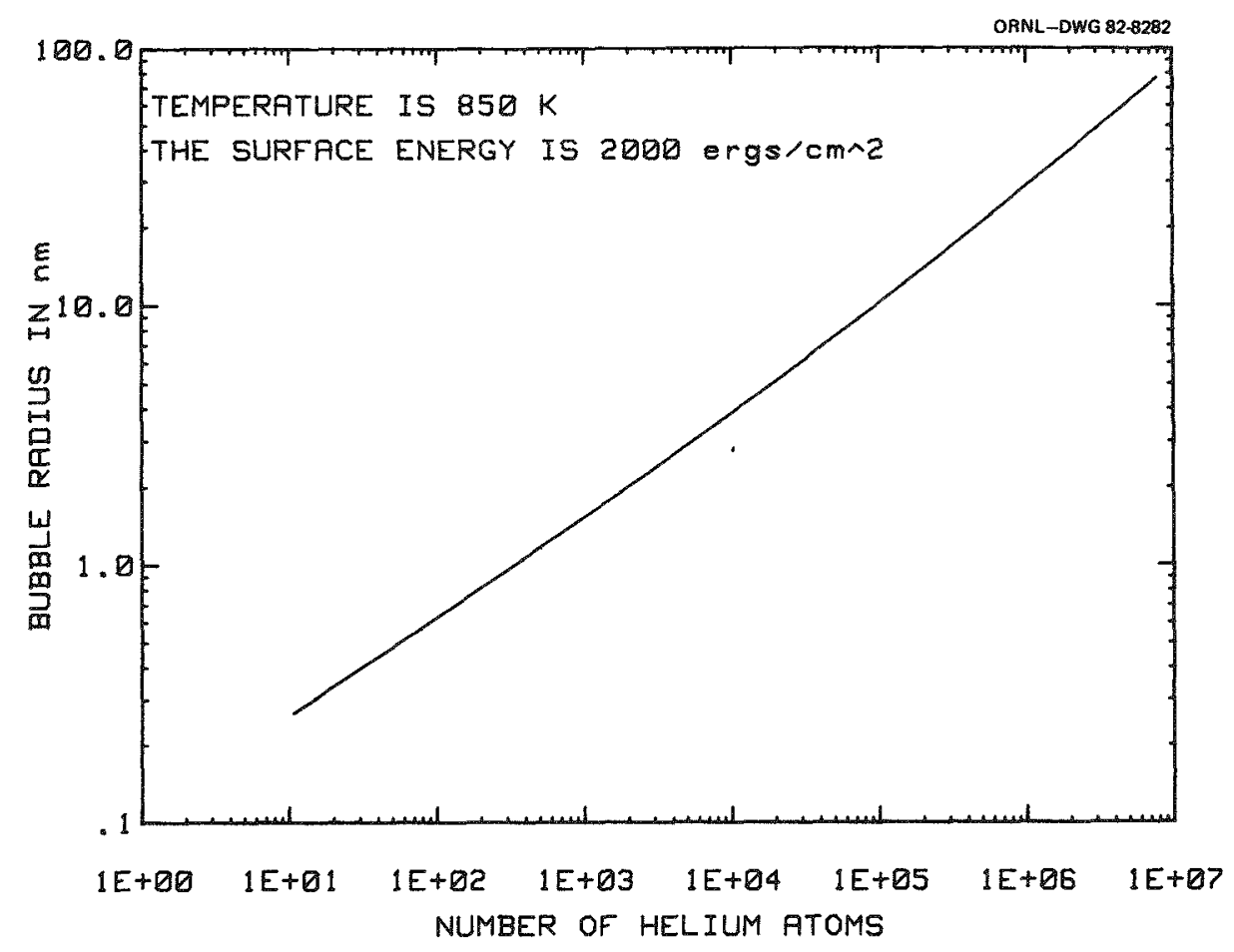

Figure G.4. Plot of the equilibrium bubble radius, req, vs the number of helium atoms in an equilibrium bubble, 荄, 


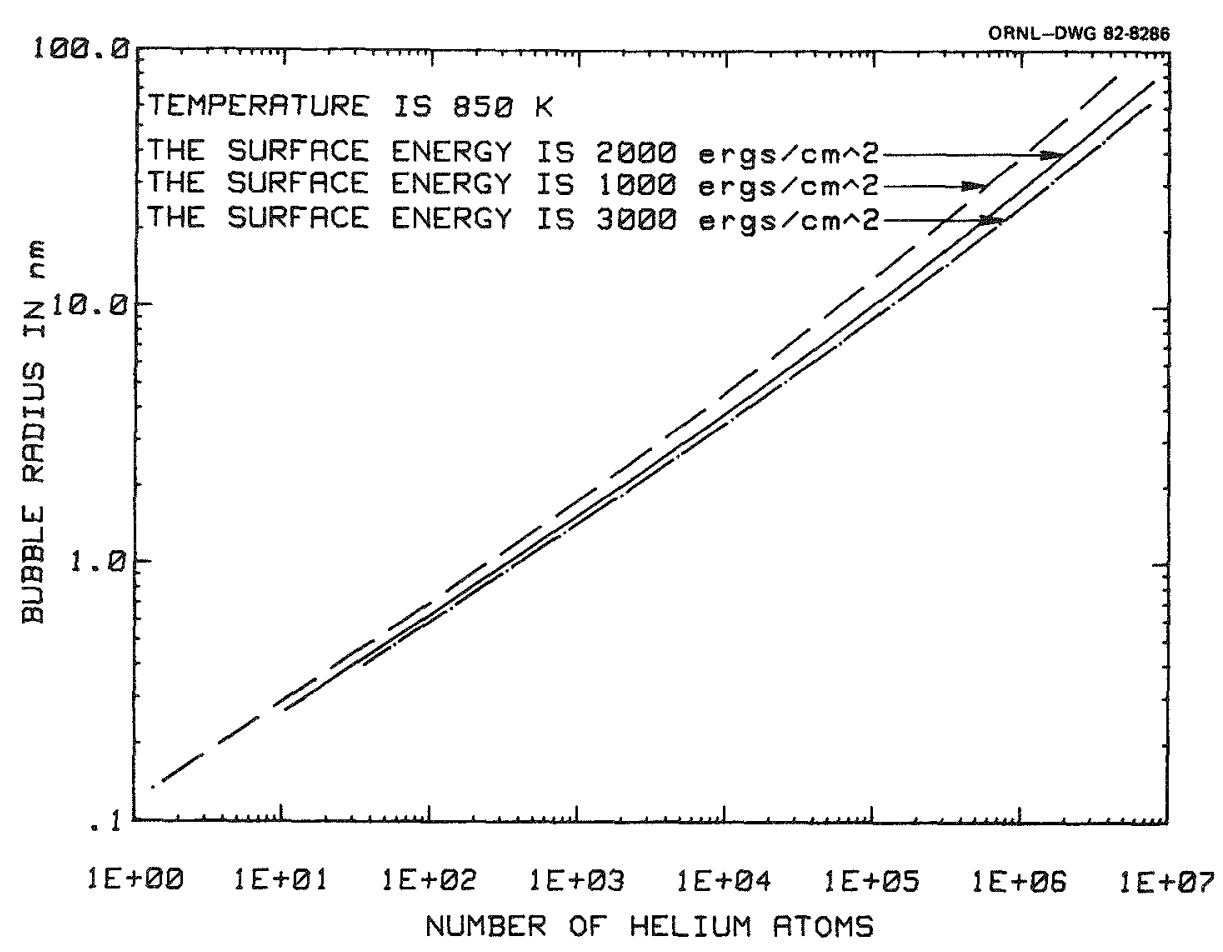

Figure G.5. A series of $r_{\text {eq }}$ vs $n_{e q}$ curves calculated by the HDEOS for a range of surface energies.

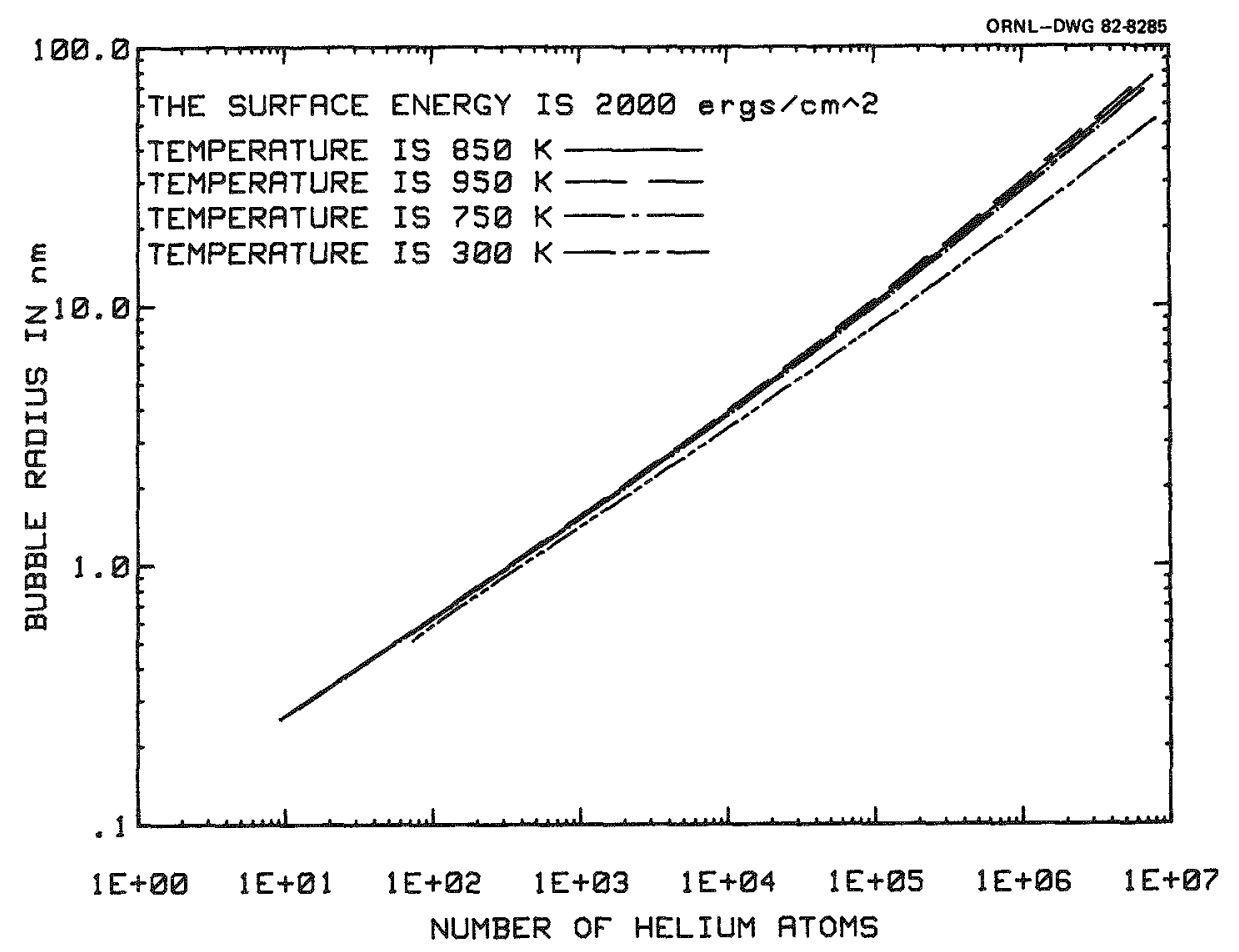

Figure G.6. A series of $r_{\text {eq }}$ vs $n_{\text {eq }}$ curves calculated by the HDEOS for a range of irradiation temperatures. 


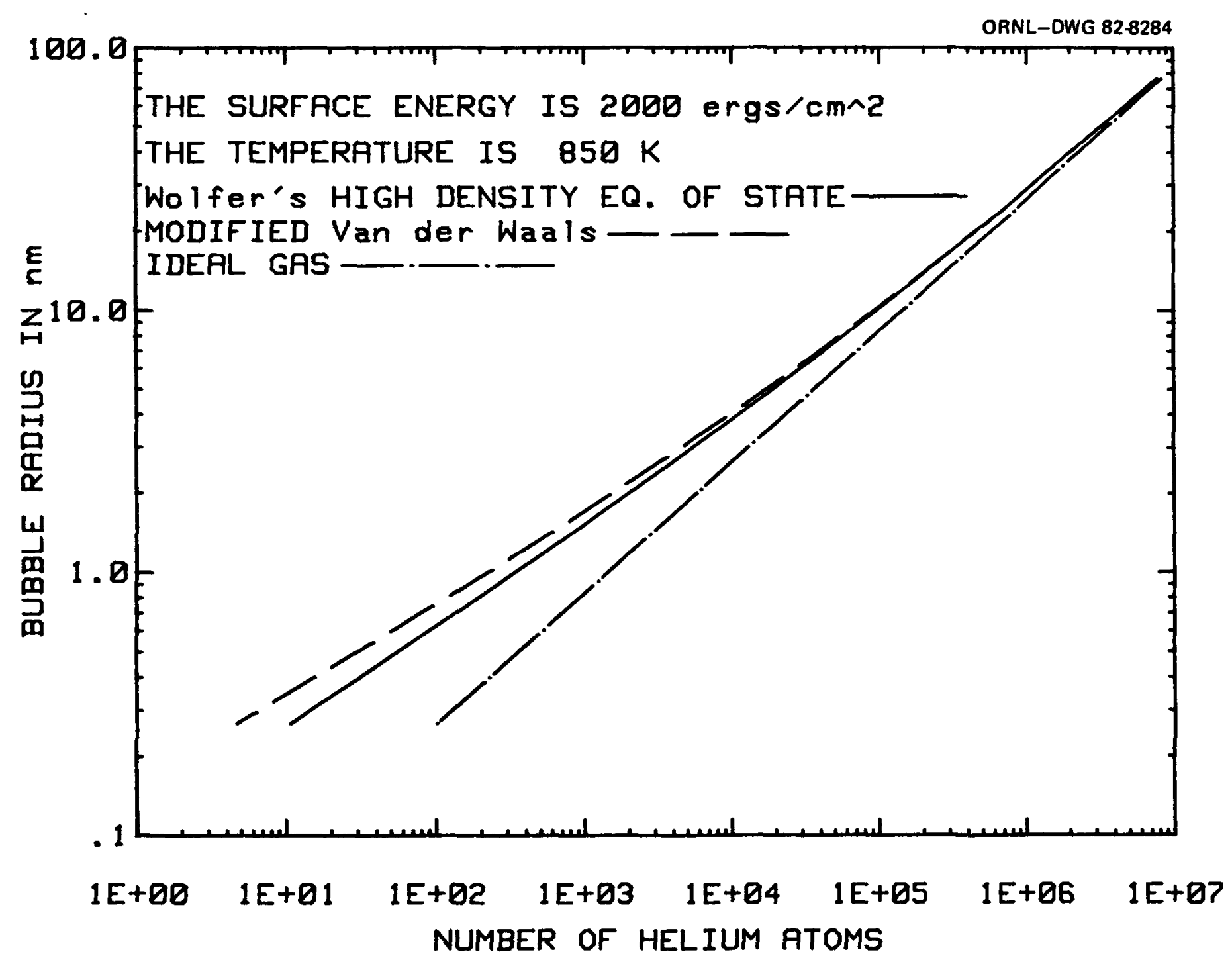

Figure G.7. $r_{\text {eq }}$ vs $n_{e q}$ curves calculated by the ideal gas equation, the modified Van der Waals EOS and the HDEOS. 
Table G.2. Numerical comparison of the $\mathrm{n}_{\mathrm{eq}}$ values calculated by the Ideal gas equation, the modified Van der Waals EOS and the HDEOS. A plot of the three $r_{e q}$ vs $n_{e q}$ curves is shown in Figure G.7.

\begin{tabular}{|c|c|c|c|}
\hline$r(n i t)$ & $\begin{array}{l}\text { Humbe } \\
\text { Hard Sphere } \\
\text { Eq. of State }\end{array}$ & $\begin{array}{l}\text { of Heliurit Ato } \\
\text { Modified } \\
\text { Van der Waals }\end{array}$ & Ideal \\
\hline 321.62 & $1.45 E+08$ & $1.44 E+08$ & $1.48 E+08$ \\
\hline 157.96 & $3.44 E+07$ & $3.40 E+07$ & $3.56 \mathrm{E}+07$ \\
\hline 103.43 & 1. $45 E+07$ & 1. $42 E+07$ & $1.53 E+07$ \\
\hline 76.19 & $7.72 E+06$ & $7.55 E+06$ & $8.29 E+06$ \\
\hline 59.86 & $4.68 E+06$ & $4.55 E+06$ & $5.12 E+0 E$ \\
\hline 48.99 & 3.DEE+DE & $2.97 E+06$ & $3.43 E+06$ \\
\hline 41.24 & $2.14 E+06$ & $2.55 E+06$ & $2.43 E+06$ \\
\hline 35.43 & $1.55 E+06$ & $1.48 E+96$ & $1.79 E+06$ \\
\hline 30.93 & $1.16 E+16$ & $1.10 E+0 \epsilon$ & 1. $37 E+06$ \\
\hline 27.33 & $8.91 E+05$ & 8.37E+05 & $1.97 E+06$ \\
\hline 24.40 & $6.97 E+05$ & $6.50 E+95$ & 8.50E+195 \\
\hline 21.96 & $5.54 E+05$ & $5.13 E+0.5$ & $6.89 E+05$ \\
\hline 19.90 & $4.47 E+05$ & 4.1BE+05 & $5.66 \mathrm{E}+05$ \\
\hline 18.14 & $3.65 E+0.5$ & $3.32 E+05$ & $4.70 E+05$ \\
\hline 16.62 & $3.90 E+05$ & $2.72 E+05$ & $3.95 E+95$ \\
\hline 15.30 & $2.50 E+05$ & $2.24 E+05$ & $3.34 E+8.5$ \\
\hline 14.13 & $2.69 E+0.5$ & $1.86 E+05$ & $2.85 E+05$ \\
\hline 13.15 & $1.77 E+0.5$ & $1.56 E+05$ & $2.45 E+0.5$ \\
\hline 12.18 & $1.56 E+05$ & $1.31 E+05$ & $2.12 E+05$ \\
\hline 11.36 & $1.28 \mathrm{E}+05$ & $1.11 E+05$ & $1.84 E+05$ \\
\hline 10.62 & $1.10 \mathrm{E}+0.5$ & $9.43 E+94$ & $1.61 \mathrm{E}+0 \mathrm{5}$ \\
\hline 9.95 & $9.45 E+04$ & 8. $0.5 E+04$ & $1.41 E+95$ \\
\hline 9.34 & a.17E+D4 & $6.90 E+D 4$ & $1.25 E+0.5$ \\
\hline 8.78 & $7.10 E+04$ & $5.94 E+04$ & $1.10 E+05$ \\
\hline 8.28 & $6.18 E+04$ & $5.12 E+54$ & $9.78 E+94$ \\
\hline 7.81 & $5.40 E+04$ & $4.44 E+04$ & $8.71 E+04$ \\
\hline 7.38 & $4.73 E+54$ & $3.85 E+04$ & $7.78 E+04$ \\
\hline 6.96 & $4.16 E+04$ & $3.35 E+04$ & $6.97 E+64$ \\
\hline$E . E$ & $3.66 E+84$ & $2.93 E+04$ & $6.25 E+54$ \\
\hline 0.28 & $3.23 E+04$ & 2. $56 \mathrm{E}+04$ & $5.62 E+04$ \\
\hline 5.36 & $2.86 E+04$ & $2.24 E+64$ & $5.57 E+64$ \\
\hline 5.67 & $2.54 E+04$ & $1.97 E+04$ & $4.58 E+04$ \\
\hline 5.39 & $2.26 E+04$ & $1.73 E+04$ & $4.15 E+04$ \\
\hline 5.13 & $2.01 E+04$ & $1.53 E+04$ & $3.77 E+04$ \\
\hline 4.80 & $1.79 E+04$ & $1.35 E+04$ & $3.42 E+104$ \\
\hline 4.67 & $1.6 G E+04$ & $1.19 E+04$ & $3.11 E+04$ \\
\hline 4.46 & $1.43 E+04$ & $1.06 \mathrm{E}+04$ & $2.84 E+94$ \\
\hline 4.26 & $1.28 E+94$ & $9.36 \mathrm{E}+03$ & $2.59 \mathrm{E}+\mathrm{D}$ \\
\hline
\end{tabular}


Table G.2. (continued)

\begin{tabular}{|c|c|c|c|}
\hline rinins & $\begin{array}{l}\text { Humber } \\
\text { Hur S Sphere } \\
\text { Eq. of State }\end{array}$ & $\begin{array}{l}\text { of Helium } \\
\text { Modified } \\
\text { Yan der Waals }\end{array}$ & Ideal \\
\hline 4.07 & $1.15 E+\overline{6} 4$ & $8.31 E+03$ & $2.37 E+04$ \\
\hline 3.89 & $1.03 E+04$ & 7. $38 E+0.3$ & 2. $17 E+\square 4$ \\
\hline 3.73 & $9.26 E+93$ & $6.57 E+03$ & $1.98 E+04$ \\
\hline 3.57 & $8.33 E+93$ & $5.86 \mathrm{E}+03$ & $1.82 E+04$ \\
\hline $5.4=$ & $7.51 E+03$ & $5.22 E+0.3$ & $1.67 E+04$ \\
\hline 3.5 & $6.77 E+93$ & $4.67 E+03$ & 1. $54 E+04$ \\
\hline 3.15 & $6.12 E+03$ & $4.17 E+03$ & $1.41 E+04$ \\
\hline 3.62 & $5.53 E+03$ & $3.73 E+93$ & $1.30 E+04$ \\
\hline 2.90 & $5.00 E+03$ & $3.34 \mathrm{E}+0.3$ & $1.2 \theta E+04$ \\
\hline 2.79 & $4.53 E+03$ & $3.00 E+03$ & $1.11 \mathrm{E}+04$ \\
\hline 2.60 & $4.11 E+03$ & $2.69 E+0.3$ & $1.02 E+04$ \\
\hline 2.58 & $3.72 E+03$ & $2.42 E+03$ & $9.47 E+03$ \\
\hline 2.48 & $3.38 E+03$ & $2.17 E+93$ & $8.76 \mathrm{E}+103$ \\
\hline 2.38 & $3.07 E+0.3$ & $1.95 E+83$ & 8. $12 E+53$ \\
\hline 2.29 & $2.79 E+03$ & $1.76 E+03$ & $7.52 E+0.3$ \\
\hline 2.21 & $2.54 E+03$ & $1.58 E+0.3$ & $6.98 \mathrm{E}+03$ \\
\hline 2.13 & $2.32 E+03$ & $1.43 E+03$ & $6.47 E+03$ \\
\hline 2.55 & $2.11 E+03$ & $1.29 E+03$ & $6.01 E+93$ \\
\hline 1.98 & $1.92 E+03$ & $1.16 \mathrm{E}+93$ & $5.59 E+0.3$ \\
\hline 1.91 & $1.76 E+63$ & $1.05 E+03$ & $5.20 E+03$ \\
\hline 1.84 & $1.60 E+03$ & $9.50 E+02$ & $4.84 E+03$ \\
\hline 1.78 & $1.47 E+03$ & $8.6 \mathrm{EE}+02$ & $4.50 E+53$ \\
\hline 1.71 & $1.34 E+03$ & $7.78 E+02$ & $4.20 E+03$ \\
\hline 1.65 & $1.23 E+03$ & $7.05 E+02$ & $3.91 E+03$ \\
\hline 1.60 & $1.12 E+03$ & $6.40 E+02$ & $3.65 E+0.3$ \\
\hline 1.54 & $1.03 E+03$ & $5.81 E+02$ & $3.41 E+03$ \\
\hline 1.49 & $9.43 E+02$ & $5.27 E+02$ & $3.18 E+03$ \\
\hline 1.74 & $8.65 E+12$ & $4.79 E+02$ & $2.97 E+03$ \\
\hline 1.49 & $7.94 E+102$ & $4.35 E+02$ & $2.78 E+03$ \\
\hline 1.35 & P. $29 \mathrm{E}+02$ & $3.96 E+02$ & $2.60 E+03$ \\
\hline 1.31 & $6.70 \mathrm{E}+02$ & $3.60 E+02$ & $2.43 E+03$ \\
\hline 1.26 & $E .16 E+02$ & $3.28 E+02$ & $2.28 E+03$ \\
\hline 1.22 & $5.66 \mathrm{E}+02$ & $2.98 E+02$ & $2.14 E+03$ \\
\hline 1.18 & $5.21 E+62$ & $2.72 E+02$ & $2.00 E+0.3$ \\
\hline 1.15 & $4.8 \Delta E+102$ & $2.48 E+02$ & $1.88 E+03$ \\
\hline 1.11 & $4.42 E+02$ & $2.26 E+02$ & $1.76 \mathrm{E}+03$ \\
\hline 1.00 & $4.07 E+02$ & $2.07 E+02$ & 1. $65 E+93$ \\
\hline 1.64 & $3.76 E+92$ & $1.89 E+02$ & $1.55 E+03$ \\
\hline 1.91 & $3.46 E+02$ & $1.73 E+82$ & $1.46 E+63$ \\
\hline
\end{tabular}


Therefore, according to these calculations, either a modified Van der Waals BOS or the HDEOS can be used to calculate $n_{\text {eq }}$ for large cavities $(r>\sim 5 \mathrm{~mm})$. For smaller cavities, however, the HDEOS probably yields more accurate values for $n_{e q}$. For the current investigation, the BDEOS was used to calculate $n_{e q}$ for a cavity radius of $\sim 11$ $\mathrm{nm}, \mathrm{T}_{\mathrm{I}}=850 \mathrm{~K}$, and for a cavity radius of $\sim 2.5 \mathrm{~mm}, \mathrm{~T}_{\mathrm{I}}=970 \mathrm{~K}$. For the $11 \mathrm{~nm}$ cavity radius, $\mathrm{n}_{\text {eq }} \sim 1.1 \times 10^{5}$ hellum atoms per cavity. For the 2.5 cavity radius, $n_{\text {eq }} \sim 3.4 \times 10^{3}$ helium atoms per cavity. 


\section{References}

[1] T. A. Gabrie1, B. L. Bishop, and F. W. Wiffen, Calculated Irradiation Response of Materials Using Fission Reactor (HFIR, ORR and EBR-II) Neutron Spectra, ORNL/TM-6361 (August 1979).

[2] T. A. Gabriel, B. L. Bishop, and F. W. Wiffen, "Calculated Atom Displacement and Gas Production Rates of Materials Using a Fusion Reactor First Wall Spectrum," Nucl. Technol. 38 (May 1978) $427-433$.

[3] G. J. Thomas and W. Bauer, "In-Situ Observation of Ion Implanted Surfaces," J. Nucl. Mater. 63 (1976) 280-284.

[4] W. A. Jesser, J. A. Horton, J. I. Bennetch, L. L. Scribner, and H.G.F. Wilsdorf, "Instrumentation for SAMI Studies," Proc. USJapan Seminar on New Applications and Extensions of the Unique Advantage of HVEM for Physical and Materials Research (1976).

[5] L. L. Schiestle Horton, Helium Ion Bombardment of 316 Stainless Steel, Masters Thesis, University of Virginia (December 1978).

[6] J. I. Bennetch, M. L. Sattler, L. L. Schiestle Horton, J. A. Horton, and W. A. Jesser, "HVEM Observations of In-Situ He-Ion Bombardments," J. Nucl. Mater. $85 \& 86$ (1979) 665-669.

[7] L. L. Horton and W. A. Jesser, A Study of Radiation Damage Produced in Thinned Specimens of 316 Stainless Steel by Energetic Helium Ions, ASTM-STP-725, ed. by D. Kramer, H. R. Brager, and J. S. Perrin (ASTM, Baltimore, MD, 1981), Pp. $642-653$.

[8] J. I. Bennetch, Microstructural Aspects of Helium Embrittlement in Stainless Steel, Ph.D. Dissertation, University of Virginia (May 1981).

[9] J. A. Horton, HVEM Studies of Helium Embrittlement, Ph.D. Dissertation, University of Virginia (December 1979).

[10] J. A. Spitznage1, F. W. Wiffen, and F. V. Nolfi, "Microstructures in 'Simulated' Fusion Irradiations," J. Nucl. Mater. 85\&86 (1979) $629-646$.

[11] R. A. Nelson, The Simulation of Void Swelling - A Critical Assessment After 10 Years, AERE-R8826 (July 1977); presented at the Annual Meeting of the American Nuclear Society, New York, NY (June 1977). 
[12] Allen N. Goland, "Experimental Evaluation of the Primary Damage Process - Neutron Energy Effects," J. Nucl. Mater. $85 \& 86$ (1979) 453-461.

[13] J. J. Holmes and J. L. Straalsund, "Irradiation Sources for Fusion Materials Development," J. Nucl. Mater. 85\&86 (1979) 447-451.

[14] E. W. Pottmeyer, Jr., "The Fusion Materlals Irradiation Test Facility at Hanford," J. Nucl. Mater. 85\&86 (1979) 463-465.

[15] F. W. Whifen and J. O. Stiegler, "Recent Progress in CTR Radiation Effects Studies," Proc. Second ANS Topical Meeting on the Technology of Controlled Nuclear Fusion, USERDA-CONF-760935-P1 (1976), pp. 135-147.

[16] E. E. Bloom, J. O. Stiegler, F. W. Wiffen, E.N.C. Dalder, T. C. Reuther, R. E. Gold, J. J. Holmes, D. L. Kummer, and F. W. Nolfi, "Alloy Development for Irradiation Performance: Program Strategy," Proc. Third Topical Meeting on the Technology of Controlzed Nuclear Fusion, DOE-CONF-780508, Vo1. 1 (1978), pp. 554-564.

[17] K. Farre11, M. B. Lewis, and N. H. Packan, "Simultaneous Bombardment with Helium, Hydrogen, and Heavy Ions to Simulate Microstructural Damage from Fission or Fusion Neutrons," Scripta Met. 12 (1978) 1121-1124.

[18] M. B. Lewis, N. H. Packan, G. F. Wells, and R. A. Buh1, "Improved Techniques for Heavy-Ion Simulation of Neutron Radiation Damage," Nucl. Instr. \& Methods, 167 (1979) 233-247.

[19] M. B. Lewis, Trans. Nucl. Sci., NS-26 (1979) 1320-1322.

[20] N. H. Packan, K. Farre11, and J. O. Stiegler, "Correlation of Neutron and Heavy Ion Damage - Part I," J. Nucl. Mater. 78, (1978) 143-155.

[21] L. K. Mansur, "Correlation of Neutron and Heavy Ion Damage Part II," J. Nucl. Mater. 78 (1978) 156-160.

[22] E. H. Lee, L. K. Mansur, and M. H. Yoo, "Spatial Variation in Void Volume During Charged Particle Bombardments - The Effects of Deposited Interstitials," $J$. Nucl. Mater. 85\&86 (1979) 577-581.

[23] A. F. Rowcliffe, E. H. Lee, and P. S. Sklad, "The Effect of Phase Instabilities on the Correlation of Nickel Ion and Neutron Irradiation Swelling in Solution Annealed 316 Stainless Steel," Proc. Suppl., International Conference on Irradiation Behavior of Metallic Materials for Fast Reactor Core Components, (June 4-8, 1979), pp. 1-7. 
[24] F. A. Garner, R. W. Powell, S. Diamond, T. Lauritzen, A. F. Rowcliffe, J. A. Sprague, and D. Keefer, "Simulation of High Fluence Swelling Behavior in Technological Materials," in Radiation Effects in Breeder Reactor Structural Materials, ed. by M. L. Blelberg and J. W. Bennett, (AIME, New York, NY, 1977), pp. 543-569.

[25] K. Farre11, "Experimental Effects of Helium on Cavity Formation During Irradiation - A Review," Rad. Eff. 53 (1980) 175-194.

[26] B. Badger and 28 co-authors, WITAMIR-I, a University of Wisconsin Tandem Mirror Reactor Design, UWFDM-400 (September 1980).

[27] B. L. Eyre, "Direct Observations of Neutron Irradiation Damage in a-Iron," Phil. Mag. 7 (1962) 2107-2113.

[28] J. S. Bryner, "A Study of Neutron Irradiation Damage in Iron by Electron-Transmission Microscopy," Acta Met. 14 (1966) 323-336.

[29] B. L. Eyre and A. F. Bartlett, "An Electron Microscope Study of Neutron Damage in Alpha-Iron," Phil. Mag. 12 (1965) 261-272.

[30] G. L. Kulcinski, B. Maste1, and J. L. Brimha11, "Formation of Voids in Iron During High Temperature Neutron Irradiation," Rad. Effects 2 (1969) 57-59.

[31] K. Farre11 and J. T. Houston, "Heterogeneous Distribution of Irradiation Voids in Iron," J. Nucl. Mater. 35 (1970) 352-355.

[32] N. Milasin, "Radiation- and Annea1-Hardening of Neutron-Irradiated Ferritic Steels and $\alpha$-Iron," Rad. Effects 15 (1972) 153-165.

[33] F. A. Smidt, Jr., and J. A. Sprague, "Property Changes Resulting from Impurity-Defect Interactions in Iron and Pressure Vessel steel Alloys," in Effects of Radiation on Substructure and Mechanical Properties of Metals and Alloys, ASTM-STP-529 (ASTM, Baltimore, $\mathrm{MD}, 1973)$, pp. 78-91.

[34] F. A. Smidt, Jr., J. A. Sprague, J. E. Westmoreland, and P. R. Malmberg, "The Effect of Alloy Additions on Void Nucleation and Growth," in Defects and Defect Clusters in B.C.C. Metals and Their AlZoys, Nucl. Met. Vol. 18, ed. by J. Arsenault (National Bureau of Standards, 1973), pp. 341-362.

[35] N. Igata, K. Watanabe, and S. Sato, "The Role of Some Alloylng Elements on Radiation Hardening in Pressure Vessel Steels," in Effects of Radiation on Substructure and Mechanical Properties of Metals and AZZoys, ASTM-STP-529 (ASTM, Baltimore, MD, 1973), pp. 63-74. 
[36] H. Kayano, H. Yoshinaga, K. Abe, and S. Morozumi, "Effects of Neutron Irradiation on Mechanical Properties of Iron-Nitrogen Alloys," J. Nucl. Sci. \& Technol. 15[3] (1978) 200-212.

[37] K. R. Garr, C. G. Rhodes, and D. Kramer, "Effects of Microstructure on Swelling and Tensile Properties of Neutron-Irradiated Types 316 and 405 Stainless Steels," in Effects of Radiation on Substructure and Mechanical Properties of Metals and Alloys, ASTMSTP-529 (ASTM, Baltimore, MD, 1973), pp. 109-118.

[38] V. I. Scherbak, V. N. Bykov, A. N. Vorobiev, and V. D. Dmitriev, "Radiation Damages in Constructional Steels and High-Nickel Alloys Irradiated with Neutrons," in Radiation Effects in Breeder Reactor Structural Materials, ed. by M. L. Bleiberg and J. W. Bennett (AIME, NY, NY, 1977), pp. 773-779.

[39] J. J. Huet, A. Delbrassine, $\mathrm{Ph}$. Van Asbroeck, and W. Vandermeulen, "Radiation Effects in Ferritic Steels," in Radiation Effects in Breeder Reactor Structural Materials, ed. by M. L. Bleiberg and J. W. Bennett (AIME, NY, NY, 1977), pp. 357-365.

[40] J. J. Huet, A. DeBremaecker, M. Snykers, Ph. Van Asbroeck, and W. Vandermeulen, "Swelling of Ferritic Steels Irradiated in Fast Reactors," in Proc. of Int. Conf. on Irr. Behavior of Met. Materials for Fast Reactor Core Components held in Corsica, France, ed. by J. Poirier and J. M. DuPouy (1979) pp. 5-9.

[41] E. A. Little, D. R. Arke11, D. R. Harries, G. R. Leuthwaite, and T. M. Williams, "Development of Ferritic-Martensitic Steels for Fast Reactor Applications," in Proc. of Int. Conf. on Irr. Behavior of Met. Materials for Fast Reactor Core Components held in Corsica, France, ed. by J. Poirier and J. M. DuPouy (1979) pp. 31-37.

[42] J. Erler, A. Maillard, G. Brun, J. Lehmann, and J. M. DuPouy, "The Behavior of Ferritic Steels under Irradiation with Fast Neutrons," in Proc. of Int. Conf. on Irr. Behavior of Met. Materials for Fast Reactor Core Components held in Corsica, France, ed. by J. Poirier and J. M. DuPouy (1979), pp. 11-16.

[43] E. A. Little and D. A. Stow, "Void Swelling in Fast Reactor Irradiated High Purity Binary Iron Chromium Alloys," in Proc. of Int. Conf. on Irr. Behavior of Met. Materials for Fast Reactor Core Components held in Corsica, France, ed. by J. Poirier and J. M. DuPouy (1979), pp. 17-24.

[44] E. A. Little and D. A. Stow, "Void Swelling in Irons and Ferritic Steels, II An Experimental Survey of Materials Irradiated in a Fast Reactor," J. Nucl. Mater. 87 (1979) 25-39. 
[45] D. S. Gelles, Microstmuctural Examination of Neutron Irradiated Simple Ferritic AZZoys, HEDL-SA-2523 (1981); submitted to the Proc. of Int. Conf. on Neutron Irrad. Effects held November 1981, Argonne, IL.

[46] R. Bullough, M. H. Wood, and E. A. Little, "A Microstructural Explanation for the Low Swelling of Ferritic Steels," in Effects of Radiation on Materials: Tenth Conf., ASTM-STP-725, ed. by David Kramer, H. R. Brager, and J. S. Perrin (ASTM, Baltimore, MD, 1981), pp. 593-609.

[47] E. A. Little, R. Bullough, and M. H. Wood, "On the Swelling Resistance of Ferritic Stee1," Proc. Roy. Society (London) A372 (1980) 565-579.

[48] D. S. Gelles, Mierostructural Examination of Several Commercial Ferpitic Alloys Irradiated to High Fluence, HEDL-SA-2338, to be presented at the Second Topical Meeting on Fusion Reactor Materials held in Seattle, WA, August 1981, proceedings to be published in a special issue of Journal of Nuclear Matemials.

[49] D. S. Gelles, "Microstructural Examination of Several Commercial Ferritic Alloys Irradiated to Very High Fluence," in Quart. Progr. Rept., Period Ending December 31, 1980, Alloy Dev. for Irr. Performance Program, DOE/ER-004515, pp. 187-211.

[50] I. M. Robertson, C. A. English, and M. L. Jenkins, "Low Dose Neutron-Irradiation Damage in $\alpha$-Iron," submitted to Proc. of Int. Conf. on Neutron Irrad. Effects held in Argonne, IL, November 1981.

[51] B. C. Masters, "Dislocation Loops in Irradiated Iron," Phil. Mag. 11 (1965) 881-893.

[52] M. L. Jenkins, C. A. English, and B. L. Eyre, "Heavy Ion Irradiation of $\alpha$ Iron," Phil. Mag. A38 (1979) 97-114.

[53] F. A. Smidt, Jr., P. R. Malmberg, J. A. Sprague, and J. E. Westmoreland, "Swelling Behavior of Commercial Ferritic Alloys, EM-12 and HT-9, as Assessed by Heavy Ion Bombardment," in Irradiation Effects on the Microstructure and Properties of Metals, ASTM-STP-611 (ASTM, Baltimore, MD, 1976), pp. 227-241.

[54] W. G. Johnston, T. Lauritzen, J. H. Rosolowski, and A. M. Turkalo, The Effect of Metallurgical Variables on Void Swelling, General Electric Technical Info. Series Report No. 76CRD019 (GE Corporate Research \& Development, Schenectady, NY, 1976). 
[55] W. G. Johnston, T. Lauritzen, J. H. Rosolowski, and A. M. Turkalo, "The Effect of Metallurgical Variables on Void Swelling," in Radiation Damage in Metals, ed. by N. L. Peterson and S. D. Harkness (American Society for Metals, Metals Park, OH, 1976), pp. 227-266.

[56] W. G. Johnson, J. H. Rosolowski, A. M. Turkalo, and T. Lauritzen, "An Experimental Survey of Swelling in Commercial $\mathrm{Fe}-\mathrm{Cu}-\mathrm{Ni}$ Alloys Bombarded with $5 \mathrm{MeV}$ Ni Ions," J. Nucl. Mater. 54 (1974) 24-40.

[57] S. Ohnuki, H. Takahashi, and T. Takeyama, "Void Swelling and Segregation of Solute in Ion-Irradiated Ferritic Steels," presented at the Second Topical Meeting on Fusion Reactor Materials held in Seattle, WA, August 1981, proceedings to be published in a special edition of Joumal of Nuclear Materials.

[58] E. Kuramoto, N. Yoshida, N. Tsukuda, K. Kitajima, N. H. Packan, M. B. Lewis, and L. K. Mansur, "Simulation Irradiation Studies on Iron," presented at the Second Topical Meeting on Fusion Reactor Materials held in Seattle, WA, August 1981, proceedings to be published in a special edition of Joumal of Nuclear Matemials.

[59] E. A. Little and B. L. Eyre, The Geometry of Dislocation Loops Generated in $\alpha$-Iron by $1 \mathrm{MeV}$ Electron Irradiation at $550^{\circ} \mathrm{C}$, AERE-R7095 (March 1972).

[60] E. A. Little, "Voids Produced in Mild Stee1 by $1 \mathrm{MeV}$ Electron Irradiation," Rad. Effects 16 (1972) 135-137.

[61] N. Yoshida, M. Kiritani, and F. Fujita, "Electron Radiation Damage of Iron in High Voltage Electron Microscope," J. Phys. Soc. (Japan) 39[1] (1975) 170-179.

[62] M. Kiritani, "Electron Radiation Damage of Metals and Nature of Point Defects by High Voltage Electron Microscopy," in Fundamental Aspects of Radiation Damage in Metals, CONF-751006-P2, ed. by M. T. Robinson and F. W. Young (NTIS, Springfield, VA, 1976) pp. 695-714.

[63] M. Kiritani, "Electron Radiation Damage of Metals and Nature of Point Defects by High Voltage Electron Microscopy," in Progress in the study of Point Defects, ed. by M. Doyama and S. Yoshida (University of Tokyo Press, Japan, 1977) pp. 247-328.

[64] M. Kiritani, H. Takata, N. Yoshida, and Y. Maehara, "Long-Range Interaction Between Radiation-Induced Point Defects and Dislocations," in Fundamental Aspects of Radiation Damage in Metals, CONF-751006-P2, ed. by M. T. Robinson and F. W. Young (NTIS, Springfield, VA, 1976) pp. 889-895. 
[65] K. Suganuma and H. Kayano, "Dislocation Loops in Electron Irradiated Ferritic Stainless Stee1," Rad. Effects 54 (1981) 81-86.

[66] E. Kuramoto, K. Futagami, and K. Kitajima, "Formation of Voids in Iron Irradiated by Electron in HVEM," in Proc. of Fifth Int. Conf. on High Voltage Electron Microscopy held in Kyoto, Japan (1977) pp. 589-592.

[67] K. Kitajima, K. Futagami, and E. Kuramoto, "Nucleation of Voids in BCC Metals," J. Nucl. Mater. 85\&86 (1979) 725-729.

[68] D. R. Arkel1 and T. M. Williams, "Void-Swelling in FV607 Ferritic Stee1 - I. Irradiation with $1 \mathrm{MeV}$ Electrons," J. Nucl. Mater. 74 (1978) 144-150.

[69] T. Takeyama and H. Takehashi, "Effect of Electron Irradiation on Precipitation of Carbon and Nitrogen in Alpha Iron," in Fundamental Aspects of Radiation Damage in Metals, CONF-751006-P2, ed. by M. T. Robinson and F. W. Young (NTIS, Springfield, VA, 1976) pp. 1100-1106.

[70] H. Takahashi, S. Ohnuki, and T. Takeyama, "Radiation-Induced Segregation at Internal Sinks in Electron Irradiated Binary Alloys," presented at the Second Topical Meeting on Fusion Reactor Materials held in Seattle, WA, August 1981, proceedings to be published in a special issue of Joumal of Nuclear Materials.

[71] B. L. Eyre and R. Bullough, "On the Formation of Interstitial Loops in Metals," Phil. Mag. 12 (1965) 31-39.

[72] M. R. Hayns and T. M. Williams, "Void-Swelling in FV607 Ferritic Stee1 - II. A Theoretical Model," J. Nucl. Mater. 74 (1978) 151162 .

[73] E. A. Little, "Void Swelling in Irons and Ferritic Steels," $J$. Nucl. Mater. 87 (1979) 11-24.

[74] J. Weertman and W. V. Green, "Void Growth Suppression by Impurity Atmospheres," in Radiation Effects on the Microstructure and Properties of Metals, ASTM-STP-611 (ASTM, Baltimore, MD, 1967) pp. 256-269.

[75] D. A. Woodford, J. P. Smith, and J. Moteff, "Observation of Helium Bubbles in an Irradiated and Annealed Austenitic Stee1,"J. Iron \& Steel Inst. (January 1969) 70-76.

[76] D. A. Woodford, J. P. Smith, and J. Moteff, "Distribution of Boron in an Austenitic Steel Inferred from the observation of Helium Gas Bubbles after Neutron Irradiation," J. Nucl. Mater. 24 (1967) $118-120$. 
[77] D. S. Gelles and F. A. Garner, "An Experimental Method to Determine the Role of Helium Neutron-Induced Microstructural Evolution," J. Nucl. Mater. 85\&86 (1979) 689-693.

[78] R. C. Rau, J. P. Smith, and J. Moteff, "Dislocation Halos in an Irradiated Nicke1-Base Alloy," Phil. Mag. 18 (1968) 209-212.

[79] P. Vela, J. Hardy, and B. Russe11, "The Behavior of Elemental Boron Particles in Copper During Neutron Irradiation," $J$. Nucl. Mater. 26 (1968) 129-131.

[80] R. C. Rau and R. L. Ladd, "Cylindrical Damage Shells in Irradiated Vanadium," J. Appl. Physics 40 (June 1969) 2899-2904.

[81] R. C. Rau, R. L. Ladd, and J. Moteff, "Radiation Damage in Vanadium," in Proc. of Twenty-Sixth Annual EMSA Meeting, ed. by C. J. Arceneaux (1968), pp. 288-289.

[82] R. C. Rau and R. L. Ladd, "Radiation Damage in Vanadium," J. NucZ. Mater. 30 (1969) 297-302.

[83] K. Farrell, J. T. Houston, A. Wolfenden, R. T. King, and A. Jostsons, "Effects of Structural Imperfections on Voids in Aluminum," in Radiation-Induced Voids in Metals, CONF-710601, ed. by J. W. Corbett and L. C. Iannie11o, (NTIS, Springfield, $\mathrm{VA}$, 1972) pp. 376-385.

[84] R. C. Rau and R. L. Ladd, "Observation of Damage Shells in Irradiated Metals," Proc. of Inter. Metallographic Society, (1968) $169-172$.

[85] W. Littmark and J. F. Ziegler, Handbook of Range Distributions for Energetic Ions in AZl Elements, ed. by J. F. Ziegler (Pergamon Press, Elmsford, NY, 1980), p. 211.

[86] J. F. Ziegler, Helium Stopping Powers and Ranges in All Elemental Matter, (Pergamon Press, Elmsford, NY, 1977).

[87] G. P. Scheidler, M. J. Makin, F. J. Minter, and W. F. Schilling, "The Effect of Irradiation Temperature on the Formation of Clusters in Neutron Irradiated Copper," in The Nature of Small Defect Clusters, ed. by M. J. Makin (HMSO, England, 1966), pp. 405-418.

[88] L. D. Hulett, Jr., T. O. Baldwin, J. C. Crump III, and F. W. Young, $\mathrm{Jr}$. , "Effect of Neutron Irradiation on Copper Crystals at High Temperatures," J. Appl. Fhysics 39 (July 1968) 3945-3954. 
[89] J. Bentley, B. L. Eyre, and M. H. Loretto, "High Temperature Neutron Irradiation Damage in Molybdenum," in Fundamental Aspects of Radiation Damage in Metals, CONF-751006-P2, ed. by

M. T. Robinson and F. W. Young, Jr. (NTIS, Springfield, VA, 1972) pp. 925-931.

[90] B. L. Eyre, "Irradiation Damage Structures Generated in Metals and Alloys During Irradiation at Elevated Temperatures," in Eundamental Aspects of Radiation Damage in Metals, CONF-751006$\mathrm{P} 2$, ed. by M. T. Robinson and F. W. Young, Jr. (NTIS, Springfield, VA, 1972), pp. 729-763.

[91] K. Farre11, Unpublished Results.

[92] J. Bentley, Ph.D. Dissertation, University of Birmingham, 1974.

[93] J. L. Brimha11 and B. Maste1, "Neutron Irradiated Molybdenum Relationship of Microstructure to Irradiation Temperature, Rad. Effects 3 (1970) 203-215.

[94] J. Bentley, B. L. Eyre, and M. H. Loretto, "Suppression of Void Formation in Neutron-Irradiated TZM," in Radiation Effects and Tritium Technology for Fusion Reactors, Vo1. I, CONF-750989, ed. by $F$. W. Wiffen and J. S. Watson (NTIS, Springfield, VA, 1976), pp. 297-311.

[95] A. G. Pard and K. R. Garr, "Damage Structure in NeutronIrradiated TZM," in Radiation Effects and Tritium Technology for Fusion Reactors, Vol. I, CONF-750989, ed. by F. W. Wiffen and J. S. Watson (NTIS, Springfield, VA, 1976), pp.312-322.

[96] V. K. Sikka and J. Moteff, "'Rafting,' in Neutron-Irradiated Tungsten," J. Nucl. Mater. 46 (1973) 217-219.

[97] D. M. Mayer and B. L. Eyre, "Neutron Irradition Damage in Molybdenum, Part I: Characterization of Small Perfect Dislocation Loops by Transmission Electron Microscopy," Phil. Mag. 23 No. 182 (1971) 409-438.

[98] L. E. Thomas and S. Lentz, "Stereoscopic Analysis of IrradiationInduced Voids," Proc. of $32^{\text {nd }}$ Annual Meeting of EMSA, (1974) $362-363$.

[99] A. F. Rowcliffe, S. Diamond, M. L. Bleiberg, J. Spitznagel, and $\mathrm{J}$. Choyke, "Swelling and Irradiation Induced Microstructural Changes in Nickel-Based Alloys," in Proc. of Reactor Structural AlZoys after Neutron or Particle Irradiation, ASTM-STP-570 (ASTM, Baltimore, MD, 1975), pp. 565-583. 
[100] S. Diamond, M. L. Bleiberg, I. M. Baron, R. Bajaj, and R. W. Chickering, "HVEM Quantitative Stereoscopy Through the Full Damage Range of an Ion-Bombarded $\mathrm{Fe}-\mathrm{Ni}-\mathrm{Cr}$ Alloy," in Radiation Effects and Tritium Technology for Fusion Reactors, ed. by J. S. Watson and F. W. Wiffen (NTIS, Springfield, VA), 1976), pp. I-207-I-229.

[101] J. H. Evans, "The Variation of Defect Damage with Depth in Molybdenum Irradiated with $2 \mathrm{MeV}$ Nitrogen Ions," Rad. Effects 8 (1971) 115-120.

[102] W. G. Johnston, J. H. Rosolowski, A. M. Turalo, and J. Lauritzen, "The Depth Distribution of Void Swelling Produced by $5 \mathrm{MeV}$ Ni Ions," $J$. Nucl. Mater. 62 (1976) 167-180.

[103] E. H. Lee and A. F. Rowcliffe, "Multiple Sectioning and Perforation Techniques for TEM Sub-Surface studies, Microstructural Science 7 ed. by LeMay, Fallon and McCall (Elsevier North-Holland, Inc., 1979), pp. 403-409.

[104] R. A. Spurling and C. G. Rhodes, "A Technique for preparing the Cross-Section of Proton-Irradiated 316 Stainless Stee1 Foils for TEM,"J.Nucl. Mater. 44 (1972) 341-344.

[105] D. W. Keefer and A. G. Pard, "Hydrogen in Proton-Irradiated Type 316 Stainless Steel and Tantalum,"J. Nucl. Mater. 47 (1973) 97-101.

[106] J. Narayan and 0. S. Den, "Depth Distribution of Self Ion Damage in Nicke1," in Proc. of the Workshop on Correlation of Neutron and Charged Particle Damage (held at Oak Ridge National Laboratory, June 8-10, 1976), pp. 356-368.

[107] G. Fenske, S. K. Das, M. Kaminsky, and G. C. Miley, "Depth Distribution of Bubbles in ${ }^{4} \mathrm{He}^{+}$-Ion Irradiated Nickel and the Mechanism of Blister Formation," J. Nucl. Mater. $76 \& 77$ (1978) 247-248.

[108] J. B. Whitley, G. L. Kulcinski, P. Wilkes, and H. V. Smith, Jr., "The Depth Dependent Damage Profile in Nickel Irradiated with Nicke1 or Copper Ions," J. Nucl. Mater. 79 (1979) 159-169.

[109] G. Fenske, S. K. Das, and M. Kaminsky, "A Technique for Determining the Depth Distribution of Cavities in He-Irradiated Nicke1," J. Nucl. Mater. 80 (1979) 373-378.

[110] N. H. Packan, K. Farre11, and J. T. Houston, "Depth Profile of Swelling in Ion-Bombarded Nickel," 1980 International Metallographic Exhibit, ed. by C.K.H. DuBose (ASM, Ohio, 1980). 
[111] K. Farre11, N. H. Packan, and J. T. Houston, "Depth Profiles of Nickel Ion Damage in Helium-Implanted Nickel," submitted to Radiation Effects.

[112] G. Fenske, S. K. Das, M. Kaminsky, and G. H. Miley, "The Effect of Dose on the Evolution of Cavities in $400-\mathrm{keV}{ }^{4} \mathrm{He}^{+}$-Ion Irradiated Nicke1," J. Nucl. Mater. $85 \& 86$ (1979) 707-711.

[113] J. B. Whitley, G. L. Kulcinski, P. Wilkes, and J. Billen, "Depth Dependent Void Swelling Rates in Self-Ion Irradiated Nickel," $J$. Nucl. Mater. 85\&86 (1979) 701-706.

[114] J. Narayan, T. S. Noggle, and O. S. Oen, "Depth Distribution in Copper Irradiated with $\mathrm{MeV} \mathrm{Ni}$ and He Ions," Fundamental Aspects of Radiation Damage in Metals, CONF-751006-P1, ed. by Mark T. Robinson and F. W. Young, Jr., (NTIS, Springfield, VA 1975), pp. 90-97.

[115] 0. S. Den, J. Narayan, and T. S. Noggle, "Ion Radiation Damage," Applications of Ion Beams to Metals, S. T. Picraux, E. P. EerNisse, and F. L. Vook, eds. (Plenum Press, New York, NY, 1974), pp. 639-650.

[116] J. Narayan, O. S. Den, and T. S. Noggle, "Ion Radiation Damage in Copper," $J_{0}$ Nucl. Mater. 71 (1977) 160-170.

[117] C. H. Henager, Jr., J. L. Brimhall, and E. P. Simonen, "The Damage Profile in Mo Bombarded with $\mathrm{Ni}^{++}$and $\mathrm{Ni}^{++}$He Ions," Rad. Effects 36 (1979) 49-55.

[118] L. J. Turner, Metals and Ceramics Division, ORNL, Private communication.

[119] I. Manning and G. P. Mueller, "Depth Distribution of Energy Deposition by Ion Bombardment," Computer Physics Communication 7 (1974) 85-94.

[120] J. F. Ziegler and W. K. Chu, "Stopping Cross Sections and Backscattering Factors for "He Ions in Matter," Atomic Data and Nuclear Data Tables, 13 (1974) 463-489.

[121] P. Sigmund, "Energy Loss of Charged Particles in Solids," in Radiation Damage Processes in Materials, ed. by C.H.S. Dupuy (Noordhoff Leyden, 1975), p. 45.

[122] P. Hvelplund and B. Fastrup, "Stopping Cross Section in Carbon of 0.2-1.5 MeV Atoms with 21 F Z. F 39," Phys. Review 165(2) (1968) $408-414$.

[123] J. Narayan, O. S. Oen, and T. S. Noggle, ORNL-5135 (1975). 
[124] L. K. Mansur and M. H. Yoo, "Advances in the Theory of Swelling in Irradiated Metals and Alloys," J. Nucl. Mater. 85\&86 (1979) $523-532$.

[125] J. R. Beeler and M. F. Beeler, "4 MeV Iron Atom Bombardment of Iron," in Applications of Ion Beams to Metals, ed. by S. T. Picraux, E. P. EerNisse, and F. L. Vook (Plenum Press, New York, 1974), pp. 651-662.

[126] H. H. Anderson and J. F. Ziegler, Hydrogen Stopping Powers and Ranges in AlZ Elements, (Pergamon Press, Elmsford, NY, 1977).

[127] E. E. Bloom, "Mechanical Properties of Materials in Fusion Reactor First-Wall and Blanket Systems," J. Nucl. Mater. 85\&86 (1979) 795-804.

[128] A.J.E. Foreman, H. S. Von Harrach, and D. K. Saldin, The TEM Contrast of Faceted Voids, AERE-R 10057 (December 1980).

[129] G. Wulff, Z. Kmistallog 34 (1901) 449.

[130] C. Herring, "The Use of Classical Macroscopic Concepts in Surface-Energy Problems," in Structure and Properties of Solid Surfaces, ed. by R. Gomer and C. S. Smith (University of Chicago Press, Chicago, Il, 1953), pp. 5-72.

[131] R. A. Nelson, D. J. Mazey, and R. S. Barnes, "The Thermal Equilibrium Shape and Size of Holes in Solids," Phil. Mag. 11 (1965) 91-111.

[132] N. H. Packan and K. Farre11, "Simulation of First Wa11 Damage: Effects of the Method of Gas Implantation," $T^{T}$. Nucl. Mater. $85 \& 86$ (1979) 677-681.

[133] K. Farrel1 and N. H. Packan, "A Helium-Induced Shift in the Temperature Dependence of Swelling," J. Nucl. Mater. 85\&86 (1979) 683-687.

[134] K. Ehrlich, R. Groß and W. Schneider, "Void Formation in Stabilized Austenitic," in Radiation Effects in Breeder Reactor Structural Materials, ed. by M. L. Bleiberg and J. W. Bennett (AIME, NY, NY, 1977), pp. 529-569.

[135] F. A. Garner, D. S. Gelles, J. J. Laidler, and W. G. Wolfer, Factors which Determine the Relative Growth Rates of Frank Faulted and Diamond Perfect Loops during Irradiation, HEDLSA-1645A (September 1978). 
[136] D. V. Edmonds and R.W.K. Honeycombe, "Precipitation in IronBase Alloys," in Precipitation Processes in Solids, ed. by K. C. Russell and H. I. Aaronson (AIME, NY, NY, 1978), pp. $121-160$.

[137] W. G. Wolfer, High Density Equation of State for Helium and Its Application to Bubbles in Solids, UWFDM-350 (University of Wisconsin Report, Madison, WI, June 1980); also published in Effects of Radiation on Materials, ASTM-STP-725, ed. by D. Kramer, H. R. Brager, and J. S. Perrin (ASTM, Baltimore, MD, 1981), pp. 201-212.

[138] W. G. Wolfer, The Role of Gas Pressure and Lateral Stress in Blistering, UWFDM-347 (University of Wisconsin Report, Madison, WI, Apri1 1980); also published in Joumal of Nuclear Matemals 93\&94 (1980), pp. 713-720.

[139] M. H. Yoo and L. K. Mansur, "The Inclusion of Mobile Helium in a Rate Theory Model of Void Swelling," $J$. Nucl. Mater. 85\&86 (1979) 571-575.

[140] J. P. Hirth and J. Lothe, Theory of Dislocations, (McGrawHill Book Co., NY, NY, 1968), p. 764 .

[141] M. R. Hayns and L. K. Mansur, "Applications of the Theory of Cavity Growth to Dual-Ion Swelling Experiments," in Effects of Radiation on Materials, ASTM-STP-725, ed. by D. Kramer, H. R. Brager, and J. S. Perrin (ASTM, Baltimore, MD, 1981), pp. 213-230.

[142] L. K. Mansur, "Void Swelling in Metals and Alloys under Irradiation: An Assessment of the Theory," Nucl. Technol. 40 (August 1978) 1-34.

[143] F. W. Wiffen, E. J. Allen, H. Farrar IV, E. E. Bloom, T. A. Gabriel, H. T. Kerr, and F. G. Perey, "The Production of Hellum During Irradiation of Nickel in Thermal Spectrum Fission Reactors," to be published in Joumal of Nuclear Materials.

[144] Information supplied by T. A. Gabriel and R. A. Lilile, Dak Ridge National Laboratory (June 4, 1981).

[145] C.K.H. DuBose and J. O. Stiegler, "Controlled Jet Polishing of Specimens for Transmission Electron Microscopy, Rev. Sei. Instr. 38(5) (May 1967) 694-695.

[146] C.K.H. DuBose and J. O. Stiegler, Semiautomatic Preparation of Specimens for Transmission Electron Microscopy, ORNL-4066 (February 1967). 
[147] K. Shirota, A. Yonezawa, K. Shibatomi, and T. Yanaka, "Magnetic Material Observation Assembly for TEM with a Eucentric Goniometer," in Proc. of the 34th Annual EMSA Meeting (1976), pp. 540-541.

[148] JEOL Publication EM-7285 (Tokyo, Japan).

[149] J. Bentley, N. J. Zaluzec, E. A. Kenik, and R. Carpenter, "Optimization of an Analytical Electron Microscope for X-Ray Microanalysis: Instrumental Problems," in Scanning Electron Microscopy II (1979), pp. 581-594.

[150] N. J. Zaluzec, "Quantitative X-Ray Microanalysis: Instrumental Considerations and Applications to Materials Science," in Introduction to Analytical Electron Microscopy, ed. by J. J. Hren, J. I. Goldstein, and D. C. Joy (Plenum Press, NY, NY, 1979), pp. 121-168.

[151] N. J. Zaluzec, "Gaussian Optics Calculations of the Parameters of a Magnetic Sector Energy Analyzer," in Proc. of Workshop on Analytical Electron Microscopy, ed. by P. J. Fejes (Cornel1 University, 1978), pp. 40-44.

[152] JEOL Instruction Manual for JEM 120C/CX, No. IEM-156-E1 (JEOL, Ltd., Tokyo, Japan), p. 22 .

[153] P. B. Hirsch, A. Howie, R. B. Nicholson, D. W. Pashley, and M. J. Whelan, Electron Microscopy of Thin Crystals (W. Clowes \& Sons, Ltd., London, England, 1969), pp. 495-497, 422-424.

[154] A. Howie and S. K. Basinski, Phil. Mag. 17 (1968) 1039.

[155] G. Schoeck, J. Appl. Physics 33 (1962) 1745.

[156] J. Topping, Errors of Observation and Their Treatment, (L. Trend and Co., Ltd., Whitstable, Great Britain, 1966), pp. 19-23.

[157] N. H. Packan and R. A. Buhl, A Multispecimen Dual-Beam Irradiation Damage Chamber, ORNL/TM-7276 (June 1980).

[158] C. H. Johnson, Nucl. Instr. \& Methods, 127 (1975) 163.

[159] I. M. Torrens and M. T. Robinson, "Computer Simulation of Atomic Displacement Cascades in Metals," in Radiation Induced Voids in Metals, CONF-710601, ed. by J. W. Corbett and L. C. Ianniel1o (NTIS, Springfield, VA, 1972), pp. 739-756.

[160] Annual Book of ASTM Standards, Part 45, Nuclear Standards, Section E-521 (ASTM, Philadelphia, PA, 1977), pp. 991-999. 
[161] Handbook of Chemistry and Physics, 54th Edition, Robert C. Weast, Editor-in-Chief (CRC Press, Cleveland, Ohio, 1973) pp. B198-199, D-157, F-109.

[162] G. L. Kulcinski, J. J. Laidler, and D. G. Doran, Radiation Effects, 7 (1971), 195.

[163] J. Lindhard, V. Nielson, M. Schraff, and P. L. Thompson, Danske Videnskab. Selskab 33, No. 10 (1963).

[164] J. Lindhard, M. Schraff, and H. E. Schiott, Danske Videnskab. Selskab 33, No. 14 (1963).

[165] M. B. Lewis, Metals and Ceramics Division, ORNL, private communication.

[166] A. Hishinuma and L. K. Mansur, "Bimodal Cavity Distribution in Neutron and Ion-Irradiated Stainless Steel," to be submitted for publication.

[167] J. R. Cost and K. Y. Chen, "The Number of Gas Atoms per Bubble of Inert Gas in a Solid," J. Nucl. Mater. 67 (1977) 265-272.

[168] R. S. Barnes, "A Theory of Swelling and Gas Release for Reactor Materials," J. Nucl. Mater. 11 (1964) 135-148.

[169] N. V. Tsederberg, V. N. Popov, and N. A. Morozova, Thermodynamic and Thermodynamic Properties of Helium, (Keter Press, Jerusalem, Israe1, 1971), p. 18 .

[170] D. E. Beck, "A New Interatomic Potential Function for Helium," Molecular Physies 14 (1968) 311-315.

[171] N. E. Carnahan and K. E. Starling, "Equation of State for NonAttracting Rigid Spheres," J. Chem. Physics 51 (1969) 635-636.

[172] J. A. Barker and D. Henderson, "Perturbation Theory and Equation of State for Fluids, II A Successful Theory of Liquids," $J$. Chem. Physics 47 (1967) 4714-4721.

[173] J. A. Barker and D. Henderson, "What is 'Liquid'? Understanding the States of Matter," Reviews of Modern Physics 48 (1976) 587-671.

[174] H. C. Anderson, D. Chandler, and J. D. Weeks, Advances in Chemical Physics, 34 (1976) 105.

[175] L. Verlet and J. J. Weiss, "Perturbation Theory for the Thermodynamic Properties of Simple Liquids," Molecular Physics 24 (1972) 1013-1024.

[176] W. G. Wolfer and B. Glasgow, University of Wisconsin, private communication. 
8

- 


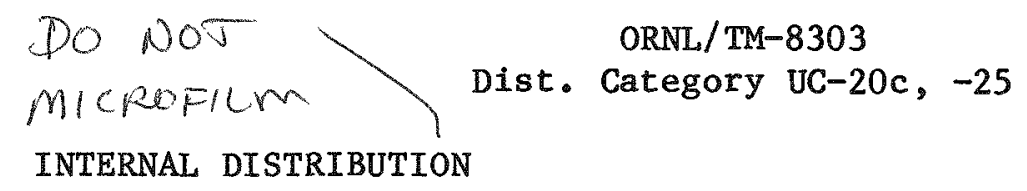

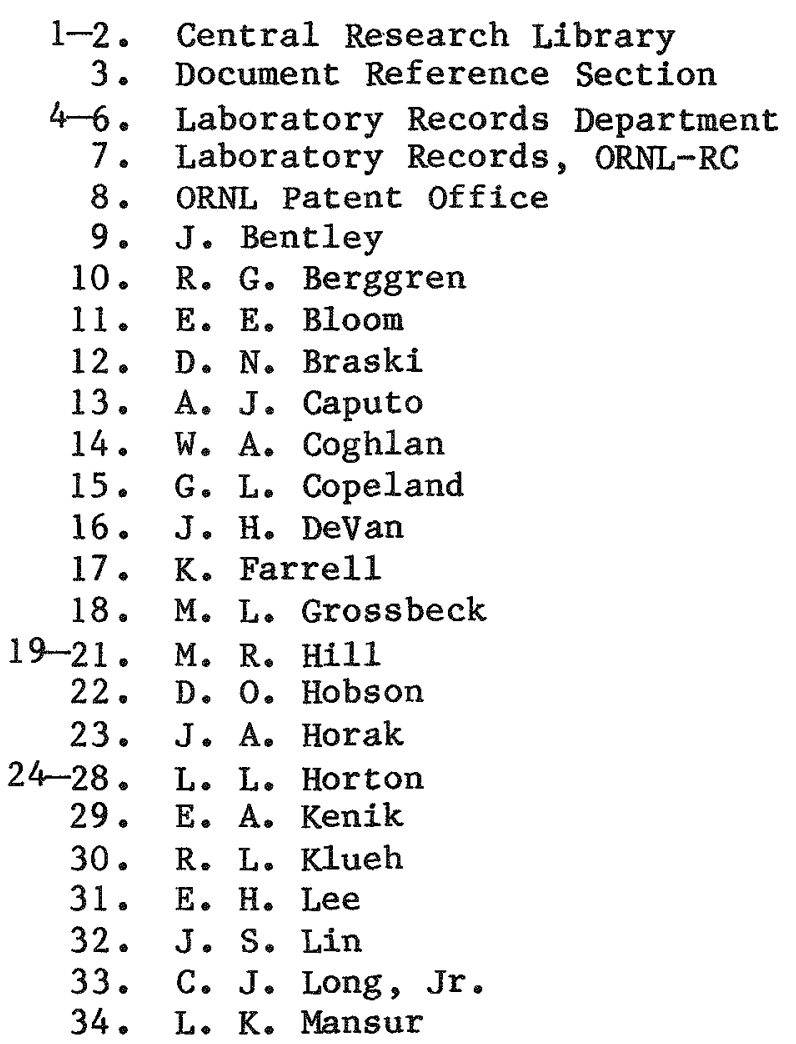

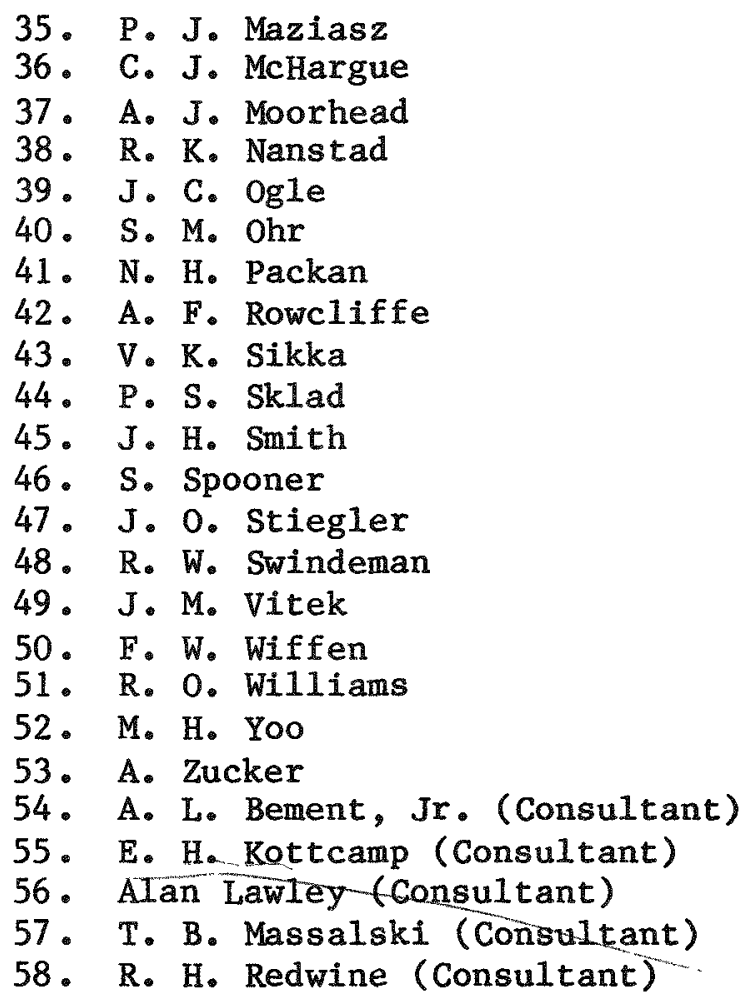

\section{EXTERNAL DISTRIBUTION}

59. J. L. Brimha11, Battelle-Pacific Northwest Laboratory, P. 0. Box 999, Richland, WA 99352

60. R. W. Carpenter, Arizona State University, Tempe, AZ 85281

61. K. Ehrlich, Institut für Reaktorwerkostoffe, Kernforschungsanlage, Jülich, GmbH, 517 Jülich 1, Postfach 1913, West Germany

62. B. Eyre, Department of Metallurgy and Material Science, The University of Liverpool, P. 0. Box 147, Liverpool, England

63. Y. Higaschiguchi, the Oarai Branch, The Institute for Iron, Steel and Other Metals, Tohoku University, Oarai, Ibaraki 311-13, Japan

64. F. A. Garner, Hanford Engineering Development Laboratory, P. O. Box 1970, Richland, WA 99352

65. D. S. Gelles, Hanford Engineering Development Laboratory, P. O. Box 1970, Richland, WA 99352

66. N. Igata, Department of Materials Science, University of Tokyo, 7-3-1 Hongo Bunkyo-ku, Tokyo, Japan 
67. S. Ishino, Department of Nuclear Engineering, University of Tokyo, Bunkyo-ku, Tokyo, Japan 113

68. M. L. Jenkins, AERE, Harwe11, Didcot, Oxon, OX11, ORB, England

69. W. Johnson, General Electric Research and Development Center, Schenectady, NY 12301

70. M. Kiritani, Department of Material Physics, Faculty of Engineering Science, Osaka University, Toyonaka, Osaka, Japan

71. E. Kuramoto, Department of Materials Science, University of Tokyo, 7-3-1 Hongo Bunkyo-ku, Tokyo, Japan

72. T. Lauritzen, General Electric Company, 319 DeGuigne Dr., Sunnyvale, CA 94086

73. E. A. Little, AERE, Harwe11, Didcot, Oxon, OX11, ORB, England

74. V. Levy, Centre d'Etudes Nucleaires de Saclay, Boite Postale No. 2, 91190 Gif-sur-Yvette, France

75. M. Loretto, Department of Physical Metallurgy and Science of Materials, University of Birmingham, Birmingham B152TT, England

76. R. E. Nygren, Argonne National Laboratory, 9700 South Cass Ave., Argonne, IL 60439

77. G. R. Odette, University of California, Santa Barbara, CA 93106

78. D. L. Porter, Idaho National Engineering Laboratory, 550 2nd Street, Idaho Falls, ID 83415

79. Y. Queré, Section d'Etúdes des Solides Irradiês, Centre de'Etûdes Nucleaires, Fontenay-aux-Roses, France

80. I. M. Robertson, Department of Metallurgy, University of Illinois, Urbana, IL 61801

81. W. Schüle, Materials Science Division, Atomic Research Center, 21020, Ispra, Italy

82. B. I. Shriver, Department of Nuclear Engr. and Engr. Physics, Reactor Bldg., University of Virginia, Charlottesville, VA 22901

83. C. L. Snead, Jr., Brookhaven National Laboratory, Upton, NY 11973

84. M. Snykers, SCK/CEN, B $2400 \mathrm{Mo1}$, Belgium

85. J. A. Spitznage1, Westinghouse Research and Development Center, 1310 Beulah Rd., Pittsburgh, PA 15235

86. J. A. Sprague, Naval Research Laboratory, Washington, DC 20375

87. T. Takeyama, Hokkaido University, Sapporo 060, Japan

88. M. Tanaka, Japan Atomic Energy Research Institute, Tokai-mura, Naka-gun, Ibaraki-ken, Japan

89. L. E. Thomas, Hanford Engineering Development Laboratory, P. O. Box 1970 , Richland, WA 99352

90. H. UIlmaier, Institute fur Festkorperförschung der Kernforschungsanlage, Jülich, D-5171, Postfach 365, West Germany

91. K. Urban, Institute für Theoret und Angew Physik der Universitat, Stuttgart, 7000 Stuttgart 80-Pfaffenwaldring 57/V1, Germany

92. J. Vander Sande, Massachusetts Institute of Technology, Cambridge, MA 02139

93. Ken Westmacot, Materials and Molecular Research Division, Lawrence Berkeley Laboratory, Berkeley, CA 94720

94. H. Wiedersich, Argonne Nationa1 Laboratory, 9700 South Cass Ave., Argonne, IL 60439 
95. W. Wolfer, Department of Nuclear Engineering, University of Wisconsin, Madison, WI 53706

96. S. Wood, Westinghouse Research and Development Center, 1310 Beulah Road, Pittsburgh, PA 15235

97-99. Department of Energy, Division of Materials Sciences, Washington, DC 20545

L. C. Ianniello

D. K. Stevens

S. Wolf

100. Department of Energy, Office of Assistant Manager for Energy Research and Development, Oak Ridge Operations office, P. O. Box E, Oak Ridge, TN 37830

101-420. Department of Energy, Technical Information Center, office of Information Services, P. 0. Box 62, Oak Ridge, TN 37830 [For distribution as shown in TID-4500 Distribution Category, UC-20c (Reactor Materials) and UC-25]. 